Key Words:

Environmental

Dosimetry

Parameters

Retention:

Permanent

\title{
LAND AND WATER USE CHARACTERISTICS AND HUMAN HEALTH INPUT PARAMETERS FOR USE IN ENVIRONMENTAL DOSIMETRY AND RISK ASSESSMENTS \\ AT THE SAVANNAH RIVER SITE
}

\author{
Author: G.T. Jannik \\ Author: D.J. Karapatakis \\ Author: P.L. Lee \\ Technical Reviewer: E.B. Farfán
}

REPORT DATE: AUGUST 6, 2010

Savannah River National Laboratory

Savannah River Nuclear Solutions

Aiken, SC 29808

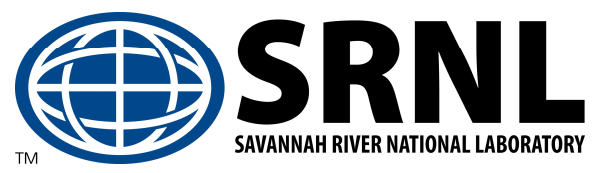




\section{DISCLAIMER}

This work was prepared under an agreement with and funded by the U.S. Government. Neither the U. S. Government or its employees, nor any of its contractors, subcontractors or their employees, makes any express or implied:

1. warranty or assumes any legal liability for the accuracy, completeness, or for the use or results of such use of any information, product, or process disclosed; or

2. representation that such use or results of such use would not infringe privately owned rights; or

3. endorsement or recommendation of any specifically identified commercial product, process, or service.

Any views and opinions of authors expressed in this work do not necessarily state or reflect those of the United States Government, or its contractors, or subcontractors.

Printed in the United States of America

Prepared for

U.S. Department of Energy 
Key Words:

Environmental

Dosimetry

Parameters

Retention:

Permanent

\title{
LAND AND WATER USE CHARACTERISTICS AND HUMAN HEALTH INPUT PARAMETERS FOR USE IN ENVIRONMENTAL DOSIMETRY AND RISK ASSESSMENTS \\ AT THE SAVANNAH RIVER SITE
}

\author{
Author: G.T. Jannik \\ Author: D.J. Karapatakis \\ Author: P.L. Lee \\ Technical Reviewer: E.B. Farfán
}

REPORT DATE: AUGUST 6, 2010

Savannah River National Laboratory

Savannah River Nuclear Solutions

Savannah River Site

Aiken, SC 29808 


\section{REVIEWS AND APPROVALS}

Tim Jannik, Author, Environmental Sciences \& Biotechnology

Date

Deno Karapatakis, Co-Author, Computational Sciences

Date

Patricia Lee, Co-Author, Computational Sciences

Date

Eduardo Farfán, Technical Reviewer, Environmental Sciences \& Biotechnology $\quad$ Date

John Gladden, Level 3 Manager, Environmental Sciences \& Biotechnology

Date

- ii - 


\section{TABLE OF CONTENTS}

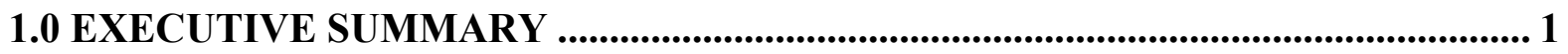

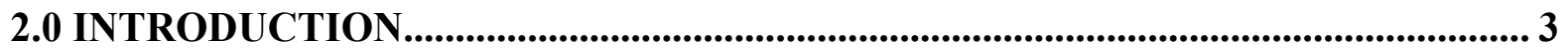

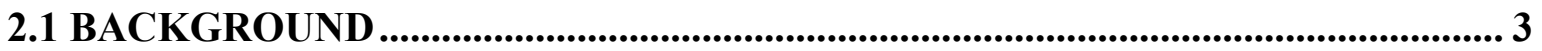

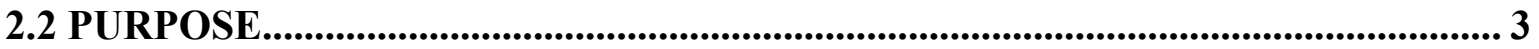

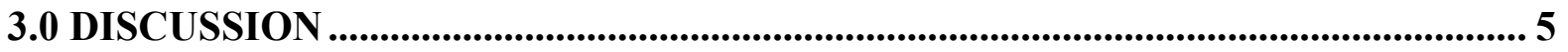

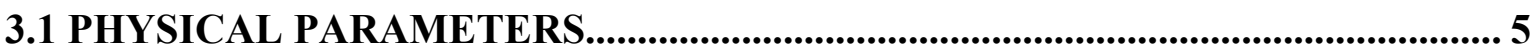

3.1.1 Agricultural Productivity and Production........................................................................ 5

3.1.2 Soil to Vegetable Transfer Factors .............................................................................. 7

3.1.3 Feed to Milk Transfer Factors ............................................................................................. 8

3.1.4 Feed to Meat Transfer Factors .............................................................................. 8

3.1.5 Water to Fish Bioaccumulation Factors......................................................................... 8

3.1.6 Water to Saltwater Invertebrates Bioaccumulation Factors .................................. 8

3.2 BEHAVIORAL PARAMETERS ..................................................................................... 9

3.2.1 Average Individual and Population Usage Parameters............................................ 9

3.2.2 Maximally Exposed Individual Usage Parameters ................................................... 10

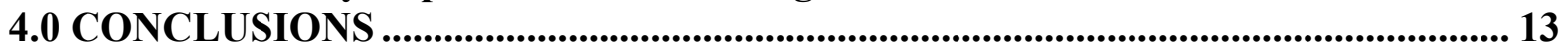

4.1 UNIT RELEASE DOSE COMPARISONS.................................................................... 13

4.1.1 LADTAP XL Maximally Exposed Individual Aquatic Dose Comparisons..... 13

4.1.2 LADTAP XL Collective Aquatic Dose Comparisons ............................................. 13

4.1.3 LADTAP XL IRRIDOSE Irrigation Maximally Exposed Individual Dose

Comparisons ........................................................................................................................ 14

4.1.4 LADTAP XL IRRIDOSE Irrigation Collective Dose Comparisons .................. 14

4.1.5 MAXDOSE-SR Maximally Exposed Individual Air Pathway Dose

Comparisons ....................................................................................................................... 14

4.1.6 POPDOSE-SR Collective Air Pathway Dose Comparisons ................................... 15

4.2 SITE ANNUAL COMPLIANCE DOSE COMPARISONS ....................................... 15

4.2.1 LADTAP XL Maximally Exposed Individual Aquatic Dose Comparisons..... 15

4.2.2 LADT AP XL Collective Aquatic Dose Comparisons ............................................ 16

4.2.3 LADTAP XL IRRIDOSE Irrigation Maximally Exposed Individual Dose

Comparisons .......................................................................................................................... 16

4.2.4 LADTAP XL IRRIDOSE Irrigation Collective Dose Comparisons .................. 16

4.2.5 MAXDOSE-SR Maximally Exposed Individual Air Pathway Dose

Comparisons ............................................................................................................................ 16

4.2.6 POPDOSE-SR Collective Air Pathway Dose Comparisons ................................. 16

5.0 SUMMARY ............................................................................................................................ 19

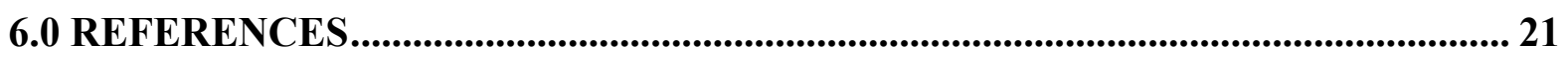

APPENDIX A UPDATED PARAMETERS TRANSFER FACTORS AND

BIOACCUMULATION FACTORS (TABLES 1-13) ............................................................. 24

APPENDIX B DOSE COMPARISONS (TABLES 14-55) ………........................................ 46 


\section{LIST OF TABLES}

Table 1. SRS Physical Parameters for Dose Calculations..................................................... 24

Table 2. Soil-to-Vegetable Transfer Factors ............................................................ 26

Table 3. Feed-to-Milk Transfer Factors ........................................................................ 30

Table 4. Feed-to-Meat Transfer Factors................................................................................. 32

Table 5. Water-to-Fish Bioaccumulation Factors..................................................................... 34

Table 6. Water to Saltwater Invertebrates Bioaccumulation Factors .............................. 36

Table 7. Vegetable Production as a Function of Distance and Sector............................... 38

Table 8. Beef Production as a Function of Distance and Sector ........................................ 39

Table 9. Milk Production as a Function of Distance and Sector ....................................... 40

Table 10. SRS Average Behavioral Parameters for Dose Calculations ........................... 41

Table 11. SRS Maximum Individual Behavioral Parameters for Dose Calculations... 42

Table 12. Average Recreational Usage Rates ....................................................................... 43

Table 13. Weighted Usage Fractions.............................................................................. 44

Table 14. LADTAP XL Maximally Exposed Individual Water Ingestion Pathway Comparison (mrem/yr) ........................................................................................................ 46

Table 15. LADTAP XL Maximally Exposed Individual Fish Ingestion Pathway Comparison (mrem/yr) .......................................................................................................49

Table 16. LADTAP XL Maximally Exposed Individual Shoreline Exposure Pathway Comparison (mrem/yr) .......................................................................................................52

Table 17. LADTAP XL Maximally Exposed Individual Swimming Exposure Pathway Comparison (mrem/yr) ......................................................................................................5 55

Table 18. LADTAP XL Maximally Exposed Individual Boating Exposure Pathway Comparison (mrem/yr) ....................................................................................................58

Table 19. LADTAP XL Maximally Exposed Individual Total of All Pathways Comparison (mrem/yr) ......................................................................................................... 61

Table 20. LADTAP XL Collective Water Ingestion Comparisons (person-rem) .......... 64

Table 21. LADTAP XL Collective Sport Fish Ingestion Comparisons (person-rem) .. 67

Table 22. LADTAP XL Collective Commercial Fish Ingestion Comparisons (person-rem) ............................................................................................................................ 70

Table 23. LADTAP XL Collective Salt Water Invertebrate Ingestion Comparisons (person-rem) ................................................................................................................... 73

Table 24. LADTAP XL Collective Shoreline Exposure Comparisons (person-rem) ... 76 Table 25. LADTAP XL Collective Swimming Exposure Comparisons (person-rem) 79

Table 26. LADTAP XL Collective Boating Exposure Comparisons (person-rem) ....... 82

Table 27. LADTAP XL Collective Total of All Pathways Comparisons (person-rem) 85

Table 28. LADTAP XL IRRIDOSE Maximally Exposed Individual Vegetable Ingestion Pathway Comparison (mrem/yr) ...................................................................... 88

Table 29. LADTAP XL IRRIDOSE Maximally Exposed Individual Milk Ingestion Pathway Comparison (mrem/yr) ........................................................................................... 91

Table 30. LADTAP XL IRRIDOSE Maximally Exposed Individual Meat Ingestion Pathway Comparison (mrem/yr) .................................................................................... 94

Table 31. LADTAP XL IRRIDOSE Maximally Exposed Individual Total Ingestion Pathway Comparison (mrem/yr) .............................................................................................. 97 
Table 32. LADTAP XL IRRIDOSE Vegetable Ingestion Pathway Collective Dose

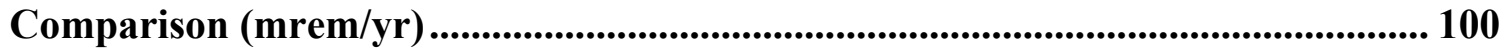

Table 33. LADTAP XL IRRIDOSE Milk Ingestion Pathway Collective Dose

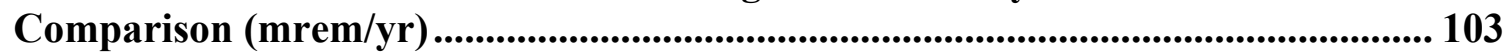

Table 34. LADTAP XL IRRIDOSE Meat Ingestion Pathway Collective Dose Comparison (mrem/yr) ............................................................................................. 105

Table 35. LADTAP XL IRRIDOSE All Pathway Collective Dose Comparison

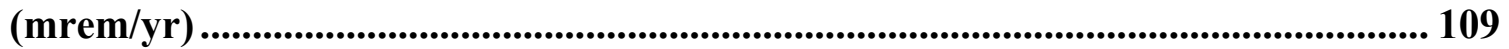

Table 36. MAXDOSE-SR Maximally Exposed Individual Ground Exposure

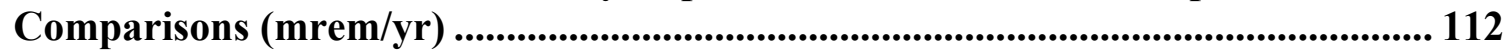

Table 37. MAXDOSE-SR Maximally Exposed Individual Plume Immersion Comparisons (mrem/yr) ..................................................................................115

Table 38. MAXDOSE-SR Maximally Exposed Individual Inhalation Pathway Comparisons (mrem/yr) ....................................................................................... 116

Table 39. MAXDOSE-SR Maximally Exposed Individual Vegetable Consumption Comparisons (mrem/yr) ..................................................................................... 119

Table 40. MAXDOSE-SR Maximally Exposed Individual Milk Consumption Comparisons (mrem/yr) 122

Table 41. MAXDOSE-SR Maximally Exposed Individual Meat Consumption Comparisons (mrem/yr) 125

Table 42. MAXDOSE-SR Maximally Exposed Individual Total All Pathways

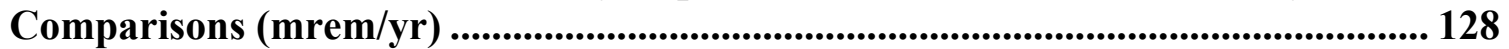

Table 43. POPDOSE-SR Collective Ground Exposure Comparisons (mrem/yr) ...... 132

Table 44. POPDOSE-SR Collective Plume Immersion Comparisons (mrem/yr)....... 135

Table 45. POPDOSE-SR Collective Inhalation Dose Comparisons (mrem/yr) .......... 136

Table 46. POPDOSE-SR Collective Vegetable Ingestion Comparisons (mrem/yr) ... 140

Table 47. POPDOSE-SR Collective Milk Ingestion Comparisons (mrem/yr) ............ 143

Table 48. POPDOSE-SR Collective Meat Ingestion Comparisons (mrem/yr) ........... 146

Table 49. POPDOSE-SR Total Collective Dose Comparisons (mrem/yr).................. 148

Table 50. LADTAP XL 2009 Annual Report Maximally Exposed Individual Dose

Comparisons (mrem) ...................................................................................................... 150

Table 51. LADTAP XL Annual Report Collective Dose Comparisons (person-rem) 151

Table 52. LADTAP XL IRRIDOSE 2009 Annual Report Maximally Exposed Individual Dose Comparisons (mrem) ............................................................................ 152

Table 53. LADTAP XL IRRIDOSE Annual Report Collective Dose Comparisons

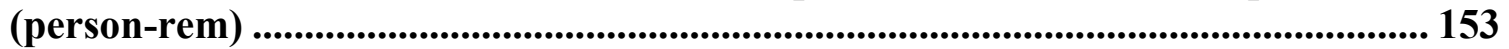

Table 54. MAXDOSE SR Annual Report Maximally Exposed Individual Dose Comparisons (mrem) ............................................................................................ 154

Table 55. POPDOSE SR Annual Report Collective Dose Comparisons (person-rem) 156 
This page intentionally left blank 


\subsection{EXECUTIVE SUMMARY}

Operations at the Savannah River Site (SRS) result in releases of small amounts of radioactive materials to the atmosphere and to the Savannah River. For regulatory compliance purposes, potential offsite radiological doses are estimated annually using computer models that follow U.S. Nuclear Regulatory Commission (NRC) Regulatory Guides. Within the regulatory guides, default values are provided for many of the dose model parameters but the use of site-specific values by the applicant is encouraged. A detailed survey of land and water use parameters was conducted in 1991 and is being updated here. These parameters include local characteristics of meat, milk and vegetable production; river recreational activities; and meat, milk and vegetable consumption rates as well as other human usage parameters required in the SRS dosimetry models. In addition, the preferred elemental bioaccumulation factors and transfer factors to be used in human health exposure calculations at SRS are documented.

Based on comparisons to the 2009 SRS environmental compliance doses, the following effects are expected in future SRS compliance dose calculations:

- Aquatic all-pathway maximally exposed individual doses may go up about 10 percent due to changes in the aquatic bioaccumulation factors

- Aquatic all-pathway collective doses may go up about 5 percent due to changes in the aquatic bioaccumulation factors that offset the reduction in average individual water consumption rates

- Irrigation pathway doses to the maximally exposed individual may go up about 40 percent due to increases in the element-specific transfer factors

- Irrigation pathway collective doses may go down about 50 percent due to changes in food productivity and production within the 50-mile radius of SRS

- Air pathway doses to the maximally exposed individual may go down about 10 percent due to the changes in food productivity in the SRS area and to the changes in element-specific transfer factors

- Air pathway collective doses may go down about 30 percent mainly due to the decrease in the inhalation rate assumed for the average individual. 
This page intentionally left blank.

Page 2 
SRNL-STI-2010-00447, REVISION 0

\subsection{INTRODUCTION}

\subsection{BACKGROUND}

Operations at the Savannah River Site (SRS) result in releases of small amounts of radioactive materials to the atmosphere and to the Savannah River. For regulatory compliance purposes, potential offsite radiological doses are estimated annually using computer models that follow U.S. Nuclear Regulatory Commission (NRC) Regulatory Guides (NRC 1977a and 1977b). Within the regulatory guides, default values are provided for many of the dose model parameters but the use of site-specific values by the applicant is encouraged (NRC 1977a). A detailed survey of land and water use parameters was conducted in Hamby (1991) and is being updated here. These parameters include local characteristics of meat, milk and vegetable production; river recreational activities; and meat, milk and vegetable consumption rates as well as other human usage parameters required in the SRS dosimetry models. In addition, the preferred elemental bioaccumulation factors and transfer factors to be used in human health exposure calculations at SRS are documented.

\subsection{PURPOSE}

The purpose of this report is to review, and update as needed, the input parameters and environmental bioaccumulation and transfer factors used in human health exposure calculations at SRS for determining compliance with applicable U.S. Department of Energy (DOE) Orders (DOE 1988 and DOE 2010) and U.S. Environmental Protection Agency (EPA) National Emission Standards for Hazardous Air Pollutants (NESHAP) regulations (EPA 2002). The reason for the update is to utilize more recent information issued, validate information currently used, and correct minor inconsistencies between other modeling efforts performed at SRS.

In Lee and Coffield (2008) many of the SRS input parameters utilized in dosimetry calculations were compared to a number of other DOE facilities and generic national/global references to establish relevance of the parameters selected and/or verify the regional differences of the U.S. Southeast. The parameters selected were applicable to SRS performance assessments (PAs) and were specifically chosen to be expected values (along with identifying a range for these values) versus the conservative specification of parameters for estimating an annual dose to the maximum exposed individual (MEI). However, because of its thoroughness and direct applicability to annual SRS dose compliance calculations, many of the parameters documented in Lee and Coffield (2008) are adopted in this report.

The intent of this report is to establish a standardized source for these parameters that is up to date with existing data and maintain it via review of any future issued national references to evaluate the need for changes as new information is released. These reviews are to be added to this document by revision. 
This page intentionally left blank.

Page 4 


\subsection{DISCUSSION}

\subsection{PHYSICAL PARAMETERS}

Physical parameters are defined as those whose value would not change if a different group of receptors were considered. For the purposes of this report, these parameters include agricultural production factors and aquatic food harvest factors. The other physical input parameters are determined by SRS's geographical location, the geophysical characteristics of the site, and the element-specific transfer and bioaccumulation factors. A summary of current and updated productivity and other physical parameters are listed in Table 1.

The International Atomic Energy Agency recently published (in Technical Reports Series No. 472) updated recommended transfer factors for a limited number of elements (IAEA 2010). With only a few exceptions (noted in the discussions to follow) these elementspecific transfer values were used in this report. For the elements not included in IAEA, the values documented in Lee and Coffield (2008) were used. The updated soil-to-vegetable, feed-to-milk, and feed-to-meat transfer factors are provided in Tables 2, 3, and 4, respectively. Updated water-to-fish bioaccumulation factors and water to saltwater invertebrates bioaccumulation factors are provided in Tables 5 and 6 , respectively.

During this updated study, the physical parameters that were determined to differ from Hamby (1991) or Lee and Coffield (2008) are discussed below. The parameters that remained the same are also documented in Tables 1 through 6 , but without additional comment.

\subsubsection{Agricultural Productivity and Production}

The updated agricultural productivity factors for vegetables, meat, and milk are weighted averages based on the 2007 U.S. Department of Agriculture (USDA) National Agricultural Statistics (USDA 2009). The annual production rates of this produce that occurs within a 50mile radius of SRS, also are based on this report. The 2002 USDA agricultural statistics were also considered to confirm agricultural production trends (USDA 2004).

\subsubsection{Vegetable Productivity and Production}

Based on USDA (2009), the South Carolina and Georgia weighted average vegetable productivity was determined to be $2.2 \mathrm{~kg} / \mathrm{m}^{2}$. This value, which includes leafy and non-leafy vegetables, is over 200 percent more than the Hamby (1991) and Lee and Coffield (2008) reported value of $0.7 \mathrm{~kg} / \mathrm{m}^{2}$. However, it is comparable to the NRC (1977) default value of $2.0 \mathrm{~kg} / \mathrm{m}^{2}$ and is essentially the same as the USDA (2004) dataset.

Based on USDA (2009), the combined leafy and non-leafy vegetable commercial production within 50 miles of SRS was estimated to be $6.7 \mathrm{E}+05 \mathrm{~kg} / \mathrm{yr}(1.4 \mathrm{E}+05 \mathrm{~kg} / \mathrm{yr}$ for leafy vegetables and $5.3 \mathrm{E}+05 \mathrm{~kg} / \mathrm{yr}$ for other vegetables). For the purposes of this report, the total combined vegetable production value was increased by a factor of 10 (revised up to $6.7 \mathrm{E}+06$ $\mathrm{kg} / \mathrm{yr}$ ) to account for unreported and non-commercial (individual use) production of vegetables in the area. This conservative factor is based on professional judgment and on 
personal discussions with County Agents and other professionals in the field. The updated production value of $6.7 \mathrm{E}+06 \mathrm{~kg} / \mathrm{yr}$ is about 85 percent less than the Hamby (1991) estimated value of $4.7 \mathrm{E}+07 \mathrm{~kg} / \mathrm{yr}$. The USDA (2004) estimated combined total was $7.1 \mathrm{E}+06 \mathrm{~kg} / \mathrm{yr}$ (including the factor of 10 for unreported production), which indicates a continuing downward trend in vegetable production in the SRS area. The updated, total combined vegetable production as a function of distance and sector is provided in Table 7.

\subsubsection{Pasture Grass Productivity}

Based on USDA (2009), the South Carolina and Georgia weighted average pasture grass productivity was determined to be $0.7 \mathrm{~kg} / \mathrm{m}^{2}$. This value is about 60 percent less than the Hamby (1991) and Lee and Coffield (2008) reported value of $1.8 \mathrm{~kg} / \mathrm{m}^{2}$. However, it is the same as the NRC (1977) default value and is essentially the same as the value estimated using the USDA (2002) dataset.

\subsubsection{Meat (Beef) Productivity and Production}

Because poultry and pork production are almost exclusively indoor operations, only beef production is considered for the meat pathway. Based on USDA (2009), the South Carolina and Georgia weighted average meat productivity was determined to be $0.01 \mathrm{~kg} / \mathrm{m}^{2}$. This value was not considered in the Hamby (1991) and Lee and Coffield (2008) reports. However, it is used in the LADTAP XL Irridose spreadsheet for population dose assessments to determine the amount of beef production on a given irrigated area. The $0.01 \mathrm{~kg} / \mathrm{m}^{2}$ value was determined based on the daily consumption rate of forage by beef cattle $(36 \mathrm{~kg} / \mathrm{d})$, the current pasture grass productivity $\left(0.7 \mathrm{~kg} / \mathrm{m}^{2}\right)$, and an assumed edible meat amount per beef cow $(200 \mathrm{~kg})$ using the following equation:

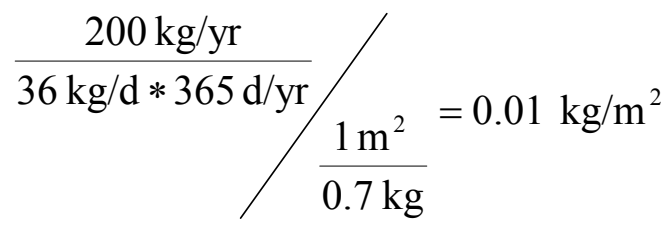

Based on USDA (2009), the commercial beef production within 50 miles of SRS was estimated to be $6.1 \mathrm{E}+06 \mathrm{~kg} / \mathrm{yr}$. For the purposes of this report, the commercial beef production value was increased by a factor of 1.25 (revised up to $7.6 \mathrm{E}+06 \mathrm{~kg} / \mathrm{yr}$ ) to account for unreported and non-commercial (individual use) production of beef in the area. Again, this factor is based on professional judgment and on personal discussions with County Agents and other professionals in the field. The updated beef production value of $7.6 \mathrm{E}+06$ $\mathrm{kg} / \mathrm{yr}$ is about 50 percent less than the Hamby (1991) estimated value of $1.5 \mathrm{E}+07 \mathrm{~kg} / \mathrm{yr}$. The USDA (2004) estimated total was $1.3 \mathrm{E}+07 \mathrm{~kg} / \mathrm{yr}$ (including the factor of 1.25 for unreported production), which indicates a continuing downward trend in beef production in the SRS area. The updated beef production as a function of distance and sector is provided in Table 8. 


\subsubsection{Milk Productivity and Production}

Based on USDA (2009), the South Carolina and Georgia weighted average milk productivity was determined to be $0.34 \mathrm{~L} / \mathrm{m}^{2}$. This value was not considered in the Hamby (1991) and Lee and Coffield (2008) reports. However, it is used in the LADTAP XL Irridose spreadsheet for population dose assessments to determine the amount of milk production on a given irrigated area. The $0.34 \mathrm{~kg} / \mathrm{m}^{2}$ value was determined based on the daily consumption rate of forage by milk cows $(52 \mathrm{~kg} / \mathrm{d})$, the current pasture grass productivity $\left(0.7 \mathrm{~kg} / \mathrm{m}^{2}\right)$, and an assumed daily milk production amount per milk cow $(25 \mathrm{~L} / \mathrm{d})$ using the following equation:

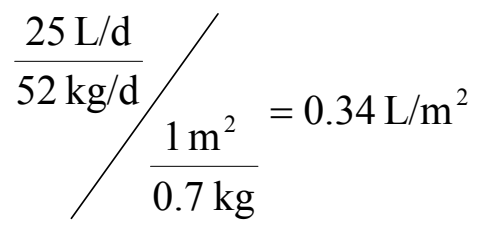

Based on USDA (2009), the commercial milk production within 50 miles of SRS was estimated to be $6.8 \mathrm{E}+07 \mathrm{~L} / \mathrm{yr}$. For the purposes of this report, the commercial milk production value was increased by a factor of 1.25 (revised up to $8.5 \mathrm{E}+06 \mathrm{~L} / \mathrm{yr}$ ) to account for unreported and non-commercial (individual use) production of milk in the area. Again, this factor is based on professional judgment and on personal discussions with County Agents and other professionals in the field. The updated milk production value of $8.5+07$ $\mathrm{L} / \mathrm{yr}$ is about 23 percent less than the Hamby (1991) estimated value of $1.1 \mathrm{E}+08 \mathrm{~L} / \mathrm{yr}$. The USDA (2004) estimated total was $1.0 \mathrm{E}+08 \mathrm{~L} / \mathrm{yr}$ (including the factor of 1.25 for unreported production), which indicates a continuing downward trend in milk production in the SRS area. The updated milk production as a function of distance and sector is provided in Table 9.

\subsubsection{Soil to Vegetable Transfer Factors}

In IAEA (2010), a selection of element-specific, soil-to-plant (vegetable) transfer factors are provided for a variety of plant groups. Based on USDA (2007), the estimated percentage breakdown of the major plant groups commercially produced in the SRS area is as follows:

- Leafy vegetables $20 \%$

- Legumes $15 \%$

- $\quad$ Tubers and Roots $10 \%$

- Non-leafy $\quad 55 \%$

These percentages were used to calculate a weighted-average element-specific transfer factor, which are documented in Table 2. For the elements not included in IAEA (2010), the values documented in Lee and Coffield (2008) were used. The updated soil-to-vegetable transfer factors are provided in the column labeled "Jannik et al." in Table 2. 


\subsubsection{Feed to Milk Transfer Factors}

In IAEA (2010) a selection of element-specific transfer coefficients to cow's milk (feed-tomilk) are provided. For the elements not included in IAEA (2010), the values documented in Lee and Coffield (2008) were used. The updated milk transfer coefficients are provided in the column labeled "Jannik et al. 2010" in Table 3.

\subsubsection{Feed to Meat Transfer Factors}

In IAEA (2010) a selection of element-specific transfer coefficients to beef (feed-to-meat) are provided. For the elements not included in IAEA (2010), the values documented in Lee and Coffield (2008) were used. The updated meat transfer coefficients are provided in the column labeled "Jannik et al. 2010" in Table 4.

\subsubsection{Water to Fish Bioaccumulation Factors}

In IAEA (2010) a selection of element-specific concentrations ratios (bioaccumulation factors) for freshwater fish tissue are provided. For the elements not included in IAEA (2010), the values documented in Lee and Coffield (2008) were used as discussed below. Exceptions to this rule, which are described below, are plutonium, cesium, and carbon. The updated fish bioaccumulation factors are provided in the column labeled "Jannik et al. 2010" in Table 5.

As discussed in section 4.0 (Conclusions), the plutonium bioaccumulation factor recommended in IAEA $(2010)$ is $21,000 \mathrm{~L} / \mathrm{kg}$. This is several orders of magnitude greater than the previous IAEA value, which (as documented in Lee and Coffield (2008) is $30 \mathrm{~L} / \mathrm{kg}$. The $21,000 \mathrm{~L} / \mathrm{kg}$ bioaccumulation value for plutonium seems unreasonable based on the professional judgement of the authors and other national experts. Therefore, the Lee and Coffield (2008) value of $30 \mathrm{~L} / \mathrm{kg}$ will be used until the IAEA (2010) value is verified or changed.

The SRS site-specific bioaccumulation factor for cesium of 3,000 L/kg (Jannik 2003) is used in this update in lieu of the IAEA (2010) value of 2,500 L/kg. The site-specific value is slightly more conservative than the IAEA (2010) value and is considered the best available data.

Also, the SRS determined bioaccumulation factor for carbon of $3 \mathrm{~L} / \mathrm{kg}$ (Hinton et al. 2009) is used in lieu of the IAEA (2010) value of 400,000 L/ kg. Hinton et al. (2009) used a systems model approach to study the carbon mass balance in an SRS stream. This study showed that most of the carbon in fish does not come from the water in which they live, which is the assumption that the IAEA referenced elemental bioaccumulation factors are based on.

\subsubsection{Water to Saltwater Invertebrates Bioaccumulation Factors}

Recommended concentration ratios for saltwater invertebrates were not addressed in IAEA (2010). Also, because the saltwater invertebrate pathway is not applicable to Performance Assessments at SRS, they were not addressed in Lee and Coffield (2008). Therefore, the values presented in PNNL (2003) were used for this update and are provided in the column 
labeled "Jannik et al." in Table 6. In PNNL (2003), saltwater bioaccumulation factors are provided for crustaceans and mollusks. To be conservative, the larger of the two values was used.

\subsection{BEHAVIORAL PARAMETERS}

Behavioral parameters are defined as those whose value depends on the receptor's behavior and the defined exposure scenario. For the purposes of this report, these parameters include the metabolic characteristics of the receptor. A summary of current and updated behavioral and metabolic parameters are listed in Table 10 for the average individual and general population and in Table 11 for the maximally exposed individual.

During this updated study, the behavioral parameters that were determined to differ from Hamby (1991) or Lee and Coffield (2008) are discussed below. The parameters that remained the same are also documented in Tables 10 and 11, but without additional comment.

\subsubsection{Average Individual and Population Usage Parameters}

Except for current updates to the recreation exposure times, all of the average individual behavioral parameters that were considered and documented in Lee and Coffield (2008), were adopted for use in this report. Of these, the only average individual parameters that differ from Hamby (1991) are the inhalation rate and drinking water consumption rate.

\subsubsection{Inhalation Rate}

As discussed in Lee and Coffield (2008), the updated average individual inhalation rate for long term exposure is $5,548 \mathrm{~m}^{3} / \mathrm{yr}$. This value is based on EPA recommendations and is about 30 percent less than the Hamby (1991) value of $8,000 \mathrm{~m}^{3} / \mathrm{yr}$.

\subsubsection{Drinking Water Consumption Rate}

As discussed in Lee and Coffield (2008), the updated average individual drinking water consumption rate for long term exposure is $337 \mathrm{~L} / \mathrm{yr}$. This value is based on EPA recommendations and is about 9 percent less than the Hamby (1991) value of $370 \mathrm{~L} / \mathrm{yr}$.

\subsubsection{Shoreline, Swimming, and Boating Exposure Time}

As shown in Table 12, the updated average individual recreational exposure usage rates for shoreline usage, swimming, and boating in the SRS area were estimated to be $10 \mathrm{hr} / \mathrm{yr}, 7$ $\mathrm{hr} / \mathrm{yr}$, and $22 \mathrm{hr} / \mathrm{yr}$, respectively. These values are based on 2005 data from the Georgia Department of Natural Resources (GDNR 2005) and the South Carolina Department of Parks and Recreation (SCDPR 2005) and on the estimated hours per occasion from Hamby (1991). The updated shoreline exposure time of $10 \mathrm{hr} / \mathrm{yr}$ is about 57 percent less than the Hamby (1991) value of $23 \mathrm{hr} / \mathrm{yr}$, but slightly more than the NRC (1977a) default value of $8.3 \mathrm{hr} / \mathrm{yr}$. The updated swimming exposure time of $7 \mathrm{hr} / \mathrm{yr}$ is about 21 percent less than the Hamby 
(1991) value of $8.9 \mathrm{hr} / \mathrm{yr}$ and the updated boating time of $22 \mathrm{hr} / \mathrm{yr}$ is about 5 percent more than the Hamby (1991) value of $21 \mathrm{hr} / \mathrm{yr}$.

Based on these revised recreational usage rates, the updated weighted population exposure times for shoreline usage, swimming, and boating were determined and are provided in Table 13. These values are based on the SC and GA state usage available fractions from Hamby (1991), the 2000 census population fraction in the SC (0.6) and GA (0.4) counties within 50 miles of SRS, and on the projected 2010 total population $(1.4 \mathrm{E}+06$ people) in those counties. The updated population shoreline exposure time is $8.22 \mathrm{E}+05$ person-hr, which is 14 percent less the Hamby (1991) value of $9.60 \mathrm{E}+05$. The updated population swimming exposure time is $2.95 \mathrm{E}+05$ person-hr, which is about 84 percent more than the Hamby (1991) value of $1.60 \mathrm{E}+05$ person-hr. The updated population boating exposure time is $3.11 \mathrm{E}+06$ person-hr, which is $183 \%$ more than the Hamby (1991) value of $1.10 \mathrm{E}+06$ person-hr.

\subsubsection{Fraction of Year Working in Garden}

As documented in Lee and Coffield (2008), the recommended fraction of time an average person works in their garden is 0.01 . This parameter was not considered in Hamby (1991).

\subsubsection{Fraction of Year Residing in Home}

As documented in Lee and Coffield (2008), the recommended fraction of time an average person stays inside of their home is 0.7. This parameter was not considered in Hamby (1991).

\subsubsection{Time Taking a Shower}

As documented in Lee and Coffield (2008), the recommended amount of time an average person takes a shower is $10 \mathrm{~min} / \mathrm{d}$. This parameter was not considered in Hamby (1991).

\subsubsection{Population Served by Downriver Drinking Water Plants}

In 2009, the operators of the three public drinking water plants that are located downriver of SRS estimated that the following populations were served:

- Beaufort-Jasper Water and Sewer Authority (Chelsea Plant)

- Beaufort-Jasper Water and Sewer Authority (Purrysburg Plant)

- City of Savannah Industrial and Domestic Water Supply Plant
77,000 people

58,000 people

26,300 people

These totals will be updated annually for use in the SRS Site Environmental Report.

\subsubsection{Maximally Exposed Individual Usage Parameters}

Except for the current updates to the recreation exposure times, all of the maximally exposed individual behavioral parameters that were considered and documented in Hamby (1991) were adopted for use in this report. All of these values remain conservative and are within the range of values documented from various other sources in Lee and Coffield (2008). 
Because of the exposure scenarios considered, Lee and Coffield (2008) did not specifically recommend maximally exposed individual parameters.

At SRS, fish consumption is an important pathway for the maximally exposed individual and for sportsmen. Though selected studies have shown that the potential for higher fish consumption rates in the Savannah River area exist (Burger 1998), the recommended maximally exposed individual fish consumption rate remains at $19 \mathrm{~kg} / \mathrm{yr}$. This value is realistically conservative and equates to the same value $(54 \mathrm{~g} / \mathrm{d}$ for $350 \mathrm{~d})$ used by EPA in their Preliminary Remediation Goals calculator for the fish consumption pathway (EPA 2010).

\subsubsection{Shoreline, Swimming, and Boating Exposure Time}

The maximally exposed individual recreation exposure times were conservatively set at twice the per capita values. In Hamby (1991), the maximally exposed individual exposure times were the same as the average individual times. The updated maximally exposed individual exposure times for shoreline, swimming, and boating are $20 \mathrm{hr} / \mathrm{yr}, 14 \mathrm{hr} / \mathrm{yr}$, and $44 \mathrm{hr} / \mathrm{yr}$, respectively. 
This page intentionally left blank.

Page 12 


\subsection{CONCLUSIONS}

\subsection{UNIT RELEASE DOSE COMPARISONS}

Dose comparisons were performed using the LADTAP XL, MAXDOSE-SR, and POPDOSE-SR dose models. The comparisons were done using the existing parameters (Hamby 1991) versus the updated physical and behavioral parameters and with the updated transfer factors and bioaccumulation factors. For these comparisons, unit releases $(1 \mathrm{Ci} / \mathrm{y})$ were assumed for each radionuclide in the dose model libraries.

\subsubsection{LADTAP XL Maximally Exposed Individual Aquatic Dose Comparisons}

The comparisons of estimated doses to the maximally exposed individual for the water ingestion, fish ingestion, shoreline exposure, swimming exposure, and boating exposure pathways are shown in Tables 14 through 18, respectively. The overall total dose comparison is provided in Table 19.

As expected, the water ingestion doses remained unchanged because there was no change in the maximally exposed individual water ingestion rate (Table 14).

The fish ingestion doses (Table 15) changed directly with the changes in the aquatic bioaccumulation factors (Table 5).

The recreation doses (shoreline, swimming, and boating; Table 16, 17, and 18, respectively) changed directly and consistently with the changes in the recreation exposure times (Table 11).

The total doses changed proportionately with the changes in the various exposure pathways (Table 19). However, because the recreation pathways are minor contributors to the total dose, the fish consumption pathway had the largest effect on changes in the total doses.

\subsubsection{LADTAP XL Collective Aquatic Dose Comparisons}

The comparisons of estimated doses to the affected downriver population for the water ingestion, sport and commercial fish ingestion, saltwater invertebrate ingestion, shoreline exposure, swimming exposure, and boating exposure pathways are shown in Tables 20 through 26, respectively. The overall total dose comparison is provided in Table 27.

The water ingestion population doses changed directly and consistently with the change in the average water consumption rate from $370 \mathrm{~L} / \mathrm{yr}$ to $337 \mathrm{~L} / \mathrm{yr}$ (Table20).

The fish and saltwater invertebrate ingestion collective doses (Tables 21, 22 and 23) changed proportionately with the changes in the sport and commercial harvests (Table 1) and the aquatic bioaccumulation factors (Tables 5 and 6).

The recreation doses (shoreline, swimming, and boating; Tables 24, 25, and 26, respectively) changed directly with the changes in the recreation exposure times (Tables 10). 
The total collective doses changed proportionately with the changes in the various exposure pathways (Table 27).

\subsubsection{LADTAP XL IRRIDOSE Irrigation Maximally Exposed Individual Dose Comparisons}

The comparisons of estimated doses to a maximally exposed individual from use of the Savannah River for irrigation of farmland downstream of SRS are shown for the vegetable, milk, and meat consumption pathways in Tables 28, 29 and 30, respectively. The overall total dose comparison is provided in Table 31.

Since all of the maximally exposed individual consumption rates for vegetables, milk, and meat remained unchanged, the only changes in the doses were caused by the changes in the elemental transfer factors documented in Tables 2, 3, and 4, respectively.

The total irrigation pathway maximally exposed individual doses changed proportionately with these changes (Table 31).

\subsubsection{LADTAP XL IRRIDOSE Irrigation Collective Dose Comparisons}

The comparisons of estimated doses to a collective population from use of the Savannah River for irrigation of farmland downstream of SRS are shown for the vegetable, milk, and meat consumption pathways are shown in Tables 32, 33, and 34, respectively. The overall total irrigation collective dose comparisons are provided in Table 35.

For this comparison, it was assumed that 1,000 acres of irrigated land is devoted to each of the three major food types (vegetables, milk, and meat). It is assumed that all of the food produced on the 1,000 acre parcels is consumed by the local population.

The vegetable consumption pathway collective doses (Table 32) changed directly with the changes in the elemental soil-to-vegetable transfer factors (Table 2) and all radionuclide collective doses increased directly with the increase in vegetable productivity from $0.7 \mathrm{~kg} / \mathrm{m}^{2}$ to $2.2 \mathrm{~kg} / \mathrm{m}^{2}$ (Table 1$)$.

The milk and meat consumption pathway collective doses (Tables 33 and 34) changed directly with the changes in the elemental feed-to-milk and feed-to-meat transfer factors (Tables 3 and 4) and all radionuclide collective doses decreased directly with the decrease in pasture grass productivity from $1.8 \mathrm{~kg} / \mathrm{m}^{2}$ to $0.7 \mathrm{~kg} / \mathrm{m}^{2}$ (Table 1 ).

The total irrigation pathway collective doses changed proportionately with the changes in the various exposure pathways (Table 35).

\subsubsection{MAXDOSE-SR Maximally Exposed Individual Air Pathway Dose Comparisons}

The comparisons of estimated doses to the maximally exposed individual for the ground exposure, plume immersion, inhalation, and vegetable, milk, and meat consumption pathways are shown in Tables 36 through 41, respectively. The overall total air pathway dose comparisons are provided in Table 42. 
As expected, the ground exposure, plume immersion, and inhalation pathway doses (Tables 36,37 , and 38) to the maximally exposed individual remained unchanged because there were no changes in the physical or behavioral usage parameters for these pathways.

Also, since all of the maximally exposed individual consumption rates for vegetables, milk, and meat remained unchanged, the only changes in the food consumption doses (Tables 39, 40 , and 41) were caused by the changes in the vegetable productivity and the elemental transfer factors documented in Tables 2,3 , and 4, respectively.

The total air pathway maximally exposed individual doses changed proportionately with these food consumption pathway changes (Table 42).

\subsubsection{POPDOSE-SR Collective Air Pathway Dose Comparisons}

The comparisons of estimated doses to the SRS 5-mile radius population for the ground exposure, plume immersion, inhalation, and vegetable, milk, and meat consumption pathways are shown in Tables 43 through 48, respectively. The overall total air pathway dose comparisons are provided in Table 49.

As expected, the ground exposure and plume immersion collective doses (Tables 43 and 44) remained unchanged because there were no changes in the physical or behavioral usage parameters for these pathways.

The inhalation pathway doses (Table 45) changed directly with the change in the average individual breathing rate from $8,000 \mathrm{~m}^{3} / \mathrm{yr}$ to $5,548 \mathrm{~m}^{3} / \mathrm{yr}$.

Since all of the average individual consumption rates for vegetables, milk, and meat remained unchanged, the changes in the food consumption collective doses (Tables 46, 47, and 48) were caused by the changes in the elemental transfer factors and by the reduced amount of vegetables, milk, and meat produced within a 50-mile radius area of SRS (Tables 7,8 , and 9 , respectively).

The total air pathway collective doses changed proportionately with the changes in these various exposure pathways (Table 49).

\subsection{SITE ANNUAL COMPLIANCE DOSE COMPARISONS}

Additional dose comparisons were performed using the LADTAP XL, MAXDOSE, and POPDOSE dose models. These comparisons were done using the existing parameters (Hamby 1991) and the 2009 SRS radiological source term as documented in the SRS site environmental report (Mamatey 2010) versus the updated physical and behavioral parameters and with the updated transfer factors and bioaccumulation factors.

\subsubsection{LADTAP XL Maximally Exposed Individual Aquatic Dose Comparisons}

The comparisons of estimated doses to the maximally exposed individual for all aquatic pathways are shown in Table 50. The overall dose using the updated parameters and transfer factors is estimated to be 0.084 mrem, which is about 10 percent more than the reported 2009 
dose of 0.077 mrem calculated using the Hamby (1991) factors. Most of the increase is attributed to the increase in the fish bioaccumulation factors for americium and plutonium.

\subsubsection{LADTAP XL Collective Aquatic Dose Comparisons}

The comparisons of estimated doses to the affected downriver population for all aquatic pathways are shown in Table 51. The overall collective dose using the updated parameters and transfer factors is estimated to be 2.33 person-rem, which is about 4 percent more than the reported 2009 dose of 2.23 person-rem calculated using the Hamby (1991) factors. Again, most of the increase is attributed to the increase in the fish bioaccumulation factors for americium and plutonium, which offset the reduction in drinking water dose due to the decrease in the average individual water consumption rate.

\subsubsection{LADTAP XL IRRIDOSE Irrigation Maximally Exposed Individual Dose Comparisons}

The comparisons of estimated doses to a maximally exposed individual from use of the Savannah River for irrigation of farmland downstream of SRS are shown in Table 52. The overall dose using the updated parameters and transfer factors is estimated to be $0.083 \mathrm{mrem}$, which is about 39 percent more than the reported 2009 dose of 0.060 mrem calculated using the Hamby (1991) factors. All of the increase is attributed to the increases in the elementspecific transfer factors (Tables 2, 3, and 4).

\subsubsection{LADTAP XL IRRIDOSE Irrigation Collective Dose Comparisons}

The comparisons of estimated doses to a collective population from use of the Savannah River for irrigation of farmland downstream of SRS are shown in Table 53. The overall collective dose using the updated parameters and transfer factors is estimated to be 2.01 person-rem, which is about 49 percent less than the reported 2009 dose of 3.94 person-rem calculated using the Hamby (1991) factors. Most of this decrease is attributed to the changes in food productivity and food production in the SRS area.

\subsubsection{MAXDOSE-SR Maximally Exposed Individual Air Pathway Dose Comparisons}

The comparisons of estimated doses to the maximally exposed individual for all air pathways are shown in Table 54. The overall dose using the updated parameters and transfer factors is estimated to be 0.0386 mrem, which is about 8 percent less than the reported 2009 dose of 0.0419 mrem calculated using the Hamby (1991) factors. Most of the decrease is attributed to the change in vegetable productivity and to changes in the element-specific transfer factors for iodine and plutonium.

\subsubsection{POPDOSE-SR Collective Air Pathway Dose Comparisons}

The comparisons of estimated doses to the population residing within 50-miles of SRS for all air pathways are shown in Table 55. The overall dose using the updated parameters and transfer factors is estimated to be 1.35 person-rem, which is about 32 percent less than the reported 2009 dose of 2.00 person-rem calculated using the Hamby (1991) factors. Most of 
the decrease is attributed to the decrease in the annual inhalation rate for the average individual and to a lesser degree to the changes in vegetable productivity and production within the 50-mile radius of SRS. 
This page intentionally left blank.

Page 18 


\subsection{SUMMARY}

A detailed survey of land and water use parameters was conducted by Hamby (1991) and has being updated in this report. These parameters include local characteristics of meat, milk and vegetable production; river recreational activities; and meat, milk and vegetable consumption rates as well as other human usage parameters required in the SRS dosimetry models. In addition, the preferred elemental bioaccumulation factors and transfer factors to be used in human health exposure calculations at SRS have been documented in this report.

Based on comparisons to the 2009 SRS environmental compliance doses that are documented in Mamatey (2010), the following effects are expected in future SRS compliance dose calculations:

- Aquatic all-pathway maximally exposed individual doses may go up about 10 percent due to changes in the aquatic bioaccumulation factors

- Aquatic all-pathway collective doses may go up about 5 percent due to changes in the aquatic bioaccumulation factors that offset the reduction in average individual water consumption rates

- Irrigation pathway doses to the maximally exposed individual may go up about 40 percent due to increases in the element-specific transfer factors

- Irrigation pathway collective doses may go down about 50 percent due to changes in food productivity and production within the 50-mile radius of SRS

- Air pathway doses to the maximally exposed individual may go down about 10 percent due to the changes in food productivity in the SRS area and to the changes in element-specific transfer factors

- Air pathway collective doses may go down about 30 percent mainly due to the decrease in the inhalation rate assumed for the average individual. 
This page intentionally left blank.

Page 20 
SRNL-STI-2010-00447, REVISION 0

\subsection{REFERENCES}

Burger 1998. J. Burger, Fishing and Risk Along the Savannah River: Possible Intervention,

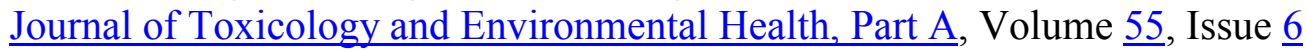
December 1998 , pages $405-419$.

DOE 1988. U.S. Department of Energy, Radiation Protection of the Public and the Environment, DOE Order 5400.5, Washington, D.C., 1988.

DOE 2010. U.S. Department of Energy, Radiation Protection of the Public and the Environment, DRAFT DOE Order 458.1, Washington, D.C., DRAFT 2010.

EPA 2002. U.S. Environmental Protection Agency, National Emission Standard for Hazardous Air Pollutants, Title 40 Code of Federal Regulations, Part 61, Subpart H, Washington, D.C., 2002.

EPA 2010. Environmental Protection Agency Risk Assessment Information System website: http://epa-prgs.ornl.gov/cgi-bin/radionuclides/rprg_search.

GDNR (2005). Personal conversations with Elizabeth Cheney, Georgia Department of Natural Resources website: http://crd.dnr.state.ga.us/

Hamby 1991. D.M. Hamby, Land and Water Use Characteristics in the Vicinity of the Savannah River Site, Westinghouse Savannah River Company Report: WSRC-RP91-17, Aiken, SC, March 1991.

Hinton et al. 2009. Hinton, T., Kaplan, D., Fletcher, D., McArthur, J., and Romanek, C., "Systems Model of Carbon Dynamics in Four Mile Branch on the Savannah River Site”, SRNS-TR-2009-00178, Rev. 1, March 25, 2009.

IAEA 2010. International Atomic Energy Agency, Handbook of Parameter Values for the Prediction of Radionuclide Transfer in Terrestrial and Freshwater Environments, IAEA Technical Reports Series No. 472, Vienna, Austria, 2010.

Mamatey 2010 A.R. Mamatey, Savannah River Site Environmental Report for 2009, Savannah River Nuclear Solutions, LLC, SRNS-STI-2010-00175, Aiken, SC, 2010.

Lee and Coffield 2008. P.L. Lee and T.W. Coffield, Baseline Parameter Update for Human Health Input and Transfer Factors for Radiological Performance Assessments at the Savannah River Site, Westinghouse Savannah River Company Report: WSRC-STI2007-00004, Rev. 4, Aiken, SC, June 2008.

NRC 1977a. U.S. Nuclear Regulatory Commission, Calculation of Annual Doses to Man from Routine Releases of Reactor Effluents for the Purpose of Evaluating Compliance with 10 CFR 50, Appendix I, Regulatory Guide 1.109, Washington, D.C., 1977. 
NRC 1977b. U.S. Nuclear Regulatory Commission, Estimating Aquatic Dispersion of Effluents from Accidental and Routine Releases for the Purpose of Implementing Appendix I, Regulatory Guide 1.113 (Rev. 1), Washington, D.C., 1977.

PNNL 2003. Pacific Northwest National Laboratory, A Compendium of Transfer Factors for Agricultural and Animal Products, PNNL-13421, Hanford, WA, 2003.

SCDPR (2005). SC 2005 Recreation Participation and Preference Study, South Carolina Department of Parks and Recreation website:

http://www.scprt.com/our-partners/tourismstatistics/researchreports.aspx

USDA 2004. U.S. Department of Agriculture, 2002 Census of Agriculture, U.S. Summary and State Data, National Agricultural Statistics Service Report AC-07-A-51, Washington, D.C., 2004.

USDA 2009. U.S. Department of Agriculture, 2007 Census of Agriculture, U.S. Summary and State Data, National Agricultural Statistics Service Report AC-07-A-51, Washington, D.C., 2009. 


\section{APPENDIX A UPDATED PARAMETERS, TRANSFER FACTORS, AND BIOACCUMULATION FACTORS (TABLES 1-13)}


SRNL-STI-2010-00447, REVISION 0

\section{APPENDIX A UPDATED PARAMETERS TRANSFER FACTORS AND BIOACCUMULATION FACTORS (TABLES 1-13)}

Table 1. SRS Physical Parameters for Dose Calculations

\begin{tabular}{|c|c|c|c|c|c|c|}
\hline \multirow[b]{2}{*}{ Parameter } & \multirow[b]{2}{*}{ Units } & 1977 & 1991 & 2008 & 2010 & \% Difference \\
\hline & & NRC Default & Hamby & $\begin{array}{l}\text { Lee and } \\
\text { Coffield }\end{array}$ & Jannik et al. & $\begin{array}{l}1991 \text { Hamby/ } \\
2010 \text { Jannik }\end{array}$ \\
\hline Beef-cow forage consumption (wet) & $\mathrm{kg} / \mathrm{d}$ & 50 & 36 & 36 & 36 & $0.00 \%$ \\
\hline Milk-cow forage consumption (wet) & $\mathrm{kg} / \mathrm{d}$ & 50 & 52 & 52 & 52 & $0.00 \%$ \\
\hline Pasture-grass exposure time to irrigation & $\mathrm{d}$ & 30 & 30 & 30 & 30 & $0.00 \%$ \\
\hline Vegetable crop exposure time to irrigation & $\mathrm{d}$ & 60 & 70 & 70 & 70 & $0.00 \%$ \\
\hline Vegetable garden productivity & $\mathrm{kg} / \mathrm{m}^{2}$ & 2 & 0.7 & 0.7 & 2.2 & $214.29 \%$ \\
\hline Agricultural productivity (produce/veg.) & $\mathrm{kg} / \mathrm{m}^{2}$ & 2 & 0.7 & 0.7 & 2.2 & $214.29 \%$ \\
\hline Agricultural productivity (pasture grass) & $\mathrm{kg} / \mathrm{m}^{2}$ & 0.7 & 1.8 & 1.8 & 0.7 & $-61.11 \%$ \\
\hline Agricultural productivity (edible beef.) & $\mathrm{kg} / \mathrm{m}^{2}$ & - & - & - & 0.01 & - \\
\hline Agricultural productivity (milk) & $\mathrm{L} / \mathrm{m}^{2}$ & - & - & - & 0.15 & - \\
\hline Irrigation rate & $\mathrm{L} / \mathrm{d} / \mathrm{m}^{2}$ & & 3.4 & 3.6 & 3.6 & $5.88 \%$ \\
\hline Transport time (feed-milk-man) & $\mathrm{d}$ & 4 & 3 & 3 & 3 & $0.00 \%$ \\
\hline Transport time (produce for avg/pop) & $\mathrm{d}$ & 14 & 14 & 6 & 6 & $-57.14 \%$ \\
\hline Transport time (produce for MEI) & $\mathrm{d}$ & 1 & 1 & $\mathrm{~N} / \mathrm{A}$ & 1 & $0.00 \%$ \\
\hline Time from slaughter to consumption & $\mathrm{d}$ & 20 & 6 & 6 & 6 & $0.00 \%$ \\
\hline Holdup time (pasture grass, forage) & $\mathrm{d}$ & 0 & 0 & 0 & 0 & $0.00 \%$ \\
\hline Holdup time (stored feed) & $\mathrm{d}$ & 90 & 90 & $\mathrm{~N} / \mathrm{A}$ & 90 & $0.00 \%$ \\
\hline Fraction of time milk-cow on pasture & - & 0.75 & 1 & 1 & 1 & $0.00 \%$ \\
\hline Fraction of time beef-cow on pasture & - & 0.75 & 1 & 1 & 1 & $0.00 \%$ \\
\hline Fraction of intake from pasture (milk cow) & - & $1(\mathrm{~b})$ & 0.56 & 0.56 & 0.56 & $0.00 \%$ \\
\hline Fraction of intake from pasture (beef-cow) & - & $1(\mathrm{~b})$ & 0.75 & 0.75 & 0.75 & $0.00 \%$ \\
\hline Fraction of veg. from local farm (avg/pop) & - & 1 & 1 & 0.308 & 0.308 & $-69.20 \%$ \\
\hline Fraction of meat from local farm (avg/pop) & - & 1 & 1 & 0.319 & 0.319 & $-68.10 \%$ \\
\hline Fraction of milk from local farm (avg/pop) & - & 1 & 1 & 0.254 & 0.254 & $-74.60 \%$ \\
\hline Fraction of veg. from local farm (MEI) & - & 1 & 1 & $\mathrm{~N} / \mathrm{A}$ & 1 & $0.00 \%$ \\
\hline Fraction of meat from local farm (MEI) & - & 1 & 1 & N/A & 1 & $0.00 \%$ \\
\hline
\end{tabular}


SRNL-STI-2010-00447, REVISION 0

Table 1. SRS Physical Parameters for Dose Calculations (continued)

\begin{tabular}{|c|c|c|c|c|c|c|}
\hline \multirow[b]{2}{*}{ Parameter } & \multirow[b]{2}{*}{ Units } & 1977 & 1991 & 2008 & 2010 & \% Difference \\
\hline & & NRC Default & Hamby & $\begin{array}{l}\text { Lee and } \\
\text { Coffield }\end{array}$ & Jannik et al. & $\begin{array}{l}1991 \text { Hamby/ } \\
2010 \text { Jannik }\end{array}$ \\
\hline Fraction of milk from local farm (MEI) & - & 1 & 1 & N/A & 1 & $0.00 \%$ \\
\hline Fraction of intake from contaminated water & - & 1 & 1 & 1 & 1 & $0.00 \%$ \\
\hline Cattle consumption rate of water (meat) & $\mathrm{L} / \mathrm{d}$ & 50 & 50 & 28 & 28 & $-44.00 \%$ \\
\hline Cattle consumption rate of water (milk) & $\mathrm{L} / \mathrm{d}$ & 60 & 60 & 50 & 50 & $-16.67 \%$ \\
\hline Buildup time in soil & $\mathrm{d}$ & 5475 & 7300 & 183 & 9125 & $25.00 \%$ \\
\hline Areal density of soil & $\mathrm{kg} / \mathrm{m}^{2}$ & 240 & 240 & 240 & 240 & $0.00 \%$ \\
\hline Soil density & $\mathrm{kg} / \mathrm{m}^{3}$ & $\mathrm{~N} / \mathrm{A}$ & $\mathrm{N} / \mathrm{A}$ & 1.6 & 1.6 & $\mathrm{~N} / \mathrm{A}$ \\
\hline Weathering removal constant & $1 / \mathrm{d}$ & 0.0495 & 0.0495 & 0.0495 & 0.0495 & $0.00 \%$ \\
\hline Fractional retention of leaves & - & 0.25 & 0.25 & 0.25 & 0.25 & $0.00 \%$ \\
\hline Depth of garden & $\mathrm{cm}$ & - & - & 15 & 15 & N/A \\
\hline Edible sport fish harvest & $\mathrm{kg} / \mathrm{yr}$ & - & $3.50 \mathrm{E}+04$ & $\mathrm{~N} / \mathrm{A}$ & $8.22 \mathrm{E}+03$ & $-76.52 \%$ \\
\hline Edible commercial fish harvest & $\mathrm{kg} / \mathrm{yr}$ & - & $2.70 \mathrm{E}+03$ & $\mathrm{~N} / \mathrm{A}$ & $5.70 \mathrm{E}+04$ & $2011.11 \%$ \\
\hline Edible commercial invertebrate harvest & $\mathrm{kg} / \mathrm{yr}$ & - & $2.45 \mathrm{E}+05$ & $\mathrm{~N} / \mathrm{A}$ & $3.80 \mathrm{E}+05$ & $55.10 \%$ \\
\hline Edible fraction of harvest - fish (whole) & - & - & 0.5 & N/A & 0.5 & $0.00 \%$ \\
\hline Edible fraction of harvest - crab (whole) & - & - & 0.14 & N/A & 0.14 & $0.00 \%$ \\
\hline Edible fraction of harvest - shrimp (tail only) & - & - & 0.9 & N/A & 0.9 & $0.00 \%$ \\
\hline Edible fraction of harvest - oysters (meat) & - & - & 1 & N/A & 1 & $0.00 \%$ \\
\hline Edible fraction of harvest - clams (meat) & - & - & 1 & N/A & 1 & $0.00 \%$ \\
\hline River dilution in estuary & - & - & 3 & $\mathrm{~N} / \mathrm{A}$ & 3 & $0.00 \%$ \\
\hline River Transport Time for Recreation & $\mathrm{d}$ & - & 1 & $\mathrm{~N} / \mathrm{A}$ & 1 & $0.00 \%$ \\
\hline River Transport Time for MEI (RM 118.8) & $\mathrm{d}$ & - & 1.5 & $\mathrm{~N} / \mathrm{A}$ & 1.5 & $0.00 \%$ \\
\hline River Transport Time for Fish & $\mathrm{d}$ & - & 2 & $\mathrm{~N} / \mathrm{A}$ & 2 & $0.00 \%$ \\
\hline River Transport Time for Irrigation & $\mathrm{d}$ & - & 2 & $\mathrm{~N} / \mathrm{A}$ & 2 & $0.00 \%$ \\
\hline River Transport Time for Drinking Water & $\mathrm{d}$ & - & 4 & N/A & 4 & $0.00 \%$ \\
\hline River Transport Time for Sport Fish & $\mathrm{d}$ & - & 10 & $\mathrm{~N} / \mathrm{A}$ & 10 & $0.00 \%$ \\
\hline River Transport Time for Commercial Fish & $\mathrm{d}$ & - & 13 & $\mathrm{~N} / \mathrm{A}$ & 13 & $0.00 \%$ \\
\hline River Transport Time for Invertibrates & $\mathrm{d}$ & - & 13 & N/A & 13 & $0.00 \%$ \\
\hline Shoreline width factor & - & 0.2 & 0.2 & $\mathrm{~N} / \mathrm{A}$ & 0.2 & $0.00 \%$ \\
\hline
\end{tabular}


SRNL-STI-2010-00447, REVISION 0

Table 2. Soil-to-Vegetable Transfer Factors

\begin{tabular}{|c|c|c|c|c|c|c|}
\hline \multirow{2}{*}{ Element } & \multirow{2}{*}{$\mathrm{Z}$} & \multirow{2}{*}{$\begin{array}{c}\text { Current SRS } \\
\text { Value }\end{array}$} & 2008 Value & 2010 Weighted Value & 2003 Weighted Value & 2010 Value \\
\hline & & & Lee and Coffield & IAEA \#472 & PNNL-13421 & Jannik, et al. \\
\hline Ac & 89 & $2.5 \mathrm{E}-03$ & $6.83 \mathrm{E}-05$ & & $6.11 \mathrm{E}-05$ & $6.11 \mathrm{E}-05$ \\
\hline $\mathrm{Ag}$ & 47 & $1.5 \mathrm{E}-01$ & $1.18 \mathrm{E}-02$ & $1.19 \mathrm{E}-04$ & & $1.19 \mathrm{E}-04$ \\
\hline $\mathrm{Al}$ & 13 & $4.0 \mathrm{E}-03$ & $1.27 \mathrm{E}-04$ & & & $1.27 \mathrm{E}-04$ \\
\hline $\mathrm{Am}$ & 95 & $2.5 \mathrm{E}-04$ & $6.83 \mathrm{E}-05$ & $7.3 \mathrm{E}-05$ & & $7.33 \mathrm{E}-05$ \\
\hline $\mathrm{Ar}$ & 18 & & $1.00 \mathrm{E}-20$ & & & $1.00 \mathrm{E}-20$ \\
\hline As & 33 & & $1.17 \mathrm{E}-03$ & & $2.73 \mathrm{E}-03$ & $2.73 \mathrm{E}-03$ \\
\hline At & 85 & & $2.93 \mathrm{E}-02$ & & & $2.93 \mathrm{E}-02$ \\
\hline $\mathrm{Au}$ & 79 & & $3.51 \mathrm{E}-03$ & & $2.64 \mathrm{E}-03$ & $2.64 \mathrm{E}-03$ \\
\hline $\mathrm{B}$ & 5 & & $3.90 \mathrm{E}-01$ & & & $3.90 \mathrm{E}-01$ \\
\hline $\mathrm{Ba}$ & 56 & $5.0 \mathrm{E}-03$ & $2.93 \mathrm{E}-03$ & $9.75 \mathrm{E}-04$ & & $9.75 \mathrm{E}-04$ \\
\hline $\mathrm{Be}$ & 4 & $4.0 \mathrm{E}-03$ & $2.93 \mathrm{E}-04$ & & $6.83 \mathrm{E}-04$ & $6.83 \mathrm{E}-04$ \\
\hline $\mathrm{Bi}$ & 83 & $1.0 \mathrm{E}-01$ & $9.75 \mathrm{E}-02$ & & $9.75 \mathrm{E}-02$ & $9.75 \mathrm{E}-02$ \\
\hline $\mathrm{Bk}$ & 97 & $5.9 \mathrm{E}-05$ & $1.00 \mathrm{E}-03$ & & & $1.00 \mathrm{E}-03$ \\
\hline $\mathrm{Br}$ & 35 & & $2.93 \mathrm{E}-01$ & & $2.93 \mathrm{E}-01$ & $2.93 \mathrm{E}-01$ \\
\hline $\mathrm{C}$ & 6 & $5.5 \mathrm{E}+00$ & $1.37 \mathrm{E}-01$ & & $1.37 \mathrm{E}-01$ & $1.37 \mathrm{E}-01$ \\
\hline $\mathrm{Ca}$ & 20 & $5.0 \mathrm{E}-01$ & $6.83 \mathrm{E}-02$ & $3.9 \mathrm{E}+00$ & & $3.90 \mathrm{E}+00$ \\
\hline $\mathrm{Cd}$ & 48 & $3.0 \mathrm{E}-01$ & $2.93 \mathrm{E}-02$ & $1.5 \mathrm{E}-01$ & & $1.49 \mathrm{E}-01$ \\
\hline $\mathrm{Ce}$ & 58 & $2.5 \mathrm{E}-03$ & $3.90 \mathrm{E}-03$ & $1.6 \mathrm{E}-03$ & & $1.63 \mathrm{E}-03$ \\
\hline $\mathrm{Cf}$ & 98 & $1.0 \mathrm{E}-03$ & $6.83 \mathrm{E}-05$ & & $6.11 \mathrm{E}-05$ & $6.11 \mathrm{E}-05$ \\
\hline $\mathrm{Cl}$ & 17 & $2.0 \mathrm{E}+01$ & $1.37 \mathrm{E}+01$ & $3.5 \mathrm{E}+00$ & & $3.49 \mathrm{E}+00$ \\
\hline $\mathrm{Cm}$ & 96 & $2.5 \mathrm{E}-03$ & $8.39 \mathrm{E}-05$ & $1.3 \mathrm{E}-04$ & & $1.27 \mathrm{E}-04$ \\
\hline $\mathrm{Co}$ & 27 & $9.4 \mathrm{E}-03$ & $1.31 \mathrm{E}-02$ & $2.5 \mathrm{E}-02$ & & $2.48 \mathrm{E}-02$ \\
\hline $\mathrm{Cr}$ & 24 & $2.5 \mathrm{E}-04$ & $8.78 \mathrm{E}-04$ & $2.0 \mathrm{E}-04$ & & $1.95 \mathrm{E}-04$ \\
\hline Cs & 55 & $1.0 \mathrm{E}-02$ & $9.00 \mathrm{E}-01$ & $6.9 \mathrm{E}-03$ & & $6.85 \mathrm{E}-03$ \\
\hline $\mathrm{Cu}$ & 29 & $1.2 \mathrm{E}-01$ & $4.88 \mathrm{E}-02$ & $1.6 \mathrm{E}-01$ & & $1.56 \mathrm{E}-01$ \\
\hline Dy & 66 & & $3.90 \mathrm{E}-03$ & & $3.90 \mathrm{E}-03$ & $3.90 \mathrm{E}-03$ \\
\hline $\mathrm{Er}$ & 68 & & $3.90 \mathrm{E}-03$ & & $3.90 \mathrm{E}-03$ & $3.90 \mathrm{E}-03$ \\
\hline Es & 99 & $5.9 \mathrm{E}-05$ & $1.00 \mathrm{E}-03$ & & & $1.00 \mathrm{E}-03$ \\
\hline
\end{tabular}

Note: $1.00 \mathrm{E}-20$ is equivalent to 0.00 . 
SRNL-STI-2010-00447, REVISION 0

Table 2. Soil-to-Vegetable Transfer Factors (continued)

\begin{tabular}{|c|c|c|c|c|c|c|}
\hline \multirow{2}{*}{ Element } & \multirow{2}{*}{$\mathrm{Z}$} & \multirow{2}{*}{$\begin{array}{c}\text { Current SRS } \\
\text { Value }\end{array}$} & 2008 Value & 2010 Weighted Value & 2003 Weighted Value & 2010 Value \\
\hline & & & Lee and Coffield & IAEA \#472 & PNNL-13421 & Jannik, et al. \\
\hline $\mathrm{Eu}$ & 63 & $2.5 \mathrm{E}-03$ & $3.90 \mathrm{E}-03$ & & $3.90 \mathrm{E}-03$ & $3.90 \mathrm{E}-03$ \\
\hline $\mathrm{F}$ & 9 & & $1.17 \mathrm{E}-03$ & & $3.65 \mathrm{E}-03$ & $3.65 \mathrm{E}-03$ \\
\hline $\mathrm{Fe}$ & 26 & $6.6 \mathrm{E}-04$ & $9.75 \mathrm{E}-03$ & $1.10 \mathrm{E}-02$ & & $1.10 \mathrm{E}-02$ \\
\hline Fm & 100 & & $2.00 \mathrm{E}-03$ & & & $2.00 \mathrm{E}-03$ \\
\hline $\mathrm{Fr}$ & 87 & & $5.85 \mathrm{E}-03$ & & & $5.85 \mathrm{E}-03$ \\
\hline $\mathrm{Ga}$ & 31 & & $7.80 \mathrm{E}-05$ & & $2.43 \mathrm{E}-04$ & $2.43 \mathrm{E}-04$ \\
\hline $\mathrm{Gd}$ & 64 & $2.0 \mathrm{E}-03$ & $3.90 \mathrm{E}-03$ & & $3.90 \mathrm{E}-03$ & $3.90 \mathrm{E}-03$ \\
\hline $\mathrm{Ge}$ & 32 & $1.0 \mathrm{E}-01$ & $1.56 \mathrm{E}-02$ & & & $1.56 \mathrm{E}-02$ \\
\hline $\mathrm{H}$ & 1 & not used & $4.80 \mathrm{E}+00$ & & & $4.80 \mathrm{E}+00$ \\
\hline $\mathrm{Ha}$ & 108 & & $2.00 \mathrm{E}-03$ & & & $2.00 \mathrm{E}-03$ \\
\hline $\mathrm{He}$ & 2 & & $1.00 \mathrm{E}-20$ & & & $1.00 \mathrm{E}-20$ \\
\hline Hf & 72 & $1.0 \mathrm{E}-04$ & $1.95 \mathrm{E}-04$ & & $1.95 \mathrm{E}-04$ & $1.95 \mathrm{E}-04$ \\
\hline $\mathrm{Hg}$ & 80 & $3.8 \mathrm{E}-01$ & $3.90 \mathrm{E}-02$ & & $9.03 \mathrm{E}-02$ & $9.03 \mathrm{E}-02$ \\
\hline Ho & 67 & $2.5 \mathrm{E}-03$ & $3.90 \mathrm{E}-03$ & & $3.90 \mathrm{E}-03$ & $3.90 \mathrm{E}-03$ \\
\hline I & 53 & $2.0 \mathrm{E}-02$ & $7.80 \mathrm{E}-03$ & $1.3 \mathrm{E}-02$ & & $1.32 \mathrm{E}-02$ \\
\hline In & 49 & $3.0 \mathrm{E}-03$ & $7.80 \mathrm{E}-05$ & & $2.43 \mathrm{E}-04$ & $2.43 \mathrm{E}-04$ \\
\hline $\mathrm{Ir}$ & 77 & $3.0 \mathrm{E}-02$ & $2.93 \mathrm{E}-03$ & & $4.76 \mathrm{E}-03$ & $4.76 \mathrm{E}-03$ \\
\hline $\mathrm{K}$ & 19 & $3.0 \mathrm{E}-01$ & $1.07 \mathrm{E}-01$ & $2.5 \mathrm{E}-01$ & & $2.54 \mathrm{E}-01$ \\
\hline $\mathrm{Kr}$ & 36 & & $1.00 \mathrm{E}-20$ & & & $1.00 \mathrm{E}-20$ \\
\hline $\mathrm{La}$ & 57 & $2.5 \mathrm{E}-03$ & $6.83 \mathrm{E}-05$ & $9.09 \mathrm{E}-04$ & & $9.09 \mathrm{E}-04$ \\
\hline $\mathrm{Li}$ & 3 & & $7.80 \mathrm{E}-04$ & & & $7.80 \mathrm{E}-04$ \\
\hline $\mathrm{Lr}$ & 103 & & $2.00 \mathrm{E}-03$ & & & $2.00 \mathrm{E}-03$ \\
\hline $\mathrm{Lu}$ & 71 & $2.5 \mathrm{E}-03$ & $7.80 \mathrm{E}-04$ & & & 7.80E-04 \\
\hline $\mathrm{Md}$ & 101 & & $2.00 \mathrm{E}-03$ & & & $2.00 \mathrm{E}-03$ \\
\hline $\mathrm{Mg}$ & 12 & & $1.07 \mathrm{E}-01$ & & $1.28 \mathrm{E}-01$ & $1.28 \mathrm{E}-01$ \\
\hline $\mathrm{Mn}$ & 25 & $2.9 \mathrm{E}-02$ & $3.90 \mathrm{E}-02$ & $6.39 \mathrm{E}-02$ & & $6.39 \mathrm{E}-02$ \\
\hline Mo & 42 & $1.2 \mathrm{E}-01$ & $1.56 \mathrm{E}-01$ & $8.7 \mathrm{E}-02$ & & $8.71 \mathrm{E}-02$ \\
\hline $\mathrm{N}$ & 7 & & $3.50 \mathrm{E}-01$ & & $7.43 \mathrm{E}-03$ & 7.43E-03 \\
\hline $\mathrm{Na}$ & 11 & $5.0 \mathrm{E}-02$ & $5.85 \mathrm{E}-02$ & $5.85 \mathrm{E}-03$ & & $5.85 \mathrm{E}-03$ \\
\hline
\end{tabular}

Note: $1.00 \mathrm{E}-20$ is equivalent to 0.00 . 
SRNL-STI-2010-00447, REVISION 0

Table 2. Soil-to-Vegetable Transfer Factors (continued)

\begin{tabular}{|c|c|c|c|c|c|c|}
\hline \multirow{2}{*}{ Element } & \multirow{2}{*}{$\mathbf{Z}$} & \multirow{2}{*}{$\begin{array}{c}\text { Current SRS } \\
\text { Value }\end{array}$} & 2008 Value & 2010 Weighted Value & 2003 Weighted Value & 2010 Value \\
\hline & & & Lee and Coffield & IAEA \#472 & PNNL-13421 & Jannik, et al. \\
\hline $\mathrm{Nb}$ & 41 & $9.4 \mathrm{E}-03$ & $4.88 \mathrm{E}-03$ & $2.2 \mathrm{E}-03$ & & $2.18 \mathrm{E}-03$ \\
\hline $\mathrm{Nd}$ & 60 & & $3.90 \mathrm{E}-03$ & $3.9 \mathrm{E}-03$ & & $3.90 \mathrm{E}-03$ \\
\hline $\mathrm{Ne}$ & 10 & & $1.00 \mathrm{E}-20$ & & & $1.00 \mathrm{E}-20$ \\
\hline $\mathrm{Ni}$ & 28 & 1.9E-02 & $1.17 \mathrm{E}-02$ & & $2.18 \mathrm{E}-02$ & $2.18 \mathrm{E}-02$ \\
\hline No & 102 & & $2.00 \mathrm{E}-03$ & & & $2.00 \mathrm{E}-03$ \\
\hline $\mathrm{Np}$ & 93 & $2.5 \mathrm{E}-03$ & $2.54 \mathrm{E}-03$ & $3.91 \mathrm{E}-03$ & & $3.91 \mathrm{E}-03$ \\
\hline $\mathrm{O}$ & 8 & & $6.00 \mathrm{E}-01$ & & & $6.00 \mathrm{E}-01$ \\
\hline Os & 76 & & $6.83 \mathrm{E}-04$ & & $6.45 \mathrm{E}-03$ & $6.45 \mathrm{E}-03$ \\
\hline $\mathrm{P}$ & 15 & $1.1 \mathrm{E}+00$ & $6.83 \mathrm{E}-01$ & $1.95 \mathrm{E}-01$ & & $1.95 \mathrm{E}-01$ \\
\hline $\mathrm{Pa}$ & 91 & $1.0 \mathrm{E}-02$ & $4.18 \mathrm{E}-04$ & & $6.11 \mathrm{E}-05$ & $6.11 \mathrm{E}-05$ \\
\hline $\mathrm{Pb}$ & 82 & $1.0 \mathrm{E}-02$ & $1.17 \mathrm{E}-03$ & $5.2 \mathrm{E}-03$ & & $5.18 \mathrm{E}-03$ \\
\hline $\mathrm{Pd}$ & 46 & $1.0 \mathrm{E}-01$ & $7.80 \mathrm{E}-03$ & & $1.28 \mathrm{E}-02$ & $1.28 \mathrm{E}-02$ \\
\hline $\mathrm{Pm}$ & 61 & $2.5 \mathrm{E}-03$ & $3.90 \mathrm{E}-03$ & $2.3 \mathrm{E}-02$ & & $2.32 \mathrm{E}-02$ \\
\hline Po & 84 & $1.0 \mathrm{E}-03$ & $1.37 \mathrm{E}-03$ & $4.3 \mathrm{E}-04$ & & $4.30 \mathrm{E}-04$ \\
\hline $\mathrm{Pr}$ & 59 & & $3.90 \mathrm{E}-03$ & $3.9 \mathrm{E}-03$ & & $3.90 \mathrm{E}-03$ \\
\hline $\mathrm{Pt}$ & 78 & $2.4 \mathrm{E}-02$ & $4.88 \mathrm{E}-03$ & & & $4.88 \mathrm{E}-03$ \\
\hline $\mathrm{Pu}$ & 94 & $2.5 \mathrm{E}-04$ & $2.15 \mathrm{E}-04$ & $2.0 \mathrm{E}-05$ & & $1.97 \mathrm{E}-05$ \\
\hline $\mathrm{Ra}$ & 88 & $4.0 \mathrm{E}-02$ & $4.64 \mathrm{E}-03$ & $1.2 \mathrm{E}-02$ & & 1.19E-02 \\
\hline $\mathrm{Rb}$ & 37 & $1.3 \mathrm{E}-01$ & $1.76 \mathrm{E}-01$ & $1.4 \mathrm{E}-01$ & & $1.39 \mathrm{E}-01$ \\
\hline $\mathrm{Re}$ & 75 & $2.1 \mathrm{E}+02$ & $1.29 \mathrm{E}+00$ & & $1.21 \mathrm{E}-01$ & $1.21 \mathrm{E}-01$ \\
\hline $\mathrm{Rf}$ & 104 & & $3.00 \mathrm{E}-03$ & & & $3.00 \mathrm{E}-03$ \\
\hline $\mathrm{Rh}$ & 45 & & $7.80 \mathrm{E}-03$ & $1.76 \mathrm{E}-01$ & & $1.76 \mathrm{E}-01$ \\
\hline $\mathrm{Rn}$ & 86 & & $1.00 \mathrm{E}-20$ & & & $1.00 \mathrm{E}-20$ \\
\hline $\mathrm{Ru}$ & 44 & $5.0 \mathrm{E}-02$ & $7.80 \mathrm{E}-03$ & $6.29 \mathrm{E}-03$ & & $6.29 \mathrm{E}-03$ \\
\hline $\mathrm{S}$ & 16 & $5.9 \mathrm{E}-01$ & $2.93 \mathrm{E}-01$ & & $2.93 \mathrm{E}-01$ & $2.93 \mathrm{E}-01$ \\
\hline $\mathrm{Sb}$ & 51 & $1.1 \mathrm{E}-02$ & $2.49 \mathrm{E}-03$ & $2.6 \mathrm{E}-04$ & & $2.61 \mathrm{E}-04$ \\
\hline Sc & 21 & & $1.95 \mathrm{E}-04$ & & $4.24 \mathrm{E}-04$ & $4.24 \mathrm{E}-04$ \\
\hline
\end{tabular}

Note: $1.00 \mathrm{E}-20$ is equivalent to 0.00 . 
SRNL-STI-2010-00447, REVISION 0

Table 2. Soil-to-Vegetable Transfer Factors (continued)

\begin{tabular}{|c|c|c|c|c|c|c|}
\hline \multirow{2}{*}{ Element } & \multirow{2}{*}{ Z } & \multirow{2}{*}{$\begin{array}{c}\text { Current SRS } \\
\text { Value }\end{array}$} & 2008 Value & 2010 Weighted Value & 2003 Weighted Value & 2010 Value \\
\hline & & & Lee and Coffield & IAEA \#472 & PNNL-13421 & Jannik, et al. \\
\hline $\mathrm{Se}$ & 34 & $1.3 \mathrm{E}+00$ & $5.14 \mathrm{E}-02$ & & $1.89 \mathrm{E}-02$ & $1.89 \mathrm{E}-02$ \\
\hline $\mathrm{Si}$ & 14 & $8.8 \mathrm{E}-02$ & $1.37 \mathrm{E}-02$ & & $2.65 \mathrm{E}-02$ & $2.65 \mathrm{E}-02$ \\
\hline $\mathrm{Sm}$ & 62 & $2.5 \mathrm{E}-03$ & $3.90 \mathrm{E}-03$ & & $3.90 \mathrm{E}-03$ & $3.90 \mathrm{E}-03$ \\
\hline $\mathrm{Sn}$ & 50 & $2.5 \mathrm{E}-03$ & $1.17 \mathrm{E}-03$ & & $2.27 \mathrm{E}-03$ & $2.27 \mathrm{E}-03$ \\
\hline $\mathrm{Sr}$ & 38 & $1.7 \mathrm{E}-02$ & $9.75 \mathrm{E}-02$ & $1.2 \mathrm{E}-01$ & & $1.23 \mathrm{E}-01$ \\
\hline $\mathrm{Ta}$ & 73 & $2.5 \mathrm{E}-03$ & $4.88 \mathrm{E}-03$ & & $4.88 \mathrm{E}-03$ & $4.88 \mathrm{E}-03$ \\
\hline $\mathrm{Tb}$ & 65 & & $3.90 \mathrm{E}-03$ & & $3.90 \mathrm{E}-03$ & $3.90 \mathrm{E}-03$ \\
\hline Tc & 43 & $2.5 \mathrm{E}-01$ & $4.68 \mathrm{E}-02$ & $1.79 \mathrm{E}+01$ & & $1.79 \mathrm{E}+01$ \\
\hline $\mathrm{Te}$ & 52 & $1.3 \mathrm{E}+00$ & $1.20 \mathrm{E}-02$ & $5.9 \mathrm{E}-02$ & & $5.85 \mathrm{E}-02$ \\
\hline Th & 90 & $4.2 \mathrm{E}-03$ & $6.44 \mathrm{E}-05$ & $3.1 \mathrm{E}-04$ & & $3.14 \mathrm{E}-04$ \\
\hline $\mathrm{Ti}$ & 22 & $1.0 \mathrm{E}-04$ & $5.85 \mathrm{E}-04$ & & & $5.85 \mathrm{E}-04$ \\
\hline $\mathrm{Tl}$ & 81 & & 7.80E-05 & & $2.43 \mathrm{E}-04$ & $2.43 \mathrm{E}-04$ \\
\hline $\mathrm{Tm}$ & 69 & & 7.80E-04 & & & 7.80E-04 \\
\hline $\mathrm{U}$ & 92 & $2.5 \mathrm{E}-03$ & $2.34 \mathrm{E}-03$ & 6.69E-03 & & $6.69 \mathrm{E}-03$ \\
\hline $\mathrm{V}$ & 23 & $1.4 \mathrm{E}-03$ & $5.85 \mathrm{E}-04$ & & & $5.85 \mathrm{E}-04$ \\
\hline $\mathrm{W}$ & 74 & & $5.00 \mathrm{E}-02$ & $2.0 \mathrm{E}-02$ & & $1.95 \mathrm{E}-02$ \\
\hline $\mathrm{Xe}$ & 54 & & $1.00 \mathrm{E}-20$ & & & $1.00 \mathrm{E}-20$ \\
\hline $\mathrm{Y}$ & 39 & $2.6 \mathrm{E}-03$ & $1.95 \mathrm{E}-03$ & $3.90 \mathrm{E}-04$ & & $3.90 \mathrm{E}-04$ \\
\hline $\mathrm{Yb}$ & 70 & & $7.80 \mathrm{E}-04$ & & & $7.80 \mathrm{E}-04$ \\
\hline $\mathrm{Zn}$ & 30 & $4.0 \mathrm{E}-01$ & $6.83 \mathrm{E}-02$ & $1.71 \mathrm{E}-01$ & & $1.71 \mathrm{E}-01$ \\
\hline $\mathrm{Zr}$ & 40 & $1.7 \mathrm{E}-04$ & $1.95 \mathrm{E}-04$ & $7.8 \mathrm{E}-04$ & & $7.80 \mathrm{E}-04$ \\
\hline
\end{tabular}

Note: $1.00 \mathrm{E}-20$ is equivalent to 0.00 . 
SRNL-STI-2010-00447, REVISION 0

Table 3. Feed-to-Milk Transfer Factors

\begin{tabular}{|c|c|c|c|c|c|}
\hline \multirow{2}{*}{ Element } & \multirow{2}{*}{$\mathbf{Z}$} & \multirow{2}{*}{$\begin{array}{c}\text { SRS } \\
\text { Current Value }\end{array}$} & 2008 Value & 2010 Value & 2010 Value \\
\hline & & & Lee and Coffield & IAEA \#472 & Jannik et al. \\
\hline Ac & 89 & $2.0 \mathrm{E}-05$ & $2.00 \mathrm{E}-05$ & & $2.00 \mathrm{E}-05$ \\
\hline $\mathrm{Ag}$ & 47 & $5.0 \mathrm{E}-02$ & $1.58 \mathrm{E}-03$ & & $1.58 \mathrm{E}-03$ \\
\hline $\mathrm{Al}$ & 13 & $2.0 \mathrm{E}-04$ & $2.06 \mathrm{E}-04$ & & $2.06 \mathrm{E}-04$ \\
\hline $\mathrm{Am}$ & 95 & $5.0 \mathrm{E}-06$ & $1.50 \mathrm{E}-06$ & $4.20 \mathrm{E}-07$ & $4.20 \mathrm{E}-07$ \\
\hline As & 33 & & $6.00 \mathrm{E}-05$ & & $6.00 \mathrm{E}-05$ \\
\hline At & 85 & & $1.03 \mathrm{E}-02$ & & $1.03 \mathrm{E}-02$ \\
\hline $\mathrm{Au}$ & 79 & & $5.50 \mathrm{E}-06$ & & $5.50 \mathrm{E}-06$ \\
\hline $\mathrm{B}$ & 5 & & $1.55 \mathrm{E}-03$ & & $1.55 \mathrm{E}-03$ \\
\hline $\mathrm{Ba}$ & 56 & $4.0 \mathrm{E}-04$ & $4.80 \mathrm{E}-04$ & $1.60 \mathrm{E}-04$ & $1.60 \mathrm{E}-04$ \\
\hline $\mathrm{Be}$ & 4 & $2.0 \mathrm{E}-06$ & $9.00 \mathrm{E}-07$ & $8.30 \mathrm{E}-07$ & $8.30 \mathrm{E}-07$ \\
\hline $\mathrm{Bi}$ & 83 & $5.0 \mathrm{E}-04$ & $5.00 \mathrm{E}-04$ & & $5.00 \mathrm{E}-04$ \\
\hline $\mathrm{Bk}$ & 97 & $4.0 \mathrm{E}-07$ & $2.00 \mathrm{E}-06$ & & $2.00 \mathrm{E}-06$ \\
\hline $\mathrm{Br}$ & 35 & & $2.00 \mathrm{E}-02$ & & $2.00 \mathrm{E}-02$ \\
\hline $\mathrm{C}$ & 6 & $1.2 \mathrm{E}-02$ & $1.20 \mathrm{E}-02$ & & $1.20 \mathrm{E}-02$ \\
\hline $\mathrm{Ca}$ & 20 & $3.0 \mathrm{E}-03$ & $3.00 \mathrm{E}-03$ & $1.00 \mathrm{E}-02$ & $1.00 \mathrm{E}-02$ \\
\hline $\mathrm{Cd}$ & 48 & $1.2 \mathrm{E}-04$ & $1.00 \mathrm{E}-03$ & $1.90 \mathrm{E}-04$ & $1.90 \mathrm{E}-04$ \\
\hline $\mathrm{Ce}$ & 58 & $6.0 \mathrm{E}-04$ & $3.00 \mathrm{E}-05$ & $2.00 \mathrm{E}-05$ & $2.00 \mathrm{E}-05$ \\
\hline $\mathrm{Cf}$ & 98 & $7.5 \mathrm{E}-07$ & $1.50 \mathrm{E}-06$ & & $1.50 \mathrm{E}-06$ \\
\hline $\mathrm{Cl}$ & 17 & $2.0 \mathrm{E}-02$ & $1.70 \mathrm{E}-02$ & & $1.70 \mathrm{E}-02$ \\
\hline $\mathrm{Cm}$ & 96 & $5.0 \mathrm{E}-06$ & $2.00 \mathrm{E}-05$ & & $2.00 \mathrm{E}-05$ \\
\hline Co & 27 & $1.0 \mathrm{E}-03$ & $3.00 \mathrm{E}-04$ & $1.10 \mathrm{E}-04$ & $1.10 \mathrm{E}-04$ \\
\hline $\mathrm{Cr}$ & 24 & $2.2 \mathrm{E}-03$ & $1.00 \mathrm{E}-05$ & $4.30 \mathrm{E}-04$ & $4.30 \mathrm{E}-04$ \\
\hline Cs & 55 & $1.2 \mathrm{E}-02$ & $7.90 \mathrm{E}-03$ & $4.60 \mathrm{E}-03$ & $4.60 \mathrm{E}-03$ \\
\hline $\mathrm{Cu}$ & 29 & $1.4 \mathrm{E}-02$ & $2.00 \mathrm{E}-03$ & & $2.00 \mathrm{E}-03$ \\
\hline Dy & 66 & & $3.00 \mathrm{E}-05$ & & $3.00 \mathrm{E}-05$ \\
\hline Er & 68 & & $3.00 \mathrm{E}-05$ & & $3.00 \mathrm{E}-05$ \\
\hline Es & 99 & $4.0 \mathrm{E}-07$ & $2.00 \mathrm{E}-06$ & & $2.00 \mathrm{E}-06$ \\
\hline $\mathrm{Eu}$ & 63 & $5.0 \mathrm{E}-06$ & $3.00 \mathrm{E}-05$ & & $3.00 \mathrm{E}-05$ \\
\hline $\mathrm{F}$ & 9 & & $1.00 \mathrm{E}-03$ & & $1.00 \mathrm{E}-03$ \\
\hline $\mathrm{Fe}$ & 26 & $1.2 \mathrm{E}-03$ & $3.00 \mathrm{E}-05$ & $3.50 \mathrm{E}-05$ & $3.50 \mathrm{E}-05$ \\
\hline $\mathrm{Fr}$ & 87 & & $2.06 \mathrm{E}-02$ & & $2.06 \mathrm{E}-02$ \\
\hline $\mathrm{Ga}$ & 31 & & $5.00 \mathrm{E}-05$ & & $5.00 \mathrm{E}-05$ \\
\hline $\mathrm{Gd}$ & 64 & $6.0 \mathrm{E}-05$ & $3.00 \mathrm{E}-05$ & & $3.00 \mathrm{E}-05$ \\
\hline $\mathrm{Ge}$ & 32 & $7.0 \mathrm{E}-02$ & 7.21E-02 & & $7.21 \mathrm{E}-02$ \\
\hline $\mathrm{H}$ & 1 & not used & $1.50 \mathrm{E}-02$ & & $1.50 \mathrm{E}-02$ \\
\hline $\mathrm{Ha}$ & 105 & & $5.00 \mathrm{E}-06$ & & $5.00 \mathrm{E}-06$ \\
\hline $\mathrm{He}$ & 2 & & $1.00 \mathrm{E}-20$ & & $1.00 \mathrm{E}-20$ \\
\hline $\mathrm{Hf}$ & 72 & $5.5 \mathrm{E}-07$ & $5.50 \mathrm{E}-07$ & & $5.50 \mathrm{E}-07$ \\
\hline $\mathrm{Hg}$ & 80 & $5.0 \mathrm{E}-04$ & $4.70 \mathrm{E}-04$ & & 4.70E-04 \\
\hline Ho & 67 & $2.0 \mathrm{E}-05$ & $3.00 \mathrm{E}-05$ & & $3.00 \mathrm{E}-05$ \\
\hline I & 53 & $6.0 \mathrm{E}-03$ & $9.00 \mathrm{E}-03$ & $5.40 \mathrm{E}-03$ & $5.40 \mathrm{E}-03$ \\
\hline In & 49 & $2.0 \mathrm{E}-04$ & 2.00E-04 & & $2.00 \mathrm{E}-04$ \\
\hline $\mathrm{Ir}$ & 77 & $2.0 \mathrm{E}-06$ & $2.00 \mathrm{E}-06$ & & $2.00 \mathrm{E}-06$ \\
\hline $\mathrm{K}$ & 19 & $7.0 \mathrm{E}-03$ & $7.20 \mathrm{E}-03$ & & $7.20 \mathrm{E}-03$ \\
\hline $\mathrm{La}$ & 57 & $5.0 \mathrm{E}-06$ & $2.00 \mathrm{E}-05$ & & $2.00 \mathrm{E}-05$ \\
\hline $\mathrm{Li}$ & 3 & & $2.06 \mathrm{E}-02$ & & $2.06 \mathrm{E}-02$ \\
\hline $\mathrm{Lr}$ & 103 & & $5.00 \mathrm{E}-06$ & & $5.00 \mathrm{E}-06$ \\
\hline $\mathrm{Lu}$ & 71 & $2.0 \mathrm{E}-05$ & $2.06 \mathrm{E}-05$ & & $2.06 \mathrm{E}-05$ \\
\hline $\mathrm{Md}$ & 101 & & $5.00 \mathrm{E}-06$ & & $5.00 \mathrm{E}-06$ \\
\hline
\end{tabular}


SRNL-STI-2010-00447, REVISION 0

Table 3. Feed-to-Milk Transfer Factors (continued)

\begin{tabular}{|c|c|c|c|c|c|}
\hline Element & $\mathbf{Z}$ & SRS Current Value & 2008 Value & 2010 Value & 2010 Value \\
\hline & & & Lee and Coffield & IAEA \#472 & Jannik et al. \\
\hline $\mathrm{Mg}$ & 12 & & $3.90 \mathrm{E}-03$ & & $3.90 \mathrm{E}-03$ \\
\hline $\mathrm{Mn}$ & 25 & $2.5 \mathrm{E}-04$ & $3.00 \mathrm{E}-05$ & $4.10 \mathrm{E}-05$ & $4.10 \mathrm{E}-05$ \\
\hline Mo & 42 & $7.5 \mathrm{E}-03$ & $1.70 \mathrm{E}-03$ & $1.10 \mathrm{E}-03$ & $1.10 \mathrm{E}-03$ \\
\hline $\mathrm{N}$ & 7 & & $2.50 \mathrm{E}-02$ & & $2.50 \mathrm{E}-02$ \\
\hline $\mathrm{Na}$ & 11 & $4.0 \mathrm{E}-02$ & $1.60 \mathrm{E}-02$ & $1.30 \mathrm{E}-02$ & $1.30 \mathrm{E}-02$ \\
\hline $\mathrm{Nb}$ & 41 & $2.5 \mathrm{E}-03$ & $3.20 \mathrm{E}-05$ & $4.10 \mathrm{E}-07$ & $4.10 \mathrm{E}-07$ \\
\hline $\mathrm{Nd}$ & 60 & & $3.00 \mathrm{E}-05$ & & $3.00 \mathrm{E}-05$ \\
\hline $\mathrm{Ni}$ & 28 & $6.7 \mathrm{E}-03$ & $1.60 \mathrm{E}-02$ & $9.50 \mathrm{E}-04$ & $9.50 \mathrm{E}-04$ \\
\hline No & 102 & & $5.00 \mathrm{E}-06$ & & $5.00 \mathrm{E}-06$ \\
\hline $\mathrm{Np}$ & 93 & $5.0 \mathrm{E}-06$ & $5.00 \mathrm{E}-06$ & & $5.00 \mathrm{E}-06$ \\
\hline Os & 76 & & $5.00 \mathrm{E}-03$ & & $5.00 \mathrm{E}-03$ \\
\hline $\mathrm{P}$ & 15 & $2.5 \mathrm{E}-02$ & $1.60 \mathrm{E}-02$ & $2.00 \mathrm{E}-02$ & $2.00 \mathrm{E}-02$ \\
\hline $\mathrm{Pa}$ & 91 & $5.0 \mathrm{E}-06$ & $5.00 \mathrm{E}-06$ & & $5.00 \mathrm{E}-06$ \\
\hline $\mathrm{Pb}$ & 82 & $3.0 \mathrm{E}-04$ & $2.60 \mathrm{E}-04$ & $1.90 \mathrm{E}-04$ & $1.90 \mathrm{E}-04$ \\
\hline $\mathrm{Pd}$ & 46 & $5.0 \mathrm{E}-03$ & $1.00 \mathrm{E}-02$ & & $1.00 \mathrm{E}-02$ \\
\hline $\mathrm{Pm}$ & 61 & $5.0 \mathrm{E}-06$ & $3.00 \mathrm{E}-05$ & & $3.00 \mathrm{E}-05$ \\
\hline Po & 84 & $3.4 \mathrm{E}-04$ & $3.40 \mathrm{E}-04$ & $2.10 \mathrm{E}-04$ & $2.10 \mathrm{E}-04$ \\
\hline $\operatorname{Pr}$ & 59 & & $3.00 \mathrm{E}-05$ & & $3.00 \mathrm{E}-05$ \\
\hline $\mathrm{Pt}$ & 78 & $5.0 \mathrm{E}-03$ & $5.15 \mathrm{E}-03$ & & $5.15 \mathrm{E}-03$ \\
\hline $\mathrm{Pu}$ & 94 & $2.0 \mathrm{E}-06$ & $1.10 \mathrm{E}-06$ & $1.00 \mathrm{E}-05$ & $1.00 \mathrm{E}-05$ \\
\hline $\mathrm{Ra}$ & 88 & $1.0 \mathrm{E}-03$ & $1.30 \mathrm{E}-03$ & $3.80 \mathrm{E}-04$ & $3.80 \mathrm{E}-04$ \\
\hline $\mathrm{Rb}$ & 37 & $3.0 \mathrm{E}-02$ & $1.20 \mathrm{E}-02$ & & $1.20 \mathrm{E}-02$ \\
\hline $\operatorname{Re}$ & 75 & $1.4 \mathrm{E}-04$ & $1.50 \mathrm{E}-03$ & & $1.50 \mathrm{E}-03$ \\
\hline $\mathrm{Rf}$ & 104 & & $2.00 \mathrm{E}-05$ & & $2.00 \mathrm{E}-05$ \\
\hline $\mathrm{Rh}$ & 45 & & $1.00 \mathrm{E}-02$ & & $1.00 \mathrm{E}-02$ \\
\hline $\mathrm{Rn}$ & 86 & & $1.00 \mathrm{E}-20$ & & $1.00 \mathrm{E}-20$ \\
\hline $\mathrm{Ru}$ & 44 & $1.0 \mathrm{E}-06$ & $3.30 \mathrm{E}-06$ & $9.40 \mathrm{E}-06$ & $9.40 \mathrm{E}-06$ \\
\hline $\mathrm{S}$ & 16 & $1.8 \mathrm{E}-02$ & $1.60 \mathrm{E}-02$ & $7.90 \mathrm{E}-03$ & $7.90 \mathrm{E}-03$ \\
\hline $\mathrm{Sb}$ & 51 & $1.5 \mathrm{E}-03$ & $2.50 \mathrm{E}-05$ & $3.80 \mathrm{E}-05$ & $3.80 \mathrm{E}-05$ \\
\hline $\mathrm{Sc}$ & 21 & & $5.00 \mathrm{E}-06$ & & $5.00 \mathrm{E}-06$ \\
\hline $\mathrm{Se}$ & 34 & $4.5 \mathrm{E}-02$ & $4.00 \mathrm{E}-03$ & 4.00E-03 & $4.00 \mathrm{E}-03$ \\
\hline $\mathrm{Si}$ & 14 & $2.0 \mathrm{E}-05$ & $2.00 \mathrm{E}-05$ & & $2.00 \mathrm{E}-05$ \\
\hline $\mathrm{Sm}$ & 62 & $5.0 \mathrm{E}-06$ & $3.00 \mathrm{E}-05$ & & $3.00 \mathrm{E}-05$ \\
\hline $\mathrm{Sn}$ & 50 & $2.5 \mathrm{E}-03$ & $1.00 \mathrm{E}-03$ & & $1.00 \mathrm{E}-03$ \\
\hline $\mathrm{Sr}$ & 38 & $8.0 \mathrm{E}-04$ & $2.80 \mathrm{E}-03$ & $1.30 \mathrm{E}-03$ & $1.30 \mathrm{E}-03$ \\
\hline Ta & 73 & $3.0 \mathrm{E}-06$ & $4.10 \mathrm{E}-07$ & & 4.10E-07 \\
\hline $\mathrm{Tb}$ & 65 & & $3.00 \mathrm{E}-05$ & & $3.00 \mathrm{E}-05$ \\
\hline Tc & 43 & $2.5 \mathrm{E}-02$ & $1.87 \mathrm{E}-03$ & & $1.87 \mathrm{E}-03$ \\
\hline $\mathrm{Te}$ & 52 & $1.0 \mathrm{E}-03$ & 4.50E-04 & $3.40 \mathrm{E}-04$ & $3.40 \mathrm{E}-04$ \\
\hline Th & 90 & $5.0 \mathrm{E}-06$ & $5.00 \mathrm{E}-06$ & & $5.00 \mathrm{E}-06$ \\
\hline $\mathrm{Ti}$ & 22 & $5.5 \mathrm{E}-07$ & $7.53 \mathrm{E}-05$ & & $7.53 \mathrm{E}-05$ \\
\hline $\mathrm{Tl}$ & 81 & & $2.00 \mathrm{E}-03$ & & $2.00 \mathrm{E}-03$ \\
\hline $\mathrm{Tm}$ & 69 & & $2.06 \mathrm{E}-05$ & & $2.06 \mathrm{E}-05$ \\
\hline $\mathrm{U}$ & 92 & $5.0 \mathrm{E}-04$ & $4.00 \mathrm{E}-04$ & $1.80 \mathrm{E}-03$ & $1.80 \mathrm{E}-03$ \\
\hline V & 23 & $2.0 \mathrm{E}-05$ & $2.06 \mathrm{E}-05$ & & $2.06 \mathrm{E}-05$ \\
\hline $\mathrm{W}$ & 74 & & $3.00 \mathrm{E}-04$ & $1.90 \mathrm{E}-04$ & $1.90 \mathrm{E}-04$ \\
\hline $\mathrm{Y}$ & 39 & $1.0 \mathrm{E}-05$ & $2.00 \mathrm{E}-05$ & & $2.00 \mathrm{E}-05$ \\
\hline $\mathrm{Yb}$ & 70 & & $2.06 \mathrm{E}-05$ & & $2.06 \mathrm{E}-05$ \\
\hline $\mathrm{Zn}$ & 30 & $3.9 \mathrm{E}-02$ & $1.00 \mathrm{E}-02$ & $2.70 \mathrm{E}-03$ & $2.70 \mathrm{E}-03$ \\
\hline $\mathrm{Zr}$ & 40 & $5.0 \mathrm{E}-06$ & $5.50 \mathrm{E}-07$ & $3.60 \mathrm{E}-06$ & $3.60 \mathrm{E}-06$ \\
\hline
\end{tabular}


SRNL-STI-2010-00447, REVISION 0

Table 4. Feed-to-Meat Transfer Factors

\begin{tabular}{|c|c|c|c|c|c|}
\hline \multirow{2}{*}{ Element } & \multirow{2}{*}{$\mathbf{Z}$} & \multirow{2}{*}{ SRS Current Value } & 2008 Value & 2010 Value & 2010 Value \\
\hline & & & Lee and Coffield & IAEA \#472 & Jannik et al. \\
\hline Ac & 89 & $2.0 \mathrm{E}-05$ & $4.00 \mathrm{E}-04$ & & $4.00 \mathrm{E}-04$ \\
\hline $\mathrm{Ag}$ & 47 & $1.7 \mathrm{E}-02$ & $3.00 \mathrm{E}-03$ & & $3.00 \mathrm{E}-03$ \\
\hline $\mathrm{Al}$ & 13 & $5.0 \mathrm{E}-04$ & $1.50 \mathrm{E}-03$ & & $1.50 \mathrm{E}-03$ \\
\hline $\mathrm{Am}$ & 95 & $2.0 \mathrm{E}-04$ & $4.00 \mathrm{E}-05$ & $5.00 \mathrm{E}-04$ & $5.00 \mathrm{E}-04$ \\
\hline As & 33 & & $2.00 \mathrm{E}-03$ & & $2.00 \mathrm{E}-03$ \\
\hline At & 85 & & $1.00 \mathrm{E}-02$ & & $1.00 \mathrm{E}-02$ \\
\hline $\mathrm{Au}$ & 79 & & $5.00 \mathrm{E}-03$ & & $5.00 \mathrm{E}-03$ \\
\hline B & 5 & & $8.00 \mathrm{E}-04$ & & $8.00 \mathrm{E}-04$ \\
\hline $\mathrm{Ba}$ & 56 & $3.2 \mathrm{E}-03$ & $2.00 \mathrm{E}-04$ & $1.40 \mathrm{E}-04$ & $1.40 \mathrm{E}-04$ \\
\hline $\mathrm{Be}$ & 4 & $1.0 \mathrm{E}-03$ & $1.00 \mathrm{E}-03$ & & $1.00 \mathrm{E}-03$ \\
\hline $\mathrm{Bi}$ & 83 & $2.0 \mathrm{E}-03$ & $4.00 \mathrm{E}-04$ & & $4.00 \mathrm{E}-04$ \\
\hline $\mathrm{Bk}$ & 97 & $2.0 \mathrm{E}-05$ & $2.50 \mathrm{E}-05$ & & $2.50 \mathrm{E}-05$ \\
\hline $\mathrm{Br}$ & 35 & & $2.50 \mathrm{E}-02$ & & $2.50 \mathrm{E}-02$ \\
\hline $\mathrm{C}$ & 6 & $3.1 \mathrm{E}-02$ & $3.10 \mathrm{E}-02$ & & $3.10 \mathrm{E}-02$ \\
\hline $\mathrm{Ca}$ & 20 & $1.6 \mathrm{E}-03$ & $2.00 \mathrm{E}-03$ & $1.30 \mathrm{E}-02$ & $1.30 \mathrm{E}-02$ \\
\hline $\mathrm{Cd}$ & 48 & $5.3 \mathrm{E}-04$ & $4.00 \mathrm{E}-04$ & $5.80 \mathrm{E}-03$ & $5.80 \mathrm{E}-03$ \\
\hline $\mathrm{Ce}$ & 58 & $1.2 \mathrm{E}-03$ & $2.00 \mathrm{E}-05$ & & $2.00 \mathrm{E}-05$ \\
\hline $\mathrm{Cf}$ & 98 & $6.0 \mathrm{E}-05$ & $4.00 \mathrm{E}-05$ & & $4.00 \mathrm{E}-05$ \\
\hline $\mathrm{Cl}$ & 17 & $6.0 \mathrm{E}-02$ & $2.00 \mathrm{E}-02$ & $1.70 \mathrm{E}-02$ & $1.70 \mathrm{E}-02$ \\
\hline $\mathrm{Cm}$ & 96 & $2.0 \mathrm{E}-04$ & $4.00 \mathrm{E}-05$ & & $4.00 \mathrm{E}-05$ \\
\hline $\mathrm{Co}$ & 27 & $1.3 \mathrm{E}-02$ & $1.00 \mathrm{E}-02$ & $4.30 \mathrm{E}-04$ & $4.30 \mathrm{E}-04$ \\
\hline $\mathrm{Cr}$ & 24 & $2.4 \mathrm{E}-03$ & $9.00 \mathrm{E}-03$ & & $9.00 \mathrm{E}-03$ \\
\hline Cs & 55 & $4.0 \mathrm{E}-03$ & $5.00 \mathrm{E}-02$ & $2.20 \mathrm{E}-02$ & $2.20 \mathrm{E}-02$ \\
\hline $\mathrm{Cu}$ & 29 & $8.0 \mathrm{E}-03$ & $9.00 \mathrm{E}-03$ & & $9.00 \mathrm{E}-03$ \\
\hline Dy & 66 & & $2.00 \mathrm{E}-05$ & & $2.00 \mathrm{E}-05$ \\
\hline Er & 68 & & $2.00 \mathrm{E}-05$ & & $2.00 \mathrm{E}-05$ \\
\hline Es & 99 & $2.0 \mathrm{E}-05$ & $2.50 \mathrm{E}-05$ & & $2.50 \mathrm{E}-05$ \\
\hline $\mathrm{Eu}$ & 63 & $4.8 \mathrm{E}-03$ & $2.00 \mathrm{E}-05$ & & $2.00 \mathrm{E}-05$ \\
\hline $\mathrm{F}$ & 9 & & $1.50 \mathrm{E}-01$ & & $1.50 \mathrm{E}-01$ \\
\hline $\mathrm{Fe}$ & 26 & $4.0 \mathrm{E}-02$ & $2.00 \mathrm{E}-02$ & $1.40 \mathrm{E}-02$ & $1.40 \mathrm{E}-02$ \\
\hline Fm & 100 & & $2.00 \mathrm{E}-04$ & & $2.00 \mathrm{E}-04$ \\
\hline $\mathrm{Fr}$ & 87 & & $2.50 \mathrm{E}-03$ & & $2.50 \mathrm{E}-03$ \\
\hline $\mathrm{Ga}$ & 31 & & $5.00 \mathrm{E}-04$ & & $5.00 \mathrm{E}-04$ \\
\hline $\mathrm{Gd}$ & 64 & $2.0 \mathrm{E}-03$ & $2.00 \mathrm{E}-05$ & & $2.00 \mathrm{E}-05$ \\
\hline $\mathrm{Ge}$ & 32 & $7.0 \mathrm{E}-01$ & $7.00 \mathrm{E}-01$ & & $7.00 \mathrm{E}-01$ \\
\hline $\mathrm{H}$ & 1 & not used & $0.00 \mathrm{E}+00$ & & $0.00 \mathrm{E}+00$ \\
\hline $\mathrm{Ha}$ & 105 & & $5.00 \mathrm{E}-06$ & & $5.00 \mathrm{E}-06$ \\
\hline $\mathrm{Hf}$ & 72 & $1.0 \mathrm{E}-06$ & $3.16 \mathrm{E}-05$ & & $3.16 \mathrm{E}-05$ \\
\hline $\mathrm{Hg}$ & 80 & $1.0 \mathrm{E}-01$ & $2.50 \mathrm{E}-01$ & & $2.50 \mathrm{E}-01$ \\
\hline Ho & 67 & $4.5 \mathrm{E}-03$ & $3.00 \mathrm{E}-04$ & & $3.00 \mathrm{E}-04$ \\
\hline $\mathrm{I}$ & 53 & $2.9 \mathrm{E}-03$ & $4.00 \mathrm{E}-02$ & $6.70 \mathrm{E}-03$ & $6.70 \mathrm{E}-03$ \\
\hline In & 49 & $4.0 \mathrm{E}-03$ & $8.00 \mathrm{E}-03$ & & $8.00 \mathrm{E}-03$ \\
\hline $\mathrm{Ir}$ & 77 & $2.0 \mathrm{E}-03$ & $1.50 \mathrm{E}-03$ & & $1.50 \mathrm{E}-03$ \\
\hline $\mathrm{K}$ & 19 & $2.0 \mathrm{E}-02$ & $2.00 \mathrm{E}-02$ & & $2.00 \mathrm{E}-02$ \\
\hline $\mathrm{La}$ & 57 & $2.0 \mathrm{E}-04$ & $2.00 \mathrm{E}-03$ & $1.30 \mathrm{E}-04$ & $1.30 \mathrm{E}-04$ \\
\hline $\mathrm{Li}$ & 3 & $4.5 \mathrm{E}-03$ & $1.00 \mathrm{E}-02$ & & $1.00 \mathrm{E}-02$ \\
\hline $\mathrm{Lr}$ & 103 & & $2.00 \mathrm{E}-04$ & & $2.00 \mathrm{E}-04$ \\
\hline $\mathrm{Lu}$ & 71 & & $4.50 \mathrm{E}-03$ & & $4.50 \mathrm{E}-03$ \\
\hline $\mathrm{Mg}$ & 12 & & $2.00 \mathrm{E}-02$ & & $2.00 \mathrm{E}-02$ \\
\hline $\mathrm{Mn}$ & 25 & $8.0 \mathrm{E}-04$ & $5.00 \mathrm{E}-04$ & $6.00 \mathrm{E}-04$ & $6.00 \mathrm{E}-04$ \\
\hline
\end{tabular}


SRNL-STI-2010-00447, REVISION 0

Table 4. Feed-to-Meat Transfer Factors (continued)

\begin{tabular}{|c|c|c|c|c|c|}
\hline \multirow{2}{*}{ Element } & \multirow{2}{*}{$\mathbf{Z}$} & \multirow{2}{*}{ SRS Current Value } & 2008 Value & 2010 Value & 2010 Value \\
\hline & & & Lee and Coffield & IAEA \#472 & Jannik et al. \\
\hline Mo & 42 & $8.0 \mathrm{E}-03$ & $1.00 \mathrm{E}-03$ & $1.00 \mathrm{E}-03$ & $1.00 \mathrm{E}-03$ \\
\hline $\mathrm{N}$ & 7 & & $7.50 \mathrm{E}-02$ & & $7.50 \mathrm{E}-02$ \\
\hline $\mathrm{Na}$ & 11 & $3.0 \mathrm{E}-02$ & $8.00 \mathrm{E}-02$ & $1.50 \mathrm{E}-02$ & $1.50 \mathrm{E}-02$ \\
\hline $\mathrm{Nb}$ & 41 & $2.8 \mathrm{E}-01$ & $2.90 \mathrm{E}-04$ & $2.60 \mathrm{E}-07$ & $2.60 \mathrm{E}-07$ \\
\hline $\mathrm{Nd}$ & 60 & & $2.00 \mathrm{E}-05$ & & $2.00 \mathrm{E}-05$ \\
\hline $\mathrm{Ni}$ & 28 & $5.3 \mathrm{E}-03$ & $5.00 \mathrm{E}-03$ & & $5.00 \mathrm{E}-03$ \\
\hline No & 102 & & $2.00 \mathrm{E}-04$ & & $2.00 \mathrm{E}-04$ \\
\hline $\mathrm{Np}$ & 93 & $2.0 \mathrm{E}-04$ & $1.00 \mathrm{E}-03$ & & $1.00 \mathrm{E}-03$ \\
\hline Os & 76 & & $4.00 \mathrm{E}-01$ & & $4.00 \mathrm{E}-01$ \\
\hline $\mathrm{P}$ & 15 & $4.6 \mathrm{E}-02$ & $5.00 \mathrm{E}-02$ & $5.50 \mathrm{E}-02$ & $5.50 \mathrm{E}-02$ \\
\hline $\mathrm{Pa}$ & 91 & $5.0 \mathrm{E}-03$ & $4.47 \mathrm{E}-04$ & & $4.47 \mathrm{E}-04$ \\
\hline $\mathrm{Pb}$ & 82 & $8.0 \mathrm{E}-04$ & $4.00 \mathrm{E}-04$ & $7.00 \mathrm{E}-04$ & $7.00 \mathrm{E}-04$ \\
\hline $\mathrm{Pd}$ & 46 & $1.0 \mathrm{E}-03$ & $4.00 \mathrm{E}-03$ & & $4.00 \mathrm{E}-03$ \\
\hline $\mathrm{Pm}$ & 61 & $4.8 \mathrm{E}-03$ & $2.00 \mathrm{E}-05$ & & $2.00 \mathrm{E}-05$ \\
\hline Po & 84 & $5.0 \mathrm{E}-03$ & $5.00 \mathrm{E}-03$ & & $5.00 \mathrm{E}-03$ \\
\hline $\operatorname{Pr}$ & 59 & & $2.00 \mathrm{E}-05$ & & $2.00 \mathrm{E}-05$ \\
\hline $\mathrm{Pt}$ & 78 & $4.0 \mathrm{E}-03$ & $4.00 \mathrm{E}-03$ & & $4.00 \mathrm{E}-03$ \\
\hline $\mathrm{Pu}$ & 94 & $1.4 \mathrm{E}-05$ & $1.00 \mathrm{E}-05$ & $1.10 \mathrm{E}-06$ & $1.10 \mathrm{E}-06$ \\
\hline $\mathrm{Ra}$ & 88 & $1.0 \mathrm{E}-03$ & $9.00 \mathrm{E}-04$ & $1.70 \mathrm{E}-03$ & $1.70 \mathrm{E}-03$ \\
\hline $\mathrm{Rb}$ & 37 & $3.1 \mathrm{E}-02$ & $1.00 \mathrm{E}-02$ & & $1.00 \mathrm{E}-02$ \\
\hline $\operatorname{Re}$ & 75 & $1.0 \mathrm{E}-04$ & $8.00 \mathrm{E}-03$ & & $8.00 \mathrm{E}-03$ \\
\hline $\mathrm{Rh}$ & 45 & & $2.00 \mathrm{E}-03$ & & $2.00 \mathrm{E}-03$ \\
\hline $\mathrm{Rn}$ & 86 & & $1.00 \mathrm{E}-20$ & & $1.00 \mathrm{E}-20$ \\
\hline $\mathrm{Ru}$ & 44 & $4.0 \mathrm{E}-01$ & $5.00 \mathrm{E}-02$ & $3.30 \mathrm{E}-03$ & $3.30 \mathrm{E}-03$ \\
\hline $\mathrm{S}$ & 16 & $1.0 \mathrm{E}-01$ & $2.00 \mathrm{E}-01$ & & $2.00 \mathrm{E}-01$ \\
\hline $\mathrm{Sb}$ & 51 & $4.0 \mathrm{E}-03$ & $1.00 \mathrm{E}-03$ & $1.20 \mathrm{E}-03$ & $1.20 \mathrm{E}-03$ \\
\hline $\mathrm{Sc}$ & 21 & & $1.50 \mathrm{E}-02$ & & $1.50 \mathrm{E}-02$ \\
\hline $\mathrm{Se}$ & 34 & $1.5 \mathrm{E}-02$ & $1.50 \mathrm{E}-02$ & & $1.50 \mathrm{E}-02$ \\
\hline $\mathrm{Si}$ & 14 & $4.0 \mathrm{E}-05$ & $4.00 \mathrm{E}-05$ & & $4.00 \mathrm{E}-05$ \\
\hline $\mathrm{Sm}$ & 62 & $5.0 \mathrm{E}-03$ & $3.16 \mathrm{E}-04$ & & $3.16 \mathrm{E}-04$ \\
\hline $\mathrm{Sn}$ & 50 & $8.0 \mathrm{E}-02$ & $8.00 \mathrm{E}-02$ & & $8.00 \mathrm{E}-02$ \\
\hline $\mathrm{Sr}$ & 38 & $6.0 \mathrm{E}-04$ & $8.00 \mathrm{E}-03$ & $1.30 \mathrm{E}-03$ & $1.30 \mathrm{E}-03$ \\
\hline $\mathrm{Ta}$ & 73 & $6.0 \mathrm{E}-04$ & $1.34 \mathrm{E}-05$ & & $1.34 \mathrm{E}-05$ \\
\hline $\mathrm{Tb}$ & 65 & & $2.00 \mathrm{E}-05$ & & $2.00 \mathrm{E}-05$ \\
\hline Tc & 43 & $4.0 \mathrm{E}-01$ & $6.32 \mathrm{E}-03$ & & $6.32 \mathrm{E}-03$ \\
\hline $\mathrm{Te}$ & 52 & 7.7E-02 & 7.00E-03 & 7.00E-03 & 7.00E-03 \\
\hline Th & 90 & $2.0 \mathrm{E}-04$ & $4.00 \mathrm{E}-05$ & $2.30 \mathrm{E}-04$ & $2.30 \mathrm{E}-04$ \\
\hline $\mathrm{Ti}$ & 22 & $1.0 \mathrm{E}-06$ & $1.73 \mathrm{E}-04$ & & $1.73 \mathrm{E}-04$ \\
\hline $\mathrm{Tl}$ & 81 & & $4.00 \mathrm{E}-02$ & & $4.00 \mathrm{E}-02$ \\
\hline $\mathrm{Tm}$ & 69 & & $4.50 \mathrm{E}-03$ & & $4.50 \mathrm{E}-03$ \\
\hline $\mathrm{U}$ & 92 & $3.4 \mathrm{E}-04$ & $3.00 \mathrm{E}-04$ & $3.90 \mathrm{E}-04$ & $3.90 \mathrm{E}-04$ \\
\hline V & 23 & $2.5 \mathrm{E}-03$ & $2.50 \mathrm{E}-03$ & & $2.50 \mathrm{E}-03$ \\
\hline $\mathrm{W}$ & 74 & & $4.00 \mathrm{E}-02$ & & $4.00 \mathrm{E}-02$ \\
\hline $\mathrm{Y}$ & 39 & $4.6 \mathrm{E}-03$ & $1.00 \mathrm{E}-03$ & & $1.00 \mathrm{E}-03$ \\
\hline $\mathrm{Yb}$ & 70 & & $4.00 \mathrm{E}-03$ & & $4.00 \mathrm{E}-03$ \\
\hline $\mathrm{Zn}$ & 30 & $3.0 \mathrm{E}-02$ & $1.00 \mathrm{E}-01$ & $1.60 \mathrm{E}-01$ & $1.60 \mathrm{E}-01$ \\
\hline $\mathrm{Zr}$ & 40 & $3.4 \mathrm{E}-02$ & $1.84 \mathrm{E}-04$ & $1.20 \mathrm{E}-06$ & $1.20 \mathrm{E}-06$ \\
\hline
\end{tabular}


SRNL-STI-2010-00447, REVISION 0

Table 5. Water-to-Fish Bioaccumulation Factors

\begin{tabular}{|c|c|c|c|c|c|}
\hline \multirow{2}{*}{ Element } & \multirow{2}{*}{$\mathbf{Z}$} & \multirow{2}{*}{ SRS Current Value } & 2008 Value & 2010 Value & 2010 Value \\
\hline & & & Lee and Coffield & IAEA \#472 & Jannik et al. \\
\hline Ac & 89 & $2.5 \mathrm{E}+01$ & $2.50 \mathrm{E}+01$ & & $2.50 \mathrm{E}+01$ \\
\hline $\mathrm{Ag}$ & 47 & $2.3 \mathrm{E}+00$ & $5.00 \mathrm{E}+00$ & $1.10 \mathrm{E}+02$ & $1.10 \mathrm{E}+02$ \\
\hline $\mathrm{Al}$ & 13 & $1.0 \mathrm{E}+01$ & $5.00 \mathrm{E}+02$ & $5.10 \mathrm{E}+01$ & $5.10 \mathrm{E}+01$ \\
\hline $\mathrm{Am}$ & 95 & $2.5 \mathrm{E}+01$ & $3.00 \mathrm{E}+01$ & $2.40 \mathrm{E}+02$ & $2.40 \mathrm{E}+02$ \\
\hline As & 33 & & $1.70 \mathrm{E}+03$ & $3.30 \mathrm{E}+02$ & $3.30 \mathrm{E}+02$ \\
\hline At & 85 & $1.5 \mathrm{E}+01$ & $1.50 \mathrm{E}+01$ & & $1.50 \mathrm{E}+01$ \\
\hline $\mathrm{Au}$ & 79 & $3.5 \mathrm{E}+01$ & $3.30 \mathrm{E}+01$ & $2.40 \mathrm{E}+02$ & $2.40 \mathrm{E}+02$ \\
\hline $\mathrm{Ba}$ & 56 & & $4.00 \mathrm{E}+00$ & $1.20 \mathrm{E}+00$ & $1.20 \mathrm{E}+00$ \\
\hline $\mathrm{Be}$ & 4 & $2.0 \mathrm{E}+00$ & $1.00 \mathrm{E}+02$ & & $1.00 \mathrm{E}+02$ \\
\hline $\mathrm{Bi}$ & 83 & $1.5 \mathrm{E}+01$ & $1.50 \mathrm{E}+01$ & & $1.50 \mathrm{E}+01$ \\
\hline $\mathrm{Bk}$ & 97 & $2.5 \mathrm{E}+01$ & $2.50 \mathrm{E}+01$ & & $2.50 \mathrm{E}+01$ \\
\hline $\mathrm{Br}$ & 35 & & $4.00 \mathrm{E}+02$ & $9.10 \mathrm{E}+01$ & $9.10 \mathrm{E}+01$ \\
\hline $\mathrm{C}$ & 6 & $4.6 \mathrm{E}+03$ & $5.00 \mathrm{E}+04$ & $4.00 \mathrm{E}+05$ & $3.00 \mathrm{E}+00$ \\
\hline $\mathrm{Ca}$ & 20 & $4.0 \mathrm{E}+01$ & $4.00 \mathrm{E}+01$ & $1.20 \mathrm{E}+01$ & $1.20 \mathrm{E}+01$ \\
\hline $\mathrm{Cd}$ & 48 & $2.0 \mathrm{E}+02$ & $2.00 \mathrm{E}+02$ & & $2.00 \mathrm{E}+02$ \\
\hline $\mathrm{Ce}$ & 58 & & $3.00 \mathrm{E}+01$ & $2.50 \mathrm{E}+01$ & $2.50 \mathrm{E}+01$ \\
\hline $\mathrm{Cf}$ & 98 & $2.5 \mathrm{E}+01$ & $2.50 \mathrm{E}+01$ & & $2.50 \mathrm{E}+01$ \\
\hline $\mathrm{Cl}$ & 17 & $5.0 \mathrm{E}+01$ & $5.00 \mathrm{E}+01$ & $4.70 \mathrm{E}+01$ & $4.70 \mathrm{E}+01$ \\
\hline $\mathrm{Cm}$ & 96 & $2.5 \mathrm{E}+01$ & $3.00 \mathrm{E}+01$ & & $3.00 \mathrm{E}+01$ \\
\hline $\mathrm{Co}$ & 27 & $5.0 \mathrm{E}+01$ & $3.00 \mathrm{E}+02$ & $7.60 \mathrm{E}+01$ & $7.60 \mathrm{E}+01$ \\
\hline $\mathrm{Cr}$ & 24 & & $4.00 \mathrm{E}+00$ & $4.00 \mathrm{E}+01$ & $4.00 \mathrm{E}+01$ \\
\hline Cs & 55 & $3.0 \mathrm{E}+03$ & $3.00 \mathrm{E}+03$ & $2.50 \mathrm{E}+03$ & $3.00 \mathrm{E}+03$ \\
\hline $\mathrm{Cu}$ & 29 & & $2.00 \mathrm{E}+02$ & $2.30 \mathrm{E}+02$ & $2.30 \mathrm{E}+02$ \\
\hline Dy & 66 & & $3.00 \mathrm{E}+01$ & $6.50 \mathrm{E}+02$ & $6.50 \mathrm{E}+02$ \\
\hline Er & 68 & & $3.00 \mathrm{E}+01$ & & $3.00 \mathrm{E}+01$ \\
\hline Es & 99 & $1.0 \mathrm{E}+01$ & $2.50 \mathrm{E}+01$ & & $2.50 \mathrm{E}+01$ \\
\hline $\mathrm{Eu}$ & 63 & & $3.00 \mathrm{E}+01$ & $1.30 \mathrm{E}+02$ & $1.30 \mathrm{E}+02$ \\
\hline $\mathrm{F}$ & 9 & & $1.00 \mathrm{E}+01$ & & $1.00 \mathrm{E}+01$ \\
\hline $\mathrm{Fe}$ & 26 & $1.0 \mathrm{E}+02$ & $2.00 \mathrm{E}+02$ & $1.70 \mathrm{E}+02$ & $1.70 \mathrm{E}+02$ \\
\hline $\mathrm{Fr}$ & 87 & $3.0 \mathrm{E}+01$ & $3.00 \mathrm{E}+01$ & & $3.00 \mathrm{E}+01$ \\
\hline $\mathrm{Ga}$ & 31 & $3.3 \mathrm{E}+02$ & $4.00 \mathrm{E}+02$ & & $4.00 \mathrm{E}+02$ \\
\hline $\mathrm{Gd}$ & 64 & $2.5 \mathrm{E}+01$ & $3.00 \mathrm{E}+01$ & & $3.00 \mathrm{E}+01$ \\
\hline $\mathrm{Ge}$ & 32 & $3.3 \mathrm{E}+03$ & $4.00 \mathrm{E}+03$ & & $4.00 \mathrm{E}+03$ \\
\hline $\mathrm{He}$ & 2 & & $1.00 \mathrm{E}+00$ & & $1.00 \mathrm{E}+00$ \\
\hline $\mathrm{H}$ & 1 & $9.0 \mathrm{E}-01$ & $1.00 \mathrm{E}+00$ & & $1.00 \mathrm{E}+00$ \\
\hline $\mathrm{Hf}$ & 72 & $3.3 \mathrm{E}+00$ & $3.00 \mathrm{E}+02$ & $1.10 \mathrm{E}+03$ & $1.10 \mathrm{E}+03$ \\
\hline $\mathrm{Hg}$ & 80 & $1.0 \mathrm{E}+03$ & $1.00 \mathrm{E}+03$ & $6.10 \mathrm{E}+03$ & $6.10 \mathrm{E}+03$ \\
\hline Ho & 67 & $2.5 \mathrm{E}+01$ & $3.00 \mathrm{E}+01$ & & $3.00 \mathrm{E}+01$ \\
\hline $\mathrm{I}$ & 53 & $1.5 \mathrm{E}+01$ & $4.00 \mathrm{E}+01$ & $3.00 \mathrm{E}+01$ & $3.00 \mathrm{E}+01$ \\
\hline In & 49 & $1.0 \mathrm{E}+04$ & $1.00 \mathrm{E}+04$ & & $1.00 \mathrm{E}+04$ \\
\hline $\mathrm{Ir}$ & 77 & $1.0 \mathrm{E}+01$ & $1.00 \mathrm{E}+01$ & & $1.00 \mathrm{E}+01$ \\
\hline $\mathrm{K}$ & 19 & $1.0 \mathrm{E}+03$ & $1.00 \mathrm{E}+03$ & $3.20 \mathrm{E}+03$ & $3.20 \mathrm{E}+03$ \\
\hline $\mathrm{La}$ & 57 & $2.5 \mathrm{E}+01$ & $3.00 \mathrm{E}+01$ & $3.70 \mathrm{E}+01$ & $3.70 \mathrm{E}+01$ \\
\hline $\mathrm{Lu}$ & 71 & $2.5 \mathrm{E}+01$ & $2.50 \mathrm{E}+01$ & & $2.50 \mathrm{E}+01$ \\
\hline $\mathrm{Mg}$ & 12 & & $5.00 \mathrm{E}+01$ & $3.70 \mathrm{E}+01$ & $3.70 \mathrm{E}+01$ \\
\hline $\mathrm{Mn}$ & 25 & $1.0 \mathrm{E}+02$ & $4.00 \mathrm{E}+02$ & $2.40 \mathrm{E}+02$ & $2.40 \mathrm{E}+02$ \\
\hline Mo & 42 & $1.0 \mathrm{E}+01$ & $1.00 \mathrm{E}+01$ & $1.90 \mathrm{E}+00$ & $1.90 \mathrm{E}+00$ \\
\hline $\mathrm{N}$ & 7 & & $2.00 \mathrm{E}+05$ & & $2.00 \mathrm{E}+05$ \\
\hline $\mathrm{Na}$ & 11 & & $2.00 \mathrm{E}+01$ & $7.60 \mathrm{E}+01$ & $7.60 \mathrm{E}+01$ \\
\hline
\end{tabular}


SRNL-STI-2010-00447, REVISION 0

Table 5. Water-to-Fish Bioaccumulation Factors (continued)

\begin{tabular}{|c|c|c|c|c|c|}
\hline \multirow{2}{*}{ Element } & \multirow{2}{*}{$\mathbf{Z}$} & \multirow{2}{*}{ SRS Current Value } & 2008 Value & 2010 Value & 2010 Value \\
\hline & & & Lee and Coffield & IAEA \#472 & Jannik et al. \\
\hline $\mathrm{Nb}$ & 41 & $3.0 \mathrm{E}+04$ & $3.00 \mathrm{E}+02$ & & $3.00 \mathrm{E}+02$ \\
\hline $\mathrm{Nd}$ & 60 & & $3.00 \mathrm{E}+01$ & & $3.00 \mathrm{E}+01$ \\
\hline $\mathrm{Ni}$ & 28 & $1.0 \mathrm{E}+02$ & $1.00 \mathrm{E}+02$ & $2.10 \mathrm{E}+01$ & $2.10 \mathrm{E}+01$ \\
\hline $\mathrm{Np}$ & 93 & $1.0 \mathrm{E}+02$ & $2.10 \mathrm{E}+01$ & & $2.10 \mathrm{E}+01$ \\
\hline $\mathrm{O}$ & 8 & & $1.00 \mathrm{E}+00$ & & $1.00 \mathrm{E}+00$ \\
\hline Os & 76 & $1.0 \mathrm{E}+05$ & $1.00 \mathrm{E}+03$ & & $1.00 \mathrm{E}+03$ \\
\hline $\mathrm{P}$ & 15 & & $5.00 \mathrm{E}+04$ & $1.40 \mathrm{E}+05$ & $1.40 \mathrm{E}+05$ \\
\hline $\mathrm{Pa}$ & 91 & $1.1 \mathrm{E}+01$ & $1.00 \mathrm{E}+01$ & & $1.00 \mathrm{E}+01$ \\
\hline $\mathrm{Pb}$ & 82 & $3.0 \mathrm{E}+02$ & $3.00 \mathrm{E}+02$ & $2.50 \mathrm{E}+01$ & $2.50 \mathrm{E}+01$ \\
\hline $\mathrm{Pd}$ & 46 & $1.0 \mathrm{E}+01$ & $1.00 \mathrm{E}+01$ & & $1.00 \mathrm{E}+01$ \\
\hline $\mathrm{Pm}$ & 61 & & $3.00 \mathrm{E}+01$ & & $3.00 \mathrm{E}+01$ \\
\hline Po & 84 & $5.0 \mathrm{E}+02$ & $5.00 \mathrm{E}+01$ & $3.60 \mathrm{E}+01$ & $3.60 \mathrm{E}+01$ \\
\hline $\operatorname{Pr}$ & 59 & & $3.00 \mathrm{E}+01$ & & $3.00 \mathrm{E}+01$ \\
\hline $\mathrm{Pt}$ & 78 & $1.0 \mathrm{E}+02$ & $3.50 \mathrm{E}+01$ & & $3.50 \mathrm{E}+01$ \\
\hline $\mathrm{Pu}$ & 94 & $3.5 \mathrm{E}+00$ & $3.00 \mathrm{E}+01$ & $2.1 \mathrm{E}+04 *$ & $3.00 \mathrm{E}+01$ \\
\hline $\mathrm{Ra}$ & 88 & $5.0 \mathrm{E}+01$ & $5.00 \mathrm{E}+01$ & $4.00 \mathrm{E}+00$ & $4.00 \mathrm{E}+00$ \\
\hline $\mathrm{Rb}$ & 37 & $2.0 \mathrm{E}+03$ & $2.00 \mathrm{E}+03$ & $4.90 \mathrm{E}+03$ & $4.90 \mathrm{E}+03$ \\
\hline $\operatorname{Re}$ & 75 & $1.2 \mathrm{E}+02$ & $1.20 \mathrm{E}+02$ & & $1.20 \mathrm{E}+02$ \\
\hline $\mathrm{Rh}$ & 45 & & $1.00 \mathrm{E}+01$ & & $1.00 \mathrm{E}+01$ \\
\hline $\mathrm{Rn}$ & 86 & $5.7 \mathrm{E}+01$ & $7.55 \mathrm{E}-10$ & & $7.55 \mathrm{E}-10$ \\
\hline $\mathrm{Ru}$ & 44 & $1.0 \mathrm{E}+01$ & $1.00 \mathrm{E}+02$ & $5.50 \mathrm{E}+01$ & $5.50 \mathrm{E}+01$ \\
\hline $\mathrm{S}$ & 16 & & $8.00 \mathrm{E}+02$ & & $8.00 \mathrm{E}+02$ \\
\hline $\mathrm{Sb}$ & 51 & $1.0 \mathrm{E}+00$ & $1.00 \mathrm{E}+02$ & $3.70 \mathrm{E}+01$ & $3.70 \mathrm{E}+01$ \\
\hline $\mathrm{Sc}$ & 21 & $1.0 \mathrm{E}+02$ & $1.00 \mathrm{E}+02$ & $1.90 \mathrm{E}+02$ & $1.90 \mathrm{E}+02$ \\
\hline $\mathrm{Se}$ & 34 & $1.7 \mathrm{E}+02$ & $1.70 \mathrm{E}+02$ & $6.00 \mathrm{E}+03$ & $6.00 \mathrm{E}+03$ \\
\hline $\mathrm{Si}$ & 14 & $2.5 \mathrm{E}+00$ & $2.00 \mathrm{E}+01$ & & $2.00 \mathrm{E}+01$ \\
\hline $\mathrm{Sm}$ & 62 & $2.5 \mathrm{E}+01$ & $3.00 \mathrm{E}+01$ & & $3.00 \mathrm{E}+01$ \\
\hline $\mathrm{Sn}$ & 50 & $3.0 \mathrm{E}+03$ & $3.00 \mathrm{E}+03$ & & $3.00 \mathrm{E}+03$ \\
\hline $\mathrm{Sr}$ & 38 & $3.0 \mathrm{E}+01$ & $6.00 \mathrm{E}+01$ & $2.90 \mathrm{E}+00$ & $2.90 \mathrm{E}+00$ \\
\hline $\mathrm{Ta}$ & 73 & $3.0 \mathrm{E}+04$ & $3.00 \mathrm{E}+02$ & & $3.00 \mathrm{E}+02$ \\
\hline $\mathrm{Tb}$ & 65 & & $3.00 \mathrm{E}+01$ & $4.10 \mathrm{E}+02$ & $4.10 \mathrm{E}+02$ \\
\hline Tc & 43 & $1.5 \mathrm{E}+01$ & $2.00 \mathrm{E}+01$ & & $2.00 \mathrm{E}+01$ \\
\hline $\mathrm{Te}$ & 52 & $4.0 \mathrm{E}+02$ & $4.00 \mathrm{E}+02$ & $1.50 \mathrm{E}+02$ & $1.50 \mathrm{E}+02$ \\
\hline Th & 90 & $3.0 \mathrm{E}+01$ & $1.00 \mathrm{E}+02$ & $6.00 \mathrm{E}+00$ & $6.00 \mathrm{E}+00$ \\
\hline $\mathrm{Ti}$ & 22 & $1.0 \mathrm{E}+03$ & $1.00 \mathrm{E}+03$ & $1.90 \mathrm{E}+02$ & $1.90 \mathrm{E}+02$ \\
\hline $\mathrm{Tl}$ & 81 & $1.0 \mathrm{E}+04$ & $1.00 \mathrm{E}+04$ & $9.00 \mathrm{E}+02$ & $9.00 \mathrm{E}+02$ \\
\hline $\mathrm{U}$ & 92 & $2.0 \mathrm{E}+00$ & $1.00 \mathrm{E}+01$ & $9.60 \mathrm{E}-01$ & $9.60 \mathrm{E}-01$ \\
\hline $\mathrm{V}$ & 23 & $1.0 \mathrm{E}+01$ & $2.00 \mathrm{E}+02$ & $9.70 \mathrm{E}+01$ & $9.70 \mathrm{E}+01$ \\
\hline W & 74 & & $1.00 \mathrm{E}+01$ & & $1.00 \mathrm{E}+01$ \\
\hline $\mathrm{Y}$ & 39 & $2.5 \mathrm{E}+01$ & $3.00 \mathrm{E}+01$ & $4.00 \mathrm{E}+01$ & $4.00 \mathrm{E}+01$ \\
\hline $\mathrm{Zn}$ & 30 & & $3.50 \mathrm{E}+02$ & $3.40 \mathrm{E}+03$ & $3.40 \mathrm{E}+03$ \\
\hline $\mathrm{Zr}$ & 40 & $3.3 \mathrm{E}+00$ & $3.00 \mathrm{E}+02$ & $2.20 \mathrm{E}+01$ & $2.20 \mathrm{E}+01$ \\
\hline
\end{tabular}

*the plutonium value of $21,000 \mathrm{~L} / \mathrm{kg}$ is considered suspect at this time. 
SRNL-STI-2010-00447, REVISION 0

Table 6. Water to Saltwater Invertebrates Bioaccumulation Factors

\begin{tabular}{|c|c|c|c|c|c|}
\hline \multirow{2}{*}{ Element } & \multirow{2}{*}{$\mathbf{Z}$} & \multirow{2}{*}{ SRS Current Value } & 2008 Value & 2003 Value & 2010 Value \\
\hline & & & Lee and Coffield & PNNL 13421 & Jannik et al. \\
\hline Ac & 89 & $1.00 \mathrm{E}+03$ & N/A & $1.00 \mathrm{E}+03$ & $1.00 \mathrm{E}+03$ \\
\hline $\mathrm{Ag}$ & 47 & $3.33 \mathrm{E}+03$ & $\mathrm{~N} / \mathrm{A}$ & $3.50 \mathrm{E}+02$ & $3.50 \mathrm{E}+02$ \\
\hline $\mathrm{Al}$ & 13 & $6.00 \mathrm{E}+01$ & N/A & & $6.00 \mathrm{E}+01$ \\
\hline $\mathrm{Am}$ & 95 & $1.00 \mathrm{E}+03$ & N/A & $3.60 \mathrm{E}+02$ & $3.60 \mathrm{E}+02$ \\
\hline As & 33 & & N/A & $3.00 \mathrm{E}+02$ & $3.00 \mathrm{E}+02$ \\
\hline $\mathrm{Ba}$ & 56 & $1.00 \mathrm{E}+02$ & N/A & $1.00 \mathrm{E}+00$ & $1.00 \mathrm{E}+00$ \\
\hline $\mathrm{Be}$ & 4 & $2.00 \mathrm{E}+02$ & N/A & $1.00 \mathrm{E}+04$ & $1.00 \mathrm{E}+04$ \\
\hline $\mathrm{Bi}$ & 83 & $1.00 \mathrm{E}+01$ & N/A & $1.00 \mathrm{E}+03$ & $1.00 \mathrm{E}+03$ \\
\hline $\mathrm{Bk}$ & 97 & $1.00 \mathrm{E}+03$ & N/A & & $1.00 \mathrm{E}+03$ \\
\hline $\mathrm{Br}$ & 35 & & N/A & $1.00 \mathrm{E}+01$ & $1.00 \mathrm{E}+01$ \\
\hline $\mathrm{C}$ & 6 & $1.40 \mathrm{E}+03$ & N/A & $2.00 \mathrm{E}+04$ & $2.00 \mathrm{E}+04$ \\
\hline $\mathrm{Ca}$ & 20 & $1.25 \mathrm{E}+01$ & N/A & $5.00 \mathrm{E}+00$ & $5.00 \mathrm{E}+00$ \\
\hline $\mathrm{Cd}$ & 48 & $2.50 \mathrm{E}+05$ & N/A & $5.00 \mathrm{E}+03$ & $5.00 \mathrm{E}+03$ \\
\hline $\mathrm{Ce}$ & 58 & $6.00 \mathrm{E}+02$ & N/A & $5.00 \mathrm{E}+02$ & $5.00 \mathrm{E}+02$ \\
\hline $\mathrm{Cf}$ & 98 & $1.00 \mathrm{E}+03$ & N/A & $5.00 \mathrm{E}+02$ & $5.00 \mathrm{E}+02$ \\
\hline $\mathrm{Cl}$ & 17 & $1.90 \mathrm{E}-02$ & N/A & $1.00 \mathrm{E}+00$ & $1.00 \mathrm{E}+00$ \\
\hline $\mathrm{Cm}$ & 96 & $1.00 \mathrm{E}+03$ & N/A & $4.60 \mathrm{E}+02$ & $4.60 \mathrm{E}+02$ \\
\hline $\mathrm{Co}$ & 27 & $1.00 \mathrm{E}+03$ & N/A & $2.00 \mathrm{E}+03$ & $2.00 \mathrm{E}+03$ \\
\hline $\mathrm{Cr}$ & 24 & $2.00 \mathrm{E}+03$ & N/A & $5.00 \mathrm{E}+02$ & $5.00 \mathrm{E}+02$ \\
\hline Cs & 55 & $2.50 \mathrm{E}+01$ & N/A & $3.00 \mathrm{E}+01$ & $3.00 \mathrm{E}+01$ \\
\hline $\mathrm{Cu}$ & 29 & $1.70 \mathrm{E}+03$ & N/A & $5.00 \mathrm{E}+03$ & $5.00 \mathrm{E}+03$ \\
\hline Dy & 66 & & N/A & $1.00 \mathrm{E}+03$ & $1.00 \mathrm{E}+03$ \\
\hline Er & 68 & & N/A & $5.00 \mathrm{E}+02$ & $5.00 \mathrm{E}+02$ \\
\hline Es & 99 & $1.00 \mathrm{E}+01$ & N/A & $7.00 \mathrm{E}+03$ & $7.00 \mathrm{E}+03$ \\
\hline $\mathrm{Eu}$ & 63 & $1.00 \mathrm{E}+03$ & N/A & $1.00 \mathrm{E}+03$ & $1.00 \mathrm{E}+03$ \\
\hline $\mathrm{F}$ & 9 & & N/A & $4.00 \mathrm{E}+00$ & $4.00 \mathrm{E}+00$ \\
\hline $\mathrm{Fe}$ & 26 & $2.00 \mathrm{E}+04$ & N/A & $5.00 \mathrm{E}+03$ & $5.00 \mathrm{E}+03$ \\
\hline $\mathrm{Fr}$ & 87 & & N/A & & $3.00 \mathrm{E}+01$ \\
\hline $\mathrm{Ga}$ & 31 & & N/A & $1.00 \mathrm{E}+04$ & $1.00 \mathrm{E}+04$ \\
\hline $\mathrm{Gd}$ & 64 & $1.00 \mathrm{E}+03$ & N/A & $2.00 \mathrm{E}+03$ & $2.00 \mathrm{E}+03$ \\
\hline $\mathrm{Ge}$ & 32 & $1.57 \mathrm{E}+04$ & N/A & & $4.00 \mathrm{E}+03$ \\
\hline $\mathrm{He}$ & 2 & & N/A & $1.00 \mathrm{E}+00$ & $1.00 \mathrm{E}+00$ \\
\hline $\mathrm{H}$ & 1 & $9.30 \mathrm{E}-01$ & N/A & & $9.30 \mathrm{E}-01$ \\
\hline $\mathrm{Hf}$ & 72 & $2.00 \mathrm{E}+01$ & N/A & $1.00 \mathrm{E}+03$ & $1.00 \mathrm{E}+03$ \\
\hline $\mathrm{Hg}$ & 80 & $3.33 \mathrm{E}+04$ & N/A & $2.00 \mathrm{E}+04$ & $2.00 \mathrm{E}+04$ \\
\hline Ho & 67 & $1.00 \mathrm{E}+03$ & N/A & $1.00 \mathrm{E}+03$ & $1.00 \mathrm{E}+03$ \\
\hline I & 53 & $5.00 \mathrm{E}+01$ & N/A & $5.00 \mathrm{E}+01$ & $5.00 \mathrm{E}+01$ \\
\hline In & 49 & & N/A & $1.00 \mathrm{E}+04$ & $1.00 \mathrm{E}+04$ \\
\hline Ir & 77 & $2.00 \mathrm{E}+03$ & N/A & $1.00 \mathrm{E}+02$ & $1.00 \mathrm{E}+02$ \\
\hline $\mathrm{K}$ & 19 & $6.58 \mathrm{E}+00$ & N/A & & $6.58 \mathrm{E}+00$ \\
\hline $\mathrm{La}$ & 57 & $1.00 \mathrm{E}+03$ & N/A & $1.00 \mathrm{E}+02$ & $1.00 \mathrm{E}+02$ \\
\hline $\mathrm{Lu}$ & 71 & $1.00 \mathrm{E}+03$ & $\mathrm{~N} / \mathrm{A}$ & & $1.00 \mathrm{E}+03$ \\
\hline $\mathrm{Mn}$ & 25 & $1.00 \mathrm{E}+04$ & N/A & $8.00 \mathrm{E}+02$ & $8.00 \mathrm{E}+02$ \\
\hline Mo & 42 & $1.00 \mathrm{E}+01$ & N/A & $2.00 \mathrm{E}+01$ & $2.00 \mathrm{E}+01$ \\
\hline $\mathrm{N}$ & 7 & & N/A & $1.00 \mathrm{E}+00$ & $1.00 \mathrm{E}+00$ \\
\hline $\mathrm{Na}$ & 11 & $1.90 \mathrm{E}-01$ & N/A & $1.00 \mathrm{E}+00$ & $1.00 \mathrm{E}+00$ \\
\hline $\mathrm{Nb}$ & 41 & $1.00 \mathrm{E}+02$ & N/A & $5.00 \mathrm{E}+01$ & $5.00 \mathrm{E}+01$ \\
\hline $\mathrm{Ni}$ & 28 & $2.50 \mathrm{E}+02$ & N/A & $5.00 \mathrm{E}+02$ & $5.00 \mathrm{E}+02$ \\
\hline $\mathrm{Np}$ & 93 & $1.00 \mathrm{E}+01$ & N/A & $1.00 \mathrm{E}+01$ & $1.00 \mathrm{E}+01$ \\
\hline
\end{tabular}


SRNL-STI-2010-00447, REVISION 0

Table 6. Water to Saltwater Invertebrates Bioaccumulation Factors (continued)

\begin{tabular}{|c|c|c|c|c|c|}
\hline \multirow{2}{*}{ Element } & \multirow{2}{*}{$\mathbf{Z}$} & \multirow{2}{*}{ SRS Current Value } & 2008 Value & 2003 Value & 2010 Value \\
\hline & & & Lee and Coffield & PNNL 13421 & Jannik et al. \\
\hline $\mathrm{P}$ & 15 & $3.00 \mathrm{E}+04$ & $\mathrm{~N} / \mathrm{A}$ & $3.80 \mathrm{E}+04$ & $3.80 \mathrm{E}+04$ \\
\hline $\mathrm{Pa}$ & 91 & $1.00 \mathrm{E}+01$ & N/A & $1.00 \mathrm{E}+01$ & $1.00 \mathrm{E}+01$ \\
\hline $\mathrm{Pb}$ & 82 & $1.00 \mathrm{E}+03$ & N/A & $1.00 \mathrm{E}+03$ & $1.00 \mathrm{E}+03$ \\
\hline $\mathrm{Pd}$ & 46 & $2.00 \mathrm{E}+03$ & N/A & $3.00 \mathrm{E}+02$ & $3.00 \mathrm{E}+02$ \\
\hline $\mathrm{Pm}$ & 61 & $1.00 \mathrm{E}+03$ & N/A & $1.00 \mathrm{E}+03$ & $1.00 \mathrm{E}+03$ \\
\hline Po & 84 & $2.00 \mathrm{E}+04$ & N/A & $5.00 \mathrm{E}+04$ & $5.00 \mathrm{E}+04$ \\
\hline $\mathrm{Pr}$ & 59 & & N/A & $1.00 \mathrm{E}+03$ & $1.00 \mathrm{E}+03$ \\
\hline $\mathrm{Pt}$ & 78 & $2.00 \mathrm{E}+03$ & N/A & & $2.00 \mathrm{E}+03$ \\
\hline $\mathrm{Pu}$ & 94 & $1.00 \mathrm{E}+02$ & N/A & $3.00 \mathrm{E}+02$ & $3.00 \mathrm{E}+02$ \\
\hline $\mathrm{Ra}$ & 88 & $1.00 \mathrm{E}+02$ & N/A & $1.00 \mathrm{E}+02$ & $1.00 \mathrm{E}+02$ \\
\hline $\mathrm{Rb}$ & 37 & $1.70 \mathrm{E}+01$ & N/A & $2.00 \mathrm{E}+02$ & $2.00 \mathrm{E}+02$ \\
\hline $\mathrm{Re}$ & 75 & $5.95 \mathrm{E}+01$ & N/A & & $5.95 \mathrm{E}+01$ \\
\hline $\mathrm{Rh}$ & 45 & & N/A & $1.00 \mathrm{E}+02$ & $1.00 \mathrm{E}+02$ \\
\hline $\mathrm{Rn}$ & 86 & & N/A & & \\
\hline $\mathrm{Ru}$ & 44 & $1.00 \mathrm{E}+03$ & N/A & $1.00 \mathrm{E}+02$ & $1.00 \mathrm{E}+02$ \\
\hline $\mathrm{S}$ & 16 & $4.40 \mathrm{E}-01$ & N/A & $1.00 \mathrm{E}+00$ & $1.00 \mathrm{E}+00$ \\
\hline $\mathrm{Sb}$ & 51 & $5.00 \mathrm{E}+00$ & N/A & $1.00 \mathrm{E}+02$ & $1.00 \mathrm{E}+02$ \\
\hline $\mathrm{Sc}$ & 21 & & N/A & $3.00 \mathrm{E}+02$ & $3.00 \mathrm{E}+02$ \\
\hline $\mathrm{Se}$ & 34 & $1.00 \mathrm{E}+03$ & N/A & $5.00 \mathrm{E}+03$ & $5.00 \mathrm{E}+03$ \\
\hline $\mathrm{Si}$ & 14 & $3.33 \mathrm{E}+01$ & N/A & $5.00 \mathrm{E}+04$ & $5.00 \mathrm{E}+04$ \\
\hline $\mathrm{Sm}$ & 62 & $1.00 \mathrm{E}+03$ & N/A & $1.00 \mathrm{E}+03$ & $1.00 \mathrm{E}+03$ \\
\hline $\mathrm{Sn}$ & 50 & $1.00 \mathrm{E}+03$ & N/A & $5.00 \mathrm{E}+04$ & $5.00 \mathrm{E}+04$ \\
\hline $\mathrm{Sr}$ & 38 & $2.00 \mathrm{E}+01$ & $\mathrm{~N} / \mathrm{A}$ & $1.00 \mathrm{E}+00$ & $1.00 \mathrm{E}+00$ \\
\hline $\mathrm{Ta}$ & 73 & $1.67 \mathrm{E}+04$ & N/A & $3.00 \mathrm{E}+03$ & $3.00 \mathrm{E}+03$ \\
\hline $\mathrm{Tb}$ & 65 & & N/A & $1.00 \mathrm{E}+03$ & $1.00 \mathrm{E}+03$ \\
\hline Tc & 43 & $5.00 \mathrm{E}+01$ & N/A & $1.00 \mathrm{E}+01$ & $1.00 \mathrm{E}+01$ \\
\hline $\mathrm{Te}$ & 52 & $1.00 \mathrm{E}+05$ & N/A & $1.00 \mathrm{E}+03$ & $1.00 \mathrm{E}+03$ \\
\hline Th & 90 & $2.00 \mathrm{E}+03$ & N/A & $1.00 \mathrm{E}+03$ & $1.00 \mathrm{E}+03$ \\
\hline $\mathrm{Ti}$ & 22 & $1.00 \mathrm{E}+03$ & N/A & & $1.00 \mathrm{E}+03$ \\
\hline $\mathrm{Tl}$ & 81 & & N/A & $1.00 \mathrm{E}+03$ & $1.00 \mathrm{E}+03$ \\
\hline $\mathrm{U}$ & 92 & $1.00 \mathrm{E}+01$ & N/A & $1.00 \mathrm{E}+01$ & $1.00 \mathrm{E}+01$ \\
\hline $\mathrm{V}$ & 23 & $5.00 \mathrm{E}+01$ & N/A & & $5.00 \mathrm{E}+01$ \\
\hline W & 74 & & N/A & $1.00 \mathrm{E}+01$ & $1.00 \mathrm{E}+01$ \\
\hline $\mathrm{Y}$ & 39 & $1.00 \mathrm{E}+03$ & N/A & $1.00 \mathrm{E}+03$ & $1.00 \mathrm{E}+03$ \\
\hline $\mathrm{Zn}$ & 30 & $5.00 \mathrm{E}+04$ & N/A & $5.00 \mathrm{E}+04$ & $5.00 \mathrm{E}+04$ \\
\hline $\mathrm{Zr}$ & 40 & $8.00 \mathrm{E}+01$ & N/A & $5.00 \mathrm{E}+01$ & $5.00 \mathrm{E}+01$ \\
\hline
\end{tabular}


Table 7. Vegetable Production as a Function of Distance and Sector 2007 Weighted Vegetable Production Grid surrounding SRS increased 10 times for unreported (home use) production (L/yr)

\begin{tabular}{|l|c|c|c|c|c|}
\hline \multicolumn{1}{|c|}{ Sector } & $\mathbf{1 0 - 2 0} \mathbf{M i}$ & $\mathbf{2 0 - 3 0 ~ M i}$ & $\mathbf{3 0 - 4 0 ~ M i}$ & $\mathbf{4 0 - 5 0 ~ M i}$ & Total \\
\hline N & $1.5 \mathrm{E}+04$ & $2.5 \mathrm{E}+04$ & $4.1 \mathrm{E}+04$ & $2.2 \mathrm{E}+05$ & $3.1 \mathrm{E}+05$ \\
\hline NNE & $1.4 \mathrm{E}+04$ & $2.5 \mathrm{E}+04$ & $2.5 \mathrm{E}+05$ & $7.9 \mathrm{E}+05$ & $1.1 \mathrm{E}+06$ \\
\hline NE & $4.2 \mathrm{E}+04$ & $4.0 \mathrm{E}+04$ & $1.3 \mathrm{E}+05$ & $4.7 \mathrm{E}+05$ & $6.8 \mathrm{E}+05$ \\
\hline ENE & $6.1 \mathrm{E}+04$ & $1.5 \mathrm{E}+05$ & $6.4 \mathrm{E}+04$ & $4.5 \mathrm{E}+04$ & $3.2 \mathrm{E}+05$ \\
\hline E & $6.8 \mathrm{E}+04$ & $2.6 \mathrm{E}+05$ & $4.4 \mathrm{E}+05$ & $2.5 \mathrm{E}+05$ & $1.0 \mathrm{E}+06$ \\
\hline ESE & $6.2 \mathrm{E}+04$ & $1.6 \mathrm{E}+05$ & $3.1 \mathrm{E}+05$ & $2.3 \mathrm{E}+05$ & $7.5 \mathrm{E}+05$ \\
\hline SE & $5.2 \mathrm{E}+04$ & $8.7 \mathrm{E}+04$ & $4.5 \mathrm{E}+04$ & $1.8 \mathrm{E}+04$ & $2.0 \mathrm{E}+05$ \\
\hline SSE & $4.1 \mathrm{E}+04$ & $6.4 \mathrm{E}+04$ & $6.7 \mathrm{E}+04$ & $2.5 \mathrm{E}+04$ & $2.0 \mathrm{E}+05$ \\
\hline S & $1.3 \mathrm{E}+04$ & $2.9 \mathrm{E}+04$ & $4.1 \mathrm{E}+04$ & $7.7 \mathrm{E}+04$ & $1.6 \mathrm{E}+05$ \\
\hline SSW & $9.8 \mathrm{E}+03$ & $1.3 \mathrm{E}+04$ & $1.4 \mathrm{E}+04$ & $8.8 \mathrm{E}+04$ & $1.2 \mathrm{E}+05$ \\
\hline SW & $1.0 \mathrm{E}+04$ & $1.5 \mathrm{E}+04$ & $1.0 \mathrm{E}+04$ & $1.2 \mathrm{E}+04$ & $4.7 \mathrm{E}+04$ \\
\hline WSW & $1.1 \mathrm{E}+04$ & $1.7 \mathrm{E}+04$ & $2.4 \mathrm{E}+04$ & $4.0 \mathrm{E}+04$ & $9.0 \mathrm{E}+04$ \\
\hline W & $9.3 \mathrm{E}+03$ & $1.3 \mathrm{E}+04$ & $2.3 \mathrm{E}+04$ & $3.1 \mathrm{E}+04$ & $7.6 \mathrm{E}+04$ \\
\hline WNW & $1.4 \mathrm{E}+04$ & $1.1 \mathrm{E}+04$ & $1.0 \mathrm{E}+04$ & $1.1 \mathrm{E}+04$ & $4.6 \mathrm{E}+04$ \\
\hline NW & $1.6 \mathrm{E}+04$ & $2.2 \mathrm{E}+04$ & $2.6 \mathrm{E}+04$ & $1.9 \mathrm{E}+04$ & $8.3 \mathrm{E}+04$ \\
\hline NNW & $1.6 \mathrm{E}+04$ & $2.5 \mathrm{E}+04$ & $3.9 \mathrm{E}+04$ & $5.6 \mathrm{E}+04$ & $1.3 \mathrm{E}+05$ \\
\hline Total & $\mathbf{4 . 5 E + 0 5}$ & $\mathbf{9 . 5 E}+\mathbf{0 5}$ & $\mathbf{1 . 5 E}+\mathbf{0 6}$ & $\mathbf{2 . 4 E + 0 6}$ & $\mathbf{5 . 3 E}+\mathbf{0 6}$ \\
\hline
\end{tabular}


Table 8. Beef Production as a Function of Distance and Sector

2007 Weighted Beef Production Grid surrounding SRS Increased 25\% for unreported (home use) production (L/yr)

\begin{tabular}{|l|l|l|l|r|c|}
\hline Sector & $\mathbf{1 0 - 2 0} \mathbf{M i}$ & $\mathbf{2 0 - 3 0} \mathbf{~ M i}$ & $\mathbf{3 0 - 4 0} \mathbf{~ M i}$ & $\mathbf{4 0 - 5 0 ~ M i}$ & \multicolumn{1}{c|}{ Total } \\
\hline N & $4.9 \mathrm{E}+04$ & $8.2 \mathrm{E}+04$ & $1.3 \mathrm{E}+05$ & $5.1 \mathrm{E}+05$ & $7.8 \mathrm{E}+05$ \\
\hline NNE & $4.7 \mathrm{E}+04$ & $8.2 \mathrm{E}+04$ & $1.0 \mathrm{E}+05$ & $1.0 \mathrm{E}+05$ & $3.3 \mathrm{E}+05$ \\
\hline NE & $4.4 \mathrm{E}+04$ & $7.9 \mathrm{E}+04$ & $1.0 \mathrm{E}+05$ & $1.0 \mathrm{E}+05$ & $3.3 \mathrm{E}+05$ \\
\hline ENE & $4.6 \mathrm{E}+04$ & $8.9 \mathrm{E}+04$ & $1.1 \mathrm{E}+05$ & $1.3 \mathrm{E}+05$ & $3.8 \mathrm{E}+05$ \\
\hline E & $5.2 \mathrm{E}+04$ & $1.0 \mathrm{E}+05$ & $1.6 \mathrm{E}+05$ & $1.5 \mathrm{E}+05$ & $4.7 \mathrm{E}+05$ \\
\hline ESE & $4.8 \mathrm{E}+04$ & $1.3 \mathrm{E}+05$ & $1.4 \mathrm{E}+05$ & $6.1 \mathrm{E}+04$ & $3.8 \mathrm{E}+05$ \\
\hline SE & $9.2 \mathrm{E}+04$ & $1.8 \mathrm{E}+05$ & $8.5 \mathrm{E}+04$ & $2.4 \mathrm{E}+04$ & $3.8 \mathrm{E}+05$ \\
\hline SSE & $9.3 \mathrm{E}+04$ & $1.5 \mathrm{E}+05$ & $1.6 \mathrm{E}+05$ & $6.2 \mathrm{E}+04$ & $4.7 \mathrm{E}+05$ \\
\hline S & $8.9 \mathrm{E}+04$ & $1.1 \mathrm{E}+05$ & $1.5 \mathrm{E}+05$ & $1.7 \mathrm{E}+05$ & $5.2 \mathrm{E}+05$ \\
\hline SSW & $1.0 \mathrm{E}+05$ & $1.3 \mathrm{E}+05$ & $1.4 \mathrm{E}+05$ & $1.2 \mathrm{E}+05$ & $4.8 \mathrm{E}+05$ \\
\hline SW & $1.0 \mathrm{E}+05$ & $1.7 \mathrm{E}+05$ & $1.8 \mathrm{E}+05$ & $1.5 \mathrm{E}+05$ & $6.0 \mathrm{E}+05$ \\
\hline WSW & $1.1 \mathrm{E}+05$ & $1.7 \mathrm{E}+05$ & $2.4 \mathrm{E}+05$ & $2.4 \mathrm{E}+05$ & $7.7 \mathrm{E}+05$ \\
\hline W & $6.3 \mathrm{E}+04$ & $1.0 \mathrm{E}+05$ & $1.7 \mathrm{E}+05$ & $1.7 \mathrm{E}+05$ & $5.0 \mathrm{E}+05$ \\
\hline WNW & $4.8 \mathrm{E}+04$ & $5.5 \mathrm{E}+04$ & $8.8 \mathrm{E}+04$ & $1.2 \mathrm{E}+05$ & $3.1 \mathrm{E}+05$ \\
\hline NW & $5.2 \mathrm{E}+04$ & $7.6 \mathrm{E}+04$ & $1.1 \mathrm{E}+05$ & $1.1 \mathrm{E}+05$ & $3.5 \mathrm{E}+05$ \\
\hline NNW & $5.1 \mathrm{E}+04$ & $8.2 \mathrm{E}+04$ & $1.3 \mathrm{E}+05$ & $2.5 \mathrm{E}+05$ & $5.2 \mathrm{E}+05$ \\
\hline \multicolumn{1}{|c|}{ Total } & $\mathbf{1 . 1 E}+\mathbf{0 6}$ & $\mathbf{1 . 8 E}+\mathbf{0 6}$ & $\mathbf{2 . 2 E + 0 6}$ & $\mathbf{2 . 5 E + 0 6}$ & $\mathbf{7 . 6 E}+\mathbf{0 6}$ \\
\hline
\end{tabular}


SRNL-STI-2010-00447, REVISION 0

Table 9. Milk Production as a Function of Distance and Sector

\begin{tabular}{|l|l|l|l|l|c|}
\hline \multicolumn{1}{|c|}{ Sector } & $\mathbf{1 0 - 2 0 ~ M i}$ & $\mathbf{2 0 - 3 0 ~ M i}$ & $\mathbf{3 0 - 4 0 ~ M i}$ & $\mathbf{4 0 - 5 0 ~ M i}$ & Total \\
\hline N & $4.4 \mathrm{E}+03$ & $4.4 \mathrm{E}+03$ & $3.0 \mathrm{E}+05$ & $3.2 \mathrm{E}+06$ & $3.5 \mathrm{E}+06$ \\
\hline NNE & $4.4 \mathrm{E}+03$ & $4.4 \mathrm{E}+03$ & $4.4 \mathrm{E}+03$ & $4.4 \mathrm{E}+03$ & $1.8 \mathrm{E}+04$ \\
\hline NE & $4.4 \mathrm{E}+03$ & $1.0 \mathrm{E}+06$ & $2.0 \mathrm{E}+06$ & $1.2 \mathrm{E}+06$ & $4.2 \mathrm{E}+06$ \\
\hline ENE & $4.4 \mathrm{E}+03$ & $1.6 \mathrm{E}+06$ & $3.8 \mathrm{E}+06$ & $4.5 \mathrm{E}+06$ & $9.9 \mathrm{E}+06$ \\
\hline E & $4.4 \mathrm{E}+03$ & $2.5 \mathrm{E}+06$ & $5.3 \mathrm{E}+06$ & $5.2 \mathrm{E}+06$ & $1.3 \mathrm{E}+07$ \\
\hline ESE & $4.4 \mathrm{E}+03$ & $9.6 \mathrm{E}+05$ & $3.0 \mathrm{E}+06$ & $7.3 \mathrm{E}+05$ & $4.7 \mathrm{E}+06$ \\
\hline SE & $4.4 \mathrm{E}+03$ & $4.4 \mathrm{E}+03$ & $4.4 \mathrm{E}+03$ & $4.4 \mathrm{E}+03$ & $1.8 \mathrm{E}+04$ \\
\hline SSE & $1.6 \mathrm{E}+05$ & $1.3 \mathrm{E}+05$ & $1.9 \mathrm{E}+05$ & $1.4 \mathrm{E}+05$ & $6.2 \mathrm{E}+05$ \\
\hline S & $1.5 \mathrm{E}+06$ & $3.8 \mathrm{E}+05$ & $4.7 \mathrm{E}+05$ & $5.5 \mathrm{E}+05$ & $2.9 \mathrm{E}+06$ \\
\hline SSW & $2.1 \mathrm{E}+06$ & $1.9 \mathrm{E}+06$ & $1.3 \mathrm{E}+06$ & $1.3 \mathrm{E}+06$ & $6.6 \mathrm{E}+06$ \\
\hline SW & $2.1 \mathrm{E}+06$ & $3.4 \mathrm{E}+06$ & $3.1 \mathrm{E}+06$ & $2.6 \mathrm{E}+06$ & $1.1 \mathrm{E}+07$ \\
\hline WSW & $2.2 \mathrm{E}+06$ & $3.5 \mathrm{E}+06$ & $4.8 \mathrm{E}+06$ & $3.8 \mathrm{E}+06$ & $1.4 \mathrm{E}+07$ \\
\hline W & $8.0 \mathrm{E}+05$ & $1.4 \mathrm{E}+06$ & $2.6 \mathrm{E}+06$ & $3.7 \mathrm{E}+06$ & $8.5 \mathrm{E}+06$ \\
\hline WNW & $4.4 \mathrm{E}+03$ & $8.9 \mathrm{E}+03$ & $6.6 \mathrm{E}+03$ & $1.5 \mathrm{E}+06$ & $1.5 \mathrm{E}+06$ \\
\hline NW & $4.4 \mathrm{E}+03$ & $7.0 \mathrm{E}+04$ & $6.1 \mathrm{E}+05$ & $4.4 \mathrm{E}+05$ & $1.1 \mathrm{E}+06$ \\
\hline NNW & $4.4 \mathrm{E}+03$ & $1.5 \mathrm{E}+04$ & $9.0 \mathrm{E}+05$ & $1.8 \mathrm{E}+06$ & $2.7 \mathrm{E}+06$ \\
\hline Total & $\mathbf{9 . 0 E}+\mathbf{0 6}$ & $\mathbf{1 . 7 E + 0 7}$ & $\mathbf{2 . 9 E}+\mathbf{0 7}$ & $\mathbf{3 . 1 E}+\mathbf{0 7}$ & $\mathbf{8 . 5 E}+\mathbf{0 7}$ \\
\hline
\end{tabular}


SRNL-STI-2010-00447, REVISION 0

Table 10. SRS Average Behavioral Parameters for Dose Calculations

\begin{tabular}{|c|c|c|c|c|c|c|}
\hline \multirow[b]{2}{*}{ Parameter } & \multirow{2}{*}{ Units } & 1977 & 1991 & 2008 & 2010 & \multirow{2}{*}{$\begin{array}{l}\text { \% Difference } \\
1991 \text { Hamby/ } \\
2010 \text { Jannik }\end{array}$} \\
\hline & & $\begin{array}{l}\text { NRC } \\
\text { Default }\end{array}$ & Hamby & $\begin{array}{l}\text { Lee and } \\
\text { Coffield }\end{array}$ & $\begin{array}{l}\text { Jannik et } \\
\text { al. }\end{array}$ & \\
\hline \multicolumn{7}{|l|}{$\begin{array}{l}\text { Average Individual and Population } \\
\text { Usage }\end{array}$} \\
\hline Inhalation Rate & $\mathrm{m}^{3} / \mathrm{yr}$ & 8,000 & 8,000 & 5,548 & 5,548 & $-30.65 \%$ \\
\hline Drinking Water consumption rate & $\mathrm{L} / \mathrm{yr}$ & 370 & 370 & 337 & 337 & $-8.92 \%$ \\
\hline Leafy Vegetable consumption rate & $\mathrm{kg} / \mathrm{yr}$ & 64 & 21 & 21 & 21 & $0.00 \%$ \\
\hline Other Produce consumption rate & $\mathrm{kg} / \mathrm{yr}$ & 190 & 163 & 163 & 163 & $0.00 \%$ \\
\hline Meat consumption rate & $\mathrm{kg} / \mathrm{yr}$ & 95 & 43 & 43 & 43 & $0.00 \%$ \\
\hline Milk consumption rate & $\mathrm{L} / \mathrm{yr}$ & 110 & 120 & 120 & 120 & $0.00 \%$ \\
\hline Fish consumption rate & $\mathrm{kg} / \mathrm{yr}$ & 6.9 & 9 & 9 & 9 & $0.00 \%$ \\
\hline Invertebrate consumption rate & $\mathrm{kg} / \mathrm{yr}$ & 1 & 2 & $\mathrm{~N} / \mathrm{A}$ & 2 & $0.00 \%$ \\
\hline Soil consumption rate & $\mathrm{kg} / \mathrm{yr}$ & - & - & 0.0365 & 0.0365 & $\mathrm{~N} / \mathrm{A}$ \\
\hline Shoreline Exposure Time & $\mathrm{hr} / \mathrm{yr}$ & 8.3 & 23.0 & 23.0 & 10.0 & $-56.52 \%$ \\
\hline Swimming Exposure Time & $\mathrm{hr} / \mathrm{yr}$ & - & 8.9 & 8.9 & 7.0 & $-21.35 \%$ \\
\hline Boating Exposure Time & $\mathrm{hr} / \mathrm{yr}$ & - & 21.0 & 21.0 & 22.0 & $4.76 \%$ \\
\hline Population Shoreline Exposure Time & per.-hr & - & $9.60 \mathrm{E}+05$ & $\mathrm{~N} / \mathrm{A}$ & $8.22 \mathrm{E}+05$ & $-14.38 \%$ \\
\hline Population Swimming Exposure Time & per.-hr & - & $1.60 \mathrm{E}+05$ & $\mathrm{~N} / \mathrm{A}$ & $2.95 \mathrm{E}+05$ & $84.38 \%$ \\
\hline Population Boating Exposure Time & per.-hr & - & $1.10 \mathrm{E}+06$ & $\mathrm{~N} / \mathrm{A}$ & $3.11 \mathrm{E}+06$ & $182.73 \%$ \\
\hline Fraction of year working in garden & - & - & - & 0.01 & 0.01 & $\mathrm{~N} / \mathrm{A}$ \\
\hline Fraction of year residing in home & - & - & - & 0.7 & 0.7 & $\mathrm{~N} / \mathrm{A}$ \\
\hline Time taking a shower & $\mathrm{min} / \mathrm{d}$ & - & - & 10 & 10 & $\mathrm{~N} / \mathrm{A}$ \\
\hline Population served - BJWSA (Chelsea) & persons & - & $5.00 \mathrm{E}+04$ & $\mathrm{~N} / \mathrm{A}$ & $7.70 \mathrm{E}+04$ & $54.00 \%$ \\
\hline Population served - BJWSA (Purrysburg) & persons & - & $\mathrm{N} / \mathrm{A}$ & $\mathrm{N} / \mathrm{A}$ & $5.80 \mathrm{E}+04$ & $\mathrm{~N} / \mathrm{A}$ \\
\hline Population served - Savannah I\&D & persons & - & $1.50 \mathrm{E}+04$ & $\mathrm{~N} / \mathrm{A}$ & $2.63 \mathrm{E}+04$ & $75.33 \%$ \\
\hline
\end{tabular}


SRNL-STI-2010-00447, REVISION 0

Table 11. SRS Maximum Individual Behavioral Parameters for Dose Calculations

\begin{tabular}{|c|c|c|c|c|c|c|}
\hline \multirow[b]{2}{*}{ Parameter } & \multirow[b]{2}{*}{ Units } & 1977 & 1991 & 2008 & 2010 & \multirow{2}{*}{$\begin{array}{l}\text { \% Difference } \\
1991 \text { Hamby/ } \\
2010 \text { Jannik }\end{array}$} \\
\hline & & $\begin{array}{c}\text { NRC } \\
\text { Default }\end{array}$ & Hamby & $\begin{array}{l}\text { Lee and } \\
\text { Coffield }\end{array}$ & $\begin{array}{c}\text { Jannik } \\
\text { et al. }\end{array}$ & \\
\hline \multicolumn{7}{|c|}{ Maximally Exposed Individual Usage } \\
\hline Inhalation Rate & $\mathrm{m}^{3} / \mathrm{yr}$ & 8,000 & 8,000 & N/A & 8,000 & $0.00 \%$ \\
\hline Drinking Water consumption rate & $\mathrm{L} / \mathrm{yr}$ & 730 & 730 & N/A & 730 & $0.00 \%$ \\
\hline Leafy Vegetable consumption rate & $\mathrm{kg} / \mathrm{yr}$ & 64 & 43 & N/A & 43 & $0.00 \%$ \\
\hline Other Produce consumption rate & $\mathrm{kg} / \mathrm{yr}$ & 520 & 276 & N/A & 276 & $0.00 \%$ \\
\hline Meat consumption rate & $\mathrm{kg} / \mathrm{yr}$ & 110 & 81 & N/A & 81 & $0.00 \%$ \\
\hline Milk consumption rate & $\mathrm{L} / \mathrm{yr}$ & 310 & 230 & N/A & 230 & $0.00 \%$ \\
\hline Fish consumption rate & $\mathrm{kg} / \mathrm{yr}$ & 21 & 19 & N/A & 19 & $0.00 \%$ \\
\hline Invertebrate consumption rate & $\mathrm{kg} / \mathrm{yr}$ & 5 & 8 & $\mathrm{~N} / \mathrm{A}$ & 8 & $0.00 \%$ \\
\hline Soil consumption rate & $\mathrm{kg} / \mathrm{yr}$ & - & - & N/A & 0.0365 & N/A \\
\hline Shoreline Exposure Time & $\mathrm{hr} / \mathrm{yr}$ & 12 & 23.0 & N/A & 20.0 & $-13.04 \%$ \\
\hline Swimming Exposure Time & $\mathrm{hr} / \mathrm{yr}$ & - & 8.9 & N/A & 14.0 & $57.30 \%$ \\
\hline Boating Exposure Time & $\mathrm{hr} / \mathrm{yr}$ & - & 21.0 & N/A & 44.0 & $109.52 \%$ \\
\hline
\end{tabular}


SRNL-STI-2010-00447, REVISION 0

Table 12. Average Recreational Usage Rates

\begin{tabular}{|l|c|c|c|c|}
\hline \multirow{2}{*}{ Survey Activity } & $\mathbf{2 0 0 5}$ & $\mathbf{2 0 0 5}$ & $\mathbf{1 9 9 1}$ & $\mathbf{2 0 1 0}$ \\
\cline { 2 - 5 } & $\begin{array}{c}\text { Percentage } \\
\text { Participating }\end{array}$ & $\begin{array}{c}\text { Average Annual } \\
\text { Frequency }\end{array}$ & $\begin{array}{c}\text { Survey Hours } \\
\text { per Occasion }\end{array}$ & $\begin{array}{c}\text { per Capita } \\
\text { Usage (hr/yr) }\end{array}$ \\
\hline & & & & \\
\hline Shoreline Usage & & & & 9.50 \\
\hline Fresh water fishing & $38 \%$ & 5.0 & 5 & $\mathbf{1 0}$ \\
\hline & & & & 3.36 \\
\hline Swimming Usage & & & 3 & 3.36 \\
\hline Lake/River Swimming & $28 \%$ & 4.0 & 3 & 7 \\
\hline Jet and Water Skiing & $28 \%$ & 4.0 & & \\
\hline & & & & \\
\hline Boating Usage & & & & \\
\hline Canoeing, kayaking, rafting & $11 \%$ & 1.0 & & 22.00 \\
\hline Boating/Sailing & $55 \%$ & 8.0 & & $\mathbf{2}$ \\
\hline
\end{tabular}

SC 2005 Recreational Participation \& Preference Study

http://www.scprt.com/our-partners/tourismstatistics/researchreports.aspx 
SRNL-STI-2010-00447, REVISION 0

Table 13. Weighted Usage Fractions

\begin{tabular}{|c|c|c|c|c|}
\hline Activity & $\begin{array}{c}\text { State Usage } \\
\text { Available } \\
\text { Fraction }\end{array}$ & $\begin{array}{c}2000 \\
\text { Population } \\
\text { Fraction* }\end{array}$ & $\begin{array}{l}\text { Weighted } \\
\text { Usage } \\
\text { Fraction }\end{array}$ & Population Usage (person-hr/yr) \\
\hline \multicolumn{5}{|l|}{ Shoreline } \\
\hline $\mathrm{SC}$, surf/bank fishing & 0.041 & 0.6 & 0.0246 & \\
\hline \multirow[t]{2}{*}{ GA, warmwater fishing } & 0.085 & 0.4 & 0.034 & \\
\hline & & & 0.059 & $8.22 \mathrm{E}+05$ \\
\hline \multicolumn{5}{|l|}{ Swimming } \\
\hline $\mathrm{SC}$, beach areas & 0.032 & 0.6 & 0.0192 & \\
\hline \multirow[t]{2}{*}{ GA, lake swimming } & 0.027 & 0.4 & 0.0108 & \\
\hline & & & 0.030 & $2.95 \mathrm{E}+05$ \\
\hline \multicolumn{5}{|l|}{ Boating } \\
\hline $\mathrm{SC}$, boat use average** & 0.13 & 0.6 & 0.078 & \\
\hline \multirow[t]{2}{*}{ GA, boating/sailing } & 0.057 & 0.4 & 0.0228 & \\
\hline & & & 0.101 & $3.11 \mathrm{E}+06$ \\
\hline
\end{tabular}

* Assumes $60 \%$ of population lives in SC and $40 \%$ of population lives in Georgia.

Based on Projected Census Data for Counties

** Average of "other fishing" and "boat ramps" fractions

Base Census 2000

50 mile Counties SC Population

50 mile Counties GA Population

Total
739,715

507,226

$1,246,941$
Projected Census 2010

832,000

571,000

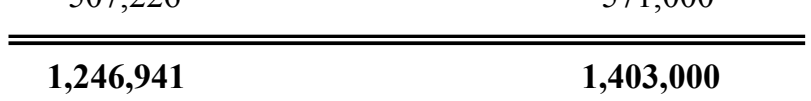




\section{APPENDIX B DOSE COMPARISONS (TABLES 14-55)}


SRNL-STI-2010-00447, REVISION 0

APPENDIX B DOSE COMPARISONS (TABLES 14-55)

Table 14. LADTAP XL Maximally Exposed Individual Water Ingestion Pathway Comparison (mrem/yr)

\begin{tabular}{|c|c|c|c|c|c|c|c|}
\hline Nuclide & $\begin{array}{l}\text { Hamby Ladtap } \\
\text { Water Pathway }\end{array}$ & $\begin{array}{l}\text { Jannik Ladtap } \\
\text { Water Pathway }\end{array}$ & $\%$ Diff & Nuclide & $\begin{array}{l}\text { Hamby Ladtap } \\
\text { Water Pathway }\end{array}$ & $\begin{array}{c}\text { Jannik Ladtap } \\
\text { Water Pathway }\end{array}$ & $\%$ Diff \\
\hline H-3 & $5.1 \mathrm{E}-06$ & $5.1 \mathrm{E}-06$ & $0.00 \%$ & Sm-146 & $1.6 \mathrm{E}-02$ & $1.6 \mathrm{E}-02$ & $0.00 \%$ \\
\hline $\mathrm{Be}-7$ & $8.8 \mathrm{E}-06$ & $8.8 \mathrm{E}-06$ & $0.00 \%$ & Sm-147 & $1.5 \mathrm{E}-02$ & $1.5 \mathrm{E}-02$ & $0.00 \%$ \\
\hline Be-10 & $3.4 \mathrm{E}-04$ & $3.4 \mathrm{E}-04$ & $0.00 \%$ & Sm-151 & $2.8 \mathrm{E}-05$ & $2.8 \mathrm{E}-05$ & $0.00 \%$ \\
\hline $\mathrm{C}-14$ & $1.7 \mathrm{E}-04$ & $1.7 \mathrm{E}-04$ & $0.00 \%$ & Pm-147 & $7.8 \mathrm{E}-05$ & $7.8 \mathrm{E}-05$ & $0.00 \%$ \\
\hline $\mathrm{Na}-22$ & $9.8 \mathrm{E}-04$ & $9.8 \mathrm{E}-04$ & $0.00 \%$ & Eu-152 & $4.9 \mathrm{E}-04$ & 4.9E-04 & $0.00 \%$ \\
\hline $\mathrm{Na}-24$ & $2.2 \mathrm{E}-05$ & $2.2 \mathrm{E}-05$ & $0.00 \%$ & Eu-154 & $7.4 \mathrm{E}-04$ & $7.4 \mathrm{E}-04$ & $0.00 \%$ \\
\hline Al-26 & $1.1 \mathrm{E}-03$ & $1.1 \mathrm{E}-03$ & $0.00 \%$ & Eu-155 & $1.1 \mathrm{E}-04$ & $1.1 \mathrm{E}-04$ & $0.00 \%$ \\
\hline P-32 & 5.9E-04 & $5.9 \mathrm{E}-04$ & $0.00 \%$ & Gd-152 & $1.2 \mathrm{E}-02$ & $1.2 \mathrm{E}-02$ & $0.00 \%$ \\
\hline $\mathrm{Si}-32$ & $1.4 \mathrm{E}-04$ & $1.4 \mathrm{E}-04$ & $0.00 \%$ & Ho-166m & $6.4 \mathrm{E}-04$ & $6.4 \mathrm{E}-04$ & $0.00 \%$ \\
\hline S-35 & $5.2 \mathrm{E}-05$ & $5.2 \mathrm{E}-05$ & $0.00 \%$ & Lu-176 & $5.4 \mathrm{E}-04$ & $5.4 \mathrm{E}-04$ & $0.00 \%$ \\
\hline $\mathrm{Cl}-36$ & $2.5 \mathrm{E}-04$ & $2.5 \mathrm{E}-04$ & $0.00 \%$ & Ta-180 & $4.8 \mathrm{E}-84$ & $4.8 \mathrm{E}-84$ & $0.00 \%$ \\
\hline $\mathrm{K}-40$ & $1.6 \mathrm{E}-03$ & $1.6 \mathrm{E}-03$ & $0.00 \%$ & Hf-182 & $1.1 \mathrm{E}-03$ & $1.1 \mathrm{E}-03$ & $0.00 \%$ \\
\hline $\mathrm{K}-43$ & $2.1 \mathrm{E}-05$ & $2.1 \mathrm{E}-05$ & $0.00 \%$ & $\mathrm{Re}-186 \mathrm{~m}$ & $2.7 \mathrm{E}-04$ & $2.7 \mathrm{E}-04$ & $0.00 \%$ \\
\hline $\mathrm{Ca}-41$ & $9.8 \mathrm{E}-05$ & $9.8 \mathrm{E}-05$ & $0.00 \%$ & Re-187 & $6.8 \mathrm{E}-07$ & $6.8 \mathrm{E}-07$ & $0.00 \%$ \\
\hline $\mathrm{Ca}-45$ & $2.4 \mathrm{E}-04$ & $2.4 \mathrm{E}-04$ & $0.00 \%$ & Ir-192m & $0.0 \mathrm{E}+00$ & $0.0 \mathrm{E}+00$ & \\
\hline $\mathrm{Ca}-47$ & $4.0 \mathrm{E}-04$ & $4.0 \mathrm{E}-04$ & $0.00 \%$ & Pt-193 & $9.0 \mathrm{E}-06$ & $9.0 \mathrm{E}-06$ & $0.00 \%$ \\
\hline Ti-44 & $1.6 \mathrm{E}-03$ & $1.6 \mathrm{E}-03$ & $0.00 \%$ & Hg-194 & $2.3 \mathrm{E}-02$ & $2.3 \mathrm{E}-02$ & $0.00 \%$ \\
\hline $\mathrm{V}-49$ & $4.4 \mathrm{E}-06$ & $4.4 \mathrm{E}-06$ & $0.00 \%$ & $\mathrm{Hg}-203$ & $8.0 \mathrm{E}-04$ & $8.0 \mathrm{E}-04$ & $0.00 \%$ \\
\hline Cr-51 & $1.0 \mathrm{E}-05$ & $1.0 \mathrm{E}-05$ & $0.00 \%$ & $\mathrm{~Pb}-202$ & $3.2 \mathrm{E}-03$ & $3.2 \mathrm{E}-03$ & $0.00 \%$ \\
\hline Mn-53 & $8.1 \mathrm{E}-06$ & $8.1 \mathrm{E}-06$ & $0.00 \%$ & $\mathrm{~Pb}-205$ & $1.2 \mathrm{E}-04$ & $1.2 \mathrm{E}-04$ & $0.00 \%$ \\
\hline $\mathrm{Mn}-54$ & $2.2 \mathrm{E}-04$ & $2.2 \mathrm{E}-04$ & $0.00 \%$ & $\mathrm{~Pb}-210$ & $4.2 \mathrm{E}-01$ & $4.2 \mathrm{E}-01$ & $0.00 \%$ \\
\hline $\mathrm{Fe}-55$ & $4.7 \mathrm{E}-05$ & $4.7 \mathrm{E}-05$ & $0.00 \%$ & Bi-207 & $4.0 \mathrm{E}-04$ & $4.0 \mathrm{E}-04$ & $0.00 \%$ \\
\hline Fe-60 & $1.2 \mathrm{E}-02$ & $1.2 \mathrm{E}-02$ & $0.00 \%$ & $\mathrm{Bi}-210$ & $3.9 \mathrm{E}-04$ & $3.9 \mathrm{E}-04$ & $0.00 \%$ \\
\hline Co-58 & $2.8 \mathrm{E}-04$ & $2.8 \mathrm{E}-04$ & $0.00 \%$ & $\mathrm{Bi}-210 \mathrm{~m}$ & $7.0 \mathrm{E}-03$ & $7.0 \mathrm{E}-03$ & $0.00 \%$ \\
\hline
\end{tabular}


SRNL-STI-2010-00447, REVISION 0

Table 14. LADTAP XL Maximally Exposed Individual Water Ingestion Pathway Comparison (mrem/yr) (continued)

\begin{tabular}{|c|c|c|c|c|c|c|c|}
\hline Nuclide & $\begin{array}{l}\text { Hamby Ladtap } \\
\text { Water Pathway }\end{array}$ & $\begin{array}{l}\text { Jannik Ladtap } \\
\text { Water Pathway }\end{array}$ & $\%$ Diff & Nuclide & $\begin{array}{l}\text { Hamby Ladtap } \\
\text { Water Pathway }\end{array}$ & $\begin{array}{l}\text { Jannik Ladtap } \\
\text { Water Pathway }\end{array}$ & $\%$ Diff \\
\hline Co-60 & $2.1 \mathrm{E}-03$ & $2.1 \mathrm{E}-03$ & $0.00 \%$ & Po-210 & $1.3 \mathrm{E}-01$ & $1.3 \mathrm{E}-01$ & $0.00 \%$ \\
\hline Ni-59 & $1.6 \mathrm{E}-05$ & $1.6 \mathrm{E}-05$ & $0.00 \%$ & Ra-223 & $4.1 \mathrm{E}-02$ & $4.1 \mathrm{E}-02$ & $0.00 \%$ \\
\hline $\mathrm{Ni}-63$ & $4.4 \mathrm{E}-05$ & $4.4 \mathrm{E}-05$ & $0.00 \%$ & $\mathrm{Ra}-224$ & $2.0 \mathrm{E}-02$ & $2.0 \mathrm{E}-02$ & $0.00 \%$ \\
\hline $\mathrm{Cu}-64$ & $4.9 \mathrm{E}-06$ & 4.9E-06 & $0.00 \%$ & Ra-225 & $2.4 \mathrm{E}-02$ & $2.4 \mathrm{E}-02$ & $0.00 \%$ \\
\hline $\mathrm{Zn}-65$ & $1.1 \mathrm{E}-03$ & $1.1 \mathrm{E}-03$ & $0.00 \%$ & Ra-226 & $9.0 \mathrm{E}-02$ & $9.0 \mathrm{E}-02$ & $0.00 \%$ \\
\hline Ge-68 & $9.0 \mathrm{E}-05$ & $9.0 \mathrm{E}-05$ & $0.00 \%$ & Ra-228 & $9.8 \mathrm{E}-02$ & $9.8 \mathrm{E}-02$ & $0.00 \%$ \\
\hline Se-75 & $7.1 \mathrm{E}-04$ & $7.1 \mathrm{E}-04$ & $0.00 \%$ & Ac-227 & $1.1 \mathrm{E}+00$ & $1.1 \mathrm{E}+00$ & $0.00 \%$ \\
\hline Se-79 & $6.8 \mathrm{E}-04$ & $6.8 \mathrm{E}-04$ & $0.00 \%$ & Th-227 & $2.8 \mathrm{E}-03$ & $2.8 \mathrm{E}-03$ & $0.00 \%$ \\
\hline $\mathrm{Rb}-87$ & $3.9 \mathrm{E}-04$ & $3.9 \mathrm{E}-04$ & $0.00 \%$ & Th-228 & $3.1 \mathrm{E}-02$ & $3.1 \mathrm{E}-02$ & $0.00 \%$ \\
\hline Sr-89 & $7.0 \mathrm{E}-04$ & $7.0 \mathrm{E}-04$ & $0.00 \%$ & Th-229 & $2.9 \mathrm{E}-01$ & $2.9 \mathrm{E}-01$ & $0.00 \%$ \\
\hline Sr-90 & $1.1 \mathrm{E}-02$ & $1.1 \mathrm{E}-02$ & $0.00 \%$ & Th-230 & $4.3 \mathrm{E}-02$ & $4.3 \mathrm{E}-02$ & $0.00 \%$ \\
\hline $\mathrm{Y}-90$ & $5.5 \mathrm{E}-04$ & $5.5 \mathrm{E}-04$ & $0.00 \%$ & Th-231 & $4.0 \mathrm{E}-05$ & $4.0 \mathrm{E}-05$ & $0.00 \%$ \\
\hline Y-91 & $7.1 \mathrm{E}-04$ & $7.1 \mathrm{E}-04$ & $0.00 \%$ & Th-232 & $2.3 \mathrm{E}-01$ & $2.3 \mathrm{E}-01$ & $0.00 \%$ \\
\hline Mo-93 & $1.1 \mathrm{E}-04$ & $1.1 \mathrm{E}-04$ & $0.00 \%$ & Th-234 & $1.0 \mathrm{E}-03$ & $1.0 \mathrm{E}-03$ & $0.00 \%$ \\
\hline Мo-99 & $2.5 \mathrm{E}-04$ & $2.5 \mathrm{E}-04$ & $0.00 \%$ & Pa-230 & $4.3 \mathrm{E}-04$ & $4.3 \mathrm{E}-04$ & $0.00 \%$ \\
\hline $\mathrm{Nb}-93 \mathrm{~m}$ & $4.3 \mathrm{E}-04$ & $4.3 \mathrm{E}-04$ & $0.00 \%$ & Pa-231 & $9.0 \mathrm{E}-01$ & $9.0 \mathrm{E}-01$ & $0.00 \%$ \\
\hline Nb-94 & $4.2 \mathrm{E}-04$ & $4.2 \mathrm{E}-04$ & $0.00 \%$ & $\mathrm{~Pa}-233$ & $2.6 \mathrm{E}-04$ & $2.6 \mathrm{E}-04$ & $0.00 \%$ \\
\hline $\mathrm{Nb}-95$ & $1.7 \mathrm{E}-04$ & $1.7 \mathrm{E}-04$ & $0.00 \%$ & U-232 & $1.1 \mathrm{E}-01$ & $1.1 \mathrm{E}-01$ & $0.00 \%$ \\
\hline Zr-93 & $1.3 \mathrm{E}-04$ & $1.3 \mathrm{E}-04$ & $0.00 \%$ & U-233 & $2.2 \mathrm{E}-02$ & $2.2 \mathrm{E}-02$ & $0.00 \%$ \\
\hline Zr-95 & $2.7 \mathrm{E}-04$ & $2.7 \mathrm{E}-04$ & $0.00 \%$ & U-234 & $2.1 \mathrm{E}-02$ & $2.1 \mathrm{E}-02$ & $0.00 \%$ \\
\hline Tc-96 & $1.7 \mathrm{E}-04$ & $1.7 \mathrm{E}-04$ & $0.00 \%$ & U-235 & $2.0 \mathrm{E}-02$ & $2.0 \mathrm{E}-02$ & $0.00 \%$ \\
\hline Tc-97 & $1.2 \mathrm{E}-05$ & $1.2 \mathrm{E}-05$ & $0.00 \%$ & U-236 & $2.0 \mathrm{E}-02$ & $2.0 \mathrm{E}-02$ & $0.00 \%$ \\
\hline Tc-98 & $3.9 \mathrm{E}-04$ & $3.9 \mathrm{E}-04$ & $0.00 \%$ & U-237 & $1.9 \mathrm{E}-04$ & $1.9 \mathrm{E}-04$ & $0.00 \%$ \\
\hline Tc-99 & $1.1 \mathrm{E}-04$ & $1.1 \mathrm{E}-04$ & $0.00 \%$ & U-238 & $1.9 \mathrm{E}-02$ & $1.9 \mathrm{E}-02$ & $0.00 \%$ \\
\hline Ru-97 & $3.7 \mathrm{E}-05$ & $3.7 \mathrm{E}-05$ & $0.00 \%$ & $\mathrm{~Np}-236$ & $6.5 \mathrm{E}-02$ & $6.5 \mathrm{E}-02$ & $0.00 \%$ \\
\hline Ru-103 & $2.1 \mathrm{E}-04$ & $2.1 \mathrm{E}-04$ & $0.00 \%$ & $\mathrm{~Np}-237$ & $3.2 \mathrm{E}-01$ & $3.2 \mathrm{E}-01$ & $0.00 \%$ \\
\hline Ru-106 & $1.7 \mathrm{E}-03$ & $1.7 \mathrm{E}-03$ & $0.00 \%$ & $\mathrm{~Np}-239$ & $1.5 \mathrm{E}-04$ & $1.5 \mathrm{E}-04$ & $0.00 \%$ \\
\hline Pd-107 & $1.1 \mathrm{E}-05$ & $1.1 \mathrm{E}-05$ & $0.00 \%$ & Am-237 & $7.5 \mathrm{E}-15$ & $7.5 \mathrm{E}-15$ & $0.00 \%$ \\
\hline Ag-108m & $6.1 \mathrm{E}-04$ & $6.1 \mathrm{E}-04$ & $0.00 \%$ & Am-241 & $3.7 \mathrm{E}-01$ & $3.7 \mathrm{E}-01$ & $0.00 \%$ \\
\hline
\end{tabular}


SRNL-STI-2010-00447, REVISION 0

Table 14. LADTAP XL Maximally Exposed Individual Water Ingestion Pathway Comparison (mrem/yr) (continued)

\begin{tabular}{|c|c|c|c|c|c|c|c|}
\hline Nuclide & $\begin{array}{l}\text { Hamby Ladtap } \\
\text { Water Pathway }\end{array}$ & $\begin{array}{l}\text { Jannik Ladtap } \\
\text { Water Pathway }\end{array}$ & $\%$ Diff & Nuclide & $\begin{array}{l}\text { Hamby Ladtap } \\
\text { Water Pathway }\end{array}$ & $\begin{array}{l}\text { Jannik Ladtap } \\
\text { Water Pathway }\end{array}$ & \% Diff \\
\hline $\mathrm{Ag}-110 \mathrm{~m}$ & $9.0 \mathrm{E}-04$ & $9.0 \mathrm{E}-04$ & $0.00 \%$ & Am-242m & $3.4 \mathrm{E}-01$ & $3.4 \mathrm{E}-01$ & $0.00 \%$ \\
\hline Cd-113 & $1.3 \mathrm{E}-02$ & $1.3 \mathrm{E}-02$ & $0.00 \%$ & Am-243 & $3.7 \mathrm{E}-01$ & $3.7 \mathrm{E}-01$ & $0.00 \%$ \\
\hline Cd-115m & $1.2 \mathrm{E}-03$ & $1.2 \mathrm{E}-03$ & $0.00 \%$ & $\mathrm{Pu}-237$ & $8.0 \mathrm{E}-05$ & $8.0 \mathrm{E}-05$ & $0.00 \%$ \\
\hline In-115 & $1.1 \mathrm{E}-02$ & $1.1 \mathrm{E}-02$ & $0.00 \%$ & $\mathrm{Pu}-238$ & $3.1 \mathrm{E}-01$ & $3.1 \mathrm{E}-01$ & $0.00 \%$ \\
\hline $\mathrm{Sb}-122$ & $3.5 \mathrm{E}-04$ & $3.5 \mathrm{E}-04$ & $0.00 \%$ & Pu-239 & $3.5 \mathrm{E}-01$ & $3.5 \mathrm{E}-01$ & $0.00 \%$ \\
\hline $\mathrm{Sb}-124$ & $7.5 \mathrm{E}-04$ & $7.5 \mathrm{E}-04$ & $0.00 \%$ & $\mathrm{Pu}-240$ & $3.5 \mathrm{E}-01$ & $3.5 \mathrm{E}-01$ & $0.00 \%$ \\
\hline Sb-125 & $2.1 \mathrm{E}-04$ & $2.1 \mathrm{E}-04$ & $0.00 \%$ & $\mathrm{Pu}-241$ & $7.0 \mathrm{E}-03$ & $7.0 \mathrm{E}-03$ & $0.00 \%$ \\
\hline Te-123 & $3.4 \mathrm{E}-04$ & $3.4 \mathrm{E}-04$ & $0.00 \%$ & $\mathrm{Pu}-242$ & $3.4 \mathrm{E}-01$ & $3.4 \mathrm{E}-01$ & $0.00 \%$ \\
\hline $\mathrm{Te}-125 \mathrm{~m}$ & $2.7 \mathrm{E}-04$ & $2.7 \mathrm{E}-04$ & $0.00 \%$ & $\mathrm{Pu}-244$ & $3.3 \mathrm{E}-01$ & $3.3 \mathrm{E}-01$ & $0.00 \%$ \\
\hline Sn-126 & $1.4 \mathrm{E}-03$ & $1.4 \mathrm{E}-03$ & $0.00 \%$ & $\mathrm{Cm}-241$ & $3.6 \mathrm{E}-04$ & $3.6 \mathrm{E}-04$ & $0.00 \%$ \\
\hline I-129 & $2.3 \mathrm{E}-02$ & $2.3 \mathrm{E}-02$ & $0.00 \%$ & $\mathrm{Cm}-242$ & $8.9 \mathrm{E}-03$ & $8.9 \mathrm{E}-03$ & $0.00 \%$ \\
\hline I-131 & $3.8 \mathrm{E}-03$ & $3.8 \mathrm{E}-03$ & $0.00 \%$ & $\mathrm{Cm}-243$ & $2.4 \mathrm{E}-01$ & $2.4 \mathrm{E}-01$ & $0.00 \%$ \\
\hline Cs-134 & $6.0 \mathrm{E}-03$ & $6.0 \mathrm{E}-03$ & $0.00 \%$ & $\mathrm{Cm}-244$ & $1.9 \mathrm{E}-01$ & $1.9 \mathrm{E}-01$ & $0.00 \%$ \\
\hline Cs-135 & $5.8 \mathrm{E}-04$ & $5.8 \mathrm{E}-04$ & $0.00 \%$ & $\mathrm{Cm}-245$ & $3.7 \mathrm{E}-01$ & $3.7 \mathrm{E}-01$ & $0.00 \%$ \\
\hline Cs-137 & $4.1 \mathrm{E}-03$ & 4.1E-03 & $0.00 \%$ & Cm-246 & $3.7 \mathrm{E}-01$ & $3.7 \mathrm{E}-01$ & $0.00 \%$ \\
\hline La-137 & $3.5 \mathrm{E}-05$ & $3.5 \mathrm{E}-05$ & $0.00 \%$ & $\mathrm{Cm}-247$ & $3.4 \mathrm{E}-01$ & $3.4 \mathrm{E}-01$ & $0.00 \%$ \\
\hline La-138 & $4.8 \mathrm{E}-04$ & $4.8 \mathrm{E}-04$ & $0.00 \%$ & Cm-248 & $1.3 \mathrm{E}+00$ & $1.3 \mathrm{E}+00$ & $0.00 \%$ \\
\hline La-140 & $3.4 \mathrm{E}-04$ & $3.4 \mathrm{E}-04$ & $0.00 \%$ & $\mathrm{Cm}-250$ & $6.4 \mathrm{E}+00$ & $6.4 \mathrm{E}+00$ & $0.00 \%$ \\
\hline Ba-140 & $6.3 \mathrm{E}-04$ & $6.3 \mathrm{E}-04$ & $0.00 \%$ & Bk-247 & $1.9 \mathrm{E}-01$ & $1.9 \mathrm{E}-01$ & $0.00 \%$ \\
\hline $\mathrm{Ce}-141$ & $2.1 \mathrm{E}-04$ & $2.1 \mathrm{E}-04$ & $0.00 \%$ & Bk-249 & $4.9 \mathrm{E}-04$ & $4.9 \mathrm{E}-04$ & $0.00 \%$ \\
\hline $\mathrm{Ce}-144$ & $1.6 \mathrm{E}-03$ & $1.6 \mathrm{E}-03$ & $0.00 \%$ & Cf-249 & $3.8 \mathrm{E}-01$ & $3.8 \mathrm{E}-01$ & $0.00 \%$ \\
\hline & & & & Cf-250 & $1.6 \mathrm{E}-01$ & $1.6 \mathrm{E}-01$ & $0.00 \%$ \\
\hline & & & & Cf-251 & $3.8 \mathrm{E}-01$ & $3.8 \mathrm{E}-01$ & $0.00 \%$ \\
\hline & & & & Cf-252 & $7.7 \mathrm{E}-02$ & $7.7 \mathrm{E}-02$ & $0.00 \%$ \\
\hline & & & & Es-253 & $1.9 \mathrm{E}-03$ & $1.9 \mathrm{E}-03$ & $0.00 \%$ \\
\hline & & & & Unidentified alpha & $3.5 \mathrm{E}-01$ & $3.5 \mathrm{E}-01$ & $0.00 \%$ \\
\hline & & & & Unidentified beta & $1.1 \mathrm{E}-02$ & $1.1 \mathrm{E}-02$ & $0.00 \%$ \\
\hline & & & & TOTAL Dose & $1.8 \mathrm{E}+01$ & $1.8 \mathrm{E}+01$ & $0.00 \%$ \\
\hline
\end{tabular}


SRNL-STI-2010-00447, REVISION 0

Table 15. LADTAP XL Maximally Exposed Individual Fish Ingestion Pathway Comparison (mrem/yr)

\begin{tabular}{|c|c|c|c|c|c|c|c|}
\hline Nuclide & $\begin{array}{l}\text { Hamby Ladtap } \\
\text { Fish Ingestion }\end{array}$ & $\begin{array}{l}\text { Jannik Ladtap } \\
\text { Fish Ingestion }\end{array}$ & $\%$ Diff & Nuclide & $\begin{array}{l}\text { Hamby Ladtap } \\
\text { Fish Ingestion }\end{array}$ & $\begin{array}{l}\text { Jannik Ladtap } \\
\text { Fish Ingestion }\end{array}$ & $\%$ Diff \\
\hline $\mathrm{H}-3$ & $1.2 \mathrm{E}-07$ & $1.3 \mathrm{E}-07$ & $11.11 \%$ & Sm-146 & $1.1 \mathrm{E}-02$ & $1.3 \mathrm{E}-02$ & $20.00 \%$ \\
\hline $\mathrm{Be}-7$ & $4.6 \mathrm{E}-07$ & $2.3 \mathrm{E}-05$ & $4900.00 \%$ & Sm-147 & $9.6 \mathrm{E}-03$ & $1.1 \mathrm{E}-02$ & $20.00 \%$ \\
\hline Be-10 & $1.8 \mathrm{E}-05$ & 8.9E-04 & $4900.00 \%$ & Sm-151 & $1.8 \mathrm{E}-05$ & $2.2 \mathrm{E}-05$ & $20.00 \%$ \\
\hline C-14 & $2.1 \mathrm{E}-02$ & $1.3 \mathrm{E}-05$ & $-99.93 \%$ & Pm-147 & $5.0 \mathrm{E}-05$ & $6.1 \mathrm{E}-05$ & $20.00 \%$ \\
\hline $\mathrm{Na}-22$ & $2.5 \mathrm{E}-03$ & $1.9 \mathrm{E}-03$ & $-24.00 \%$ & Eu-152 & $3.2 \mathrm{E}-04$ & $1.7 \mathrm{E}-03$ & $420.00 \%$ \\
\hline $\mathrm{Na}-24$ & $3.2 \mathrm{E}-05$ & $2.5 \mathrm{E}-05$ & $-24.00 \%$ & Eu-154 & $4.8 \mathrm{E}-04$ & $2.5 \mathrm{E}-03$ & $420.00 \%$ \\
\hline Al-26 & $2.8 \mathrm{E}-04$ & $1.4 \mathrm{E}-03$ & $410.00 \%$ & Eu-155 & $6.9 \mathrm{E}-05$ & $3.6 \mathrm{E}-04$ & $420.00 \%$ \\
\hline $\mathrm{P}-32$ & $1.5 \mathrm{E}+00$ & $2.1 \mathrm{E}+00$ & $40.00 \%$ & Gd-152 & $8.0 \mathrm{E}-03$ & $9.6 \mathrm{E}-03$ & $20.00 \%$ \\
\hline $\mathrm{Si}-32$ & $9.0 \mathrm{E}-06$ & $7.2 \mathrm{E}-05$ & $700.00 \%$ & Ho-166m & $4.1 \mathrm{E}-04$ & $5.0 \mathrm{E}-04$ & $20.00 \%$ \\
\hline S-35 & $1.0 \mathrm{E}-03$ & $1.1 \mathrm{E}-03$ & $6.67 \%$ & Lu-176 & $3.5 \mathrm{E}-04$ & $3.5 \mathrm{E}-04$ & $0.00 \%$ \\
\hline $\mathrm{Cl}-36$ & $3.2 \mathrm{E}-04$ & $3.0 \mathrm{E}-04$ & $-6.00 \%$ & Ta-180 & 9.9E-108 & $9.9 \mathrm{E}-110$ & $-99.00 \%$ \\
\hline $\mathrm{K}-40$ & $4.0 \mathrm{E}-02$ & $1.3 \mathrm{E}-01$ & $220.00 \%$ & Hf-182 & 9.9E-05 & $3.3 \mathrm{E}-02$ & $32933.03 \%$ \\
\hline $\mathrm{K}-43$ & $3.7 \mathrm{E}-04$ & $1.2 \mathrm{E}-03$ & $220.00 \%$ & Re-186m & $8.4 \mathrm{E}-04$ & $8.4 \mathrm{E}-04$ & $0.84 \%$ \\
\hline $\mathrm{Ca}-41$ & $1.0 \mathrm{E}-04$ & $3.1 \mathrm{E}-05$ & $-70.00 \%$ & Re-187 & $2.1 \mathrm{E}-06$ & $2.1 \mathrm{E}-06$ & $0.84 \%$ \\
\hline $\mathrm{Ca}-45$ & $2.5 \mathrm{E}-04$ & $7.6 \mathrm{E}-05$ & $-70.00 \%$ & Ir-192m & $0.0 \mathrm{E}+00$ & $0.0 \mathrm{E}+00$ & \#DIV/0! \\
\hline $\mathrm{Ca}-47$ & $3.9 \mathrm{E}-04$ & $1.2 \mathrm{E}-04$ & $-70.00 \%$ & Pt-193 & $2.3 \mathrm{E}-05$ & $8.2 \mathrm{E}-06$ & $-65.00 \%$ \\
\hline Ti-44 & $4.0 \mathrm{E}-02$ & $7.7 \mathrm{E}-03$ & $-81.00 \%$ & Hg-194 & $6.0 \mathrm{E}-01$ & $3.6 \mathrm{E}+00$ & $510.00 \%$ \\
\hline V-49 & $1.1 \mathrm{E}-06$ & $1.1 \mathrm{E}-05$ & $870.00 \%$ & $\mathrm{Hg}-203$ & $2.1 \mathrm{E}-02$ & $1.3 \mathrm{E}-01$ & $510.00 \%$ \\
\hline $\mathrm{Cr}-51$ & $5.3 \mathrm{E}-05$ & $1.1 \mathrm{E}-05$ & $-80.00 \%$ & $\mathrm{~Pb}-202$ & $2.5 \mathrm{E}-02$ & $2.1 \mathrm{E}-03$ & $-91.67 \%$ \\
\hline Mn-53 & $2.1 \mathrm{E}-05$ & $5.1 \mathrm{E}-05$ & $140.00 \%$ & $\mathrm{~Pb}-205$ & $9.6 \mathrm{E}-04$ & $8.0 \mathrm{E}-05$ & $-91.67 \%$ \\
\hline $\mathrm{Mn}-54$ & $2.3 \mathrm{E}-03$ & $1.4 \mathrm{E}-03$ & $-40.00 \%$ & $\mathrm{~Pb}-210$ & $3.3 \mathrm{E}+00$ & $2.7 \mathrm{E}-01$ & $-91.67 \%$ \\
\hline $\mathrm{Fe}-55$ & $1.2 \mathrm{E}-04$ & $2.1 \mathrm{E}-04$ & $70.00 \%$ & Bi-207 & $1.6 \mathrm{E}-04$ & $1.6 \mathrm{E}-04$ & $0.00 \%$ \\
\hline Fe-60 & $3.2 \mathrm{E}-02$ & $5.4 \mathrm{E}-02$ & $70.00 \%$ & Bi-210 & $1.4 \mathrm{E}-04$ & $1.4 \mathrm{E}-04$ & $0.00 \%$ \\
\hline Co-58 & $3.7 \mathrm{E}-04$ & $5.5 \mathrm{E}-04$ & $52.00 \%$ & $\mathrm{Bi}-210 \mathrm{~m}$ & $2.7 \mathrm{E}-03$ & $2.7 \mathrm{E}-03$ & $0.00 \%$ \\
\hline Co-60 & $2.8 \mathrm{E}-03$ & $4.2 \mathrm{E}-03$ & $52.00 \%$ & Po-210 & $1.7 \mathrm{E}+00$ & $1.2 \mathrm{E}-01$ & $-92.80 \%$ \\
\hline Ni-59 & $4.3 \mathrm{E}-05$ & $8.9 \mathrm{E}-06$ & $-79.00 \%$ & Ra-223 & $5.2 \mathrm{E}-02$ & $4.1 \mathrm{E}-03$ & $-92.00 \%$ \\
\hline Ni-63 & $1.1 \mathrm{E}-04$ & $2.4 \mathrm{E}-05$ & $-79.00 \%$ & Ra-224 & $2.4 \mathrm{E}-02$ & $1.9 \mathrm{E}-03$ & $-92.00 \%$ \\
\hline $\mathrm{Cu}-64$ & $1.3 \mathrm{E}-05$ & $1.5 \mathrm{E}-05$ & $15.00 \%$ & Ra-225 & $3.0 \mathrm{E}-02$ & $2.4 \mathrm{E}-03$ & $-92.00 \%$ \\
\hline
\end{tabular}


SRNL-STI-2010-00447, REVISION 0

Table 15. LADTAP XL Maximally Exposed Individual Fish Ingestion Pathway Comparison (mrem/yr) (continued)

\begin{tabular}{|c|c|c|c|c|c|c|c|}
\hline Nuclide & $\begin{array}{c}\text { Hamby Ladtap } \\
\text { Fish Ingestion }\end{array}$ & $\begin{array}{l}\text { Jannik Ladtap } \\
\text { Fish Ingestion }\end{array}$ & $\%$ Diff & Nuclide & $\begin{array}{c}\text { Hamby Ladtap } \\
\text { Fish Ingestion }\end{array}$ & $\begin{array}{c}\text { Jannik Ladtap } \\
\text { Fish Ingestion }\end{array}$ & $\%$ Diff \\
\hline $\mathrm{Zn}-65$ & 5.9E-02 & $1.0 \mathrm{E}-01$ & $70.00 \%$ & Ra-226 & $1.2 \mathrm{E}-01$ & $9.4 \mathrm{E}-03$ & $-92.00 \%$ \\
\hline Ge-68 & $7.8 \mathrm{E}-03$ & $9.3 \mathrm{E}-03$ & $20.12 \%$ & Ra-228 & $1.3 \mathrm{E}-01$ & $1.0 \mathrm{E}-02$ & $-92.00 \%$ \\
\hline Se-75 & $3.1 \mathrm{E}-03$ & $1.1 \mathrm{E}-01$ & $3429.41 \%$ & Ac-227 & $7.4 \mathrm{E}-01$ & 7.4E-01 & $0.00 \%$ \\
\hline Se-79 & $3.0 \mathrm{E}-03$ & $1.1 \mathrm{E}-01$ & $3429.41 \%$ & Th-227 & $2.1 \mathrm{E}-03$ & $4.3 \mathrm{E}-04$ & $-80.00 \%$ \\
\hline $\mathrm{Rb}-87$ & $2.0 \mathrm{E}-02$ & $5.0 \mathrm{E}-02$ & $145.00 \%$ & Th-228 & $2.4 \mathrm{E}-02$ & $4.8 \mathrm{E}-03$ & $-80.00 \%$ \\
\hline Sr-89 & $5.4 \mathrm{E}-04$ & $5.2 \mathrm{E}-05$ & $-90.33 \%$ & Th-229 & $2.2 \mathrm{E}-01$ & $4.5 \mathrm{E}-02$ & $-80.00 \%$ \\
\hline Sr-90 & $8.3 \mathrm{E}-03$ & $8.0 \mathrm{E}-04$ & $-90.33 \%$ & Th-230 & $3.4 \mathrm{E}-02$ & $6.8 \mathrm{E}-03$ & $-80.00 \%$ \\
\hline Y-90 & $3.2 \mathrm{E}-04$ & $5.1 \mathrm{E}-04$ & $60.00 \%$ & Th-231 & $2.3 \mathrm{E}-05$ & $4.5 \mathrm{E}-06$ & $-80.00 \%$ \\
\hline Y-91 & $4.6 \mathrm{E}-04$ & 7.4E-04 & $60.00 \%$ & Th-232 & $1.8 \mathrm{E}-01$ & $3.6 \mathrm{E}-02$ & $-80.00 \%$ \\
\hline Mo-93 & $2.8 \mathrm{E}-05$ & $5.3 \mathrm{E}-06$ & $-81.00 \%$ & Th-234 & $7.8 \mathrm{E}-04$ & $1.6 \mathrm{E}-04$ & $-80.00 \%$ \\
\hline Mo-99 & $5.7 \mathrm{E}-05$ & $1.1 \mathrm{E}-05$ & $-81.00 \%$ & $\mathrm{~Pa}-230$ & $1.2 \mathrm{E}-04$ & $1.1 \mathrm{E}-04$ & $-11.50 \%$ \\
\hline $\mathrm{Nb}-93 \mathrm{~m}$ & $3.4 \mathrm{E}-01$ & $3.4 \mathrm{E}-03$ & $-99.00 \%$ & $\mathrm{~Pa}-231$ & $2.6 \mathrm{E}-01$ & $2.3 \mathrm{E}-01$ & $-11.50 \%$ \\
\hline $\mathrm{Nb}-94$ & $3.3 \mathrm{E}-01$ & $3.3 \mathrm{E}-03$ & $-99.00 \%$ & $\mathrm{~Pa}-233$ & $7.5 \mathrm{E}-05$ & $6.7 \mathrm{E}-05$ & $-11.50 \%$ \\
\hline $\mathrm{Nb}-95$ & $1.3 \mathrm{E}-01$ & $1.3 \mathrm{E}-03$ & $-99.00 \%$ & U-232 & $2.8 \mathrm{E}-02$ & $2.7 \mathrm{E}-03$ & $-90.40 \%$ \\
\hline Zr-93 & $1.1 \mathrm{E}-05$ & $7.5 \mathrm{E}-05$ & $566.67 \%$ & U-233 & $1.1 \mathrm{E}-03$ & $5.5 \mathrm{E}-04$ & $-52.00 \%$ \\
\hline Zr-95 & $2.3 \mathrm{E}-05$ & $1.6 \mathrm{E}-04$ & $566.67 \%$ & U-234 & $1.1 \mathrm{E}-03$ & $5.3 \mathrm{E}-04$ & $-52.00 \%$ \\
\hline Tc-96 & $6.2 \mathrm{E}-05$ & $8.3 \mathrm{E}-05$ & $33.33 \%$ & U-235 & $1.1 \mathrm{E}-03$ & $5.1 \mathrm{E}-04$ & $-52.00 \%$ \\
\hline Tc-97 & $4.8 \mathrm{E}-06$ & $6.4 \mathrm{E}-06$ & $33.33 \%$ & U-236 & $1.1 \mathrm{E}-03$ & $5.1 \mathrm{E}-04$ & $-52.00 \%$ \\
\hline Tc-98 & $1.5 \mathrm{E}-04$ & $2.0 \mathrm{E}-04$ & $33.33 \%$ & U-237 & $9.3 \mathrm{E}-06$ & $4.5 \mathrm{E}-06$ & $-52.00 \%$ \\
\hline Tc-99 & $4.1 \mathrm{E}-05$ & $5.5 \mathrm{E}-05$ & $33.33 \%$ & U-238 & $9.8 \mathrm{E}-04$ & $4.7 \mathrm{E}-04$ & $-52.00 \%$ \\
\hline Ru-97 & $8.4 \mathrm{E}-06$ & $4.6 \mathrm{E}-05$ & $450.00 \%$ & $\mathrm{~Np}-236$ & $1.7 \mathrm{E}-02$ & $3.5 \mathrm{E}-02$ & $110.00 \%$ \\
\hline Ru-103 & $5.5 \mathrm{E}-05$ & $3.0 \mathrm{E}-04$ & $450.00 \%$ & $\mathrm{~Np}-237$ & $8.3 \mathrm{E}-02$ & $1.7 \mathrm{E}-01$ & $110.00 \%$ \\
\hline Ru-106 & $4.5 \mathrm{E}-04$ & $2.4 \mathrm{E}-03$ & $450.00 \%$ & $\mathrm{~Np}-239$ & $3.4 \mathrm{E}-05$ & $7.2 \mathrm{E}-05$ & $110.00 \%$ \\
\hline Pd-107 & $3.0 \mathrm{E}-06$ & $3.0 \mathrm{E}-06$ & $0.00 \%$ & Am-237 & $5.3 \mathrm{E}-18$ & $5.1 \mathrm{E}-17$ & $860.00 \%$ \\
\hline Ag-108m & $3.7 \mathrm{E}-05$ & $1.8 \mathrm{E}-03$ & $4661.90 \%$ & Am-241 & $2.4 \mathrm{E}-01$ & $2.3 \mathrm{E}+00$ & $860.00 \%$ \\
\hline Ag-110m & $5.4 \mathrm{E}-05$ & $2.6 \mathrm{E}-03$ & $4682.61 \%$ & Am-242m & $2.2 \mathrm{E}-01$ & $2.1 \mathrm{E}+00$ & $860.00 \%$ \\
\hline Cd-113 & $6.8 \mathrm{E}-02$ & $6.8 \mathrm{E}-02$ & $0.00 \%$ & Am-243 & $2.4 \mathrm{E}-01$ & $2.3 \mathrm{E}+00$ & $860.00 \%$ \\
\hline
\end{tabular}


SRNL-STI-2010-00447, REVISION 0

Table 15. LADTAP XL Maximally Exposed Individual Fish Ingestion Pathway Comparison (mrem/yr) (continued)

\begin{tabular}{|c|c|c|c|c|c|c|c|}
\hline Nuclide & $\begin{array}{l}\text { Hamby Ladtap } \\
\text { Fish Ingestion }\end{array}$ & $\begin{array}{l}\text { Jannik Ladtap } \\
\text { Fish Ingestion }\end{array}$ & $\%$ Diff & Nuclide & $\begin{array}{c}\text { Hamby Ladtap } \\
\text { Fish Ingestion }\end{array}$ & $\begin{array}{l}\text { Jannik Ladtap } \\
\text { Fish Ingestion }\end{array}$ & $\%$ Diff \\
\hline Cd-115m & $6.2 \mathrm{E}-03$ & $6.2 \mathrm{E}-03$ & $0.00 \%$ & $\mathrm{Pu}-237$ & $7.2 \mathrm{E}-06$ & $6.2 \mathrm{E}-05$ & $757.14 \%$ \\
\hline In-115 & $0.0 \mathrm{E}+00$ & $3.0 \mathrm{E}+00$ & $0.00 \%$ & $\mathrm{Pu}-238$ & $2.8 \mathrm{E}-02$ & $2.4 \mathrm{E}-01$ & $757.14 \%$ \\
\hline Sb-122 & 8.0E-06 & $3.0 \mathrm{E}-04$ & $3600.00 \%$ & Pu-239 & $3.2 \mathrm{E}-02$ & $2.7 \mathrm{E}-01$ & $757.14 \%$ \\
\hline $\mathrm{Sb}-124$ & $1.9 \mathrm{E}-05$ & 7.2E-04 & $3600.00 \%$ & $\mathrm{Pu}-240$ & $3.2 \mathrm{E}-02$ & $2.7 \mathrm{E}-01$ & $757.14 \%$ \\
\hline Sb-125 & $5.5 \mathrm{E}-06$ & $2.0 \mathrm{E}-04$ & $3600.00 \%$ & $\mathrm{Pu}-241$ & $6.4 \mathrm{E}-04$ & $5.5 \mathrm{E}-03$ & $757.14 \%$ \\
\hline Te-123 & $0.0 \mathrm{E}+00$ & $1.3 \mathrm{E}-03$ & $0.00 \%$ & $\mathrm{Pu}-242$ & $3.1 \mathrm{E}-02$ & $2.6 \mathrm{E}-01$ & $757.14 \%$ \\
\hline Te- $125 \mathrm{~m}$ & $2.8 \mathrm{E}-03$ & $1.1 \mathrm{E}-03$ & $-62.50 \%$ & $\mathrm{Pu}-244$ & $3.0 \mathrm{E}-02$ & $2.6 \mathrm{E}-01$ & $757.14 \%$ \\
\hline Sn-126 & $1.1 \mathrm{E}-01$ & $1.1 \mathrm{E}-01$ & $0.00 \%$ & $\mathrm{Cm}-241$ & $2.3 \mathrm{E}-04$ & $2.8 \mathrm{E}-04$ & $20.00 \%$ \\
\hline I-129 & $8.9 \mathrm{E}-03$ & $1.8 \mathrm{E}-02$ & $100.00 \%$ & $\mathrm{Cm}-242$ & $5.8 \mathrm{E}-03$ & $7.0 \mathrm{E}-03$ & $20.00 \%$ \\
\hline I-131 & $1.4 \mathrm{E}-03$ & $2.8 \mathrm{E}-03$ & $100.00 \%$ & Cm-243 & $1.5 \mathrm{E}-01$ & $1.9 \mathrm{E}-01$ & $20.00 \%$ \\
\hline Cs-134 & $4.7 \mathrm{E}-01$ & $4.7 \mathrm{E}-01$ & $0.00 \%$ & Cm-244 & $1.2 \mathrm{E}-01$ & $1.5 \mathrm{E}-01$ & $20.00 \%$ \\
\hline Cs-135 & $4.5 \mathrm{E}-02$ & $4.5 \mathrm{E}-02$ & $0.00 \%$ & Cm-245 & $2.4 \mathrm{E}-01$ & $2.9 \mathrm{E}-01$ & $20.00 \%$ \\
\hline Cs-137 & $3.2 \mathrm{E}-01$ & $3.2 \mathrm{E}-01$ & $0.00 \%$ & Cm-246 & $2.4 \mathrm{E}-01$ & $2.9 \mathrm{E}-01$ & $20.00 \%$ \\
\hline La-137 & $2.3 \mathrm{E}-05$ & $3.4 \mathrm{E}-05$ & $48.00 \%$ & $\mathrm{Cm}-247$ & $2.2 \mathrm{E}-01$ & $2.6 \mathrm{E}-01$ & $20.00 \%$ \\
\hline La-138 & $3.1 \mathrm{E}-04$ & $4.6 \mathrm{E}-04$ & $48.00 \%$ & Cm-248 & $8.5 \mathrm{E}-01$ & $1.0 \mathrm{E}+00$ & $20.00 \%$ \\
\hline La-140 & $1.8 \mathrm{E}-04$ & $2.7 \mathrm{E}-04$ & $48.00 \%$ & $\mathrm{Cm}-250$ & $4.1 \mathrm{E}+00$ & $5.0 \mathrm{E}+00$ & $20.00 \%$ \\
\hline Ba-140 & $6.4 \mathrm{E}-05$ & $1.9 \mathrm{E}-05$ & $-70.00 \%$ & Bk-247 & $1.2 \mathrm{E}-01$ & $1.2 \mathrm{E}-01$ & $0.00 \%$ \\
\hline $\mathrm{Ce}-141$ & $5.3 \mathrm{E}-06$ & $1.3 \mathrm{E}-04$ & $2400.00 \%$ & Bk-249 & $3.2 \mathrm{E}-04$ & $3.2 \mathrm{E}-04$ & $0.00 \%$ \\
\hline \multirow[t]{8}{*}{ Ce-144 } & $4.2 \mathrm{E}-05$ & $1.1 \mathrm{E}-03$ & $2400.00 \%$ & Cf-249 & $2.4 \mathrm{E}-01$ & $2.4 \mathrm{E}-01$ & $0.00 \%$ \\
\hline & & & & Cf-250 & $1.0 \mathrm{E}-01$ & $1.0 \mathrm{E}-01$ & $0.00 \%$ \\
\hline & & & & Cf-251 & $2.4 \mathrm{E}-01$ & $2.4 \mathrm{E}-01$ & $0.00 \%$ \\
\hline & & & & Cf-252 & $5.0 \mathrm{E}-02$ & $5.0 \mathrm{E}-02$ & $0.00 \%$ \\
\hline & & & & Es-253 & $4.8 \mathrm{E}-04$ & $1.2 \mathrm{E}-03$ & $150.00 \%$ \\
\hline & & & & $\begin{array}{c}\text { Unidentified } \\
\text { alpha }\end{array}$ & $3.2 \mathrm{E}-02$ & $2.7 \mathrm{E}-01$ & $757.14 \%$ \\
\hline & & & & $\begin{array}{c}\text { Unidentified } \\
\text { beta }\end{array}$ & $8.3 \mathrm{E}-03$ & $8.0 \mathrm{E}-04$ & $-90.33 \%$ \\
\hline & & & & TOTAL Dose & $1.9 \mathrm{E}+01$ & $2.8 \mathrm{E}+01$ & $51.91 \%$ \\
\hline
\end{tabular}


Table 16. LADTAP XL Maximally Exposed Individual Shoreline Exposure Pathway Comparison (mrem/yr)

\begin{tabular}{|c|c|c|c|c|c|c|c|}
\hline Nuclide & $\begin{array}{c}\text { Hamby Ladtap } \\
\text { Shoreline Pathway }\end{array}$ & $\begin{array}{c}\text { Jannik Ladtap } \\
\text { Shoreline Pathway }\end{array}$ & $\%$ Diff & Nuclide & $\begin{array}{c}\text { Hamby Ladtap } \\
\text { Shoreline Pathway }\end{array}$ & $\begin{array}{c}\text { Jannik Ladtap } \\
\text { Shoreline Pathway }\end{array}$ & $\%$ Diff \\
\hline $\mathrm{H}-3$ & $0.0 \mathrm{E}+00$ & $0.0 \mathrm{E}+00$ & & Sm-146 & $0.0 \mathrm{E}+00$ & $0.0 \mathrm{E}+00$ & \\
\hline $\mathrm{Be}-7$ & $1.6 \mathrm{E}-06$ & $1.4 \mathrm{E}-06$ & $-13.04 \%$ & Sm-147 & $0.0 \mathrm{E}+00$ & $0.0 \mathrm{E}+00$ & \\
\hline Be-10 & $0.0 \mathrm{E}+00$ & $0.0 \mathrm{E}+00$ & & Sm-151 & $2.7 \mathrm{E}-08$ & 2.3E-08 & $-13.04 \%$ \\
\hline C-14 & $0.0 \mathrm{E}+00$ & $0.0 \mathrm{E}+00$ & & Pm-147 & $2.3 \mathrm{E}-09$ & $2.0 \mathrm{E}-09$ & $-13.04 \%$ \\
\hline $\mathrm{Na}-22$ & $1.2 \mathrm{E}-03$ & $1.0 \mathrm{E}-03$ & $-13.04 \%$ & Eu-152 & $2.8 \mathrm{E}-03$ & $2.4 \mathrm{E}-03$ & $-13.04 \%$ \\
\hline $\mathrm{Na}-24$ & $4.3 \mathrm{E}-07$ & $3.7 \mathrm{E}-07$ & $-13.04 \%$ & Eu-154 & $2.2 \mathrm{E}-03$ & $1.9 \mathrm{E}-03$ & $-13.04 \%$ \\
\hline Al-26 & $1.5 \mathrm{E}-02$ & $1.3 \mathrm{E}-02$ & $-13.04 \%$ & Eu-155 & $7.6 \mathrm{E}-05$ & $6.6 \mathrm{E}-05$ & $-13.04 \%$ \\
\hline P-32 & $0.0 \mathrm{E}+00$ & $0.0 \mathrm{E}+00$ & & Gd-152 & $0.0 \mathrm{E}+00$ & $0.0 \mathrm{E}+00$ & \\
\hline Si-32 & $0.0 \mathrm{E}+00$ & $0.0 \mathrm{E}+00$ & & Ho-166m & $9.7 \mathrm{E}-03$ & $8.4 \mathrm{E}-03$ & $-13.04 \%$ \\
\hline S-35 & $0.0 \mathrm{E}+00$ & $0.0 \mathrm{E}+00$ & & Lu-176 & $0.0 \mathrm{E}+00$ & $0.0 \mathrm{E}+00$ & \\
\hline Cl-36 & $2.8 \mathrm{E}-10$ & $2.4 \mathrm{E}-10$ & $-13.04 \%$ & Ta-180 & $0.0 \mathrm{E}+00$ & $0.0 \mathrm{E}+00$ & \\
\hline K-40 & $8.2 \mathrm{E}-04$ & $7.1 \mathrm{E}-04$ & $-13.04 \%$ & Hf-182 & $0.0 \mathrm{E}+00$ & $0.0 \mathrm{E}+00$ & \\
\hline $\mathrm{K}-43$ & $2.6 \mathrm{E}-07$ & $2.3 \mathrm{E}-07$ & $-13.04 \%$ & Re-186m & $0.0 \mathrm{E}+00$ & $0.0 \mathrm{E}+00$ & \\
\hline $\mathrm{Ca}-41$ & $1.2 \mathrm{E}-07$ & $1.1 \mathrm{E}-07$ & $-13.04 \%$ & Re-187 & $0.0 \mathrm{E}+00$ & $0.0 \mathrm{E}+00$ & \\
\hline $\mathrm{Ca}-45$ & $2.1 \mathrm{E}-14$ & $1.8 \mathrm{E}-14$ & $-13.04 \%$ & Ir-192m & $0.0 \mathrm{E}+00$ & $0.0 \mathrm{E}+00$ & \\
\hline $\mathrm{Ca}-47$ & $2.2 \mathrm{E}-06$ & $1.9 \mathrm{E}-06$ & $-13.04 \%$ & Pt-193 & $2.5 \mathrm{E}-06$ & $2.2 \mathrm{E}-06$ & $-13.04 \%$ \\
\hline Ti-44 & $7.6 \mathrm{E}-04$ & $6.6 \mathrm{E}-04$ & $-13.04 \%$ & Hg-194 & $0.0 \mathrm{E}+00$ & $0.0 \mathrm{E}+00$ & \\
\hline $\mathrm{V}-49$ & $1.5 \mathrm{E}-08$ & $1.3 \mathrm{E}-08$ & $-13.04 \%$ & $\mathrm{Hg}-203$ & $6.7 \mathrm{E}-06$ & 5.9E-06 & $-13.04 \%$ \\
\hline Cr-51 & $5.4 \mathrm{E}-07$ & $4.7 \mathrm{E}-07$ & $-13.04 \%$ & $\mathrm{~Pb}-202$ & $0.0 \mathrm{E}+00$ & $0.0 \mathrm{E}+00$ & \\
\hline Mn-53 & 9.4E-07 & $8.2 \mathrm{E}-07$ & $-13.04 \%$ & $\mathrm{~Pb}-205$ & $4.5 \mathrm{E}-06$ & $3.9 \mathrm{E}-06$ & $-13.04 \%$ \\
\hline Mn-54 & $1.5 \mathrm{E}-04$ & $1.3 \mathrm{E}-04$ & $-13.04 \%$ & $\mathrm{~Pb}-210$ & $1.0 \mathrm{E}-05$ & 8.9E-06 & $-13.04 \%$ \\
\hline $\mathrm{Fe}-55$ & $1.3 \mathrm{E}-07$ & $1.1 \mathrm{E}-07$ & $-13.04 \%$ & Bi-207 & $6.1 \mathrm{E}-03$ & $5.3 \mathrm{E}-03$ & $-13.04 \%$ \\
\hline $\mathrm{Fe}-60$ & $0.0 \mathrm{E}+00$ & $0.0 \mathrm{E}+00$ & & $\mathrm{Bi}-210$ & $0.0 \mathrm{E}+00$ & $0.0 \mathrm{E}+00$ & \\
\hline Co-58 & $4.1 \mathrm{E}-05$ & $3.5 \mathrm{E}-05$ & $-13.04 \%$ & $\mathrm{Bi}-210 \mathrm{~m}$ & $0.0 \mathrm{E}+00$ & $0.0 \mathrm{E}+00$ & \\
\hline Co-60 & $2.6 \mathrm{E}-03$ & $2.2 \mathrm{E}-03$ & $-13.04 \%$ & Po-210 & $7.0 \mathrm{E}-10$ & $6.1 \mathrm{E}-10$ & $-13.04 \%$ \\
\hline Ni-59 & $2.5 \mathrm{E}-06$ & $2.2 \mathrm{E}-06$ & $-13.04 \%$ & Ra-223 & $9.6 \mathrm{E}-07$ & $8.4 \mathrm{E}-07$ & $-13.04 \%$ \\
\hline Ni-63 & $0.0 \mathrm{E}+00$ & $0.0 \mathrm{E}+00$ & & Ra-224 & $1.9 \mathrm{E}-08$ & $1.7 \mathrm{E}-08$ & $-13.04 \%$ \\
\hline $\mathrm{Cu}-64$ & $1.6 \mathrm{E}-08$ & $1.4 \mathrm{E}-08$ & $-13.04 \%$ & Ra-225 & $6.3 \mathrm{E}-08$ & $5.5 \mathrm{E}-08$ & $-13.04 \%$ \\
\hline
\end{tabular}


SRNL-STI-2010-00447, REVISION 0

Table 16. LADTAP XL Maximally Exposed Individual Shoreline Exposure Pathway Comparison (mrem/yr) (continued)

\begin{tabular}{|c|c|c|c|c|c|c|c|}
\hline Nuclide & $\begin{array}{c}\text { Hamby Ladtap } \\
\text { Shoreline Pathway }\end{array}$ & $\begin{array}{c}\text { Jannik Ladtap } \\
\text { Shoreline Pathway }\end{array}$ & $\%$ Diff & Nuclide & $\begin{array}{c}\text { Hamby Ladtap } \\
\text { Shoreline Pathway }\end{array}$ & $\begin{array}{c}\text { Jannik Ladtap } \\
\text { Shoreline Pathway }\end{array}$ & $\%$ Diff \\
\hline $\mathrm{Zn}-65$ & $7.8 \mathrm{E}-05$ & $6.8 \mathrm{E}-05$ & $-13.04 \%$ & $\mathrm{Ra}-226$ & $4.5 \mathrm{E}-05$ & $3.9 \mathrm{E}-05$ & $-13.04 \%$ \\
\hline Ge-68 & $1.9 \mathrm{E}-07$ & $1.7 \mathrm{E}-07$ & $-13.04 \%$ & Ra-228 & $8.2 \mathrm{E}-13$ & $7.1 \mathrm{E}-13$ & $-13.04 \%$ \\
\hline $\mathrm{Se}-75$ & $3.0 \mathrm{E}-05$ & $2.6 \mathrm{E}-05$ & $-13.04 \%$ & Ac-227 & $7.1 \mathrm{E}-07$ & $6.2 \mathrm{E}-07$ & $-13.04 \%$ \\
\hline Se-79 & $0.0 \mathrm{E}+00$ & $0.0 \mathrm{E}+00$ & & Th-227 & $1.3 \mathrm{E}-06$ & $1.1 \mathrm{E}-06$ & $-13.04 \%$ \\
\hline $\mathrm{Rb}-87$ & $0.0 \mathrm{E}+00$ & $0.0 \mathrm{E}+00$ & & Th-228 & $1.1 \mathrm{E}-06$ & $9.9 \mathrm{E}-07$ & $-13.04 \%$ \\
\hline Sr-89 & $4.0 \mathrm{E}-09$ & $3.5 \mathrm{E}-09$ & $-13.04 \%$ & Th-229 & $6.2 \mathrm{E}-04$ & $5.4 \mathrm{E}-04$ & $-13.04 \%$ \\
\hline Sr-90 & $0.0 \mathrm{E}+00$ & $0.0 \mathrm{E}+00$ & & Th-230 & $5.4 \mathrm{E}-06$ & $4.7 \mathrm{E}-06$ & $-13.04 \%$ \\
\hline $\mathrm{Y}-90$ & $0.0 \mathrm{E}+00$ & $0.0 \mathrm{E}+00$ & & Th-231 & $6.2 \mathrm{E}-09$ & $5.4 \mathrm{E}-09$ & $-13.04 \%$ \\
\hline Y-91 & $1.1 \mathrm{E}-07$ & $9.8 \mathrm{E}-08$ & $-13.04 \%$ & Th-232 & $4.0 \mathrm{E}-06$ & $3.4 \mathrm{E}-06$ & $-13.04 \%$ \\
\hline Мo-93 & $3.4 \mathrm{E}-05$ & $3.0 \mathrm{E}-05$ & $-13.04 \%$ & Th-234 & $1.4 \mathrm{E}-07$ & $1.2 \mathrm{E}-07$ & $-13.04 \%$ \\
\hline Мo-99 & $3.7 \mathrm{E}-07$ & $3.2 \mathrm{E}-07$ & $-13.04 \%$ & $\mathrm{~Pa}-230$ & $6.6 \mathrm{E}-06$ & $5.7 \mathrm{E}-06$ & $-13.04 \%$ \\
\hline $\mathrm{Nb}-93 \mathrm{~m}$ & $2.6 \mathrm{E}-06$ & $2.3 \mathrm{E}-06$ & $-13.04 \%$ & $\mathrm{~Pa}-231$ & $2.1 \mathrm{E}-04$ & $1.9 \mathrm{E}-04$ & $-13.04 \%$ \\
\hline $\mathrm{Nb}-94$ & $9.5 \mathrm{E}-03$ & $8.2 \mathrm{E}-03$ & $-13.04 \%$ & $\mathrm{~Pa}-233$ & $3.7 \mathrm{E}-06$ & $3.2 \mathrm{E}-06$ & $-13.04 \%$ \\
\hline $\mathrm{Nb}-95$ & $1.6 \mathrm{E}-05$ & $1.4 \mathrm{E}-05$ & $-13.04 \%$ & U-232 & $5.1 \mathrm{E}-06$ & $4.4 \mathrm{E}-06$ & $-13.04 \%$ \\
\hline $\mathrm{Zr}-93$ & $0.0 \mathrm{E}+00$ & $0.0 \mathrm{E}+00$ & & U-233 & $3.0 \mathrm{E}-06$ & $2.6 \mathrm{E}-06$ & $-13.04 \%$ \\
\hline $\mathrm{Zr}-95$ & $5.7 \mathrm{E}-05$ & $4.9 \mathrm{E}-05$ & $-13.04 \%$ & U-234 & $4.8 \mathrm{E}-06$ & $4.2 \mathrm{E}-06$ & $-13.04 \%$ \\
\hline Tc-96 & $5.4 \mathrm{E}-06$ & $4.7 \mathrm{E}-06$ & $-13.04 \%$ & U-235 & $1.0 \mathrm{E}-03$ & $8.8 \mathrm{E}-04$ & $-13.04 \%$ \\
\hline Tc-97 & $3.8 \mathrm{E}-05$ & $3.3 \mathrm{E}-05$ & $-13.04 \%$ & U-236 & $4.4 \mathrm{E}-06$ & $3.8 \mathrm{E}-06$ & $-13.04 \%$ \\
\hline Tc-98 & $8.4 \mathrm{E}-03$ & $7.3 \mathrm{E}-03$ & $-13.04 \%$ & U-237 & $5.7 \mathrm{E}-07$ & 5.0E-07 & $-13.04 \%$ \\
\hline Tc-99 & $3.7 \mathrm{E}-09$ & $3.2 \mathrm{E}-09$ & $-13.04 \%$ & U-238 & $3.8 \mathrm{E}-06$ & $3.3 \mathrm{E}-06$ & $-13.04 \%$ \\
\hline Ru-97 & $3.5 \mathrm{E}-07$ & $3.0 \mathrm{E}-07$ & $-13.04 \%$ & $\mathrm{~Np}-236$ & $9.4 \mathrm{E}-04$ & $8.2 \mathrm{E}-04$ & $-13.04 \%$ \\
\hline Ru-103 & $1.1 \mathrm{E}-05$ & 9.9E-06 & $-13.04 \%$ & $\mathrm{~Np}-237$ & $1.9 \mathrm{E}-04$ & $1.7 \mathrm{E}-04$ & $-13.04 \%$ \\
\hline Ru-106 & $4.5 \mathrm{E}-05$ & $4.0 \mathrm{E}-05$ & $-13.04 \%$ & $\mathrm{~Np}-239$ & $2.0 \mathrm{E}-07$ & $1.7 \mathrm{E}-07$ & $-13.04 \%$ \\
\hline Pd-107 & $0.0 \mathrm{E}+00$ & $0.0 \mathrm{E}+00$ & & Am-237 & $0.0 \mathrm{E}+00$ & $0.0 \mathrm{E}+00$ & \\
\hline Ag-108m & 8.9E-03 & $7.7 \mathrm{E}-03$ & $-13.04 \%$ & Am-241 & $1.7 \mathrm{E}-04$ & $1.5 \mathrm{E}-04$ & $-13.04 \%$ \\
\hline Ag-110m & 3.9E-04 & $3.4 \mathrm{E}-04$ & $-13.04 \%$ & Am-242m & $1.5 \mathrm{E}-05$ & $1.3 \mathrm{E}-05$ & $-13.04 \%$ \\
\hline Cd-113 & $0.0 \mathrm{E}+00$ & $0.0 \mathrm{E}+00$ & & Am-243 & $3.9 \mathrm{E}-04$ & $3.4 \mathrm{E}-04$ & $-13.04 \%$ \\
\hline
\end{tabular}


SRNL-STI-2010-00447, REVISION 0

Table 16. LADTAP XL Maximally Exposed Individual Shoreline Exposure Pathway Comparison (mrem/yr) (continued)

\begin{tabular}{|c|c|c|c|c|c|c|c|}
\hline Nuclide & $\begin{array}{c}\text { Hamby Ladtap } \\
\text { Shoreline Pathway }\end{array}$ & $\begin{array}{c}\text { Jannik Ladtap } \\
\text { Shoreline Pathway }\end{array}$ & $\%$ Diff & Nuclide & $\begin{array}{c}\text { Hamby Ladtap } \\
\text { Shoreline Pathway }\end{array}$ & $\begin{array}{c}\text { Jannik Ladtap } \\
\text { Shoreline Pathway }\end{array}$ & $\%$ Diff \\
\hline $\mathrm{Cd}-115 \mathrm{~m}$ & $5.4 \mathrm{E}-07$ & $4.7 \mathrm{E}-07$ & $-13.04 \%$ & $\mathrm{Pu}-237$ & $1.6 \mathrm{E}-06$ & $1.4 \mathrm{E}-06$ & $-13.04 \%$ \\
\hline In-115 & $0.0 \mathrm{E}+00$ & $0.0 \mathrm{E}+00$ & & $\mathrm{Pu}-238$ & $4.4 \mathrm{E}-06$ & $3.8 \mathrm{E}-06$ & $-13.04 \%$ \\
\hline $\mathrm{Sb}-122$ & $5.6 \mathrm{E}-07$ & $4.9 \mathrm{E}-07$ & $-13.04 \%$ & $\mathrm{Pu}-239$ & $2.2 \mathrm{E}-06$ & $2.0 \mathrm{E}-06$ & $-13.04 \%$ \\
\hline Sb-124 & $6.2 \mathrm{E}-05$ & $5.4 \mathrm{E}-05$ & $-13.04 \%$ & $\mathrm{Pu}-240$ & $4.9 \mathrm{E}-06$ & $4.2 \mathrm{E}-06$ & $-13.04 \%$ \\
\hline $\mathrm{Sb}-125$ & $2.6 \mathrm{E}-04$ & $2.3 \mathrm{E}-04$ & $-13.04 \%$ & $\mathrm{Pu}-241$ & $0.0 \mathrm{E}+00$ & $0.0 \mathrm{E}+00$ & \\
\hline Te-123 & $4.2 \mathrm{E}-05$ & $3.7 \mathrm{E}-05$ & $-13.04 \%$ & $\mathrm{Pu}-242$ & $4.1 \mathrm{E}-06$ & $3.5 \mathrm{E}-06$ & $-13.04 \%$ \\
\hline $\mathrm{Te}-125 \mathrm{~m}$ & $7.1 \mathrm{E}-07$ & $6.2 \mathrm{E}-07$ & $-13.04 \%$ & $\mathrm{Pu}-244$ & $3.5 \mathrm{E}-06$ & $3.0 \mathrm{E}-06$ & $-13.04 \%$ \\
\hline Sn-126 & $3.7 \mathrm{E}-04$ & $3.2 \mathrm{E}-04$ & $-13.04 \%$ & $\mathrm{Cm}-241$ & $0.0 \mathrm{E}+00$ & $0.0 \mathrm{E}+00$ & \\
\hline I-129 & $1.3 \mathrm{E}-04$ & $1.1 \mathrm{E}-04$ & $-13.04 \%$ & Cm-242 & $8.9 \mathrm{E}-08$ & $7.8 \mathrm{E}-08$ & $-13.04 \%$ \\
\hline I-131 & $1.8 \mathrm{E}-06$ & $1.5 \mathrm{E}-06$ & $-13.04 \%$ & $\mathrm{Cm}-243$ & $5.6 \mathrm{E}-04$ & $4.8 \mathrm{E}-04$ & $-13.04 \%$ \\
\hline Cs-134 & $7.0 \mathrm{E}-04$ & $6.1 \mathrm{E}-04$ & $-13.04 \%$ & $\mathrm{Cm}-244$ & $2.5 \mathrm{E}-06$ & $2.2 \mathrm{E}-06$ & $-13.04 \%$ \\
\hline Cs-135 & $0.0 \mathrm{E}+00$ & $0.0 \mathrm{E}+00$ & & $\mathrm{Cm}-245$ & $5.1 \mathrm{E}-04$ & 4.4E-04 & $-13.04 \%$ \\
\hline Cs-137 & $2.2 \mathrm{E}-03$ & $2.0 \mathrm{E}-03$ & $-13.04 \%$ & $\mathrm{Cm}-246$ & $4.4 \mathrm{E}-06$ & $3.8 \mathrm{E}-06$ & $-13.04 \%$ \\
\hline La-137 & $0.0 \mathrm{E}+00$ & $0.0 \mathrm{E}+00$ & & $\mathrm{Cm}-247$ & $2.0 \mathrm{E}-03$ & $1.7 \mathrm{E}-03$ & $-13.04 \%$ \\
\hline La-138 & $0.0 \mathrm{E}+00$ & $0.0 \mathrm{E}+00$ & & $\mathrm{Cm}-248$ & $3.5 \mathrm{E}-06$ & $3.0 \mathrm{E}-06$ & $-13.04 \%$ \\
\hline La-140 & $1.4 \mathrm{E}-06$ & $1.2 \mathrm{E}-06$ & $-13.04 \%$ & $\mathrm{Cm}-250$ & $0.0 \mathrm{E}+00$ & $0.0 \mathrm{E}+00$ & \\
\hline $\mathrm{Ba}-140$ & $1.7 \mathrm{E}-05$ & $1.4 \mathrm{E}-05$ & $-13.04 \%$ & Bk-247 & $0.0 \mathrm{E}+00$ & $0.0 \mathrm{E}+00$ & \\
\hline $\mathrm{Ce}-141$ & $1.6 \mathrm{E}-06$ & $1.4 \mathrm{E}-06$ & $-13.04 \%$ & Bk-249 & $0.0 \mathrm{E}+00$ & $0.0 \mathrm{E}+00$ & \\
\hline Ce-144 & $8.5 \mathrm{E}-06$ & 7.4E-06 & $-13.04 \%$ & Cf-249 & $2.0 \mathrm{E}-03$ & $1.8 \mathrm{E}-03$ & $-13.04 \%$ \\
\hline & & & & Cf-250 & $1.7 \mathrm{E}-06$ & $1.5 \mathrm{E}-06$ & $-13.04 \%$ \\
\hline & & & & Cf-251 & $8.0 \mathrm{E}-04$ & $6.9 \mathrm{E}-04$ & $-13.04 \%$ \\
\hline & & & & Cf-252 & $3.6 \mathrm{E}-07$ & $3.1 \mathrm{E}-07$ & $-13.04 \%$ \\
\hline & & & & Es-253 & 8.3E-09 & 7.2E-09 & $-13.04 \%$ \\
\hline & & & & Unidentified alpha & $2.2 \mathrm{E}-06$ & $2.0 \mathrm{E}-06$ & $-13.04 \%$ \\
\hline & & & & Unidentified beta & $0.0 \mathrm{E}+00$ & $0.0 \mathrm{E}+00$ & \\
\hline & & & & TOTAL Dose & 8.2E-02 & $7.1 \mathrm{E}-02$ & $-13.04 \%$ \\
\hline
\end{tabular}


Table 17. LADTAP XL Maximally Exposed Individual Swimming Exposure Pathway Comparison (mrem/yr)

\begin{tabular}{|c|c|c|c|c|c|c|c|}
\hline Nuclide & $\begin{array}{c}\text { Hamby Ladtap } \\
\text { Swimming Pathway }\end{array}$ & $\begin{array}{c}\text { Jannik Ladtap } \\
\text { Swimming Pathway }\end{array}$ & $\%$ Diff & Nuclide & $\begin{array}{c}\text { Hamby Ladtap } \\
\text { Swimming Pathway }\end{array}$ & $\begin{array}{c}\text { Jannik Ladtap } \\
\text { Swimming Pathway }\end{array}$ & $\%$ Diff \\
\hline H-3 & $2.2 \mathrm{E}-09$ & $3.5 \mathrm{E}-09$ & $57.30 \%$ & $\mathrm{Sm}-146$ & $0.0 \mathrm{E}+00$ & $0.0 \mathrm{E}+00$ & \\
\hline $\mathrm{Be}-7$ & $6.1 \mathrm{E}-08$ & $9.6 \mathrm{E}-08$ & $57.30 \%$ & Sm-147 & $0.0 \mathrm{E}+00$ & $0.0 \mathrm{E}+00$ & \\
\hline $\mathrm{Be}-10$ & $0.0 \mathrm{E}+00$ & $0.0 \mathrm{E}+00$ & & Sm-151 & $1.3 \mathrm{E}-12$ & $2.1 \mathrm{E}-12$ & $57.30 \%$ \\
\hline C-14 & $0.0 \mathrm{E}+00$ & $0.0 \mathrm{E}+00$ & & Pm-147 & $4.7 \mathrm{E}-12$ & $7.4 \mathrm{E}-12$ & $57.30 \%$ \\
\hline $\mathrm{Na}-22$ & $2.8 \mathrm{E}-06$ & $4.3 \mathrm{E}-06$ & $57.30 \%$ & Eu-152 & $1.5 \mathrm{E}-06$ & $2.3 \mathrm{E}-06$ & $57.30 \%$ \\
\hline $\mathrm{Na}-24$ & $1.9 \mathrm{E}-06$ & $3.0 \mathrm{E}-06$ & $57.30 \%$ & Eu-154 & $1.6 \mathrm{E}-06$ & $2.5 \mathrm{E}-06$ & $57.30 \%$ \\
\hline Al-26 & $3.6 \mathrm{E}-06$ & $5.6 \mathrm{E}-06$ & $57.30 \%$ & Eu-155 & $7.6 \mathrm{E}-08$ & $1.2 \mathrm{E}-07$ & $57.30 \%$ \\
\hline $\mathrm{P}-32$ & $0.0 \mathrm{E}+00$ & $0.0 \mathrm{E}+00$ & & Gd-152 & $0.0 \mathrm{E}+00$ & $0.0 \mathrm{E}+00$ & \\
\hline Si-32 & $0.0 \mathrm{E}+00$ & $0.0 \mathrm{E}+00$ & & Ho-166m & $3.6 \mathrm{E}-08$ & $5.7 \mathrm{E}-08$ & $57.30 \%$ \\
\hline S-35 & $0.0 \mathrm{E}+00$ & $0.0 \mathrm{E}+00$ & & Lu-176 & $0.0 \mathrm{E}+00$ & $0.0 \mathrm{E}+00$ & \\
\hline $\mathrm{Cl}-36$ & $1.2 \mathrm{E}-14$ & $1.9 \mathrm{E}-14$ & $57.30 \%$ & Ta-180 & $0.0 \mathrm{E}+00$ & $0.0 \mathrm{E}+00$ & \\
\hline $\mathrm{K}-40$ & $2.0 \mathrm{E}-07$ & $3.1 \mathrm{E}-07$ & $57.30 \%$ & Hf-182 & $0.0 \mathrm{E}+00$ & $0.0 \mathrm{E}+00$ & \\
\hline $\mathrm{K}-43$ & $5.7 \mathrm{E}-07$ & $9.0 \mathrm{E}-07$ & $57.30 \%$ & Re-186m & $0.0 \mathrm{E}+00$ & $0.0 \mathrm{E}+00$ & \\
\hline $\mathrm{Ca}-41$ & $4.4 \mathrm{E}-12$ & $6.9 \mathrm{E}-12$ & $57.30 \%$ & Re-187 & $0.0 \mathrm{E}+00$ & $0.0 \mathrm{E}+00$ & \\
\hline $\mathrm{Ca}-45$ & $2.4 \mathrm{E}-17$ & $3.7 \mathrm{E}-17$ & $57.30 \%$ & Ir-192m & $0.0 \mathrm{E}+00$ & $0.0 \mathrm{E}+00$ & \\
\hline Ca-47 & $1.2 \mathrm{E}-06$ & $1.8 \mathrm{E}-06$ & $57.30 \%$ & Pt-193 & $6.3 \mathrm{E}-11$ & $1.0 \mathrm{E}-10$ & $57.30 \%$ \\
\hline $\mathrm{Ti}-44$ & $1.8 \mathrm{E}-07$ & $2.9 \mathrm{E}-07$ & $57.30 \%$ & Hg-194 & $0.0 \mathrm{E}+00$ & $0.0 \mathrm{E}+00$ & \\
\hline $\mathrm{V}-49$ & $1.3 \mathrm{E}-11$ & $2.1 \mathrm{E}-11$ & $57.30 \%$ & Hg-203 & $2.8 \mathrm{E}-07$ & $4.5 \mathrm{E}-07$ & $57.30 \%$ \\
\hline Cr-51 & $3.8 \mathrm{E}-08$ & $6.1 \mathrm{E}-08$ & $57.30 \%$ & $\mathrm{~Pb}-202$ & $0.0 \mathrm{E}+00$ & $0.0 \mathrm{E}+00$ & \\
\hline $\mathrm{Mn}-53$ & $2.5 \mathrm{E}-11$ & $3.9 \mathrm{E}-11$ & $57.30 \%$ & $\mathrm{~Pb}-205$ & $8.3 \mathrm{E}-11$ & $1.3 \mathrm{E}-10$ & $57.30 \%$ \\
\hline $\mathrm{Mn}-54$ & $1.1 \mathrm{E}-06$ & $1.7 \mathrm{E}-06$ & $57.30 \%$ & $\mathrm{~Pb}-210$ & 1.9E-09 & $3.0 \mathrm{E}-09$ & $57.30 \%$ \\
\hline $\mathrm{Fe}-55$ & $3.3 \mathrm{E}-11$ & $5.1 \mathrm{E}-11$ & $57.30 \%$ & Bi-207 & $2.0 \mathrm{E}-06$ & $3.1 \mathrm{E}-06$ & $57.30 \%$ \\
\hline Fe-60 & $0.0 \mathrm{E}+00$ & $0.0 \mathrm{E}+00$ & & $\mathrm{Bi}-210$ & $0.0 \mathrm{E}+00$ & $0.0 \mathrm{E}+00$ & \\
\hline Co-58 & $1.2 \mathrm{E}-06$ & $1.9 \mathrm{E}-06$ & $57.30 \%$ & $\mathrm{Bi}-210 \mathrm{~m}$ & $0.0 \mathrm{E}+00$ & $0.0 \mathrm{E}+00$ & \\
\hline Co-60 & $4.3 \mathrm{E}-06$ & $6.8 \mathrm{E}-06$ & $57.30 \%$ & Po-210 & $1.1 \mathrm{E}-11$ & $1.7 \mathrm{E}-11$ & $57.30 \%$ \\
\hline $\mathrm{Ni}-59$ & $5.5 \mathrm{E}-11$ & $8.7 \mathrm{E}-11$ & $57.30 \%$ & Ra-223 & $1.6 \mathrm{E}-07$ & $2.5 \mathrm{E}-07$ & $57.30 \%$ \\
\hline Ni-63 & $0.0 \mathrm{E}+00$ & $0.0 \mathrm{E}+00$ & & Ra-224 & $1.0 \mathrm{E}-08$ & $1.6 \mathrm{E}-08$ & $57.30 \%$ \\
\hline $\mathrm{Cu}-64$ & $6.3 \mathrm{E}-08$ & 9.9E-08 & $57.30 \%$ & Ra-225 & $9.3 \mathrm{E}-09$ & $1.5 \mathrm{E}-08$ & $57.30 \%$ \\
\hline
\end{tabular}


SRNL-STI-2010-00447, REVISION 0

Table 17. LADTAP XL Maximally Exposed Individual Swimming Exposure Pathway Comparison (mrem/yr) (continued)

\begin{tabular}{|c|c|c|c|c|c|c|c|}
\hline Nuclide & $\begin{array}{c}\text { Hamby Ladtap } \\
\text { Swimming Pathway }\end{array}$ & $\begin{array}{c}\text { Jannik Ladtap } \\
\text { Swimming Pathway }\end{array}$ & $\%$ Diff & Nuclide & $\begin{array}{c}\text { Hamby Ladtap } \\
\text { Swimming Pathway }\end{array}$ & $\begin{array}{c}\text { Jannik Ladtap } \\
\text { Swimming Pathway }\end{array}$ & $\%$ Diff \\
\hline Zn-65 & $7.4 \mathrm{E}-07$ & $1.2 \mathrm{E}-06$ & $57.30 \%$ & Ra-226 & $8.7 \mathrm{E}-09$ & $1.4 \mathrm{E}-08$ & $57.30 \%$ \\
\hline Ge-68 & $1.3 \mathrm{E}-10$ & $2.0 \mathrm{E}-10$ & $57.30 \%$ & Ra-228 & $9.1 \mathrm{E}-17$ & $1.4 \mathrm{E}-16$ & $57.30 \%$ \\
\hline Se-75 & $4.9 \mathrm{E}-07$ & $7.8 \mathrm{E}-07$ & $57.30 \%$ & Ac- 227 & $1.6 \mathrm{E}-10$ & $2.6 \mathrm{E}-10$ & $57.30 \%$ \\
\hline Se-79 & $0.0 \mathrm{E}+00$ & $0.0 \mathrm{E}+00$ & & Th-227 & $1.3 \mathrm{E}-07$ & $2.0 \mathrm{E}-07$ & $57.30 \%$ \\
\hline $\mathrm{Rb}-87$ & $0.0 \mathrm{E}+00$ & $0.0 \mathrm{E}+00$ & & Th-228 & $2.6 \mathrm{E}-09$ & 4.1E-09 & $57.30 \%$ \\
\hline Sr-89 & $1.7 \mathrm{E}-10$ & $2.7 \mathrm{E}-10$ & $57.30 \%$ & Th-229 & $1.1 \mathrm{E}-07$ & $1.8 \mathrm{E}-07$ & $57.30 \%$ \\
\hline Sr-90 & $0.0 \mathrm{E}+00$ & $0.0 \mathrm{E}+00$ & & Th-230 & $5.3 \mathrm{E}-10$ & $8.3 \mathrm{E}-10$ & $57.30 \%$ \\
\hline$Y-90$ & $0.0 \mathrm{E}+00$ & $0.0 \mathrm{E}+00$ & & Th-231 & $8.1 \mathrm{E}-09$ & $1.3 \mathrm{E}-08$ & $57.30 \%$ \\
\hline Y-91 & $4.6 \mathrm{E}-09$ & 7.2E-09 & $57.30 \%$ & Th-232 & $2.6 \mathrm{E}-10$ & $4.0 \mathrm{E}-10$ & $57.30 \%$ \\
\hline Мo-93 & $1.1 \mathrm{E}-09$ & $1.8 \mathrm{E}-09$ & $57.30 \%$ & Th-234 & $1.0 \mathrm{E}-08$ & $1.6 \mathrm{E}-08$ & $57.30 \%$ \\
\hline Mo-99 & $2.7 \mathrm{E}-07$ & $4.2 \mathrm{E}-07$ & $57.30 \%$ & $\mathrm{~Pa}-230$ & $8.1 \mathrm{E}-07$ & $1.3 \mathrm{E}-06$ & $57.30 \%$ \\
\hline $\mathrm{Nb}-93 \mathrm{~m}$ & $2.0 \mathrm{E}-10$ & $3.1 \mathrm{E}-10$ & $57.30 \%$ & $\mathrm{~Pa}-231$ & $3.8 \mathrm{E}-08$ & $6.0 \mathrm{E}-08$ & $57.30 \%$ \\
\hline $\mathrm{Nb}-94$ & $2.0 \mathrm{E}-06$ & $3.2 \mathrm{E}-06$ & $57.30 \%$ & $\mathrm{~Pa}-233$ & $2.6 \mathrm{E}-07$ & $4.1 \mathrm{E}-07$ & $57.30 \%$ \\
\hline $\mathrm{Nb}-95$ & $9.5 \mathrm{E}-07$ & $1.5 \mathrm{E}-06$ & $57.30 \%$ & U-232 & $3.6 \mathrm{E}-10$ & $5.7 \mathrm{E}-10$ & $57.30 \%$ \\
\hline Zr-93 & $0.0 \mathrm{E}+00$ & $0.0 \mathrm{E}+00$ & & U-233 & $3.1 \mathrm{E}-10$ & $4.9 \mathrm{E}-10$ & $57.30 \%$ \\
\hline Zr-95 & $9.2 \mathrm{E}-07$ & $1.5 \mathrm{E}-06$ & $57.30 \%$ & U-234 & $2.1 \mathrm{E}-10$ & $3.3 \mathrm{E}-10$ & $57.30 \%$ \\
\hline Tc-96 & $2.7 \mathrm{E}-06$ & $4.3 \mathrm{E}-06$ & $57.30 \%$ & U-235 & $2.0 \mathrm{E}-07$ & $3.1 \mathrm{E}-07$ & $57.30 \%$ \\
\hline Tc-97 & $1.4 \mathrm{E}-09$ & $2.2 \mathrm{E}-09$ & $57.30 \%$ & U-236 & $1.7 \mathrm{E}-10$ & $2.7 \mathrm{E}-10$ & $57.30 \%$ \\
\hline Tc-98 & $1.8 \mathrm{E}-06$ & $2.8 \mathrm{E}-06$ & $57.30 \%$ & U-237 & $1.6 \mathrm{E}-07$ & $2.5 \mathrm{E}-07$ & $57.30 \%$ \\
\hline Tc-99 & $7.0 \mathrm{E}-13$ & $1.1 \mathrm{E}-12$ & $57.30 \%$ & U-238 & $1.5 \mathrm{E}-10$ & $2.3 \mathrm{E}-10$ & $57.30 \%$ \\
\hline Ru-97 & $2.3 \mathrm{E}-07$ & $3.6 \mathrm{E}-07$ & $57.30 \%$ & $\mathrm{~Np}-236$ & $1.7 \mathrm{E}-07$ & $2.7 \mathrm{E}-07$ & $57.30 \%$ \\
\hline Ru-103 & $5.9 \mathrm{E}-07$ & $9.2 \mathrm{E}-07$ & $57.30 \%$ & $\mathrm{~Np}-237$ & $3.0 \mathrm{E}-08$ & $4.8 \mathrm{E}-08$ & $57.30 \%$ \\
\hline Ru-106 & $2.6 \mathrm{E}-07$ & 4.1E-07 & $57.30 \%$ & Np-239 & $1.6 \mathrm{E}-07$ & $2.5 \mathrm{E}-07$ & $57.30 \%$ \\
\hline Pd-107 & $0.0 \mathrm{E}+00$ & $0.0 \mathrm{E}+00$ & & Am-237 & $0.0 \mathrm{E}+00$ & $0.0 \mathrm{E}+00$ & \\
\hline Ag-108m & $2.0 \mathrm{E}-06$ & $3.2 \mathrm{E}-06$ & $57.30 \%$ & Am-241 & $2.7 \mathrm{E}-08$ & $4.2 \mathrm{E}-08$ & $57.30 \%$ \\
\hline Ag-110m & $3.5 \mathrm{E}-06$ & $5.5 \mathrm{E}-06$ & $57.30 \%$ & Am-242m & $6.6 \mathrm{E}-10$ & $1.0 \mathrm{E}-09$ & $57.30 \%$ \\
\hline Cd-113 & $0.0 \mathrm{E}+00$ & $0.0 \mathrm{E}+00$ & & Am-243 & $6.9 \mathrm{E}-08$ & $1.1 \mathrm{E}-07$ & $57.30 \%$ \\
\hline
\end{tabular}


SRNL-STI-2010-00447, REVISION 0

Table 17. LADTAP XL Maximally Exposed Individual Swimming Exposure Pathway Comparison (mrem/yr) (continued)

\begin{tabular}{|c|c|c|c|c|c|c|c|}
\hline Nuclide & $\begin{array}{c}\text { Hamby Ladtap } \\
\text { Swimming Pathway }\end{array}$ & $\begin{array}{c}\text { Jannik Ladtap } \\
\text { Swimming Pathway }\end{array}$ & $\%$ Diff & Nuclide & $\begin{array}{c}\text { Hamby Ladtap } \\
\text { Swimming Pathway }\end{array}$ & $\begin{array}{c}\text { Jannik Ladtap } \\
\text { Swimming Pathway }\end{array}$ & $\%$ Diff \\
\hline Cd-115m & $2.8 \mathrm{E}-08$ & $4.4 \mathrm{E}-08$ & $57.30 \%$ & $\mathrm{Pu}-237$ & $6.2 \mathrm{E}-08$ & $9.8 \mathrm{E}-08$ & $57.30 \%$ \\
\hline In-115 & $0.0 \mathrm{E}+00$ & $0.0 \mathrm{E}+00$ & & $\mathrm{Pu}-238$ & $1.3 \mathrm{E}-10$ & $2.0 \mathrm{E}-10$ & $57.30 \%$ \\
\hline Sb-122 & $4.3 \mathrm{E}-07$ & $6.7 \mathrm{E}-07$ & $57.30 \%$ & Pu-239 & $1.1 \mathrm{E}-10$ & $1.7 \mathrm{E}-10$ & $57.30 \%$ \\
\hline Sb-124 & $2.4 \mathrm{E}-06$ & $3.8 \mathrm{E}-06$ & $57.30 \%$ & $\mathrm{Pu}-240$ & $1.3 \mathrm{E}-10$ & $2.0 \mathrm{E}-10$ & $57.30 \%$ \\
\hline Sb-125 & $5.3 \mathrm{E}-07$ & $8.3 \mathrm{E}-07$ & $57.30 \%$ & $\mathrm{Pu}-241$ & $0.0 \mathrm{E}+00$ & $0.0 \mathrm{E}+00$ & \\
\hline Te-123 & $4.4 \mathrm{E}-09$ & 7.0E-09 & $57.30 \%$ & $\mathrm{Pu}-242$ & $1.1 \mathrm{E}-10$ & $1.7 \mathrm{E}-10$ & $57.30 \%$ \\
\hline Te- $125 \mathrm{~m}$ & $1.4 \mathrm{E}-08$ & $2.2 \mathrm{E}-08$ & $57.30 \%$ & $\mathrm{Pu}-244$ & $7.8 \mathrm{E}-11$ & $1.2 \mathrm{E}-10$ & $57.30 \%$ \\
\hline Sn-126 & $6.5 \mathrm{E}-08$ & $1.0 \mathrm{E}-07$ & $57.30 \%$ & $\mathrm{Cm}-241$ & $0.0 \mathrm{E}+00$ & $0.0 \mathrm{E}+00$ & \\
\hline I-129 & $1.2 \mathrm{E}-08$ & $1.9 \mathrm{E}-08$ & $57.30 \%$ & $\mathrm{Cm}-242$ & $1.4 \mathrm{E}-10$ & $2.2 \mathrm{E}-10$ & $57.30 \%$ \\
\hline I-131 & $4.4 \mathrm{E}-07$ & $6.8 \mathrm{E}-07$ & $57.30 \%$ & $\mathrm{Cm}-243$ & $1.6 \mathrm{E}-07$ & $2.6 \mathrm{E}-07$ & $57.30 \%$ \\
\hline Cs-134 & $2.0 \mathrm{E}-06$ & $3.1 \mathrm{E}-06$ & $57.30 \%$ & Cm-244 & $1.2 \mathrm{E}-10$ & $1.9 \mathrm{E}-10$ & $57.30 \%$ \\
\hline Cs-135 & $0.0 \mathrm{E}+00$ & $0.0 \mathrm{E}+00$ & & $\mathrm{Cm}-245$ & $9.3 \mathrm{E}-08$ & $1.5 \mathrm{E}-07$ & $57.30 \%$ \\
\hline Cs-137 & $7.1 \mathrm{E}-07$ & $1.1 \mathrm{E}-06$ & $57.30 \%$ & $\mathrm{Cm}-246$ & $1.0 \mathrm{E}-10$ & $1.6 \mathrm{E}-10$ & $57.30 \%$ \\
\hline La-137 & $0.0 \mathrm{E}+00$ & $0.0 \mathrm{E}+00$ & & $\mathrm{Cm}-247$ & $3.9 \mathrm{E}-07$ & $6.2 \mathrm{E}-07$ & $57.30 \%$ \\
\hline La-138 & $0.0 \mathrm{E}+00$ & $0.0 \mathrm{E}+00$ & & $\mathrm{Cm}-248$ & $8.9 \mathrm{E}-11$ & $1.4 \mathrm{E}-10$ & $57.30 \%$ \\
\hline La-140 & $2.0 \mathrm{E}-06$ & $3.1 \mathrm{E}-06$ & $57.30 \%$ & $\mathrm{Cm}-250$ & $0.0 \mathrm{E}+00$ & $0.0 \mathrm{E}+00$ & \\
\hline Ba-140 & $3.1 \mathrm{E}-06$ & $4.9 \mathrm{E}-06$ & $57.30 \%$ & Bk-247 & $0.0 \mathrm{E}+00$ & $0.0 \mathrm{E}+00$ & \\
\hline $\mathrm{Ce}-141$ & $9.7 \mathrm{E}-08$ & $1.5 \mathrm{E}-07$ & $57.30 \%$ & Bk-249 & $0.0 \mathrm{E}+00$ & $0.0 \mathrm{E}+00$ & \\
\hline $\mathrm{Ce}-144$ & $6.7 \mathrm{E}-08$ & $1.1 \mathrm{E}-07$ & $57.30 \%$ & Cf-249 & $4.1 \mathrm{E}-07$ & $6.4 \mathrm{E}-07$ & $57.30 \%$ \\
\hline & & & & Cf-250 & $1.2 \mathrm{E}-10$ & $1.9 \mathrm{E}-10$ & $57.30 \%$ \\
\hline & & & & Cf-251 & $1.5 \mathrm{E}-07$ & $2.4 \mathrm{E}-07$ & $57.30 \%$ \\
\hline & & & & Cf-252 & $1.1 \mathrm{E}-10$ & $1.7 \mathrm{E}-10$ & $57.30 \%$ \\
\hline & & & & Es-253 & $4.2 \mathrm{E}-10$ & $6.6 \mathrm{E}-10$ & $57.30 \%$ \\
\hline & & & & Unidentified alpha & $1.1 \mathrm{E}-10$ & $1.7 \mathrm{E}-10$ & $57.30 \%$ \\
\hline & & & & Unidentified beta & $0.0 \mathrm{E}+00$ & $0.0 \mathrm{E}+00$ & \\
\hline & & & & TOTAL Dose & $5.5 \mathrm{E}-05$ & 8.6E-05 & $57.30 \%$ \\
\hline
\end{tabular}


SRNL-STI-2010-00447, REVISION 0

Table 18. LADTAP XL Maximally Exposed Individual Boating Exposure Pathway Comparison (mrem/yr)

\begin{tabular}{|c|c|c|c|c|c|c|c|}
\hline Nuclide & $\begin{array}{l}\text { Hamby Ladtap } \\
\text { Boating Pathway }\end{array}$ & $\begin{array}{l}\text { Jannik Ladtap } \\
\text { Boating Pathway }\end{array}$ & $\%$ Diff & Nuclide & $\begin{array}{l}\text { Hamby Ladtap } \\
\text { Boating Pathway }\end{array}$ & $\begin{array}{l}\text { Jannik Ladtap } \\
\text { Boating Pathway }\end{array}$ & $\%$ Diff \\
\hline H-3 & $0.0 \mathrm{E}+00$ & $0.0 \mathrm{E}+00$ & & Sm-146 & $0.0 \mathrm{E}+00$ & $0.0 \mathrm{E}+00$ & \\
\hline $\mathrm{Be}-7$ & $7.2 \mathrm{E}-08$ & $1.5 \mathrm{E}-07$ & $109.52 \%$ & Sm-147 & $0.0 \mathrm{E}+00$ & $0.0 \mathrm{E}+00$ & \\
\hline Be-10 & $0.0 \mathrm{E}+00$ & $0.0 \mathrm{E}+00$ & & Sm-151 & $1.6 \mathrm{E}-12$ & $3.3 \mathrm{E}-12$ & $109.52 \%$ \\
\hline C-14 & $0.0 \mathrm{E}+00$ & $0.0 \mathrm{E}+00$ & & Pm-147 & $5.6 \mathrm{E}-12$ & $1.2 \mathrm{E}-11$ & $109.52 \%$ \\
\hline $\mathrm{Na}-22$ & $3.3 \mathrm{E}-06$ & $6.8 \mathrm{E}-06$ & $109.52 \%$ & Eu-152 & $1.7 \mathrm{E}-06$ & $3.6 \mathrm{E}-06$ & $109.52 \%$ \\
\hline $\mathrm{Na}-24$ & $2.3 \mathrm{E}-06$ & $4.8 \mathrm{E}-06$ & $109.52 \%$ & Eu-154 & $1.9 \mathrm{E}-06$ & $4.0 \mathrm{E}-06$ & $109.52 \%$ \\
\hline Al-26 & $4.2 \mathrm{E}-06$ & $8.8 \mathrm{E}-06$ & $109.52 \%$ & Eu-155 & $9.0 \mathrm{E}-08$ & $1.9 \mathrm{E}-07$ & $109.52 \%$ \\
\hline P-32 & $0.0 \mathrm{E}+00$ & $0.0 \mathrm{E}+00$ & & Gd-152 & $0.0 \mathrm{E}+00$ & $0.0 \mathrm{E}+00$ & \\
\hline Si-32 & $0.0 \mathrm{E}+00$ & $0.0 \mathrm{E}+00$ & & Ho-166m & $4.2 \mathrm{E}-08$ & 8.9E-08 & $109.52 \%$ \\
\hline S-35 & $0.0 \mathrm{E}+00$ & $0.0 \mathrm{E}+00$ & & Lu-176 & $0.0 \mathrm{E}+00$ & $0.0 \mathrm{E}+00$ & \\
\hline Cl-36 & $1.4 \mathrm{E}-14$ & $2.9 \mathrm{E}-14$ & $109.52 \%$ & Ta-180 & $0.0 \mathrm{E}+00$ & $0.0 \mathrm{E}+00$ & \\
\hline $\mathrm{K}-40$ & $2.4 \mathrm{E}-07$ & 4.9E-07 & $109.52 \%$ & Hf-182 & $0.0 \mathrm{E}+00$ & $0.0 \mathrm{E}+00$ & \\
\hline $\mathrm{K}-43$ & $6.7 \mathrm{E}-07$ & $1.4 \mathrm{E}-06$ & $109.52 \%$ & Re-186m & $0.0 \mathrm{E}+00$ & $0.0 \mathrm{E}+00$ & \\
\hline $\mathrm{Ca}-41$ & $5.2 \mathrm{E}-12$ & $1.1 \mathrm{E}-11$ & $109.52 \%$ & Re-187 & $0.0 \mathrm{E}+00$ & $0.0 \mathrm{E}+00$ & \\
\hline $\mathrm{Ca}-45$ & $2.8 \mathrm{E}-17$ & $5.9 \mathrm{E}-17$ & $109.52 \%$ & Ir-192m & $0.0 \mathrm{E}+00$ & $0.0 \mathrm{E}+00$ & \\
\hline $\mathrm{Ca}-47$ & $1.4 \mathrm{E}-06$ & $2.9 \mathrm{E}-06$ & $109.52 \%$ & Pt-193 & $7.5 \mathrm{E}-11$ & $1.6 \mathrm{E}-10$ & $109.52 \%$ \\
\hline Ti-44 & $2.2 \mathrm{E}-07$ & $4.5 \mathrm{E}-07$ & $109.52 \%$ & Hg-194 & $0.0 \mathrm{E}+00$ & $0.0 \mathrm{E}+00$ & \\
\hline $\mathrm{V}-49$ & $1.6 \mathrm{E}-11$ & $3.3 \mathrm{E}-11$ & $109.52 \%$ & $\mathrm{Hg}-203$ & $3.3 \mathrm{E}-07$ & 7.0E-07 & $109.52 \%$ \\
\hline Cr-51 & $4.5 \mathrm{E}-08$ & $9.5 \mathrm{E}-08$ & $109.52 \%$ & $\mathrm{~Pb}-202$ & $0.0 \mathrm{E}+00$ & $0.0 \mathrm{E}+00$ & \\
\hline Mn-53 & $2.9 \mathrm{E}-11$ & $6.1 \mathrm{E}-11$ & $109.52 \%$ & $\mathrm{~Pb}-205$ & $9.8 \mathrm{E}-11$ & $2.0 \mathrm{E}-10$ & $109.52 \%$ \\
\hline Mn-54 & $1.3 \mathrm{E}-06$ & $2.6 \mathrm{E}-06$ & $109.52 \%$ & $\mathrm{~Pb}-210$ & $2.3 \mathrm{E}-09$ & 4.7E-09 & $109.52 \%$ \\
\hline $\mathrm{Fe}-55$ & $3.8 \mathrm{E}-11$ & $8.0 \mathrm{E}-11$ & $109.52 \%$ & Bi-207 & $2.3 \mathrm{E}-06$ & $4.9 \mathrm{E}-06$ & $109.52 \%$ \\
\hline Fe-60 & $0.0 \mathrm{E}+00$ & $0.0 \mathrm{E}+00$ & & $\mathrm{Bi}-210$ & $0.0 \mathrm{E}+00$ & $0.0 \mathrm{E}+00$ & \\
\hline Co-58 & $1.4 \mathrm{E}-06$ & $3.0 \mathrm{E}-06$ & $109.52 \%$ & $\mathrm{Bi}-210 \mathrm{~m}$ & $0.0 \mathrm{E}+00$ & $0.0 \mathrm{E}+00$ & \\
\hline Co-60 & $5.1 \mathrm{E}-06$ & $1.1 \mathrm{E}-05$ & $109.52 \%$ & Po-210 & $1.3 \mathrm{E}-11$ & $2.7 \mathrm{E}-11$ & $109.52 \%$ \\
\hline Ni-59 & $6.5 \mathrm{E}-11$ & $1.4 \mathrm{E}-10$ & $109.52 \%$ & Ra-223 & $1.9 \mathrm{E}-07$ & $4.0 \mathrm{E}-07$ & $109.52 \%$ \\
\hline Ni-63 & $0.0 \mathrm{E}+00$ & $0.0 \mathrm{E}+00$ & & Ra-224 & $1.2 \mathrm{E}-08$ & $2.6 \mathrm{E}-08$ & $109.52 \%$ \\
\hline $\mathrm{Cu}-64$ & 7.5E-08 & $1.6 \mathrm{E}-07$ & $109.52 \%$ & Ra-225 & $1.1 \mathrm{E}-08$ & $2.3 \mathrm{E}-08$ & $109.52 \%$ \\
\hline
\end{tabular}


SRNL-STI-2010-00447, REVISION 0

Table 18. LADTAP XL Maximally Exposed Individual Boating Exposure Pathway Comparison (mrem/yr) (continued)

\begin{tabular}{|c|c|c|c|c|c|c|c|}
\hline Nuclide & $\begin{array}{c}\text { Hamby Ladtap } \\
\text { Boating Pathway }\end{array}$ & $\begin{array}{c}\text { Jannik Ladtap } \\
\text { Boating Pathway }\end{array}$ & $\%$ Diff & Nuclide & $\begin{array}{c}\text { Hamby Ladtap } \\
\text { Boating Pathway }\end{array}$ & $\begin{array}{c}\text { Jannik Ladtap } \\
\text { Boating Pathway }\end{array}$ & $\%$ Diff \\
\hline $\mathrm{Zn}-65$ & $8.8 \mathrm{E}-07$ & $1.8 \mathrm{E}-06$ & $109.52 \%$ & Ra-226 & $1.0 \mathrm{E}-08$ & $2.1 \mathrm{E}-08$ & $109.52 \%$ \\
\hline Ge-68 & $1.5 \mathrm{E}-10$ & $3.2 \mathrm{E}-10$ & $109.52 \%$ & Ra-228 & $1.1 \mathrm{E}-16$ & $2.3 \mathrm{E}-16$ & $109.52 \%$ \\
\hline $\mathrm{Se}-75$ & $5.8 \mathrm{E}-07$ & $1.2 \mathrm{E}-06$ & $109.52 \%$ & Ac-227 & $1.9 \mathrm{E}-10$ & $4.0 \mathrm{E}-10$ & $109.52 \%$ \\
\hline Se-79 & $0.0 \mathrm{E}+00$ & $0.0 \mathrm{E}+00$ & & Th-227 & $1.5 \mathrm{E}-07$ & $3.1 \mathrm{E}-07$ & $109.52 \%$ \\
\hline $\mathrm{Rb}-87$ & $0.0 \mathrm{E}+00$ & $0.0 \mathrm{E}+00$ & & Th-228 & $3.0 \mathrm{E}-09$ & $6.4 \mathrm{E}-09$ & $109.52 \%$ \\
\hline Sr-89 & $2.1 \mathrm{E}-10$ & $4.3 \mathrm{E}-10$ & $109.52 \%$ & Th-229 & $1.3 \mathrm{E}-07$ & $2.8 \mathrm{E}-07$ & $109.52 \%$ \\
\hline Sr-90 & $0.0 \mathrm{E}+00$ & $0.0 \mathrm{E}+00$ & & Th-230 & $6.2 \mathrm{E}-10$ & $1.3 \mathrm{E}-09$ & $109.52 \%$ \\
\hline $\mathrm{Y}-90$ & $0.0 \mathrm{E}+00$ & $0.0 \mathrm{E}+00$ & & Th-231 & $9.6 \mathrm{E}-09$ & $2.0 \mathrm{E}-08$ & $109.52 \%$ \\
\hline Y-91 & $5.4 \mathrm{E}-09$ & $1.1 \mathrm{E}-08$ & $109.52 \%$ & Th-232 & $3.0 \mathrm{E}-10$ & $6.4 \mathrm{E}-10$ & $109.52 \%$ \\
\hline Mo-93 & $1.3 \mathrm{E}-09$ & $2.8 \mathrm{E}-09$ & $109.52 \%$ & Th-234 & $1.2 \mathrm{E}-08$ & $2.5 \mathrm{E}-08$ & $109.52 \%$ \\
\hline Mo-99 & $3.2 \mathrm{E}-07$ & $6.7 \mathrm{E}-07$ & $109.52 \%$ & $\mathrm{~Pa}-230$ & $9.6 \mathrm{E}-07$ & $2.0 \mathrm{E}-06$ & $109.52 \%$ \\
\hline $\mathrm{Nb}-93 \mathrm{~m}$ & $2.3 \mathrm{E}-10$ & $4.9 \mathrm{E}-10$ & $109.52 \%$ & $\mathrm{~Pa}-231$ & $4.5 \mathrm{E}-08$ & 9.4E-08 & $109.52 \%$ \\
\hline $\mathrm{Nb}-94$ & $2.4 \mathrm{E}-06$ & $5.0 \mathrm{E}-06$ & $109.52 \%$ & $\mathrm{~Pa}-233$ & $3.1 \mathrm{E}-07$ & $6.5 \mathrm{E}-07$ & $109.52 \%$ \\
\hline $\mathrm{Nb}-95$ & $1.1 \mathrm{E}-06$ & $2.4 \mathrm{E}-06$ & $109.52 \%$ & U-232 & $4.3 \mathrm{E}-10$ & $9.0 \mathrm{E}-10$ & $109.52 \%$ \\
\hline Zr-93 & $0.0 \mathrm{E}+00$ & $0.0 \mathrm{E}+00$ & & U-233 & $3.7 \mathrm{E}-10$ & $7.8 \mathrm{E}-10$ & $109.52 \%$ \\
\hline Zr-95 & $1.1 \mathrm{E}-06$ & $2.3 \mathrm{E}-06$ & $109.52 \%$ & U-234 & $2.5 \mathrm{E}-10$ & $5.2 \mathrm{E}-10$ & $109.52 \%$ \\
\hline Tc-96 & $3.2 \mathrm{E}-06$ & $6.7 \mathrm{E}-06$ & $109.52 \%$ & U-235 & $2.3 \mathrm{E}-07$ & $4.8 \mathrm{E}-07$ & $109.52 \%$ \\
\hline Tc-97 & $1.6 \mathrm{E}-09$ & $3.4 \mathrm{E}-09$ & $109.52 \%$ & U-236 & $2.0 \mathrm{E}-10$ & $4.2 \mathrm{E}-10$ & $109.52 \%$ \\
\hline Tc-98 & $2.1 \mathrm{E}-06$ & 4.4E-06 & $109.52 \%$ & U-237 & $1.9 \mathrm{E}-07$ & $3.9 \mathrm{E}-07$ & $109.52 \%$ \\
\hline Tc-99 & $8.3 \mathrm{E}-13$ & $1.7 \mathrm{E}-12$ & $109.52 \%$ & U-238 & $1.7 \mathrm{E}-10$ & $3.6 \mathrm{E}-10$ & $109.52 \%$ \\
\hline Ru-97 & $2.7 \mathrm{E}-07$ & $5.7 \mathrm{E}-07$ & $109.52 \%$ & $\mathrm{~Np}-236$ & $2.0 \mathrm{E}-07$ & $4.2 \mathrm{E}-07$ & $109.52 \%$ \\
\hline Ru-103 & 6.9E-07 & $1.5 \mathrm{E}-06$ & $109.52 \%$ & Np-237 & $3.6 \mathrm{E}-08$ & 7.5E-08 & $109.52 \%$ \\
\hline Ru-106 & $3.1 \mathrm{E}-07$ & $6.4 \mathrm{E}-07$ & $109.52 \%$ & $\mathrm{~Np}-239$ & $1.9 \mathrm{E}-07$ & $4.0 \mathrm{E}-07$ & $109.52 \%$ \\
\hline Pd-107 & $0.0 \mathrm{E}+00$ & $0.0 \mathrm{E}+00$ & & Am-237 & $0.0 \mathrm{E}+00$ & $0.0 \mathrm{E}+00$ & \\
\hline Ag-108m & $2.4 \mathrm{E}-06$ & $5.0 \mathrm{E}-06$ & $109.52 \%$ & Am-241 & $3.1 \mathrm{E}-08$ & $6.6 \mathrm{E}-08$ & $109.52 \%$ \\
\hline Ag-110m & $4.1 \mathrm{E}-06$ & $8.6 \mathrm{E}-06$ & $109.52 \%$ & Am-242m & $7.8 \mathrm{E}-10$ & $1.6 \mathrm{E}-09$ & $109.52 \%$ \\
\hline Cd-113 & $0.0 \mathrm{E}+00$ & $0.0 \mathrm{E}+00$ & & Am-243 & $8.2 \mathrm{E}-08$ & $1.7 \mathrm{E}-07$ & $109.52 \%$ \\
\hline
\end{tabular}


SRNL-STI-2010-00447, REVISION 0

Table 18. LADTAP XL Maximally Exposed Individual Boating Exposure Pathway Comparison (mrem/yr) (continued)

\begin{tabular}{|c|c|c|c|c|c|c|c|}
\hline Nuclide & $\begin{array}{c}\text { Hamby Ladtap } \\
\text { Boating Pathway }\end{array}$ & $\begin{array}{c}\text { Jannik Ladtap } \\
\text { Boating Pathway }\end{array}$ & $\%$ Diff & Nuclide & $\begin{array}{c}\text { Hamby Ladtap } \\
\text { Boating Pathway }\end{array}$ & $\begin{array}{c}\text { Jannik Ladtap } \\
\text { Boating Pathway }\end{array}$ & $\%$ Diff \\
\hline Cd-115m & $3.3 \mathrm{E}-08$ & 6.9E-08 & $109.52 \%$ & $\mathrm{Pu}-237$ & 7.3E-08 & $1.5 \mathrm{E}-07$ & $109.52 \%$ \\
\hline In-115 & $0.0 \mathrm{E}+00$ & $0.0 \mathrm{E}+00$ & & $\mathrm{Pu}-238$ & $1.5 \mathrm{E}-10$ & $3.1 \mathrm{E}-10$ & $109.52 \%$ \\
\hline Sb-122 & $5.0 \mathrm{E}-07$ & $1.1 \mathrm{E}-06$ & $109.52 \%$ & Pu-239 & $1.3 \mathrm{E}-10$ & $2.7 \mathrm{E}-10$ & $109.52 \%$ \\
\hline Sb-124 & $2.9 \mathrm{E}-06$ & $6.0 \mathrm{E}-06$ & $109.52 \%$ & $\mathrm{Pu}-240$ & $1.5 \mathrm{E}-10$ & $3.1 \mathrm{E}-10$ & $109.52 \%$ \\
\hline Sb-125 & $6.2 \mathrm{E}-07$ & $1.3 \mathrm{E}-06$ & $109.52 \%$ & $\mathrm{Pu}-241$ & $0.0 \mathrm{E}+00$ & $0.0 \mathrm{E}+00$ & \\
\hline Te-123 & $5.2 \mathrm{E}-09$ & $1.1 \mathrm{E}-08$ & $109.52 \%$ & $\mathrm{Pu}-242$ & $1.3 \mathrm{E}-10$ & $2.6 \mathrm{E}-10$ & $109.52 \%$ \\
\hline Te-125m & $1.6 \mathrm{E}-08$ & $3.4 \mathrm{E}-08$ & $109.52 \%$ & $\mathrm{Pu}-244$ & $9.2 \mathrm{E}-11$ & $1.9 \mathrm{E}-10$ & $109.52 \%$ \\
\hline Sn-126 & 7.7E-08 & $1.6 \mathrm{E}-07$ & $109.52 \%$ & Cm-241 & $0.0 \mathrm{E}+00$ & $0.0 \mathrm{E}+00$ & \\
\hline I-129 & $1.4 \mathrm{E}-08$ & $3.0 \mathrm{E}-08$ & $109.52 \%$ & $\mathrm{Cm}-242$ & $1.7 \mathrm{E}-10$ & $3.5 \mathrm{E}-10$ & $109.52 \%$ \\
\hline $\mathrm{I}-131$ & $5.1 \mathrm{E}-07$ & $1.1 \mathrm{E}-06$ & $109.52 \%$ & $\mathrm{Cm}-243$ & $1.9 \mathrm{E}-07$ & $4.0 \mathrm{E}-07$ & $109.52 \%$ \\
\hline Cs-134 & $2.3 \mathrm{E}-06$ & $4.9 \mathrm{E}-06$ & $109.52 \%$ & $\mathrm{Cm}-244$ & $1.4 \mathrm{E}-10$ & $3.0 \mathrm{E}-10$ & $109.52 \%$ \\
\hline Cs-135 & $0.0 \mathrm{E}+00$ & $0.0 \mathrm{E}+00$ & & $\mathrm{Cm}-245$ & $1.1 \mathrm{E}-07$ & $2.3 \mathrm{E}-07$ & $109.52 \%$ \\
\hline Cs-137 & $8.3 \mathrm{E}-07$ & $1.7 \mathrm{E}-06$ & $109.52 \%$ & $\mathrm{Cm}-246$ & $1.2 \mathrm{E}-10$ & $2.5 \mathrm{E}-10$ & $109.52 \%$ \\
\hline La-137 & $0.0 \mathrm{E}+00$ & $0.0 \mathrm{E}+00$ & & $\mathrm{Cm}-247$ & $4.6 \mathrm{E}-07$ & 9.7E-07 & $109.52 \%$ \\
\hline La-138 & $0.0 \mathrm{E}+00$ & $0.0 \mathrm{E}+00$ & & $\mathrm{Cm}-248$ & $1.1 \mathrm{E}-10$ & $2.2 \mathrm{E}-10$ & $109.52 \%$ \\
\hline La-140 & $2.4 \mathrm{E}-06$ & $4.9 \mathrm{E}-06$ & $109.52 \%$ & $\mathrm{Cm}-250$ & $0.0 \mathrm{E}+00$ & $0.0 \mathrm{E}+00$ & \\
\hline $\mathrm{Ba}-140$ & $3.6 \mathrm{E}-06$ & $7.6 \mathrm{E}-06$ & $109.52 \%$ & Bk-247 & $0.0 \mathrm{E}+00$ & $0.0 \mathrm{E}+00$ & \\
\hline Ce-141 & $1.1 \mathrm{E}-07$ & $2.4 \mathrm{E}-07$ & $109.52 \%$ & Bk-249 & $0.0 \mathrm{E}+00$ & $0.0 \mathrm{E}+00$ & \\
\hline \multirow[t]{8}{*}{ Ce-144 } & 7.9E-08 & $1.7 \mathrm{E}-07$ & $109.52 \%$ & Cf-249 & $4.8 \mathrm{E}-07$ & $1.0 \mathrm{E}-06$ & $109.52 \%$ \\
\hline & & & & Cf-250 & $1.4 \mathrm{E}-10$ & $3.0 \mathrm{E}-10$ & $109.52 \%$ \\
\hline & & & & Cf-251 & $1.8 \mathrm{E}-07$ & $3.7 \mathrm{E}-07$ & $109.52 \%$ \\
\hline & & & & Cf-252 & $1.3 \mathrm{E}-10$ & $2.7 \mathrm{E}-10$ & $109.52 \%$ \\
\hline & & & & Es-253 & $4.9 \mathrm{E}-10$ & $1.0 \mathrm{E}-09$ & $109.52 \%$ \\
\hline & & & & Unidentified alpha & $1.3 \mathrm{E}-10$ & $2.7 \mathrm{E}-10$ & $109.52 \%$ \\
\hline & & & & Unidentified beta & $0.0 \mathrm{E}+00$ & $0.0 \mathrm{E}+00$ & \\
\hline & & & & TOTAL Dose & $6.4 \mathrm{E}-05$ & 1.3E-04 & $109.52 \%$ \\
\hline
\end{tabular}


Table 19. LADTAP XL Maximally Exposed Individual Total of All Pathways Comparison (mrem/yr)

\begin{tabular}{|c|c|c|c|c|c|c|c|}
\hline Nuclide & $\begin{array}{l}\text { Hamby Ladtap } \\
\text { Total Pathway }\end{array}$ & $\begin{array}{l}\text { Jannik Ladtap } \\
\text { Total Pathway }\end{array}$ & $\%$ Diff & Nuclide & $\begin{array}{l}\text { Hamby Ladtap } \\
\text { Total Pathway }\end{array}$ & $\begin{array}{l}\text { Jannik Ladtap } \\
\text { Total Pathway }\end{array}$ & $\%$ Diff \\
\hline $\mathrm{H}-3$ & $5.3 \mathrm{E}-06$ & $5.29 \mathrm{E}-06$ & $0.28 \%$ & Sm-146 & $2.7 \mathrm{E}-02$ & $2.91 \mathrm{E}-02$ & $7.88 \%$ \\
\hline $\mathrm{Be}-7$ & $1.1 \mathrm{E}-05$ & $3.33 \mathrm{E}-05$ & $202.17 \%$ & Sm-147 & $2.4 \mathrm{E}-02$ & $2.62 \mathrm{E}-02$ & $7.88 \%$ \\
\hline Be-10 & $3.6 \mathrm{E}-04$ & $1.24 \mathrm{E}-03$ & $242.45 \%$ & Sm-151 & $4.6 \mathrm{E}-05$ & $4.95 \mathrm{E}-05$ & $7.87 \%$ \\
\hline C-14 & $2.1 \mathrm{E}-02$ & $1.85 \mathrm{E}-04$ & $-99.11 \%$ & Pm-147 & $1.3 \mathrm{E}-04$ & $1.38 \mathrm{E}-04$ & $7.88 \%$ \\
\hline $\mathrm{Na}-22$ & $4.7 \mathrm{E}-03$ & $3.95 \mathrm{E}-03$ & $-16.14 \%$ & Eu-152 & $3.6 \mathrm{E}-03$ & $4.60 \mathrm{E}-03$ & $26.86 \%$ \\
\hline $\mathrm{Na}-24$ & $5.9 \mathrm{E}-05$ & $5.44 \mathrm{E}-05$ & $-7.18 \%$ & Eu-154 & $3.4 \mathrm{E}-03$ & $5.17 \mathrm{E}-03$ & $51.24 \%$ \\
\hline Al-26 & $1.6 \mathrm{E}-02$ & $1.54 \mathrm{E}-02$ & $-4.93 \%$ & Eu-155 & $2.5 \mathrm{E}-04$ & $5.32 \mathrm{E}-04$ & $111.63 \%$ \\
\hline P-32 & $1.5 \mathrm{E}+00$ & $2.08 \mathrm{E}+00$ & $39.98 \%$ & Gd-152 & $2.0 \mathrm{E}-02$ & $2.18 \mathrm{E}-02$ & $7.88 \%$ \\
\hline $\mathrm{Si}-32$ & $1.5 \mathrm{E}-04$ & $2.11 \mathrm{E}-04$ & $42.77 \%$ & Ho-166m & $1.1 \mathrm{E}-02$ & $9.57 \mathrm{E}-03$ & $-11.00 \%$ \\
\hline S-35 & $1.1 \mathrm{E}-03$ & $1.14 \mathrm{E}-03$ & $6.34 \%$ & Lu-176 & 8.9E-04 & $8.90 \mathrm{E}-04$ & $0.00 \%$ \\
\hline Cl-36 & $5.6 \mathrm{E}-04$ & $5.45 \mathrm{E}-04$ & $-3.39 \%$ & Ta-180 & $4.8 \mathrm{E}-84$ & $4.84 \mathrm{E}-84$ & $0.00 \%$ \\
\hline $\mathrm{K}-40$ & $4.3 \mathrm{E}-02$ & $1.32 \mathrm{E}-01$ & $207.58 \%$ & Hf-182 & $1.2 \mathrm{E}-03$ & $3.39 \mathrm{E}-02$ & $2626.69 \%$ \\
\hline $\mathrm{K}-43$ & $4.0 \mathrm{E}-04$ & $1.22 \mathrm{E}-03$ & $207.84 \%$ & Re-186m & $1.1 \mathrm{E}-03$ & $1.11 \mathrm{E}-03$ & $0.64 \%$ \\
\hline $\mathrm{Ca}-41$ & $2.0 \mathrm{E}-04$ & $1.29 \mathrm{E}-04$ & $-35.69 \%$ & Re-187 & $2.8 \mathrm{E}-06$ & $2.80 \mathrm{E}-06$ & $0.64 \%$ \\
\hline $\mathrm{Ca}-45$ & $5.0 \mathrm{E}-04$ & $3.20 \mathrm{E}-04$ & $-35.67 \%$ & Ir-192m & $0.0 \mathrm{E}+00$ & $0.00 \mathrm{E}+00$ & $0.00 \%$ \\
\hline $\mathrm{Ca}-47$ & $8.0 \mathrm{E}-04$ & $5.26 \mathrm{E}-04$ & $-33.93 \%$ & Pt-193 & $3.5 \mathrm{E}-05$ & $1.94 \mathrm{E}-05$ & $-44.48 \%$ \\
\hline $\mathrm{Ti}-44$ & $4.3 \mathrm{E}-02$ & 9.89E-03 & $-76.84 \%$ & $\mathrm{Hg}-194$ & $6.2 \mathrm{E}-01$ & $3.66 \mathrm{E}+00$ & $491.13 \%$ \\
\hline$V-49$ & $5.6 \mathrm{E}-06$ & $1.55 \mathrm{E}-05$ & $179.02 \%$ & $\mathrm{Hg}-203$ & $2.1 \mathrm{E}-02$ & $1.27 \mathrm{E}-01$ & $490.82 \%$ \\
\hline $\mathrm{Cr}-51$ & $6.3 \mathrm{E}-05$ & $2.14 \mathrm{E}-05$ & $-66.31 \%$ & $\mathrm{~Pb}-202$ & $2.8 \mathrm{E}-02$ & $5.26 \mathrm{E}-03$ & $-81.26 \%$ \\
\hline $\mathrm{Mn}-53$ & $3.0 \mathrm{E}-05$ & $5.95 \mathrm{E}-05$ & $97.57 \%$ & $\mathrm{~Pb}-205$ & $1.1 \mathrm{E}-03$ & $2.06 \mathrm{E}-04$ & $-80.98 \%$ \\
\hline $\mathrm{Mn}-54$ & $2.7 \mathrm{E}-03$ & $1.73 \mathrm{E}-03$ & $-35.03 \%$ & $\mathrm{~Pb}-210$ & $3.7 \mathrm{E}+00$ & $6.88 \mathrm{E}-01$ & $-81.26 \%$ \\
\hline $\mathrm{Fe}-55$ & $1.7 \mathrm{E}-04$ & $2.57 \mathrm{E}-04$ & $50.52 \%$ & Bi-207 & $6.7 \mathrm{E}-03$ & $5.87 \mathrm{E}-03$ & $-11.89 \%$ \\
\hline $\mathrm{Fe}-60$ & $4.4 \mathrm{E}-02$ & $6.65 \mathrm{E}-02$ & $50.57 \%$ & Bi-210 & $5.3 \mathrm{E}-04$ & $5.35 \mathrm{E}-04$ & $0.00 \%$ \\
\hline Co-58 & $6.9 \mathrm{E}-04$ & $8.77 \mathrm{E}-04$ & $27.06 \%$ & Bi-210m & $9.8 \mathrm{E}-03$ & $9.77 \mathrm{E}-03$ & $0.00 \%$ \\
\hline Co-60 & $7.5 \mathrm{E}-03$ & $8.56 \mathrm{E}-03$ & $14.92 \%$ & Po-210 & $1.8 \mathrm{E}+00$ & $2.51 \mathrm{E}-01$ & $-86.16 \%$ \\
\hline Ni-59 & $6.1 \mathrm{E}-05$ & $2.74 \mathrm{E}-05$ & $-55.30 \%$ & Ra-223 & $9.3 \mathrm{E}-02$ & $4.52 \mathrm{E}-02$ & $-51.34 \%$ \\
\hline Ni-63 & $1.6 \mathrm{E}-04$ & $6.83 \mathrm{E}-05$ & $-57.07 \%$ & Ra-224 & 4.4E-02 & $2.22 \mathrm{E}-02$ & $-49.85 \%$ \\
\hline $\mathrm{Cu}-64$ & $1.8 \mathrm{E}-05$ & $2.05 \mathrm{E}-05$ & $11.49 \%$ & Ra-225 & $5.4 \mathrm{E}-02$ & $2.60 \mathrm{E}-02$ & $-51.49 \%$ \\
\hline
\end{tabular}


SRNL-STI-2010-00447, REVISION 0

Table 19. LADTAP XL Maximally Exposed Individual Total of All Pathways Comparison (mrem/yr) (continued)

\begin{tabular}{|c|c|c|c|c|c|c|c|}
\hline Nuclide & $\begin{array}{l}\text { Hamby Ladtap } \\
\text { Total Pathway }\end{array}$ & $\begin{array}{l}\text { Jannik Ladtap } \\
\text { Total Pathway }\end{array}$ & $\%$ Diff & Nuclide & $\begin{array}{l}\text { Hamby Ladtap } \\
\text { Total Pathway }\end{array}$ & $\begin{array}{l}\text { Jannik Ladtap } \\
\text { Total Pathway }\end{array}$ & $\%$ Diff \\
\hline $\mathrm{Zn}-65$ & $6.0 \mathrm{E}-02$ & $1.02 \mathrm{E}-01$ & $68.57 \%$ & Ra-226 & $2.1 \mathrm{E}-01$ & $9.93 \mathrm{E}-02$ & $-52.02 \%$ \\
\hline Ge-68 & $7.8 \mathrm{E}-03$ & $9.41 \mathrm{E}-03$ & $19.89 \%$ & Ra-228 & $2.3 \mathrm{E}-01$ & $1.08 \mathrm{E}-01$ & $-52.02 \%$ \\
\hline Se-75 & $3.9 \mathrm{E}-03$ & $1.12 \mathrm{E}-01$ & $2773.16 \%$ & Ac-227 & $1.9 \mathrm{E}+00$ & $1.89 \mathrm{E}+00$ & $0.00 \%$ \\
\hline Se-79 & $3.7 \mathrm{E}-03$ & $1.07 \mathrm{E}-01$ & $2797.22 \%$ & Th-227 & $4.9 \mathrm{E}-03$ & $3.21 \mathrm{E}-03$ & $-34.70 \%$ \\
\hline Rb-87 & $2.1 \mathrm{E}-02$ & $5.04 \mathrm{E}-02$ & $142.27 \%$ & Th-228 & $5.5 \mathrm{E}-02$ & $3.59 \mathrm{E}-02$ & $-35.07 \%$ \\
\hline Sr-89 & $1.2 \mathrm{E}-03$ & $7.49 \mathrm{E}-04$ & $-39.46 \%$ & Th-229 & $5.1 \mathrm{E}-01$ & $3.31 \mathrm{E}-01$ & $-35.05 \%$ \\
\hline Sr-90 & $1.9 \mathrm{E}-02$ & $1.14 \mathrm{E}-02$ & $-39.61 \%$ & Th-230 & 7.7E-02 & $5.01 \mathrm{E}-02$ & $-35.08 \%$ \\
\hline Y-90 & 8.7E-04 & $1.06 \mathrm{E}-03$ & $21.82 \%$ & Th-231 & $6.3 \mathrm{E}-05$ & $4.45 \mathrm{E}-05$ & $-28.80 \%$ \\
\hline Y-91 & $1.2 \mathrm{E}-03$ & $1.45 \mathrm{E}-03$ & $23.56 \%$ & Th-232 & $4.1 \mathrm{E}-01$ & $2.65 \mathrm{E}-01$ & $-35.08 \%$ \\
\hline Mo-93 & $1.7 \mathrm{E}-04$ & $1.41 \mathrm{E}-04$ & $-15.98 \%$ & Th-234 & $1.8 \mathrm{E}-03$ & $1.17 \mathrm{E}-03$ & $-34.79 \%$ \\
\hline Mo-99 & $3.0 \mathrm{E}-04$ & $2.59 \mathrm{E}-04$ & $-14.92 \%$ & $\mathrm{~Pa}-230$ & $5.6 \mathrm{E}-04$ & $5.50 \mathrm{E}-04$ & $-2.42 \%$ \\
\hline $\mathrm{Nb}-93 \mathrm{~m}$ & $3.4 \mathrm{E}-01$ & $3.82 \mathrm{E}-03$ & $-98.87 \%$ & $\mathrm{~Pa}-231$ & $1.2 \mathrm{E}+00$ & $1.13 \mathrm{E}+00$ & $-2.62 \%$ \\
\hline $\mathrm{Nb}-94$ & $3.4 \mathrm{E}-01$ & $1.19 \mathrm{E}-02$ & $-96.45 \%$ & $\mathrm{~Pa}-233$ & $3.4 \mathrm{E}-04$ & $3.30 \mathrm{E}-04$ & $-2.55 \%$ \\
\hline $\mathrm{Nb}-95$ & $1.4 \mathrm{E}-01$ & $1.54 \mathrm{E}-03$ & $-98.86 \%$ & U-232 & $1.3 \mathrm{E}-01$ & $1.09 \mathrm{E}-01$ & $-18.67 \%$ \\
\hline Zr-93 & $1.4 \mathrm{E}-04$ & $2.06 \mathrm{E}-04$ & $44.82 \%$ & U-233 & $2.3 \mathrm{E}-02$ & $2.26 \mathrm{E}-02$ & $-2.57 \%$ \\
\hline $\mathrm{Zr}-95$ & $3.6 \mathrm{E}-04$ & $4.82 \mathrm{E}-04$ & $35.63 \%$ & U-234 & $2.2 \mathrm{E}-02$ & $2.18 \mathrm{E}-02$ & $-2.58 \%$ \\
\hline Tc-96 & $2.5 \mathrm{E}-04$ & $2.72 \mathrm{E}-04$ & $10.19 \%$ & U-235 & $2.3 \mathrm{E}-02$ & $2.18 \mathrm{E}-02$ & $-3.04 \%$ \\
\hline Tc-97 & $5.5 \mathrm{E}-05$ & $5.17 \mathrm{E}-05$ & $-6.09 \%$ & U-236 & $2.2 \mathrm{E}-02$ & $2.09 \mathrm{E}-02$ & $-2.58 \%$ \\
\hline Tc-98 & $9.0 \mathrm{E}-03$ & $7.95 \mathrm{E}-03$ & $-11.64 \%$ & U-237 & $2.0 \mathrm{E}-04$ & $1.95 \mathrm{E}-04$ & $-2.33 \%$ \\
\hline Tc-99 & $1.5 \mathrm{E}-04$ & $1.62 \mathrm{E}-04$ & $9.36 \%$ & U-238 & $2.0 \mathrm{E}-02$ & $1.93 \mathrm{E}-02$ & $-2.57 \%$ \\
\hline Ru-97 & $4.6 \mathrm{E}-05$ & $8.42 \mathrm{E}-05$ & $83.71 \%$ & $\mathrm{~Np}-236$ & $8.2 \mathrm{E}-02$ & $1.01 \mathrm{E}-01$ & $22.31 \%$ \\
\hline $\mathrm{Ru}-103$ & $2.8 \mathrm{E}-04$ & $5.32 \mathrm{E}-04$ & $88.02 \%$ & $\mathrm{~Np}-237$ & $4.0 \mathrm{E}-01$ & $4.93 \mathrm{E}-01$ & $22.70 \%$ \\
\hline $\mathrm{Ru}-106$ & $2.2 \mathrm{E}-03$ & $4.20 \mathrm{E}-03$ & $90.68 \%$ & $\mathrm{~Np}-239$ & $1.9 \mathrm{E}-04$ & $2.25 \mathrm{E}-04$ & $20.26 \%$ \\
\hline Pd-107 & $1.4 \mathrm{E}-05$ & $1.44 \mathrm{E}-05$ & $0.00 \%$ & Am-237 & $7.5 \mathrm{E}-15$ & $7.57 \mathrm{E}-15$ & $0.60 \%$ \\
\hline Ag-108m & $9.5 \mathrm{E}-03$ & $1.01 \mathrm{E}-02$ & $5.93 \%$ & Am-241 & $6.1 \mathrm{E}-01$ & $2.67 \mathrm{E}+00$ & $338.90 \%$ \\
\hline $\mathrm{Ag}-110 \mathrm{~m}$ & $1.3 \mathrm{E}-03$ & $3.81 \mathrm{E}-03$ & $182.39 \%$ & Am-242m & $5.7 \mathrm{E}-01$ & $2.49 \mathrm{E}+00$ & $338.99 \%$ \\
\hline $\mathrm{Cd}-113$ & $8.1 \mathrm{E}-02$ & $8.12 \mathrm{E}-02$ & $0.00 \%$ & Am-243 & $6.1 \mathrm{E}-01$ & $2.67 \mathrm{E}+00$ & $338.78 \%$ \\
\hline
\end{tabular}


SRNL-STI-2010-00447, REVISION 0

Table 19. LADTAP XL Maximally Exposed Individual Total of All Pathways Comparison (mrem/yr) (continued)

\begin{tabular}{|c|c|c|c|c|c|c|c|}
\hline Nuclide & $\begin{array}{l}\text { Hamby Ladtap } \\
\text { Total Pathway }\end{array}$ & $\begin{array}{l}\text { Jannik Ladtap } \\
\text { Total Pathway }\end{array}$ & $\%$ Diff & Nuclide & $\begin{array}{l}\text { Hamby Ladtap } \\
\text { Total Pathway }\end{array}$ & $\begin{array}{l}\text { Jannik Ladtap } \\
\text { Total Pathway }\end{array}$ & $\%$ Diff \\
\hline Cd-115m & $7.4 \mathrm{E}-03$ & 7.39E-03 & $0.00 \%$ & $\mathrm{Pu}-237$ & $8.9 \mathrm{E}-05$ & $1.43 \mathrm{E}-04$ & $61.47 \%$ \\
\hline In-115 & $1.1 \mathrm{E}-02$ & $2.99 \mathrm{E}+00$ & $26027.40 \%$ & $\mathrm{Pu}-238$ & $3.4 \mathrm{E}-01$ & $5.53 \mathrm{E}-01$ & $63.21 \%$ \\
\hline Sb-122 & $3.6 \mathrm{E}-04$ & $6.50 \mathrm{E}-04$ & $80.46 \%$ & $\mathrm{Pu}-239$ & $3.8 \mathrm{E}-01$ & $6.26 \mathrm{E}-01$ & $63.21 \%$ \\
\hline Sb-124 & $8.3 \mathrm{E}-04$ & $1.53 \mathrm{E}-03$ & $83.10 \%$ & $\mathrm{Pu}-240$ & $3.8 \mathrm{E}-01$ & $6.26 \mathrm{E}-01$ & $63.21 \%$ \\
\hline Sb-125 & $4.8 \mathrm{E}-04$ & $6.45 \mathrm{E}-04$ & $34.62 \%$ & $\mathrm{Pu}-241$ & $7.7 \mathrm{E}-03$ & $1.25 \mathrm{E}-02$ & $63.21 \%$ \\
\hline Te-123 & $3.8 \mathrm{E}-04$ & $1.68 \mathrm{E}-03$ & $345.19 \%$ & $\mathrm{Pu}-242$ & $3.7 \mathrm{E}-01$ & $5.97 \mathrm{E}-01$ & $63.21 \%$ \\
\hline Te-125m & $3.1 \mathrm{E}-03$ & $1.33 \mathrm{E}-03$ & $-56.98 \%$ & $\mathrm{Pu}-244$ & $3.6 \mathrm{E}-01$ & $5.82 \mathrm{E}-01$ & $63.21 \%$ \\
\hline Sn-126 & $1.1 \mathrm{E}-01$ & $1.10 \mathrm{E}-01$ & $-0.04 \%$ & Cm-241 & $6.0 \mathrm{E}-04$ & $6.46 \mathrm{E}-04$ & $7.83 \%$ \\
\hline I-129 & $3.2 \mathrm{E}-02$ & 4.09E-02 & $27.91 \%$ & $\mathrm{Cm}-242$ & $1.5 \mathrm{E}-02$ & $1.59 \mathrm{E}-02$ & $7.87 \%$ \\
\hline $\mathrm{I}-131$ & $5.2 \mathrm{E}-03$ & $6.66 \mathrm{E}-03$ & $27.21 \%$ & $\mathrm{Cm}-243$ & $3.9 \mathrm{E}-01$ & $4.23 \mathrm{E}-01$ & $7.85 \%$ \\
\hline Cs-134 & $4.8 \mathrm{E}-01$ & $4.78 \mathrm{E}-01$ & $-0.02 \%$ & $\mathrm{Cm}-244$ & $3.1 \mathrm{E}-01$ & $3.35 \mathrm{E}-01$ & $7.88 \%$ \\
\hline Cs-135 & $4.6 \mathrm{E}-02$ & $4.59 \mathrm{E}-02$ & $0.00 \%$ & $\mathrm{Cm}-245$ & $6.1 \mathrm{E}-01$ & $6.55 \mathrm{E}-01$ & $7.87 \%$ \\
\hline Cs-137 & $3.3 \mathrm{E}-01$ & $3.25 \mathrm{E}-01$ & $-0.09 \%$ & $\mathrm{Cm}-246$ & $6.1 \mathrm{E}-01$ & $6.55 \mathrm{E}-01$ & $7.88 \%$ \\
\hline La-137 & $5.8 \mathrm{E}-05$ & $6.90 \mathrm{E}-05$ & $18.92 \%$ & $\mathrm{Cm}-247$ & $5.6 \mathrm{E}-01$ & $5.99 \mathrm{E}-01$ & $7.81 \%$ \\
\hline La-138 & $8.0 \mathrm{E}-04$ & $9.47 \mathrm{E}-04$ & $18.92 \%$ & $\mathrm{Cm}-248$ & $2.2 \mathrm{E}+00$ & $2.33 \mathrm{E}+00$ & $7.88 \%$ \\
\hline La-140 & $5.2 \mathrm{E}-04$ & $6.13 \mathrm{E}-04$ & $17.11 \%$ & $\mathrm{Cm}-250$ & $1.0 \mathrm{E}+01$ & $1.13 \mathrm{E}+01$ & $7.88 \%$ \\
\hline Ba-140 & $7.2 \mathrm{E}-04$ & $6.79 \mathrm{E}-04$ & $-5.73 \%$ & Bk-247 & $3.1 \mathrm{E}-01$ & $3.10 \mathrm{E}-01$ & $0.00 \%$ \\
\hline Ce-141 & $2.1 \mathrm{E}-04$ & $3.40 \mathrm{E}-04$ & $59.71 \%$ & Bk-249 & $8.1 \mathrm{E}-04$ & $8.07 \mathrm{E}-04$ & $0.00 \%$ \\
\hline Ce-144 & $1.7 \mathrm{E}-03$ & $2.69 \mathrm{E}-03$ & $60.44 \%$ & Cf-249 & $6.2 \mathrm{E}-01$ & $6.22 \mathrm{E}-01$ & $-0.04 \%$ \\
\hline & & & & Cf- 250 & $2.6 \mathrm{E}-01$ & $2.56 \mathrm{E}-01$ & $0.00 \%$ \\
\hline & & & & Cf-251 & $6.2 \mathrm{E}-01$ & $6.21 \mathrm{E}-01$ & $-0.02 \%$ \\
\hline & & & & Cf-252 & $1.3 \mathrm{E}-01$ & $1.27 \mathrm{E}-01$ & $0.00 \%$ \\
\hline & & & & Es-253 & $2.3 \mathrm{E}-03$ & $3.06 \mathrm{E}-03$ & $30.56 \%$ \\
\hline & & & & Unidentified alpha & $3.8 \mathrm{E}-01$ & $6.3 \mathrm{E}-01$ & $63.21 \%$ \\
\hline & & & & Unidentified beta & $1.9 \mathrm{E}-02$ & $1.1 \mathrm{E}-02$ & $-39.61 \%$ \\
\hline & & & & TOTAL Dose & $3.7 \mathrm{E}+01$ & $4.6 \mathrm{E}+01$ & $26.61 \%$ \\
\hline
\end{tabular}


Table 20. LADTAP XL Collective Water Ingestion Comparisons (person-rem)

\begin{tabular}{|c|c|c|c|c|c|c|c|}
\hline Nuclide & $\begin{array}{c}\text { Hamby } \\
\text { Ladtap } \\
\text { Total Water }\end{array}$ & $\begin{array}{c}\text { Jannik } \\
\text { Ladtap } \\
\text { Total Water }\end{array}$ & $\%$ Diff & Nuclide & $\begin{array}{c}\text { Hamby } \\
\text { Ladtap } \\
\text { Total Water }\end{array}$ & $\begin{array}{c}\text { Jannik } \\
\text { Ladtap } \\
\text { Total Water }\end{array}$ & $\%$ Diff \\
\hline $\mathrm{H}-3$ & 4.2E-04 & $3.8 \mathrm{E}-04$ & $-8.92 \%$ & Sm-146 & $1.3 \mathrm{E}+00$ & $1.2 \mathrm{E}+00$ & $-8.92 \%$ \\
\hline $\mathrm{Be}-7$ & $7.0 \mathrm{E}-04$ & $6.4 \mathrm{E}-04$ & $-8.92 \%$ & Sm-147 & $1.2 \mathrm{E}+00$ & $1.1 \mathrm{E}+00$ & $-8.92 \%$ \\
\hline Be-10 & $2.8 \mathrm{E}-02$ & $2.6 \mathrm{E}-02$ & $-8.92 \%$ & Sm-151 & $2.3 \mathrm{E}-03$ & $2.1 \mathrm{E}-03$ & $-8.92 \%$ \\
\hline C-14 & $1.4 \mathrm{E}-02$ & $1.3 \mathrm{E}-02$ & $-8.92 \%$ & Pm-147 & $6.3 \mathrm{E}-03$ & $5.8 \mathrm{E}-03$ & $-8.92 \%$ \\
\hline $\mathrm{Na}-22$ & $8.0 \mathrm{E}-02$ & $7.3 \mathrm{E}-02$ & $-8.92 \%$ & Eu-152 & $4.0 \mathrm{E}-02$ & $3.6 \mathrm{E}-02$ & $-8.92 \%$ \\
\hline $\mathrm{Na}-24$ & $1.1 \mathrm{E}-04$ & $1.0 \mathrm{E}-04$ & $-8.92 \%$ & Eu-154 & $6.1 \mathrm{E}-02$ & $5.5 \mathrm{E}-02$ & $-8.92 \%$ \\
\hline Al-26 & $8.7 \mathrm{E}-02$ & $7.9 \mathrm{E}-02$ & $-8.92 \%$ & Eu-155 & 8.7E-03 & 7.9E-03 & $-8.92 \%$ \\
\hline $\mathrm{P}-32$ & $4.2 \mathrm{E}-02$ & $3.9 \mathrm{E}-02$ & $-8.92 \%$ & Gd-152 & $1.0 \mathrm{E}+00$ & $9.1 \mathrm{E}-01$ & $-8.92 \%$ \\
\hline Si-32 & $1.1 \mathrm{E}-02$ & $1.0 \mathrm{E}-02$ & $-8.92 \%$ & Ho-166m & $5.2 \mathrm{E}-02$ & $4.7 \mathrm{E}-02$ & $-8.92 \%$ \\
\hline S-35 & $4.2 \mathrm{E}-03$ & $3.8 \mathrm{E}-03$ & $-8.92 \%$ & Lu-176 & $4.4 \mathrm{E}-02$ & $4.0 \mathrm{E}-02$ & $-8.92 \%$ \\
\hline Cl-36 & $2.0 \mathrm{E}-02$ & $1.8 \mathrm{E}-02$ & $-8.92 \%$ & Ta-180 & $4.9 \mathrm{E}-215$ & $4.4 \mathrm{E}-215$ & $-8.92 \%$ \\
\hline $\mathrm{K}-40$ & $1.3 \mathrm{E}-01$ & $1.2 \mathrm{E}-01$ & $-8.92 \%$ & Hf-182 & $9.4 \mathrm{E}-02$ & $8.5 \mathrm{E}-02$ & $-8.92 \%$ \\
\hline $\mathrm{K}-43$ & $2.6 \mathrm{E}-04$ & $2.4 \mathrm{E}-04$ & $-8.92 \%$ & Re-186m & $2.2 \mathrm{E}-02$ & $2.0 \mathrm{E}-02$ & $-8.92 \%$ \\
\hline $\mathrm{Ca}-41$ & $8.0 \mathrm{E}-03$ & $7.3 \mathrm{E}-03$ & $-8.92 \%$ & Re-187 & $5.5 \mathrm{E}-05$ & $5.1 \mathrm{E}-05$ & $-8.92 \%$ \\
\hline $\mathrm{Ca}-45$ & $2.0 \mathrm{E}-02$ & $1.8 \mathrm{E}-02$ & $-8.92 \%$ & Ir-192m & $0.0 \mathrm{E}+00$ & $0.0 \mathrm{E}+00$ & $0.00 \%$ \\
\hline $\mathrm{Ca}-47$ & $2.2 \mathrm{E}-02$ & $2.0 \mathrm{E}-02$ & $-8.92 \%$ & Pt-193 & $7.3 \mathrm{E}-04$ & $6.7 \mathrm{E}-04$ & $-8.92 \%$ \\
\hline Ti-44 & $1.3 \mathrm{E}-01$ & $1.2 \mathrm{E}-01$ & $-8.92 \%$ & Hg-194 & $1.9 \mathrm{E}+00$ & $1.7 \mathrm{E}+00$ & $-8.92 \%$ \\
\hline V-49 & $3.6 \mathrm{E}-04$ & $3.3 \mathrm{E}-04$ & $-8.92 \%$ & $\mathrm{Hg}-203$ & $6.3 \mathrm{E}-02$ & $5.7 \mathrm{E}-02$ & $-8.92 \%$ \\
\hline $\mathrm{Cr}-51$ & 7.9E-04 & $7.2 \mathrm{E}-04$ & $-8.92 \%$ & $\mathrm{~Pb}-202$ & $2.6 \mathrm{E}-01$ & $2.4 \mathrm{E}-01$ & $-8.92 \%$ \\
\hline Mn-53 & $6.6 \mathrm{E}-04$ & $6.0 \mathrm{E}-04$ & $-8.92 \%$ & $\mathrm{~Pb}-205$ & $1.0 \mathrm{E}-02$ & $9.1 \mathrm{E}-03$ & $-8.92 \%$ \\
\hline Mn-54 & $1.8 \mathrm{E}-02$ & $1.6 \mathrm{E}-02$ & $-8.92 \%$ & $\mathrm{~Pb}-210$ & $3.4 \mathrm{E}+01$ & $3.1 \mathrm{E}+01$ & $-8.92 \%$ \\
\hline $\mathrm{Fe}-55$ & $3.9 \mathrm{E}-03$ & $3.5 \mathrm{E}-03$ & $-8.92 \%$ & Bi-207 & $3.3 \mathrm{E}-02$ & $3.0 \mathrm{E}-02$ & $-8.92 \%$ \\
\hline $\mathrm{Fe}-60$ & $1.0 \mathrm{E}+00$ & $9.1 \mathrm{E}-01$ & $-8.92 \%$ & Bi-210 & $2.3 \mathrm{E}-02$ & $2.1 \mathrm{E}-02$ & $-8.92 \%$ \\
\hline Co-58 & $2.2 \mathrm{E}-02$ & $2.0 \mathrm{E}-02$ & $-8.92 \%$ & $\mathrm{Bi}-210 \mathrm{~m}$ & $5.7 \mathrm{E}-01$ & $5.2 \mathrm{E}-01$ & $-8.92 \%$ \\
\hline Co-60 & $1.7 \mathrm{E}-01$ & $1.6 \mathrm{E}-01$ & $-8.92 \%$ & Po-210 & $1.0 \mathrm{E}+01$ & $9.5 \mathrm{E}+00$ & $-8.92 \%$ \\
\hline Ni-59 & $1.3 \mathrm{E}-03$ & $1.2 \mathrm{E}-03$ & $-8.92 \%$ & Ra-223 & $2.9 \mathrm{E}+00$ & $2.6 \mathrm{E}+00$ & $-8.92 \%$ \\
\hline $\mathrm{Ni}-63$ & $3.6 \mathrm{E}-03$ & $3.3 \mathrm{E}-03$ & $-8.92 \%$ & $\mathrm{Ra}-224$ & $1.0 \mathrm{E}+00$ & $9.3 \mathrm{E}-01$ & $-8.92 \%$ \\
\hline $\mathrm{Cu}-64$ & $1.5 \mathrm{E}-05$ & $1.4 \mathrm{E}-05$ & $-8.92 \%$ & Ra-225 & $1.7 \mathrm{E}+00$ & $1.6 \mathrm{E}+00$ & $-8.92 \%$ \\
\hline
\end{tabular}


Table 20. LADTAP XL Collective Water Ingestion Comparisons (person-rem) (continued)

\begin{tabular}{|c|c|c|c|c|c|c|c|}
\hline Nuclide & $\begin{array}{c}\text { Hamby } \\
\text { Ladtap } \\
\text { Total Water }\end{array}$ & $\begin{array}{c}\text { Jannik } \\
\text { Ladtap } \\
\text { Total Water }\end{array}$ & $\%$ Diff & Nuclide & $\begin{array}{c}\text { Hamby } \\
\text { Ladtap } \\
\text { Total Water }\end{array}$ & $\begin{array}{c}\text { Jannik } \\
\text { Ladtap } \\
\text { Total Water }\end{array}$ & $\%$ Diff \\
\hline Zn-65 & $9.2 \mathrm{E}-02$ & $8.4 \mathrm{E}-02$ & $-8.92 \%$ & Ra-226 & $7.4 \mathrm{E}+00$ & $6.7 \mathrm{E}+00$ & $-8.92 \%$ \\
\hline Ge-68 & 7.3E-03 & $6.6 \mathrm{E}-03$ & $-8.92 \%$ & Ra-228 & $8.0 \mathrm{E}+00$ & $7.3 \mathrm{E}+00$ & $-8.92 \%$ \\
\hline Se-75 & $5.7 \mathrm{E}-02$ & $5.2 \mathrm{E}-02$ & $-8.92 \%$ & Ac-227 & $9.4 \mathrm{E}+01$ & $8.5 \mathrm{E}+01$ & $-8.92 \%$ \\
\hline Se-79 & $5.5 \mathrm{E}-02$ & $5.1 \mathrm{E}-02$ & $-8.92 \%$ & Th-227 & $2.1 \mathrm{E}-01$ & $1.9 \mathrm{E}-01$ & $-8.92 \%$ \\
\hline $\mathrm{Rb}-87$ & $3.2 \mathrm{E}-02$ & $2.9 \mathrm{E}-02$ & $-8.92 \%$ & Th-228 & $2.5 \mathrm{E}+00$ & $2.3 \mathrm{E}+00$ & $-8.92 \%$ \\
\hline Sr-89 & $5.5 \mathrm{E}-02$ & $5.0 \mathrm{E}-02$ & $-8.92 \%$ & Th-229 & $2.3 \mathrm{E}+01$ & $2.1 \mathrm{E}+01$ & $-8.92 \%$ \\
\hline Sr-90 & $8.7 \mathrm{E}-01$ & $7.9 \mathrm{E}-01$ & $-8.92 \%$ & Th-230 & $3.5 \mathrm{E}+00$ & $3.2 \mathrm{E}+00$ & $-8.92 \%$ \\
\hline $\mathrm{Y}-90$ & $2.4 \mathrm{E}-02$ & $2.2 \mathrm{E}-02$ & $-8.92 \%$ & Th-231 & $6.4 \mathrm{E}-04$ & $5.8 \mathrm{E}-04$ & $-8.92 \%$ \\
\hline Y-91 & $5.7 \mathrm{E}-02$ & $5.2 \mathrm{E}-02$ & $-8.92 \%$ & Th-232 & $1.9 \mathrm{E}+01$ & $1.7 \mathrm{E}+01$ & $-8.92 \%$ \\
\hline Mo-93 & $8.7 \mathrm{E}-03$ & 7.9E-03 & $-8.92 \%$ & Th-234 & 7.7E-02 & $7.1 \mathrm{E}-02$ & $-8.92 \%$ \\
\hline Мo-99 & $1.1 \mathrm{E}-02$ & $9.8 \mathrm{E}-03$ & $-8.92 \%$ & $\mathrm{~Pa}-230$ & $3.2 \mathrm{E}-02$ & $2.9 \mathrm{E}-02$ & $-8.92 \%$ \\
\hline $\mathrm{Nb}-93 \mathrm{~m}$ & $3.5 \mathrm{E}-02$ & $3.2 \mathrm{E}-02$ & $-8.92 \%$ & Pa-231 & $7.4 \mathrm{E}+01$ & $6.7 \mathrm{E}+01$ & $-8.92 \%$ \\
\hline $\mathrm{Nb}-94$ & $3.4 \mathrm{E}-02$ & $3.1 \mathrm{E}-02$ & $-8.92 \%$ & $\mathrm{~Pa}-233$ & $2.0 \mathrm{E}-02$ & $1.8 \mathrm{E}-02$ & $-8.92 \%$ \\
\hline $\mathrm{Nb}-95$ & $1.4 \mathrm{E}-02$ & $1.2 \mathrm{E}-02$ & $-8.92 \%$ & U-232 & $8.7 \mathrm{E}+00$ & $7.9 \mathrm{E}+00$ & $-8.92 \%$ \\
\hline Zr-93 & $1.1 \mathrm{E}-02$ & $9.7 \mathrm{E}-03$ & $-8.92 \%$ & U-233 & $1.8 \mathrm{E}+00$ & $1.6 \mathrm{E}+00$ & $-8.92 \%$ \\
\hline Zr-95 & $2.2 \mathrm{E}-02$ & $2.0 \mathrm{E}-02$ & $-8.92 \%$ & U-234 & $1.7 \mathrm{E}+00$ & $1.6 \mathrm{E}+00$ & $-8.92 \%$ \\
\hline Tc-96 & $9.5 \mathrm{E}-03$ & $8.6 \mathrm{E}-03$ & $-8.92 \%$ & U-235 & $1.7 \mathrm{E}+00$ & $1.5 \mathrm{E}+00$ & $-8.92 \%$ \\
\hline Tc-97 & $1.0 \mathrm{E}-03$ & $9.1 \mathrm{E}-04$ & $-8.92 \%$ & U-236 & $1.7 \mathrm{E}+00$ & $1.5 \mathrm{E}+00$ & $-8.92 \%$ \\
\hline Tc-98 & $3.2 \mathrm{E}-02$ & $2.9 \mathrm{E}-02$ & $-8.92 \%$ & U-237 & $1.2 \mathrm{E}-02$ & $1.1 \mathrm{E}-02$ & $-8.92 \%$ \\
\hline Tc-99 & $8.7 \mathrm{E}-03$ & $7.9 \mathrm{E}-03$ & $-8.92 \%$ & U-238 & $1.5 \mathrm{E}+00$ & $1.4 \mathrm{E}+00$ & $-8.92 \%$ \\
\hline Ru-97 & $1.6 \mathrm{E}-03$ & $1.5 \mathrm{E}-03$ & $-8.92 \%$ & $\mathrm{~Np}-236$ & $5.3 \mathrm{E}+00$ & $4.8 \mathrm{E}+00$ & $-8.92 \%$ \\
\hline Ru-103 & $1.7 \mathrm{E}-02$ & $1.5 \mathrm{E}-02$ & $-8.92 \%$ & Np-237 & $2.6 \mathrm{E}+01$ & $2.4 \mathrm{E}+01$ & $-8.92 \%$ \\
\hline $\mathrm{Ru}-106$ & $1.4 \mathrm{E}-01$ & $1.3 \mathrm{E}-01$ & $-8.92 \%$ & $\mathrm{~Np}-239$ & $6.0 \mathrm{E}-03$ & $5.4 \mathrm{E}-03$ & $-8.92 \%$ \\
\hline Pd-107 & $9.4 \mathrm{E}-04$ & $8.5 \mathrm{E}-04$ & $-8.92 \%$ & Am-237 & $8.8 \mathrm{E}-28$ & $8.1 \mathrm{E}-28$ & $-8.92 \%$ \\
\hline Ag-108m & $5.0 \mathrm{E}-02$ & $4.6 \mathrm{E}-02$ & $-8.92 \%$ & Am-241 & $3.0 \mathrm{E}+01$ & $2.7 \mathrm{E}+01$ & $-8.92 \%$ \\
\hline Ag-110m & $7.3 \mathrm{E}-02$ & $6.6 \mathrm{E}-02$ & $-8.92 \%$ & Am-242m & $2.8 \mathrm{E}+01$ & $2.6 \mathrm{E}+01$ & $-8.92 \%$ \\
\hline $\mathrm{Cd}-113$ & $1.1 \mathrm{E}+00$ & $9.7 \mathrm{E}-01$ & $-8.92 \%$ & Am-243 & $3.0 \mathrm{E}+01$ & $2.7 \mathrm{E}+01$ & $-8.92 \%$ \\
\hline
\end{tabular}


SRNL-STI-2010-00447, REVISION 0

Table 20. LADTAP XL Collective Water Ingestion Comparisons (person-rem) (continued)

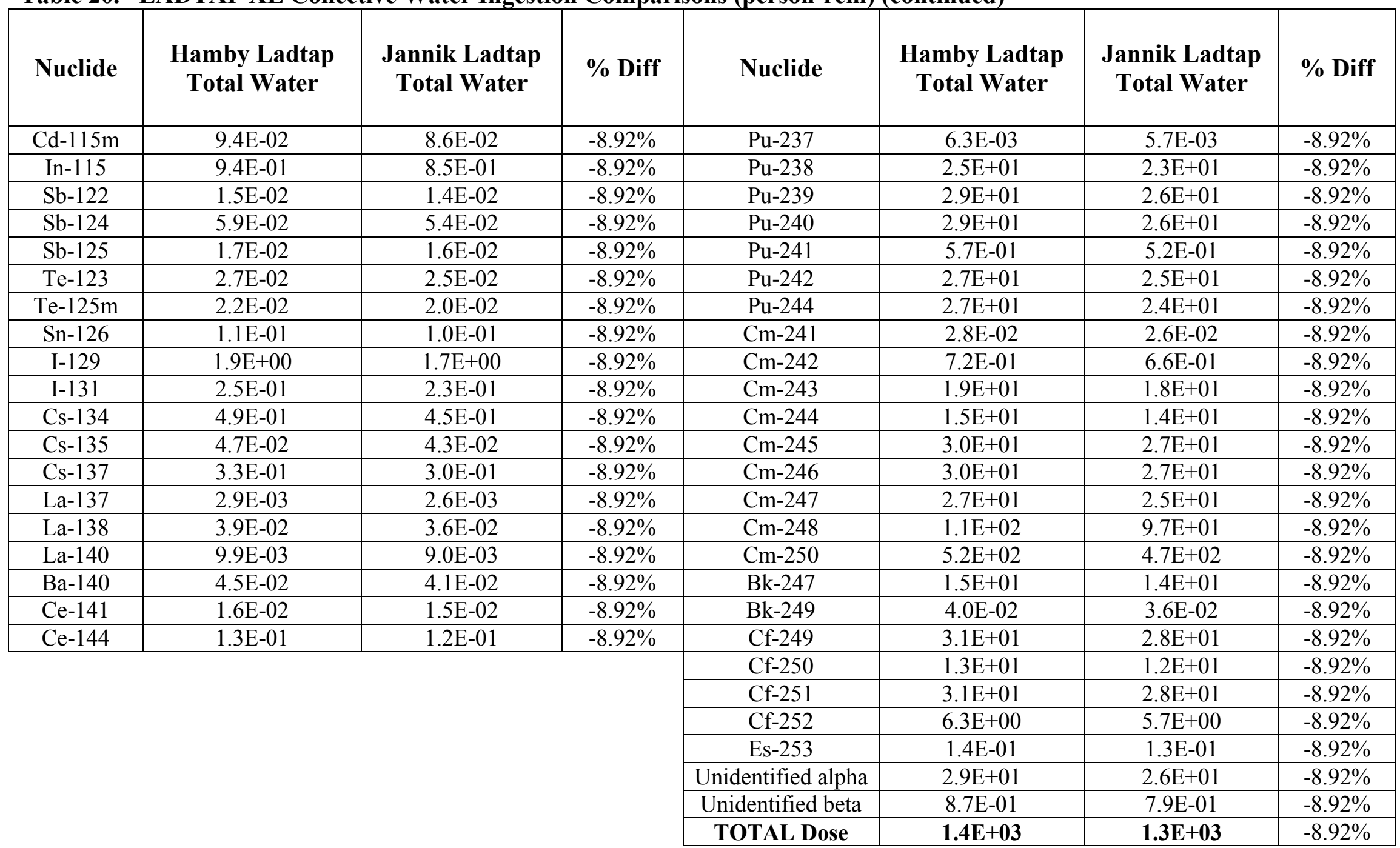


Table 21. LADTAP XL Collective Sport Fish Ingestion Comparisons (person-rem)

\begin{tabular}{|c|c|c|c|c|c|c|c|}
\hline Nuclide & $\begin{array}{c}\text { Hamby } \\
\text { Ladtap } \\
\text { Sport fish } \\
\end{array}$ & $\begin{array}{c}\text { Jannik } \\
\text { Ladtap } \\
\text { Sport fish } \\
\end{array}$ & $\%$ Diff & Nuclide & $\begin{array}{c}\text { Hamby } \\
\text { Ladtap } \\
\text { Sport fish } \\
\end{array}$ & $\begin{array}{c}\text { Jannik } \\
\text { Ladtap } \\
\text { Sport fish } \\
\end{array}$ & $\%$ Diff \\
\hline H-3 & $2.2 \mathrm{E}-07$ & $5.8 \mathrm{E}-08$ & $-283.21 \%$ & Sm-146 & $2.0 \mathrm{E}-02$ & $5.5 \mathrm{E}-03$ & $-254.83 \%$ \\
\hline $\mathrm{Be}-7$ & $7.6 \mathrm{E}-07$ & 8.9E-06 & $91.48 \%$ & Sm-147 & $1.8 \mathrm{E}-02$ & $5.0 \mathrm{E}-03$ & $-254.83 \%$ \\
\hline $\mathrm{Be}-10$ & $3.3 \mathrm{E}-05$ & $3.9 \mathrm{E}-04$ & $91.48 \%$ & Sm-151 & $3.3 \mathrm{E}-05$ & $9.4 \mathrm{E}-06$ & $-254.83 \%$ \\
\hline $\mathrm{C}-14$ & $3.8 \mathrm{E}-02$ & $5.8 \mathrm{E}-06$ & $-652779.16 \%$ & Pm-147 & $9.2 \mathrm{E}-05$ & $2.6 \mathrm{E}-05$ & $-254.83 \%$ \\
\hline $\mathrm{Na}-22$ & 4.7E-03 & $8.3 \mathrm{E}-04$ & $-460.25 \%$ & Eu-152 & 5.9E-04 & $7.2 \mathrm{E}-04$ & $18.12 \%$ \\
\hline $\mathrm{Na}-24$ & 8.3E-09 & $1.5 \mathrm{E}-09$ & $-460.25 \%$ & Eu-154 & 8.9E-04 & $1.1 \mathrm{E}-03$ & $18.12 \%$ \\
\hline Al-26 & $5.1 \mathrm{E}-04$ & $6.1 \mathrm{E}-04$ & $16.51 \%$ & Eu-155 & $1.3 \mathrm{E}-04$ & $1.5 \mathrm{E}-04$ & $18.12 \%$ \\
\hline $\mathrm{P}-32$ & $1.9 \mathrm{E}+00$ & $6.1 \mathrm{E}-01$ & $-204.14 \%$ & Gd-152 & $1.5 \mathrm{E}-02$ & $4.1 \mathrm{E}-03$ & $-254.83 \%$ \\
\hline $\mathrm{Si}-32$ & $1.7 \mathrm{E}-05$ & $3.1 \mathrm{E}-05$ & $46.78 \%$ & Ho-166m & $7.6 \mathrm{E}-04$ & $2.2 \mathrm{E}-04$ & $-254.83 \%$ \\
\hline $\mathrm{S}-35$ & $1.8 \mathrm{E}-03$ & $4.4 \mathrm{E}-04$ & $-299.18 \%$ & Lu-176 & $6.5 \mathrm{E}-04$ & $1.5 \mathrm{E}-04$ & $-325.79 \%$ \\
\hline $\mathrm{Cl}-36$ & 5.9E-04 & $1.3 \mathrm{E}-04$ & $-352.97 \%$ & Ta-180 & $0.0 \mathrm{E}+00$ & $0.0 \mathrm{E}+00$ & \\
\hline $\mathrm{K}-40$ & 7.4E-02 & $5.6 \mathrm{E}-02$ & $-33.06 \%$ & Hf-182 & $1.8 \mathrm{E}-04$ & $1.4 \mathrm{E}-02$ & $98.71 \%$ \\
\hline $\mathrm{K}-43$ & $1.8 \mathrm{E}-06$ & $1.3 \mathrm{E}-06$ & $-33.06 \%$ & Re-186m & $1.5 \mathrm{E}-03$ & $3.6 \mathrm{E}-04$ & $-322.24 \%$ \\
\hline $\mathrm{Ca}-41$ & 1.9E-04 & $1.3 \mathrm{E}-05$ & $-1319.30 \%$ & $\mathrm{Re}-187$ & $3.9 \mathrm{E}-06$ & $9.2 \mathrm{E}-07$ & $-322.24 \%$ \\
\hline $\mathrm{Ca}-45$ & $4.5 \mathrm{E}-04$ & $3.2 \mathrm{E}-05$ & $-1319.30 \%$ & Ir-192m & $0.0 \mathrm{E}+00$ & $0.0 \mathrm{E}+00$ & \\
\hline $\mathrm{Ca}-47$ & $2.1 \mathrm{E}-04$ & $1.5 \mathrm{E}-05$ & $-1319.30 \%$ & Pt-193 & $4.3 \mathrm{E}-05$ & $3.5 \mathrm{E}-06$ & $-1116.55 \%$ \\
\hline Ti-44 & 7.4E-02 & $3.3 \mathrm{E}-03$ & $-2141.00 \%$ & Hg-194 & $1.1 \mathrm{E}+00$ & $1.6 \mathrm{E}+00$ & $30.20 \%$ \\
\hline $\mathrm{V}-49$ & $2.1 \mathrm{E}-06$ & $4.7 \mathrm{E}-06$ & $56.10 \%$ & $\mathrm{Hg}-203$ & $3.4 \mathrm{E}-02$ & $4.8 \mathrm{E}-02$ & $30.20 \%$ \\
\hline Cr-51 & 7.9E-05 & $3.7 \mathrm{E}-06$ & $-2028.95 \%$ & $\mathrm{~Pb}-202$ & $4.6 \mathrm{E}-02$ & $9.0 \mathrm{E}-04$ & $-5009.49 \%$ \\
\hline $\mathrm{Mn}-53$ & $3.9 \mathrm{E}-05$ & $2.2 \mathrm{E}-05$ & $-77.41 \%$ & $\mathrm{~Pb}-205$ & $1.8 \mathrm{E}-03$ & $3.5 \mathrm{E}-05$ & $-5009.49 \%$ \\
\hline $\mathrm{Mn}-54$ & $4.1 \mathrm{E}-03$ & $5.8 \mathrm{E}-04$ & $-609.65 \%$ & $\mathrm{~Pb}-210$ & $6.0 \mathrm{E}+00$ & $1.2 \mathrm{E}-01$ & $-5009.49 \%$ \\
\hline $\mathrm{Fe}-55$ & $2.3 \mathrm{E}-04$ & $9.0 \mathrm{E}-05$ & $-150.47 \%$ & $\mathrm{Bi}-207$ & $2.9 \mathrm{E}-04$ & $6.8 \mathrm{E}-05$ & $-325.79 \%$ \\
\hline Fe-60 & $5.9 \mathrm{E}-02$ & $2.3 \mathrm{E}-02$ & $-150.47 \%$ & $\mathrm{Bi}-210$ & $8.7 E-05$ & $2.0 \mathrm{E}-05$ & $-325.79 \%$ \\
\hline Co-58 & $6.2 \mathrm{E}-04$ & $2.2 \mathrm{E}-04$ & $-180.13 \%$ & $\mathrm{Bi}-210 \mathrm{~m}$ & $5.1 \mathrm{E}-03$ & $1.2 \mathrm{E}-03$ & $-325.79 \%$ \\
\hline Co-60 & $5.1 \mathrm{E}-03$ & $1.8 \mathrm{E}-03$ & $-180.13 \%$ & Po-210 & $3.0 \mathrm{E}+00$ & $5.0 \mathrm{E}-02$ & $-5813.76 \%$ \\
\hline $\mathrm{Ni}-59$ & $7.8 \mathrm{E}-05$ & $3.9 \mathrm{E}-06$ & $-1927.58 \%$ & Ra-223 & $5.9 \mathrm{E}-02$ & $1.1 \mathrm{E}-03$ & $-5222.38 \%$ \\
\hline $\mathrm{Ni}-63$ & $2.1 \mathrm{E}-04$ & $1.0 \mathrm{E}-05$ & $-1927.58 \%$ & $\mathrm{Ra}-224$ & $9.5 \mathrm{E}-03$ & $1.8 \mathrm{E}-04$ & $-5222.38 \%$ \\
\hline $\mathrm{Cu}-64$ & $6.9 \mathrm{E}-10$ & $1.9 \mathrm{E}-10$ & $-270.25 \%$ & $\mathrm{Ra}-225$ & $3.8 \mathrm{E}-02$ & $7.1 \mathrm{E}-04$ & $-5222.38 \%$ \\
\hline
\end{tabular}


SRNL-STI-2010-00447, REVISION 0

Table 21. LADTAP XL Collective Sport Fish Ingestion Comparisons (person-rem) (continued)

\begin{tabular}{|c|c|c|c|c|c|c|c|}
\hline Nuclide & $\begin{array}{c}\text { Hamby Ladtap } \\
\text { Sport fish }\end{array}$ & $\begin{array}{c}\text { Jannik Ladtap } \\
\text { Sport fish }\end{array}$ & $\%$ Diff & Nuclide & $\begin{array}{c}\text { Hamby Ladtap } \\
\text { Sport fish }\end{array}$ & $\begin{array}{c}\text { Jannik Ladtap } \\
\text { Sport fish }\end{array}$ & $\%$ Diff \\
\hline $\mathrm{Zn}-65$ & $1.1 \mathrm{E}-01$ & $4.3 \mathrm{E}-02$ & $-150.47 \%$ & Ra-226 & $2.2 \mathrm{E}-01$ & $4.0 \mathrm{E}-03$ & $-5222.38 \%$ \\
\hline Ge-68 & $1.4 \mathrm{E}-02$ & $4.0 \mathrm{E}-03$ & $-254.47 \%$ & $\mathrm{Ra}-228$ & $2.3 \mathrm{E}-01$ & $4.4 \mathrm{E}-03$ & $-5222.38 \%$ \\
\hline $\mathrm{Se}-75$ & $5.5 \mathrm{E}-03$ & $4.6 \mathrm{E}-02$ & $87.94 \%$ & Ac- 227 & $1.4 \mathrm{E}+00$ & $3.2 \mathrm{E}-01$ & $-325.79 \%$ \\
\hline Se-79 & $5.5 \mathrm{E}-03$ & $4.6 \mathrm{E}-02$ & $87.94 \%$ & Th-227 & $2.9 \mathrm{E}-03$ & $1.4 \mathrm{E}-04$ & $-2028.95 \%$ \\
\hline $\mathrm{Rb}-87$ & $3.8 \mathrm{E}-02$ & $2.2 \mathrm{E}-02$ & $-73.79 \%$ & Th-228 & $4.4 \mathrm{E}-02$ & $2.1 \mathrm{E}-03$ & $-2028.95 \%$ \\
\hline Sr-89 & 8.9E-04 & $2.0 \mathrm{E}-05$ & $-4304.73 \%$ & Th-229 & $4.1 \mathrm{E}-01$ & $1.9 \mathrm{E}-02$ & $-2028.95 \%$ \\
\hline Sr-90 & $1.5 \mathrm{E}-02$ & $3.5 \mathrm{E}-04$ & $-4304.73 \%$ & Th-230 & $6.2 \mathrm{E}-02$ & $2.9 \mathrm{E}-03$ & $-2028.95 \%$ \\
\hline $\mathrm{Y}-90$ & $7.3 \mathrm{E}-05$ & $2.8 \mathrm{E}-05$ & $-166.12 \%$ & Th-231 & $2.3 \mathrm{E}-07$ & $1.1 \mathrm{E}-08$ & $-2028.95 \%$ \\
\hline Y-91 & 7.7E-04 & $2.9 \mathrm{E}-04$ & $-166.12 \%$ & Th-232 & $3.3 \mathrm{E}-01$ & $1.5 \mathrm{E}-02$ & $-2028.95 \%$ \\
\hline Mo-93 & $5.1 \mathrm{E}-05$ & $2.3 \mathrm{E}-06$ & $-2141.00 \%$ & Th-234 & $1.1 \mathrm{E}-03$ & $5.4 \mathrm{E}-05$ & $-2028.95 \%$ \\
\hline Mo-99 & $1.4 \mathrm{E}-05$ & $6.2 \mathrm{E}-07$ & $-2141.00 \%$ & $\mathrm{~Pa}-230$ & $1.7 \mathrm{E}-04$ & $3.5 \mathrm{E}-05$ & $-381.14 \%$ \\
\hline $\mathrm{Nb}-93 \mathrm{~m}$ & $6.2 \mathrm{E}-01$ & $1.5 \mathrm{E}-03$ & $-42479.08 \%$ & $\mathrm{~Pa}-231$ & 4.9E-01 & $1.0 \mathrm{E}-01$ & $-381.14 \%$ \\
\hline $\mathrm{Nb}-94$ & $6.0 \mathrm{E}-01$ & $1.4 \mathrm{E}-03$ & $-42479.08 \%$ & $\mathrm{~Pa}-233$ & $1.1 \mathrm{E}-04$ & $2.3 \mathrm{E}-05$ & $-381.14 \%$ \\
\hline $\mathrm{Nb}-95$ & $2.1 \mathrm{E}-01$ & $5.0 \mathrm{E}-04$ & $-42479.08 \%$ & U-232 & $5.1 \mathrm{E}-02$ & $1.1 \mathrm{E}-03$ & $-4335.32 \%$ \\
\hline Zr-93 & $2.1 \mathrm{E}-05$ & $3.2 \mathrm{E}-05$ & $36.13 \%$ & U-233 & $2.1 \mathrm{E}-03$ & $2.4 \mathrm{E}-04$ & $-787.06 \%$ \\
\hline Zr-95 & $3.9 \mathrm{E}-05$ & $6.2 \mathrm{E}-05$ & $36.13 \%$ & U-234 & $2.0 \mathrm{E}-03$ & $2.3 \mathrm{E}-04$ & $-787.06 \%$ \\
\hline Tc-96 & $3.2 \mathrm{E}-05$ & $9.9 \mathrm{E}-06$ & $-219.34 \%$ & U-235 & $2.0 \mathrm{E}-03$ & $2.2 \mathrm{E}-04$ & $-787.06 \%$ \\
\hline Tc-97 & $8.8 \mathrm{E}-06$ & $2.8 \mathrm{E}-06$ & $-219.34 \%$ & U-236 & $2.0 \mathrm{E}-03$ & $2.2 \mathrm{E}-04$ & $-787.06 \%$ \\
\hline Tc-98 & $2.8 \mathrm{E}-04$ & $8.8 \mathrm{E}-05$ & $-219.34 \%$ & U-237 & $7.6 \mathrm{E}-06$ & $8.5 \mathrm{E}-07$ & $-787.06 \%$ \\
\hline Tc-99 & 7.6E-05 & $2.4 \mathrm{E}-05$ & $-219.34 \%$ & U-238 & $1.8 \mathrm{E}-03$ & $2.0 \mathrm{E}-04$ & $-787.06 \%$ \\
\hline Ru-97 & $2.3 \mathrm{E}-06$ & $3.0 \mathrm{E}-06$ & $22.58 \%$ & $\mathrm{~Np}-236$ & $3.1 \mathrm{E}-02$ & $1.5 \mathrm{E}-02$ & $-102.76 \%$ \\
\hline $\mathrm{Ru}-103$ & $8.9 \mathrm{E}-05$ & $1.1 \mathrm{E}-04$ & $22.58 \%$ & $\mathrm{~Np}-237$ & $1.5 \mathrm{E}-01$ & $7.5 \mathrm{E}-02$ & $-102.76 \%$ \\
\hline Ru-106 & $8.1 \mathrm{E}-04$ & $1.0 \mathrm{E}-03$ & $22.58 \%$ & $\mathrm{~Np}-239$ & $5.9 \mathrm{E}-06$ & $2.9 \mathrm{E}-06$ & $-102.76 \%$ \\
\hline Pd-107 & $5.5 \mathrm{E}-06$ & $1.3 \mathrm{E}-06$ & $-325.79 \%$ & Am-237 & $3.1 \mathrm{E}-65$ & $7.0 \mathrm{E}-65$ & $55.65 \%$ \\
\hline Ag-108m & $6.8 \mathrm{E}-05$ & $7.6 \mathrm{E}-04$ & $91.06 \%$ & Am-241 & $4.4 \mathrm{E}-01$ & $9.9 \mathrm{E}-01$ & $55.65 \%$ \\
\hline Ag-110m & $9.6 \mathrm{E}-05$ & $1.1 \mathrm{E}-03$ & $91.10 \%$ & Am-242m & $4.1 \mathrm{E}-01$ & $9.3 \mathrm{E}-01$ & $55.65 \%$ \\
\hline $\mathrm{Cd}-113$ & $1.3 \mathrm{E}-01$ & $2.9 \mathrm{E}-02$ & $-325.79 \%$ & Am-243 & 4.4E-01 & 9.9E-01 & $55.65 \%$ \\
\hline
\end{tabular}

\section{(continued)}


SRNL-STI-2010-00447, REVISION 0

\begin{tabular}{|c|c|c|c|c|c|c|c|}
\hline Nuclide & $\begin{array}{c}\text { Hamby Ladtap } \\
\text { Sport fish }\end{array}$ & $\begin{array}{c}\text { Jannik Ladtap } \\
\text { Sport fish }\end{array}$ & $\%$ Diff & Nuclide & $\begin{array}{l}\text { Hamby Ladtap } \\
\text { Sport fish }\end{array}$ & $\begin{array}{c}\text { Jannik Ladtap } \\
\text { Sport fish }\end{array}$ & $\%$ Diff \\
\hline Cd-115m & $1.0 \mathrm{E}-02$ & $2.4 \mathrm{E}-03$ & $-325.79 \%$ & $\mathrm{Pu}-237$ & $1.2 \mathrm{E}-05$ & $2.4 \mathrm{E}-05$ & $50.32 \%$ \\
\hline In-115 & $0.0 \mathrm{E}+00$ & $1.3 \mathrm{E}+00$ & $100.00 \%$ & $\mathrm{Pu}-238$ & $5.2 \mathrm{E}-02$ & $1.0 \mathrm{E}-01$ & $50.32 \%$ \\
\hline Sb-122 & $1.9 \mathrm{E}-06$ & $1.7 \mathrm{E}-05$ & $88.49 \%$ & Pu-239 & $5.9 \mathrm{E}-02$ & $1.2 \mathrm{E}-01$ & $50.32 \%$ \\
\hline Sb-124 & $3.2 \mathrm{E}-05$ & $2.8 \mathrm{E}-04$ & $88.49 \%$ & $\mathrm{Pu}-240$ & 5.9E-02 & $1.2 \mathrm{E}-01$ & $50.32 \%$ \\
\hline Sb-125 & $1.0 \mathrm{E}-05$ & $8.8 \mathrm{E}-05$ & $88.49 \%$ & $\mathrm{Pu}-241$ & $1.2 \mathrm{E}-03$ & $2.4 \mathrm{E}-03$ & $50.32 \%$ \\
\hline Te-123 & $0.0 \mathrm{E}+00$ & $5.7 \mathrm{E}-04$ & $100.00 \%$ & $\mathrm{Pu}-242$ & $5.6 \mathrm{E}-02$ & $1.1 \mathrm{E}-01$ & $50.32 \%$ \\
\hline $\mathrm{Te}-125 \mathrm{~m}$ & $4.7 \mathrm{E}-03$ & $4.2 \mathrm{E}-04$ & $-1035.44 \%$ & $\mathrm{Pu}-244$ & $5.5 \mathrm{E}-02$ & $1.1 \mathrm{E}-01$ & $50.32 \%$ \\
\hline $\mathrm{Sn}-126$ & $2.0 \mathrm{E}-01$ & $4.7 \mathrm{E}-02$ & $-325.79 \%$ & $\mathrm{Cm}-241$ & $3.6 \mathrm{E}-04$ & $1.0 \mathrm{E}-04$ & $-254.83 \%$ \\
\hline I-129 & $1.6 \mathrm{E}-02$ & 7.7E-03 & $-112.90 \%$ & $\mathrm{Cm}-242$ & $1.0 \mathrm{E}-02$ & $2.9 \mathrm{E}-03$ & $-254.83 \%$ \\
\hline I-131 & $1.3 \mathrm{E}-03$ & $6.2 \mathrm{E}-04$ & $-112.90 \%$ & $\mathrm{Cm}-243$ & $2.8 \mathrm{E}-01$ & $8.0 \mathrm{E}-02$ & $-254.83 \%$ \\
\hline Cs-134 & $8.6 \mathrm{E}-01$ & $2.0 \mathrm{E}-01$ & $-325.79 \%$ & Cm-244 & 2.3E-01 & $6.3 \mathrm{E}-02$ & $-254.83 \%$ \\
\hline Cs-135 & $8.3 \mathrm{E}-02$ & $2.0 \mathrm{E}-02$ & $-325.79 \%$ & Cm-245 & $4.4 \mathrm{E}-01$ & $1.2 \mathrm{E}-01$ & $-254.83 \%$ \\
\hline Cs-137 & 5.9E-01 & $1.4 \mathrm{E}-01$ & $-325.79 \%$ & $\mathrm{Cm}-246$ & $4.4 \mathrm{E}-01$ & $1.2 \mathrm{E}-01$ & $-254.83 \%$ \\
\hline La-137 & $4.2 \mathrm{E}-05$ & $1.5 \mathrm{E}-05$ & $-187.70 \%$ & $\mathrm{Cm}-247$ & $4.0 \mathrm{E}-01$ & $1.1 \mathrm{E}-01$ & $-254.83 \%$ \\
\hline La-138 & $5.8 \mathrm{E}-04$ & $2.0 \mathrm{E}-04$ & $-187.70 \%$ & $\mathrm{Cm}-248$ & $1.6 \mathrm{E}+00$ & $4.4 \mathrm{E}-01$ & $-254.83 \%$ \\
\hline La-140 & $1.2 \mathrm{E}-05$ & $4.2 \mathrm{E}-06$ & $-187.70 \%$ & $\mathrm{Cm}-250$ & $7.6 \mathrm{E}+00$ & $2.1 \mathrm{E}+00$ & $-254.83 \%$ \\
\hline $\mathrm{Ba}-140$ & 7.7E-05 & $5.4 \mathrm{E}-06$ & $-1319.30 \%$ & Bk-247 & $2.3 \mathrm{E}-01$ & $5.3 \mathrm{E}-02$ & $-325.79 \%$ \\
\hline $\mathrm{Ce}-141$ & $8.2 \mathrm{E}-06$ & $4.8 \mathrm{E}-05$ & $82.97 \%$ & Bk-249 & $5.8 \mathrm{E}-04$ & $1.4 \mathrm{E}-04$ & $-325.79 \%$ \\
\hline \multirow[t]{8}{*}{$\mathrm{Ce}-144$} & $7.6 \mathrm{E}-05$ & $4.5 \mathrm{E}-04$ & $82.97 \%$ & Cf-249 & $4.5 \mathrm{E}-01$ & $1.1 \mathrm{E}-01$ & $-325.79 \%$ \\
\hline & & & & Cf-250 & $1.9 \mathrm{E}-01$ & $4.4 \mathrm{E}-02$ & $-325.79 \%$ \\
\hline & & & & Cf-251 & $4.5 \mathrm{E}-01$ & $1.1 \mathrm{E}-01$ & $-325.79 \%$ \\
\hline & & & & Cf-252 & $9.1 \mathrm{E}-02$ & $2.1 \mathrm{E}-02$ & $-325.79 \%$ \\
\hline & & & & Es-253 & $6.7 \mathrm{E}-04$ & $3.9 \mathrm{E}-04$ & $-70.32 \%$ \\
\hline & & & & Unidentified alpha & $5.9 \mathrm{E}-02$ & $1.2 \mathrm{E}-01$ & $50.32 \%$ \\
\hline & & & & Unidentified beta & $1.5 \mathrm{E}-02$ & $3.5 \mathrm{E}-04$ & $-4304.73 \%$ \\
\hline & & & & TOTAL Dose & $3.3 \mathrm{E}+01$ & $1.2 \mathrm{E}+01$ & $-177.83 \%$ \\
\hline
\end{tabular}


SRNL-STI-2010-00447, REVISION 0

Table 22. LADTAP XL Collective Commercial Fish Ingestion Comparisons (person-rem)

\begin{tabular}{|c|c|c|c|c|c|c|c|}
\hline Nuclide & $\begin{array}{l}\text { Hamby Ladtap } \\
\text { Commercial Fish }\end{array}$ & $\begin{array}{l}\text { Jannik Ladtap } \\
\text { Commercial Fish }\end{array}$ & $\%$ Diff & Nuclide & $\begin{array}{l}\text { Hamby Ladtap } \\
\text { Commercial Fish }\end{array}$ & $\begin{array}{l}\text { Jannik Ladtap } \\
\text { Commercial Fish }\end{array}$ & $\%$ Diff \\
\hline $\mathrm{H}-3$ & $1.7 \mathrm{E}-08$ & $4.0 \mathrm{E}-07$ & $95.74 \%$ & $\mathrm{Sm}-146$ & $1.5 \mathrm{E}-03$ & $3.8 \mathrm{E}-02$ & $96.05 \%$ \\
\hline $\mathrm{Be}-7$ & $5.6 \mathrm{E}-08$ & 5.9E-05 & $99.91 \%$ & Sm-147 & $1.4 \mathrm{E}-03$ & $3.4 \mathrm{E}-02$ & $96.05 \%$ \\
\hline Be-10 & $2.5 \mathrm{E}-06$ & $2.7 \mathrm{E}-03$ & $99.91 \%$ & Sm-151 & $2.6 \mathrm{E}-06$ & $6.5 \mathrm{E}-05$ & $96.05 \%$ \\
\hline $\mathrm{C}-14$ & $2.9 \mathrm{E}-03$ & $4.0 \mathrm{E}-05$ & $-7163.16 \%$ & Pm-147 & 7.1E-06 & $1.8 \mathrm{E}-04$ & $96.05 \%$ \\
\hline $\mathrm{Na}-22$ & $3.6 \mathrm{E}-04$ & $5.8 \mathrm{E}-03$ & $93.77 \%$ & Eu-152 & $4.5 \mathrm{E}-05$ & $5.0 \mathrm{E}-03$ & $99.09 \%$ \\
\hline $\mathrm{Na}-24$ & 2.3E-11 & $3.7 \mathrm{E}-10$ & $93.77 \%$ & Eu-154 & $6.9 \mathrm{E}-05$ & 7.5E-03 & $99.09 \%$ \\
\hline Al-26 & $3.9 \mathrm{E}-05$ & $4.2 \mathrm{E}-03$ & $99.07 \%$ & Eu-155 & $9.8 \mathrm{E}-06$ & $1.1 \mathrm{E}-03$ & $99.09 \%$ \\
\hline P-32 & $1.2 \mathrm{E}-01$ & $3.7 \mathrm{E}+00$ & $96.62 \%$ & Gd-152 & $1.1 \mathrm{E}-03$ & 2.9E-02 & $96.05 \%$ \\
\hline $\mathrm{Si}-32$ & $1.3 \mathrm{E}-06$ & $2.2 \mathrm{E}-04$ & $99.41 \%$ & Ho-166m & $5.9 \mathrm{E}-05$ & $1.5 \mathrm{E}-03$ & $96.05 \%$ \\
\hline S-35 & $1.3 \mathrm{E}-04$ & $3.0 \mathrm{E}-03$ & $95.56 \%$ & Lu-176 & $5.0 \mathrm{E}-05$ & $1.1 \mathrm{E}-03$ & $95.26 \%$ \\
\hline $\mathrm{Cl}-36$ & $4.5 \mathrm{E}-05$ & $9.0 \mathrm{E}-04$ & $94.96 \%$ & Ta-180 & $0.0 \mathrm{E}+00$ & $0.0 \mathrm{E}+00$ & \\
\hline $\mathrm{K}-40$ & $5.7 \mathrm{E}-03$ & $3.9 \mathrm{E}-01$ & $98.52 \%$ & Hf-182 & $1.4 \mathrm{E}-05$ & $9.8 \mathrm{E}-02$ & $99.99 \%$ \\
\hline $\mathrm{K}-43$ & $1.4 \mathrm{E}-08$ & $9.8 \mathrm{E}-07$ & $98.52 \%$ & Re-186m & $1.2 \mathrm{E}-04$ & $2.5 \mathrm{E}-03$ & $95.30 \%$ \\
\hline $\mathrm{Ca}-41$ & $1.5 \mathrm{E}-05$ & $9.2 \mathrm{E}-05$ & $84.21 \%$ & Re-187 & $3.0 \mathrm{E}-07$ & $6.4 \mathrm{E}-06$ & $95.30 \%$ \\
\hline $\mathrm{Ca}-45$ & $3.4 \mathrm{E}-05$ & $2.2 \mathrm{E}-04$ & $84.21 \%$ & Ir-192m & $0.0 \mathrm{E}+00$ & $0.0 \mathrm{E}+00$ & \\
\hline $\mathrm{Ca}-47$ & $1.0 \mathrm{E}-05$ & $6.5 \mathrm{E}-05$ & $84.21 \%$ & Pt-193 & $3.3 \mathrm{E}-06$ & $2.5 \mathrm{E}-05$ & $86.47 \%$ \\
\hline $\mathrm{Ti}-44$ & $5.7 \mathrm{E}-03$ & $2.3 \mathrm{E}-02$ & $75.07 \%$ & Hg-194 & $8.5 \mathrm{E}-02$ & $1.1 \mathrm{E}+01$ & $99.22 \%$ \\
\hline $\mathrm{V}-49$ & $1.6 \mathrm{E}-07$ & $3.3 \mathrm{E}-05$ & $99.51 \%$ & $\mathrm{Hg}-203$ & $2.5 \mathrm{E}-03$ & $3.2 \mathrm{E}-01$ & $99.22 \%$ \\
\hline $\mathrm{Cr}-51$ & 5.7E-06 & $2.4 \mathrm{E}-05$ & $76.32 \%$ & $\mathrm{~Pb}-202$ & $3.5 \mathrm{E}-03$ & $6.2 \mathrm{E}-03$ & $43.16 \%$ \\
\hline $\mathrm{Mn}-53$ & $3.0 \mathrm{E}-06$ & $1.5 \mathrm{E}-04$ & $98.03 \%$ & $\mathrm{~Pb}-205$ & $1.4 \mathrm{E}-04$ & $2.4 \mathrm{E}-04$ & $43.16 \%$ \\
\hline Mn-54 & $3.2 \mathrm{E}-04$ & $4.0 \mathrm{E}-03$ & $92.11 \%$ & $\mathrm{~Pb}-210$ & 4.6E-01 & $8.1 \mathrm{E}-01$ & $43.16 \%$ \\
\hline $\mathrm{Fe}-55$ & $1.7 \mathrm{E}-05$ & $6.2 \mathrm{E}-04$ & $97.21 \%$ & Bi-207 & $2.2 \mathrm{E}-05$ & 4.7E-04 & $95.26 \%$ \\
\hline $\mathrm{Fe}-60$ & $4.5 \mathrm{E}-03$ & $1.6 \mathrm{E}-01$ & $97.21 \%$ & $\mathrm{Bi}-210$ & 4.4E-06 & $9.4 \mathrm{E}-05$ & $95.26 \%$ \\
\hline Co-58 & 4.7E-05 & $1.5 \mathrm{E}-03$ & $96.88 \%$ & $\mathrm{Bi}-210 \mathrm{~m}$ & $3.9 \mathrm{E}-04$ & $8.2 \mathrm{E}-03$ & $95.26 \%$ \\
\hline Co-60 & $3.9 \mathrm{E}-04$ & $1.3 \mathrm{E}-02$ & $96.88 \%$ & Po-210 & $2.3 \mathrm{E}-01$ & $3.4 \mathrm{E}-01$ & $34.21 \%$ \\
\hline Ni-59 & $6.0 \mathrm{E}-06$ & $2.7 \mathrm{E}-05$ & $77.44 \%$ & Ra-223 & $3.8 \mathrm{E}-03$ & $6.4 \mathrm{E}-03$ & $40.79 \%$ \\
\hline Ni-63 & $1.6 \mathrm{E}-05$ & 7.2E-05 & $77.44 \%$ & Ra-224 & 4.1E-04 & $7.0 \mathrm{E}-04$ & $40.79 \%$ \\
\hline $\mathrm{Cu}-64$ & $1.0 \mathrm{E}-12$ & $2.5 \mathrm{E}-11$ & $95.88 \%$ & Ra-225 & $2.5 \mathrm{E}-03$ & $4.3 \mathrm{E}-03$ & $40.79 \%$ \\
\hline
\end{tabular}


SRNL-STI-2010-00447, REVISION 0

Table 22. LADTAP XL Collective Commercial Fish Ingestion Comparisons (person-rem) (continued)

\begin{tabular}{|c|c|c|c|c|c|c|c|}
\hline Nuclide & $\begin{array}{l}\text { Hamby Ladtap } \\
\text { Commercial Fish }\end{array}$ & $\begin{array}{l}\text { Jannik Ladtap } \\
\text { Commercial Fish }\end{array}$ & $\%$ Diff & Nuclide & $\begin{array}{l}\text { Hamby Ladtap } \\
\text { Commercial Fish }\end{array}$ & $\begin{array}{l}\text { Jannik Ladtap } \\
\text { Commercial Fish }\end{array}$ & $\%$ Diff \\
\hline Zn-65 & $8.2 \mathrm{E}-03$ & 2.9E-01 & $97.21 \%$ & Ra-226 & $1.7 \mathrm{E}-02$ & $2.8 \mathrm{E}-02$ & $40.79 \%$ \\
\hline Ge-68 & $1.1 \mathrm{E}-03$ & 2.7E-02 & $96.06 \%$ & Ra-228 & $1.8 \mathrm{E}-02$ & $3.1 \mathrm{E}-02$ & $40.79 \%$ \\
\hline Se-75 & $4.2 \mathrm{E}-04$ & $3.1 \mathrm{E}-01$ & $99.87 \%$ & Ac- 227 & $1.1 \mathrm{E}-01$ & $2.2 \mathrm{E}+00$ & $95.26 \%$ \\
\hline Se-79 & 4.3E-04 & $3.2 \mathrm{E}-01$ & $99.87 \%$ & Th-227 & $2.0 \mathrm{E}-04$ & $8.5 \mathrm{E}-04$ & $76.32 \%$ \\
\hline $\mathrm{Rb}-87$ & 2.9E-03 & $1.5 \mathrm{E}-01$ & $98.07 \%$ & Th-228 & $3.4 \mathrm{E}-03$ & $1.4 \mathrm{E}-02$ & $76.32 \%$ \\
\hline Sr-89 & $6.6 \mathrm{E}-05$ & $1.3 \mathrm{E}-04$ & $51.00 \%$ & Th-229 & $3.2 \mathrm{E}-02$ & $1.3 \mathrm{E}-01$ & $76.32 \%$ \\
\hline Sr-90 & $1.2 \mathrm{E}-03$ & $2.4 \mathrm{E}-03$ & $51.00 \%$ & Th-230 & $4.8 \mathrm{E}-03$ & $2.0 \mathrm{E}-02$ & $76.32 \%$ \\
\hline $\mathrm{Y}-90$ & $2.6 \mathrm{E}-06$ & $8.8 \mathrm{E}-05$ & $97.04 \%$ & Th-231 & $2.5 \mathrm{E}-09$ & $1.0 \mathrm{E}-08$ & $76.32 \%$ \\
\hline Y-91 & $5.8 \mathrm{E}-05$ & $1.9 \mathrm{E}-03$ & $97.04 \%$ & Th-232 & $2.5 \mathrm{E}-02$ & $1.1 \mathrm{E}-01$ & $76.32 \%$ \\
\hline Mo-93 & $3.9 \mathrm{E}-06$ & $1.6 \mathrm{E}-05$ & $75.07 \%$ & Th-234 & $8.1 \mathrm{E}-05$ & $3.4 \mathrm{E}-04$ & $76.32 \%$ \\
\hline Mo-99 & $5.0 \mathrm{E}-07$ & $2.0 \mathrm{E}-06$ & $75.07 \%$ & $\mathrm{~Pa}-230$ & $1.1 \mathrm{E}-05$ & $2.1 \mathrm{E}-04$ & $94.65 \%$ \\
\hline $\mathrm{Nb}-93 \mathrm{~m}$ & $4.8 \mathrm{E}-02$ & $1.0 \mathrm{E}-02$ & $-373.68 \%$ & $\mathrm{~Pa}-231$ & $3.8 \mathrm{E}-02$ & $7.0 \mathrm{E}-01$ & $94.65 \%$ \\
\hline $\mathrm{Nb}-94$ & 4.6E-02 & $9.8 \mathrm{E}-03$ & $-373.68 \%$ & Pa-233 & $8.1 \mathrm{E}-06$ & $1.5 \mathrm{E}-04$ & $94.65 \%$ \\
\hline $\mathrm{Nb}-95$ & $1.5 \mathrm{E}-02$ & $3.3 \mathrm{E}-03$ & $-373.68 \%$ & U-232 & $3.9 \mathrm{E}-03$ & 8.0E-03 & $50.66 \%$ \\
\hline Zr-93 & $1.6 \mathrm{E}-06$ & $2.2 \mathrm{E}-04$ & $99.29 \%$ & U-233 & $1.6 \mathrm{E}-04$ & $1.7 \mathrm{E}-03$ & $90.13 \%$ \\
\hline Zr-95 & 2.9E-06 & $4.1 \mathrm{E}-04$ & $99.29 \%$ & U-234 & $1.6 \mathrm{E}-04$ & $1.6 \mathrm{E}-03$ & $90.13 \%$ \\
\hline Tc-96 & $1.5 \mathrm{E}-06$ & $4.3 \mathrm{E}-05$ & $96.45 \%$ & U-235 & $1.5 \mathrm{E}-04$ & $1.5 \mathrm{E}-03$ & $90.13 \%$ \\
\hline Tc-97 & $6.8 \mathrm{E}-07$ & $1.9 \mathrm{E}-05$ & $96.45 \%$ & U-236 & $1.5 \mathrm{E}-04$ & $1.5 \mathrm{E}-03$ & $90.13 \%$ \\
\hline Tc-98 & $2.2 \mathrm{E}-05$ & $6.1 \mathrm{E}-04$ & $96.45 \%$ & U-237 & $4.3 \mathrm{E}-07$ & $4.3 \mathrm{E}-06$ & $90.13 \%$ \\
\hline Tc-99 & 5.9E-06 & $1.7 \mathrm{E}-04$ & $96.45 \%$ & U-238 & $1.4 \mathrm{E}-04$ & $1.4 \mathrm{E}-03$ & $90.13 \%$ \\
\hline Ru-97 & 8.7E-08 & $1.0 \mathrm{E}-05$ & $99.14 \%$ & $\mathrm{~Np}-236$ & $2.4 \mathrm{E}-03$ & $1.1 \mathrm{E}-01$ & $97.74 \%$ \\
\hline Ru-103 & $6.5 \mathrm{E}-06$ & 7.5E-04 & $99.14 \%$ & Np-237 & $1.2 \mathrm{E}-02$ & $5.2 \mathrm{E}-01$ & $97.74 \%$ \\
\hline Ru-106 & $6.2 \mathrm{E}-05$ & 7.2E-03 & $99.14 \%$ & Np-239 & $1.9 \mathrm{E}-07$ & $8.4 \mathrm{E}-06$ & $97.74 \%$ \\
\hline Pd-107 & 4.2E-07 & 8.9E-06 & $95.26 \%$ & Am-237 & $3.7 \mathrm{E}-84$ & 7.5E-82 & $99.51 \%$ \\
\hline Ag-108m & $5.2 \mathrm{E}-06$ & $5.3 \mathrm{E}-03$ & $99.90 \%$ & Am-241 & $3.4 \mathrm{E}-02$ & $6.9 \mathrm{E}+00$ & $99.51 \%$ \\
\hline
\end{tabular}


SRNL-STI-2010-00447, REVISION 0

Table 22. LADTAP XL Collective Commercial Fish Ingestion Comparisons (person-rem) (continued)

\begin{tabular}{|c|c|c|c|c|c|c|c|}
\hline Nuclide & $\begin{array}{l}\text { Hamby Ladtap } \\
\text { Commercial Fish }\end{array}$ & $\begin{array}{l}\text { Jannik Ladtap } \\
\text { Commercial Fish }\end{array}$ & $\%$ Diff & Nuclide & $\begin{array}{l}\text { Hamby Ladtap } \\
\text { Commercial Fish }\end{array}$ & $\begin{array}{l}\text { Jannik Ladtap } \\
\text { Commercial Fish }\end{array}$ & $\%$ Diff \\
\hline Ag-110m & 7.4E-06 & $7.4 \mathrm{E}-03$ & $99.90 \%$ & Am-242m & $3.2 \mathrm{E}-02$ & $6.4 \mathrm{E}+00$ & $99.51 \%$ \\
\hline Cd-113 & $9.7 \mathrm{E}-03$ & $2.0 \mathrm{E}-01$ & $95.26 \%$ & Am-243 & $3.4 \mathrm{E}-02$ & $6.9 \mathrm{E}+00$ & $99.51 \%$ \\
\hline $\mathrm{Cd}-115 \mathrm{~m}$ & 7.4E-04 & $1.6 \mathrm{E}-02$ & $95.26 \%$ & $\mathrm{Pu}-237$ & $8.7 \mathrm{E}-07$ & $1.6 \mathrm{E}-04$ & $99.45 \%$ \\
\hline In-115 & $0.0 \mathrm{E}+00$ & $8.9 \mathrm{E}+00$ & $100.00 \%$ & $\mathrm{Pu}-238$ & $4.0 \mathrm{E}-03$ & 7.3E-01 & $99.45 \%$ \\
\hline Sb-122 & $6.8 \mathrm{E}-08$ & $5.3 \mathrm{E}-05$ & $99.87 \%$ & $\mathrm{Pu}-239$ & $4.5 \mathrm{E}-03$ & $8.2 \mathrm{E}-01$ & $99.45 \%$ \\
\hline $\mathrm{Sb}-124$ & $2.4 \mathrm{E}-06$ & $1.9 \mathrm{E}-03$ & $99.87 \%$ & $\mathrm{Pu}-240$ & $4.5 \mathrm{E}-03$ & 8.2E-01 & $99.45 \%$ \\
\hline $\mathrm{Sb}-125$ & $7.8 \mathrm{E}-07$ & $6.1 \mathrm{E}-04$ & $99.87 \%$ & $\mathrm{Pu}-241$ & $9.1 \mathrm{E}-05$ & $1.6 \mathrm{E}-02$ & $99.45 \%$ \\
\hline Te-123 & $0.0 \mathrm{E}+00$ & $3.9 \mathrm{E}-03$ & $100.00 \%$ & $\mathrm{Pu}-242$ & $4.3 \mathrm{E}-03$ & 7.9E-01 & $99.45 \%$ \\
\hline Te- $125 \mathrm{~m}$ & $3.5 \mathrm{E}-04$ & $2.8 \mathrm{E}-03$ & $87.37 \%$ & $\mathrm{Pu}-244$ & 4.2E-03 & 7.7E-01 & $99.45 \%$ \\
\hline Sn-126 & $1.5 \mathrm{E}-02$ & 3.3E-01 & $95.26 \%$ & $\mathrm{Cm}-241$ & $2.6 \mathrm{E}-05$ & $6.7 \mathrm{E}-04$ & $96.05 \%$ \\
\hline I-129 & $1.3 \mathrm{E}-03$ & $5.4 \mathrm{E}-02$ & $97.63 \%$ & $\mathrm{Cm}-242$ & 7.9E-04 & $2.0 \mathrm{E}-02$ & $96.05 \%$ \\
\hline I-131 & $7.8 \mathrm{E}-05$ & $3.3 \mathrm{E}-03$ & $97.63 \%$ & $\mathrm{Cm}-243$ & $2.2 \mathrm{E}-02$ & $5.5 \mathrm{E}-01$ & $96.05 \%$ \\
\hline Cs-134 & $6.6 \mathrm{E}-02$ & $1.4 \mathrm{E}+00$ & $95.26 \%$ & $\mathrm{Cm}-244$ & $1.7 \mathrm{E}-02$ & 4.4E-01 & $96.05 \%$ \\
\hline Cs-135 & $6.4 \mathrm{E}-03$ & $1.4 \mathrm{E}-01$ & $95.26 \%$ & $\mathrm{Cm}-245$ & $3.4 \mathrm{E}-02$ & 8.6E-01 & $96.05 \%$ \\
\hline Cs-137 & $4.5 \mathrm{E}-02$ & $9.6 \mathrm{E}-01$ & $95.26 \%$ & $\mathrm{Cm}-246$ & $3.4 \mathrm{E}-02$ & $8.6 \mathrm{E}-01$ & $96.05 \%$ \\
\hline La-137 & $3.2 \mathrm{E}-06$ & $1.0 \mathrm{E}-04$ & $96.80 \%$ & $\mathrm{Cm}-247$ & $3.1 \mathrm{E}-02$ & 7.9E-01 & $96.05 \%$ \\
\hline La-138 & $4.5 \mathrm{E}-05$ & $1.4 \mathrm{E}-03$ & $96.80 \%$ & $\mathrm{Cm}-248$ & $1.2 \mathrm{E}-01$ & $3.1 \mathrm{E}+00$ & $96.05 \%$ \\
\hline La-140 & 2.7E-07 & $8.5 \mathrm{E}-06$ & $96.80 \%$ & $\mathrm{Cm}-250$ & 5.9E-01 & $1.5 \mathrm{E}+01$ & $96.05 \%$ \\
\hline $\mathrm{Ba}-140$ & $5.0 \mathrm{E}-06$ & $3.2 \mathrm{E}-05$ & $84.21 \%$ & Bk-247 & $1.7 \mathrm{E}-02$ & $3.7 \mathrm{E}-01$ & $95.26 \%$ \\
\hline $\mathrm{Ce}-141$ & $6.0 \mathrm{E}-07$ & $3.1 \mathrm{E}-04$ & $99.81 \%$ & Bk-249 & 4.4E-05 & $9.3 \mathrm{E}-04$ & $95.26 \%$ \\
\hline $\mathrm{Ce}-144$ & 5.9E-06 & $3.1 \mathrm{E}-03$ & $99.81 \%$ & Cf-249 & $3.5 \mathrm{E}-02$ & 7.3E-01 & $95.26 \%$ \\
\hline & & & & Cf -250 & $1.4 \mathrm{E}-02$ & $3.0 \mathrm{E}-01$ & $95.26 \%$ \\
\hline & & & & Cf-251 & $3.5 \mathrm{E}-02$ & 7.3E-01 & $95.26 \%$ \\
\hline & & & & Cf-252 & 7.0E-03 & $1.5 \mathrm{E}-01$ & $95.26 \%$ \\
\hline & & & & Es-253 & 4.7E-05 & $2.5 \mathrm{E}-03$ & $98.11 \%$ \\
\hline & & & & $\begin{array}{c}\text { Unidentified } \\
\text { alpha }\end{array}$ & 4.5E-03 & $8.2 \mathrm{E}-01$ & $99.45 \%$ \\
\hline & & & & Unidentified beta & $1.2 \mathrm{E}-03$ & $2.4 \mathrm{E}-03$ & $51.00 \%$ \\
\hline & & & & TOTAL Dose & $2.6 \mathrm{E}+00$ & $8.3 \mathrm{E}+01$ & $96.92 \%$ \\
\hline
\end{tabular}


SRNL-STI-2010-00447, REVISION 0

Table 23. LADTAP XL Collective Salt Water Invertebrate Ingestion Comparisons (person-rem)

\begin{tabular}{|c|c|c|c|c|c|c|c|}
\hline Nuclide & $\begin{array}{l}\text { Hamby Ladtap } \\
\text { Invertebrates }\end{array}$ & $\begin{array}{l}\text { Jannik Ladtap } \\
\text { Invertebrates }\end{array}$ & $\%$ Diff & Nuclide & $\begin{array}{c}\text { Hamby Ladtap } \\
\text { Invertebrates }\end{array}$ & $\begin{array}{l}\text { Jannik Ladtap } \\
\text { Invertebrates }\end{array}$ & $\%$ Diff \\
\hline H-3 & $5.3 \mathrm{E}-07$ & $8.3 \mathrm{E}-07$ & $35.53 \%$ & $\mathrm{Sm}-146$ & $1.8 \mathrm{E}+00$ & $2.8 \mathrm{E}+00$ & $35.53 \%$ \\
\hline $\mathrm{Be}-7$ & $1.7 \mathrm{E}-04$ & $1.3 \mathrm{E}-02$ & $98.71 \%$ & Sm-147 & $1.6 \mathrm{E}+00$ & $2.6 \mathrm{E}+00$ & $35.53 \%$ \\
\hline Be-10 & $7.7 \mathrm{E}-03$ & $6.0 \mathrm{E}-01$ & $98.71 \%$ & Sm-151 & $3.1 \mathrm{E}-03$ & $4.8 \mathrm{E}-03$ & $35.53 \%$ \\
\hline C-14 & $2.7 \mathrm{E}-02$ & $6.0 \mathrm{E}-01$ & $95.49 \%$ & Pm-147 & $8.6 \mathrm{E}-03$ & $1.3 \mathrm{E}-02$ & $35.53 \%$ \\
\hline $\mathrm{Na}-22$ & $2.1 \mathrm{E}-05$ & $1.7 \mathrm{E}-04$ & $87.75 \%$ & Eu-152 & $5.5 \mathrm{E}-02$ & $8.5 \mathrm{E}-02$ & $35.53 \%$ \\
\hline $\mathrm{Na}-24$ & $1.3 \mathrm{E}-12$ & $1.1 \mathrm{E}-11$ & $87.75 \%$ & Eu-154 & $8.3 \mathrm{E}-02$ & $1.3 \mathrm{E}-01$ & $35.53 \%$ \\
\hline Al-26 & $7.1 \mathrm{E}-03$ & $1.1 \mathrm{E}-02$ & $35.53 \%$ & Eu-155 & $1.2 \mathrm{E}-02$ & $1.8 \mathrm{E}-02$ & $35.53 \%$ \\
\hline P-32 & $1.1 \mathrm{E}+00$ & $2.2 \mathrm{E}+00$ & $49.10 \%$ & Gd-152 & $1.4 \mathrm{E}+00$ & $4.3 \mathrm{E}+00$ & $67.76 \%$ \\
\hline Si-32 & $5.2 \mathrm{E}-04$ & $1.2 \mathrm{E}+00$ & $99.96 \%$ & Ho-166m & $7.1 \mathrm{E}-02$ & $1.1 \mathrm{E}-01$ & $35.53 \%$ \\
\hline S-35 & $2.4 \mathrm{E}-06$ & $8.3 \mathrm{E}-06$ & $71.63 \%$ & Lu-176 & $6.0 \mathrm{E}-02$ & $9.4 \mathrm{E}-02$ & $35.53 \%$ \\
\hline Cl-36 & $5.2 \mathrm{E}-07$ & $4.3 \mathrm{E}-05$ & $98.78 \%$ & Ta-180 & $0.0 \mathrm{E}+00$ & $0.0 \mathrm{E}+00$ & \\
\hline K-40 & $1.1 \mathrm{E}-03$ & $1.8 \mathrm{E}-03$ & $35.53 \%$ & Hf-182 & $2.6 \mathrm{E}-03$ & $2.0 \mathrm{E}-01$ & $98.71 \%$ \\
\hline $\mathrm{K}-43$ & $2.9 \mathrm{E}-09$ & $4.5 \mathrm{E}-09$ & $35.33 \%$ & Re-186m & $1.8 \mathrm{E}-03$ & $2.8 \mathrm{E}-03$ & $35.53 \%$ \\
\hline $\mathrm{Ca}-41$ & $1.4 \mathrm{E}-04$ & $8.5 \mathrm{E}-05$ & $-61.18 \%$ & Re-187 & $4.5 \mathrm{E}-06$ & $7.0 \mathrm{E}-06$ & $35.53 \%$ \\
\hline $\mathrm{Ca}-45$ & $3.4 \mathrm{E}-04$ & $2.0 \mathrm{E}-04$ & $-67.63 \%$ & Ir-192m & $0.0 \mathrm{E}+00$ & $0.0 \mathrm{E}+00$ & \\
\hline $\mathrm{Ca}-47$ & $1.0 \mathrm{E}-04$ & $6.0 \mathrm{E}-05$ & $-67.63 \%$ & Pt-193 & $2.0 \mathrm{E}-03$ & $3.1 \mathrm{E}-03$ & $35.53 \%$ \\
\hline Ti-44 & $1.7 \mathrm{E}-01$ & $2.7 \mathrm{E}-01$ & $35.53 \%$ & Hg-194 & $8.5 \mathrm{E}+01$ & $7.9 \mathrm{E}+01$ & $-7.35 \%$ \\
\hline $\mathrm{V}-49$ & $2.4 \mathrm{E}-05$ & $3.7 \mathrm{E}-05$ & $35.53 \%$ & $\mathrm{Hg}-203$ & $2.5 \mathrm{E}+00$ & $2.3 \mathrm{E}+00$ & $-6.38 \%$ \\
\hline Cr-51 & $1.7 \mathrm{E}-03$ & $6.7 \mathrm{E}-04$ & $-157.89 \%$ & $\mathrm{~Pb}-202$ & $3.6 \mathrm{E}-01$ & $5.5 \mathrm{E}-01$ & $35.53 \%$ \\
\hline $\mathrm{Mn}-53$ & $9.1 \mathrm{E}-03$ & $1.1 \mathrm{E}-03$ & $-705.92 \%$ & $\mathrm{~Pb}-205$ & $1.4 \mathrm{E}-02$ & $2.1 \mathrm{E}-02$ & $35.53 \%$ \\
\hline Mn-54 & $9.6 \mathrm{E}-03$ & $3.0 \mathrm{E}-02$ & $67.76 \%$ & $\mathrm{~Pb}-210$ & $4.7 \mathrm{E}+01$ & $7.2 \mathrm{E}+01$ & $35.53 \%$ \\
\hline Fe-55 & $1.1 \mathrm{E}-01$ & $4.1 \mathrm{E}-02$ & $-157.89 \%$ & Bi-207 & $4.5 \mathrm{E}-04$ & $6.9 \mathrm{E}-02$ & $99.36 \%$ \\
\hline Fe-60 & $2.7 \mathrm{E}+01$ & $1.1 \mathrm{E}+01$ & $-157.89 \%$ & $\mathrm{Bi}-210$ & $8.9 \mathrm{E}-05$ & $1.4 \mathrm{E}-02$ & $99.36 \%$ \\
\hline Co-58 & $2.8 \mathrm{E}-02$ & $8.7 \mathrm{E}-02$ & $67.76 \%$ & $\mathrm{Bi}-210 \mathrm{~m}$ & $7.9 \mathrm{E}-03$ & $1.2 \mathrm{E}+00$ & $99.36 \%$ \\
\hline Co-60 & $2.4 \mathrm{E}-01$ & $7.3 \mathrm{E}-01$ & $67.76 \%$ & Po-210 & $2.7 \mathrm{E}+02$ & $1.1 \mathrm{E}+03$ & $74.21 \%$ \\
\hline Ni-59 & $4.6 \mathrm{E}-04$ & $1.4 \mathrm{E}-03$ & $67.76 \%$ & Ra-223 & $2.3 \mathrm{E}-01$ & $3.5 \mathrm{E}-01$ & $35.53 \%$ \\
\hline Ni-63 & $1.2 \mathrm{E}-03$ & $3.8 \mathrm{E}-03$ & $67.76 \%$ & Ra-224 & $2.5 \mathrm{E}-02$ & $3.9 \mathrm{E}-02$ & $35.53 \%$ \\
\hline $\mathrm{Cu}-64$ & $2.7 \mathrm{E}-10$ & $1.2 \mathrm{E}-09$ & $78.08 \%$ & Ra-225 & $1.5 \mathrm{E}-01$ & $2.4 \mathrm{E}-01$ & $35.53 \%$ \\
\hline
\end{tabular}


SRNL-STI-2010-00447, REVISION 0

Table 23. LADTAP XL Collective Salt Water Invertebrate Ingestion Comparisons (person-rem) (continued)

\begin{tabular}{|c|c|c|c|c|c|c|c|}
\hline Nuclide & $\begin{array}{l}\text { Hamby Ladtap } \\
\text { Invertebrates }\end{array}$ & $\begin{array}{l}\text { Jannik Ladtap } \\
\text { Invertebrates }\end{array}$ & $\%$ Diff & Nuclide & $\begin{array}{l}\text { Hamby Ladtap } \\
\text { Invertebrates }\end{array}$ & $\begin{array}{c}\text { Jannik Ladtap } \\
\text { Invertebrates }\end{array}$ & $\%$ Diff \\
\hline $\mathrm{Zn}-65$ & $6.2 \mathrm{E}+00$ & $9.6 \mathrm{E}+00$ & $35.53 \%$ & Ra-226 & $1.0 \mathrm{E}+00$ & $1.6 \mathrm{E}+00$ & $35.53 \%$ \\
\hline Ge-68 & $1.5 \mathrm{E}-01$ & $6.0 \mathrm{E}-02$ & $-153.06 \%$ & Ra-228 & $1.1 \mathrm{E}+00$ & $1.7 \mathrm{E}+00$ & $35.53 \%$ \\
\hline Se-75 & $7.5 \mathrm{E}-02$ & $5.8 \mathrm{E}-01$ & $87.11 \%$ & Ac- 227 & $1.3 \mathrm{E}+02$ & $2.0 \mathrm{E}+02$ & $35.53 \%$ \\
\hline Se-79 & $7.6 \mathrm{E}-02$ & $5.9 \mathrm{E}-01$ & $87.11 \%$ & Th-227 & $4.1 \mathrm{E}-01$ & $3.2 \mathrm{E}-01$ & $-28.95 \%$ \\
\hline $\mathrm{Rb}-87$ & $7.5 \mathrm{E}-04$ & $1.4 \mathrm{E}-02$ & $94.52 \%$ & Th-228 & $6.9 \mathrm{E}+00$ & $5.3 \mathrm{E}+00$ & $-28.95 \%$ \\
\hline Sr-89 & $1.3 \mathrm{E}-03$ & $1.0 \mathrm{E}-04$ & $-1189.47 \%$ & Th-229 & $6.4 \mathrm{E}+01$ & $5.0 \mathrm{E}+01$ & $-28.95 \%$ \\
\hline Sr-90 & $2.4 \mathrm{E}-02$ & $1.8 \mathrm{E}-03$ & $-1189.47 \%$ & Th-230 & $9.7 \mathrm{E}+00$ & $7.5 \mathrm{E}+00$ & $-28.95 \%$ \\
\hline Y-90 & $3.2 \mathrm{E}-03$ & $4.9 \mathrm{E}-03$ & $35.53 \%$ & Th-231 & $5.0 \mathrm{E}-06$ & $3.9 \mathrm{E}-06$ & $-28.95 \%$ \\
\hline Y-91 & $7.0 \mathrm{E}-02$ & $1.1 \mathrm{E}-01$ & $35.53 \%$ & Th-232 & $5.1 \mathrm{E}+01$ & $4.0 \mathrm{E}+01$ & $-28.95 \%$ \\
\hline Мо-93 & $1.2 \mathrm{E}-04$ & $3.7 \mathrm{E}-04$ & $67.76 \%$ & Th-234 & $1.6 \mathrm{E}-01$ & $1.3 \mathrm{E}-01$ & $-28.95 \%$ \\
\hline Мo-99 & $1.5 \mathrm{E}-05$ & $4.7 \mathrm{E}-05$ & $67.76 \%$ & $\mathrm{~Pa}-230$ & $3.1 \mathrm{E}-04$ & $4.7 \mathrm{E}-04$ & $35.53 \%$ \\
\hline $\mathrm{Nb}-93 \mathrm{~m}$ & $4.8 \mathrm{E}-03$ & $3.8 \mathrm{E}-03$ & $-28.95 \%$ & $\mathrm{~Pa}-231$ & $1.0 \mathrm{E}+00$ & $1.6 \mathrm{E}+00$ & $35.53 \%$ \\
\hline $\mathrm{Nb}-94$ & 4.7E-03 & $3.6 \mathrm{E}-03$ & $-28.95 \%$ & $\mathrm{~Pa}-233$ & $2.2 \mathrm{E}-04$ & $3.4 \mathrm{E}-04$ & $35.53 \%$ \\
\hline $\mathrm{Nb}-95$ & $1.6 \mathrm{E}-03$ & $1.2 \mathrm{E}-03$ & $-28.95 \%$ & U-232 & $1.2 \mathrm{E}-01$ & $1.8 \mathrm{E}-01$ & $35.53 \%$ \\
\hline Zr-93 & $1.2 \mathrm{E}-03$ & $1.1 \mathrm{E}-03$ & $-3.16 \%$ & U-233 & $2.5 \mathrm{E}-02$ & $3.8 \mathrm{E}-02$ & $35.53 \%$ \\
\hline $\mathrm{Zr}-95$ & $2.2 \mathrm{E}-03$ & $2.1 \mathrm{E}-03$ & $-3.16 \%$ & U-234 & $2.4 \mathrm{E}-02$ & $3.7 \mathrm{E}-02$ & $35.53 \%$ \\
\hline Tc-96 & $1.5 \mathrm{E}-04$ & $4.7 \mathrm{E}-05$ & $-222.37 \%$ & U-235 & $2.3 \mathrm{E}-02$ & $3.5 \mathrm{E}-02$ & $35.53 \%$ \\
\hline Tc-97 & $6.9 \mathrm{E}-05$ & $2.1 \mathrm{E}-05$ & $-222.37 \%$ & U-236 & $2.3 \mathrm{E}-02$ & $3.5 \mathrm{E}-02$ & $35.53 \%$ \\
\hline Tc-98 & $2.2 \mathrm{E}-03$ & $6.8 \mathrm{E}-04$ & $-222.37 \%$ & U-237 & $6.5 \mathrm{E}-05$ & $1.0 \mathrm{E}-04$ & $35.53 \%$ \\
\hline Tc-99 & 5.9E-04 & $1.8 \mathrm{E}-04$ & $-222.37 \%$ & U-238 & $2.1 \mathrm{E}-02$ & $3.3 \mathrm{E}-02$ & $35.53 \%$ \\
\hline Ru-97 & $5.2 \mathrm{E}-04$ & $4.1 \mathrm{E}-05$ & $-1189.47 \%$ & $\mathrm{~Np}-236$ & $7.2 \mathrm{E}-02$ & $1.1 \mathrm{E}-01$ & $35.53 \%$ \\
\hline Ru-103 & $2.0 \mathrm{E}-02$ & $3.0 \mathrm{E}-03$ & $-544.74 \%$ & $\mathrm{~Np}-237$ & $3.6 \mathrm{E}-01$ & $5.5 \mathrm{E}-01$ & $35.53 \%$ \\
\hline Ru-106 & $1.9 \mathrm{E}-01$ & $2.9 \mathrm{E}-02$ & $-544.74 \%$ & $\mathrm{~Np}-239$ & $5.7 \mathrm{E}-06$ & 8.9E-06 & $35.53 \%$ \\
\hline Pd-107 & $2.6 \mathrm{E}-03$ & $6.0 \mathrm{E}-04$ & $-329.82 \%$ & Am-237 & $4.5 \mathrm{E}-81$ & $2.5 \mathrm{E}-81$ & $-79.09 \%$ \\
\hline Ag-108m & $2.3 \mathrm{E}-01$ & $3.7 \mathrm{E}-02$ & $-513.42 \%$ & Am-241 & $4.1 \mathrm{E}+01$ & $2.3 \mathrm{E}+01$ & $-79.09 \%$ \\
\hline
\end{tabular}


SRNL-STI-2010-00447, REVISION 0

Table 23. LADTAP XL Collective Salt Water Invertebrate Ingestion Comparisons (person-rem) (continued)

\begin{tabular}{|c|c|c|c|c|c|c|c|}
\hline Nuclide & $\begin{array}{c}\text { Hamby Ladtap } \\
\text { Invertebrates }\end{array}$ & $\begin{array}{c}\text { Jannik Ladtap } \\
\text { Invertebrates }\end{array}$ & $\%$ Diff & Nuclide & $\begin{array}{c}\text { Hamby Ladtap } \\
\text { Invertebrates }\end{array}$ & $\begin{array}{c}\text { Jannik Ladtap } \\
\text { Invertebrates }\end{array}$ & $\%$ Diff \\
\hline Ag-110m & $3.2 \mathrm{E}-01$ & $5.3 \mathrm{E}-02$ & $-507.89 \%$ & Am-242m & $3.8 \mathrm{E}+01$ & $2.1 \mathrm{E}+01$ & $-79.09 \%$ \\
\hline Cd-113 & $3.7 \mathrm{E}+02$ & $1.1 \mathrm{E}+01$ & $-3123.68 \%$ & Am-243 & $4.1 \mathrm{E}+01$ & $2.3 \mathrm{E}+01$ & $-79.09 \%$ \\
\hline $\mathrm{Cd}-115 \mathrm{~m}$ & $2.8 \mathrm{E}+01$ & 8.7E-01 & $-3123.68 \%$ & $\mathrm{Pu}-237$ & $7.5 \mathrm{E}-04$ & $3.5 \mathrm{E}-03$ & $78.51 \%$ \\
\hline In-115 & $0.0 \mathrm{E}+00$ & $2.0 \mathrm{E}+01$ & $100.00 \%$ & $\mathrm{Pu}-238$ & $6.9 \mathrm{E}+00$ & $1.6 \mathrm{E}+01$ & $57.02 \%$ \\
\hline $\mathrm{Sb}-122$ & $1.0 \mathrm{E}-05$ & $3.2 \mathrm{E}-04$ & $96.78 \%$ & Pu-239 & $7.9 \mathrm{E}+00$ & $1.8 \mathrm{E}+01$ & $57.02 \%$ \\
\hline $\mathrm{Sb}-124$ & $3.7 \mathrm{E}-04$ & $1.1 \mathrm{E}-02$ & $96.78 \%$ & $\mathrm{Pu}-240$ & $7.9 \mathrm{E}+00$ & $1.8 \mathrm{E}+01$ & $57.02 \%$ \\
\hline $\mathrm{Sb}-125$ & $1.2 \mathrm{E}-04$ & $3.7 \mathrm{E}-03$ & $96.78 \%$ & $\mathrm{Pu}-241$ & $1.6 \mathrm{E}-01$ & $3.7 \mathrm{E}-01$ & $57.02 \%$ \\
\hline Te-123 & $3.7 \mathrm{E}+00$ & $5.8 \mathrm{E}-02$ & $-6347.37 \%$ & $\mathrm{Pu}-242$ & $7.5 \mathrm{E}+00$ & $1.7 \mathrm{E}+01$ & $57.02 \%$ \\
\hline Te-125m & $2.7 \mathrm{E}+00$ & $4.1 \mathrm{E}-02$ & $-6347.37 \%$ & $\mathrm{Pu}-244$ & $3.7 \mathrm{E}+00$ & $1.7 \mathrm{E}+01$ & $78.51 \%$ \\
\hline Sn-126 & $1.6 \mathrm{E}-01$ & $1.2 \mathrm{E}+01$ & $98.71 \%$ & $\mathrm{Cm}-241$ & $3.2 \mathrm{E}-02$ & $2.3 \mathrm{E}-02$ & $-40.16 \%$ \\
\hline I-129 & $1.3 \mathrm{E}-01$ & $2.0 \mathrm{E}-01$ & $35.53 \%$ & $\mathrm{Cm}-242$ & $9.5 \mathrm{E}-01$ & $6.8 \mathrm{E}-01$ & $-40.16 \%$ \\
\hline $\mathrm{I}-131$ & 7.9E-03 & $1.2 \mathrm{E}-02$ & $35.53 \%$ & $\mathrm{Cm}-243$ & $2.6 \mathrm{E}+01$ & $1.9 \mathrm{E}+01$ & $-40.16 \%$ \\
\hline Cs-134 & $1.7 \mathrm{E}-02$ & $3.1 \mathrm{E}-02$ & $46.27 \%$ & Cm-244 & $2.1 \mathrm{E}+01$ & $1.5 \mathrm{E}+01$ & $-40.16 \%$ \\
\hline Cs-135 & $1.6 \mathrm{E}-03$ & $3.0 \mathrm{E}-03$ & $46.27 \%$ & $\mathrm{Cm}-245$ & $4.1 \mathrm{E}+01$ & $2.9 \mathrm{E}+01$ & $-40.16 \%$ \\
\hline Cs-137 & $1.1 \mathrm{E}-02$ & $2.1 \mathrm{E}-02$ & $46.27 \%$ & Cm-246 & $4.1 \mathrm{E}+01$ & $2.9 \mathrm{E}+01$ & $-40.16 \%$ \\
\hline La-137 & $3.9 \mathrm{E}-03$ & $6.1 \mathrm{E}-04$ & $-544.74 \%$ & $\mathrm{Cm}-247$ & $3.7 \mathrm{E}+01$ & $2.7 \mathrm{E}+01$ & $-40.16 \%$ \\
\hline La-138 & $5.4 \mathrm{E}-02$ & $8.4 \mathrm{E}-03$ & $-544.74 \%$ & Cm-248 & $1.5 \mathrm{E}+02$ & $1.0 \mathrm{E}+02$ & $-40.16 \%$ \\
\hline La-140 & $3.3 \mathrm{E}-04$ & $5.1 \mathrm{E}-05$ & $-544.74 \%$ & $\mathrm{Cm}-250$ & $7.1 \mathrm{E}+02$ & $5.1 \mathrm{E}+02$ & $-40.16 \%$ \\
\hline Ba-140 & $3.8 \mathrm{E}-03$ & $5.9 \mathrm{E}-05$ & $-6347.37 \%$ & Bk-247 & $2.1 \mathrm{E}+01$ & $3.3 \mathrm{E}+01$ & $35.53 \%$ \\
\hline $\mathrm{Ce}-141$ & $1.1 \mathrm{E}-02$ & $1.4 \mathrm{E}-02$ & $22.63 \%$ & Bk-249 & $5.3 \mathrm{E}-02$ & $8.3 \mathrm{E}-02$ & $35.53 \%$ \\
\hline \multirow[t]{8}{*}{ Ce-144 } & $1.1 \mathrm{E}-01$ & $1.4 \mathrm{E}-01$ & $22.63 \%$ & Cf-249 & $4.2 \mathrm{E}+01$ & $3.3 \mathrm{E}+01$ & $-28.95 \%$ \\
\hline & & & & Cf-250 & $1.7 \mathrm{E}+01$ & $1.3 \mathrm{E}+01$ & $-28.95 \%$ \\
\hline & & & & Cf-251 & $4.2 \mathrm{E}+01$ & $3.3 \mathrm{E}+01$ & $-28.95 \%$ \\
\hline & & & & Cf-252 & $8.5 \mathrm{E}+00$ & $6.6 \mathrm{E}+00$ & $-28.95 \%$ \\
\hline & & & & Es-253 & $1.4 \mathrm{E}-03$ & $1.5 \mathrm{E}+00$ & $99.91 \%$ \\
\hline & & & & $\begin{array}{l}\text { Unidentified } \\
\text { alpha }\end{array}$ & $7.9 \mathrm{E}+00$ & $1.8 \mathrm{E}+01$ & $57.02 \%$ \\
\hline & & & & $\begin{array}{l}\text { Unidentified } \\
\text { beta }\end{array}$ & $2.4 \mathrm{E}-02$ & $1.8 \mathrm{E}-03$ & \#\#\#\#\#\#\#\# \\
\hline & & & & $\begin{array}{l}\text { TOTAL } \\
\text { Dose }\end{array}$ & $2.4 \mathrm{E}+03$ & $2.6 \mathrm{E}+03$ & $7.55 \%$ \\
\hline
\end{tabular}


SRNL-STI-2010-00447, REVISION 0

Table 24. LADTAP XL Collective Shoreline Exposure Comparisons (person-rem)

\begin{tabular}{|c|c|c|c|c|c|c|c|}
\hline Nuclide & $\begin{array}{c}\text { Hamby Ladtap } \\
\text { Shoreline Pathway }\end{array}$ & $\begin{array}{c}\text { Jannik Ladtap } \\
\text { Shoreline Pathway }\end{array}$ & $\%$ Diff & Nuclide & $\begin{array}{c}\text { Hamby Ladtap } \\
\text { Shoreline Pathway }\end{array}$ & $\begin{array}{c}\text { Jannik Ladtap } \\
\text { Shoreline Pathway }\end{array}$ & $\%$ Diff \\
\hline $\mathrm{H}-3$ & $0.0 \mathrm{E}+00$ & $0.0 \mathrm{E}+00$ & & Sm-146 & $0.0 \mathrm{E}+00$ & $0.0 \mathrm{E}+00$ & \\
\hline $\mathrm{Be}-7$ & $6.7 \mathrm{E}-05$ & $5.7 \mathrm{E}-05$ & $-16.79 \%$ & Sm-147 & $0.0 \mathrm{E}+00$ & $0.0 \mathrm{E}+00$ & \\
\hline Be-10 & $0.0 \mathrm{E}+00$ & $0.0 \mathrm{E}+00$ & & Sm-151 & $1.1 \mathrm{E}-06$ & $9.5 \mathrm{E}-07$ & $-16.79 \%$ \\
\hline C-14 & $0.0 \mathrm{E}+00$ & $0.0 \mathrm{E}+00$ & & Pm-147 & $9.6 \mathrm{E}-08$ & $8.2 \mathrm{E}-08$ & $-16.79 \%$ \\
\hline $\mathrm{Na}-22$ & $4.9 \mathrm{E}-02$ & $4.2 \mathrm{E}-02$ & $-16.79 \%$ & Eu-152 & $1.2 \mathrm{E}-01$ & $1.0 \mathrm{E}-01$ & $-16.79 \%$ \\
\hline $\mathrm{Na}-24$ & $1.8 \mathrm{E}-05$ & $1.5 \mathrm{E}-05$ & $-16.79 \%$ & Eu-154 & $9.1 \mathrm{E}-02$ & $7.8 \mathrm{E}-02$ & $-16.79 \%$ \\
\hline Al-26 & $6.2 \mathrm{E}-01$ & $5.3 \mathrm{E}-01$ & $-16.79 \%$ & Eu-155 & $3.2 \mathrm{E}-03$ & $2.7 \mathrm{E}-03$ & $-16.79 \%$ \\
\hline P-32 & $0.0 \mathrm{E}+00$ & $0.0 \mathrm{E}+00$ & & Gd-152 & $0.0 \mathrm{E}+00$ & $0.0 \mathrm{E}+00$ & \\
\hline $\mathrm{Si}-32$ & $0.0 \mathrm{E}+00$ & $0.0 \mathrm{E}+00$ & & Ho-166m & $4.1 \mathrm{E}-01$ & $3.5 \mathrm{E}-01$ & $-16.79 \%$ \\
\hline S-35 & $0.0 \mathrm{E}+00$ & $0.0 \mathrm{E}+00$ & & Lu-176 & $0.0 \mathrm{E}+00$ & $0.0 \mathrm{E}+00$ & \\
\hline $\mathrm{Cl}-36$ & $1.2 \mathrm{E}-08$ & 9.9E-09 & $-16.79 \%$ & Ta-180 & $0.0 \mathrm{E}+00$ & $0.0 \mathrm{E}+00$ & \\
\hline K-40 & $3.4 \mathrm{E}-02$ & $2.9 \mathrm{E}-02$ & $-16.79 \%$ & Hf-182 & $0.0 \mathrm{E}+00$ & $0.0 \mathrm{E}+00$ & \\
\hline $\mathrm{Ca}-41$ & $5.1 \mathrm{E}-06$ & $4.4 \mathrm{E}-06$ & $-16.79 \%$ & Re-187 & $0.0 \mathrm{E}+00$ & $0.0 \mathrm{E}+00$ & \\
\hline $\mathrm{Ca}-45$ & $8.6 \mathrm{E}-13$ & $7.4 \mathrm{E}-13$ & $-16.79 \%$ & Ir-192m & $0.0 \mathrm{E}+00$ & $0.0 \mathrm{E}+00$ & \\
\hline $\mathrm{Ca}-47$ & $9.2 \mathrm{E}-05$ & 7.9E-05 & $-16.79 \%$ & Pt-193 & $1.1 \mathrm{E}-04$ & $9.1 \mathrm{E}-05$ & $-16.79 \%$ \\
\hline Ti-44 & $3.2 \mathrm{E}-02$ & $2.7 \mathrm{E}-02$ & $-16.79 \%$ & $\mathrm{Hg}-194$ & $0.0 \mathrm{E}+00$ & $0.0 \mathrm{E}+00$ & \\
\hline $\mathrm{V}-49$ & $6.1 \mathrm{E}-07$ & $5.2 \mathrm{E}-07$ & $-16.79 \%$ & $\mathrm{Hg}-203$ & $2.8 \mathrm{E}-04$ & $2.4 \mathrm{E}-04$ & $-16.79 \%$ \\
\hline Cr-51 & $2.3 \mathrm{E}-05$ & $1.9 \mathrm{E}-05$ & $-16.79 \%$ & $\mathrm{~Pb}-202$ & $0.0 \mathrm{E}+00$ & $0.0 \mathrm{E}+00$ & \\
\hline Mn-53 & $3.9 \mathrm{E}-05$ & $3.4 \mathrm{E}-05$ & $-16.79 \%$ & $\mathrm{~Pb}-205$ & $1.9 \mathrm{E}-04$ & $1.6 \mathrm{E}-04$ & $-16.79 \%$ \\
\hline Mn-54 & $6.4 \mathrm{E}-03$ & $5.5 \mathrm{E}-03$ & $-16.79 \%$ & $\mathrm{~Pb}-210$ & $4.3 \mathrm{E}-04$ & $3.6 \mathrm{E}-04$ & $-16.79 \%$ \\
\hline $\mathrm{Fe}-55$ & $5.3 \mathrm{E}-06$ & $4.5 \mathrm{E}-06$ & $-16.79 \%$ & Bi-207 & $2.5 \mathrm{E}-01$ & $2.2 \mathrm{E}-01$ & $-16.79 \%$ \\
\hline $\mathrm{Fe}-60$ & $0.0 \mathrm{E}+00$ & $0.0 \mathrm{E}+00$ & & $\mathrm{Bi}-210$ & $0.0 \mathrm{E}+00$ & $0.0 \mathrm{E}+00$ & \\
\hline Co-58 & $1.7 \mathrm{E}-03$ & $1.5 \mathrm{E}-03$ & $-16.79 \%$ & $\mathrm{Bi}-210 \mathrm{~m}$ & $0.0 \mathrm{E}+00$ & $0.0 \mathrm{E}+00$ & \\
\hline Co-60 & $1.1 \mathrm{E}-01$ & $9.1 \mathrm{E}-02$ & $-16.79 \%$ & Po-210 & 2.9E-08 & $2.5 \mathrm{E}-08$ & $-16.79 \%$ \\
\hline Ni-59 & $1.0 \mathrm{E}-04$ & $8.8 \mathrm{E}-05$ & $-16.79 \%$ & $\mathrm{Ra}-223$ & $4.0 \mathrm{E}-05$ & $3.4 \mathrm{E}-05$ & $-16.79 \%$ \\
\hline Ni-63 & $0.0 \mathrm{E}+00$ & $0.0 \mathrm{E}+00$ & & Ra-224 & $8.1 \mathrm{E}-07$ & $6.9 \mathrm{E}-07$ & $-16.79 \%$ \\
\hline $\mathrm{Cu}-64$ & $6.8 \mathrm{E}-07$ & $5.8 \mathrm{E}-07$ & $-16.79 \%$ & Ra-225 & $2.6 \mathrm{E}-06$ & $2.3 \mathrm{E}-06$ & $-16.79 \%$ \\
\hline
\end{tabular}


SRNL-STI-2010-00447, REVISION 0

Table 24. LADTAP XL Collective Shoreline Exposure Comparisons (person-rem) (continued)

\begin{tabular}{|c|c|c|c|c|c|c|c|}
\hline Nuclide & $\begin{array}{c}\text { Hamby Ladtap } \\
\text { Shoreline Pathway }\end{array}$ & $\begin{array}{c}\text { Jannik Ladtap } \\
\text { Shoreline Pathway }\end{array}$ & $\%$ Diff & Nuclide & $\begin{array}{c}\text { Hamby Ladtap } \\
\text { Shoreline Pathway }\end{array}$ & $\begin{array}{c}\text { Jannik Ladtap } \\
\text { Shoreline Pathway }\end{array}$ & $\%$ Diff \\
\hline $\mathrm{Zn}-65$ & $3.3 \mathrm{E}-03$ & $2.8 \mathrm{E}-03$ & $-16.79 \%$ & Ra-226 & $1.9 \mathrm{E}-03$ & $1.6 \mathrm{E}-03$ & $-16.79 \%$ \\
\hline Ge-68 & $8.1 \mathrm{E}-06$ & $6.9 \mathrm{E}-06$ & $-16.79 \%$ & Ra-228 & $3.4 \mathrm{E}-11$ & $2.9 \mathrm{E}-11$ & $-16.79 \%$ \\
\hline Se-75 & $1.3 \mathrm{E}-03$ & $1.1 \mathrm{E}-03$ & $-16.79 \%$ & Ac- 227 & $3.0 \mathrm{E}-05$ & $2.5 \mathrm{E}-05$ & $-16.79 \%$ \\
\hline Se-79 & $0.0 \mathrm{E}+00$ & $0.0 \mathrm{E}+00$ & & Th-227 & $5.2 \mathrm{E}-05$ & $4.5 \mathrm{E}-05$ & $-16.79 \%$ \\
\hline $\mathrm{Rb}-87$ & $0.0 \mathrm{E}+00$ & $0.0 \mathrm{E}+00$ & & Th-228 & $4.7 \mathrm{E}-05$ & $4.1 \mathrm{E}-05$ & $-16.79 \%$ \\
\hline Sr-89 & $1.7 \mathrm{E}-07$ & $1.4 \mathrm{E}-07$ & $-16.79 \%$ & Th-229 & $2.6 \mathrm{E}-02$ & $2.2 \mathrm{E}-02$ & $-16.79 \%$ \\
\hline Sr-90 & $0.0 \mathrm{E}+00$ & $0.0 \mathrm{E}+00$ & & Th-230 & $2.3 \mathrm{E}-04$ & $1.9 \mathrm{E}-04$ & $-16.79 \%$ \\
\hline$Y-90$ & $0.0 \mathrm{E}+00$ & $0.0 \mathrm{E}+00$ & & Th-231 & $2.6 \mathrm{E}-07$ & $2.2 \mathrm{E}-07$ & $-16.79 \%$ \\
\hline Y-91 & $4.7 \mathrm{E}-06$ & $4.0 \mathrm{E}-06$ & $-16.79 \%$ & Th-232 & $1.7 \mathrm{E}-04$ & $1.4 \mathrm{E}-04$ & $-16.79 \%$ \\
\hline Мо-93 & $1.4 \mathrm{E}-03$ & $1.2 \mathrm{E}-03$ & $-16.79 \%$ & Th-234 & $5.8 \mathrm{E}-06$ & $5.0 \mathrm{E}-06$ & $-16.79 \%$ \\
\hline Мо-99 & $1.5 \mathrm{E}-05$ & $1.3 \mathrm{E}-05$ & $-16.79 \%$ & $\mathrm{~Pa}-230$ & $2.7 \mathrm{E}-04$ & $2.4 \mathrm{E}-04$ & $-16.79 \%$ \\
\hline $\mathrm{Nb}-93 \mathrm{~m}$ & $1.1 \mathrm{E}-04$ & $9.3 \mathrm{E}-05$ & $-16.79 \%$ & $\mathrm{~Pa}-231$ & 8.9E-03 & $7.6 \mathrm{E}-03$ & $-16.79 \%$ \\
\hline $\mathrm{Nb}-94$ & $3.9 \mathrm{E}-01$ & $3.4 \mathrm{E}-01$ & $-16.79 \%$ & $\mathrm{~Pa}-233$ & $1.5 \mathrm{E}-04$ & $1.3 \mathrm{E}-04$ & $-16.79 \%$ \\
\hline $\mathrm{Nb}-95$ & $6.5 \mathrm{E}-04$ & $5.6 \mathrm{E}-04$ & $-16.79 \%$ & U-232 & $2.1 \mathrm{E}-04$ & $1.8 \mathrm{E}-04$ & $-16.79 \%$ \\
\hline Zr-93 & $0.0 \mathrm{E}+00$ & $0.0 \mathrm{E}+00$ & & U-233 & $1.2 \mathrm{E}-04$ & $1.1 \mathrm{E}-04$ & $-16.79 \%$ \\
\hline Zr-95 & $2.4 \mathrm{E}-03$ & $2.0 \mathrm{E}-03$ & $-16.79 \%$ & U-234 & $2.0 \mathrm{E}-04$ & $1.7 \mathrm{E}-04$ & $-16.79 \%$ \\
\hline Tc-96 & $2.3 \mathrm{E}-04$ & $1.9 \mathrm{E}-04$ & $-16.79 \%$ & U-235 & $4.2 \mathrm{E}-02$ & $3.6 \mathrm{E}-02$ & $-16.79 \%$ \\
\hline Tc-97 & $1.6 \mathrm{E}-03$ & $1.4 \mathrm{E}-03$ & $-16.79 \%$ & U-236 & $1.8 \mathrm{E}-04$ & $1.6 \mathrm{E}-04$ & $-16.79 \%$ \\
\hline Tc-98 & $3.5 \mathrm{E}-01$ & $3.0 \mathrm{E}-01$ & $-16.79 \%$ & U-237 & $2.4 \mathrm{E}-05$ & $2.1 \mathrm{E}-05$ & $-16.79 \%$ \\
\hline Tc-99 & $1.6 \mathrm{E}-07$ & $1.3 \mathrm{E}-07$ & $-16.79 \%$ & U-238 & $1.6 \mathrm{E}-04$ & $1.4 \mathrm{E}-04$ & $-16.79 \%$ \\
\hline Ru-97 & $1.5 \mathrm{E}-05$ & $1.2 \mathrm{E}-05$ & $-16.79 \%$ & $\mathrm{~Np}-236$ & $3.9 \mathrm{E}-02$ & $3.4 \mathrm{E}-02$ & $-16.79 \%$ \\
\hline Ru-103 & $4.8 \mathrm{E}-04$ & $4.1 \mathrm{E}-04$ & $-16.79 \%$ & Np-237 & $8.0 \mathrm{E}-03$ & $6.9 \mathrm{E}-03$ & $-16.79 \%$ \\
\hline Ru-106 & $1.9 \mathrm{E}-03$ & $1.6 \mathrm{E}-03$ & $-16.79 \%$ & $\mathrm{~Np}-239$ & $8.2 \mathrm{E}-06$ & $7.1 \mathrm{E}-06$ & $-16.79 \%$ \\
\hline Pd-107 & $0.0 \mathrm{E}+00$ & $0.0 \mathrm{E}+00$ & & Am-237 & $0.0 \mathrm{E}+00$ & $0.0 \mathrm{E}+00$ & \\
\hline Ag-108m & $3.7 \mathrm{E}-01$ & $3.2 \mathrm{E}-01$ & $-16.79 \%$ & Am-241 & $7.2 \mathrm{E}-03$ & $6.2 \mathrm{E}-03$ & $-16.79 \%$ \\
\hline
\end{tabular}


SRNL-STI-2010-00447, REVISION 0

Table 24. LADTAP XL Collective Shoreline Exposure Comparisons (person-rem) (continued)

\begin{tabular}{|c|c|c|c|c|c|c|c|}
\hline Nuclide & $\begin{array}{c}\text { Hamby Ladtap } \\
\text { Shoreline Pathway }\end{array}$ & $\begin{array}{c}\text { Jannik Ladtap } \\
\text { Shoreline Pathway }\end{array}$ & $\%$ Diff & Nuclide & $\begin{array}{c}\text { Hamby Ladtap } \\
\text { Shoreline Pathway }\end{array}$ & $\begin{array}{c}\text { Jannik Ladtap } \\
\text { Shoreline Pathway }\end{array}$ & $\%$ Diff \\
\hline Ag-110m & $1.6 \mathrm{E}-02$ & $1.4 \mathrm{E}-02$ & $-16.79 \%$ & Am-242m & $6.3 \mathrm{E}-04$ & $5.4 \mathrm{E}-04$ & $-16.79 \%$ \\
\hline Cd-113 & $0.0 \mathrm{E}+00$ & $0.0 \mathrm{E}+00$ & & Am-243 & $1.6 \mathrm{E}-02$ & $1.4 \mathrm{E}-02$ & $-16.79 \%$ \\
\hline Cd-115m & $2.3 \mathrm{E}-05$ & $1.9 \mathrm{E}-05$ & $-16.79 \%$ & $\mathrm{Pu}-237$ & $6.5 \mathrm{E}-05$ & $5.6 \mathrm{E}-05$ & $-16.79 \%$ \\
\hline In-115 & $0.0 \mathrm{E}+00$ & $0.0 \mathrm{E}+00$ & & $\mathrm{Pu}-238$ & $1.8 \mathrm{E}-04$ & $1.6 \mathrm{E}-04$ & $-16.79 \%$ \\
\hline $\mathrm{Sb}-122$ & $2.3 \mathrm{E}-05$ & $2.0 \mathrm{E}-05$ & $-16.79 \%$ & $\mathrm{Pu}-239$ & $9.4 \mathrm{E}-05$ & $8.0 \mathrm{E}-05$ & $-16.79 \%$ \\
\hline Sb-124 & $2.6 \mathrm{E}-03$ & $2.2 \mathrm{E}-03$ & $-16.79 \%$ & $\mathrm{Pu}-240$ & $2.0 \mathrm{E}-04$ & $1.7 \mathrm{E}-04$ & $-16.79 \%$ \\
\hline Sb-125 & $1.1 \mathrm{E}-02$ & $9.3 \mathrm{E}-03$ & $-16.79 \%$ & $\mathrm{Pu}-241$ & $0.0 \mathrm{E}+00$ & $0.0 \mathrm{E}+00$ & \\
\hline Te-123 & $1.8 \mathrm{E}-03$ & $1.5 \mathrm{E}-03$ & $-16.79 \%$ & $\mathrm{Pu}-242$ & $1.7 \mathrm{E}-04$ & $1.4 \mathrm{E}-04$ & $-16.79 \%$ \\
\hline $\mathrm{Te}-125 \mathrm{~m}$ & $3.0 \mathrm{E}-05$ & $2.5 \mathrm{E}-05$ & $-16.79 \%$ & $\mathrm{Pu}-244$ & $1.4 \mathrm{E}-04$ & $1.2 \mathrm{E}-04$ & $-16.79 \%$ \\
\hline Sn-126 & $1.5 \mathrm{E}-02$ & $1.3 \mathrm{E}-02$ & $-16.79 \%$ & $\mathrm{Cm}-241$ & $0.0 \mathrm{E}+00$ & $0.0 \mathrm{E}+00$ & \\
\hline I-129 & $5.5 \mathrm{E}-03$ & $4.7 \mathrm{E}-03$ & $-16.79 \%$ & $\mathrm{Cm}-242$ & $3.7 \mathrm{E}-06$ & $3.2 \mathrm{E}-06$ & $-16.79 \%$ \\
\hline $\mathrm{I}-131$ & $7.3 \mathrm{E}-05$ & $6.3 \mathrm{E}-05$ & $-16.79 \%$ & $\mathrm{Cm}-243$ & $2.3 \mathrm{E}-02$ & $2.0 \mathrm{E}-02$ & $-16.79 \%$ \\
\hline Cs-134 & $2.9 \mathrm{E}-02$ & $2.5 \mathrm{E}-02$ & $-16.79 \%$ & $\mathrm{Cm}-244$ & $1.1 \mathrm{E}-04$ & $9.0 \mathrm{E}-05$ & $-16.79 \%$ \\
\hline Cs-135 & $0.0 \mathrm{E}+00$ & $0.0 \mathrm{E}+00$ & & $\mathrm{Cm}-245$ & $2.1 \mathrm{E}-02$ & $1.8 \mathrm{E}-02$ & $-16.79 \%$ \\
\hline Cs-137 & $9.4 \mathrm{E}-02$ & $8.0 \mathrm{E}-02$ & $-16.79 \%$ & $\mathrm{Cm}-246$ & $1.8 \mathrm{E}-04$ & $1.6 \mathrm{E}-04$ & $-16.79 \%$ \\
\hline La-137 & $0.0 \mathrm{E}+00$ & $0.0 \mathrm{E}+00$ & & $\mathrm{Cm}-247$ & $8.3 \mathrm{E}-02$ & $7.1 \mathrm{E}-02$ & $-16.79 \%$ \\
\hline La-138 & $0.0 \mathrm{E}+00$ & $0.0 \mathrm{E}+00$ & & Cm-248 & $1.5 \mathrm{E}-04$ & $1.3 \mathrm{E}-04$ & $-16.79 \%$ \\
\hline La-140 & $5.8 \mathrm{E}-05$ & $5.0 \mathrm{E}-05$ & $-16.79 \%$ & $\mathrm{Cm}-250$ & $0.0 \mathrm{E}+00$ & $0.0 \mathrm{E}+00$ & \\
\hline Ba-140 & $6.9 \mathrm{E}-04$ & $5.9 \mathrm{E}-04$ & $-16.79 \%$ & Bk-247 & $0.0 \mathrm{E}+00$ & $0.0 \mathrm{E}+00$ & \\
\hline Ce-141 & $6.9 \mathrm{E}-05$ & $5.9 \mathrm{E}-05$ & $-16.79 \%$ & Bk-249 & $0.0 \mathrm{E}+00$ & $0.0 \mathrm{E}+00$ & \\
\hline \multirow[t]{8}{*}{ Ce-144 } & $3.5 \mathrm{E}-04$ & $3.0 \mathrm{E}-04$ & $-16.79 \%$ & Cf-249 & $8.4 \mathrm{E}-02$ & $7.2 \mathrm{E}-02$ & $-16.79 \%$ \\
\hline & & & & Cf-250 & $7.1 \mathrm{E}-05$ & $6.0 \mathrm{E}-05$ & $-16.79 \%$ \\
\hline & & & & Cf-251 & $3.3 \mathrm{E}-02$ & $2.8 \mathrm{E}-02$ & $-16.79 \%$ \\
\hline & & & & Cf-252 & $1.5 \mathrm{E}-05$ & $1.3 \mathrm{E}-05$ & $-16.79 \%$ \\
\hline & & & & Es-253 & $3.5 \mathrm{E}-07$ & $3.0 \mathrm{E}-07$ & $-16.79 \%$ \\
\hline & & & & Unidentified alpha & $9.4 \mathrm{E}-05$ & $8.0 \mathrm{E}-05$ & $-16.79 \%$ \\
\hline & & & & Unidentified beta & $0.0 \mathrm{E}+00$ & $0.0 \mathrm{E}+00$ & \\
\hline & & & & TOTAL Dose & $3.4 \mathrm{E}+00$ & $2.9 \mathrm{E}+00$ & $-16.79 \%$ \\
\hline
\end{tabular}


SRNL-STI-2010-00447, REVISION 0

Table 25. LADTAP XL Collective Swimming Exposure Comparisons (person-rem)

\begin{tabular}{|c|c|c|c|c|c|c|c|}
\hline Nuclide & $\begin{array}{c}\text { Hamby Ladtap } \\
\text { Swimming }\end{array}$ & $\begin{array}{c}\text { Jannik Ladtap } \\
\text { Swimming }\end{array}$ & $\%$ Diff & Nuclide & $\begin{array}{c}\text { Hamby Ladtap } \\
\text { Swimming }\end{array}$ & $\begin{array}{c}\text { Jannik Ladtap } \\
\text { Swimming }\end{array}$ & $\%$ Diff \\
\hline $\mathrm{H}-3$ & $4.0 \mathrm{E}-08$ & $7.3 \mathrm{E}-08$ & $45.76 \%$ & Sm-146 & $0.0 \mathrm{E}+00$ & $0.0 \mathrm{E}+00$ & \\
\hline $\mathrm{Be}-7$ & $1.1 \mathrm{E}-06$ & $2.0 \mathrm{E}-06$ & $45.76 \%$ & Sm-147 & $0.0 \mathrm{E}+00$ & $0.0 \mathrm{E}+00$ & \\
\hline Be-10 & $0.0 \mathrm{E}+00$ & $0.0 \mathrm{E}+00$ & & Sm-151 & $2.4 \mathrm{E}-11$ & $4.4 \mathrm{E}-11$ & $45.76 \%$ \\
\hline C-14 & $0.0 \mathrm{E}+00$ & $0.0 \mathrm{E}+00$ & & Pm-147 & $8.5 \mathrm{E}-11$ & $1.6 \mathrm{E}-10$ & $45.76 \%$ \\
\hline $\mathrm{Na}-22$ & $5.0 \mathrm{E}-05$ & $9.2 \mathrm{E}-05$ & $45.76 \%$ & Eu-152 & $2.6 \mathrm{E}-05$ & $4.8 \mathrm{E}-05$ & $45.76 \%$ \\
\hline $\mathrm{Na}-24$ & $3.5 \mathrm{E}-05$ & $6.4 \mathrm{E}-05$ & $45.76 \%$ & Eu-154 & $2.9 \mathrm{E}-05$ & $5.3 \mathrm{E}-05$ & $45.76 \%$ \\
\hline Al-26 & $6.4 \mathrm{E}-05$ & $1.2 \mathrm{E}-04$ & $45.76 \%$ & Eu-155 & $1.4 \mathrm{E}-06$ & $2.5 \mathrm{E}-06$ & $45.76 \%$ \\
\hline P-32 & $0.0 \mathrm{E}+00$ & $0.0 \mathrm{E}+00$ & & Gd-152 & $0.0 \mathrm{E}+00$ & $0.0 \mathrm{E}+00$ & \\
\hline Si-32 & $0.0 \mathrm{E}+00$ & $0.0 \mathrm{E}+00$ & & Ho-166m & $6.5 \mathrm{E}-07$ & $1.2 \mathrm{E}-06$ & $45.76 \%$ \\
\hline S-35 & $0.0 \mathrm{E}+00$ & $0.0 \mathrm{E}+00$ & & Lu-176 & $0.0 \mathrm{E}+00$ & $0.0 \mathrm{E}+00$ & \\
\hline $\mathrm{Cl}-36$ & $2.1 \mathrm{E}-13$ & $3.9 \mathrm{E}-13$ & $45.76 \%$ & Ta-180 & $0.0 \mathrm{E}+00$ & $0.0 \mathrm{E}+00$ & \\
\hline $\mathrm{K}-40$ & $3.6 \mathrm{E}-06$ & $6.6 \mathrm{E}-06$ & $45.76 \%$ & Hf-182 & $0.0 \mathrm{E}+00$ & $0.0 \mathrm{E}+00$ & \\
\hline K-43 & $1.0 \mathrm{E}-05$ & $1.9 \mathrm{E}-05$ & $45.76 \%$ & Re-186m & $0.0 \mathrm{E}+00$ & $0.0 \mathrm{E}+00$ & \\
\hline Ca-41 & $7.9 \mathrm{E}-11$ & $1.5 \mathrm{E}-10$ & $45.76 \%$ & Re-187 & $0.0 \mathrm{E}+00$ & $0.0 \mathrm{E}+00$ & \\
\hline Ca-45 & $4.3 \mathrm{E}-16$ & $7.8 \mathrm{E}-16$ & $45.76 \%$ & Ir-192m & $0.0 \mathrm{E}+00$ & $0.0 \mathrm{E}+00$ & \\
\hline $\mathrm{Ca}-47$ & $2.1 \mathrm{E}-05$ & $3.9 \mathrm{E}-05$ & $45.76 \%$ & Pt-193 & $1.1 \mathrm{E}-09$ & $2.1 \mathrm{E}-09$ & $45.76 \%$ \\
\hline Ti-44 & $3.3 \mathrm{E}-06$ & $6.1 \mathrm{E}-06$ & $45.76 \%$ & $\mathrm{Hg}-194$ & $0.0 \mathrm{E}+00$ & $0.0 \mathrm{E}+00$ & \\
\hline $\mathrm{V}-49$ & $2.4 \mathrm{E}-10$ & $4.4 \mathrm{E}-10$ & $45.76 \%$ & $\mathrm{Hg}-203$ & $5.1 \mathrm{E}-06$ & $9.4 \mathrm{E}-06$ & $45.76 \%$ \\
\hline $\mathrm{Cr}-51$ & $6.9 \mathrm{E}-07$ & $1.3 \mathrm{E}-06$ & $45.76 \%$ & $\mathrm{~Pb}-202$ & $0.0 \mathrm{E}+00$ & $0.0 \mathrm{E}+00$ & \\
\hline $\mathrm{Mn}-53$ & $4.4 \mathrm{E}-10$ & $8.2 \mathrm{E}-10$ & $45.76 \%$ & $\mathrm{~Pb}-205$ & $1.5 \mathrm{E}-09$ & $2.7 \mathrm{E}-09$ & $45.76 \%$ \\
\hline $\mathrm{Mn}-54$ & $1.9 \mathrm{E}-05$ & $3.5 \mathrm{E}-05$ & $45.76 \%$ & $\mathrm{~Pb}-210$ & $3.4 \mathrm{E}-08$ & $6.3 \mathrm{E}-08$ & $45.76 \%$ \\
\hline Fe-55 & $5.8 \mathrm{E}-10$ & $1.1 \mathrm{E}-09$ & $45.76 \%$ & Bi-207 & $3.5 \mathrm{E}-05$ & $6.5 \mathrm{E}-05$ & $45.76 \%$ \\
\hline Fe-60 & $0.0 \mathrm{E}+00$ & $0.0 \mathrm{E}+00$ & & Bi-210 & $0.0 \mathrm{E}+00$ & $0.0 \mathrm{E}+00$ & \\
\hline Co-58 & $2.2 \mathrm{E}-05$ & $4.1 \mathrm{E}-05$ & $45.76 \%$ & $\mathrm{Bi}-210 \mathrm{~m}$ & $0.0 \mathrm{E}+00$ & $0.0 \mathrm{E}+00$ & \\
\hline Co-60 & 7.7E-05 & $1.4 \mathrm{E}-04$ & $45.76 \%$ & Po-210 & $1.9 \mathrm{E}-10$ & $3.6 \mathrm{E}-10$ & $45.76 \%$ \\
\hline $\mathrm{Ni}-59$ & $9.9 \mathrm{E}-10$ & $1.8 \mathrm{E}-09$ & $45.76 \%$ & Ra-223 & $2.9 \mathrm{E}-06$ & $5.4 \mathrm{E}-06$ & $45.76 \%$ \\
\hline Ni-63 & $0.0 \mathrm{E}+00$ & $0.0 \mathrm{E}+00$ & & Ra-224 & $1.9 \mathrm{E}-07$ & $3.5 \mathrm{E}-07$ & $45.76 \%$ \\
\hline $\mathrm{Cu}-64$ & $1.1 \mathrm{E}-06$ & $2.1 \mathrm{E}-06$ & $45.76 \%$ & $\mathrm{Ra}-225$ & $1.7 \mathrm{E}-07$ & $3.1 \mathrm{E}-07$ & $45.76 \%$ \\
\hline
\end{tabular}


SRNL-STI-2010-00447, REVISION 0

Table 25. LADTAP XL Collective Swimming Exposure Comparisons (person-rem) (continued)

\begin{tabular}{|c|c|c|c|c|c|c|c|}
\hline Nuclide & $\begin{array}{c}\text { Hamby Ladtap } \\
\text { Swimming }\end{array}$ & $\begin{array}{c}\text { Jannik Ladtap } \\
\text { Swimming }\end{array}$ & $\%$ Diff & Nuclide & $\begin{array}{c}\text { Hamby Ladtap } \\
\text { Swimming }\end{array}$ & $\begin{array}{c}\text { Jannik Ladtap } \\
\text { Swimming }\end{array}$ & $\%$ Diff \\
\hline $\mathrm{Zn}-65$ & $1.3 \mathrm{E}-05$ & $2.5 \mathrm{E}-05$ & $45.76 \%$ & Ra-226 & $1.6 \mathrm{E}-07$ & $2.9 \mathrm{E}-07$ & $45.76 \%$ \\
\hline Ge-68 & $2.3 \mathrm{E}-09$ & $4.3 \mathrm{E}-09$ & $45.76 \%$ & Ra-228 & $1.6 \mathrm{E}-15$ & $3.0 \mathrm{E}-15$ & $45.76 \%$ \\
\hline Se-75 & 8.9E-06 & $1.6 \mathrm{E}-05$ & $45.76 \%$ & Ac-227 & 2.9E-09 & $5.4 \mathrm{E}-09$ & $45.76 \%$ \\
\hline Se-79 & $0.0 \mathrm{E}+00$ & $0.0 \mathrm{E}+00$ & & Th-227 & $2.3 \mathrm{E}-06$ & $4.2 \mathrm{E}-06$ & $45.76 \%$ \\
\hline $\mathrm{Rb}-87$ & $0.0 \mathrm{E}+00$ & $0.0 \mathrm{E}+00$ & & Th-228 & $4.6 \mathrm{E}-08$ & $8.6 \mathrm{E}-08$ & $45.76 \%$ \\
\hline Sr-89 & $3.1 \mathrm{E}-09$ & $5.8 \mathrm{E}-09$ & $45.76 \%$ & Th-229 & $2.0 \mathrm{E}-06$ & $3.7 \mathrm{E}-06$ & $45.76 \%$ \\
\hline Sr-90 & $0.0 \mathrm{E}+00$ & $0.0 \mathrm{E}+00$ & & Th-230 & $9.5 \mathrm{E}-09$ & $1.7 \mathrm{E}-08$ & $45.76 \%$ \\
\hline $\mathrm{Y}-90$ & $0.0 \mathrm{E}+00$ & $0.0 \mathrm{E}+00$ & & Th-231 & $1.5 \mathrm{E}-07$ & $2.7 \mathrm{E}-07$ & $45.76 \%$ \\
\hline Y-91 & $8.2 \mathrm{E}-08$ & $1.5 \mathrm{E}-07$ & $45.76 \%$ & Th-232 & 4.6E-09 & $8.5 \mathrm{E}-09$ & $45.76 \%$ \\
\hline Mo-93 & $2.0 \mathrm{E}-08$ & $3.7 \mathrm{E}-08$ & $45.76 \%$ & Th-234 & $1.8 \mathrm{E}-07$ & $3.3 \mathrm{E}-07$ & $45.76 \%$ \\
\hline Mo-99 & $4.8 \mathrm{E}-06$ & $8.9 \mathrm{E}-06$ & $45.76 \%$ & $\mathrm{~Pa}-230$ & $1.5 \mathrm{E}-05$ & $2.7 \mathrm{E}-05$ & $45.76 \%$ \\
\hline $\mathrm{Nb}-93 \mathrm{~m}$ & 3.6E-09 & $6.6 \mathrm{E}-09$ & $45.76 \%$ & $\mathrm{~Pa}-231$ & $6.8 \mathrm{E}-07$ & $1.3 \mathrm{E}-06$ & $45.76 \%$ \\
\hline Nb-94 & $3.6 \mathrm{E}-05$ & $6.7 \mathrm{E}-05$ & $45.76 \%$ & $\mathrm{~Pa}-233$ & $4.7 \mathrm{E}-06$ & $8.7 \mathrm{E}-06$ & $45.76 \%$ \\
\hline $\mathrm{Nb}-95$ & $1.7 \mathrm{E}-05$ & $3.2 \mathrm{E}-05$ & $45.76 \%$ & U-232 & $6.5 \mathrm{E}-09$ & $1.2 \mathrm{E}-08$ & $45.76 \%$ \\
\hline Zr-93 & $0.0 \mathrm{E}+00$ & $0.0 \mathrm{E}+00$ & & U-233 & 5.6E-09 & $1.0 \mathrm{E}-08$ & $45.76 \%$ \\
\hline Zr-95 & $1.7 \mathrm{E}-05$ & $3.1 \mathrm{E}-05$ & $45.76 \%$ & U-234 & $3.8 \mathrm{E}-09$ & 7.0E-09 & $45.76 \%$ \\
\hline Tc-96 & $4.9 \mathrm{E}-05$ & $9.0 \mathrm{E}-05$ & $45.76 \%$ & U-235 & $3.5 \mathrm{E}-06$ & $6.5 \mathrm{E}-06$ & $45.76 \%$ \\
\hline Tc-97 & $2.5 \mathrm{E}-08$ & $4.6 \mathrm{E}-08$ & $45.76 \%$ & U-236 & $3.1 \mathrm{E}-09$ & $5.7 \mathrm{E}-09$ & $45.76 \%$ \\
\hline Tc-98 & $3.2 \mathrm{E}-05$ & $5.8 \mathrm{E}-05$ & $45.76 \%$ & U-237 & $2.8 \mathrm{E}-06$ & $5.2 \mathrm{E}-06$ & $45.76 \%$ \\
\hline Tc-99 & $1.3 \mathrm{E}-11$ & $2.3 \mathrm{E}-11$ & $45.76 \%$ & U-238 & 2.6E-09 & 4.9E-09 & $45.76 \%$ \\
\hline Ru-97 & $4.1 \mathrm{E}-06$ & $7.6 \mathrm{E}-06$ & $45.76 \%$ & $\mathrm{~Np}-236$ & $3.1 \mathrm{E}-06$ & $5.7 \mathrm{E}-06$ & $45.76 \%$ \\
\hline $\mathrm{Ru}-103$ & $1.1 \mathrm{E}-05$ & $1.9 \mathrm{E}-05$ & $45.76 \%$ & $\mathrm{~Np}-237$ & $5.5 \mathrm{E}-07$ & $1.0 \mathrm{E}-06$ & $45.76 \%$ \\
\hline Ru-106 & $4.7 \mathrm{E}-06$ & $8.6 \mathrm{E}-06$ & $45.76 \%$ & $\mathrm{~Np}-239$ & $2.9 \mathrm{E}-06$ & $5.3 \mathrm{E}-06$ & $45.76 \%$ \\
\hline Pd-107 & $0.0 \mathrm{E}+00$ & $0.0 \mathrm{E}+00$ & & Am-237 & $0.0 \mathrm{E}+00$ & $0.0 \mathrm{E}+00$ & \\
\hline Ag-108m & $3.6 \mathrm{E}-05$ & $6.7 \mathrm{E}-05$ & $45.76 \%$ & Am-241 & 4.8E-07 & $8.8 \mathrm{E}-07$ & $45.76 \%$ \\
\hline
\end{tabular}


SRNL-STI-2010-00447, REVISION 0

Table 25. LADTAP XL Collective Swimming Exposure Comparisons (person-rem) (continued)

\begin{tabular}{|c|c|c|c|c|c|c|c|}
\hline Nuclide & $\begin{array}{c}\text { Hamby Ladtap } \\
\text { Swimming }\end{array}$ & $\begin{array}{c}\text { Jannik Ladtap } \\
\text { Swimming }\end{array}$ & $\%$ Diff & Nuclide & $\begin{array}{c}\text { Hamby Ladtap } \\
\text { Swimming }\end{array}$ & $\begin{array}{c}\text { Jannik Ladtap } \\
\text { Swimming }\end{array}$ & $\%$ Diff \\
\hline Ag-110m & $6.3 \mathrm{E}-05$ & $1.2 \mathrm{E}-04$ & $45.76 \%$ & $\mathrm{Am}-242 \mathrm{~m}$ & $1.2 \mathrm{E}-08$ & $2.2 \mathrm{E}-08$ & $45.76 \%$ \\
\hline Cd-113 & $0.0 \mathrm{E}+00$ & $0.0 \mathrm{E}+00$ & & Am-243 & $1.2 \mathrm{E}-06$ & $2.3 \mathrm{E}-06$ & $45.76 \%$ \\
\hline Cd-115m & $5.0 \mathrm{E}-07$ & $9.2 \mathrm{E}-07$ & $45.76 \%$ & $\mathrm{Pu}-237$ & $1.1 \mathrm{E}-06$ & $2.1 \mathrm{E}-06$ & $45.76 \%$ \\
\hline In-115 & $0.0 \mathrm{E}+00$ & $0.0 \mathrm{E}+00$ & & $\mathrm{Pu}-238$ & $2.3 \mathrm{E}-09$ & $4.2 \mathrm{E}-09$ & $45.76 \%$ \\
\hline Sb-122 & $7.7 \mathrm{E}-06$ & $1.4 \mathrm{E}-05$ & $45.76 \%$ & $\mathrm{Pu}-239$ & $2.0 \mathrm{E}-09$ & 3.7E-09 & $45.76 \%$ \\
\hline Sb-124 & $4.3 \mathrm{E}-05$ & $8.0 \mathrm{E}-05$ & $45.76 \%$ & $\mathrm{Pu}-240$ & $2.2 \mathrm{E}-09$ & 4.1E-09 & $45.76 \%$ \\
\hline Sb-125 & $9.5 \mathrm{E}-06$ & $1.7 \mathrm{E}-05$ & $45.76 \%$ & $\mathrm{Pu}-241$ & $0.0 \mathrm{E}+00$ & $0.0 \mathrm{E}+00$ & \\
\hline Te-123 & $8.0 \mathrm{E}-08$ & $1.5 \mathrm{E}-07$ & $45.76 \%$ & $\mathrm{Pu}-242$ & 1.9E-09 & $3.5 \mathrm{E}-09$ & $45.76 \%$ \\
\hline Te-125m & $2.5 \mathrm{E}-07$ & $4.6 \mathrm{E}-07$ & $45.76 \%$ & $\mathrm{Pu}-244$ & $1.4 \mathrm{E}-09$ & $2.6 \mathrm{E}-09$ & $45.76 \%$ \\
\hline Sn-126 & $1.2 \mathrm{E}-06$ & $2.2 \mathrm{E}-06$ & $45.76 \%$ & $\mathrm{Cm}-241$ & $0.0 \mathrm{E}+00$ & $0.0 \mathrm{E}+00$ & \\
\hline I-129 & $2.2 \mathrm{E}-07$ & $4.0 \mathrm{E}-07$ & $45.76 \%$ & $\mathrm{Cm}-242$ & $2.5 \mathrm{E}-09$ & 4.7E-09 & $45.76 \%$ \\
\hline $\mathrm{I}-131$ & $7.8 \mathrm{E}-06$ & $1.4 \mathrm{E}-05$ & $45.76 \%$ & $\mathrm{Cm}-243$ & $2.9 \mathrm{E}-06$ & $5.4 \mathrm{E}-06$ & $45.76 \%$ \\
\hline Cs-134 & $3.5 \mathrm{E}-05$ & $6.5 \mathrm{E}-05$ & $45.76 \%$ & $\mathrm{Cm}-244$ & $2.2 \mathrm{E}-09$ & $4.0 \mathrm{E}-09$ & $45.76 \%$ \\
\hline Cs-135 & $0.0 \mathrm{E}+00$ & $0.0 \mathrm{E}+00$ & & $\mathrm{Cm}-245$ & $1.7 \mathrm{E}-06$ & $3.1 \mathrm{E}-06$ & $45.76 \%$ \\
\hline Cs-137 & $1.3 \mathrm{E}-05$ & $2.3 \mathrm{E}-05$ & $45.76 \%$ & $\mathrm{Cm}-246$ & $1.8 \mathrm{E}-09$ & $3.4 \mathrm{E}-09$ & $45.76 \%$ \\
\hline La-137 & $0.0 \mathrm{E}+00$ & $0.0 \mathrm{E}+00$ & & $\mathrm{Cm}-247$ & $7.1 \mathrm{E}-06$ & $1.3 \mathrm{E}-05$ & $45.76 \%$ \\
\hline La-138 & $0.0 \mathrm{E}+00$ & $0.0 \mathrm{E}+00$ & & $\mathrm{Cm}-248$ & $1.6 \mathrm{E}-09$ & $3.0 \mathrm{E}-09$ & $45.76 \%$ \\
\hline La-140 & $3.6 \mathrm{E}-05$ & $6.6 \mathrm{E}-05$ & $45.76 \%$ & $\mathrm{Cm}-250$ & $0.0 \mathrm{E}+00$ & $0.0 \mathrm{E}+00$ & \\
\hline Ba-140 & $5.6 \mathrm{E}-05$ & $1.0 \mathrm{E}-04$ & $45.76 \%$ & Bk-247 & $0.0 \mathrm{E}+00$ & $0.0 \mathrm{E}+00$ & \\
\hline Ce-141 & $1.7 \mathrm{E}-06$ & $3.2 \mathrm{E}-06$ & $45.76 \%$ & Bk-249 & $0.0 \mathrm{E}+00$ & $0.0 \mathrm{E}+00$ & \\
\hline \multirow[t]{8}{*}{ Ce-144 } & $1.2 \mathrm{E}-06$ & $2.2 \mathrm{E}-06$ & $45.76 \%$ & Cf-249 & $7.3 \mathrm{E}-06$ & $1.4 \mathrm{E}-05$ & $45.76 \%$ \\
\hline & & & & Cf-250 & $2.2 \mathrm{E}-09$ & $4.1 \mathrm{E}-09$ & $45.76 \%$ \\
\hline & & & & Cf-251 & $2.7 \mathrm{E}-06$ & $5.0 \mathrm{E}-06$ & $45.76 \%$ \\
\hline & & & & Cf-252 & $1.9 \mathrm{E}-09$ & $3.6 \mathrm{E}-09$ & $45.76 \%$ \\
\hline & & & & Es-253 & $7.5 \mathrm{E}-09$ & $1.4 \mathrm{E}-08$ & $45.76 \%$ \\
\hline & & & & Unidentified alpha & $2.0 \mathrm{E}-09$ & $3.7 \mathrm{E}-09$ & $45.76 \%$ \\
\hline & & & & Unidentified beta & $0.0 \mathrm{E}+00$ & $0.0 \mathrm{E}+00$ & \\
\hline & & & & TOTAL Dose & 9.8E-04 & 1.8E-03 & $45.76 \%$ \\
\hline
\end{tabular}


SRNL-STI-2010-00447, REVISION 0

Table 26. LADTAP XL Collective Boating Exposure Comparisons (person-rem)

\begin{tabular}{|c|c|c|c|c|c|c|c|}
\hline Nuclide & $\begin{array}{l}\text { Hamby Ladtap } \\
\text { Boating Pathway }\end{array}$ & $\begin{array}{l}\text { Jannik Ladtap } \\
\text { Boating Pathway }\end{array}$ & $\%$ Diff & Nuclide & $\begin{array}{l}\text { Hamby Ladtap } \\
\text { Boating Pathway }\end{array}$ & $\begin{array}{l}\text { Jannik Ladtap } \\
\text { Boating Pathway }\end{array}$ & $\%$ Diff \\
\hline $\mathrm{H}-3$ & $0.0 \mathrm{E}+00$ & $0.0 \mathrm{E}+00$ & & Sm-146 & $0.0 \mathrm{E}+00$ & $0.0 \mathrm{E}+00$ & \\
\hline $\mathrm{Be}-7$ & $3.8 \mathrm{E}-06$ & $1.1 \mathrm{E}-05$ & $182.73 \%$ & Sm-147 & $0.0 \mathrm{E}+00$ & $0.0 \mathrm{E}+00$ & \\
\hline Be-10 & $0.0 \mathrm{E}+00$ & $0.0 \mathrm{E}+00$ & & Sm-151 & $8.3 \mathrm{E}-11$ & $2.3 \mathrm{E}-10$ & $182.73 \%$ \\
\hline C-14 & $0.0 \mathrm{E}+00$ & $0.0 \mathrm{E}+00$ & & Pm-147 & $2.9 \mathrm{E}-10$ & $8.2 \mathrm{E}-10$ & $182.73 \%$ \\
\hline $\mathrm{Na}-22$ & $1.7 \mathrm{E}-04$ & $4.8 \mathrm{E}-04$ & $182.73 \%$ & Eu-152 & $9.0 \mathrm{E}-05$ & $2.5 \mathrm{E}-04$ & $182.73 \%$ \\
\hline $\mathrm{Na}-24$ & $1.2 \mathrm{E}-04$ & $3.4 \mathrm{E}-04$ & $182.73 \%$ & Eu-154 & $9.9 \mathrm{E}-05$ & $2.8 \mathrm{E}-04$ & $182.73 \%$ \\
\hline Al-26 & $2.2 \mathrm{E}-04$ & $6.2 \mathrm{E}-04$ & $182.73 \%$ & Eu-155 & $4.7 \mathrm{E}-06$ & $1.3 \mathrm{E}-05$ & $182.73 \%$ \\
\hline P-32 & $0.0 \mathrm{E}+00$ & $0.0 \mathrm{E}+00$ & & Gd-152 & $0.0 \mathrm{E}+00$ & $0.0 \mathrm{E}+00$ & \\
\hline Si-32 & $0.0 \mathrm{E}+00$ & $0.0 \mathrm{E}+00$ & & Ho-166m & $2.2 \mathrm{E}-06$ & $6.3 \mathrm{E}-06$ & $182.73 \%$ \\
\hline S-35 & $0.0 \mathrm{E}+00$ & $0.0 \mathrm{E}+00$ & & Lu-176 & $0.0 \mathrm{E}+00$ & $0.0 \mathrm{E}+00$ & \\
\hline $\mathrm{Cl}-36$ & $7.3 \mathrm{E}-13$ & $2.1 \mathrm{E}-12$ & $182.73 \%$ & Ta-180 & $0.0 \mathrm{E}+00$ & $0.0 \mathrm{E}+00$ & \\
\hline $\mathrm{K}-40$ & $1.2 \mathrm{E}-05$ & $3.5 \mathrm{E}-05$ & $182.73 \%$ & Hf-182 & $0.0 \mathrm{E}+00$ & $0.0 \mathrm{E}+00$ & \\
\hline $\mathrm{K}-43$ & $3.5 \mathrm{E}-05$ & $1.0 \mathrm{E}-04$ & $64.63 \%$ & Re-186m & $0.0 \mathrm{E}+00$ & $0.0 \mathrm{E}+00$ & \\
\hline $\mathrm{Ca}-41$ & $2.7 \mathrm{E}-10$ & $7.7 \mathrm{E}-10$ & $182.73 \%$ & Re-187 & $0.0 \mathrm{E}+00$ & $0.0 \mathrm{E}+00$ & \\
\hline $\mathrm{Ca}-45$ & $1.5 \mathrm{E}-15$ & $4.1 \mathrm{E}-15$ & $182.73 \%$ & Ir-192m & $0.0 \mathrm{E}+00$ & $0.0 \mathrm{E}+00$ & \\
\hline $\mathrm{Ca}-47$ & $7.2 \mathrm{E}-05$ & $2.0 \mathrm{E}-04$ & $182.73 \%$ & Pt-193 & $3.9 \mathrm{E}-09$ & $1.1 \mathrm{E}-08$ & $182.73 \%$ \\
\hline Ti-44 & $1.1 \mathrm{E}-05$ & $3.2 \mathrm{E}-05$ & $182.73 \%$ & Hg-194 & $0.0 \mathrm{E}+00$ & $0.0 \mathrm{E}+00$ & \\
\hline $\mathrm{V}-49$ & $8.2 \mathrm{E}-10$ & $2.3 \mathrm{E}-09$ & $182.73 \%$ & $\mathrm{Hg}-203$ & $1.8 \mathrm{E}-05$ & $5.0 \mathrm{E}-05$ & $182.73 \%$ \\
\hline $\mathrm{Cr}-51$ & $2.4 \mathrm{E}-06$ & $6.7 \mathrm{E}-06$ & $182.73 \%$ & $\mathrm{~Pb}-202$ & $0.0 \mathrm{E}+00$ & $0.0 \mathrm{E}+00$ & \\
\hline $\mathrm{Mn}-53$ & $1.5 \mathrm{E}-09$ & $4.3 \mathrm{E}-09$ & $182.73 \%$ & $\mathrm{~Pb}-205$ & $5.1 \mathrm{E}-09$ & $1.4 \mathrm{E}-08$ & $182.73 \%$ \\
\hline Mn-54 & $6.6 \mathrm{E}-05$ & $1.9 \mathrm{E}-04$ & $182.73 \%$ & $\mathrm{~Pb}-210$ & $1.2 \mathrm{E}-07$ & $3.3 \mathrm{E}-07$ & $182.73 \%$ \\
\hline $\mathrm{Fe}-55$ & $2.0 \mathrm{E}-09$ & 5.7E-09 & $182.73 \%$ & Bi-207 & $1.2 \mathrm{E}-04$ & $3.4 \mathrm{E}-04$ & $182.73 \%$ \\
\hline Fe-60 & $0.0 \mathrm{E}+00$ & $0.0 \mathrm{E}+00$ & & $\mathrm{Bi}-210$ & $0.0 \mathrm{E}+00$ & $0.0 \mathrm{E}+00$ & \\
\hline Co-58 & $7.6 \mathrm{E}-05$ & $2.1 \mathrm{E}-04$ & $182.73 \%$ & $\mathrm{Bi}-210 \mathrm{~m}$ & $0.0 \mathrm{E}+00$ & $0.0 \mathrm{E}+00$ & \\
\hline Co-60 & $2.7 \mathrm{E}-04$ & $7.5 \mathrm{E}-04$ & $182.73 \%$ & Po-210 & $6.7 \mathrm{E}-10$ & $1.9 \mathrm{E}-09$ & $182.73 \%$ \\
\hline Ni-59 & $3.4 \mathrm{E}-09$ & $9.6 \mathrm{E}-09$ & $182.73 \%$ & Ra-223 & $1.0 \mathrm{E}-05$ & $2.8 \mathrm{E}-05$ & $182.73 \%$ \\
\hline Ni-63 & $0.0 \mathrm{E}+00$ & $0.0 \mathrm{E}+00$ & & Ra-224 & $6.4 \mathrm{E}-07$ & $1.8 \mathrm{E}-06$ & $182.73 \%$ \\
\hline $\mathrm{Cu}-64$ & $3.9 \mathrm{E}-06$ & $1.1 \mathrm{E}-05$ & $182.73 \%$ & Ra-225 & $5.8 \mathrm{E}-07$ & $1.6 \mathrm{E}-06$ & $182.73 \%$ \\
\hline
\end{tabular}


SRNL-STI-2010-00447, REVISION 0

Table 26. LADTAP XL Collective Boating Exposure Comparisons (person-rem) (continued)

\begin{tabular}{|c|c|c|c|c|c|c|c|}
\hline Nuclide & $\begin{array}{c}\text { Hamby Ladtap } \\
\text { Boating Pathway }\end{array}$ & $\begin{array}{c}\text { Jannik Ladtap } \\
\text { Boating Pathway }\end{array}$ & $\%$ Diff & Nuclide & $\begin{array}{c}\text { Hamby Ladtap } \\
\text { Boating Pathway }\end{array}$ & $\begin{array}{c}\text { Jannik Ladtap } \\
\text { Boating Pathway }\end{array}$ & $\%$ Diff \\
\hline Zn-65 & $4.6 \mathrm{E}-05$ & $1.3 \mathrm{E}-04$ & $182.73 \%$ & Ra-226 & 5.4E-07 & $1.5 \mathrm{E}-06$ & $182.73 \%$ \\
\hline Ge-68 & $8.0 \mathrm{E}-09$ & $2.3 \mathrm{E}-08$ & $182.73 \%$ & Ra-228 & $5.6 \mathrm{E}-15$ & $1.6 \mathrm{E}-14$ & $182.73 \%$ \\
\hline Se-75 & $3.1 \mathrm{E}-05$ & $8.6 \mathrm{E}-05$ & $182.73 \%$ & Ac-227 & $1.0 \mathrm{E}-08$ & 2.9E-08 & $182.73 \%$ \\
\hline Se-79 & $0.0 \mathrm{E}+00$ & $0.0 \mathrm{E}+00$ & & Th-227 & 7.9E-06 & $2.2 \mathrm{E}-05$ & $182.73 \%$ \\
\hline $\mathrm{Rb}-87$ & $0.0 \mathrm{E}+00$ & $0.0 \mathrm{E}+00$ & & Th-228 & $1.6 \mathrm{E}-07$ & $4.5 \mathrm{E}-07$ & $182.73 \%$ \\
\hline Sr-89 & $1.1 \mathrm{E}-08$ & $3.0 \mathrm{E}-08$ & $182.73 \%$ & Th-229 & $7.0 \mathrm{E}-06$ & $2.0 \mathrm{E}-05$ & $182.73 \%$ \\
\hline Sr-90 & $0.0 \mathrm{E}+00$ & $0.0 \mathrm{E}+00$ & & Th-230 & $3.3 \mathrm{E}-08$ & 9.2E-08 & $182.73 \%$ \\
\hline $\mathrm{Y}-90$ & $0.0 \mathrm{E}+00$ & $0.0 \mathrm{E}+00$ & & Th-231 & $5.0 \mathrm{E}-07$ & $1.4 \mathrm{E}-06$ & $182.73 \%$ \\
\hline Y-91 & $2.8 \mathrm{E}-07$ & $8.0 \mathrm{E}-07$ & $182.73 \%$ & Th-232 & $1.6 \mathrm{E}-08$ & $4.5 \mathrm{E}-08$ & $182.73 \%$ \\
\hline Mo-93 & $6.9 \mathrm{E}-08$ & $1.9 \mathrm{E}-07$ & $182.73 \%$ & Th-234 & $6.2 \mathrm{E}-07$ & $1.8 \mathrm{E}-06$ & $64.63 \%$ \\
\hline Mo-99 & $1.7 \mathrm{E}-05$ & $4.7 \mathrm{E}-05$ & $182.73 \%$ & $\mathrm{~Pa}-230$ & $5.0 \mathrm{E}-05$ & $1.4 \mathrm{E}-04$ & $182.73 \%$ \\
\hline $\mathrm{Nb}-93 \mathrm{~m}$ & $1.2 \mathrm{E}-08$ & $3.5 \mathrm{E}-08$ & $182.73 \%$ & $\mathrm{~Pa}-231$ & $2.3 \mathrm{E}-06$ & $6.6 \mathrm{E}-06$ & $182.73 \%$ \\
\hline $\mathrm{Nb}-94$ & $1.2 \mathrm{E}-04$ & $3.5 \mathrm{E}-04$ & $182.73 \%$ & $\mathrm{~Pa}-233$ & $1.6 \mathrm{E}-05$ & $4.6 \mathrm{E}-05$ & $182.73 \%$ \\
\hline $\mathrm{Nb}-95$ & $5.9 \mathrm{E}-05$ & $1.7 \mathrm{E}-04$ & $182.73 \%$ & U-232 & $2.2 \mathrm{E}-08$ & $6.4 \mathrm{E}-08$ & $182.73 \%$ \\
\hline Zr-93 & $0.0 \mathrm{E}+00$ & $0.0 \mathrm{E}+00$ & & U-233 & $1.9 \mathrm{E}-08$ & $5.5 \mathrm{E}-08$ & $182.73 \%$ \\
\hline Zr-95 & $5.7 \mathrm{E}-05$ & $1.6 \mathrm{E}-04$ & $182.73 \%$ & U-234 & $1.3 \mathrm{E}-08$ & $3.7 \mathrm{E}-08$ & $182.73 \%$ \\
\hline Tc-96 & $1.7 \mathrm{E}-04$ & $4.8 \mathrm{E}-04$ & $182.73 \%$ & U-235 & $1.2 \mathrm{E}-05$ & $3.4 \mathrm{E}-05$ & $182.73 \%$ \\
\hline Tc-97 & $8.6 \mathrm{E}-08$ & $2.4 \mathrm{E}-07$ & $182.73 \%$ & U-236 & $1.1 \mathrm{E}-08$ & $3.0 \mathrm{E}-08$ & $182.73 \%$ \\
\hline Tc-98 & $1.1 \mathrm{E}-04$ & $3.1 \mathrm{E}-04$ & $182.73 \%$ & U-237 & $9.7 \mathrm{E}-06$ & $2.7 \mathrm{E}-05$ & $182.73 \%$ \\
\hline Tc-99 & $4.3 \mathrm{E}-11$ & $1.2 \mathrm{E}-10$ & $182.73 \%$ & U-238 & $9.1 \mathrm{E}-09$ & $2.6 \mathrm{E}-08$ & $182.73 \%$ \\
\hline Ru-97 & $1.4 \mathrm{E}-05$ & $4.0 \mathrm{E}-05$ & $182.73 \%$ & $\mathrm{~Np}-236$ & $1.1 \mathrm{E}-05$ & $3.0 \mathrm{E}-05$ & $182.73 \%$ \\
\hline $\mathrm{Ru}-103$ & $3.6 \mathrm{E}-05$ & $1.0 \mathrm{E}-04$ & $182.73 \%$ & $\mathrm{~Np}-237$ & $1.9 \mathrm{E}-06$ & $5.3 \mathrm{E}-06$ & $182.73 \%$ \\
\hline $\mathrm{Ru}-106$ & $1.6 \mathrm{E}-05$ & $4.5 \mathrm{E}-05$ & $182.73 \%$ & $\mathrm{~Np}-239$ & 9.9E-06 & $2.8 \mathrm{E}-05$ & $182.73 \%$ \\
\hline Pd-107 & $0.0 \mathrm{E}+00$ & $0.0 \mathrm{E}+00$ & & Am-237 & $0.0 \mathrm{E}+00$ & $0.0 \mathrm{E}+00$ & \\
\hline Ag-108m & $1.2 \mathrm{E}-04$ & $3.5 \mathrm{E}-04$ & $182.73 \%$ & Am-241 & $1.6 \mathrm{E}-06$ & $4.6 \mathrm{E}-06$ & $182.73 \%$ \\
\hline
\end{tabular}


SRNL-STI-2010-00447, REVISION 0

Table 26. LADTAP XL Collective Boating Exposure Comparisons (person-rem) (continued)

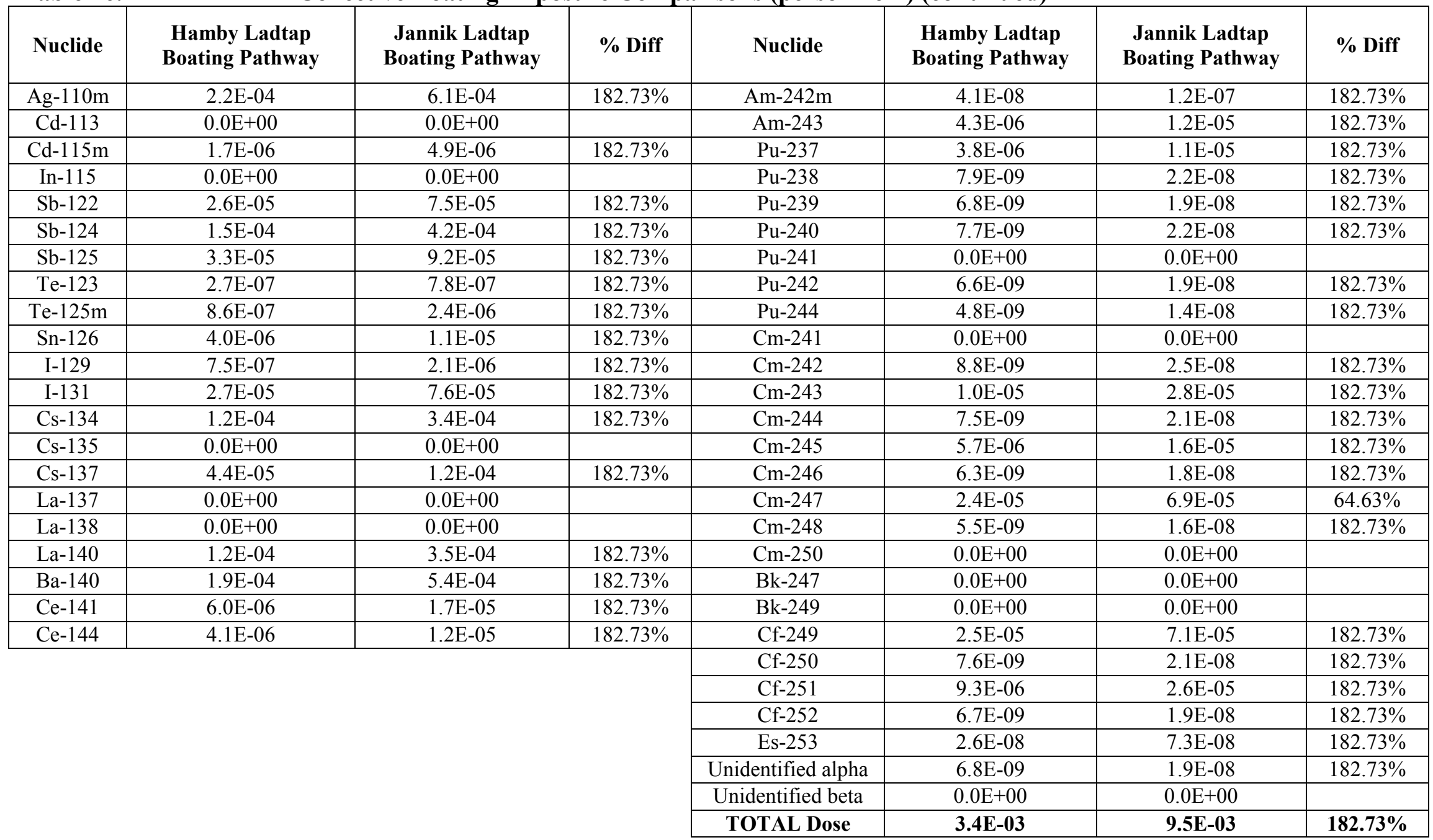


SRNL-STI-2010-00447, REVISION 0

Table 27. LADTAP XL Collective Total of All Pathways Comparisons (person-rem)

\begin{tabular}{|c|c|c|c|c|c|c|c|}
\hline Nuclide & $\begin{array}{c}\text { Hamby Ladtap } \\
\text { All Pathways }\end{array}$ & $\begin{array}{c}\text { Jannik Ladtap } \\
\text { All Pathways }\end{array}$ & $\%$ Diff & Nuclide & $\begin{array}{c}\text { Hamby Ladtap } \\
\text { All Pathways }\end{array}$ & $\begin{array}{c}\text { Jannik Ladtap } \\
\text { All Pathways }\end{array}$ & $\%$ Diff \\
\hline H-3 & $4.2 \mathrm{E}-04$ & $3.8 \mathrm{E}-04$ & $-8.77 \%$ & Sm-146 & $3.2 \mathrm{E}+00$ & $4.1 \mathrm{E}+00$ & $28.60 \%$ \\
\hline Be-7 & $9.4 \mathrm{E}-04$ & $1.4 \mathrm{E}-02$ & $1383.70 \%$ & Sm-147 & $2.9 \mathrm{E}+00$ & $3.7 \mathrm{E}+00$ & $28.60 \%$ \\
\hline $\mathrm{Be}-10$ & $3.6 \mathrm{E}-02$ & $6.2 \mathrm{E}-01$ & $1644.71 \%$ & Sm-151 & $5.4 \mathrm{E}-03$ & $7.0 \mathrm{E}-03$ & $28.59 \%$ \\
\hline C-14 & $8.2 \mathrm{E}-02$ & $6.1 \mathrm{E}-01$ & $644.86 \%$ & Pm-147 & $1.5 \mathrm{E}-02$ & $1.9 \mathrm{E}-02$ & $28.49 \%$ \\
\hline $\mathrm{Na}-22$ & $1.3 \mathrm{E}-01$ & $1.2 \mathrm{E}-01$ & $-9.01 \%$ & Eu-152 & $2.1 \mathrm{E}-01$ & $2.3 \mathrm{E}-01$ & $7.01 \%$ \\
\hline $\mathrm{Na}-24$ & $2.8 \mathrm{E}-04$ & $5.2 \mathrm{E}-04$ & $83.26 \%$ & Eu-154 & $2.4 \mathrm{E}-01$ & $2.7 \mathrm{E}-01$ & $14.86 \%$ \\
\hline Al-26 & $7.2 \mathrm{E}-01$ & $6.3 \mathrm{E}-01$ & $-12.34 \%$ & Eu-155 & $2.4 \mathrm{E}-02$ & $3.0 \mathrm{E}-02$ & $26.84 \%$ \\
\hline $\mathrm{P}-32$ & $3.1 \mathrm{E}+00$ & $6.5 \mathrm{E}+00$ & $107.11 \%$ & Gd-152 & $2.4 \mathrm{E}+00$ & $5.2 \mathrm{E}+00$ & $117.62 \%$ \\
\hline $\mathrm{Si}-32$ & $1.2 \mathrm{E}-02$ & $1.2 \mathrm{E}+00$ & $10122.75 \%$ & Ho-166m & $5.3 \mathrm{E}-01$ & $5.1 \mathrm{E}-01$ & $-4.28 \%$ \\
\hline S-35 & $6.1 \mathrm{E}-03$ & 7.3E-03 & $19.13 \%$ & Lu-176 & $1.1 \mathrm{E}-01$ & $1.3 \mathrm{E}-01$ & $28.37 \%$ \\
\hline $\mathrm{Cl}-36$ & $2.1 \mathrm{E}-02$ & $1.9 \mathrm{E}-02$ & $-6.53 \%$ & Ta-180 & $4.9 \mathrm{E}-215$ & $4.4 \mathrm{E}-215$ & $-8.92 \%$ \\
\hline $\mathrm{K}-40$ & $2.4 \mathrm{E}-01$ & 5.9E-01 & $143.69 \%$ & Hf-182 & $9.6 \mathrm{E}-02$ & $4.0 \mathrm{E}-01$ & $311.41 \%$ \\
\hline K-43 & $3.2 \mathrm{E}-04$ & $3.7 \mathrm{E}-04$ & $15.12 \%$ & Re-186m & $2.6 \mathrm{E}-02$ & $2.6 \mathrm{E}-02$ & $1.01 \%$ \\
\hline $\mathrm{Ca}-41$ & $8.4 \mathrm{E}-03$ & $7.5 \mathrm{E}-03$ & $-10.35 \%$ & Re-187 & $6.4 \mathrm{E}-05$ & $6.5 \mathrm{E}-05$ & $1.01 \%$ \\
\hline $\mathrm{Ca}-45$ & $2.1 \mathrm{E}-02$ & $1.8 \mathrm{E}-02$ & $-10.37 \%$ & Ir-192m & $0.0 \mathrm{E}+00$ & $0.0 \mathrm{E}+00$ & $0.00 \%$ \\
\hline $\mathrm{Ca}-47$ & $2.3 \mathrm{E}-02$ & $2.1 \mathrm{E}-02$ & $-8.92 \%$ & Pt-193 & $2.9 \mathrm{E}-03$ & $3.9 \mathrm{E}-03$ & $34.80 \%$ \\
\hline $\mathrm{Ti}-44$ & $4.1 \mathrm{E}-01$ & $4.4 \mathrm{E}-01$ & $6.30 \%$ & Hg-194 & $8.8 \mathrm{E}+01$ & $9.4 \mathrm{E}+01$ & $5.99 \%$ \\
\hline $\mathrm{V}-49$ & $3.8 \mathrm{E}-04$ & $4.0 \mathrm{E}-04$ & $4.23 \%$ & $\mathrm{Hg}-203$ & $2.6 \mathrm{E}+00$ & $2.8 \mathrm{E}+00$ & $6.89 \%$ \\
\hline $\mathrm{Cr}-51$ & $2.6 \mathrm{E}-03$ & $1.4 \mathrm{E}-03$ & $-45.03 \%$ & $\mathrm{~Pb}-202$ & $6.7 \mathrm{E}-01$ & $8.0 \mathrm{E}-01$ & $19.65 \%$ \\
\hline $\mathrm{Mn}-53$ & $9.8 \mathrm{E}-03$ & $1.9 \mathrm{E}-03$ & $-80.27 \%$ & $\mathrm{~Pb}-205$ & $2.6 \mathrm{E}-02$ & $3.1 \mathrm{E}-02$ & $19.40 \%$ \\
\hline Mn-54 & $3.8 \mathrm{E}-02$ & $5.6 \mathrm{E}-02$ & $46.64 \%$ & $\mathrm{~Pb}-210$ & $8.7 \mathrm{E}+01$ & $1.0 \mathrm{E}+02$ & $19.64 \%$ \\
\hline Fe-55 & $1.1 \mathrm{E}-01$ & $4.5 \mathrm{E}-02$ & $-58.80 \%$ & Bi-207 & $2.9 \mathrm{E}-01$ & $3.2 \mathrm{E}-01$ & $10.38 \%$ \\
\hline Fe-60 & $2.8 \mathrm{E}+01$ & $1.2 \mathrm{E}+01$ & $-58.82 \%$ & Bi-210 & $2.3 \mathrm{E}-02$ & $3.5 \mathrm{E}-02$ & $51.52 \%$ \\
\hline Co-58 & $5.3 \mathrm{E}-02$ & $1.1 \mathrm{E}-01$ & $109.52 \%$ & $\mathrm{Bi}-210 \mathrm{~m}$ & 5.9E-01 & $1.8 \mathrm{E}+00$ & $198.06 \%$ \\
\hline Co-60 & $5.2 \mathrm{E}-01$ & $1.0 \mathrm{E}+00$ & $91.10 \%$ & Po-210 & $2.9 \mathrm{E}+02$ & $1.1 \mathrm{E}+03$ & $272.77 \%$ \\
\hline Ni-59 & $2.0 \mathrm{E}-03$ & $2.8 \mathrm{E}-03$ & $39.03 \%$ & Ra-223 & $3.2 \mathrm{E}+00$ & $3.0 \mathrm{E}+00$ & $-5.87 \%$ \\
\hline Ni-63 & $5.1 \mathrm{E}-03$ & $7.2 \mathrm{E}-03$ & $41.96 \%$ & Ra-224 & $1.1 \mathrm{E}+00$ & $9.7 \mathrm{E}-01$ & $-8.18 \%$ \\
\hline $\mathrm{Cu}-64$ & $2.1 \mathrm{E}-05$ & $2.8 \mathrm{E}-05$ & $31.70 \%$ & Ra-225 & $1.9 \mathrm{E}+00$ & $1.8 \mathrm{E}+00$ & $-5.43 \%$ \\
\hline
\end{tabular}


SRNL-STI-2010-00447, REVISION 0

Table 27. LADTAP XL Collective Total of All Pathways Comparisons (person-rem) (continued)

\begin{tabular}{|c|c|c|c|c|c|c|c|}
\hline Nuclide & $\begin{array}{c}\text { Hamby Ladtap } \\
\text { All Pathways }\end{array}$ & $\begin{array}{c}\text { Jannik Ladtap } \\
\text { All Pathways }\end{array}$ & $\%$ Diff & Nuclide & $\begin{array}{c}\text { Hamby Ladtap } \\
\text { All Pathways }\end{array}$ & $\begin{array}{c}\text { Jannik Ladtap } \\
\text { All Pathways }\end{array}$ & $\%$ Diff \\
\hline $\mathrm{Zn}-65$ & $6.4 \mathrm{E}+00$ & $1.0 \mathrm{E}+01$ & $56.60 \%$ & Ra-226 & $8.6 \mathrm{E}+00$ & $8.3 \mathrm{E}+00$ & $-3.51 \%$ \\
\hline Ge-68 & $1.8 \mathrm{E}-01$ & $9.8 \mathrm{E}-02$ & $-43.97 \%$ & Ra-228 & $9.4 \mathrm{E}+00$ & $9.0 \mathrm{E}+00$ & $-3.53 \%$ \\
\hline Se-75 & $1.4 \mathrm{E}-01$ & 9.9E-01 & $610.98 \%$ & Ac-227 & $2.2 \mathrm{E}+02$ & $2.9 \mathrm{E}+02$ & $28.36 \%$ \\
\hline Se-79 & $1.4 \mathrm{E}-01$ & $1.0 \mathrm{E}+00$ & $630.26 \%$ & Th-227 & $6.2 \mathrm{E}-01$ & $5.1 \mathrm{E}-01$ & $-18.13 \%$ \\
\hline Rb-87 & $7.3 \mathrm{E}-02$ & $2.1 \mathrm{E}-01$ & $192.57 \%$ & Th-228 & $9.4 \mathrm{E}+00$ & $7.6 \mathrm{E}+00$ & $-19.04 \%$ \\
\hline Sr-89 & $5.7 \mathrm{E}-02$ & $5.0 \mathrm{E}-02$ & $-12.11 \%$ & Th-229 & $8.8 \mathrm{E}+01$ & $7.1 \mathrm{E}+01$ & $-19.06 \%$ \\
\hline Sr-90 & $9.1 \mathrm{E}-01$ & $8.0 \mathrm{E}-01$ & $-12.44 \%$ & Th-230 & $1.3 \mathrm{E}+01$ & $1.1 \mathrm{E}+01$ & $-19.06 \%$ \\
\hline $\mathrm{Y}-90$ & $2.7 \mathrm{E}-02$ & $2.7 \mathrm{E}-02$ & $-1.25 \%$ & Th-231 & $6.5 \mathrm{E}-04$ & $5.9 \mathrm{E}-04$ & $-8.88 \%$ \\
\hline Y-91 & $1.3 \mathrm{E}-01$ & $1.6 \mathrm{E}-01$ & $27.33 \%$ & Th-232 & $7.0 \mathrm{E}+01$ & $5.7 \mathrm{E}+01$ & $-19.06 \%$ \\
\hline Mo-93 & $1.0 \mathrm{E}-02$ & $9.5 \mathrm{E}-03$ & $-7.45 \%$ & Th-234 & $2.4 \mathrm{E}-01$ & $2.0 \mathrm{E}-01$ & $-18.35 \%$ \\
\hline Mo-99 & $1.1 \mathrm{E}-02$ & 9.9E-03 & $-8.38 \%$ & $\mathrm{~Pa}-230$ & $3.3 \mathrm{E}-02$ & $3.0 \mathrm{E}-02$ & $-7.77 \%$ \\
\hline $\mathrm{Nb}-93 \mathrm{~m}$ & $7.1 \mathrm{E}-01$ & $4.8 \mathrm{E}-02$ & $-93.29 \%$ & $\mathrm{~Pa}-231$ & $7.5 \mathrm{E}+01$ & $6.9 \mathrm{E}+01$ & $-7.63 \%$ \\
\hline $\mathrm{Nb}-94$ & $1.1 \mathrm{E}+00$ & $3.8 \mathrm{E}-01$ & $-64.41 \%$ & $\mathrm{~Pa}-233$ & $2.0 \mathrm{E}-02$ & $1.9 \mathrm{E}-02$ & $-7.79 \%$ \\
\hline $\mathrm{Nb}-95$ & $2.4 \mathrm{E}-01$ & $1.8 \mathrm{E}-02$ & $-92.57 \%$ & U-232 & $8.9 \mathrm{E}+00$ & $8.1 \mathrm{E}+00$ & $-8.52 \%$ \\
\hline Zr-93 & $1.2 \mathrm{E}-02$ & $1.1 \mathrm{E}-02$ & $-6.35 \%$ & U-233 & $1.8 \mathrm{E}+00$ & $1.7 \mathrm{E}+00$ & $-8.07 \%$ \\
\hline Zr-95 & $2.6 \mathrm{E}-02$ & $2.5 \mathrm{E}-02$ & $-6.80 \%$ & U-234 & $1.8 \mathrm{E}+00$ & $1.6 \mathrm{E}+00$ & $-8.07 \%$ \\
\hline Tc-96 & $1.0 \mathrm{E}-02$ & $9.5 \mathrm{E}-03$ & $-6.08 \%$ & U-235 & $1.7 \mathrm{E}+00$ & $1.6 \mathrm{E}+00$ & $-8.22 \%$ \\
\hline Tc-97 & $2.7 \mathrm{E}-03$ & $2.3 \mathrm{E}-03$ & $-13.20 \%$ & U-236 & $1.7 \mathrm{E}+00$ & $1.6 \mathrm{E}+00$ & $-8.07 \%$ \\
\hline Tc-98 & $3.9 \mathrm{E}-01$ & $3.3 \mathrm{E}-01$ & $-14.06 \%$ & U-237 & $1.2 \mathrm{E}-02$ & $1.1 \mathrm{E}-02$ & $-8.43 \%$ \\
\hline Tc-99 & $9.4 \mathrm{E}-03$ & $8.3 \mathrm{E}-03$ & $-11.50 \%$ & U-238 & $1.6 \mathrm{E}+00$ & $1.4 \mathrm{E}+00$ & $-8.07 \%$ \\
\hline Ru-97 & $2.2 \mathrm{E}-03$ & $1.6 \mathrm{E}-03$ & $-26.87 \%$ & $\mathrm{~Np}-236$ & $5.4 \mathrm{E}+00$ & $5.1 \mathrm{E}+00$ & $-6.43 \%$ \\
\hline Ru-103 & $3.7 \mathrm{E}-02$ & $2.0 \mathrm{E}-02$ & $-46.70 \%$ & Np-237 & $2.7 \mathrm{E}+01$ & $2.5 \mathrm{E}+01$ & $-6.38 \%$ \\
\hline Ru-106 & $3.3 \mathrm{E}-01$ & $1.7 \mathrm{E}-01$ & $-49.66 \%$ & $\mathrm{~Np}-239$ & $6.0 \mathrm{E}-03$ & $5.5 \mathrm{E}-03$ & $-8.41 \%$ \\
\hline Pd-107 & $3.5 \mathrm{E}-03$ & $1.5 \mathrm{E}-03$ & $-58.36 \%$ & Am-237 & $8.8 \mathrm{E}-28$ & $8.1 \mathrm{E}-28$ & $-8.92 \%$ \\
\hline Ag-108m & $6.5 \mathrm{E}-01$ & $4.1 \mathrm{E}-01$ & $-37.38 \%$ & Am-241 & $7.2 \mathrm{E}+01$ & $5.8 \mathrm{E}+01$ & $-18.75 \%$ \\
\hline
\end{tabular}


SRNL-STI-2010-00447, REVISION 0

Table 27. LADTAP XL Collective Total of All Pathways Comparisons (person-rem) (continued)

\begin{tabular}{|c|c|c|c|c|c|c|c|}
\hline Nuclide & $\begin{array}{c}\text { Hamby Ladtap } \\
\text { All Pathways }\end{array}$ & $\begin{array}{c}\text { Jannik Ladtap } \\
\text { All Pathways }\end{array}$ & $\%$ Diff & Nuclide & $\begin{array}{c}\text { Hamby Ladtap } \\
\text { All Pathways }\end{array}$ & $\begin{array}{c}\text { Jannik Ladtap } \\
\text { All Pathways }\end{array}$ & $\%$ Diff \\
\hline Ag-110m & $4.1 \mathrm{E}-01$ & $1.4 \mathrm{E}-01$ & $-65.29 \%$ & Am-242m & $6.7 \mathrm{E}+01$ & $5.4 \mathrm{E}+01$ & $-18.75 \%$ \\
\hline $\mathrm{Cd}-113$ & $3.7 \mathrm{E}+02$ & $1.3 \mathrm{E}+01$ & $-96.58 \%$ & Am-243 & $7.2 \mathrm{E}+01$ & $5.8 \mathrm{E}+01$ & $-18.75 \%$ \\
\hline $\mathrm{Cd}-115 \mathrm{~m}$ & $2.8 \mathrm{E}+01$ & $9.7 \mathrm{E}-01$ & $-96.54 \%$ & $\mathrm{Pu}-237$ & $7.1 \mathrm{E}-03$ & $9.5 \mathrm{E}-03$ & $32.93 \%$ \\
\hline In-115 & 9.4E-01 & $3.1 \mathrm{E}+01$ & $3206.29 \%$ & $\mathrm{Pu}-238$ & $3.2 \mathrm{E}+01$ & $4.0 \mathrm{E}+01$ & $23.85 \%$ \\
\hline Sb-122 & $1.5 \mathrm{E}-02$ & $1.4 \mathrm{E}-02$ & $-6.05 \%$ & $\mathrm{Pu}-239$ & $3.7 \mathrm{E}+01$ & $4.5 \mathrm{E}+01$ & $23.86 \%$ \\
\hline Sb-124 & $6.3 \mathrm{E}-02$ & 7.0E-02 & $12.44 \%$ & $\mathrm{Pu}-240$ & $3.7 \mathrm{E}+01$ & $4.5 \mathrm{E}+01$ & $23.86 \%$ \\
\hline Sb-125 & $2.8 \mathrm{E}-02$ & $3.0 \mathrm{E}-02$ & $4.17 \%$ & $\mathrm{Pu}-241$ & 7.3E-01 & $9.1 \mathrm{E}-01$ & $23.83 \%$ \\
\hline Te-123 & $3.8 \mathrm{E}+00$ & 8.9E-02 & $-97.64 \%$ & $\mathrm{Pu}-242$ & $3.5 \mathrm{E}+01$ & $4.3 \mathrm{E}+01$ & $23.86 \%$ \\
\hline Te- $125 \mathrm{~m}$ & $2.7 \mathrm{E}+00$ & $6.4 \mathrm{E}-02$ & $-97.61 \%$ & $\mathrm{Pu}-244$ & $3.0 \mathrm{E}+01$ & $4.2 \mathrm{E}+01$ & $38.74 \%$ \\
\hline Sn-126 & $5.0 \mathrm{E}-01$ & $1.3 \mathrm{E}+01$ & $2410.47 \%$ & Cm-241 & $6.1 \mathrm{E}-02$ & $4.9 \mathrm{E}-02$ & $-18.64 \%$ \\
\hline I-129 & $2.0 \mathrm{E}+00$ & $2.0 \mathrm{E}+00$ & $-2.65 \%$ & $\mathrm{Cm}-242$ & $1.7 \mathrm{E}+00$ & $1.4 \mathrm{E}+00$ & $-19.31 \%$ \\
\hline $\mathrm{I}-131$ & $2.6 \mathrm{E}-01$ & $2.4 \mathrm{E}-01$ & $-5.93 \%$ & Cm-243 & $4.6 \mathrm{E}+01$ & $3.7 \mathrm{E}+01$ & $-19.47 \%$ \\
\hline Cs-134 & $1.5 \mathrm{E}+00$ & $2.1 \mathrm{E}+00$ & $43.67 \%$ & Cm-244 & $3.7 \mathrm{E}+01$ & $2.9 \mathrm{E}+01$ & $-19.47 \%$ \\
\hline Cs-135 & $1.4 \mathrm{E}-01$ & $2.0 \mathrm{E}-01$ & $45.19 \%$ & Cm-245 & $7.2 \mathrm{E}+01$ & $5.8 \mathrm{E}+01$ & $-19.47 \%$ \\
\hline Cs-137 & $1.1 \mathrm{E}+00$ & $1.5 \mathrm{E}+00$ & $39.95 \%$ & Cm-246 & $7.2 \mathrm{E}+01$ & $5.8 \mathrm{E}+01$ & $-19.47 \%$ \\
\hline La-137 & $6.9 \mathrm{E}-03$ & $3.3 \mathrm{E}-03$ & $-51.20 \%$ & $\mathrm{Cm}-247$ & $6.5 \mathrm{E}+01$ & $5.3 \mathrm{E}+01$ & $-19.47 \%$ \\
\hline La-138 & $9.4 \mathrm{E}-02$ & $4.6 \mathrm{E}-02$ & $-51.20 \%$ & Cm-248 & $2.5 \mathrm{E}+02$ & $2.1 \mathrm{E}+02$ & $-19.47 \%$ \\
\hline La-140 & $1.0 \mathrm{E}-02$ & $9.5 \mathrm{E}-03$ & $-8.72 \%$ & $\mathrm{Cm}-250$ & $1.2 \mathrm{E}+03$ & $1.0 \mathrm{E}+03$ & $-19.47 \%$ \\
\hline $\mathrm{Ba}-140$ & $5.0 \mathrm{E}-02$ & $4.2 \mathrm{E}-02$ & $-15.03 \%$ & Bk-247 & $3.7 \mathrm{E}+01$ & $4.7 \mathrm{E}+01$ & $28.37 \%$ \\
\hline $\mathrm{Ce}-141$ & $2.7 \mathrm{E}-02$ & $2.9 \mathrm{E}-02$ & $7.81 \%$ & Bk-249 & $9.4 \mathrm{E}-02$ & $1.2 \mathrm{E}-01$ & $28.06 \%$ \\
\hline \multirow[t]{8}{*}{ Ce-144 } & $2.4 \mathrm{E}-01$ & $2.6 \mathrm{E}-01$ & $9.50 \%$ & Cf-249 & $7.3 \mathrm{E}+01$ & $6.2 \mathrm{E}+01$ & $-16.14 \%$ \\
\hline & & & & Cf-250 & $3.0 \mathrm{E}+01$ & $2.5 \mathrm{E}+01$ & $-16.14 \%$ \\
\hline & & & & Cf-251 & $7.3 \mathrm{E}+01$ & $6.1 \mathrm{E}+01$ & $-16.14 \%$ \\
\hline & & & & Cf-252 & $1.5 \mathrm{E}+01$ & $1.2 \mathrm{E}+01$ & $-16.12 \%$ \\
\hline & & & & Es-253 & $1.4 \mathrm{E}-01$ & $1.7 \mathrm{E}+00$ & $1070.73 \%$ \\
\hline & & & & Unidentified alpha & $3.7 \mathrm{E}+01$ & $4.5 \mathrm{E}+01$ & $23.86 \%$ \\
\hline & & & & Unidentified beta & $9.1 \mathrm{E}-01$ & $8.0 \mathrm{E}-01$ & $-12.44 \%$ \\
\hline & & & & TOTAL Dose & $3.9 \mathrm{E}+03$ & $4.0 \mathrm{E}+03$ & $3.27 \%$ \\
\hline
\end{tabular}


SRNL-STI-2010-00447, REVISION 0

Table 28. LADTAP XL IRRIDOSE Maximally Exposed Individual Vegetable Ingestion Pathway Comparison (mrem/yr)

\begin{tabular}{|c|c|c|c|c|c|c|c|}
\hline Nuclide & $\begin{array}{c}\text { Hamby Ladtap } \\
\text { Veg }\end{array}$ & $\begin{array}{c}\text { Jannik Ladtap } \\
\text { Veg }\end{array}$ & $\%$ Diff & Nuclide & $\begin{array}{c}\text { Hamby Ladtap } \\
\text { Veg }\end{array}$ & $\begin{array}{c}\text { Jannik Ladtap } \\
\text { Veg }\end{array}$ & $\%$ Diff \\
\hline $\mathrm{H}-3$ & $2.25 \mathrm{E}-06$ & $2.25 \mathrm{E}-06$ & $0.00 \%$ & Sm-146 & $1.72 \mathrm{E}-01$ & $6.10 \mathrm{E}-02$ & $-64.45 \%$ \\
\hline $\mathrm{Be}-7$ & $7.25 \mathrm{E}-05$ & $2.44 \mathrm{E}-05$ & $-66.31 \%$ & Sm-147 & $1.54 \mathrm{E}-01$ & 5.49E-02 & $-64.45 \%$ \\
\hline $\mathrm{Be}-10$ & $3.63 \mathrm{E}-03$ & $1.22 \mathrm{E}-03$ & $-66.50 \%$ & Sm-151 & $2.91 \mathrm{E}-04$ & $1.03 \mathrm{E}-04$ & $-64.62 \%$ \\
\hline C-14 & 4.44E-02 & $2.00 \mathrm{E}-03$ & $-95.50 \%$ & Pm-147 & $7.96 \mathrm{E}-04$ & $2.84 \mathrm{E}-04$ & $-64.34 \%$ \\
\hline $\mathrm{Na}-22$ & $1.04 \mathrm{E}-02$ & $3.43 \mathrm{E}-03$ & $-67.15 \%$ & Eu-152 & $5.11 \mathrm{E}-03$ & $1.78 \mathrm{E}-03$ & $-65.27 \%$ \\
\hline $\mathrm{Na}-24$ & $1.88 \mathrm{E}-06$ & $6.32 \mathrm{E}-07$ & $-66.32 \%$ & Eu-154 & 7.73E-03 & $2.67 \mathrm{E}-03$ & $-65.51 \%$ \\
\hline Al-26 & $1.12 \mathrm{E}-02$ & $3.73 \mathrm{E}-03$ & $-66.81 \%$ & Eu-155 & $1.10 \mathrm{E}-03$ & $3.76 \mathrm{E}-04$ & $-65.81 \%$ \\
\hline P-32 & $3.02 \mathrm{E}-03$ & $1.01 \mathrm{E}-03$ & $-66.69 \%$ & Gd-152 & $1.28 \mathrm{E}-01$ & $4.58 \mathrm{E}-02$ & $-64.37 \%$ \\
\hline $\mathrm{Si}-32$ & $1.98 \mathrm{E}-03$ & $7.01 \mathrm{E}-04$ & $-64.69 \%$ & Нo-166m & $6.69 \mathrm{E}-03$ & $2.38 \mathrm{E}-03$ & $-64.46 \%$ \\
\hline S-35 & 4.94E-04 & $1.71 \mathrm{E}-04$ & $-65.40 \%$ & Lu-176 & $5.66 \mathrm{E}-03$ & $1.91 \mathrm{E}-03$ & $-66.23 \%$ \\
\hline Cl-36 & $2.24 \mathrm{E}-01$ & $5.20 \mathrm{E}-02$ & $-76.80 \%$ & Ta-180 & $3.77 \mathrm{E}-166$ & $1.27 \mathrm{E}-166$ & $-66.31 \%$ \\
\hline K-40 & $3.72 \mathrm{E}-02$ & $2.90 \mathrm{E}-02$ & $-22.06 \%$ & Hf-182 & $1.19 \mathrm{E}-02$ & $4.02 \mathrm{E}-03$ & $-66.21 \%$ \\
\hline K-43 & $4.55 \mathrm{E}-06$ & $1.54 \mathrm{E}-06$ & $-66.10 \%$ & Re-186m & $2.56 \mathrm{E}+00$ & $2.90 \mathrm{E}-03$ & $-99.89 \%$ \\
\hline $\mathrm{Ca}-41$ & $3.24 \mathrm{E}-03$ & $2.32 \mathrm{E}-02$ & $617.88 \%$ & Re-187 & $6.45 \mathrm{E}-03$ & $7.28 \mathrm{E}-06$ & $-99.89 \%$ \\
\hline $\mathrm{Ca}-45$ & $2.52 \mathrm{E}-03$ & $2.26 \mathrm{E}-03$ & $-10.11 \%$ & Ir-192m & $0.00 \mathrm{E}+00$ & $0.00 \mathrm{E}+00$ & \#DIV/0! \\
\hline $\mathrm{Ca}-47$ & $8.46 \mathrm{E}-04$ & $3.36 \mathrm{E}-04$ & $-60.24 \%$ & Pt-193 & $1.02 \mathrm{E}-04$ & $3.36 \mathrm{E}-05$ & $-66.95 \%$ \\
\hline Ti-44 & $1.61 \mathrm{E}-02$ & $5.47 \mathrm{E}-03$ & $-66.04 \%$ & Hg-194 & $6.26 \mathrm{E}-01$ & $2.02 \mathrm{E}-01$ & $-67.77 \%$ \\
\hline $\mathrm{V}-49$ & 4.39E-05 & $1.48 \mathrm{E}-05$ & $-66.31 \%$ & $\mathrm{Hg}-203$ & $6.49 \mathrm{E}-03$ & $2.18 \mathrm{E}-03$ & $-66.47 \%$ \\
\hline Cr-51 & $6.98 \mathrm{E}-05$ & $2.35 \mathrm{E}-05$ & $-66.31 \%$ & $\mathrm{~Pb}-202$ & $3.45 \mathrm{E}-02$ & $1.21 \mathrm{E}-02$ & $-64.86 \%$ \\
\hline Mn-53 & $9.46 \mathrm{E}-05$ & $5.92 \mathrm{E}-05$ & $-37.42 \%$ & $\mathrm{~Pb}-205$ & $1.33 \mathrm{E}-03$ & $4.67 \mathrm{E}-04$ & $-64.86 \%$ \\
\hline $\mathrm{Mn}-54$ & $2.21 \mathrm{E}-03$ & 7.79E-04 & $-64.71 \%$ & $\mathrm{~Pb}-210$ & $4.46 \mathrm{E}+00$ & $1.55 \mathrm{E}+00$ & $-65.36 \%$ \\
\hline $\mathrm{Fe}-55$ & $4.85 \mathrm{E}-04$ & $1.68 \mathrm{E}-04$ & $-65.34 \%$ & $\mathrm{Bi}-207$ & $5.64 \mathrm{E}-03$ & $3.22 \mathrm{E}-03$ & $-42.83 \%$ \\
\hline $\mathrm{Fe}-60$ & $1.28 \mathrm{E}-01$ & $5.10 \mathrm{E}-02$ & $-60.10 \%$ & Bi-210 & $9.02 \mathrm{E}-04$ & $3.05 \mathrm{E}-04$ & $-66.20 \%$ \\
\hline Co-58 & $2.45 \mathrm{E}-03$ & $8.29 \mathrm{E}-04$ & $-66.14 \%$ & $\mathrm{Bi}-210 \mathrm{~m}$ & $1.05 \mathrm{E}-01$ & $6.56 \mathrm{E}-02$ & $-37.40 \%$ \\
\hline Co-60 & $2.22 \mathrm{E}-02$ & $8.30 \mathrm{E}-03$ & $-62.63 \%$ & Po-210 & $1.23 \mathrm{E}+00$ & $4.13 \mathrm{E}-01$ & $-66.31 \%$ \\
\hline $\mathrm{Ni}-59$ & $1.84 \mathrm{E}-04$ & $7.85 \mathrm{E}-05$ & $-57.29 \%$ & $\mathrm{Ra}-223$ & $1.81 \mathrm{E}-01$ & $6.09 \mathrm{E}-02$ & $-66.31 \%$ \\
\hline $\mathrm{Ni}-63$ & 4.94E-04 & $2.07 \mathrm{E}-04$ & $-58.02 \%$ & $\mathrm{Ra}-224$ & $3.35 \mathrm{E}-02$ & $1.13 \mathrm{E}-02$ & $-66.31 \%$ \\
\hline $\mathrm{Cu}-64$ & $2.70 \mathrm{E}-07$ & $9.13 \mathrm{E}-08$ & $-66.16 \%$ & Ra-225 & $1.21 \mathrm{E}-01$ & $4.09 \mathrm{E}-02$ & $-66.31 \%$ \\
\hline
\end{tabular}


SRNL-STI-2010-00447, REVISION 0

(continued)

\begin{tabular}{|c|c|c|c|c|c|c|c|}
\hline Nuclide & $\begin{array}{c}\text { Hamby Ladtap } \\
\text { Veg }\end{array}$ & $\begin{array}{c}\text { Jannik Ladtap } \\
\text { Veg }\end{array}$ & $\%$ Diff & Nuclide & $\begin{array}{c}\text { Hamby Ladtap } \\
\text { Veg }\end{array}$ & $\begin{array}{c}\text { Jannik Ladtap } \\
\text { Veg }\end{array}$ & $\%$ Diff \\
\hline $\mathrm{Zn}-65$ & $1.22 \mathrm{E}-02$ & $4.22 \mathrm{E}-03$ & $-65.37 \%$ & Ra-226 & $1.10 \mathrm{E}+00$ & $3.78 \mathrm{E}-01$ & $-65.49 \%$ \\
\hline Ge-68 & $9.11 \mathrm{E}-04$ & $3.03 \mathrm{E}-04$ & $-66.74 \%$ & Ra-228 & $1.08 \mathrm{E}+00$ & $3.63 \mathrm{E}-01$ & $-66.36 \%$ \\
\hline $\mathrm{Se}-75$ & $7.63 \mathrm{E}-03$ & $2.25 \mathrm{E}-03$ & $-70.45 \%$ & Ac- 227 & $1.20 \mathrm{E}+01$ & $4.00 \mathrm{E}+00$ & $-66.56 \%$ \\
\hline Se-79 & $4.69 \mathrm{E}-02$ & $3.14 \mathrm{E}-03$ & $-93.30 \%$ & Th-227 & $1.61 \mathrm{E}-02$ & $5.43 \mathrm{E}-03$ & $-66.31 \%$ \\
\hline $\mathrm{Rb}-87$ & $6.38 \mathrm{E}-03$ & $4.64 \mathrm{E}-03$ & $-27.31 \%$ & Th-228 & $3.17 \mathrm{E}-01$ & $1.07 \mathrm{E}-01$ & $-66.38 \%$ \\
\hline Sr-89 & $5.67 \mathrm{E}-03$ & $1.95 \mathrm{E}-03$ & $-65.63 \%$ & Th-229 & $3.03 \mathrm{E}+00$ & $1.01 \mathrm{E}+00$ & $-66.74 \%$ \\
\hline Sr-90 & $1.17 \mathrm{E}-01$ & $9.59 \mathrm{E}-02$ & $-17.83 \%$ & Th-230 & $4.58 \mathrm{E}-01$ & $1.52 \mathrm{E}-01$ & $-66.74 \%$ \\
\hline Y-90 & $6.46 \mathrm{E}-04$ & $2.18 \mathrm{E}-04$ & $-66.31 \%$ & Th-231 & $1.14 \mathrm{E}-05$ & $3.84 \mathrm{E}-06$ & $-66.31 \%$ \\
\hline Y-91 & $6.00 \mathrm{E}-03$ & $2.02 \mathrm{E}-03$ & $-66.31 \%$ & Th-232 & $2.42 \mathrm{E}+00$ & $8.05 \mathrm{E}-01$ & $-66.74 \%$ \\
\hline Mo-93 & $1.68 \mathrm{E}-03$ & $9.24 \mathrm{E}-04$ & $-44.95 \%$ & Th-234 & $6.58 \mathrm{E}-03$ & $2.22 \mathrm{E}-03$ & $-66.31 \%$ \\
\hline Mo-99 & $2.98 \mathrm{E}-04$ & $1.01 \mathrm{E}-04$ & $-66.24 \%$ & $\mathrm{~Pa}-230$ & $2.41 \mathrm{E}-03$ & $8.11 \mathrm{E}-04$ & $-66.32 \%$ \\
\hline $\mathrm{Nb}-93 \mathrm{~m}$ & $4.60 \mathrm{E}-03$ & $1.54 \mathrm{E}-03$ & $-66.46 \%$ & $\mathrm{~Pa}-231$ & $9.74 \mathrm{E}+00$ & $3.15 \mathrm{E}+00$ & $-67.68 \%$ \\
\hline $\mathrm{Nb}-94$ & $4.51 \mathrm{E}-03$ & $1.51 \mathrm{E}-03$ & $-66.43 \%$ & $\mathrm{~Pa}-233$ & $1.75 \mathrm{E}-03$ & $5.91 \mathrm{E}-04$ & $-66.32 \%$ \\
\hline $\mathrm{Nb}-95$ & $1.29 \mathrm{E}-03$ & 4.34E-04 & $-66.31 \%$ & U-232 & $1.11 \mathrm{E}+00$ & 4.09E-01 & $-63.25 \%$ \\
\hline Zr-93 & $1.36 \mathrm{E}-03$ & $4.64 \mathrm{E}-04$ & $-65.89 \%$ & U-233 & $2.32 \mathrm{E}-01$ & $8.61 \mathrm{E}-02$ & $-62.86 \%$ \\
\hline Zr-95 & $2.33 \mathrm{E}-03$ & $7.86 \mathrm{E}-04$ & $-66.31 \%$ & U-234 & $2.23 \mathrm{E}-01$ & 8.29E-02 & $-62.86 \%$ \\
\hline Tc-96 & $3.45 \mathrm{E}-04$ & $2.15 \mathrm{E}-04$ & $-37.68 \%$ & U-235 & $2.15 \mathrm{E}-01$ & 7.97E-02 & $-62.86 \%$ \\
\hline Tc-97 & $2.66 \mathrm{E}-04$ & $1.32 \mathrm{E}-02$ & $4846.48 \%$ & U-236 & $2.15 \mathrm{E}-01$ & 7.97E-02 & $-62.86 \%$ \\
\hline Tc-98 & $8.51 \mathrm{E}-03$ & $4.21 \mathrm{E}-01$ & $4846.48 \%$ & U-237 & 5.64E-04 & $1.90 \mathrm{E}-04$ & $-66.30 \%$ \\
\hline Tc-99 & $2.30 \mathrm{E}-03$ & $1.14 \mathrm{E}-01$ & $4846.37 \%$ & U-238 & $1.97 \mathrm{E}-01$ & 7.33E-02 & $-62.86 \%$ \\
\hline Ru-97 & $4.70 \mathrm{E}-05$ & $1.58 \mathrm{E}-05$ & $-66.32 \%$ & $\mathrm{~Np}-236$ & $6.78 \mathrm{E}-01$ & $2.41 \mathrm{E}-01$ & $-64.45 \%$ \\
\hline Ru-103 & $1.64 \mathrm{E}-03$ & $5.53 \mathrm{E}-04$ & $-66.36 \%$ & $\mathrm{~Np}-237$ & $3.35 \mathrm{E}+00$ & $1.19 \mathrm{E}+00$ & $-64.45 \%$ \\
\hline $\mathrm{Ru}-106$ & $1.74 \mathrm{E}-02$ & $5.81 \mathrm{E}-03$ & $-66.64 \%$ & $\mathrm{~Np}-239$ & $1.51 \mathrm{E}-04$ & $5.08 \mathrm{E}-05$ & $-66.31 \%$ \\
\hline $\mathrm{Pd}-107$ & $1.71 \mathrm{E}-04$ & $4.88 \mathrm{E}-05$ & $-71.37 \%$ & Am-237 & $3.62 \mathrm{E}-25$ & $1.22 \mathrm{E}-25$ & $-66.31 \%$ \\
\hline Ag-108m & $1.03 \mathrm{E}-02$ & $2.15 \mathrm{E}-03$ & $-79.15 \%$ & Am-241 & $3.82 \mathrm{E}+00$ & $1.29 \mathrm{E}+00$ & $-66.30 \%$ \\
\hline Ag-110m & $9.12 \mathrm{E}-03$ & $2.97 \mathrm{E}-03$ & $-67.41 \%$ & Am-242m & $3.57 \mathrm{E}+00$ & $1.20 \mathrm{E}+00$ & $-66.31 \%$ \\
\hline
\end{tabular}

(continued) 
SRNL-STI-2010-00447, REVISION 0

\begin{tabular}{|c|c|c|c|c|c|c|c|}
\hline Nuclide & $\begin{array}{c}\text { Hamby Ladtap } \\
\text { Veg }\end{array}$ & $\begin{array}{c}\text { Jannik Ladtap } \\
\text { Veg }\end{array}$ & $\%$ Diff & Nuclide & $\begin{array}{c}\text { Hamby Ladtap } \\
\text { Veg }\end{array}$ & $\begin{array}{c}\text { Jannik Ladtap } \\
\text { Veg }\end{array}$ & $\%$ Diff \\
\hline Cd-113 & $3.08 \mathrm{E}-01$ & $1.64 \mathrm{E}-01$ & $-46.83 \%$ & Am-243 & $3.82 \mathrm{E}+00$ & $1.29 \mathrm{E}+00$ & $-66.30 \%$ \\
\hline Cd-115m & $9.59 \mathrm{E}-03$ & $3.26 \mathrm{E}-03$ & $-66.04 \%$ & $\mathrm{Pu}-237$ & $6.33 \mathrm{E}-04$ & $2.13 \mathrm{E}-04$ & $-66.31 \%$ \\
\hline In-115 & $1.20 \mathrm{E}-01$ & $4.02 \mathrm{E}-02$ & $-66.60 \%$ & $\mathrm{Pu}-238$ & $3.23 \mathrm{E}+00$ & $1.09 \mathrm{E}+00$ & $-66.33 \%$ \\
\hline Sb-122 & $4.15 \mathrm{E}-04$ & $1.40 \mathrm{E}-04$ & $-66.31 \%$ & $\mathrm{Pu}-239$ & $3.65 \mathrm{E}+00$ & $1.23 \mathrm{E}+00$ & $-66.34 \%$ \\
\hline Sb-124 & $6.30 \mathrm{E}-03$ & $2.12 \mathrm{E}-03$ & $-66.33 \%$ & $\mathrm{Pu}-240$ & $3.65 \mathrm{E}+00$ & $1.23 \mathrm{E}+00$ & $-66.34 \%$ \\
\hline Sb-125 & $2.20 \mathrm{E}-03$ & 7.33E-04 & $-66.60 \%$ & $\mathrm{Pu}-241$ & 7.29E-02 & $2.45 \mathrm{E}-02$ & $-66.33 \%$ \\
\hline Te-123 & $2.32 \mathrm{E}-02$ & $2.35 \mathrm{E}-03$ & $-89.88 \%$ & $\mathrm{Pu}-242$ & $3.48 \mathrm{E}+00$ & $1.17 \mathrm{E}+00$ & $-66.34 \%$ \\
\hline $\mathrm{Te}-125 \mathrm{~m}$ & $2.47 \mathrm{E}-03$ & 7.79E-04 & $-68.44 \%$ & $\mathrm{Pu}-244$ & $3.40 \mathrm{E}+00$ & $1.14 \mathrm{E}+00$ & $-66.34 \%$ \\
\hline Sn-126 & $1.46 \mathrm{E}-02$ & $5.05 \mathrm{E}-03$ & $-65.38 \%$ & $\mathrm{Cm}-241$ & $2.63 \mathrm{E}-03$ & $8.87 \mathrm{E}-04$ & $-66.31 \%$ \\
\hline I-129 & $2.58 \mathrm{E}-01$ & $9.81 \mathrm{E}-02$ & $-62.03 \%$ & $\mathrm{Cm}-242$ & $8.56 \mathrm{E}-02$ & $2.88 \mathrm{E}-02$ & $-66.32 \%$ \\
\hline $\mathrm{I}-131$ & $1.31 \mathrm{E}-02$ & $4.41 \mathrm{E}-03$ & $-66.30 \%$ & $\mathrm{Cm}-243$ & $2.48 \mathrm{E}+00$ & $8.30 \mathrm{E}-01$ & $-66.54 \%$ \\
\hline Cs-134 & $6.20 \mathrm{E}-02$ & $2.11 \mathrm{E}-02$ & $-66.06 \%$ & $\mathrm{Cm}-244$ & $1.96 \mathrm{E}+00$ & $6.57 \mathrm{E}-01$ & $-66.52 \%$ \\
\hline Cs-135 & $6.29 \mathrm{E}-03$ & $2.27 \mathrm{E}-03$ & $-63.93 \%$ & $\mathrm{Cm}-245$ & $3.86 \mathrm{E}+00$ & $1.29 \mathrm{E}+00$ & $-66.60 \%$ \\
\hline Cs-137 & 4.39E-02 & $1.56 \mathrm{E}-02$ & $-64.54 \%$ & $\mathrm{Cm}-246$ & $3.86 \mathrm{E}+00$ & $1.29 \mathrm{E}+00$ & $-66.60 \%$ \\
\hline La-137 & $3.69 \mathrm{E}-04$ & $1.25 \mathrm{E}-04$ & $-66.15 \%$ & $\mathrm{Cm}-247$ & $3.52 \mathrm{E}+00$ & $1.18 \mathrm{E}+00$ & $-66.60 \%$ \\
\hline La-138 & $5.06 \mathrm{E}-03$ & $1.71 \mathrm{E}-03$ & $-66.15 \%$ & $\mathrm{Cm}-248$ & $1.37 \mathrm{E}+01$ & $4.59 \mathrm{E}+00$ & $-66.60 \%$ \\
\hline La-140 & $2.09 \mathrm{E}-04$ & $7.05 \mathrm{E}-05$ & $-66.31 \%$ & $\mathrm{Cm}-250$ & $6.67 \mathrm{E}+01$ & $2.23 \mathrm{E}+01$ & $-66.60 \%$ \\
\hline Ba-140 & $2.98 \mathrm{E}-03$ & $1.01 \mathrm{E}-03$ & $-66.31 \%$ & Bk-247 & $1.95 \mathrm{E}+00$ & $6.69 \mathrm{E}-01$ & $-65.75 \%$ \\
\hline Ce-141 & $1.48 \mathrm{E}-03$ & $5.00 \mathrm{E}-04$ & $-66.31 \%$ & Bk-249 & $4.87 \mathrm{E}-03$ & $1.64 \mathrm{E}-03$ & $-66.28 \%$ \\
\hline $\mathrm{Ce}-144$ & \multirow[t]{8}{*}{$1.62 \mathrm{E}-02$} & \multirow[t]{8}{*}{$5.45 \mathrm{E}-03$} & \multirow[t]{8}{*}{$-66.29 \%$} & Cf-249 & $3.92 \mathrm{E}+00$ & $1.32 \mathrm{E}+00$ & $-66.42 \%$ \\
\hline & & & & Cf-250 & $1.61 \mathrm{E}+00$ & $5.42 \mathrm{E}-01$ & $-66.38 \%$ \\
\hline & & & & Cf-251 & $3.92 \mathrm{E}+00$ & $1.32 \mathrm{E}+00$ & $-66.42 \%$ \\
\hline & & & & Cf-252 & $7.87 \mathrm{E}-01$ & $2.65 \mathrm{E}-01$ & $-66.33 \%$ \\
\hline & & & & Es-253 & $1.12 \mathrm{E}-02$ & $3.79 \mathrm{E}-03$ & $-66.31 \%$ \\
\hline & & & & Unidentified alpha & $3.65 \mathrm{E}+00$ & $1.23 \mathrm{E}+00$ & $-66.34 \%$ \\
\hline & & & & Unidentified beta & $1.17 \mathrm{E}-01$ & $9.58 \mathrm{E}-02$ & $-17.93 \%$ \\
\hline & & & & TOTAL Dose & $1.9 \mathrm{E}+02$ & $6.32 \mathrm{E}+01$ & $-66.51 \%$ \\
\hline
\end{tabular}


SRNL-STI-2010-00447, REVISION 0

Table 29. LADTAP XL IRRIDOSE Maximally Exposed Individual Milk Ingestion Pathway Comparison (mrem/yr)

\begin{tabular}{|c|c|c|c|c|c|c|c|}
\hline Nuclide & $\begin{array}{c}\text { Hamby Ladtap } \\
\text { Milk }\end{array}$ & $\begin{array}{c}\text { Jannik Ladtap } \\
\text { Milk }\end{array}$ & $\%$ Diff & Nuclide & $\begin{array}{c}\text { Hamby } \\
\text { Ladtap } \\
\text { Milk }\end{array}$ & $\begin{array}{c}\text { Jannik } \\
\text { Ladtap } \\
\text { Milk }\end{array}$ & $\%$ Diff \\
\hline $\mathrm{H}-3$ & $1.62 \mathrm{E}-06$ & $1.62 \mathrm{E}-06$ & $0.00 \%$ & Sm-146 & $1.18 \mathrm{E}-05$ & $1.73 \mathrm{E}-04$ & $1373.22 \%$ \\
\hline $\mathrm{Be}-7$ & $2.09 \mathrm{E}-09$ & $2.11 \mathrm{E}-09$ & $0.96 \%$ & Sm-147 & $1.06 \mathrm{E}-05$ & $1.56 \mathrm{E}-04$ & $1373.22 \%$ \\
\hline Be-10 & $1.01 \mathrm{E}-07$ & $9.87 \mathrm{E}-08$ & $-1.91 \%$ & Sm-151 & $2.00 \mathrm{E}-08$ & $2.94 \mathrm{E}-07$ & $1373.07 \%$ \\
\hline C-14 & $1.95 \mathrm{E}-02$ & $1.34 \mathrm{E}-03$ & $-93.11 \%$ & Pm-147 & $5.40 \mathrm{E}-08$ & $8.13 \mathrm{E}-07$ & $1404.53 \%$ \\
\hline $\mathrm{Na}-22$ & $6.05 \mathrm{E}-03$ & $4.37 \mathrm{E}-03$ & $-27.67 \%$ & Eu-152 & $3.49 \mathrm{E}-07$ & $5.14 \mathrm{E}-06$ & $1373.33 \%$ \\
\hline Al-26 & $3.12 \mathrm{E}-05$ & $7.56 \mathrm{E}-05$ & $142.57 \%$ & Eu-155 & $7.46 \mathrm{E}-08$ & $1.10 \mathrm{E}-06$ & $1375.13 \%$ \\
\hline $\mathrm{P}-32$ & $1.22 \mathrm{E}-03$ & $2.18 \mathrm{E}-03$ & $78.15 \%$ & Gd-152 & $1.05 \mathrm{E}-04$ & $1.30 \mathrm{E}-04$ & $23.50 \%$ \\
\hline $\mathrm{Si}-32$ & 7.94E-07 & $1.12 \mathrm{E}-06$ & $40.93 \%$ & Ho-166m & $1.84 \mathrm{E}-06$ & $6.76 \mathrm{E}-06$ & $268.30 \%$ \\
\hline S-35 & $1.35 \mathrm{E}-04$ & $1.32 \mathrm{E}-04$ & $-2.51 \%$ & Lu-176 & $1.55 \mathrm{E}-06$ & $3.85 \mathrm{E}-06$ & $147.90 \%$ \\
\hline $\mathrm{Cl}-36$ & $1.67 \mathrm{E}-01$ & $3.40 \mathrm{E}-02$ & $-79.60 \%$ & Ta-180 & $0.00 \mathrm{E}+00$ & $0.00 \mathrm{E}+00$ & $0.00 \%$ \\
\hline $\mathrm{K}-40$ & $7.05 \mathrm{E}-03$ & $1.02 \mathrm{E}-02$ & $44.96 \%$ & Hf-182 & $8.81 \mathrm{E}-08$ & $2.17 \mathrm{E}-07$ & $146.73 \%$ \\
\hline K-43 & $3.08 \mathrm{E}-07$ & $4.66 \mathrm{E}-07$ & $51.54 \%$ & Re-186m & $1.34 \mathrm{E}-02$ & $2.49 \mathrm{E}-04$ & $-98.14 \%$ \\
\hline $\mathrm{Ca}-45$ & $1.17 \mathrm{E}-04$ & $1.34 \mathrm{E}-03$ & $1049.06 \%$ & Ir-192m & $0.00 \mathrm{E}+00$ & $0.00 \mathrm{E}+00$ & $0.00 \%$ \\
\hline $\mathrm{Ca}-47$ & $4.09 \mathrm{E}-05$ & $2.97 \mathrm{E}-04$ & $625.74 \%$ & Pt-193 & $7.88 \mathrm{E}-06$ & $1.64 \mathrm{E}-05$ & $108.05 \%$ \\
\hline $\mathrm{Ti}-44$ & $1.19 \mathrm{E}-07$ & $4.04 \mathrm{E}-05$ & $33733.73 \%$ & Hg-194 & $8.87 \mathrm{E}-03$ & $5.85 \mathrm{E}-03$ & $-34.03 \%$ \\
\hline $\mathrm{V}-49$ & $1.20 \mathrm{E}-08$ & $3.03 \mathrm{E}-08$ & $153.34 \%$ & $\mathrm{Hg}-203$ & $4.84 \mathrm{E}-05$ & $1.06 \mathrm{E}-04$ & $118.83 \%$ \\
\hline $\mathrm{Cr}-51$ & $2.30 \mathrm{E}-06$ & $1.08 \mathrm{E}-06$ & $-53.04 \%$ & $\mathrm{~Pb}-202$ & $1.50 \mathrm{E}-04$ & $2.16 \mathrm{E}-04$ & $44.06 \%$ \\
\hline Mn-53 & $3.82 \mathrm{E}-07$ & $1.62 \mathrm{E}-07$ & $-57.63 \%$ & $\mathrm{~Pb}-205$ & $5.77 \mathrm{E}-06$ & $8.31 \mathrm{E}-06$ & $44.06 \%$ \\
\hline $\mathrm{Mn}-54$ & $7.63 \mathrm{E}-06$ & $3.08 \mathrm{E}-06$ & $-59.68 \%$ & $\mathrm{~Pb}-210$ & $1.90 \mathrm{E}-02$ & $2.79 \mathrm{E}-02$ & $46.66 \%$ \\
\hline $\mathrm{Fe}-55$ & $7.88 \mathrm{E}-06$ & $5.72 \mathrm{E}-07$ & $-92.74 \%$ & Bi-207 & $5.57 \mathrm{E}-05$ & $1.03 \mathrm{E}-04$ & $85.09 \%$ \\
\hline Fe-60 & $2.07 \mathrm{E}-03$ & $1.59 \mathrm{E}-04$ & $-92.35 \%$ & Bi-210 & $7.26 \mathrm{E}-06$ & $1.54 \mathrm{E}-05$ & $112.40 \%$ \\
\hline Co-58 & $3.48 \mathrm{E}-05$ & $9.34 \mathrm{E}-06$ & $-73.13 \%$ & $\mathrm{Bi}-210 \mathrm{~m}$ & $1.09 \mathrm{E}-03$ & $1.98 \mathrm{E}-03$ & $82.24 \%$ \\
\hline Co-60 & $3.07 \mathrm{E}-04$ & $8.40 \mathrm{E}-05$ & $-72.68 \%$ & Po-210 & $5.78 \mathrm{E}-03$ & $8.76 \mathrm{E}-03$ & $51.56 \%$ \\
\hline Ni-59 & $1.88 \mathrm{E}-05$ & $6.12 \mathrm{E}-06$ & $-67.54 \%$ & Ra-223 & $2.88 \mathrm{E}-03$ & $2.52 \mathrm{E}-03$ & $-12.35 \%$ \\
\hline $\mathrm{Ni}-63$ & $5.02 \mathrm{E}-05$ & $1.63 \mathrm{E}-05$ & $-67.46 \%$ & Ra-224 & $5.29 \mathrm{E}-04$ & $4.06 \mathrm{E}-04$ & $-23.22 \%$ \\
\hline $\mathrm{Cu}-64$ & $1.73 \mathrm{E}-08$ & $3.14 \mathrm{E}-09$ & $-81.85 \%$ & $\mathrm{Ra}-225$ & $1.90 \mathrm{E}-03$ & $1.69 \mathrm{E}-03$ & $-11.02 \%$ \\
\hline
\end{tabular}


SRNL-STI-2010-00447, REVISION 0

Table 29. LADTAP XL IRRIDOSE Maximally Exposed Individual Milk Ingestion Pathway Comparison (mrem/yr)

(continued)

\begin{tabular}{|c|c|c|c|c|c|c|c|}
\hline Nuclide & $\begin{array}{c}\text { Hamby Ladtap } \\
\text { Milk }\end{array}$ & $\begin{array}{c}\text { Jannik Ladtap } \\
\text { Milk }\end{array}$ & $\%$ Diff & Nuclide & $\begin{array}{c}\text { Hamby } \\
\text { Ladtap } \\
\text { Milk } \\
\end{array}$ & $\begin{array}{c}\text { Jannik } \\
\text { Ladtap } \\
\text { Milk } \\
\end{array}$ & $\%$ Diff \\
\hline $\mathrm{Zn}-65$ & 7.42E-03 & $1.06 \mathrm{E}-03$ & $-85.66 \%$ & Ra-226 & $1.86 \mathrm{E}-02$ & $1.27 \mathrm{E}-02$ & $-31.92 \%$ \\
\hline Ge-68 & $9.09 \mathrm{E}-04$ & $2.16 \mathrm{E}-03$ & $137.89 \%$ & Ra-228 & $1.62 \mathrm{E}-02$ & $1.31 \mathrm{E}-02$ & $-18.89 \%$ \\
\hline Se-75 & $5.80 \mathrm{E}-03$ & $9.08 \mathrm{E}-04$ & $-84.33 \%$ & Ac-227 & $3.27 \mathrm{E}-03$ & 7.89E-03 & $141.35 \%$ \\
\hline Se-79 & $7.15 \mathrm{E}-02$ & $1.05 \mathrm{E}-03$ & $-98.53 \%$ & Th-227 & $1.24 \mathrm{E}-06$ & $2.95 \mathrm{E}-06$ & $137.07 \%$ \\
\hline $\mathrm{Rb}-87$ & $4.24 \mathrm{E}-03$ & 3.09E-03 & $-27.03 \%$ & Th-228 & $2.15 \mathrm{E}-05$ & $5.27 \mathrm{E}-05$ & $144.79 \%$ \\
\hline Sr-89 & $6.55 \mathrm{E}-05$ & $2.60 \mathrm{E}-04$ & $297.22 \%$ & Th-229 & $2.10 \mathrm{E}-04$ & $4.94 \mathrm{E}-04$ & $135.26 \%$ \\
\hline Sr-90 & $1.38 \mathrm{E}-03$ & 7.62E-03 & $451.82 \%$ & Th-230 & $3.18 \mathrm{E}-05$ & 7.49E-05 & $135.25 \%$ \\
\hline$Y-90$ & $9.85 \mathrm{E}-08$ & $3.76 \mathrm{E}-07$ & $282.10 \%$ & Th-231 & $6.12 \mathrm{E}-10$ & $9.35 \mathrm{E}-10$ & $52.96 \%$ \\
\hline Y-91 & $8.58 \mathrm{E}-07$ & $4.18 \mathrm{E}-06$ & $387.23 \%$ & Th-232 & $1.68 \mathrm{E}-04$ & $3.96 \mathrm{E}-04$ & $135.25 \%$ \\
\hline Мо-93 & $2.73 \mathrm{E}-04$ & $6.31 \mathrm{E}-05$ & $-76.90 \%$ & Th-234 & 4.99E-07 & $1.19 \mathrm{E}-06$ & $139.22 \%$ \\
\hline Мо-99 & $3.42 \mathrm{E}-05$ & $9.62 \mathrm{E}-06$ & $-71.86 \%$ & $\mathrm{~Pa}-230$ & $1.87 \mathrm{E}-07$ & $4.42 \mathrm{E}-07$ & $136.26 \%$ \\
\hline $\mathrm{Nb}-93 \mathrm{~m}$ & $1.62 \mathrm{E}-04$ & $6.16 \mathrm{E}-08$ & $-99.96 \%$ & $\mathrm{~Pa}-231$ & $7.05 \mathrm{E}-04$ & $1.55 \mathrm{E}-03$ & $120.14 \%$ \\
\hline $\mathrm{Nb}-94$ & $1.62 \mathrm{E}-04$ & 5.98E-08 & $-99.96 \%$ & $\mathrm{~Pa}-233$ & $1.32 \mathrm{E}-07$ & $3.16 \mathrm{E}-07$ & $139.91 \%$ \\
\hline $\mathrm{Nb}-95$ & $4.75 \mathrm{E}-05$ & $1.88 \mathrm{E}-08$ & $-99.96 \%$ & U-232 & 7.63E-03 & $6.85 \mathrm{E}-02$ & $797.78 \%$ \\
\hline Zr-93 & $9.16 \mathrm{E}-08$ & $1.63 \mathrm{E}-07$ & $78.17 \%$ & U-233 & $1.59 \mathrm{E}-03$ & $1.43 \mathrm{E}-02$ & $799.58 \%$ \\
\hline Zr-95 & $1.66 \mathrm{E}-07$ & $2.92 \mathrm{E}-07$ & $75.66 \%$ & U-234 & $1.53 \mathrm{E}-03$ & $1.38 \mathrm{E}-02$ & $799.58 \%$ \\
\hline Tc-96 & $1.38 \mathrm{E}-04$ & $2.64 \mathrm{E}-05$ & $-80.88 \%$ & U-235 & $1.47 \mathrm{E}-03$ & $1.32 \mathrm{E}-02$ & $799.58 \%$ \\
\hline Tc-97 & $1.73 \mathrm{E}-04$ & $9.27 \mathrm{E}-04$ & $436.95 \%$ & U-236 & $1.47 \mathrm{E}-03$ & $1.32 \mathrm{E}-02$ & $799.58 \%$ \\
\hline Tc-98 & $5.53 \mathrm{E}-03$ & $2.97 \mathrm{E}-02$ & $436.95 \%$ & U-237 & $4.56 \mathrm{E}-06$ & $3.62 \mathrm{E}-05$ & $694.15 \%$ \\
\hline Tc-99 & $1.50 \mathrm{E}-03$ & 8.04E-03 & $436.95 \%$ & U-238 & $1.35 \mathrm{E}-03$ & $1.22 \mathrm{E}-02$ & $799.58 \%$ \\
\hline Ru-97 & $7.25 \mathrm{E}-10$ & $1.32 \mathrm{E}-08$ & $1723.19 \%$ & $\mathrm{~Np}-236$ & $4.65 \mathrm{E}-05$ & $1.14 \mathrm{E}-04$ & $145.55 \%$ \\
\hline Ru-103 & $2.41 \mathrm{E}-08$ & $5.47 \mathrm{E}-07$ & $2164.85 \%$ & $\mathrm{~Np}-237$ & $2.30 \mathrm{E}-04$ & $5.64 \mathrm{E}-04$ & $145.55 \%$ \\
\hline Ru-106 & $2.44 \mathrm{E}-07$ & $5.42 \mathrm{E}-06$ & $2120.47 \%$ & $\mathrm{~Np}-239$ & $1.12 \mathrm{E}-08$ & $2.09 \mathrm{E}-08$ & $85.91 \%$ \\
\hline Pd-107 & $1.77 \mathrm{E}-05$ & $4.28 \mathrm{E}-05$ & $141.71 \%$ & Am-237 & $1.22 \mathrm{E}-39$ & $9.09 \mathrm{E}-41$ & $-92.54 \%$ \\
\hline Ag-108m & $1.17 \mathrm{E}-02$ & $3.35 \mathrm{E}-04$ & $-97.13 \%$ & Am-241 & $2.58 \mathrm{E}-04$ & $5.33 \mathrm{E}-05$ & $-79.33 \%$ \\
\hline
\end{tabular}


SRNL-STI-2010-00447, REVISION 0

Table 29. LADTAP XL IRRIDOSE Maximally Exposed Individual Milk Ingestion Pathway Comparison (mrem/yr)

(continued)

\begin{tabular}{|c|c|c|c|c|c|c|c|}
\hline Nuclide & $\begin{array}{c}\text { Hamby } \\
\text { Ladtap } \\
\text { Milk } \\
\end{array}$ & $\begin{array}{c}\text { Jannik } \\
\text { Ladtap } \\
\text { Milk } \\
\end{array}$ & $\%$ Diff & Nuclide & $\begin{array}{c}\text { Hamby } \\
\text { Ladtap } \\
\text { Milk }\end{array}$ & $\begin{array}{c}\text { Jannik } \\
\text { Ladtap } \\
\text { Milk } \\
\end{array}$ & $\%$ Diff \\
\hline $\mathrm{Ag}-110 \mathrm{~m}$ & $6.59 \mathrm{E}-03$ & $4.69 \mathrm{E}-04$ & $-92.88 \%$ & Am-242m & $2.41 \mathrm{E}-04$ & $4.97 \mathrm{E}-05$ & $-79.33 \%$ \\
\hline Cd-113 & 9.92E-04 & $1.70 \mathrm{E}-03$ & $70.97 \%$ & Am-243 & $2.58 \mathrm{E}-04$ & $5.33 \mathrm{E}-05$ & $-79.33 \%$ \\
\hline $\mathrm{Cd}-115 \mathrm{~m}$ & $1.71 \mathrm{E}-05$ & $6.38 \mathrm{E}-05$ & $273.57 \%$ & $\mathrm{Pu}-237$ & $1.84 \mathrm{E}-08$ & $2.23 \mathrm{E}-07$ & $1113.98 \%$ \\
\hline In-115 & $3.32 \mathrm{E}-04$ & $7.91 \mathrm{E}-04$ & $138.48 \%$ & $\mathrm{Pu}-238$ & $8.71 \mathrm{E}-05$ & $1.07 \mathrm{E}-03$ & $1130.27 \%$ \\
\hline Sb-122 & $9.50 \mathrm{E}-06$ & $4.61 \mathrm{E}-07$ & $-95.15 \%$ & $\mathrm{Pu}-239$ & $9.86 \mathrm{E}-05$ & $1.21 \mathrm{E}-03$ & $1130.03 \%$ \\
\hline $\mathrm{Sb}-124$ & $1.35 \mathrm{E}-04$ & $8.34 \mathrm{E}-06$ & $-93.83 \%$ & $\mathrm{Pu}-240$ & $9.86 \mathrm{E}-05$ & $1.21 \mathrm{E}-03$ & $1130.04 \%$ \\
\hline Sb-125 & $4.53 \mathrm{E}-05$ & $2.76 \mathrm{E}-06$ & $-93.91 \%$ & $\mathrm{Pu}-241$ & $1.97 \mathrm{E}-06$ & $2.42 \mathrm{E}-05$ & $1131.13 \%$ \\
\hline Te-123 & $7.85 \mathrm{E}-04$ & $5.43 \mathrm{E}-05$ & $-93.09 \%$ & $\mathrm{Pu}-242$ & $9.40 \mathrm{E}-05$ & $1.16 \mathrm{E}-03$ & $1130.03 \%$ \\
\hline $\mathrm{Te}-125 \mathrm{~m}$ & $3.94 \mathrm{E}-05$ & $2.72 \mathrm{E}-05$ & $-30.85 \%$ & $\mathrm{Pu}-244$ & $9.17 \mathrm{E}-05$ & $1.13 \mathrm{E}-03$ & $1130.03 \%$ \\
\hline Sn-126 & $5.00 \mathrm{E}-04$ & $4.86 \mathrm{E}-04$ & $-2.80 \%$ & $\mathrm{Cm}-241$ & $1.95 \mathrm{E}-07$ & $1.88 \mathrm{E}-06$ & $864.92 \%$ \\
\hline I-129 & 2.39E-02 & $4.63 \mathrm{E}-02$ & $94.04 \%$ & $\mathrm{Cm}-242$ & 5.91E-06 & $5.80 \mathrm{E}-05$ & $881.60 \%$ \\
\hline $\mathrm{I}-131$ & $1.27 \mathrm{E}-03$ & $2.56 \mathrm{E}-03$ & $102.05 \%$ & $\mathrm{Cm}-243$ & $1.70 \mathrm{E}-04$ & $1.63 \mathrm{E}-03$ & $864.28 \%$ \\
\hline Cs-134 & $1.02 \mathrm{E}-02$ & $9.51 \mathrm{E}-03$ & $-6.70 \%$ & $\mathrm{Cm}-244$ & $1.34 \mathrm{E}-04$ & $1.30 \mathrm{E}-03$ & $866.82 \%$ \\
\hline Cs-135 & $1.09 \mathrm{E}-03$ & $9.62 \mathrm{E}-04$ & $-11.89 \%$ & $\mathrm{Cm}-245$ & $2.65 \mathrm{E}-04$ & $2.54 \mathrm{E}-03$ & $858.67 \%$ \\
\hline Cs-137 & $7.52 \mathrm{E}-03$ & $6.70 \mathrm{E}-03$ & $-10.89 \%$ & Cm-246 & $2.65 \mathrm{E}-04$ & $2.54 \mathrm{E}-03$ & $858.69 \%$ \\
\hline La-137 & $2.53 \mathrm{E}-08$ & $2.44 \mathrm{E}-07$ & $863.52 \%$ & $\mathrm{Cm}-247$ & $2.41 \mathrm{E}-04$ & $2.31 \mathrm{E}-03$ & $858.65 \%$ \\
\hline La-138 & $3.47 \mathrm{E}-07$ & $3.35 \mathrm{E}-06$ & $863.52 \%$ & $\mathrm{Cm}-248$ & $9.42 \mathrm{E}-04$ & $9.03 \mathrm{E}-03$ & $858.65 \%$ \\
\hline La-140 & $1.42 \mathrm{E}-08$ & 9.79E-08 & $588.11 \%$ & $\mathrm{Cm}-250$ & 4.57E-03 & 4.39E-02 & $858.68 \%$ \\
\hline $\mathrm{Ba}-140$ & $1.89 \mathrm{E}-05$ & $1.76 \mathrm{E}-05$ & $-6.95 \%$ & Bk-247 & $1.05 \mathrm{E}-05$ & $1.31 \mathrm{E}-04$ & $1140.69 \%$ \\
\hline Ce-141 & $1.32 \mathrm{E}-05$ & $1.06 \mathrm{E}-06$ & $-91.96 \%$ & Bk-249 & $2.66 \mathrm{E}-08$ & $3.27 \mathrm{E}-07$ & $1131.22 \%$ \\
\hline Ce-144 & $1.33 \mathrm{E}-04$ & $1.09 \mathrm{E}-05$ & $-91.81 \%$ & Cf-249 & $3.99 \mathrm{E}-05$ & $1.95 \mathrm{E}-04$ & $387.83 \%$ \\
\hline & & & & Cf-250 & $1.64 \mathrm{E}-05$ & $8.02 \mathrm{E}-05$ & $389.81 \%$ \\
\hline & & & & Cf-251 & $3.99 \mathrm{E}-05$ & $1.95 \mathrm{E}-04$ & $387.77 \%$ \\
\hline & & & & Cf-252 & 7.99E-06 & $3.93 \mathrm{E}-05$ & $391.97 \%$ \\
\hline & & & & Es-253 & $6.90 \mathrm{E}-08$ & $8.21 \mathrm{E}-07$ & $1089.83 \%$ \\
\hline & & & & $\begin{array}{c}\text { Unidentified } \\
\text { alpha }\end{array}$ & $9.86 \mathrm{E}-05$ & $1.21 \mathrm{E}-03$ & $1130.03 \%$ \\
\hline & & & & Unidentified beta & $1.38 \mathrm{E}-03$ & $7.62 \mathrm{E}-03$ & $451.42 \%$ \\
\hline & & & & TOTAL Dose & 4.86E-01 & 4.89E-01 & $0.65 \%$ \\
\hline
\end{tabular}


SRNL-STI-2010-00447, REVISION 0

Table 30. LADTAP XL IRRIDOSE Maximally Exposed Individual Meat Ingestion Pathway Comparison (mrem/yr)

\begin{tabular}{|c|c|c|c|c|c|c|c|}
\hline Nuclide & $\begin{array}{c}\text { Hamby Ladtap } \\
\text { Meat }\end{array}$ & $\begin{array}{c}\text { Jannik Ladtap } \\
\text { Meat }\end{array}$ & $\%$ Diff & Nuclide & $\begin{array}{c}\text { Hamby Ladtap } \\
\text { Meat }\end{array}$ & $\begin{array}{c}\text { Jannik Ladtap } \\
\text { Meat }\end{array}$ & $\%$ Diff \\
\hline H-3 & $5.7 \mathrm{E}-07$ & $5.7 \mathrm{E}-07$ & $0.00 \%$ & Sm-146 & $2.9 \mathrm{E}-03$ & $4.4 \mathrm{E}-04$ & $-85.00 \%$ \\
\hline $\mathrm{Be}-7$ & $2.5 \mathrm{E}-07$ & $5.9 \mathrm{E}-07$ & $133.57 \%$ & Sm-147 & $2.7 \mathrm{E}-03$ & $4.0 \mathrm{E}-04$ & $-85.00 \%$ \\
\hline Be-10 & $1.3 \mathrm{E}-05$ & $2.9 \mathrm{E}-05$ & $128.30 \%$ & Sm-151 & $5.0 \mathrm{E}-06$ & $7.5 \mathrm{E}-07$ & $-85.01 \%$ \\
\hline $\mathrm{C}-14$ & $1.2 \mathrm{E}-02$ & $8.4 \mathrm{E}-04$ & $-93.15 \%$ & Pm-147 & $1.3 \mathrm{E}-05$ & $1.3 \mathrm{E}-07$ & $-98.99 \%$ \\
\hline $\mathrm{Na}-22$ & $1.1 \mathrm{E}-03$ & $1.2 \mathrm{E}-03$ & $7.62 \%$ & Eu-152 & $8.4 \mathrm{E}-05$ & $8.3 \mathrm{E}-07$ & $-99.01 \%$ \\
\hline $\mathrm{Na}-24$ & $3.4 \mathrm{E}-09$ & $1.8 \mathrm{E}-09$ & $-47.50 \%$ & Eu-154 & $1.3 \mathrm{E}-04$ & $1.2 \mathrm{E}-06$ & $-99.01 \%$ \\
\hline Al-26 & $1.9 \mathrm{E}-05$ & $1.3 \mathrm{E}-04$ & $582.43 \%$ & Eu-155 & $1.8 \mathrm{E}-05$ & $1.8 \mathrm{E}-07$ & $-99.01 \%$ \\
\hline P-32 & $4.9 \mathrm{E}-04$ & $1.2 \mathrm{E}-03$ & $152.77 \%$ & Gd-152 & $8.8 \mathrm{E}-04$ & $2.1 \mathrm{E}-05$ & $-97.62 \%$ \\
\hline $\mathrm{Si}-32$ & $3.9 \mathrm{E}-07$ & $5.4 \mathrm{E}-07$ & $38.01 \%$ & Ho-166m & $1.0 \mathrm{E}-04$ & $1.6 \mathrm{E}-05$ & $-84.19 \%$ \\
\hline S-35 & $1.8 \mathrm{E}-04$ & 7.9E-04 & $328.92 \%$ & Lu-176 & $8.8 \mathrm{E}-05$ & $2.0 \mathrm{E}-04$ & $132.38 \%$ \\
\hline $\mathrm{Cl}-36$ & $1.2 \mathrm{E}-01$ & $8.3 \mathrm{E}-03$ & $-93.20 \%$ & Тa-180 & $0.0 \mathrm{E}+00$ & $0.0 \mathrm{E}+00$ & $0.00 \%$ \\
\hline $\mathrm{K}-40$ & $4.9 \mathrm{E}-03$ & $6.9 \mathrm{E}-03$ & $39.64 \%$ & Hf-182 & $4.0 \mathrm{E}-08$ & $3.0 \mathrm{E}-06$ & $7427.31 \%$ \\
\hline $\mathrm{K}-43$ & $2.6 \mathrm{E}-08$ & $3.1 \mathrm{E}-08$ & $20.67 \%$ & Re-186m & $2.3 \mathrm{E}-03$ & $3.2 \mathrm{E}-04$ & $-86.22 \%$ \\
\hline $\mathrm{Ca}-41$ & $3.8 \mathrm{E}-05$ & $2.8 \mathrm{E}-03$ & $7351.98 \%$ & Re-187 & $5.9 \mathrm{E}-06$ & $8.1 \mathrm{E}-07$ & $-86.22 \%$ \\
\hline $\mathrm{Ca}-45$ & $1.5 \mathrm{E}-05$ & $4.2 \mathrm{E}-04$ & $2620.99 \%$ & Ir-192m & $0.0 \mathrm{E}+00$ & $0.0 \mathrm{E}+00$ & $0.00 \%$ \\
\hline $\mathrm{Ca}-47$ & $3.6 \mathrm{E}-06$ & $5.8 \mathrm{E}-05$ & $1518.77 \%$ & Pt-193 & $1.6 \mathrm{E}-06$ & $3.1 \mathrm{E}-06$ & $95.97 \%$ \\
\hline $\mathrm{Ti}-44$ & $5.4 \mathrm{E}-08$ & $2.2 \mathrm{E}-05$ & $41212.62 \%$ & Hg-194 & $4.3 \mathrm{E}-01$ & $7.5 \mathrm{E}-01$ & $73.62 \%$ \\
\hline $\mathrm{V}-49$ & $3.7 \mathrm{E}-07$ & $8.8 \mathrm{E}-07$ & $137.11 \%$ & $\mathrm{Hg}-203$ & $2.3 \mathrm{E}-03$ & $1.3 \mathrm{E}-02$ & $459.22 \%$ \\
\hline Cr-51 & 5.9E-07 & $5.1 \mathrm{E}-06$ & $761.21 \%$ & Pb-202 & $1.0 \mathrm{E}-04$ & $1.9 \mathrm{E}-04$ & $92.61 \%$ \\
\hline $\mathrm{Mn}-53$ & $3.0 \mathrm{E}-07$ & $5.7 \mathrm{E}-07$ & $88.73 \%$ & $\mathrm{~Pb}-205$ & $3.8 \mathrm{E}-06$ & $7.4 \mathrm{E}-06$ & $92.61 \%$ \\
\hline Mn-54 & $6.1 \mathrm{E}-06$ & $1.1 \mathrm{E}-05$ & $77.89 \%$ & $\mathrm{~Pb}-210$ & $1.3 \mathrm{E}-02$ & $2.5 \mathrm{E}-02$ & $95.94 \%$ \\
\hline Fe-55 & $6.6 \mathrm{E}-05$ & $5.5 \mathrm{E}-05$ & $-15.97 \%$ & Bi-207 & $5.5 \mathrm{E}-05$ & $2.0 \mathrm{E}-05$ & $-63.70 \%$ \\
\hline Fe-60 & $1.7 \mathrm{E}-02$ & $1.5 \mathrm{E}-02$ & $-11.38 \%$ & $\mathrm{Bi}-210$ & $5.0 \mathrm{E}-06$ & $1.9 \mathrm{E}-06$ & $-61.02 \%$ \\
\hline Co-58 & $1.1 \mathrm{E}-04$ & $8.6 \mathrm{E}-06$ & $-92.23 \%$ & $\mathrm{Bi}-210 \mathrm{~m}$ & $1.1 \mathrm{E}-03$ & $3.8 \mathrm{E}-04$ & $-64.19 \%$ \\
\hline Co-60 & $1.0 \mathrm{E}-03$ & $7.9 \mathrm{E}-05$ & $-92.07 \%$ & Po-210 & $2.1 \mathrm{E}-02$ & $5.0 \mathrm{E}-02$ & $136.29 \%$ \\
\hline $\mathrm{Ni}-59$ & $3.7 \mathrm{E}-06$ & $7.8 \mathrm{E}-06$ & $109.65 \%$ & Ra-223 & $6.1 \mathrm{E}-04$ & $2.3 \mathrm{E}-03$ & $269.81 \%$ \\
\hline Ni-63 & $9.9 \mathrm{E}-06$ & $2.1 \mathrm{E}-05$ & $110.05 \%$ & Ra-224 & $7.8 \mathrm{E}-05$ & $2.4 \mathrm{E}-04$ & $210.08 \%$ \\
\hline $\mathrm{Cu}-64$ & $5.5 \mathrm{E}-11$ & $6.1 \mathrm{E}-11$ & $11.71 \%$ & Ra-225 & $4.2 \mathrm{E}-04$ & $1.6 \mathrm{E}-03$ & $277.37 \%$ \\
\hline
\end{tabular}


SRNL-STI-2010-00447, REVISION 0

Table 30. LADTAP XL IRRIDOSE Maximally Exposed Individual Meat Ingestion Pathway Comparison (mrem/yr) (continued)

\begin{tabular}{|c|c|c|c|c|c|c|c|}
\hline Nuclide & $\begin{array}{c}\text { Hamby Ladtap } \\
\text { Meat }\end{array}$ & $\begin{array}{c}\text { Jannik Ladtap } \\
\text { Meat }\end{array}$ & $\%$ Diff & Nuclide & $\begin{array}{c}\text { Hamby Ladtap } \\
\text { Meat }\end{array}$ & $\begin{array}{c}\text { Jannik Ladtap } \\
\text { Meat }\end{array}$ & $\%$ Diff \\
\hline $\mathrm{Zn}-65$ & $1.4 \mathrm{E}-03$ & $1.5 \mathrm{E}-02$ & $970.58 \%$ & $\mathrm{Ra}-226$ & $4.6 \mathrm{E}-03$ & $1.4 \mathrm{E}-02$ & $196.60 \%$ \\
\hline Ge-68 & $2.3 \mathrm{E}-03$ & $5.0 \mathrm{E}-03$ & $123.03 \%$ & Ra-228 & $4.0 \mathrm{E}-03$ & $1.4 \mathrm{E}-02$ & $251.57 \%$ \\
\hline Se-75 & $4.7 \mathrm{E}-04$ & $8.1 \mathrm{E}-04$ & $71.13 \%$ & Ac-227 & $8.2 \mathrm{E}-04$ & $3.8 \mathrm{E}-02$ & $4559.24 \%$ \\
\hline Se-79 & $5.8 \mathrm{E}-03$ & $9.5 \mathrm{E}-04$ & $-83.62 \%$ & Th-227 & $1.1 \mathrm{E}-05$ & $2.9 \mathrm{E}-05$ & $159.40 \%$ \\
\hline $\mathrm{Rb}-87$ & $1.1 \mathrm{E}-03$ & $6.3 \mathrm{E}-04$ & $-42.04 \%$ & Th-228 & $2.2 \mathrm{E}-04$ & $5.8 \mathrm{E}-04$ & $171.53 \%$ \\
\hline Sr-89 & $1.2 \mathrm{E}-05$ & $6.0 \mathrm{E}-05$ & $408.44 \%$ & Th-229 & $2.1 \mathrm{E}-03$ & $5.5 \mathrm{E}-03$ & $161.34 \%$ \\
\hline Sr-90 & $2.6 \mathrm{E}-04$ & $1.8 \mathrm{E}-03$ & $614.81 \%$ & Th-230 & $3.2 \mathrm{E}-04$ & $8.3 \mathrm{E}-04$ & $161.33 \%$ \\
\hline Y-90 & $5.5 \mathrm{E}-06$ & $2.0 \mathrm{E}-06$ & $-63.18 \%$ & Th-231 & $9.5 \mathrm{E}-10$ & $1.4 \mathrm{E}-09$ & $45.61 \%$ \\
\hline Y-91 & $9.6 \mathrm{E}-05$ & $4.9 \mathrm{E}-05$ & $-49.13 \%$ & Th-232 & $1.7 \mathrm{E}-03$ & $4.4 \mathrm{E}-03$ & $161.33 \%$ \\
\hline Mo-93 & $7.2 \mathrm{E}-05$ & $1.4 \mathrm{E}-05$ & $-80.64 \%$ & Th-234 & $4.6 \mathrm{E}-06$ & $1.2 \mathrm{E}-05$ & $162.58 \%$ \\
\hline Мo-99 & $4.5 \mathrm{E}-06$ & $9.7 \mathrm{E}-07$ & $-78.70 \%$ & $\mathrm{~Pa}-230$ & $4.2 \mathrm{E}-05$ & $8.4 \mathrm{E}-06$ & $-79.91 \%$ \\
\hline $\mathrm{Nb}-93 \mathrm{~m}$ & $4.5 \mathrm{E}-03$ & $9.4 \mathrm{E}-09$ & $-100.00 \%$ & $\mathrm{~Pa}-231$ & $1.8 \mathrm{E}-01$ & $3.4 \mathrm{E}-02$ & $-80.95 \%$ \\
\hline $\mathrm{Nb}-94$ & $4.5 \mathrm{E}-03$ & $9.2 \mathrm{E}-09$ & $-100.00 \%$ & $\mathrm{~Pa}-233$ & $3.1 \mathrm{E}-05$ & $6.3 \mathrm{E}-06$ & $-79.49 \%$ \\
\hline $\mathrm{Nb}-95$ & $1.3 \mathrm{E}-03$ & $2.7 \mathrm{E}-09$ & $-100.00 \%$ & U-232 & $1.3 \mathrm{E}-03$ & $3.6 \mathrm{E}-03$ & $176.24 \%$ \\
\hline Zr-93 & $1.6 \mathrm{E}-04$ & $1.3 \mathrm{E}-08$ & $-99.99 \%$ & U-233 & $2.7 \mathrm{E}-04$ & $7.5 \mathrm{E}-04$ & $176.83 \%$ \\
\hline Zr-95 & $2.7 \mathrm{E}-04$ & $2.3 \mathrm{E}-08$ & $-99.99 \%$ & U-234 & $2.6 \mathrm{E}-04$ & $7.2 \mathrm{E}-04$ & $176.83 \%$ \\
\hline Tc-96 & $3.6 \mathrm{E}-04$ & $1.3 \mathrm{E}-05$ & $-96.30 \%$ & U-235 & $2.5 \mathrm{E}-04$ & $6.9 \mathrm{E}-04$ & $176.83 \%$ \\
\hline Tc-97 & $6.8 \mathrm{E}-04$ & $7.6 \mathrm{E}-04$ & $12.67 \%$ & U-236 & $2.5 \mathrm{E}-04$ & $6.9 \mathrm{E}-04$ & $176.83 \%$ \\
\hline Tc-98 & $2.2 \mathrm{E}-02$ & $2.4 \mathrm{E}-02$ & $12.67 \%$ & U-237 & $5.9 \mathrm{E}-07$ & $1.4 \mathrm{E}-06$ & $134.95 \%$ \\
\hline Tc-99 & $5.9 \mathrm{E}-03$ & $6.6 \mathrm{E}-03$ & $12.67 \%$ & U-238 & $2.3 \mathrm{E}-04$ & $6.4 \mathrm{E}-04$ & $176.83 \%$ \\
\hline Ru-97 & $3.7 \mathrm{E}-05$ & $5.3 \mathrm{E}-07$ & $-98.57 \%$ & $\mathrm{~Np}-236$ & $4.7 \mathrm{E}-04$ & $5.5 \mathrm{E}-03$ & $1085.61 \%$ \\
\hline Ru-103 & $2.3 \mathrm{E}-03$ & $4.4 \mathrm{E}-05$ & $-98.09 \%$ & $\mathrm{~Np}-237$ & $2.3 \mathrm{E}-03$ & $2.7 \mathrm{E}-02$ & $1085.61 \%$ \\
\hline Ru-106 & $2.4 \mathrm{E}-02$ & $4.6 \mathrm{E}-04$ & $-98.12 \%$ & Np-239 & $4.9 \mathrm{E}-08$ & $4.0 \mathrm{E}-07$ & $717.11 \%$ \\
\hline Pd-107 & $8.7 \mathrm{E}-07$ & $4.1 \mathrm{E}-06$ & $373.63 \%$ & Am-237 & $2.2 \mathrm{E}-56$ & $3.4 \mathrm{E}-56$ & $53.07 \%$ \\
\hline $\begin{array}{c}\text { Ag- } \\
108 \mathrm{~m}\end{array}$ & $9.8 \mathrm{E}-04$ & $1.5 \mathrm{E}-04$ & $-84.30 \%$ & Am-241 & $2.6 \mathrm{E}-03$ & $1.5 \mathrm{E}-02$ & $493.58 \%$ \\
\hline
\end{tabular}


SRNL-STI-2010-00447, REVISION 0

Table 30. LADTAP XL IRRIDOSE Maximally Exposed Individual Meat Ingestion Pathway Comparison (mrem/yr) (continued)

\begin{tabular}{|c|c|c|c|c|c|c|c|}
\hline Nuclide & $\begin{array}{c}\text { Hamby Ladtap } \\
\text { Meat }\end{array}$ & $\begin{array}{c}\text { Jannik Ladtap } \\
\text { Meat }\end{array}$ & $\%$ Diff & Nuclide & $\begin{array}{c}\text { Hamby Ladtap } \\
\text { Meat }\end{array}$ & $\begin{array}{c}\text { annik Ladtap } \\
\text { Meat }\end{array}$ & $\%$ Diff \\
\hline Ag-110m & $5.6 \mathrm{E}-04$ & $2.1 \mathrm{E}-04$ & $-61.61 \%$ & Am-242m & $2.4 \mathrm{E}-03$ & $1.4 \mathrm{E}-02$ & $493.61 \%$ \\
\hline Cd-113 & $1.1 \mathrm{E}-03$ & $1.3 \mathrm{E}-02$ & $1069.34 \%$ & Am-243 & $2.6 \mathrm{E}-03$ & $1.5 \mathrm{E}-02$ & $493.56 \%$ \\
\hline Cd-115m & $1.8 \mathrm{E}-05$ & $4.5 \mathrm{E}-04$ & $2379.61 \%$ & $\mathrm{Pu}-237$ & $3.1 \mathrm{E}-08$ & $5.7 \mathrm{E}-09$ & $-81.70 \%$ \\
\hline In-115 & $1.7 \mathrm{E}-03$ & $7.6 \mathrm{E}-03$ & $360.57 \%$ & $\mathrm{Pu}-238$ & $1.5 \mathrm{E}-04$ & $2.8 \mathrm{E}-05$ & $-81.35 \%$ \\
\hline Sb-122 & $3.1 \mathrm{E}-06$ & $1.6 \mathrm{E}-06$ & $-49.04 \%$ & $\mathrm{Pu}-239$ & $1.7 \mathrm{E}-04$ & $3.2 \mathrm{E}-05$ & $-81.35 \%$ \\
\hline $\mathrm{Sb}-124$ & $8.8 \mathrm{E}-05$ & $6.1 \mathrm{E}-05$ & $-29.86 \%$ & $\mathrm{Pu}-240$ & $1.7 \mathrm{E}-04$ & $3.2 \mathrm{E}-05$ & $-81.35 \%$ \\
\hline $\mathrm{Sb}-125$ & $3.0 \mathrm{E}-05$ & $2.1 \mathrm{E}-05$ & $-30.41 \%$ & $\mathrm{Pu}-241$ & $3.4 \mathrm{E}-06$ & $6.4 \mathrm{E}-07$ & $-81.34 \%$ \\
\hline Te-123 & $1.5 \mathrm{E}-02$ & $2.7 \mathrm{E}-04$ & $-98.17 \%$ & $\mathrm{Pu}-242$ & $1.6 \mathrm{E}-04$ & $3.1 \mathrm{E}-05$ & $-81.35 \%$ \\
\hline Te-125m & $7.3 \mathrm{E}-04$ & $1.3 \mathrm{E}-04$ & $-82.15 \%$ & $\mathrm{Pu}-244$ & $1.6 \mathrm{E}-04$ & $3.0 \mathrm{E}-05$ & $-81.35 \%$ \\
\hline Sn-126 & $4.0 \mathrm{E}-03$ & $9.4 \mathrm{E}-03$ & $134.64 \%$ & $\mathrm{Cm}-241$ & $1.8 \mathrm{E}-06$ & $8.5 \mathrm{E}-07$ & $-53.82 \%$ \\
\hline I-129 & $2.9 \mathrm{E}-03$ & $1.4 \mathrm{E}-02$ & $383.38 \%$ & $\mathrm{Cm}-242$ & $5.9 \mathrm{E}-05$ & $2.8 \mathrm{E}-05$ & $-52.72 \%$ \\
\hline $\mathrm{I}-131$ & $1.2 \mathrm{E}-04$ & $5.9 \mathrm{E}-04$ & $384.52 \%$ & $\mathrm{Cm}-243$ & $1.7 \mathrm{E}-03$ & $7.9 \mathrm{E}-04$ & $-53.46 \%$ \\
\hline Cs-134 & $8.5 \mathrm{E}-04$ & $1.1 \mathrm{E}-02$ & $1191.62 \%$ & $\mathrm{Cm}-244$ & $1.3 \mathrm{E}-03$ & $6.3 \mathrm{E}-04$ & $-53.34 \%$ \\
\hline Cs-135 & $9.1 \mathrm{E}-05$ & $1.1 \mathrm{E}-03$ & $1123.59 \%$ & $\mathrm{Cm}-245$ & $2.7 \mathrm{E}-03$ & $1.2 \mathrm{E}-03$ & $-53.72 \%$ \\
\hline Cs-137 & $6.3 \mathrm{E}-04$ & $7.7 \mathrm{E}-03$ & $1136.71 \%$ & $\mathrm{Cm}-246$ & $2.7 \mathrm{E}-03$ & $1.2 \mathrm{E}-03$ & $-53.72 \%$ \\
\hline La-137 & $2.5 \mathrm{E}-07$ & $3.8 \mathrm{E}-07$ & $51.17 \%$ & $\mathrm{Cm}-247$ & $2.4 \mathrm{E}-03$ & $1.1 \mathrm{E}-03$ & $-53.72 \%$ \\
\hline La-138 & $3.5 \mathrm{E}-06$ & $5.3 \mathrm{E}-06$ & $51.17 \%$ & $\mathrm{Cm}-248$ & $9.4 \mathrm{E}-03$ & 4.4E-03 & $-53.72 \%$ \\
\hline La-140 & $4.5 \mathrm{E}-08$ & 4.3E-08 & $-4.04 \%$ & $\mathrm{Cm}-250$ & $4.6 \mathrm{E}-02$ & $2.1 \mathrm{E}-02$ & $-53.72 \%$ \\
\hline $\mathrm{Ba}-140$ & $3.3 \mathrm{E}-05$ & $3.1 \mathrm{E}-06$ & $-90.38 \%$ & Bk-247 & $1.3 \mathrm{E}-04$ & $3.9 \mathrm{E}-04$ & $199.24 \%$ \\
\hline $\mathrm{Ce}-141$ & $6.2 \mathrm{E}-06$ & $2.4 \mathrm{E}-07$ & $-96.15 \%$ & Bk-249 & $3.3 \mathrm{E}-07$ & $9.8 \mathrm{E}-07$ & $196.71 \%$ \\
\hline $\mathrm{Ce}-144$ & $6.6 \mathrm{E}-05$ & $2.6 \mathrm{E}-06$ & $-96.05 \%$ & Cf-249 & $8.0 \mathrm{E}-04$ & $1.3 \mathrm{E}-03$ & $56.92 \%$ \\
\hline & & & & Cf-250 & $3.3 \mathrm{E}-04$ & $5.2 \mathrm{E}-04$ & $57.53 \%$ \\
\hline & & & & Cf-251 & 8.0E-04 & $1.3 \mathrm{E}-03$ & $56.90 \%$ \\
\hline & & & & Cf-252 & $1.6 \mathrm{E}-04$ & $2.5 \mathrm{E}-04$ & $58.17 \%$ \\
\hline & & & & Es-253 & 7.9E-07 & $2.2 \mathrm{E}-06$ & $183.36 \%$ \\
\hline & & & & Unidentified alpha & $1.7 \mathrm{E}-04$ & $3.2 \mathrm{E}-05$ & $-81.35 \%$ \\
\hline & & & & Unidentified beta & $2.6 \mathrm{E}-04$ & $1.8 \mathrm{E}-03$ & $614.28 \%$ \\
\hline & & & & TOTAL Dose & $1.0 \mathrm{E}+00$ & $1.2 \mathrm{E}+00$ & $21.59 \%$ \\
\hline
\end{tabular}


SRNL-STI-2010-00447, REVISION 0

Table 31. LADTAP XL IRRIDOSE Maximally Exposed Individual Total Ingestion Pathway Comparison (mrem/yr)

\begin{tabular}{|c|c|c|c|c|c|c|c|}
\hline Nuclide & $\begin{array}{c}\text { Hamby Ladtap } \\
\text { Total }\end{array}$ & $\begin{array}{c}\text { Jannik Ladtap } \\
\text { Total }\end{array}$ & $\%$ Diff & Nuclide & $\begin{array}{c}\text { Hamby Ladtap } \\
\text { Total }\end{array}$ & $\begin{array}{c}\text { Jannik Ladtap } \\
\text { Total }\end{array}$ & $\%$ Diff \\
\hline H-3 & $4.4 \mathrm{E}-06$ & $4.4 \mathrm{E}-06$ & $0.00 \%$ & Sm-146 & $1.7 \mathrm{E}-01$ & $6.2 \mathrm{E}-02$ & $-64.70 \%$ \\
\hline $\mathrm{Be}-7$ & $7.3 \mathrm{E}-05$ & $2.5 \mathrm{E}-05$ & $-65.62 \%$ & Sm-147 & $1.6 \mathrm{E}-01$ & $5.5 \mathrm{E}-02$ & $-64.70 \%$ \\
\hline Be-10 & $3.6 \mathrm{E}-03$ & $1.2 \mathrm{E}-03$ & $-65.82 \%$ & Sm-151 & $3.0 \mathrm{E}-04$ & $1.0 \mathrm{E}-04$ & $-64.87 \%$ \\
\hline C-14 & 7.6E-02 & $4.2 \mathrm{E}-03$ & $-94.51 \%$ & Pm-147 & $8.1 \mathrm{E}-04$ & $2.8 \mathrm{E}-04$ & $-64.80 \%$ \\
\hline $\mathrm{Na}-22$ & $1.8 \mathrm{E}-02$ & $9.0 \mathrm{E}-03$ & $-48.81 \%$ & Eu-152 & $5.2 \mathrm{E}-03$ & $1.8 \mathrm{E}-03$ & $-65.72 \%$ \\
\hline $\mathrm{Na}-24$ & $2.3 \mathrm{E}-06$ & $8.3 \mathrm{E}-07$ & $-64.46 \%$ & Eu-154 & $7.9 \mathrm{E}-03$ & $2.7 \mathrm{E}-03$ & $-65.96 \%$ \\
\hline Al-26 & $1.1 \mathrm{E}-02$ & $3.9 \mathrm{E}-03$ & $-65.11 \%$ & Eu-155 & $1.1 \mathrm{E}-03$ & $3.8 \mathrm{E}-04$ & $-66.24 \%$ \\
\hline P-32 & 4.7E-03 & $4.4 \mathrm{E}-03$ & $-6.49 \%$ & Gd-152 & $1.3 \mathrm{E}-01$ & $4.6 \mathrm{E}-02$ & $-64.53 \%$ \\
\hline Si-32 & $2.0 \mathrm{E}-03$ & 7.0E-04 & $-64.62 \%$ & Ho-166m & $6.8 \mathrm{E}-03$ & $2.4 \mathrm{E}-03$ & $-64.67 \%$ \\
\hline S-35 & $8.1 \mathrm{E}-04$ & $1.1 \mathrm{E}-03$ & $33.91 \%$ & Lu-176 & $5.8 \mathrm{E}-03$ & $2.1 \mathrm{E}-03$ & $-63.15 \%$ \\
\hline Cl-36 & $5.1 \mathrm{E}-01$ & $9.4 \mathrm{E}-02$ & $-81.61 \%$ & Ta-180 & $0.0 \mathrm{E}+00$ & $0.0 \mathrm{E}+00$ & $0.00 \%$ \\
\hline K-40 & 4.9E-02 & $4.6 \mathrm{E}-02$ & $-6.26 \%$ & Hf-182 & $1.2 \mathrm{E}-02$ & $4.0 \mathrm{E}-03$ & $-66.19 \%$ \\
\hline $\mathrm{K}-43$ & 4.9E-06 & $2.0 \mathrm{E}-06$ & $-58.24 \%$ & Re-186m & $2.6 \mathrm{E}+00$ & $3.5 \mathrm{E}-03$ & $-99.87 \%$ \\
\hline $\mathrm{Ca}-41$ & $3.6 \mathrm{E}-03$ & $3.5 \mathrm{E}-02$ & $881.25 \%$ & Re-187 & $6.5 \mathrm{E}-03$ & $8.7 \mathrm{E}-06$ & $-99.87 \%$ \\
\hline $\mathrm{Ca}-45$ & $2.6 \mathrm{E}-03$ & $4.0 \mathrm{E}-03$ & $51.77 \%$ & Ir-192m & $0.0 \mathrm{E}+00$ & $0.0 \mathrm{E}+00$ & $0.00 \%$ \\
\hline $\mathrm{Ca}-47$ & 8.9E-04 & $6.9 \mathrm{E}-04$ & $-22.38 \%$ & Pt-193 & $1.1 \mathrm{E}-04$ & $5.3 \mathrm{E}-05$ & $-52.26 \%$ \\
\hline Ti-44 & $1.6 \mathrm{E}-02$ & $5.5 \mathrm{E}-03$ & $-65.65 \%$ & $\mathrm{Hg}-194$ & $1.1 \mathrm{E}+00$ & $9.6 \mathrm{E}-01$ & $-10.00 \%$ \\
\hline $\mathrm{V}-49$ & $4.4 \mathrm{E}-05$ & $1.6 \mathrm{E}-05$ & $-64.53 \%$ & $\mathrm{Hg}-203$ & 8.9E-03 & $1.5 \mathrm{E}-02$ & $72.37 \%$ \\
\hline Cr-51 & $7.3 \mathrm{E}-05$ & $3.0 \mathrm{E}-05$ & $-59.20 \%$ & $\mathrm{~Pb}-202$ & $3.5 \mathrm{E}-02$ & $1.3 \mathrm{E}-02$ & $-63.94 \%$ \\
\hline $\mathrm{Mn}-53$ & $9.5 \mathrm{E}-05$ & $6.0 \mathrm{E}-05$ & $-37.10 \%$ & $\mathrm{~Pb}-205$ & $1.3 \mathrm{E}-03$ & $4.8 \mathrm{E}-04$ & $-63.94 \%$ \\
\hline Mn-54 & $2.2 \mathrm{E}-03$ & 7.9E-04 & $-64.30 \%$ & $\mathrm{~Pb}-210$ & $4.5 \mathrm{E}+00$ & $1.6 \mathrm{E}+00$ & $-64.43 \%$ \\
\hline Fe-55 & $5.6 \mathrm{E}-04$ & $2.2 \mathrm{E}-04$ & $-59.92 \%$ & Bi-207 & $5.7 \mathrm{E}-03$ & $3.3 \mathrm{E}-03$ & $-41.79 \%$ \\
\hline Fe-60 & $1.5 \mathrm{E}-01$ & $6.6 \mathrm{E}-02$ & $-54.82 \%$ & $\mathrm{Bi}-210$ & $9.1 \mathrm{E}-04$ & $3.2 \mathrm{E}-04$ & $-64.75 \%$ \\
\hline Co-58 & $2.6 \mathrm{E}-03$ & $8.5 \mathrm{E}-04$ & $-67.35 \%$ & $\mathrm{Bi}-210 \mathrm{~m}$ & $1.1 \mathrm{E}-01$ & $6.8 \mathrm{E}-02$ & $-36.45 \%$ \\
\hline Co-60 & $2.4 \mathrm{E}-02$ & $8.5 \mathrm{E}-03$ & $-64.01 \%$ & Po-210 & $1.3 \mathrm{E}+00$ & $4.7 \mathrm{E}-01$ & $-62.37 \%$ \\
\hline Ni-59 & $2.1 \mathrm{E}-04$ & $9.2 \mathrm{E}-05$ & $-55.22 \%$ & Ra-223 & $1.8 \mathrm{E}-01$ & $6.6 \mathrm{E}-02$ & $-64.35 \%$ \\
\hline Ni-63 & $5.5 \mathrm{E}-04$ & $2.4 \mathrm{E}-04$ & $-55.87 \%$ & Ra-224 & $3.4 \mathrm{E}-02$ & $1.2 \mathrm{E}-02$ & $-65.01 \%$ \\
\hline $\mathrm{Cu}-64$ & $2.9 \mathrm{E}-07$ & $9.5 \mathrm{E}-08$ & $-67.09 \%$ & Ra-225 & $1.2 \mathrm{E}-01$ & $4.4 \mathrm{E}-02$ & $-64.29 \%$ \\
\hline
\end{tabular}


SRNL-STI-2010-00447, REVISION 0

Table 31. LADTAP XL IRRIDOSE Maximally Exposed Individual Total Ingestion Pathway Comparison (mrem/yr) (continued)

\begin{tabular}{|c|c|c|c|c|c|c|c|}
\hline Nuclide & $\begin{array}{c}\text { Hamby Ladtap } \\
\text { Total }\end{array}$ & $\begin{array}{c}\text { Jannik Ladtap } \\
\text { Total }\end{array}$ & $\%$ Diff & Nuclide & $\begin{array}{c}\text { Hamby Ladtap } \\
\text { Total }\end{array}$ & $\begin{array}{c}\text { Jannik Ladtap } \\
\text { Total }\end{array}$ & $\%$ Diff \\
\hline $\mathrm{Zn}-65$ & $2.1 \mathrm{E}-02$ & $2.0 \mathrm{E}-02$ & $-3.00 \%$ & Ra-226 & $1.1 \mathrm{E}+00$ & $4.0 \mathrm{E}-01$ & $-63.85 \%$ \\
\hline Ge-68 & $4.1 \mathrm{E}-03$ & $7.5 \mathrm{E}-03$ & $83.94 \%$ & Ra-228 & $1.1 \mathrm{E}+00$ & $3.9 \mathrm{E}-01$ & $-64.50 \%$ \\
\hline Se-75 & $1.4 \mathrm{E}-02$ & $4.0 \mathrm{E}-03$ & $-71.42 \%$ & Ac- 227 & $1.2 \mathrm{E}+01$ & $4.0 \mathrm{E}+00$ & $-66.18 \%$ \\
\hline Se-79 & $1.2 \mathrm{E}-01$ & $5.1 \mathrm{E}-03$ & $-95.86 \%$ & Th-227 & $1.6 \mathrm{E}-02$ & $5.5 \mathrm{E}-03$ & $-66.14 \%$ \\
\hline $\mathrm{Rb}-87$ & $1.2 \mathrm{E}-02$ & $8.4 \mathrm{E}-03$ & $-28.57 \%$ & Th-228 & $3.2 \mathrm{E}-01$ & $1.1 \mathrm{E}-01$ & $-66.20 \%$ \\
\hline Sr-89 & $5.7 \mathrm{E}-03$ & $2.3 \mathrm{E}-03$ & $-60.52 \%$ & Th-229 & $3.0 \mathrm{E}+00$ & $1.0 \mathrm{E}+00$ & $-66.56 \%$ \\
\hline Sr-90 & $1.2 \mathrm{E}-01$ & $1.1 \mathrm{E}-01$ & $-10.97 \%$ & Th-230 & $4.6 \mathrm{E}-01$ & $1.5 \mathrm{E}-01$ & $-66.56 \%$ \\
\hline $\mathrm{Y}-90$ & $6.5 \mathrm{E}-04$ & $2.2 \mathrm{E}-04$ & $-66.23 \%$ & Th-231 & $1.1 \mathrm{E}-05$ & $3.8 \mathrm{E}-06$ & $-66.30 \%$ \\
\hline Y-91 & $6.1 \mathrm{E}-03$ & $2.1 \mathrm{E}-03$ & $-65.98 \%$ & Th-232 & $2.4 \mathrm{E}+00$ & $8.1 \mathrm{E}-01$ & $-66.56 \%$ \\
\hline Mo-93 & $2.0 \mathrm{E}-03$ & $1.0 \mathrm{E}-03$ & $-50.53 \%$ & Th-234 & $6.6 \mathrm{E}-03$ & $2.2 \mathrm{E}-03$ & $-66.14 \%$ \\
\hline Mo-99 & $3.4 \mathrm{E}-04$ & $1.1 \mathrm{E}-04$ & $-66.98 \%$ & $\mathrm{~Pa}-230$ & $2.5 \mathrm{E}-03$ & $8.2 \mathrm{E}-04$ & $-66.54 \%$ \\
\hline $\mathrm{Nb}-93 \mathrm{~m}$ & $9.3 \mathrm{E}-03$ & $1.5 \mathrm{E}-03$ & $-83.40 \%$ & $\mathrm{~Pa}-231$ & $9.9 \mathrm{E}+00$ & $3.2 \mathrm{E}+00$ & $-67.90 \%$ \\
\hline $\mathrm{Nb}-94$ & $9.2 \mathrm{E}-03$ & $1.5 \mathrm{E}-03$ & $-83.57 \%$ & $\mathrm{~Pa}-233$ & $1.8 \mathrm{E}-03$ & $6.0 \mathrm{E}-04$ & $-66.53 \%$ \\
\hline Nb-95 & $2.6 \mathrm{E}-03$ & $4.3 \mathrm{E}-04$ & $-83.30 \%$ & U-232 & $1.1 \mathrm{E}+00$ & $4.8 \mathrm{E}-01$ & $-57.12 \%$ \\
\hline Zr-93 & $1.5 \mathrm{E}-03$ & $4.6 \mathrm{E}-04$ & $-69.39 \%$ & U-233 & $2.3 \mathrm{E}-01$ & $1.0 \mathrm{E}-01$ & $-56.71 \%$ \\
\hline Zr-95 & $2.6 \mathrm{E}-03$ & 7.9E-04 & $-69.85 \%$ & U-234 & $2.2 \mathrm{E}-01$ & $9.7 \mathrm{E}-02$ & $-56.71 \%$ \\
\hline Tc-96 & $8.4 \mathrm{E}-04$ & $2.5 \mathrm{E}-04$ & $-69.65 \%$ & U-235 & $2.2 \mathrm{E}-01$ & $9.4 \mathrm{E}-02$ & $-56.71 \%$ \\
\hline Tc-97 & $1.1 \mathrm{E}-03$ & $1.5 \mathrm{E}-02$ & $1228.93 \%$ & U-236 & $2.2 \mathrm{E}-01$ & $9.4 \mathrm{E}-02$ & $-56.71 \%$ \\
\hline Tc-98 & $3.6 \mathrm{E}-02$ & $4.7 \mathrm{E}-01$ & $1228.93 \%$ & U-237 & $5.7 \mathrm{E}-04$ & $2.3 \mathrm{E}-04$ & $-60.00 \%$ \\
\hline Tc-99 & $9.7 \mathrm{E}-03$ & $1.3 \mathrm{E}-01$ & $1228.91 \%$ & U-238 & $2.0 \mathrm{E}-01$ & $8.6 \mathrm{E}-02$ & $-56.71 \%$ \\
\hline Ru-97 & $8.4 \mathrm{E}-05$ & $1.6 \mathrm{E}-05$ & $-80.60 \%$ & $\mathrm{~Np}-236$ & $6.8 \mathrm{E}-01$ & $2.5 \mathrm{E}-01$ & $-63.64 \%$ \\
\hline Ru-103 & $3.9 \mathrm{E}-03$ & $6.0 \mathrm{E}-04$ & $-84.87 \%$ & $\mathrm{~Np}-237$ & $3.3 \mathrm{E}+00$ & $1.2 \mathrm{E}+00$ & $-63.64 \%$ \\
\hline Ru-106 & $4.2 \mathrm{E}-02$ & $6.3 \mathrm{E}-03$ & $-84.95 \%$ & $\mathrm{~Np}-239$ & $1.5 \mathrm{E}-04$ & $5.1 \mathrm{E}-05$ & $-66.04 \%$ \\
\hline Pd-107 & $1.9 \mathrm{E}-04$ & $9.6 \mathrm{E}-05$ & $-49.38 \%$ & Am-237 & $3.6 \mathrm{E}-25$ & $1.2 \mathrm{E}-25$ & $-66.31 \%$ \\
\hline Ag-108m & $2.3 \mathrm{E}-02$ & $2.6 \mathrm{E}-03$ & $-88.51 \%$ & Am-241 & $3.8 \mathrm{E}+00$ & $1.3 \mathrm{E}+00$ & $-65.93 \%$ \\
\hline
\end{tabular}


SRNL-STI-2010-00447, REVISION 0

Table 31. LADTAP XL IRRIDOSE Maximally Exposed Individual Total Ingestion Pathway Comparison (mrem/yr) (continued)

\begin{tabular}{|c|c|c|c|c|c|c|c|}
\hline Nuclide & $\begin{array}{c}\text { Hamby Ladtap } \\
\text { Total }\end{array}$ & $\begin{array}{c}\text { Jannik Ladtap } \\
\text { Total }\end{array}$ & $\%$ Diff & Nuclide & $\begin{array}{c}\text { Hamby Ladtap } \\
\text { Total }\end{array}$ & $\begin{array}{c}\text { Jannik Ladtap } \\
\text { Total }\end{array}$ & $\%$ Diff \\
\hline Ag-110m & $1.6 \mathrm{E}-02$ & $3.7 \mathrm{E}-03$ & $-77.53 \%$ & Am-242m & $3.6 \mathrm{E}+00$ & $1.2 \mathrm{E}+00$ & $-65.93 \%$ \\
\hline Cd-113 & $3.1 \mathrm{E}-01$ & $1.8 \mathrm{E}-01$ & $-42.58 \%$ & Am-243 & $3.8 \mathrm{E}+00$ & $1.3 \mathrm{E}+00$ & $-65.93 \%$ \\
\hline Cd-115m & $9.6 \mathrm{E}-03$ & $3.8 \mathrm{E}-03$ & $-60.84 \%$ & $\mathrm{Pu}-237$ & $6.3 \mathrm{E}-04$ & $2.1 \mathrm{E}-04$ & $-66.28 \%$ \\
\hline In-115 & $1.2 \mathrm{E}-01$ & $4.9 \mathrm{E}-02$ & $-60.25 \%$ & $\mathrm{Pu}-238$ & $3.2 \mathrm{E}+00$ & $1.1 \mathrm{E}+00$ & $-66.30 \%$ \\
\hline Sb-122 & $4.3 \mathrm{E}-04$ & $1.4 \mathrm{E}-04$ & $-66.83 \%$ & $\mathrm{Pu}-239$ & $3.7 \mathrm{E}+00$ & $1.2 \mathrm{E}+00$ & $-66.30 \%$ \\
\hline Sb-124 & $6.5 \mathrm{E}-03$ & $2.2 \mathrm{E}-03$ & $-66.41 \%$ & $\mathrm{Pu}-240$ & $3.7 \mathrm{E}+00$ & $1.2 \mathrm{E}+00$ & $-66.30 \%$ \\
\hline Sb-125 & $2.3 \mathrm{E}-03$ & $7.6 \mathrm{E}-04$ & $-66.66 \%$ & $\mathrm{Pu}-241$ & $7.3 \mathrm{E}-02$ & $2.5 \mathrm{E}-02$ & $-66.30 \%$ \\
\hline Te-123 & $3.9 \mathrm{E}-02$ & $2.7 \mathrm{E}-03$ & $-93.10 \%$ & $\mathrm{Pu}-242$ & $3.5 \mathrm{E}+00$ & $1.2 \mathrm{E}+00$ & $-66.30 \%$ \\
\hline Te-125m & $3.2 \mathrm{E}-03$ & $9.4 \mathrm{E}-04$ & $-71.08 \%$ & $\mathrm{Pu}-244$ & $3.4 \mathrm{E}+00$ & $1.1 \mathrm{E}+00$ & $-66.30 \%$ \\
\hline Sn-126 & $1.9 \mathrm{E}-02$ & $1.5 \mathrm{E}-02$ & $-21.75 \%$ & $\mathrm{Cm}-241$ & $2.6 \mathrm{E}-03$ & 8.9E-04 & $-66.24 \%$ \\
\hline I-129 & $2.9 \mathrm{E}-01$ & $1.6 \mathrm{E}-01$ & $-44.48 \%$ & $\mathrm{Cm}-242$ & $8.6 \mathrm{E}-02$ & $2.9 \mathrm{E}-02$ & $-66.25 \%$ \\
\hline $\mathrm{I}-131$ & $1.4 \mathrm{E}-02$ & $7.6 \mathrm{E}-03$ & $-47.78 \%$ & $\mathrm{Cm}-243$ & $2.5 \mathrm{E}+00$ & $8.3 \mathrm{E}-01$ & $-66.47 \%$ \\
\hline Cs-134 & 7.3E-02 & $4.2 \mathrm{E}-02$ & $-43.17 \%$ & $\mathrm{Cm}-244$ & $2.0 \mathrm{E}+00$ & $6.6 \mathrm{E}-01$ & $-66.45 \%$ \\
\hline Cs-135 & $7.5 \mathrm{E}-03$ & $4.3 \mathrm{E}-03$ & $-41.88 \%$ & Cm-245 & $3.9 \mathrm{E}+00$ & $1.3 \mathrm{E}+00$ & $-66.53 \%$ \\
\hline Cs-137 & $5.2 \mathrm{E}-02$ & $3.0 \mathrm{E}-02$ & $-42.33 \%$ & $\mathrm{Cm}-246$ & $3.9 \mathrm{E}+00$ & $1.3 \mathrm{E}+00$ & $-66.53 \%$ \\
\hline La-137 & $3.7 \mathrm{E}-04$ & $1.3 \mathrm{E}-04$ & $-66.01 \%$ & $\mathrm{Cm}-247$ & $3.5 \mathrm{E}+00$ & $1.2 \mathrm{E}+00$ & $-66.53 \%$ \\
\hline La-138 & $5.1 \mathrm{E}-03$ & $1.7 \mathrm{E}-03$ & $-66.01 \%$ & Cm-248 & $1.4 \mathrm{E}+01$ & $4.6 \mathrm{E}+00$ & $-66.53 \%$ \\
\hline La-140 & $2.1 \mathrm{E}-04$ & $7.1 \mathrm{E}-05$ & $-66.25 \%$ & $\mathrm{Cm}-250$ & $6.7 \mathrm{E}+01$ & $2.2 \mathrm{E}+01$ & $-66.53 \%$ \\
\hline Ba-140 & $3.0 \mathrm{E}-03$ & $1.0 \mathrm{E}-03$ & $-66.20 \%$ & Bk-247 & $2.0 \mathrm{E}+00$ & $6.7 \mathrm{E}-01$ & $-65.72 \%$ \\
\hline $\mathrm{Ce}-141$ & $1.5 \mathrm{E}-03$ & $5.0 \mathrm{E}-04$ & $-66.66 \%$ & Bk-249 & 4.9E-03 & $1.6 \mathrm{E}-03$ & $-66.26 \%$ \\
\hline \multirow[t]{8}{*}{$\mathrm{Ce}-144$} & $1.6 \mathrm{E}-02$ & $5.5 \mathrm{E}-03$ & $-66.61 \%$ & Cf-249 & $3.9 \mathrm{E}+00$ & $1.3 \mathrm{E}+00$ & $-66.39 \%$ \\
\hline & & & & Cf-250 & $1.6 \mathrm{E}+00$ & $5.4 \mathrm{E}-01$ & $-66.35 \%$ \\
\hline & & & & Cf-251 & $3.9 \mathrm{E}+00$ & $1.3 \mathrm{E}+00$ & $-66.39 \%$ \\
\hline & & & & Cf-252 & 7.9E-01 & $2.7 \mathrm{E}-01$ & $-66.30 \%$ \\
\hline & & & & Es-253 & $1.1 \mathrm{E}-02$ & $3.8 \mathrm{E}-03$ & $-66.28 \%$ \\
\hline & & & & Unidentified alpha & $3.7 \mathrm{E}+00$ & $1.2 \mathrm{E}+00$ & $-66.30 \%$ \\
\hline & & & & Unidentified beta & $1.2 \mathrm{E}-01$ & $1.1 \mathrm{E}-01$ & $-11.07 \%$ \\
\hline & & & & TOTAL Dose & $1.9 \mathrm{E}+02$ & $6.5 \mathrm{E}+01$ & $-65.87 \%$ \\
\hline
\end{tabular}


Table 32. LADTAP XL IRRIDOSE Vegetable Ingestion Pathway Collective Dose Comparison (person-rem/yr)

\begin{tabular}{|c|c|c|c|c|c|c|c|}
\hline Nuclide & $\begin{array}{c}\text { Hamby Ladtap } \\
\text { Veg }\end{array}$ & $\begin{array}{c}\text { Jannik Ladtap } \\
\text { Veg }\end{array}$ & $\%$ Diff & Nuclide & $\begin{array}{c}\text { Hamby Ladtap } \\
\text { Veg }\end{array}$ & $\begin{array}{c}\text { Jannik } \\
\text { Ladtap } \\
\text { Veg }\end{array}$ & $\%$ Diff \\
\hline H-3 & $2.00 \mathrm{E}-05$ & $6.28 \mathrm{E}-05$ & $214.25 \%$ & Sm-146 & $1.52 \mathrm{E}+00$ & $1.70 \mathrm{E}+00$ & $11.71 \%$ \\
\hline $\mathrm{Be}-7$ & $5.44 \mathrm{E}-04$ & $6.39 \mathrm{E}-04$ & $17.46 \%$ & Sm-147 & $1.37 \mathrm{E}+00$ & $1.53 \mathrm{E}+00$ & $11.71 \%$ \\
\hline Be-10 & $3.22 \mathrm{E}-02$ & $3.39 \mathrm{E}-02$ & $5.27 \%$ & Sm-151 & $2.59 \mathrm{E}-03$ & $2.88 \mathrm{E}-03$ & $11.19 \%$ \\
\hline $\mathrm{C}-14$ & $3.94 \mathrm{E}-01$ & $5.58 \mathrm{E}-02$ & $-85.84 \%$ & Pm-147 & $7.00 \mathrm{E}-03$ & $7.89 \mathrm{E}-03$ & $12.71 \%$ \\
\hline $\mathrm{Na}-22$ & $9.19 \mathrm{E}-02$ & $9.54 \mathrm{E}-02$ & $3.83 \%$ & Eu-152 & $4.53 \mathrm{E}-02$ & $4.95 \mathrm{E}-02$ & $9.25 \%$ \\
\hline $\mathrm{Na}-24$ & $9.01 \mathrm{E}-12$ & $6.85 \mathrm{E}-08$ & $760467.65 \%$ & Eu-154 & $6.85 \mathrm{E}-02$ & $7.43 \mathrm{E}-02$ & $8.56 \%$ \\
\hline Al-26 & $9.97 \mathrm{E}-02$ & $1.04 \mathrm{E}-01$ & $4.28 \%$ & Eu-155 & $9.71 \mathrm{E}-03$ & $1.05 \mathrm{E}-02$ & $7.78 \%$ \\
\hline $\mathrm{P}-32$ & $1.43 \mathrm{E}-02$ & $2.20 \mathrm{E}-02$ & $54.31 \%$ & Gd-152 & $1.14 \mathrm{E}+00$ & $1.28 \mathrm{E}+00$ & $11.96 \%$ \\
\hline $\mathrm{Si}-32$ & $1.76 \mathrm{E}-02$ & $1.96 \mathrm{E}-02$ & $10.98 \%$ & Нo-166m & $5.94 \mathrm{E}-02$ & $6.64 \mathrm{E}-02$ & $11.67 \%$ \\
\hline S-35 & $3.96 \mathrm{E}-03$ & $4.59 \mathrm{E}-03$ & $15.86 \%$ & Lu-176 & $5.03 \mathrm{E}-02$ & $5.34 \mathrm{E}-02$ & $6.13 \%$ \\
\hline $\mathrm{Cl}-36$ & $1.99 \mathrm{E}+00$ & $1.45 \mathrm{E}+00$ & $-27.08 \%$ & Ta-180 & $0.00 \mathrm{E}+00$ & $0.00 \mathrm{E}+00$ & $0.00 \%$ \\
\hline $\mathrm{K}-40$ & $3.30 \mathrm{E}-01$ & $8.09 \mathrm{E}-01$ & $144.93 \%$ & Hf-182 & $1.06 \mathrm{E}-01$ & $1.12 \mathrm{E}-01$ & $6.18 \%$ \\
\hline K-43 & $2.48 \mathrm{E}-09$ & $1.03 \mathrm{E}-06$ & $41520.29 \%$ & Re-186m & $2.28 \mathrm{E}+01$ & $8.08 \mathrm{E}-02$ & $-99.64 \%$ \\
\hline $\mathrm{Ca}-41$ & $2.87 \mathrm{E}-02$ & $6.48 \mathrm{E}-01$ & $2155.91 \%$ & Re-187 & $5.72 \mathrm{E}-02$ & $2.03 \mathrm{E}-04$ & $-99.64 \%$ \\
\hline $\mathrm{Ca}-45$ & $2.12 \mathrm{E}-02$ & $6.18 \mathrm{E}-02$ & $192.14 \%$ & Ir-192m & $0.00 \mathrm{E}+00$ & $0.00 \mathrm{E}+00$ & $0.00 \%$ \\
\hline $\mathrm{Ca}-47$ & $1.03 \mathrm{E}-03$ & $4.37 \mathrm{E}-03$ & $324.90 \%$ & Pt-193 & $9.04 \mathrm{E}-04$ & 9.39E-04 & $3.88 \%$ \\
\hline Ti-44 & $1.43 \mathrm{E}-01$ & $1.53 \mathrm{E}-01$ & $6.75 \%$ & $\mathrm{Hg}-194$ & $5.56 \mathrm{E}+00$ & $5.63 \mathrm{E}+00$ & $1.29 \%$ \\
\hline $\mathrm{V}-49$ & $3.79 \mathrm{E}-04$ & $4.09 \mathrm{E}-04$ & $7.68 \%$ & $\mathrm{Hg}-203$ & $4.75 \mathrm{E}-02$ & $5.64 \mathrm{E}-02$ & $18.70 \%$ \\
\hline Cr-51 & $4.48 \mathrm{E}-04$ & $5.79 \mathrm{E}-04$ & $29.31 \%$ & $\mathrm{~Pb}-202$ & $3.07 \mathrm{E}-01$ & $3.39 \mathrm{E}-01$ & $10.43 \%$ \\
\hline $\mathrm{Mn}-53$ & $8.41 \mathrm{E}-04$ & $1.65 \mathrm{E}-03$ & $96.64 \%$ & $\mathrm{~Pb}-205$ & $1.18 \mathrm{E}-02$ & $1.30 \mathrm{E}-02$ & $10.43 \%$ \\
\hline Mn-54 & $1.90 \mathrm{E}-02$ & $2.15 \mathrm{E}-02$ & $12.89 \%$ & $\mathrm{~Pb}-210$ & $3.96 \mathrm{E}+01$ & $4.31 \mathrm{E}+01$ & $8.93 \%$ \\
\hline $\mathrm{Fe}-55$ & $4.27 \mathrm{E}-03$ & $4.68 \mathrm{E}-03$ & $9.54 \%$ & $\mathrm{Bi}-207$ & $5.00 \mathrm{E}-02$ & 8.99E-02 & $79.73 \%$ \\
\hline Fe-60 & $1.13 \mathrm{E}+00$ & $1.42 \mathrm{E}+00$ & $25.40 \%$ & Bi-210 & $1.33 \mathrm{E}-03$ & $4.26 \mathrm{E}-03$ & $221.05 \%$ \\
\hline Co-58 & $1.91 \mathrm{E}-02$ & $2.20 \mathrm{E}-02$ & $15.07 \%$ & $\mathrm{Bi}-210 \mathrm{~m}$ & $9.30 \mathrm{E}-01$ & $1.83 \mathrm{E}+00$ & $96.73 \%$ \\
\hline Co-60 & $1.96 \mathrm{E}-01$ & $2.31 \mathrm{E}-01$ & $17.76 \%$ & Po-210 & $1.02 \mathrm{E}+01$ & $1.13 \mathrm{E}+01$ & $10.20 \%$ \\
\hline $\mathrm{Ni}-59$ & $1.63 \mathrm{E}-03$ & $2.19 \mathrm{E}-03$ & $34.22 \%$ & $\mathrm{Ra}-223$ & $7.30 \mathrm{E}-01$ & $1.25 \mathrm{E}+00$ & $71.92 \%$ \\
\hline Ni-63 & $4.38 \mathrm{E}-03$ & $5.78 \mathrm{E}-03$ & $31.93 \%$ & Ra-224 & $2.47 \mathrm{E}-02$ & $1.21 \mathrm{E}-01$ & $389.63 \%$ \\
\hline $\mathrm{Cu}-64$ & $9.63 \mathrm{E}-14$ & $3.65 \mathrm{E}-09$ & $3785338.19 \%$ & Ra-225 & $5.86 \mathrm{E}-01$ & $9.02 \mathrm{E}-01$ & $53.97 \%$ \\
\hline
\end{tabular}


SRNL-STI-2010-00447, REVISION 0

Table 32. LADTAP XL IRRIDOSE Vegetable Ingestion Pathway Collective Dose Comparison (person-rem/yr) (continued)

\begin{tabular}{|c|c|c|c|c|c|c|c|}
\hline Nuclide & $\begin{array}{c}\text { Hamby Ladtap } \\
\text { Veg }\end{array}$ & $\begin{array}{c}\text { Jannik Ladtap } \\
\text { Veg }\end{array}$ & $\%$ Diff & Nuclide & $\begin{array}{c}\text { Hamby Ladtap } \\
\text { Veg }\end{array}$ & $\begin{array}{c}\text { Jannik Ladtap } \\
\text { Veg }\end{array}$ & $\%$ Diff \\
\hline Zn-65 & $1.04 \mathrm{E}-01$ & $1.16 \mathrm{E}-01$ & $11.33 \%$ & Ra-226 & $9.73 \mathrm{E}+00$ & $1.06 \mathrm{E}+01$ & $8.44 \%$ \\
\hline Ge-68 & $7.85 \mathrm{E}-03$ & $8.36 \mathrm{E}-03$ & $6.54 \%$ & Ra-228 & $9.54 \mathrm{E}+00$ & $1.01 \mathrm{E}+01$ & $5.98 \%$ \\
\hline Se-75 & $6.28 \mathrm{E}-02$ & $6.11 \mathrm{E}-02$ & $-2.75 \%$ & Ac-227 & $1.06 \mathrm{E}+02$ & $1.12 \mathrm{E}+02$ & $5.17 \%$ \\
\hline Se-79 & $4.17 \mathrm{E}-01$ & $8.77 \mathrm{E}-02$ & $-78.95 \%$ & Th-227 & $8.84 \mathrm{E}-02$ & $1.26 \mathrm{E}-01$ & $42.35 \%$ \\
\hline $\mathrm{Rb}-87$ & $5.67 \mathrm{E}-02$ & $1.29 \mathrm{E}-01$ & $128.42 \%$ & Th-228 & $2.78 \mathrm{E}+00$ & $2.96 \mathrm{E}+00$ & $6.50 \%$ \\
\hline Sr-89 & $4.21 \mathrm{E}-02$ & $5.08 \mathrm{E}-02$ & $20.51 \%$ & Th-229 & $2.69 \mathrm{E}+01$ & $2.81 \mathrm{E}+01$ & $4.53 \%$ \\
\hline Sr-90 & $1.04 \mathrm{E}+00$ & $2.67 \mathrm{E}+00$ & $158.34 \%$ & Th-230 & $4.07 \mathrm{E}+00$ & $4.25 \mathrm{E}+00$ & $4.53 \%$ \\
\hline $\mathrm{Y}-90$ & $1.98 \mathrm{E}-04$ & $1.66 \mathrm{E}-03$ & $740.66 \%$ & Th-231 & $2.12 \mathrm{E}-08$ & $4.11 \mathrm{E}-06$ & $19353.17 \%$ \\
\hline Y-91 & $4.57 \mathrm{E}-02$ & $5.31 \mathrm{E}-02$ & $16.34 \%$ & Th-232 & $2.15 \mathrm{E}+01$ & $2.25 \mathrm{E}+01$ & $4.53 \%$ \\
\hline Mo-93 & $1.49 \mathrm{E}-02$ & $2.58 \mathrm{E}-02$ & $72.98 \%$ & Th-234 & $4.02 \mathrm{E}-02$ & $5.36 \mathrm{E}-02$ & $33.24 \%$ \\
\hline Мo-99 & $9.99 \mathrm{E}-05$ & $7.96 \mathrm{E}-04$ & $696.59 \%$ & Pa-230 & $1.27 \mathrm{E}-02$ & $1.86 \mathrm{E}-02$ & $45.56 \%$ \\
\hline $\mathrm{Nb}-93 \mathrm{~m}$ & $4.08 \mathrm{E}-02$ & $4.30 \mathrm{E}-02$ & $5.51 \%$ & $\mathrm{~Pa}-231$ & $8.65 \mathrm{E}+01$ & $8.79 \mathrm{E}+01$ & $1.56 \%$ \\
\hline Nb-94 & $4.00 \mathrm{E}-02$ & $4.22 \mathrm{E}-02$ & $5.50 \%$ & $\mathrm{~Pa}-233$ & $1.12 \mathrm{E}-02$ & $1.45 \mathrm{E}-02$ & $29.96 \%$ \\
\hline Nb-95 & $8.84 \mathrm{E}-03$ & $1.10 \mathrm{E}-02$ & $24.03 \%$ & U-232 & $9.89 \mathrm{E}+00$ & $1.14 \mathrm{E}+01$ & $15.52 \%$ \\
\hline Zr-93 & $1.21 \mathrm{E}-02$ & $1.29 \mathrm{E}-02$ & $7.20 \%$ & U-233 & $2.06 \mathrm{E}+00$ & $2.40 \mathrm{E}+00$ & $16.71 \%$ \\
\hline $\mathrm{Zr}-95$ & $1.80 \mathrm{E}-02$ & $2.08 \mathrm{E}-02$ & $15.44 \%$ & U-234 & $1.98 \mathrm{E}+00$ & $2.31 \mathrm{E}+00$ & $16.71 \%$ \\
\hline Tc-96 & $3.77 \mathrm{E}-04$ & $2.68 \mathrm{E}-03$ & $610.00 \%$ & U-235 & $1.91 \mathrm{E}+00$ & $2.22 \mathrm{E}+00$ & $16.71 \%$ \\
\hline Tc-97 & $2.36 \mathrm{E}-03$ & $3.67 \mathrm{E}-01$ & $15444.16 \%$ & U-236 & $1.91 \mathrm{E}+00$ & $2.22 \mathrm{E}+00$ & $16.71 \%$ \\
\hline Tc-98 & $7.56 \mathrm{E}-02$ & $1.17 \mathrm{E}+01$ & $15444.17 \%$ & U-237 & $1.31 \mathrm{E}-03$ & $3.17 \mathrm{E}-03$ & $141.42 \%$ \\
\hline Tc-99 & $2.05 \mathrm{E}-02$ & $3.18 \mathrm{E}+00$ & $15443.83 \%$ & U-238 & $1.75 \mathrm{E}+00$ & $2.05 \mathrm{E}+00$ & $16.71 \%$ \\
\hline Ru-97 & $1.87 \mathrm{E}-05$ & $1.34 \mathrm{E}-04$ & $615.87 \%$ & $\mathrm{~Np}-236$ & $6.02 \mathrm{E}+00$ & $6.73 \mathrm{E}+00$ & $11.73 \%$ \\
\hline Ru-103 & $1.16 \mathrm{E}-02$ & $1.41 \mathrm{E}-02$ & $21.71 \%$ & $\mathrm{~Np}-237$ & $2.97 \mathrm{E}+01$ & $3.32 \mathrm{E}+01$ & $11.73 \%$ \\
\hline Ru-106 & $1.51 \mathrm{E}-01$ & $1.61 \mathrm{E}-01$ & $6.43 \%$ & $\mathrm{~Np}-239$ & $2.89 \mathrm{E}-05$ & $3.24 \mathrm{E}-04$ & $1021.41 \%$ \\
\hline Pd-107 & $1.51 \mathrm{E}-03$ & $1.36 \mathrm{E}-03$ & $-10.03 \%$ & Am-237 & $0.00 \mathrm{E}+00$ & $0.00 \mathrm{E}+00$ & $0.00 \%$ \\
\hline $\mathrm{Ag}-108 \mathrm{~m}$ & $9.15 \mathrm{E}-02$ & $6.00 \mathrm{E}-02$ & $-34.46 \%$ & Am-241 & $3.40 \mathrm{E}+01$ & $3.60 \mathrm{E}+01$ & $5.89 \%$ \\
\hline
\end{tabular}


SRNL-STI-2010-00447, REVISION 0

Table 32. LADTAP XL IRRIDOSE Vegetable Ingestion Pathway Collective Dose Comparison (person-rem/yr) (continued)

\begin{tabular}{|c|c|c|c|c|c|c|c|}
\hline Nuclide & $\begin{array}{c}\text { Hamby Ladtap } \\
\text { Veg }\end{array}$ & $\begin{array}{c}\text { Jannik Ladtap } \\
\text { Veg }\end{array}$ & $\%$ Diff & Nuclide & $\begin{array}{c}\text { Hamby Ladtap } \\
\text { Veg }\end{array}$ & $\begin{array}{c}\text { Jannik Ladtap } \\
\text { Veg }\end{array}$ & $\%$ Diff \\
\hline Ag-110m & $7.81 \mathrm{E}-02$ & $8.18 \mathrm{E}-02$ & $4.70 \%$ & Am-242m & $3.17 \mathrm{E}+01$ & $3.36 \mathrm{E}+01$ & $5.90 \%$ \\
\hline Cd-113 & $2.73 \mathrm{E}+00$ & $4.56 \mathrm{E}+00$ & $67.09 \%$ & Am-243 & $3.40 \mathrm{E}+01$ & $3.60 \mathrm{E}+01$ & $5.89 \%$ \\
\hline $\mathrm{Cd}-115 \mathrm{~m}$ & $6.97 \mathrm{E}-02$ & $8.42 \mathrm{E}-02$ & $20.82 \%$ & $\mathrm{Pu}-237$ & $4.60 \mathrm{E}-03$ & $5.51 \mathrm{E}-03$ & $19.65 \%$ \\
\hline In-115 & $1.07 \mathrm{E}+00$ & $1.12 \mathrm{E}+00$ & $4.95 \%$ & $\mathrm{Pu}-238$ & $2.87 \mathrm{E}+01$ & $3.03 \mathrm{E}+01$ & $5.81 \%$ \\
\hline Sb-122 & $1.32 \mathrm{E}-04$ & $1.08 \mathrm{E}-03$ & $720.62 \%$ & $\mathrm{Pu}-239$ & $3.25 \mathrm{E}+01$ & $3.43 \mathrm{E}+01$ & $5.79 \%$ \\
\hline $\mathrm{Sb}-124$ & $4.82 \mathrm{E}-02$ & $5.59 \mathrm{E}-02$ & $16.00 \%$ & $\mathrm{Pu}-240$ & $3.25 \mathrm{E}+01$ & $3.43 \mathrm{E}+01$ & $5.79 \%$ \\
\hline Sb-125 & $1.93 \mathrm{E}-02$ & $2.04 \mathrm{E}-02$ & $5.55 \%$ & $\mathrm{Pu}-241$ & $6.46 \mathrm{E}-01$ & $6.84 \mathrm{E}-01$ & $5.93 \%$ \\
\hline Te-123 & $2.06 \mathrm{E}-01$ & $6.55 \mathrm{E}-02$ & $-68.19 \%$ & $\mathrm{Pu}-242$ & $3.09 \mathrm{E}+01$ & $3.27 \mathrm{E}+01$ & $5.79 \%$ \\
\hline Te-125m & $1.88 \mathrm{E}-02$ & $2.05 \mathrm{E}-02$ & $9.10 \%$ & $\mathrm{Pu}-244$ & $3.02 \mathrm{E}+01$ & $3.19 \mathrm{E}+01$ & $5.79 \%$ \\
\hline Sn-126 & $1.30 \mathrm{E}-01$ & $1.41 \mathrm{E}-01$ & $8.80 \%$ & $\mathrm{Cm}-241$ & $1.78 \mathrm{E}-02$ & $2.23 \mathrm{E}-02$ & $25.35 \%$ \\
\hline I-129 & $2.29 \mathrm{E}+00$ & $2.74 \mathrm{E}+00$ & $19.33 \%$ & $\mathrm{Cm}-242$ & $7.20 \mathrm{E}-01$ & $7.88 \mathrm{E}-01$ & $9.50 \%$ \\
\hline I-131 & $3.79 \mathrm{E}-02$ & $8.00 \mathrm{E}-02$ & $111.07 \%$ & $\mathrm{Cm}-243$ & $2.20 \mathrm{E}+01$ & $2.31 \mathrm{E}+01$ & $5.19 \%$ \\
\hline Cs-134 & $5.44 \mathrm{E}-01$ & $5.85 \mathrm{E}-01$ & $7.46 \%$ & Cm-244 & $1.74 \mathrm{E}+01$ & $1.83 \mathrm{E}+01$ & $5.31 \%$ \\
\hline Cs-135 & $5.59 \mathrm{E}-02$ & $6.33 \mathrm{E}-02$ & $13.34 \%$ & Cm-245 & $3.43 \mathrm{E}+01$ & $3.60 \mathrm{E}+01$ & $4.96 \%$ \\
\hline Cs-137 & $3.89 \mathrm{E}-01$ & $4.34 \mathrm{E}-01$ & $11.48 \%$ & Cm-246 & $3.43 \mathrm{E}+01$ & $3.60 \mathrm{E}+01$ & $4.96 \%$ \\
\hline La-137 & $3.28 \mathrm{E}-03$ & $3.49 \mathrm{E}-03$ & $6.36 \%$ & $\mathrm{Cm}-247$ & $3.12 \mathrm{E}+01$ & $3.28 \mathrm{E}+01$ & $4.96 \%$ \\
\hline La-138 & $4.50 \mathrm{E}-02$ & $4.78 \mathrm{E}-02$ & $6.36 \%$ & $\mathrm{Cm}-248$ & $1.22 \mathrm{E}+02$ & $1.28 \mathrm{E}+02$ & $4.96 \%$ \\
\hline La-140 & $8.66 \mathrm{E}-06$ & $2.49 \mathrm{E}-04$ & $2781.91 \%$ & $\mathrm{Cm}-250$ & $5.92 \mathrm{E}+02$ & $6.21 \mathrm{E}+02$ & $4.96 \%$ \\
\hline Ba-140 & $1.31 \mathrm{E}-02$ & $2.14 \mathrm{E}-02$ & $63.33 \%$ & Bk-247 & $1.73 \mathrm{E}+01$ & $1.87 \mathrm{E}+01$ & $7.64 \%$ \\
\hline Ce-141 & 9.99E-03 & $1.25 \mathrm{E}-02$ & $25.55 \%$ & Bk-249 & $4.21 \mathrm{E}-02$ & $4.53 \mathrm{E}-02$ & $7.81 \%$ \\
\hline Ce-144 & $1.39 \mathrm{E}-01$ & $1.50 \mathrm{E}-01$ & $8.03 \%$ & Cf-249 & $3.48 \mathrm{E}+01$ & $3.67 \mathrm{E}+01$ & $5.53 \%$ \\
\hline & & & & Cf-250 & $1.43 \mathrm{E}+01$ & $1.51 \mathrm{E}+01$ & $5.77 \%$ \\
\hline & & & & Cf-251 & $3.48 \mathrm{E}+01$ & $3.68 \mathrm{E}+01$ & $5.52 \%$ \\
\hline & & & & Cf-252 & $6.92 \mathrm{E}+00$ & $7.37 \mathrm{E}+00$ & $6.41 \%$ \\
\hline & & & & Es-253 & $6.43 \mathrm{E}-02$ & $8.93 \mathrm{E}-02$ & $38.82 \%$ \\
\hline & & & & Unidentified alpha & $3.25 \mathrm{E}+01$ & $3.43 \mathrm{E}+01$ & $5.79 \%$ \\
\hline & & & & Unidentified beta & $1.04 \mathrm{E}+00$ & $2.67 \mathrm{E}+00$ & $158.04 \%$ \\
\hline & & & & TOTAL Dose & $1.7 E+03$ & $1.76 \mathrm{E}+03$ & $5.35 \%$ \\
\hline
\end{tabular}


SRNL-STI-2010-00447, REVISION 0

Table 33. LADTAP XL IRRIDOSE Milk Ingestion Pathway Collective Dose Comparison (person-rem/yr)

\begin{tabular}{|c|c|c|c|c|c|c|c|}
\hline Nuclide & $\begin{array}{c}\text { Hamby Ladtap } \\
\text { Milk }\end{array}$ & $\begin{array}{c}\text { Jannik Ladtap } \\
\text { Milk }\end{array}$ & $\%$ Diff & Nuclide & $\begin{array}{c}\text { Hamby Ladtap } \\
\text { Milk }\end{array}$ & $\begin{array}{c}\text { Jannik Ladtap } \\
\text { Milk }\end{array}$ & $\%$ Diff \\
\hline $\mathrm{H}-3$ & $2.5 \mathrm{E}-05$ & $9.7 \mathrm{E}-06$ & $-60.69 \%$ & Sm-146 & $1.8 \mathrm{E}-04$ & $1.0 \mathrm{E}-03$ & $479.16 \%$ \\
\hline $\mathrm{Be}-7$ & $3.2 \mathrm{E}-08$ & $1.3 \mathrm{E}-08$ & $-60.31 \%$ & Sm-147 & $1.6 \mathrm{E}-04$ & $9.3 \mathrm{E}-04$ & $479.16 \%$ \\
\hline Be-10 & $1.5 \mathrm{E}-06$ & 5.9E-07 & $-61.44 \%$ & Sm-151 & $3.0 \mathrm{E}-07$ & $1.8 \mathrm{E}-06$ & $479.10 \%$ \\
\hline $\mathrm{C}-14$ & $3.0 \mathrm{E}-01$ & $8.0 \mathrm{E}-03$ & $-97.29 \%$ & Pm-147 & $8.2 \mathrm{E}-07$ & $4.9 \mathrm{E}-06$ & $491.46 \%$ \\
\hline $\mathrm{Na}-22$ & $9.2 \mathrm{E}-02$ & $2.6 \mathrm{E}-02$ & $-71.57 \%$ & Eu-152 & $5.3 \mathrm{E}-06$ & $3.1 \mathrm{E}-05$ & $479.20 \%$ \\
\hline $\mathrm{Na}-24$ & $6.9 \mathrm{E}-06$ & $1.2 \mathrm{E}-06$ & $-83.06 \%$ & Eu-154 & $8.0 \mathrm{E}-06$ & $4.6 \mathrm{E}-05$ & $479.43 \%$ \\
\hline Al-26 & $4.7 \mathrm{E}-04$ & $4.5 \mathrm{E}-04$ & $-4.64 \%$ & Eu-155 & $1.1 \mathrm{E}-06$ & $6.6 \mathrm{E}-06$ & $479.91 \%$ \\
\hline $\mathrm{P}-32$ & $1.9 \mathrm{E}-02$ & $1.3 \mathrm{E}-02$ & $-29.97 \%$ & Gd-152 & $1.6 \mathrm{E}-03$ & $7.8 \mathrm{E}-04$ & $-51.45 \%$ \\
\hline $\mathrm{Si}-32$ & $1.2 \mathrm{E}-05$ & $6.7 \mathrm{E}-06$ & $-44.60 \%$ & Ho- $166 \mathrm{~m}$ & $2.8 \mathrm{E}-05$ & $4.0 \mathrm{E}-05$ & $44.79 \%$ \\
\hline S-35 & $2.1 \mathrm{E}-03$ & 7.9E-04 & $-61.67 \%$ & Lu-176 & $2.4 \mathrm{E}-05$ & $2.3 \mathrm{E}-05$ & $-2.54 \%$ \\
\hline Cl-36 & $2.5 \mathrm{E}+00$ & $2.0 \mathrm{E}-01$ & $-91.98 \%$ & Ta-180 & $3.5 \mathrm{E}-273$ & $1.6 \mathrm{E}-274$ & $-95.49 \%$ \\
\hline $\mathrm{K}-40$ & $1.1 \mathrm{E}-01$ & $6.1 \mathrm{E}-02$ & $-43.01 \%$ & Hf-182 & $1.3 \mathrm{E}-06$ & $1.3 \mathrm{E}-06$ & $-3.00 \%$ \\
\hline $\mathrm{K}-43$ & 4.7E-06 & $2.8 \mathrm{E}-06$ & $-40.43 \%$ & Re-186m & $2.0 \mathrm{E}-01$ & $1.5 \mathrm{E}-03$ & $-99.27 \%$ \\
\hline $\mathrm{Ca}-41$ & $4.4 \mathrm{E}-03$ & $5.3 \mathrm{E}-02$ & $1106.95 \%$ & Re-187 & $5.1 \mathrm{E}-04$ & $3.8 \mathrm{E}-06$ & $-99.27 \%$ \\
\hline Ca-45 & $1.8 \mathrm{E}-03$ & $8.0 \mathrm{E}-03$ & $351.72 \%$ & Ir-192m & $0.0 \mathrm{E}+00$ & $0.0 \mathrm{E}+00$ & \#DIV/0! \\
\hline $\mathrm{Ca}-47$ & $6.2 \mathrm{E}-04$ & $1.8 \mathrm{E}-03$ & $185.31 \%$ & Pt-193 & $1.2 \mathrm{E}-04$ & $9.8 \mathrm{E}-05$ & $-18.21 \%$ \\
\hline $\mathrm{Ti}-44$ & $1.8 \mathrm{E}-06$ & $2.4 \mathrm{E}-04$ & $13200.83 \%$ & Hg-194 & $1.3 \mathrm{E}-01$ & $3.5 \mathrm{E}-02$ & $-74.07 \%$ \\
\hline $\mathrm{V}-49$ & $1.8 \mathrm{E}-07$ & $1.8 \mathrm{E}-07$ & $-0.41 \%$ & $\mathrm{Hg}-203$ & $7.4 \mathrm{E}-04$ & $6.3 \mathrm{E}-04$ & $-13.97 \%$ \\
\hline $\mathrm{Cr}-51$ & $3.5 \mathrm{E}-05$ & $6.5 \mathrm{E}-06$ & $-81.54 \%$ & $\mathrm{~Pb}-202$ & $2.3 \mathrm{E}-03$ & $1.3 \mathrm{E}-03$ & $-43.37 \%$ \\
\hline Mn-53 & $5.8 \mathrm{E}-06$ & $9.7 \mathrm{E}-07$ & $-83.34 \%$ & $\mathrm{~Pb}-205$ & $8.8 \mathrm{E}-05$ & $5.0 \mathrm{E}-05$ & $-43.37 \%$ \\
\hline Mn-54 & $1.2 \mathrm{E}-04$ & $1.8 \mathrm{E}-05$ & $-84.15 \%$ & $\mathrm{~Pb}-210$ & $2.9 \mathrm{E}-01$ & $1.7 \mathrm{E}-01$ & $-42.35 \%$ \\
\hline $\mathrm{Fe}-55$ & $1.2 \mathrm{E}-04$ & $3.4 \mathrm{E}-06$ & $-97.15 \%$ & Bi-207 & $8.5 \mathrm{E}-04$ & $6.2 \mathrm{E}-04$ & $-27.24 \%$ \\
\hline Fe-60 & $3.2 \mathrm{E}-02$ & $9.5 \mathrm{E}-04$ & $-96.99 \%$ & $\mathrm{Bi}-210$ & $1.1 \mathrm{E}-04$ & $9.2 \mathrm{E}-05$ & $-16.50 \%$ \\
\hline Co-58 & $5.3 \mathrm{E}-04$ & $5.6 \mathrm{E}-05$ & $-89.44 \%$ & $\mathrm{Bi}-210 \mathrm{~m}$ & $1.7 \mathrm{E}-02$ & $1.2 \mathrm{E}-02$ & $-28.36 \%$ \\
\hline Co-60 & $4.7 \mathrm{E}-03$ & $5.0 \mathrm{E}-04$ & $-89.26 \%$ & Po-210 & $8.8 \mathrm{E}-02$ & $5.2 \mathrm{E}-02$ & $-40.42 \%$ \\
\hline Ni-59 & $2.9 \mathrm{E}-04$ & $3.7 \mathrm{E}-05$ & $-87.24 \%$ & Ra-223 & $4.4 \mathrm{E}-02$ & $1.5 \mathrm{E}-02$ & $-65.54 \%$ \\
\hline Ni-63 & $7.6 \mathrm{E}-04$ & $9.8 \mathrm{E}-05$ & $-87.21 \%$ & Ra-224 & $8.0 \mathrm{E}-03$ & $2.4 \mathrm{E}-03$ & $-69.81 \%$ \\
\hline $\mathrm{Cu}-64$ & $2.6 \mathrm{E}-07$ & $1.9 \mathrm{E}-08$ & $-92.86 \%$ & Ra-225 & $2.9 \mathrm{E}-02$ & $1.0 \mathrm{E}-02$ & $-65.02 \%$ \\
\hline
\end{tabular}


SRNL-STI-2010-00447, REVISION 0

Table 33. LADTAP XL IRRIDOSE Milk Ingestion Pathway Collective Dose Comparison (person-rem/yr) (continued)

\begin{tabular}{|c|c|c|c|c|c|c|c|}
\hline Nuclide & $\begin{array}{c}\text { Hamby Ladtap } \\
\text { Milk }\end{array}$ & $\begin{array}{c}\text { Jannik Ladtap } \\
\text { Milk }\end{array}$ & $\%$ Diff & Nuclide & $\begin{array}{c}\text { Hamby Ladtap } \\
\text { Milk }\end{array}$ & $\begin{array}{c}\text { Jannik Ladtap } \\
\text { Milk }\end{array}$ & $\%$ Diff \\
\hline $\mathrm{Zn}-65$ & $1.1 \mathrm{E}-01$ & $6.4 \mathrm{E}-03$ & $-94.36 \%$ & Ra-226 & $2.8 \mathrm{E}-01$ & $7.6 \mathrm{E}-02$ & $-73.23 \%$ \\
\hline Ge-68 & $1.4 \mathrm{E}-02$ & $1.3 \mathrm{E}-02$ & $-6.48 \%$ & Ra-228 & $2.5 \mathrm{E}-01$ & $7.8 \mathrm{E}-02$ & $-68.11 \%$ \\
\hline Se-75 & $8.8 \mathrm{E}-02$ & $5.4 \mathrm{E}-03$ & $-93.84 \%$ & Ac-227 & $5.0 \mathrm{E}-02$ & $4.7 \mathrm{E}-02$ & $-5.12 \%$ \\
\hline Se-79 & $1.1 \mathrm{E}+00$ & $6.3 \mathrm{E}-03$ & $-99.42 \%$ & Th-227 & $1.9 \mathrm{E}-05$ & $1.8 \mathrm{E}-05$ & $-6.80 \%$ \\
\hline $\mathrm{Rb}-87$ & $6.4 \mathrm{E}-02$ & $1.9 \mathrm{E}-02$ & $-71.31 \%$ & Th-228 & $3.3 \mathrm{E}-04$ & $3.2 \mathrm{E}-04$ & $-3.77 \%$ \\
\hline Sr-89 & $1.0 \mathrm{E}-03$ & $1.6 \mathrm{E}-03$ & $56.16 \%$ & Th-229 & $3.2 \mathrm{E}-03$ & $3.0 \mathrm{E}-03$ & $-7.51 \%$ \\
\hline Sr-90 & $2.1 \mathrm{E}-02$ & $4.6 \mathrm{E}-02$ & $116.93 \%$ & Th-230 & $4.8 \mathrm{E}-04$ & $4.5 \mathrm{E}-04$ & $-7.52 \%$ \\
\hline $\mathrm{Y}-90$ & $1.5 \mathrm{E}-06$ & $2.3 \mathrm{E}-06$ & $50.21 \%$ & Th-231 & $9.3 \mathrm{E}-09$ & $5.6 \mathrm{E}-09$ & $-39.87 \%$ \\
\hline Y-91 & $1.3 \mathrm{E}-05$ & $2.5 \mathrm{E}-05$ & $91.54 \%$ & Th-232 & $2.6 \mathrm{E}-03$ & $2.4 \mathrm{E}-03$ & $-7.52 \%$ \\
\hline Mo-93 & 4.2E-03 & $3.8 \mathrm{E}-04$ & $-90.92 \%$ & Th-234 & $7.6 \mathrm{E}-06$ & $7.1 \mathrm{E}-06$ & $-5.96 \%$ \\
\hline Mo-99 & $5.2 \mathrm{E}-04$ & $5.8 \mathrm{E}-05$ & $-88.94 \%$ & Pa-230 & $2.8 \mathrm{E}-06$ & $2.6 \mathrm{E}-06$ & $-7.12 \%$ \\
\hline $\mathrm{Nb}-93 \mathrm{~m}$ & $2.5 \mathrm{E}-03$ & $3.7 \mathrm{E}-07$ & $-99.99 \%$ & $\mathrm{~Pa}-231$ & $1.1 \mathrm{E}-02$ & $9.3 \mathrm{E}-03$ & $-13.46 \%$ \\
\hline $\mathrm{Nb}-94$ & $2.5 \mathrm{E}-03$ & $3.6 \mathrm{E}-07$ & $-99.99 \%$ & $\mathrm{~Pa}-233$ & $2.0 \mathrm{E}-06$ & $1.9 \mathrm{E}-06$ & $-5.69 \%$ \\
\hline $\mathrm{Nb}-95$ & $7.2 \mathrm{E}-04$ & $1.1 \mathrm{E}-07$ & $-99.98 \%$ & U-232 & $1.2 \mathrm{E}-01$ & $4.1 \mathrm{E}-01$ & $252.94 \%$ \\
\hline Zr-93 & $1.4 \mathrm{E}-06$ & $9.8 \mathrm{E}-07$ & $-29.96 \%$ & U-233 & $2.4 \mathrm{E}-02$ & $8.6 \mathrm{E}-02$ & $253.65 \%$ \\
\hline Zr-95 & $2.5 \mathrm{E}-06$ & $1.7 \mathrm{E}-06$ & $-30.94 \%$ & U-234 & $2.3 \mathrm{E}-02$ & $8.2 \mathrm{E}-02$ & $253.65 \%$ \\
\hline Tc-96 & $2.1 \mathrm{E}-03$ & $1.6 \mathrm{E}-04$ & $-92.49 \%$ & U-235 & $2.2 \mathrm{E}-02$ & $7.9 \mathrm{E}-02$ & $253.65 \%$ \\
\hline Tc-97 & $2.6 \mathrm{E}-03$ & $5.5 \mathrm{E}-03$ & $111.09 \%$ & U-236 & $2.2 \mathrm{E}-02$ & $7.9 \mathrm{E}-02$ & $253.65 \%$ \\
\hline Tc-98 & $8.4 \mathrm{E}-02$ & $1.8 \mathrm{E}-01$ & $111.09 \%$ & U-237 & $6.9 \mathrm{E}-05$ & $2.2 \mathrm{E}-04$ & $212.20 \%$ \\
\hline Tc-99 & $2.3 \mathrm{E}-02$ & $4.8 \mathrm{E}-02$ & $111.09 \%$ & U-238 & $2.1 \mathrm{E}-02$ & $7.3 \mathrm{E}-02$ & $253.65 \%$ \\
\hline Ru-97 & $1.1 \mathrm{E}-08$ & 7.9E-08 & $616.74 \%$ & $\mathrm{~Np}-236$ & $7.1 \mathrm{E}-04$ & $6.8 \mathrm{E}-04$ & $-3.47 \%$ \\
\hline Ru-103 & $3.7 \mathrm{E}-07$ & $3.3 \mathrm{E}-06$ & $790.37 \%$ & $\mathrm{~Np}-237$ & $3.5 \mathrm{E}-03$ & $3.4 \mathrm{E}-03$ & $-3.47 \%$ \\
\hline Ru-106 & $3.7 \mathrm{E}-06$ & $3.2 \mathrm{E}-05$ & $772.92 \%$ & $\mathrm{~Np}-239$ & $1.7 \mathrm{E}-07$ & $1.2 \mathrm{E}-07$ & $-26.91 \%$ \\
\hline Pd-107 & $2.7 \mathrm{E}-04$ & $2.6 \mathrm{E}-04$ & $-4.98 \%$ & Am-237 & $1.9 \mathrm{E}-38$ & $5.4 \mathrm{E}-40$ & $-97.07 \%$ \\
\hline Ag-108m & $1.8 \mathrm{E}-01$ & $2.0 \mathrm{E}-03$ & $-98.87 \%$ & Am-241 & $3.9 \mathrm{E}-03$ & $3.2 \mathrm{E}-04$ & $-91.87 \%$ \\
\hline
\end{tabular}


SRNL-STI-2010-00447, REVISION 0

Table 33. LADTAP XL IRRIDOSE Milk Ingestion Pathway Collective Dose Comparison (person-rem/yr) (continued)

\begin{tabular}{|c|c|c|c|c|c|c|c|}
\hline Nuclide & $\begin{array}{c}\text { Hamby Ladtap } \\
\text { Milk }\end{array}$ & $\begin{array}{c}\text { Jannik Ladtap } \\
\text { Milk }\end{array}$ & $\%$ Diff & Nuclide & $\begin{array}{c}\text { Hamby Ladtap } \\
\text { Milk }\end{array}$ & $\begin{array}{c}\text { Jannik Ladtap } \\
\text { Milk }\end{array}$ & $\%$ Diff \\
\hline Ag-110m & $1.0 \mathrm{E}-01$ & $2.8 \mathrm{E}-03$ & $-97.20 \%$ & Am-242m & $3.7 \mathrm{E}-03$ & $3.0 \mathrm{E}-04$ & $-91.87 \%$ \\
\hline Cd-113 & $1.5 \mathrm{E}-02$ & $1.0 \mathrm{E}-02$ & $-32.79 \%$ & Am-243 & $3.9 \mathrm{E}-03$ & $3.2 \mathrm{E}-04$ & $-91.87 \%$ \\
\hline Cd-115m & $2.6 \mathrm{E}-04$ & $3.8 \mathrm{E}-04$ & $46.86 \%$ & $\mathrm{Pu}-237$ & $2.8 \mathrm{E}-07$ & $1.3 \mathrm{E}-06$ & $377.24 \%$ \\
\hline In-115 & $5.0 \mathrm{E}-03$ & $4.7 \mathrm{E}-03$ & $-6.25 \%$ & $\mathrm{Pu}-238$ & $1.3 \mathrm{E}-03$ & $6.4 \mathrm{E}-03$ & $383.65 \%$ \\
\hline Sb-122 & $1.4 \mathrm{E}-04$ & $2.8 \mathrm{E}-06$ & $-98.09 \%$ & $\mathrm{Pu}-239$ & $1.5 \mathrm{E}-03$ & $7.3 \mathrm{E}-03$ & $383.56 \%$ \\
\hline Sb-124 & $2.1 \mathrm{E}-03$ & $5.0 \mathrm{E}-05$ & $-97.58 \%$ & $\mathrm{Pu}-240$ & $1.5 \mathrm{E}-03$ & $7.3 \mathrm{E}-03$ & $383.56 \%$ \\
\hline Sb-125 & $6.9 \mathrm{E}-04$ & $1.6 \mathrm{E}-05$ & $-97.61 \%$ & $\mathrm{Pu}-241$ & $3.0 \mathrm{E}-05$ & $1.4 \mathrm{E}-04$ & $383.99 \%$ \\
\hline Te-123 & $1.2 \mathrm{E}-02$ & $3.2 \mathrm{E}-04$ & $-97.28 \%$ & $\mathrm{Pu}-242$ & $1.4 \mathrm{E}-03$ & $6.9 \mathrm{E}-03$ & $383.55 \%$ \\
\hline Te-125m & $6.0 \mathrm{E}-04$ & $1.6 \mathrm{E}-04$ & $-72.82 \%$ & $\mathrm{Pu}-244$ & $1.4 \mathrm{E}-03$ & $6.7 \mathrm{E}-03$ & $383.55 \%$ \\
\hline Sn-126 & $7.6 \mathrm{E}-03$ & $2.9 \mathrm{E}-03$ & $-61.79 \%$ & $\mathrm{Cm}-241$ & $3.0 \mathrm{E}-06$ & $1.1 \mathrm{E}-05$ & $279.33 \%$ \\
\hline I-129 & $3.6 \mathrm{E}-01$ & $2.8 \mathrm{E}-01$ & $-23.72 \%$ & $\mathrm{Cm}-242$ & $9.0 \mathrm{E}-05$ & $3.5 \mathrm{E}-04$ & $285.89 \%$ \\
\hline $\mathrm{I}-131$ & $1.9 \mathrm{E}-02$ & $1.5 \mathrm{E}-02$ & $-20.57 \%$ & $\mathrm{Cm}-243$ & $2.6 \mathrm{E}-03$ & $9.8 \mathrm{E}-03$ & $279.08 \%$ \\
\hline Cs-134 & $1.6 \mathrm{E}-01$ & $5.7 \mathrm{E}-02$ & $-63.32 \%$ & $\mathrm{Cm}-244$ & $2.0 \mathrm{E}-03$ & $7.8 \mathrm{E}-03$ & $280.08 \%$ \\
\hline Cs-135 & $1.7 \mathrm{E}-02$ & $5.8 \mathrm{E}-03$ & $-65.36 \%$ & $\mathrm{Cm}-245$ & $4.0 \mathrm{E}-03$ & $1.5 \mathrm{E}-02$ & $276.88 \%$ \\
\hline Cs-137 & $1.1 \mathrm{E}-01$ & $4.0 \mathrm{E}-02$ & $-64.97 \%$ & $\mathrm{Cm}-246$ & $4.0 \mathrm{E}-03$ & $1.5 \mathrm{E}-02$ & $276.88 \%$ \\
\hline La-137 & $3.9 \mathrm{E}-07$ & $1.5 \mathrm{E}-06$ & $278.78 \%$ & $\mathrm{Cm}-247$ & $3.7 \mathrm{E}-03$ & $1.4 \mathrm{E}-02$ & $276.87 \%$ \\
\hline La-138 & $5.3 \mathrm{E}-06$ & $2.0 \mathrm{E}-05$ & $278.78 \%$ & $\mathrm{Cm}-248$ & $1.4 \mathrm{E}-02$ & $5.4 \mathrm{E}-02$ & $276.87 \%$ \\
\hline La-140 & $2.2 \mathrm{E}-07$ & $5.9 \mathrm{E}-07$ & $170.51 \%$ & $\mathrm{Cm}-250$ & $7.0 \mathrm{E}-02$ & $2.6 \mathrm{E}-01$ & $276.88 \%$ \\
\hline $\mathrm{Ba}-140$ & $2.9 \mathrm{E}-04$ & $1.1 \mathrm{E}-04$ & $-63.42 \%$ & Bk-247 & $1.6 \mathrm{E}-04$ & $7.8 \mathrm{E}-04$ & $387.74 \%$ \\
\hline $\mathrm{Ce}-141$ & $2.0 \mathrm{E}-04$ & $6.3 \mathrm{E}-06$ & $-96.84 \%$ & Bk-249 & $4.0 \mathrm{E}-07$ & $2.0 \mathrm{E}-06$ & $384.02 \%$ \\
\hline \multirow[t]{8}{*}{$\mathrm{Ce}-144$} & $2.0 \mathrm{E}-03$ & $6.5 \mathrm{E}-05$ & $-96.78 \%$ & Cf-249 & $6.1 \mathrm{E}-04$ & $1.2 \mathrm{E}-03$ & $91.78 \%$ \\
\hline & & & & Cf-250 & $2.5 \mathrm{E}-04$ & $4.8 \mathrm{E}-04$ & $92.56 \%$ \\
\hline & & & & Cf-251 & $6.1 \mathrm{E}-04$ & $1.2 \mathrm{E}-03$ & $91.75 \%$ \\
\hline & & & & Cf-252 & $1.2 \mathrm{E}-04$ & $2.4 \mathrm{E}-04$ & $93.40 \%$ \\
\hline & & & & Es-253 & $1.0 \mathrm{E}-06$ & $4.9 \mathrm{E}-06$ & $367.75 \%$ \\
\hline & & & & Unidentified alpha & $1.5 \mathrm{E}-03$ & $7.3 \mathrm{E}-03$ & $383.56 \%$ \\
\hline & & & & Unidentified beta & $2.1 \mathrm{E}-02$ & $4.6 \mathrm{E}-02$ & $116.77 \%$ \\
\hline & & & & TOTAL Dose & $7.4 \mathrm{E}+00$ & $2.9 \mathrm{E}+00$ & $-60.43 \%$ \\
\hline
\end{tabular}

Table 34. LADTAP XL IRRIDOSE Meat Ingestion Pathway Collective Dose Comparison (person-rem/yr) 
SRNL-STI-2010-00447, REVISION 0

\begin{tabular}{|c|c|c|c|c|c|c|c|}
\hline Nuclide & $\begin{array}{c}\text { Hamby Ladtap } \\
\text { Meat }\end{array}$ & $\begin{array}{c}\text { Jannik Ladtap } \\
\text { Meat }\end{array}$ & $\%$ Diff & Nuclide & $\begin{array}{c}\text { Hamby Ladtap } \\
\text { Meat }\end{array}$ & $\begin{array}{c}\text { Jannik Ladtap } \\
\text { Meat }\end{array}$ & $\%$ Diff \\
\hline H-3 & $7.8 \mathrm{E}-07$ & $2.9 \mathrm{E}-07$ & $-63.21 \%$ & Sm-146 & $4.0 \mathrm{E}-03$ & $2.2 \mathrm{E}-04$ & $-94.48 \%$ \\
\hline $\mathrm{Be}-7$ & $3.4 \mathrm{E}-07$ & $2.9 \mathrm{E}-07$ & $-14.07 \%$ & Sm-147 & $3.6 \mathrm{E}-03$ & $2.0 \mathrm{E}-04$ & $-94.48 \%$ \\
\hline Be-10 & $1.7 \mathrm{E}-05$ & $1.4 \mathrm{E}-05$ & $-16.01 \%$ & Sm-151 & $6.8 \mathrm{E}-06$ & $3.7 \mathrm{E}-07$ & $-94.48 \%$ \\
\hline C-14 & $1.7 \mathrm{E}-02$ & $4.2 \mathrm{E}-04$ & $-97.48 \%$ & Pm-147 & $1.8 \mathrm{E}-05$ & $6.5 \mathrm{E}-08$ & $-99.63 \%$ \\
\hline $\mathrm{Na}-22$ & $1.5 \mathrm{E}-03$ & $6.1 \mathrm{E}-04$ & $-60.41 \%$ & Eu-152 & $1.1 \mathrm{E}-04$ & 4.1E-07 & $-99.64 \%$ \\
\hline $\mathrm{Na}-24$ & 4.7E-09 & $9.0 \mathrm{E}-10$ & $-80.68 \%$ & Eu-154 & $1.7 \mathrm{E}-04$ & $6.2 \mathrm{E}-07$ & $-99.64 \%$ \\
\hline Al-26 & $2.6 \mathrm{E}-05$ & $6.6 \mathrm{E}-05$ & $151.06 \%$ & Eu-155 & $2.4 \mathrm{E}-05$ & 8.9E-08 & $-99.64 \%$ \\
\hline $\mathrm{P}-32$ & $6.7 \mathrm{E}-04$ & $6.2 \mathrm{E}-04$ & $-7.01 \%$ & Gd-152 & $1.2 \mathrm{E}-03$ & $1.0 \mathrm{E}-05$ & $-99.12 \%$ \\
\hline $\mathrm{Si}-32$ & $5.3 \mathrm{E}-07$ & $2.7 \mathrm{E}-07$ & $-49.23 \%$ & Ho-166m & $1.4 \mathrm{E}-04$ & $8.2 \mathrm{E}-06$ & $-94.18 \%$ \\
\hline S-35 & $2.5 \mathrm{E}-04$ & $3.9 \mathrm{E}-04$ & $57.80 \%$ & Lu-176 & $1.2 \mathrm{E}-04$ & $1.0 \mathrm{E}-04$ & $-14.51 \%$ \\
\hline $\mathrm{Cl}-36$ & $1.7 \mathrm{E}-01$ & $4.1 \mathrm{E}-03$ & $-97.50 \%$ & Тa-180 & $0.0 \mathrm{E}+00$ & $0.0 \mathrm{E}+00$ & $0.00 \%$ \\
\hline $\mathrm{K}-40$ & $6.7 \mathrm{E}-03$ & $3.4 \mathrm{E}-03$ & $-48.63 \%$ & Hf-182 & $5.4 \mathrm{E}-08$ & $1.5 \mathrm{E}-06$ & $2669.23 \%$ \\
\hline K-43 & $3.5 \mathrm{E}-08$ & $1.6 \mathrm{E}-08$ & $-55.61 \%$ & Re-186m & $3.2 \mathrm{E}-03$ & $1.6 \mathrm{E}-04$ & $-94.93 \%$ \\
\hline $\mathrm{Ca}-41$ & $5.1 \mathrm{E}-05$ & $1.4 \mathrm{E}-03$ & $2641.52 \%$ & Re-187 & $8.0 \mathrm{E}-06$ & $4.1 \mathrm{E}-07$ & $-94.93 \%$ \\
\hline $\mathrm{Ca}-45$ & $2.1 \mathrm{E}-05$ & $2.1 \mathrm{E}-04$ & $901.03 \%$ & Ir-192m & $0.0 \mathrm{E}+00$ & $0.0 \mathrm{E}+00$ & $0.00 \%$ \\
\hline $\mathrm{Ca}-47$ & $4.9 \mathrm{E}-06$ & $2.9 \mathrm{E}-05$ & $495.53 \%$ & Pt-193 & $2.1 \mathrm{E}-06$ & $1.5 \mathrm{E}-06$ & $-27.91 \%$ \\
\hline Ti-44 & 7.4E-08 & $1.1 \mathrm{E}-05$ & $15098.54 \%$ & Hg-194 & $5.9 \mathrm{E}-01$ & $3.8 \mathrm{E}-01$ & $-36.13 \%$ \\
\hline $\mathrm{V}-49$ & $5.1 \mathrm{E}-07$ & $4.4 \mathrm{E}-07$ & $-12.77 \%$ & $\mathrm{Hg}-203$ & $3.2 \mathrm{E}-03$ & $6.5 \mathrm{E}-03$ & $105.73 \%$ \\
\hline $\mathrm{Cr}-51$ & $8.0 \mathrm{E}-07$ & $2.5 \mathrm{E}-06$ & $216.83 \%$ & $\mathrm{~Pb}-202$ & $1.4 \mathrm{E}-04$ & $9.6 \mathrm{E}-05$ & $-29.14 \%$ \\
\hline Mn-53 & $4.1 \mathrm{E}-07$ & $2.9 \mathrm{E}-07$ & $-30.57 \%$ & $\mathrm{~Pb}-205$ & $5.2 \mathrm{E}-06$ & $3.7 \mathrm{E}-06$ & $-29.14 \%$ \\
\hline $\mathrm{Mn}-54$ & $8.3 \mathrm{E}-06$ & $5.4 \mathrm{E}-06$ & $-34.55 \%$ & $\mathrm{~Pb}-210$ & $1.7 \mathrm{E}-02$ & $1.2 \mathrm{E}-02$ & $-27.92 \%$ \\
\hline $\mathrm{Fe}-55$ & $8.9 \mathrm{E}-05$ & $2.8 \mathrm{E}-05$ & $-69.09 \%$ & Bi-207 & $7.5 \mathrm{E}-05$ & $1.0 \mathrm{E}-05$ & $-86.65 \%$ \\
\hline Fe-60 & $2.4 \mathrm{E}-02$ & $7.7 \mathrm{E}-03$ & $-67.40 \%$ & Bi-210 & $6.8 \mathrm{E}-06$ & $9.7 \mathrm{E}-07$ & $-85.66 \%$ \\
\hline Co-58 & $1.5 \mathrm{E}-04$ & $4.3 \mathrm{E}-06$ & $-97.14 \%$ & $\mathrm{Bi}-210 \mathrm{~m}$ & $1.5 \mathrm{E}-03$ & $1.9 \mathrm{E}-04$ & $-86.83 \%$ \\
\hline Co-60 & $1.4 \mathrm{E}-03$ & $4.0 \mathrm{E}-05$ & $-97.08 \%$ & Po-210 & $2.9 \mathrm{E}-02$ & $2.5 \mathrm{E}-02$ & $-13.07 \%$ \\
\hline Ni-59 & $5.0 \mathrm{E}-06$ & $3.9 \mathrm{E}-06$ & $-22.87 \%$ & Ra-223 & $8.3 \mathrm{E}-04$ & $1.1 \mathrm{E}-03$ & $36.05 \%$ \\
\hline $\mathrm{Ni}-63$ & $1.3 \mathrm{E}-05$ & $1.0 \mathrm{E}-05$ & $-22.73 \%$ & Ra-224 & $1.1 \mathrm{E}-04$ & $1.2 \mathrm{E}-04$ & $14.08 \%$ \\
\hline $\mathrm{Cu}-64$ & $7.4 \mathrm{E}-11$ & $3.1 \mathrm{E}-11$ & $-58.90 \%$ & Ra-225 & $5.7 \mathrm{E}-04$ & $7.9 \mathrm{E}-04$ & $38.83 \%$ \\
\hline
\end{tabular}

Table 34. LADTAP XL IRRIDOSE Meat Ingestion Pathway Collective Dose Comparison (person-rem/yr) (continued) 
SRNL-STI-2010-00447, REVISION 0

\begin{tabular}{|c|c|c|c|c|c|c|c|}
\hline Nuclide & $\begin{array}{c}\text { Hamby Ladtap } \\
\text { Meat }\end{array}$ & $\begin{array}{c}\text { Jannik Ladtap } \\
\text { Meat }\end{array}$ & $\%$ Diff & Nuclide & $\begin{array}{c}\text { Hamby Ladtap } \\
\text { Meat }\end{array}$ & $\begin{array}{c}\text { Jannik Ladtap } \\
\text { Meat }\end{array}$ & $\%$ Diff \\
\hline $\mathrm{Zn}-65$ & $1.9 \mathrm{E}-03$ & $7.6 \mathrm{E}-03$ & $293.85 \%$ & Ra-226 & $6.3 \mathrm{E}-03$ & $6.9 \mathrm{E}-03$ & $9.12 \%$ \\
\hline Ge-68 & $3.1 \mathrm{E}-03$ & $2.5 \mathrm{E}-03$ & $-17.95 \%$ & Ra-228 & $5.5 \mathrm{E}-03$ & $7.1 \mathrm{E}-03$ & $29.34 \%$ \\
\hline Se-75 & $6.4 \mathrm{E}-04$ & $4.0 \mathrm{E}-04$ & $-37.04 \%$ & Ac- 227 & $1.1 \mathrm{E}-03$ & $1.9 \mathrm{E}-02$ & $1614.09 \%$ \\
\hline Se-79 & 7.9E-03 & $4.8 \mathrm{E}-04$ & $-93.97 \%$ & Th-227 & $1.5 \mathrm{E}-05$ & $1.5 \mathrm{E}-05$ & $-4.57 \%$ \\
\hline $\mathrm{Rb}-87$ & $1.5 \mathrm{E}-03$ & $3.1 \mathrm{E}-04$ & $-78.68 \%$ & Th-228 & $2.9 \mathrm{E}-04$ & $2.9 \mathrm{E}-04$ & $-0.11 \%$ \\
\hline Sr-89 & $1.6 \mathrm{E}-05$ & $3.0 \mathrm{E}-05$ & $87.05 \%$ & Th-229 & $2.9 \mathrm{E}-03$ & $2.7 \mathrm{E}-03$ & $-3.86 \%$ \\
\hline Sr-90 & $3.5 \mathrm{E}-04$ & $9.2 \mathrm{E}-04$ & $162.97 \%$ & Th-230 & $4.3 \mathrm{E}-04$ & $4.2 \mathrm{E}-04$ & $-3.86 \%$ \\
\hline Y-90 & $7.5 \mathrm{E}-06$ & $1.0 \mathrm{E}-06$ & $-86.45 \%$ & Th-231 & $1.3 \mathrm{E}-09$ & $6.9 \mathrm{E}-10$ & $-46.43 \%$ \\
\hline Y-91 & $1.3 \mathrm{E}-04$ & $2.4 \mathrm{E}-05$ & $-81.29 \%$ & Th-232 & $2.3 \mathrm{E}-03$ & $2.2 \mathrm{E}-03$ & $-3.86 \%$ \\
\hline Mo-93 & $9.8 \mathrm{E}-05$ & $6.9 \mathrm{E}-06$ & $-92.88 \%$ & Th-234 & $6.3 \mathrm{E}-06$ & $6.1 \mathrm{E}-06$ & $-3.40 \%$ \\
\hline Mo-99 & $6.2 \mathrm{E}-06$ & $4.8 \mathrm{E}-07$ & $-92.16 \%$ & $\mathrm{~Pa}-230$ & $5.7 \mathrm{E}-05$ & $4.2 \mathrm{E}-06$ & $-92.61 \%$ \\
\hline $\mathrm{Nb}-93 \mathrm{~m}$ & $6.2 \mathrm{E}-03$ & $4.7 \mathrm{E}-09$ & $-100.00 \%$ & Pa-231 & $2.4 \mathrm{E}-01$ & $1.7 \mathrm{E}-02$ & $-92.99 \%$ \\
\hline Nb-94 & $6.2 \mathrm{E}-03$ & $4.6 \mathrm{E}-09$ & $-100.00 \%$ & $\mathrm{~Pa}-233$ & $4.2 \mathrm{E}-05$ & $3.2 \mathrm{E}-06$ & $-92.46 \%$ \\
\hline $\mathrm{Nb}-95$ & $1.7 \mathrm{E}-03$ & $1.4 \mathrm{E}-09$ & $-100.00 \%$ & U-232 & $1.8 \mathrm{E}-03$ & $1.8 \mathrm{E}-03$ & $1.63 \%$ \\
\hline Zr-93 & $2.1 \mathrm{E}-04$ & $6.6 \mathrm{E}-09$ & $-100.00 \%$ & U-233 & $3.7 \mathrm{E}-04$ & $3.7 \mathrm{E}-04$ & $1.84 \%$ \\
\hline Zr-95 & $3.7 \mathrm{E}-04$ & $1.1 \mathrm{E}-08$ & $-100.00 \%$ & U-234 & $3.5 \mathrm{E}-04$ & $3.6 \mathrm{E}-04$ & $1.84 \%$ \\
\hline Tc-96 & $4.8 \mathrm{E}-04$ & $6.6 \mathrm{E}-06$ & $-98.64 \%$ & U-235 & $3.4 \mathrm{E}-04$ & $3.5 \mathrm{E}-04$ & $1.84 \%$ \\
\hline Tc-97 & $9.2 \mathrm{E}-04$ & $3.8 \mathrm{E}-04$ & $-58.55 \%$ & U-236 & $3.4 \mathrm{E}-04$ & $3.5 \mathrm{E}-04$ & $1.84 \%$ \\
\hline Tc-98 & $2.9 \mathrm{E}-02$ & $1.2 \mathrm{E}-02$ & $-58.55 \%$ & U-237 & 8.0E-07 & $6.9 \mathrm{E}-07$ & $-13.56 \%$ \\
\hline Tc-99 & $8.0 \mathrm{E}-03$ & $3.3 \mathrm{E}-03$ & $-58.55 \%$ & U-238 & $3.1 \mathrm{E}-04$ & $3.2 \mathrm{E}-04$ & $1.84 \%$ \\
\hline Ru-97 & $5.1 \mathrm{E}-05$ & $2.7 \mathrm{E}-07$ & $-99.48 \%$ & $\mathrm{~Np}-236$ & $6.3 \mathrm{E}-04$ & $2.8 \mathrm{E}-03$ & $336.18 \%$ \\
\hline Ru-103 & $3.1 \mathrm{E}-03$ & $2.2 \mathrm{E}-05$ & $-99.30 \%$ & $\mathrm{~Np}-237$ & $3.1 \mathrm{E}-03$ & $1.4 \mathrm{E}-02$ & $336.18 \%$ \\
\hline Ru-106 & $3.3 \mathrm{E}-02$ & $2.3 \mathrm{E}-04$ & $-99.31 \%$ & $\mathrm{~Np}-239$ & $6.7 \mathrm{E}-08$ & $2.0 \mathrm{E}-07$ & $200.61 \%$ \\
\hline Pd-107 & $1.2 \mathrm{E}-06$ & $2.1 \mathrm{E}-06$ & $74.24 \%$ & Am-237 & $3.0 \mathrm{E}-56$ & $1.7 \mathrm{E}-56$ & $-43.69 \%$ \\
\hline $\mathrm{Ag}-108 \mathrm{~m}$ & $1.3 \mathrm{E}-03$ & 7.7E-05 & $-94.22 \%$ & Am-241 & $3.5 \mathrm{E}-03$ & 7.7E-03 & $118.37 \%$ \\
\hline
\end{tabular}


SRNL-STI-2010-00447, REVISION 0

Table 34. LADTAP XL IRRIDOSE Meat Ingestion Pathway Collective Dose Comparison (person-rem/yr) (continued)

\begin{tabular}{|c|c|c|c|c|c|c|c|}
\hline Nuclide & $\begin{array}{c}\text { Hamby Ladtap } \\
\text { Meat }\end{array}$ & $\begin{array}{c}\text { Jannik Ladtap } \\
\text { Meat }\end{array}$ & $\%$ Diff & Nuclide & $\begin{array}{c}\text { Hamby Ladtap } \\
\text { Meat }\end{array}$ & $\begin{array}{c}\text { Jannik Ladtap } \\
\text { Meat }\end{array}$ & $\%$ Diff \\
\hline Ag-110m & $7.5 \mathrm{E}-04$ & $1.1 \mathrm{E}-04$ & $-85.88 \%$ & Am-242m & $3.3 \mathrm{E}-03$ & $7.2 \mathrm{E}-03$ & $118.39 \%$ \\
\hline Cd-113 & $1.5 \mathrm{E}-03$ & $6.3 \mathrm{E}-03$ & $330.19 \%$ & Am-243 & $3.5 \mathrm{E}-03$ & 7.7E-03 & $118.37 \%$ \\
\hline Cd-115m & $2.5 \mathrm{E}-05$ & $2.2 \mathrm{E}-04$ & $812.23 \%$ & $\mathrm{Pu}-237$ & $4.2 \mathrm{E}-08$ & $2.8 \mathrm{E}-09$ & $-93.27 \%$ \\
\hline In-115 & $2.3 \mathrm{E}-03$ & $3.8 \mathrm{E}-03$ & $69.44 \%$ & $\mathrm{Pu}-238$ & $2.1 \mathrm{E}-04$ & $1.4 \mathrm{E}-05$ & $-93.14 \%$ \\
\hline Sb-122 & $4.2 \mathrm{E}-06$ & 7.9E-07 & $-81.25 \%$ & $\mathrm{Pu}-239$ & $2.3 \mathrm{E}-04$ & $1.6 \mathrm{E}-05$ & $-93.14 \%$ \\
\hline Sb-124 & $1.2 \mathrm{E}-04$ & $3.1 \mathrm{E}-05$ & $-74.20 \%$ & $\mathrm{Pu}-240$ & $2.3 \mathrm{E}-04$ & $1.6 \mathrm{E}-05$ & $-93.14 \%$ \\
\hline Sb-125 & $4.1 \mathrm{E}-05$ & $1.0 \mathrm{E}-05$ & $-74.40 \%$ & $\mathrm{Pu}-241$ & $4.7 \mathrm{E}-06$ & $3.2 \mathrm{E}-07$ & $-93.13 \%$ \\
\hline Te-123 & $2.0 \mathrm{E}-02$ & $1.4 \mathrm{E}-04$ & $-99.33 \%$ & $\mathrm{Pu}-242$ & $2.2 \mathrm{E}-04$ & $1.5 \mathrm{E}-05$ & $-93.14 \%$ \\
\hline Te-125m & $9.9 \mathrm{E}-04$ & $6.5 \mathrm{E}-05$ & $-93.43 \%$ & $\mathrm{Pu}-244$ & $2.2 \mathrm{E}-04$ & $1.5 \mathrm{E}-05$ & $-93.14 \%$ \\
\hline Sn-126 & $5.4 \mathrm{E}-03$ & $4.7 \mathrm{E}-03$ & $-13.68 \%$ & $\mathrm{Cm}-241$ & $2.5 \mathrm{E}-06$ & $4.3 \mathrm{E}-07$ & $-83.01 \%$ \\
\hline $\mathrm{I}-129$ & $3.9 \mathrm{E}-03$ & $6.9 \mathrm{E}-03$ & $77.83 \%$ & $\mathrm{Cm}-242$ & $8.0 \mathrm{E}-05$ & $1.4 \mathrm{E}-05$ & $-82.61 \%$ \\
\hline $\mathrm{I}-131$ & $1.6 \mathrm{E}-04$ & $2.9 \mathrm{E}-04$ & $78.25 \%$ & $\mathrm{Cm}-243$ & $2.3 \mathrm{E}-03$ & $3.9 \mathrm{E}-04$ & $-82.88 \%$ \\
\hline Cs-134 & $1.2 \mathrm{E}-03$ & $5.5 \mathrm{E}-03$ & $375.18 \%$ & $\mathrm{Cm}-244$ & $1.8 \mathrm{E}-03$ & $3.1 \mathrm{E}-04$ & $-82.83 \%$ \\
\hline Cs-135 & $1.2 \mathrm{E}-04$ & $5.6 \mathrm{E}-04$ & $350.15 \%$ & $\mathrm{Cm}-245$ & $3.6 \mathrm{E}-03$ & $6.1 \mathrm{E}-04$ & $-82.97 \%$ \\
\hline Cs-137 & $8.5 \mathrm{E}-04$ & $3.9 \mathrm{E}-03$ & $354.98 \%$ & $\mathrm{Cm}-246$ & $3.6 \mathrm{E}-03$ & $6.1 \mathrm{E}-04$ & $-82.97 \%$ \\
\hline La-137 & $3.4 \mathrm{E}-07$ & $1.9 \mathrm{E}-07$ & $-44.39 \%$ & $\mathrm{Cm}-247$ & $3.3 \mathrm{E}-03$ & $5.6 \mathrm{E}-04$ & $-82.97 \%$ \\
\hline La-138 & 4.7E-06 & $2.6 \mathrm{E}-06$ & $-44.39 \%$ & $\mathrm{Cm}-248$ & $1.3 \mathrm{E}-02$ & $2.2 \mathrm{E}-03$ & $-82.97 \%$ \\
\hline La-140 & $6.0 \mathrm{E}-08$ & $2.1 \mathrm{E}-08$ & $-64.70 \%$ & $\mathrm{Cm}-250$ & $6.2 \mathrm{E}-02$ & $1.1 \mathrm{E}-02$ & $-82.97 \%$ \\
\hline $\mathrm{Ba}-140$ & $4.4 \mathrm{E}-05$ & $1.6 \mathrm{E}-06$ & $-96.46 \%$ & Bk-247 & $1.8 \mathrm{E}-04$ & $2.0 \mathrm{E}-04$ & $10.09 \%$ \\
\hline $\mathrm{Ce}-141$ & $8.5 \mathrm{E}-06$ & $1.2 \mathrm{E}-07$ & $-98.58 \%$ & Bk-249 & $4.5 \mathrm{E}-07$ & 4.9E-07 & $9.16 \%$ \\
\hline $\mathrm{Ce}-144$ & $9.0 \mathrm{E}-05$ & $1.3 \mathrm{E}-06$ & $-98.55 \%$ & Cf-249 & $1.1 \mathrm{E}-03$ & $6.3 \mathrm{E}-04$ & $-42.27 \%$ \\
\hline & & & & Cf-250 & $4.5 \mathrm{E}-04$ & $2.6 \mathrm{E}-04$ & $-42.05 \%$ \\
\hline & & & & Cf-251 & $1.1 \mathrm{E}-03$ & $6.3 \mathrm{E}-04$ & $-42.28 \%$ \\
\hline & & & & Cf-252 & $2.2 \mathrm{E}-04$ & $1.3 \mathrm{E}-04$ & $-41.81 \%$ \\
\hline & & & & Es-253 & $1.1 \mathrm{E}-06$ & $1.1 \mathrm{E}-06$ & $4.25 \%$ \\
\hline & & & & Unidentified alpha & $2.3 \mathrm{E}-04$ & $1.6 \mathrm{E}-05$ & $-93.14 \%$ \\
\hline & & & & Unidentified beta & $3.5 \mathrm{E}-04$ & $9.2 \mathrm{E}-04$ & $162.78 \%$ \\
\hline & & & & TOTAL Dose & $1.4 \mathrm{E}+00$ & $6.2 \mathrm{E}-01$ & $-55.27 \%$ \\
\hline
\end{tabular}


SRNL-STI-2010-00447, REVISION 0

Table 35. LADTAP XL IRRIDOSE All Pathway Collective Dose Comparison (person-rem/yr)

\begin{tabular}{|c|c|c|c|c|c|c|c|}
\hline Nuclide & $\begin{array}{c}\text { Hamby Ladtap } \\
\text { Total }\end{array}$ & $\begin{array}{c}\text { Jannik Ladtap } \\
\text { Total }\end{array}$ & $\%$ Diff & Nuclide & $\begin{array}{c}\text { Hamby Ladtap } \\
\text { Total }\end{array}$ & $\begin{array}{c}\text { Jannik Ladtap } \\
\text { Total }\end{array}$ & $\%$ Diff \\
\hline $\mathrm{H}-3$ & $4.5 \mathrm{E}-05$ & 7.3E-05 & $60.19 \%$ & Sm-146 & $1.5 \mathrm{E}+00$ & $1.7 \mathrm{E}+00$ & $11.49 \%$ \\
\hline $\mathrm{Be}-7$ & $5.4 \mathrm{E}-04$ & $6.4 \mathrm{E}-04$ & $17.44 \%$ & Sm-147 & $1.4 \mathrm{E}+00$ & $1.5 \mathrm{E}+00$ & $11.49 \%$ \\
\hline Be-10 & $3.2 \mathrm{E}-02$ & $3.4 \mathrm{E}-02$ & $5.26 \%$ & Sm-151 & $2.6 \mathrm{E}-03$ & $2.9 \mathrm{E}-03$ & $10.97 \%$ \\
\hline $\mathrm{C}-14$ & $7.1 \mathrm{E}-01$ & $6.4 \mathrm{E}-02$ & $-90.91 \%$ & Pm-147 & $7.0 \mathrm{E}-03$ & $7.9 \mathrm{E}-03$ & $12.48 \%$ \\
\hline $\mathrm{Na}-22$ & $1.9 \mathrm{E}-01$ & $1.2 \mathrm{E}-01$ & $-34.11 \%$ & Eu-152 & $4.5 \mathrm{E}-02$ & $5.0 \mathrm{E}-02$ & $9.03 \%$ \\
\hline $\mathrm{Na}-24$ & $6.9 \mathrm{E}-06$ & $1.2 \mathrm{E}-06$ & $-82.07 \%$ & Eu-154 & $6.9 \mathrm{E}-02$ & $7.4 \mathrm{E}-02$ & $8.34 \%$ \\
\hline Al-26 & $1.0 \mathrm{E}-01$ & $1.0 \mathrm{E}-01$ & $4.28 \%$ & Eu-155 & $9.7 \mathrm{E}-03$ & $1.0 \mathrm{E}-02$ & $7.56 \%$ \\
\hline P-32 & $3.4 \mathrm{E}-02$ & $3.6 \mathrm{E}-02$ & $6.37 \%$ & Gd-152 & $1.1 \mathrm{E}+00$ & $1.3 \mathrm{E}+00$ & $11.75 \%$ \\
\hline $\mathrm{Si}-32$ & $1.8 \mathrm{E}-02$ & $2.0 \mathrm{E}-02$ & $10.94 \%$ & Нo-166m & $6.0 \mathrm{E}-02$ & $6.6 \mathrm{E}-02$ & $11.44 \%$ \\
\hline S-35 & $6.3 \mathrm{E}-03$ & $5.8 \mathrm{E}-03$ & $-7.90 \%$ & Lu-176 & $5.0 \mathrm{E}-02$ & $5.4 \mathrm{E}-02$ & $6.07 \%$ \\
\hline $\mathrm{Cl}-36$ & $4.7 \mathrm{E}+00$ & $1.7 \mathrm{E}+00$ & $-64.66 \%$ & Ta-180 & $0.0 \mathrm{E}+00$ & $0.0 \mathrm{E}+00$ & $0.00 \%$ \\
\hline $\mathrm{K}-40$ & $4.4 \mathrm{E}-01$ & $8.7 \mathrm{E}-01$ & $96.64 \%$ & Hf-182 & $1.1 \mathrm{E}-01$ & $1.1 \mathrm{E}-01$ & $6.18 \%$ \\
\hline K-43 & 4.7E-06 & $3.8 \mathrm{E}-06$ & $-18.67 \%$ & Re-186m & $2.3 \mathrm{E}+01$ & $8.2 \mathrm{E}-02$ & $-99.64 \%$ \\
\hline $\mathrm{Ca}-41$ & $3.3 \mathrm{E}-02$ & $7.0 \mathrm{E}-01$ & $2017.03 \%$ & Re-187 & $5.8 \mathrm{E}-02$ & $2.1 \mathrm{E}-04$ & $-99.64 \%$ \\
\hline $\mathrm{Ca}-45$ & $2.3 \mathrm{E}-02$ & 7.0E-02 & $205.12 \%$ & Ir-192m & $0.0 \mathrm{E}+00$ & $0.0 \mathrm{E}+00$ & $0.00 \%$ \\
\hline $\mathrm{Ca}-47$ & $1.7 \mathrm{E}-03$ & $6.2 \mathrm{E}-03$ & $272.92 \%$ & Pt-193 & $1.0 \mathrm{E}-03$ & $1.0 \mathrm{E}-03$ & $1.24 \%$ \\
\hline Ti-44 & $1.4 \mathrm{E}-01$ & $1.5 \mathrm{E}-01$ & $6.93 \%$ & $\mathrm{Hg}-194$ & $6.3 \mathrm{E}+00$ & $6.0 \mathrm{E}+00$ & $-3.85 \%$ \\
\hline $\mathrm{V}-49$ & $3.8 \mathrm{E}-04$ & $4.1 \mathrm{E}-04$ & $7.65 \%$ & $\mathrm{Hg}-203$ & $5.1 \mathrm{E}-02$ & $6.4 \mathrm{E}-02$ & $23.57 \%$ \\
\hline $\mathrm{Cr}-51$ & $4.8 \mathrm{E}-04$ & 5.9E-04 & $21.59 \%$ & $\mathrm{~Pb}-202$ & $3.1 \mathrm{E}-01$ & $3.4 \mathrm{E}-01$ & $10.02 \%$ \\
\hline Mn-53 & $8.5 \mathrm{E}-04$ & $1.7 \mathrm{E}-03$ & $95.34 \%$ & $\mathrm{~Pb}-205$ & $1.2 \mathrm{E}-02$ & $1.3 \mathrm{E}-02$ & $10.02 \%$ \\
\hline Mn-54 & $1.9 \mathrm{E}-02$ & $2.2 \mathrm{E}-02$ & $12.28 \%$ & $\mathrm{~Pb}-210$ & $4.0 \mathrm{E}+01$ & $4.3 \mathrm{E}+01$ & $8.54 \%$ \\
\hline Fe-55 & $4.5 \mathrm{E}-03$ & $4.7 \mathrm{E}-03$ & $5.12 \%$ & Bi-207 & $5.1 \mathrm{E}-02$ & $9.1 \mathrm{E}-02$ & $77.70 \%$ \\
\hline $\mathrm{Fe}-60$ & $1.2 \mathrm{E}+00$ & $1.4 \mathrm{E}+00$ & $20.32 \%$ & Bi-210 & $1.4 \mathrm{E}-03$ & $4.4 \mathrm{E}-03$ & $201.44 \%$ \\
\hline Co-58 & $2.0 \mathrm{E}-02$ & $2.2 \mathrm{E}-02$ & $11.43 \%$ & $\mathrm{Bi}-210 \mathrm{~m}$ & $9.5 \mathrm{E}-01$ & $1.8 \mathrm{E}+00$ & $94.27 \%$ \\
\hline Co-60 & $2.0 \mathrm{E}-01$ & $2.3 \mathrm{E}-01$ & $14.52 \%$ & Po-210 & $1.0 \mathrm{E}+01$ & $1.1 \mathrm{E}+01$ & $9.71 \%$ \\
\hline Ni-59 & $1.9 \mathrm{E}-03$ & $2.2 \mathrm{E}-03$ & $15.97 \%$ & Ra-223 & 7.7E-01 & $1.3 \mathrm{E}+00$ & $64.10 \%$ \\
\hline $\mathrm{Ni}-63$ & $5.2 \mathrm{E}-03$ & $5.9 \mathrm{E}-03$ & $14.14 \%$ & $\mathrm{Ra}-224$ & $3.3 \mathrm{E}-02$ & $1.2 \mathrm{E}-01$ & $275.83 \%$ \\
\hline $\mathrm{Cu}-64$ & $2.6 \mathrm{E}-07$ & $2.2 \mathrm{E}-08$ & $-91.47 \%$ & Ra-225 & $6.2 \mathrm{E}-01$ & $9.1 \mathrm{E}-01$ & $48.35 \%$ \\
\hline
\end{tabular}


SRNL-STI-2010-00447, REVISION 0

Table 35. LADTAP XL IRRIDOSE All Pathway Collective Dose Comparison (person-rem/yr) (continued)

\begin{tabular}{|c|c|c|c|c|c|c|c|}
\hline Nuclide & $\begin{array}{c}\text { Hamby Ladtap } \\
\text { Total }\end{array}$ & $\begin{array}{c}\text { Jannik Ladtap } \\
\text { Total }\end{array}$ & $\%$ Diff & Nuclide & $\begin{array}{c}\text { Hamby Ladtap } \\
\text { Total }\end{array}$ & $\begin{array}{c}\text { Jannik Ladtap } \\
\text { Total }\end{array}$ & $\%$ Diff \\
\hline $\mathrm{Zn}-65$ & $2.2 \mathrm{E}-01$ & $1.3 \mathrm{E}-01$ & $-40.64 \%$ & $\mathrm{Ra}-226$ & $1.0 \mathrm{E}+01$ & $1.1 \mathrm{E}+01$ & $6.13 \%$ \\
\hline Ge-68 & $2.5 \mathrm{E}-02$ & $2.4 \mathrm{E}-02$ & $-3.77 \%$ & Ra-228 & $9.8 \mathrm{E}+00$ & $1.0 \mathrm{E}+01$ & $4.13 \%$ \\
\hline Se-75 & $1.5 \mathrm{E}-01$ & $6.7 \mathrm{E}-02$ & $-55.87 \%$ & Ac-227 & $1.1 \mathrm{E}+02$ & $1.1 \mathrm{E}+02$ & $5.18 \%$ \\
\hline Se-79 & $1.5 \mathrm{E}+00$ & $9.4 \mathrm{E}-02$ & $-93.76 \%$ & Th-227 & $8.8 \mathrm{E}-02$ & $1.3 \mathrm{E}-01$ & $42.33 \%$ \\
\hline $\mathrm{Rb}-87$ & $1.2 \mathrm{E}-01$ & $1.5 \mathrm{E}-01$ & $20.89 \%$ & Th-228 & $2.8 \mathrm{E}+00$ & $3.0 \mathrm{E}+00$ & $6.50 \%$ \\
\hline Sr-89 & $4.3 \mathrm{E}-02$ & $5.2 \mathrm{E}-02$ & $21.36 \%$ & Th-229 & $2.7 \mathrm{E}+01$ & $2.8 \mathrm{E}+01$ & $4.53 \%$ \\
\hline Sr-90 & $1.1 \mathrm{E}+00$ & $2.7 \mathrm{E}+00$ & $157.52 \%$ & Th-230 & $4.1 \mathrm{E}+00$ & $4.3 \mathrm{E}+00$ & $4.52 \%$ \\
\hline $\mathrm{Y}-90$ & $2.1 \mathrm{E}-04$ & $1.7 \mathrm{E}-03$ & $705.70 \%$ & Th-231 & $3.2 \mathrm{E}-08$ & $4.1 \mathrm{E}-06$ & $12878.43 \%$ \\
\hline Y-91 & $4.6 \mathrm{E}-02$ & $5.3 \mathrm{E}-02$ & $16.08 \%$ & Th-232 & $2.1 \mathrm{E}+01$ & $2.2 \mathrm{E}+01$ & $4.52 \%$ \\
\hline Мo-93 & $1.9 \mathrm{E}-02$ & $2.6 \mathrm{E}-02$ & $36.58 \%$ & Th-234 & $4.0 \mathrm{E}-02$ & $5.4 \mathrm{E}-02$ & $33.23 \%$ \\
\hline Mo-99 & $6.3 \mathrm{E}-04$ & $8.5 \mathrm{E}-04$ & $36.33 \%$ & $\mathrm{~Pa}-230$ & $1.3 \mathrm{E}-02$ & $1.9 \mathrm{E}-02$ & $44.93 \%$ \\
\hline $\mathrm{Nb}-93 \mathrm{~m}$ & 4.9E-02 & $4.3 \mathrm{E}-02$ & $-12.90 \%$ & $\mathrm{~Pa}-231$ & $8.7 \mathrm{E}+01$ & $8.8 \mathrm{E}+01$ & $1.30 \%$ \\
\hline $\mathrm{Nb}-94$ & 4.9E-02 & $4.2 \mathrm{E}-02$ & $-13.23 \%$ & $\mathrm{~Pa}-233$ & $1.1 \mathrm{E}-02$ & $1.5 \mathrm{E}-02$ & $29.49 \%$ \\
\hline $\mathrm{Nb}-95$ & $1.1 \mathrm{E}-02$ & $1.1 \mathrm{E}-02$ & $-2.78 \%$ & U-232 & $1.0 \mathrm{E}+01$ & $1.2 \mathrm{E}+01$ & $18.27 \%$ \\
\hline Zr-93 & $1.2 \mathrm{E}-02$ & $1.3 \mathrm{E}-02$ & $5.35 \%$ & U-233 & $2.1 \mathrm{E}+00$ & $2.5 \mathrm{E}+00$ & $19.46 \%$ \\
\hline $\mathrm{Zr}-95$ & $1.8 \mathrm{E}-02$ & $2.1 \mathrm{E}-02$ & $13.09 \%$ & U-234 & $2.0 \mathrm{E}+00$ & $2.4 \mathrm{E}+00$ & $19.46 \%$ \\
\hline Tc-96 & $3.0 \mathrm{E}-03$ & $2.8 \mathrm{E}-03$ & $-3.93 \%$ & U-235 & $1.9 \mathrm{E}+00$ & $2.3 \mathrm{E}+00$ & $19.46 \%$ \\
\hline Tc-97 & $5.9 \mathrm{E}-03$ & $3.7 \mathrm{E}-01$ & $6210.03 \%$ & U-236 & $1.9 \mathrm{E}+00$ & $2.3 \mathrm{E}+00$ & $19.46 \%$ \\
\hline Tc-98 & $1.9 \mathrm{E}-01$ & $1.2 \mathrm{E}+01$ & $6210.03 \%$ & U-237 & $1.4 \mathrm{E}-03$ & $3.4 \mathrm{E}-03$ & $144.88 \%$ \\
\hline Tc-99 & $5.1 \mathrm{E}-02$ & $3.2 \mathrm{E}+00$ & $6209.92 \%$ & U-238 & $1.8 \mathrm{E}+00$ & $2.1 \mathrm{E}+00$ & $19.46 \%$ \\
\hline Ru-97 & $7.0 \mathrm{E}-05$ & $1.3 \mathrm{E}-04$ & $92.94 \%$ & $\mathrm{~Np}-236$ & $6.0 \mathrm{E}+00$ & $6.7 \mathrm{E}+00$ & $11.76 \%$ \\
\hline Ru-103 & $1.5 \mathrm{E}-02$ & $1.4 \mathrm{E}-02$ & $-3.96 \%$ & $\mathrm{~Np}-237$ & $3.0 \mathrm{E}+01$ & $3.3 \mathrm{E}+01$ & $11.76 \%$ \\
\hline Ru-106 & $1.8 \mathrm{E}-01$ & $1.6 \mathrm{E}-01$ & $-12.51 \%$ & Np-239 & $2.9 \mathrm{E}-05$ & $3.2 \mathrm{E}-04$ & $1013.38 \%$ \\
\hline Pd-107 & $1.8 \mathrm{E}-03$ & $1.6 \mathrm{E}-03$ & $-9.21 \%$ & Am-237 & $1.9 \mathrm{E}-38$ & $5.4 \mathrm{E}-40$ & $-97.07 \%$ \\
\hline Ag-108m & $2.7 \mathrm{E}-01$ & $6.2 \mathrm{E}-02$ & $-77.05 \%$ & Am-241 & $3.4 \mathrm{E}+01$ & $3.6 \mathrm{E}+01$ & $5.89 \%$ \\
\hline
\end{tabular}


SRNL-STI-2010-00447, REVISION 0

Table 35. LADTAP XL IRRIDOSE All Pathway Collective Dose Comparison (person-rem/yr) (continued)

\begin{tabular}{|c|c|c|c|c|c|c|c|}
\hline Nuclide & $\begin{array}{c}\text { Hamby Ladtap } \\
\text { Total }\end{array}$ & $\begin{array}{c}\text { Jannik Ladtap } \\
\text { Total }\end{array}$ & $\%$ Diff & Nuclide & $\begin{array}{c}\text { Hamby Ladtap } \\
\text { Total }\end{array}$ & $\begin{array}{c}\text { Jannik Ladtap } \\
\text { Total }\end{array}$ & $\%$ Diff \\
\hline Ag-110m & $1.8 \mathrm{E}-01$ & 8.5E-02 & $-52.72 \%$ & Am-242m & $3.2 \mathrm{E}+01$ & $3.4 \mathrm{E}+01$ & $5.90 \%$ \\
\hline Cd-113 & $2.7 \mathrm{E}+00$ & $4.6 \mathrm{E}+00$ & $66.68 \%$ & Am-243 & $3.4 \mathrm{E}+01$ & $3.6 \mathrm{E}+01$ & $5.89 \%$ \\
\hline $\mathrm{Cd}-115 \mathrm{~m}$ & $7.0 \mathrm{E}-02$ & $8.5 \mathrm{E}-02$ & $21.20 \%$ & $\mathrm{Pu}-237$ & $4.6 \mathrm{E}-03$ & $5.5 \mathrm{E}-03$ & $19.67 \%$ \\
\hline In-115 & $1.1 \mathrm{E}+00$ & $1.1 \mathrm{E}+00$ & $5.03 \%$ & $\mathrm{Pu}-238$ & $2.9 \mathrm{E}+01$ & $3.0 \mathrm{E}+01$ & $5.83 \%$ \\
\hline Sb-122 & $2.8 \mathrm{E}-04$ & $1.1 \mathrm{E}-03$ & $287.32 \%$ & $\mathrm{Pu}-239$ & $3.2 \mathrm{E}+01$ & $3.4 \mathrm{E}+01$ & $5.81 \%$ \\
\hline $\mathrm{Sb}-124$ & $5.0 \mathrm{E}-02$ & $5.6 \mathrm{E}-02$ & $11.15 \%$ & $\mathrm{Pu}-240$ & $3.2 \mathrm{E}+01$ & $3.4 \mathrm{E}+01$ & $5.81 \%$ \\
\hline $\mathrm{Sb}-125$ & $2.0 \mathrm{E}-02$ & $2.0 \mathrm{E}-02$ & $1.84 \%$ & $\mathrm{Pu}-241$ & $6.5 \mathrm{E}-01$ & $6.8 \mathrm{E}-01$ & $5.94 \%$ \\
\hline Te-123 & $2.4 \mathrm{E}-01$ & $6.6 \mathrm{E}-02$ & $-72.28 \%$ & $\mathrm{Pu}-242$ & $3.1 \mathrm{E}+01$ & $3.3 \mathrm{E}+01$ & $5.81 \%$ \\
\hline Te- $125 \mathrm{~m}$ & $2.0 \mathrm{E}-02$ & $2.1 \mathrm{E}-02$ & $1.69 \%$ & $\mathrm{Pu}-244$ & $3.0 \mathrm{E}+01$ & $3.2 \mathrm{E}+01$ & $5.81 \%$ \\
\hline Sn-126 & $1.4 \mathrm{E}-01$ & $1.5 \mathrm{E}-01$ & $4.17 \%$ & $\mathrm{Cm}-241$ & $1.8 \mathrm{E}-02$ & $2.2 \mathrm{E}-02$ & $25.38 \%$ \\
\hline I-129 & $2.7 \mathrm{E}+00$ & $3.0 \mathrm{E}+00$ & $13.55 \%$ & $\mathrm{Cm}-242$ & 7.2E-01 & 7.9E-01 & $9.52 \%$ \\
\hline $\mathrm{I}-131$ & $5.7 \mathrm{E}-02$ & $9.6 \mathrm{E}-02$ & $66.71 \%$ & $\mathrm{Cm}-243$ & $2.2 \mathrm{E}+01$ & $2.3 \mathrm{E}+01$ & $5.21 \%$ \\
\hline Cs-134 & $7.0 \mathrm{E}-01$ & $6.5 \mathrm{E}-01$ & $-7.61 \%$ & $\mathrm{Cm}-244$ & $1.7 \mathrm{E}+01$ & $1.8 \mathrm{E}+01$ & $5.33 \%$ \\
\hline Cs-135 & $7.3 \mathrm{E}-02$ & $7.0 \mathrm{E}-02$ & $-4.10 \%$ & $\mathrm{Cm}-245$ & $3.4 \mathrm{E}+01$ & $3.6 \mathrm{E}+01$ & $4.98 \%$ \\
\hline Cs-137 & $5.0 \mathrm{E}-01$ & $4.8 \mathrm{E}-01$ & $-5.27 \%$ & $\mathrm{Cm}-246$ & $3.4 \mathrm{E}+01$ & $3.6 \mathrm{E}+01$ & $4.98 \%$ \\
\hline La-137 & $3.3 \mathrm{E}-03$ & $3.5 \mathrm{E}-03$ & $6.38 \%$ & $\mathrm{Cm}-247$ & $3.1 \mathrm{E}+01$ & $3.3 \mathrm{E}+01$ & $4.98 \%$ \\
\hline La-138 & $4.5 \mathrm{E}-02$ & $4.8 \mathrm{E}-02$ & $6.38 \%$ & $\mathrm{Cm}-248$ & $1.2 \mathrm{E}+02$ & $1.3 \mathrm{E}+02$ & $4.98 \%$ \\
\hline La-140 & 8.9E-06 & $2.5 \mathrm{E}-04$ & $2699.37 \%$ & $\mathrm{Cm}-250$ & $5.9 \mathrm{E}+02$ & $6.2 \mathrm{E}+02$ & $4.98 \%$ \\
\hline $\mathrm{Ba}-140$ & $1.3 \mathrm{E}-02$ & $2.2 \mathrm{E}-02$ & $60.09 \%$ & Bk-247 & $1.7 \mathrm{E}+01$ & $1.9 \mathrm{E}+01$ & $7.65 \%$ \\
\hline $\mathrm{Ce}-141$ & $1.0 \mathrm{E}-02$ & $1.3 \mathrm{E}-02$ & $23.04 \%$ & Bk-249 & 4.2E-02 & $4.5 \mathrm{E}-02$ & $7.82 \%$ \\
\hline $\mathrm{Ce}-144$ & $1.4 \mathrm{E}-01$ & $1.5 \mathrm{E}-01$ & $6.46 \%$ & Cf-249 & $3.5 \mathrm{E}+01$ & $3.7 \mathrm{E}+01$ & $5.53 \%$ \\
\hline & & & & Cf-250 & $1.4 \mathrm{E}+01$ & $1.5 \mathrm{E}+01$ & $5.77 \%$ \\
\hline & & & & Cf-251 & $3.5 \mathrm{E}+01$ & $3.7 \mathrm{E}+01$ & $5.52 \%$ \\
\hline & & & & Cf-252 & $6.9 \mathrm{E}+00$ & $7.4 \mathrm{E}+00$ & $6.41 \%$ \\
\hline & & & & Es-253 & $6.4 \mathrm{E}-02$ & $8.9 \mathrm{E}-02$ & $38.82 \%$ \\
\hline & & & & Unidentified alpha & $3.2 \mathrm{E}+01$ & $3.4 \mathrm{E}+01$ & $5.81 \%$ \\
\hline & & & & Unidentified beta & $1.1 \mathrm{E}+00$ & $2.7 \mathrm{E}+00$ & $157.22 \%$ \\
\hline & & & & TOTAL Dose & $1.7 E+03$ & $1.8 \mathrm{E}+03$ & $5.01 \%$ \\
\hline
\end{tabular}


Table 36. MAXDOSE-SR Maximally Exposed Individual Ground Exposure Comparisons (mrem/yr)

\begin{tabular}{|c|c|c|c|}
\hline \multicolumn{4}{|c|}{ Maxdose Ground Pathway } \\
\hline Nuclide & Hamby & Jannik & $\%$ Diff \\
\hline $\mathrm{Ag}-110 \mathrm{M}$ & 9.89E-03 & $9.89 \mathrm{E}-03$ & $0.00 \%$ \\
\hline Am-241 & $1.40 \mathrm{E}-04$ & $1.40 \mathrm{E}-04$ & $0.00 \%$ \\
\hline Am-243 & $3.10 \mathrm{E}-04$ & $3.10 \mathrm{E}-04$ & $0.00 \%$ \\
\hline Ba-139 & $2.16 \mathrm{E}-08$ & $2.16 \mathrm{E}-08$ & $0.00 \%$ \\
\hline Ba-140 & $1.10 \mathrm{E}-03$ & $1.10 \mathrm{E}-03$ & $0.00 \%$ \\
\hline Ba-141 & $9.80 \mathrm{E}-10$ & $9.80 \mathrm{E}-10$ & $0.00 \%$ \\
\hline Ba-142 & $7.73 \mathrm{E}-12$ & $7.73 \mathrm{E}-12$ & $0.00 \%$ \\
\hline $\mathrm{Be}-7$ & $9.24 \mathrm{E}-05$ & $9.24 \mathrm{E}-05$ & $0.00 \%$ \\
\hline $\mathrm{Br}-83$ & $1.31 \mathrm{E}-08$ & $1.31 \mathrm{E}-08$ & $0.00 \%$ \\
\hline $\mathrm{Br}-84$ & $4.02 \mathrm{E}-08$ & $4.02 \mathrm{E}-08$ & $0.00 \%$ \\
\hline Ce-141 & $1.03 \mathrm{E}-04$ & $1.03 \mathrm{E}-04$ & $0.00 \%$ \\
\hline $\mathrm{Ce}-143$ & $1.38 \mathrm{E}-05$ & $1.38 \mathrm{E}-05$ & $0.00 \%$ \\
\hline Ce-144 & $1.93 \mathrm{E}-04$ & $1.93 \mathrm{E}-04$ & $0.00 \%$ \\
\hline Cf-252 & $2.79 \mathrm{E}-06$ & $2.79 \mathrm{E}-06$ & $0.00 \%$ \\
\hline $\mathrm{Cm}-242$ & $5.87 \mathrm{E}-06$ & $5.87 \mathrm{E}-06$ & $0.00 \%$ \\
\hline $\mathrm{Cm}-243$ & $6.83 \mathrm{E}-04$ & $6.83 \mathrm{E}-04$ & $0.00 \%$ \\
\hline $\mathrm{Cm}-244$ & $3.86 \mathrm{E}-06$ & $3.86 \mathrm{E}-06$ & $0.00 \%$ \\
\hline $\mathrm{Cm}-246$ & $3.45 \mathrm{E}-06$ & $3.45 \mathrm{E}-06$ & $0.00 \%$ \\
\hline $\mathrm{Cm}-248$ & $2.76 \mathrm{E}-06$ & $2.76 \mathrm{E}-06$ & $0.00 \%$ \\
\hline Co-57 & $5.36 \mathrm{E}-04$ & $5.36 \mathrm{E}-04$ & $0.00 \%$ \\
\hline Co-58 & $2.18 \mathrm{E}-03$ & $2.18 \mathrm{E}-03$ & $0.00 \%$ \\
\hline Co-60 & $1.03 \mathrm{E}-02$ & $1.03 \mathrm{E}-02$ & $0.00 \%$ \\
\hline Cr-51 & $3.47 \mathrm{E}-05$ & $3.47 \mathrm{E}-05$ & $0.00 \%$ \\
\hline Cs-134 & $6.82 \mathrm{E}-03$ & $6.82 \mathrm{E}-03$ & $0.00 \%$ \\
\hline Cs-137 & $2.70 \mathrm{E}-03$ & $2.70 \mathrm{E}-03$ & $0.00 \%$ \\
\hline Cs-138 & $5.76 \mathrm{E}-08$ & $5.76 \mathrm{E}-08$ & $0.00 \%$ \\
\hline Eu-152 & $5.16 \mathrm{E}-03$ & $5.16 \mathrm{E}-03$ & $0.00 \%$ \\
\hline Eu-154 & $5.57 \mathrm{E}-03$ & $5.57 \mathrm{E}-03$ & $0.00 \%$ \\
\hline Eu-155 & $3.24 \mathrm{E}-04$ & $3.24 \mathrm{E}-04$ & $0.00 \%$ \\
\hline $\mathrm{Fe}-55$ & $9.70 \mathrm{E}-07$ & $9.70 \mathrm{E}-07$ & $0.00 \%$ \\
\hline Fe-59 & $1.72 \mathrm{E}-03$ & $1.72 \mathrm{E}-03$ & $0.00 \%$ \\
\hline Hf-181 & $8.62 \mathrm{E}-04$ & $8.62 \mathrm{E}-04$ & $0.00 \%$ \\
\hline $\mathrm{I}-129$ & $1.03 \mathrm{E}-04$ & $1.03 \mathrm{E}-04$ & $0.00 \%$ \\
\hline $\mathrm{I}-131$ & $1.20 \mathrm{E}-04$ & $1.20 \mathrm{E}-04$ & $0.00 \%$ \\
\hline $\mathrm{I}-132$ & $3.65 \mathrm{E}-06$ & $3.65 \mathrm{E}-06$ & $0.00 \%$ \\
\hline I-133 & $1.97 \mathrm{E}-05$ & $1.97 \mathrm{E}-05$ & $0.00 \%$ \\
\hline $\mathrm{I}-134$ & $4.24 \mathrm{E}-07$ & $4.24 \mathrm{E}-07$ & $0.00 \%$ \\
\hline $\mathrm{I}-135$ & $1.12 \mathrm{E}-05$ & $1.12 \mathrm{E}-05$ & $0.00 \%$ \\
\hline La-140 & $1.27 \mathrm{E}-04$ & $1.27 \mathrm{E}-04$ & $0.00 \%$ \\
\hline La-141 & $1.45 \mathrm{E}-07$ & $1.45 \mathrm{E}-07$ & $0.00 \%$ \\
\hline La-142 & $1.76 \mathrm{E}-06$ & $1.76 \mathrm{E}-06$ & $0.00 \%$ \\
\hline
\end{tabular}


SRNL-STI-2010-00447, REVISION 0

Table 36. MAXDOSE-SR Maximally Exposed Individual Ground Exposure Comparisons (mrem/yr) (continued)

\begin{tabular}{|l|r|r|c|}
\hline \multicolumn{4}{|c|}{ Maxdose Ground Pathway } \\
\hline Nuclide & Hamby & Jannik & \% Diff \\
\hline Mn-54 & $3.25 \mathrm{E}-03$ & $3.25 \mathrm{E}-03$ & $0.00 \%$ \\
\hline Mn-56 & $3.10 \mathrm{E}-06$ & $3.10 \mathrm{E}-06$ & $0.00 \%$ \\
\hline Mo-99 & $2.89 \mathrm{E}-05$ & $2.89 \mathrm{E}-05$ & $0.00 \%$ \\
\hline Na-24 & $7.32 \mathrm{E}-05$ & $7.32 \mathrm{E}-05$ & $0.00 \%$ \\
\hline Nb-95 & $9.80 \mathrm{E}-04$ & $9.80 \mathrm{E}-04$ & $0.00 \%$ \\
\hline Nb-97 & $2.72 \mathrm{E}-07$ & $2.72 \mathrm{E}-07$ & $0.00 \%$ \\
\hline Ni-59 & $1.96 \mathrm{E}-06$ & $1.96 \mathrm{E}-06$ & $0.00 \%$ \\
\hline Ni-65 & $9.28 \mathrm{E}-07$ & $9.28 \mathrm{E}-07$ & $0.00 \%$ \\
\hline Np-237 & $1.52 \mathrm{E}-04$ & $1.52 \mathrm{E}-04$ & $0.00 \%$ \\
\hline Np-238 & $4.08 \mathrm{E}-05$ & $4.08 \mathrm{E}-05$ & $0.00 \%$ \\
\hline Np-239 & $1.63 \mathrm{E}-05$ & $1.63 \mathrm{E}-05$ & $0.00 \%$ \\
\hline Os-185 & $1.88 \mathrm{E}-03$ & $1.88 \mathrm{E}-03$ & $0.00 \%$ \\
\hline P-32 & $0.00 \mathrm{E}+00$ & $0.00 \mathrm{E}+00$ & \\
\hline Pa-233 & $2.34 \mathrm{E}-04$ & $2.34 \mathrm{E}-04$ & $0.00 \%$ \\
\hline Pd-109 & $1.29 \mathrm{E}-08$ & $1.29 \mathrm{E}-08$ & $0.00 \%$ \\
\hline Pm-147 & $1.80 \mathrm{E}-08$ & $1.80 \mathrm{E}-08$ & $0.00 \%$ \\
\hline Pm-148 & $1.06 \mathrm{E}-04$ & $1.06 \mathrm{E}-04$ & $0.00 \%$ \\
\hline Pm-151 & $1.47 \mathrm{E}-05$ & $1.47 \mathrm{E}-05$ & $0.00 \%$ \\
\hline Pr-144 & $2.16 \mathrm{E}-11$ & $2.16 \mathrm{E}-11$ & $0.00 \%$ \\
\hline Pu-238 & $4.02 \mathrm{E}-06$ & $4.02 \mathrm{E}-06$ & $0.00 \%$ \\
\hline Pu-239 & $1.78 \mathrm{E}-06$ & $1.78 \mathrm{E}-06$ & $0.00 \%$ \\
\hline Pu-240 & $3.85 \mathrm{E}-06$ & $3.85 \mathrm{E}-06$ & $0.00 \%$ \\
\hline Pu-242 & $3.21 \mathrm{E}-06$ & $3.21 \mathrm{E}-06$ & $0.00 \%$ \\
\hline Ra-224 & $1.47 \mathrm{E}-06$ & $1.47 \mathrm{E}-06$ & $0.00 \%$ \\
\hline Ra-228 & $3.04 \mathrm{E}-12$ & $3.04 \mathrm{E}-12$ & $0.00 \%$ \\
\hline Rb-86 & $6.22 \mathrm{E}-05$ & $6.22 \mathrm{E}-05$ & $0.00 \%$ \\
\hline Rb-88 & $5.36 \mathrm{E}-10$ & $5.36 \mathrm{E}-10$ & $0.00 \%$ \\
\hline Rb-89 & $5.08 \mathrm{E}-10$ & $5.08 \mathrm{E}-10$ & $0.00 \%$ \\
\hline Rh-105 & $4.35 \mathrm{E}-06$ & $4.35 \mathrm{E}-06$ & $0.00 \%$ \\
\hline Ru-103 & $7.07 \mathrm{E}-04$ & $7.07 \mathrm{E}-04$ & $0.00 \%$ \\
\hline Ru-105 & $3.64 \mathrm{E}-06$ & $3.64 \mathrm{E}-06$ & $0.00 \%$ \\
\hline Ru-106 & $8.36 \mathrm{E}-04$ & $8.36 \mathrm{E}-04$ & $0.00 \%$ \\
\hline Sb-122 & $4.44 \mathrm{E}-05$ & $4.44 \mathrm{E}-05$ & $0.00 \%$ \\
\hline Sb-124 & $3.46 \mathrm{E}-03$ & $3.46 \mathrm{E}-03$ & $0.00 \%$ \\
\hline Sb-125 & $1.98 \mathrm{E}-03$ & $1.98 \mathrm{E}-03$ & $0.00 \%$ \\
\hline Sb-126 & $1.29 \mathrm{E}-03$ & $1.29 \mathrm{E}-03$ & $0.00 \%$ \\
\hline Sb-126M & $2.33 \mathrm{E}-09$ & $2.33 \mathrm{E}-09$ & $0.00 \%$ \\
\hline Se-75 & $1.25 \mathrm{E}-03$ & $1.25 \mathrm{E}-03$ & $0.00 \%$ \\
\hline Sm-151 & $2.44 \mathrm{E}-08$ & $0.00 \%$ \\
\hline Sn-123 & $1.96 \mathrm{E}-05$ & $0.00 \%$ \\
\hline Sn-126 04 & $0.00 \%$ \\
\hline
\end{tabular}


SRNL-STI-2010-00447, REVISION 0

Table 36. MAXDOSE-SR Maximally Exposed Individual Ground Exposure Comparisons (mrem/yr) (continued)

\begin{tabular}{|l|r|r|c|}
\hline \multicolumn{4}{|c|}{ Maxdose Ground Pathway } \\
\hline Nuclide & Hamby & Jannik & $\mathbf{\% ~ D i f f ~}$ \\
\hline Sr-89 & $2.33 \mathrm{E}-07$ & $2.33 \mathrm{E}-07$ & $0.00 \%$ \\
\hline Sr-90 & $0.00 \mathrm{E}+00$ & $0.00 \mathrm{E}+00$ & \\
\hline Sr-91 & $8.18 \mathrm{E}-06$ & $8.18 \mathrm{E}-06$ & $0.00 \%$ \\
\hline Sr-92 & $2.53 \mathrm{E}-06$ & $2.53 \mathrm{E}-06$ & $0.00 \%$ \\
\hline Tc-101 & $5.47 \mathrm{E}-11$ & $5.47 \mathrm{E}-11$ & $0.00 \%$ \\
\hline Tc-99 & $2.94 \mathrm{E}-09$ & $2.94 \mathrm{E}-09$ & $0.00 \%$ \\
\hline Tc-99 M & $1.01 \mathrm{E}-06$ & $1.01 \mathrm{E}-06$ & $0.00 \%$ \\
\hline Te-125M & $4.02 \mathrm{E}-05$ & $4.02 \mathrm{E}-05$ & $0.00 \%$ \\
\hline Te-127 & $6.07 \mathrm{E}-08$ & $6.07 \mathrm{E}-08$ & $0.00 \%$ \\
\hline Te-127M & $1.86 \mathrm{E}-05$ & $1.86 \mathrm{E}-05$ & $0.00 \%$ \\
\hline Te-129 & $2.07 \mathrm{E}-08$ & $2.07 \mathrm{E}-08$ & $0.00 \%$ \\
\hline Te-129M & $4.46 \mathrm{E}-05$ & $4.46 \mathrm{E}-05$ & $0.00 \%$ \\
\hline Te-131 & $3.38 \mathrm{E}-09$ & $3.38 \mathrm{E}-09$ & $0.00 \%$ \\
\hline Te-131M & $6.16 \mathrm{E}-05$ & $6.16 \mathrm{E}-05$ & $0.00 \%$ \\
\hline Te-132 & $2.93 \mathrm{E}-05$ & $2.93 \mathrm{E}-05$ & $0.00 \%$ \\
\hline Te-134 & $6.87 \mathrm{E}-08$ & $6.87 \mathrm{E}-08$ & $0.00 \%$ \\
\hline Th-228 & $1.19 \mathrm{E}-05$ & $1.19 \mathrm{E}-05$ & $0.00 \%$ \\
\hline Th-230 & $4.28 \mathrm{E}-06$ & $4.28 \mathrm{E}-06$ & $0.00 \%$ \\
\hline Th-232 & $3.13 \mathrm{E}-06$ & $3.13 \mathrm{E}-06$ & $0.00 \%$ \\
\hline Th-234 & $8.94 \mathrm{E}-06$ & $8.94 \mathrm{E}-06$ & $0.00 \%$ \\
\hline U-232 & $4.85 \mathrm{E}-06$ & $4.85 \mathrm{E}-06$ & $0.00 \%$ \\
\hline U-233 & $2.35 \mathrm{E}-06$ & $2.35 \mathrm{E}-06$ & $0.00 \%$ \\
\hline U-234 & $3.79 \mathrm{E}-06$ & $3.79 \mathrm{E}-06$ & $0.00 \%$ \\
\hline U-235 & $8.03 \mathrm{E}-04$ & $8.03 \mathrm{E}-04$ & $0.00 \%$ \\
\hline U-236 & $3.45 \mathrm{E}-06$ & $3.45 \mathrm{E}-06$ & $0.00 \%$ \\
\hline U-237 & $3.99 \mathrm{E}-05$ & $3.99 \mathrm{E}-05$ & $0.00 \%$ \\
\hline U-238 & $3.03 \mathrm{E}-06$ & $3.03 \mathrm{E}-06$ & $0.00 \%$ \\
\hline W-187 & $1.69 \mathrm{E}-05$ & $1.69 \mathrm{E}-05$ & $0.00 \%$ \\
\hline Y-90 & $0.00 \mathrm{E}+00$ & $0.00 \mathrm{E}+00$ & \\
\hline Y-91 & $6.36 \mathrm{E}-06$ & $6.36 \mathrm{E}-06$ & $0.00 \%$ \\
\hline Y-91M & $7.57 \mathrm{E}-08$ & $7.57 \mathrm{E}-08$ & $0.00 \%$ \\
\hline Y-92 & $7.90 \mathrm{E}-07$ & $7.90 \mathrm{E}-07$ & $0.00 \%$ \\
\hline Y-93 & $1.11 \mathrm{E}-06$ & $1.11 \mathrm{E}-06$ & $0.00 \%$ \\
\hline Zn-65 & $2.01 \mathrm{E}-03$ & $2.01 \mathrm{E}-03$ & $0.00 \%$ \\
\hline Zr-95 & $3.18 \mathrm{E}-03$ & $3.18 \mathrm{E}-03$ & $0.00 \%$ \\
\hline Zr-97 & & $4.08 \mathrm{E}-06$ & $0.00 \%$ \\
\hline TOTAL* & & $\mathbf{7 . 1 6 E - 0 2}$ & $\mathbf{0 . 0 0 \%}$ \\
\hline & & & \\
\hline
\end{tabular}


SRNL-STI-2010-00447, REVISION 0

Table 37. MAXDOSE-SR Maximally Exposed Individual Plume Immersion Comparisons (mrem/yr)

\begin{tabular}{|l|c|c|c|}
\hline \multicolumn{4}{|c|}{ Maxdose Plume Pathway } \\
\hline \multicolumn{1}{|c|}{ Nuclide } & Hamby & Jannik & \% Diff \\
\hline Ar-41 & $1.57 \mathrm{E}-06$ & $1.57 \mathrm{E}-06$ & $0.00 \%$ \\
\hline $\mathrm{Kr}-83 \mathrm{M}$ & $1.12 \mathrm{E}-10$ & $1.12 \mathrm{E}-10$ & $0.00 \%$ \\
\hline $\mathrm{Kr}-85$ & $7.27 \mathrm{E}-09$ & $7.27 \mathrm{E}-09$ & $0.00 \%$ \\
\hline $\mathrm{Kr}-85 \mathrm{M}$ & $3.51 \mathrm{E}-07$ & $3.51 \mathrm{E}-07$ & $0.00 \%$ \\
\hline $\mathrm{Kr}-87$ & $6.82 \mathrm{E}-07$ & $6.82 \mathrm{E}-07$ & $0.00 \%$ \\
\hline $\mathrm{Kr}-88$ & $5.05 \mathrm{E}-06$ & $5.05 \mathrm{E}-06$ & $0.00 \%$ \\
\hline $\mathrm{Kr}-89$ & $6.03 \mathrm{E}-21$ & $6.03 \mathrm{E}-21$ & $0.00 \%$ \\
\hline $\mathrm{Xe}-133$ & $1.13 \mathrm{E}-07$ & $1.13 \mathrm{E}-07$ & $0.00 \%$ \\
\hline $\mathrm{Xe}-133 \mathrm{M}$ & $9.34 \mathrm{E}-08$ & $9.34 \mathrm{E}-08$ & $0.00 \%$ \\
\hline $\mathrm{Xe}-135$ & $6.62 \mathrm{E}-07$ & $6.62 \mathrm{E}-07$ & $0.00 \%$ \\
\hline $\mathrm{Xe}-135 \mathrm{M}$ & $1.03 \mathrm{E}-09$ & $1.03 \mathrm{E}-09$ & $0.00 \%$ \\
\hline $\mathrm{Xe}-137$ & $2.88 \mathrm{E}-19$ & $2.88 \mathrm{E}-19$ & $0.00 \%$ \\
\hline Xe-138 & $1.64 \mathrm{E}-09$ & $1.64 \mathrm{E}-09$ & $0.00 \%$ \\
\hline Xe-131M & $2.77 \mathrm{E}-08$ & $2.77 \mathrm{E}-08$ & $0.00 \%$ \\
\hline \multirow{2}{*}{ Total } & $\mathbf{8 . 5 6 E - 0 6}$ & $\mathbf{8 . 5 6 E}-06$ & $\mathbf{- 0 . 0 1 \%}$ \\
\hline
\end{tabular}


Table 38. MAXDOSE-SR Maximally Exposed Individual Inhalation Pathway Comparisons (mrem/yr)

\begin{tabular}{|c|c|c|c|}
\hline \multicolumn{4}{|c|}{ Maxdose Inhalation Pathway } \\
\hline Nuclide & Hamby & Jannik & \% Diff \\
\hline Ag-110M & $2.71 \mathrm{E}-04$ & $2.71 \mathrm{E}-04$ & $0.00 \%$ \\
\hline Am-241 & $2.65 \mathrm{E}+00$ & $2.65 \mathrm{E}+00$ & $0.00 \%$ \\
\hline Am-243 & $2.65 \mathrm{E}+00$ & $2.65 \mathrm{E}+00$ & $0.00 \%$ \\
\hline Ba-139 & $2.13 \mathrm{E}-07$ & $2.13 \mathrm{E}-07$ & $0.00 \%$ \\
\hline Ba-140 & $1.83 \mathrm{E}-05$ & $1.83 \mathrm{E}-05$ & $0.00 \%$ \\
\hline Ba-141 & $8.79 \mathrm{E}-10$ & $8.79 \mathrm{E}-10$ & $0.00 \%$ \\
\hline Ba-142 & $5.82 \mathrm{E}-12$ & $5.82 \mathrm{E}-12$ & $0.00 \%$ \\
\hline $\mathrm{Be}-7$ & $1.38 \mathrm{E}-06$ & $1.38 \mathrm{E}-06$ & $0.00 \%$ \\
\hline $\mathrm{Br}-83$ & $1.89 \mathrm{E}-07$ & $1.89 \mathrm{E}-07$ & $0.00 \%$ \\
\hline $\mathrm{Br}-84$ & $1.37 \mathrm{E}-08$ & $1.37 \mathrm{E}-08$ & $0.00 \%$ \\
\hline Ce-141 & 4.33E-05 & 4.33E-05 & $0.00 \%$ \\
\hline Ce-143 & $1.55 \mathrm{E}-05$ & $1.55 \mathrm{E}-05$ & $0.00 \%$ \\
\hline Ce-144 & $1.79 \mathrm{E}-03$ & $1.79 \mathrm{E}-03$ & $0.00 \%$ \\
\hline Cf-252 & $6.64 \mathrm{E}-01$ & 6.64E-01 & $0.00 \%$ \\
\hline $\mathrm{Cm}-242$ & $8.68 \mathrm{E}-02$ & $8.68 \mathrm{E}-02$ & $0.00 \%$ \\
\hline $\mathrm{Cm}-243$ & $1.79 \mathrm{E}+00$ & $1.79 \mathrm{E}+00$ & $0.00 \%$ \\
\hline $\mathrm{Cm}-244$ & $1.38 \mathrm{E}+00$ & $1.38 \mathrm{E}+00$ & $0.00 \%$ \\
\hline $\mathrm{Cm}-246$ & $2.76 \mathrm{E}+00$ & $2.76 \mathrm{E}+00$ & $0.00 \%$ \\
\hline $\mathrm{Cm}-248$ & $9.70 \mathrm{E}+00$ & $9.70 \mathrm{E}+00$ & $0.00 \%$ \\
\hline Co-57 & $3.83 \mathrm{E}-05$ & $3.83 \mathrm{E}-05$ & $0.00 \%$ \\
\hline Co-58 & $3.62 \mathrm{E}-05$ & $3.62 \mathrm{E}-05$ & $0.00 \%$ \\
\hline Co-60 & $7.66 \mathrm{E}-04$ & $7.66 \mathrm{E}-04$ & $0.00 \%$ \\
\hline Cr-51 & $1.32 \mathrm{E}-06$ & $1.32 \mathrm{E}-06$ & $0.00 \%$ \\
\hline Cs-134 & $2.40 \mathrm{E}-04$ & $2.40 \mathrm{E}-04$ & $0.00 \%$ \\
\hline Cs-135 & $2.30 \mathrm{E}-05$ & $2.30 \mathrm{E}-05$ & $0.00 \%$ \\
\hline Cs-137 & $1.63 \mathrm{E}-04$ & $1.63 \mathrm{E}-04$ & $0.00 \%$ \\
\hline Cs-138 & $1.45 \mathrm{E}-08$ & $1.45 \mathrm{E}-08$ & $0.00 \%$ \\
\hline Eu-152 & $1.12 \mathrm{E}-03$ & $1.12 \mathrm{E}-03$ & $0.00 \%$ \\
\hline Eu-154 & $1.33 \mathrm{E}-03$ & $1.33 \mathrm{E}-03$ & $0.00 \%$ \\
\hline Eu-155 & $1.99 \mathrm{E}-04$ & 1.99E-04 & $0.00 \%$ \\
\hline $\mathrm{Fe}-55$ & $1.33 \mathrm{E}-05$ & $1.33 \mathrm{E}-05$ & $0.00 \%$ \\
\hline $\mathrm{Fe}-59$ & $7.65 \mathrm{E}-05$ & $7.65 \mathrm{E}-05$ & $0.00 \%$ \\
\hline $\mathrm{H}-3$ & 7.05E-07 & 7.05E-07 & $0.00 \%$ \\
\hline Hf-181 & $6.63 \mathrm{E}-05$ & $6.63 \mathrm{E}-05$ & $0.00 \%$ \\
\hline I-129 & 9.19E-04 & 9.19E-04 & $0.00 \%$ \\
\hline I-131 & $1.62 \mathrm{E}-04$ & $1.62 \mathrm{E}-04$ & $0.00 \%$ \\
\hline $\mathrm{I}-132$ & $7.55 \mathrm{E}-07$ & $7.55 \mathrm{E}-07$ & $0.00 \%$ \\
\hline I-133 & $2.52 \mathrm{E}-05$ & $2.52 \mathrm{E}-05$ & $0.00 \%$ \\
\hline I-134 & $6.81 \mathrm{E}-08$ & $6.81 \mathrm{E}-08$ & $0.00 \%$ \\
\hline $\mathrm{I}-135$ & 4.24E-06 & 4.24E-06 & $0.00 \%$ \\
\hline La-140 & $2.15 \mathrm{E}-05$ & $2.15 \mathrm{E}-05$ & $0.00 \%$ \\
\hline La-141 & $1.72 \mathrm{E}-06$ & $1.72 \mathrm{E}-06$ & $0.00 \%$ \\
\hline La-142 & 3.39E-07 & 3.39E-07 & $0.00 \%$ \\
\hline
\end{tabular}


SRNL-STI-2010-00447, REVISION 0

Table 38. MAXDOSE-SR Maximally Exposed Individual Inhalation Pathway Comparisons (mrem/yr) (continued)

\begin{tabular}{|c|c|c|c|}
\hline \multicolumn{4}{|c|}{ Maxdose Inhalation Pathway } \\
\hline Nuclide & Hamby & Jannik & $\%$ Diff \\
\hline Mn-54 & $3.27 \mathrm{E}-05$ & $3.27 \mathrm{E}-05$ & $0.00 \%$ \\
\hline Mn-56 & $8.21 \mathrm{E}-07$ & $8.21 \mathrm{E}-07$ & $0.00 \%$ \\
\hline Mo-99 & $1.79 \mathrm{E}-05$ & $1.79 \mathrm{E}-05$ & $0.00 \%$ \\
\hline $\mathrm{Na}-24$ & $4.29 \mathrm{E}-06$ & $4.29 \mathrm{E}-06$ & $0.00 \%$ \\
\hline $\mathrm{Nb}-95$ & $2.29 \mathrm{E}-05$ & $2.29 \mathrm{E}-05$ & $0.00 \%$ \\
\hline $\mathrm{Nb}-97$ & 7.81E-08 & 7.81E-08 & $0.00 \%$ \\
\hline Ni-59 & $6.64 \mathrm{E}-06$ & $6.64 \mathrm{E}-06$ & $0.00 \%$ \\
\hline $\mathrm{Ni}-63$ & $1.53 \mathrm{E}-05$ & $1.53 \mathrm{E}-05$ & $0.00 \%$ \\
\hline $\mathrm{Ni}-65$ & $5.15 \mathrm{E}-07$ & $5.15 \mathrm{E}-07$ & $0.00 \%$ \\
\hline $\mathrm{Np}-237$ & $2.50 \mathrm{E}+00$ & $2.50 \mathrm{E}+00$ & $0.00 \%$ \\
\hline $\mathrm{Np}-238$ & $1.53 \mathrm{E}-04$ & $1.53 \mathrm{E}-04$ & $0.00 \%$ \\
\hline $\mathrm{Np}-239$ & $1.09 \mathrm{E}-05$ & $1.09 \mathrm{E}-05$ & $0.00 \%$ \\
\hline Os-185 & $5.10 \mathrm{E}-05$ & $5.10 \mathrm{E}-05$ & $0.00 \%$ \\
\hline $\begin{array}{l}\mathrm{P}-32 \\
\end{array}$ & $6.60 \mathrm{E}-05$ & $6.60 \mathrm{E}-05$ & $0.00 \%$ \\
\hline Pa-233 & $4.38 \mathrm{E}-05$ & $4.38 \mathrm{E}-05$ & $0.00 \%$ \\
\hline Pd-109 & 4.90E-06 & 4.90E-06 & $0.00 \%$ \\
\hline Pm-147 & $1.74 \mathrm{E}-04$ & 1.74E-04 & $0.00 \%$ \\
\hline Pm-148 & $5.03 \mathrm{E}-05$ & $5.03 \mathrm{E}-05$ & $0.00 \%$ \\
\hline Pm-151 & $7.65 \mathrm{E}-06$ & $7.65 \mathrm{E}-06$ & $0.00 \%$ \\
\hline Pr-144 & $3.50 \mathrm{E}-10$ & $3.50 \mathrm{E}-10$ & $0.00 \%$ \\
\hline $\mathrm{Pu}-238$ & $2.35 \mathrm{E}+00$ & $2.35 \mathrm{E}+00$ & $0.00 \%$ \\
\hline $\mathrm{Pu}-239$ & $2.60 \mathrm{E}+00$ & $2.60 \mathrm{E}+00$ & $0.00 \%$ \\
\hline $\mathrm{Pu}-240$ & $2.60 \mathrm{E}+00$ & $2.60 \mathrm{E}+00$ & $0.00 \%$ \\
\hline $\mathrm{Pu}-241$ & $5.11 \mathrm{E}-02$ & $5.11 \mathrm{E}-02$ & $0.00 \%$ \\
\hline $\mathrm{Pu}-242$ & $2.45 \mathrm{E}+00$ & $2.45 \mathrm{E}+00$ & $0.00 \%$ \\
\hline Ra-224 & $1.45 \mathrm{E}-02$ & $1.45 \mathrm{E}-02$ & $0.00 \%$ \\
\hline Ra-228 & $2.14 \mathrm{E}-02$ & $2.14 \mathrm{E}-02$ & $0.00 \%$ \\
\hline Rb-86 & $3.36 \mathrm{E}-05$ & $3.36 \mathrm{E}-05$ & $0.00 \%$ \\
\hline $\mathrm{Rb}-87$ & $1.68 \mathrm{E}-05$ & $1.68 \mathrm{E}-05$ & $0.00 \%$ \\
\hline $\mathrm{Rb}-88$ & $8.23 \mathrm{E}-10$ & $8.23 \mathrm{E}-10$ & $0.00 \%$ \\
\hline $\mathrm{Rb}-89$ & $1.29 \mathrm{E}-10$ & $1.29 \mathrm{E}-10$ & $0.00 \%$ \\
\hline Rh-105 & $4.31 \mathrm{E}-06$ & $4.31 \mathrm{E}-06$ & $0.00 \%$ \\
\hline Rh-106M & $4.38 \mathrm{E}-07$ & $4.38 \mathrm{E}-07$ & $0.00 \%$ \\
\hline $\mathrm{Ru}-103$ & $3.97 \mathrm{E}-05$ & $3.97 \mathrm{E}-05$ & $0.00 \%$ \\
\hline Ru-105 & $1.38 \mathrm{E}-06$ & $1.38 \mathrm{E}-06$ & $0.00 \%$ \\
\hline Ru-106 & $2.25 \mathrm{E}-03$ & $2.25 \mathrm{E}-03$ & $0.00 \%$ \\
\hline S-35 & $1.17 \mathrm{E}-05$ & $1.17 \mathrm{E}-05$ & $0.00 \%$ \\
\hline Sb-122 & $2.33 \mathrm{E}-05$ & $2.33 \mathrm{E}-05$ & $0.00 \%$ \\
\hline Sb-124 & $1.07 \mathrm{E}-04$ & $1.07 \mathrm{E}-04$ & $0.00 \%$ \\
\hline Sb-125 & $5.00 \mathrm{E}-05$ & $5.00 \mathrm{E}-05$ & $0.00 \%$ \\
\hline Sb-126 & $5.07 \mathrm{E}-05$ & $5.07 \mathrm{E}-05$ & $0.00 \%$ \\
\hline
\end{tabular}


SRNL-STI-2010-00447, REVISION 0

Table 38. MAXDOSE-SR Maximally Exposed Individual Inhalation Pathway Comparisons (mrem/yr) (continued)

\begin{tabular}{|c|c|c|c|}
\hline \multicolumn{4}{|c|}{ Maxdose Inhalation Pathway } \\
\hline Nuclide & Hamby & Jannik & \% Diff \\
\hline Sb-126M & $4.19 \mathrm{E}-10$ & $4.19 \mathrm{E}-10$ & $0.00 \%$ \\
\hline Se-75 & $4.18 \mathrm{E}-05$ & $4.18 \mathrm{E}-05$ & $0.00 \%$ \\
\hline Se-79 & $4.54 \mathrm{E}-05$ & $4.54 \mathrm{E}-05$ & $0.00 \%$ \\
\hline Sm-151 & $1.48 \mathrm{E}-04$ & $1.48 \mathrm{E}-04$ & $0.00 \%$ \\
\hline Sn-123 & $1.53 \mathrm{E}-04$ & $1.53 \mathrm{E}-04$ & $0.00 \%$ \\
\hline Sn-126 & $4.39 \mathrm{E}-04$ & $4.39 \mathrm{E}-04$ & $0.00 \%$ \\
\hline Sr-89 & $1.89 \mathrm{E}-04$ & $1.89 \mathrm{E}-04$ & $0.00 \%$ \\
\hline Sr-90 & $6.64 \mathrm{E}-03$ & $6.64 \mathrm{E}-03$ & $0.00 \%$ \\
\hline Sr-91 & $5.88 \mathrm{E}-06$ & $5.88 \mathrm{E}-06$ & $0.00 \%$ \\
\hline Sr-92 & $1.99 \mathrm{E}-06$ & $1.99 \mathrm{E}-06$ & $0.00 \%$ \\
\hline Tc-101 & $3.32 \mathrm{E}-11$ & $3.32 \mathrm{E}-11$ & $0.00 \%$ \\
\hline Tc-99 & $3.83 \mathrm{E}-05$ & $3.83 \mathrm{E}-05$ & $0.00 \%$ \\
\hline Tc-99 M & $1.20 \mathrm{E}-07$ & $1.20 \mathrm{E}-07$ & $0.00 \%$ \\
\hline Te-125M & $3.42 \mathrm{E}-05$ & $3.42 \mathrm{E}-05$ & $0.00 \%$ \\
\hline Te-127 & $1.22 \mathrm{E}-06$ & $1.22 \mathrm{E}-06$ & $0.00 \%$ \\
\hline Te-127M & $9.69 \mathrm{E}-05$ & $9.69 \mathrm{E}-05$ & $0.00 \%$ \\
\hline Te-129 & $8.00 \mathrm{E}-08$ & $8.00 \mathrm{E}-08$ & $0.00 \%$ \\
\hline Te-129M & $1.02 \mathrm{E}-04$ & $1.02 \mathrm{E}-04$ & $0.00 \%$ \\
\hline Te-131 & $6.07 \mathrm{E}-09$ & $6.07 \mathrm{E}-09$ & $0.00 \%$ \\
\hline Te-131M & $2.64 \mathrm{E}-05$ & $2.64 \mathrm{E}-05$ & $0.00 \%$ \\
\hline Te-132 & $3.84 \mathrm{E}-05$ & $3.84 \mathrm{E}-05$ & $0.00 \%$ \\
\hline Te-134 & $3.58 \mathrm{E}-08$ & $3.58 \mathrm{E}-08$ & $0.00 \%$ \\
\hline Th-228 & $1.58 \mathrm{E}+00$ & $1.58 \mathrm{E}+00$ & $0.00 \%$ \\
\hline Th-230 & $1.63 \mathrm{E}+00$ & $1.63 \mathrm{E}+00$ & $0.00 \%$ \\
\hline Th-232 & $8.17 \mathrm{E}+00$ & $8.17 \mathrm{E}+00$ & $0.00 \%$ \\
\hline Th-234 & $1.68 \mathrm{E}-04$ & $1.68 \mathrm{E}-04$ & $0.00 \%$ \\
\hline U-232 & $3.42 \mathrm{E}+00$ & $3.42 \mathrm{E}+00$ & $0.00 \%$ \\
\hline U-233 & $6.64 \mathrm{E}-01$ & $6.64 \mathrm{E}-01$ & $0.00 \%$ \\
\hline U-234 & $6.64 \mathrm{E}-01$ & $6.64 \mathrm{E}-01$ & $0.00 \%$ \\
\hline U-235 & $6.13 \mathrm{E}-01$ & $6.13 \mathrm{E}-01$ & $0.00 \%$ \\
\hline U-236 & $6.13 \mathrm{E}-01$ & $6.13 \mathrm{E}-01$ & $0.00 \%$ \\
\hline U-237 & $1.67 \mathrm{E}-05$ & $1.67 \mathrm{E}-05$ & $0.00 \%$ \\
\hline U-238 & $6.13 \mathrm{E}-01$ & $6.13 \mathrm{E}-01$ & $0.00 \%$ \\
\hline W-187 & $2.50 \mathrm{E}-06$ & $2.50 \mathrm{E}-06$ & $0.00 \%$ \\
\hline Y-90 & $4.07 \mathrm{E}-05$ & $4.07 \mathrm{E}-05$ & $0.00 \%$ \\
\hline Y-91 & $2.24 \mathrm{E}-04$ & $2.24 \mathrm{E}-04$ & $0.00 \%$ \\
\hline Y-91M & $1.71 \mathrm{E}-08$ & $1.71 \mathrm{E}-08$ & $0.00 \%$ \\
\hline Y-92 & $1.88 \mathrm{E}-06$ & $1.88 \mathrm{E}-06$ & $0.00 \%$ \\
\hline Y-93-65 & $8.93 \mathrm{E}-06$ & $8.93 \mathrm{E}-06$ & $0.00 \%$ \\
\hline Tr-93 05 & $1.63 \mathrm{E}-03$ & $9.19 \mathrm{E}-05$ & $0.00 \%$ \\
\hline $\mathrm{F}-05$ & $1.83 \mathrm{E}-03$ & 0.05 & $0.00 \%$ \\
\hline 5.23E+01 & $\mathbf{0 . 0 0 \%}$ \\
\hline
\end{tabular}


Table 39. MAXDOSE-SR Maximally Exposed Individual Vegetable Consumption Comparisons (mrem/yr)

\begin{tabular}{|c|c|c|c|}
\hline \multicolumn{4}{|c|}{ Maxdose Vegetation Pathway } \\
\hline Nuclide & Hamby & Jannik & $\%$ Diff \\
\hline Ag-110M & $4.83 \mathrm{E}-03$ & $1.59 \mathrm{E}-03$ & $-67.08 \%$ \\
\hline Am-241 & $2.34 \mathrm{E}+00$ & 7.44E-01 & $-68.21 \%$ \\
\hline Am-243 & $2.34 \mathrm{E}+00$ & 7.44E-01 & $-68.21 \%$ \\
\hline Ba-139 & $2.11 \mathrm{E}-13$ & $6.73 \mathrm{E}-14$ & $-68.10 \%$ \\
\hline Ba-140 & 4.13E-04 & $1.32 \mathrm{E}-04$ & $-68.04 \%$ \\
\hline Ba-141 & $7.43 \mathrm{E}-35$ & $2.36 \mathrm{E}-35$ & $-68.24 \%$ \\
\hline $\mathrm{Be}-7$ & $2.52 \mathrm{E}-05$ & $8.02 \mathrm{E}-06$ & $-68.17 \%$ \\
\hline $\mathrm{Br}-83$ & $2.16 \mathrm{E}-11$ & $7.02 \mathrm{E}-12$ & $-67.50 \%$ \\
\hline $\mathrm{Br}-84$ & $1.61 \mathrm{E}-23$ & $5.23 \mathrm{E}-24$ & $-67.52 \%$ \\
\hline $\mathrm{C}-14$ & $3.42 \mathrm{E}-04$ & $3.42 \mathrm{E}-04$ & $0.00 \%$ \\
\hline Ce-141 & $3.84 \mathrm{E}-04$ & $1.22 \mathrm{E}-04$ & $-68.23 \%$ \\
\hline Ce-143 & $1.97 \mathrm{E}-05$ & $6.27 \mathrm{E}-06$ & $-68.17 \%$ \\
\hline Ce-144 & 8.83E-03 & $2.81 \mathrm{E}-03$ & $-68.18 \%$ \\
\hline Cf-252 & $4.65 \mathrm{E}-01$ & $1.48 \mathrm{E}-01$ & $-68.17 \%$ \\
\hline $\mathrm{Cm}-242$ & $4.31 \mathrm{E}-02$ & $1.37 \mathrm{E}-02$ & $-68.21 \%$ \\
\hline $\mathrm{Cm}-243$ & $1.50 \mathrm{E}+00$ & $4.78 \mathrm{E}-01$ & $-68.13 \%$ \\
\hline $\mathrm{Cm}-244$ & $1.19 \mathrm{E}+00$ & $3.78 \mathrm{E}-01$ & $-68.24 \%$ \\
\hline $\mathrm{Cm}-246$ & $2.34 \mathrm{E}+00$ & $7.45 \mathrm{E}-01$ & $-68.16 \%$ \\
\hline $\mathrm{Cm}-248$ & $8.32 \mathrm{E}+00$ & $2.65 \mathrm{E}+00$ & $-68.15 \%$ \\
\hline Co-57 & $4.82 \mathrm{E}-04$ & $1.54 \mathrm{E}-04$ & $-68.05 \%$ \\
\hline Co-58 & $9.78 \mathrm{E}-04$ & $3.12 \mathrm{E}-04$ & $-68.10 \%$ \\
\hline Co-60 & $1.32 \mathrm{E}-02$ & $4.21 \mathrm{E}-03$ & $-68.11 \%$ \\
\hline $\mathrm{Cr}-51$ & $1.62 \mathrm{E}-05$ & $5.15 \mathrm{E}-06$ & $-68.21 \%$ \\
\hline Cs-134 & $3.62 \mathrm{E}-02$ & $1.15 \mathrm{E}-02$ & $-68.23 \%$ \\
\hline Cs-135 & $3.70 \mathrm{E}-03$ & $1.18 \mathrm{E}-03$ & $-68.11 \%$ \\
\hline Cs-137 & $2.59 \mathrm{E}-02$ & $8.27 \mathrm{E}-03$ & $-68.07 \%$ \\
\hline Cs-138 & $2.78 \mathrm{E}-23$ & $8.83 \mathrm{E}-24$ & $-68.24 \%$ \\
\hline Eu-152 & $3.09 \mathrm{E}-03$ & 9.84E-04 & $-68.16 \%$ \\
\hline Eu-154 & $4.66 \mathrm{E}-03$ & $1.48 \mathrm{E}-03$ & $-68.24 \%$ \\
\hline Eu-155 & $6.59 \mathrm{E}-04$ & $2.10 \mathrm{E}-04$ & $-68.13 \%$ \\
\hline $\mathrm{Fe}-55$ & $2.87 \mathrm{E}-04$ & $9.15 \mathrm{E}-05$ & $-68.12 \%$ \\
\hline $\mathrm{Fe}-59$ & $1.32 \mathrm{E}-03$ & $4.19 \mathrm{E}-04$ & $-68.26 \%$ \\
\hline $\mathrm{H}-3$ & 4.91E-07 & $4.91 \mathrm{E}-07$ & $0.00 \%$ \\
\hline Hf-181 & $8.21 \mathrm{E}-04$ & $2.61 \mathrm{E}-04$ & $-68.21 \%$ \\
\hline I-129 & $7.28 \mathrm{E}-01$ & 2.32E-01 & $-68.13 \%$ \\
\hline $\mathrm{I}-131$ & 8.27E-03 & $2.63 \mathrm{E}-03$ & $-68.20 \%$ \\
\hline I-132 & $5.73 \mathrm{E}-10$ & $1.82 \mathrm{E}-10$ & $-68.24 \%$ \\
\hline $\mathrm{I}-133$ & $1.10 \mathrm{E}-04$ & $3.49 \mathrm{E}-05$ & $-68.27 \%$ \\
\hline $\mathrm{I}-134$ & $1.52 \mathrm{E}-16$ & $4.83 \mathrm{E}-17$ & $-68.22 \%$ \\
\hline I-135 & $1.07 \mathrm{E}-06$ & 3.39E-07 & $-68.32 \%$ \\
\hline La-140 & $4.75 \mathrm{E}-05$ & $1.51 \mathrm{E}-05$ & $-68.21 \%$ \\
\hline
\end{tabular}


Table 39. MAXDOSE-SR Maximally Exposed Individual Vegetable Consumption Comparisons (mrem/yr) (continued)

\begin{tabular}{|c|c|c|c|}
\hline \multicolumn{4}{|c|}{ Maxdose Vegetation Pathway } \\
\hline Nuclide & Hamby & Jannik & \% Diff \\
\hline La-141 & $1.35 \mathrm{E}-08$ & $4.31 \mathrm{E}-09$ & $-68.07 \%$ \\
\hline La-142 & $1.61 \mathrm{E}-12$ & $5.13 \mathrm{E}-13$ & $-68.14 \%$ \\
\hline Mn-54 & $1.21 \mathrm{E}-03$ & $3.89 \mathrm{E}-04$ & $-67.85 \%$ \\
\hline $\mathrm{Mn}-56$ & $4.91 \mathrm{E}-10$ & $1.56 \mathrm{E}-10$ & $-68.23 \%$ \\
\hline Mo-99 & $3.29 \mathrm{E}-05$ & $1.05 \mathrm{E}-05$ & $-68.09 \%$ \\
\hline $\mathrm{Na}-24$ & $1.60 \mathrm{E}-06$ & $5.11 \mathrm{E}-07$ & $-68.06 \%$ \\
\hline $\mathrm{Nb}-95$ & $3.51 \mathrm{E}-04$ & $1.12 \mathrm{E}-04$ & $-68.09 \%$ \\
\hline $\mathrm{Nb}-97$ & $1.60 \mathrm{E}-14$ & $5.09 \mathrm{E}-15$ & $-68.19 \%$ \\
\hline $\mathrm{Ni}-59$ & $1.04 \mathrm{E}-04$ & $3.33 \mathrm{E}-05$ & $-67.98 \%$ \\
\hline $\mathrm{Ni}-63$ & $2.81 \mathrm{E}-04$ & $8.99 \mathrm{E}-05$ & $-68.01 \%$ \\
\hline $\mathrm{Ni}-65$ & $2.71 \mathrm{E}-10$ & $8.62 \mathrm{E}-11$ & $-68.19 \%$ \\
\hline Np-237 & $2.03 \mathrm{E}+00$ & $6.46 \mathrm{E}-01$ & $-68.18 \%$ \\
\hline $\mathrm{Np}-238$ & $2.83 \mathrm{E}-05$ & $9.01 \mathrm{E}-06$ & $-68.16 \%$ \\
\hline Np-239 & $2.75 \mathrm{E}-05$ & $8.77 \mathrm{E}-06$ & $-68.11 \%$ \\
\hline Os-185 & $6.77 \mathrm{E}-04$ & $2.17 \mathrm{E}-04$ & $-67.95 \%$ \\
\hline P-32 & $4.44 \mathrm{E}-04$ & $1.51 \mathrm{E}-04$ & $-65.99 \%$ \\
\hline $\mathrm{Pa}-233$ & $3.98 \mathrm{E}-04$ & $1.27 \mathrm{E}-04$ & $-68.09 \%$ \\
\hline Pd-109 & $2.01 \mathrm{E}-06$ & $7.37 \mathrm{E}-07$ & $-63.33 \%$ \\
\hline Pm-147 & $4.70 \mathrm{E}-04$ & $1.50 \mathrm{E}-04$ & $-68.09 \%$ \\
\hline Pm-148 & $2.09 \mathrm{E}-04$ & $6.66 \mathrm{E}-05$ & $-68.13 \%$ \\
\hline Pm-151 & $1.03 \mathrm{E}-05$ & $3.29 \mathrm{E}-06$ & $-68.06 \%$ \\
\hline Pr-144 & $1.11 \mathrm{E}-36$ & $3.52 \mathrm{E}-37$ & $-68.29 \%$ \\
\hline $\mathrm{Pu}-238$ & $1.97 \mathrm{E}+00$ & $6.28 \mathrm{E}-01$ & $-68.12 \%$ \\
\hline Pu-239 & $2.23 \mathrm{E}+00$ & $7.11 \mathrm{E}-01$ & $-68.12 \%$ \\
\hline $\mathrm{Pu}-240$ & $2.23 \mathrm{E}+00$ & $7.11 \mathrm{E}-01$ & $-68.12 \%$ \\
\hline $\mathrm{Pu}-241$ & $4.43 \mathrm{E}-02$ & $1.41 \mathrm{E}-02$ & $-68.17 \%$ \\
\hline $\mathrm{Pu}-242$ & $2.13 \mathrm{E}+00$ & $6.78 \mathrm{E}-01$ & $-68.17 \%$ \\
\hline $\mathrm{Ra}-224$ & $5.06 \mathrm{E}-03$ & $1.61 \mathrm{E}-03$ & $-68.18 \%$ \\
\hline Ra-228 & $6.10 \mathrm{E}-01$ & $1.94 \mathrm{E}-01$ & $-68.20 \%$ \\
\hline $\mathrm{Rb}-86$ & 7.29E-04 & $2.34 \mathrm{E}-04$ & $-67.90 \%$ \\
\hline $\mathrm{Rb}-87$ & $2.54 \mathrm{E}-03$ & 8.38E-04 & $-67.01 \%$ \\
\hline $\mathrm{Rb}-88$ & $1.38 \mathrm{E}-35$ & $4.40 \mathrm{E}-36$ & $-68.12 \%$ \\
\hline Rb-89 & $1.14 \mathrm{E}-40$ & $3.65 \mathrm{E}-41$ & $-67.98 \%$ \\
\hline Rh-105 & $8.69 \mathrm{E}-06$ & $3.79 \mathrm{E}-06$ & $-56.39 \%$ \\
\hline Rh-106M & $8.91 \mathrm{E}-11$ & $3.81 \mathrm{E}-11$ & $-57.24 \%$ \\
\hline Ru-103 & $4.87 \mathrm{E}-04$ & $1.56 \mathrm{E}-04$ & $-67.97 \%$ \\
\hline Ru-105 & $1.85 \mathrm{E}-08$ & $5.88 \mathrm{E}-09$ & $-68.22 \%$ \\
\hline Ru-106 & $9.68 \mathrm{E}-03$ & $3.12 \mathrm{E}-03$ & $-67.77 \%$ \\
\hline S-35 & $2.11 \mathrm{E}-04$ & $7.40 \mathrm{E}-05$ & $-64.93 \%$ \\
\hline Sb-122 & $7.02 \mathrm{E}-05$ & $2.23 \mathrm{E}-05$ & $-68.23 \%$ \\
\hline Sb-124 & $2.33 \mathrm{E}-03$ & 7.44E-04 & $-68.07 \%$ \\
\hline Sb-125 & $1.29 \mathrm{E}-03$ & $4.12 \mathrm{E}-04$ & $-68.06 \%$ \\
\hline Sb-126 & $4.56 \mathrm{E}-04$ & $1.45 \mathrm{E}-04$ & $-68.20 \%$ \\
\hline
\end{tabular}


Table 39. MAXDOSE-SR Maximally Exposed Individual Vegetable Consumption Comparisons (mrem/yr) (continued)

\begin{tabular}{|c|c|c|c|}
\hline \multicolumn{4}{|c|}{ Maxdose Vegetation Pathway } \\
\hline Nuclide & Hamby & Jannik & $\%$ Diff \\
\hline Sb-126M & $2.82 \mathrm{E}-34$ & $8.98 \mathrm{E}-35$ & $-68.16 \%$ \\
\hline Se-75 & $3.50 \mathrm{E}-03$ & $1.37 \mathrm{E}-03$ & $-60.86 \%$ \\
\hline Se-79 & $5.08 \mathrm{E}-03$ & $2.14 \mathrm{E}-03$ & $-57.87 \%$ \\
\hline Sm-151 & $1.77 \mathrm{E}-04$ & $5.62 \mathrm{E}-05$ & $-68.25 \%$ \\
\hline Sn-123 & $2.81 \mathrm{E}-03$ & $8.94 \mathrm{E}-04$ & $-68.19 \%$ \\
\hline Sn-126 & $8.84 \mathrm{E}-03$ & $2.81 \mathrm{E}-03$ & $-68.21 \%$ \\
\hline Sr-89 & $1.92 \mathrm{E}-03$ & $6.12 \mathrm{E}-04$ & $-68.13 \%$ \\
\hline Sr-90 & $6.74 \mathrm{E}-02$ & $2.16 \mathrm{E}-02$ & $-67.95 \%$ \\
\hline Sr-91 & $1.07 \mathrm{E}-06$ & $3.41 \mathrm{E}-07$ & $-68.13 \%$ \\
\hline $\mathrm{Sr}-92$ & $1.52 \mathrm{E}-09$ & $4.85 \mathrm{E}-10$ & $-68.09 \%$ \\
\hline Tc-101 & $2.84 \mathrm{E}-43$ & $9.11 \mathrm{E}-44$ & $-67.92 \%$ \\
\hline Tc-99 & $6.99 \mathrm{E}-04$ & $2.38 \mathrm{E}-04$ & $-65.95 \%$ \\
\hline Tc-99 M & $4.48 \mathrm{E}-09$ & $1.44 \mathrm{E}-09$ & $-67.86 \%$ \\
\hline Te-125M & $9.04 \mathrm{E}-04$ & $3.38 \mathrm{E}-04$ & $-62.61 \%$ \\
\hline Te-127 & $2.41 \mathrm{E}-07$ & 7.97E-08 & $-66.93 \%$ \\
\hline Te-127M & $3.02 \mathrm{E}-03$ & $1.18 \mathrm{E}-03$ & $-60.93 \%$ \\
\hline Te-129 & $7.30 \mathrm{E}-15$ & $2.42 \mathrm{E}-15$ & $-66.85 \%$ \\
\hline Te-129M & $1.61 \mathrm{E}-03$ & $5.77 \mathrm{E}-04$ & $-64.16 \%$ \\
\hline Te-131 & $1.40 \mathrm{E}-27$ & $4.62 \mathrm{E}-28$ & $-67.00 \%$ \\
\hline Te-131M & $6.18 \mathrm{E}-05$ & $2.05 \mathrm{E}-05$ & $-66.83 \%$ \\
\hline Te-132 & $1.03 \mathrm{E}-04$ & $3.44 \mathrm{E}-05$ & $-66.60 \%$ \\
\hline Te-134 & $1.14 \mathrm{E}-19$ & $3.78 \mathrm{E}-20$ & $-66.84 \%$ \\
\hline Th-228 & $1.85 \mathrm{E}-01$ & $5.89 \mathrm{E}-02$ & $-68.16 \%$ \\
\hline Th-230 & $2.76 \mathrm{E}-01$ & $8.78 \mathrm{E}-02$ & $-68.19 \%$ \\
\hline Th-232 & $1.46 \mathrm{E}+00$ & $4.64 \mathrm{E}-01$ & $-68.22 \%$ \\
\hline Th-234 & $1.37 \mathrm{E}-03$ & $4.37 \mathrm{E}-04$ & $-68.10 \%$ \\
\hline U-232 & $6.75 \mathrm{E}-01$ & $2.15 \mathrm{E}-01$ & $-68.15 \%$ \\
\hline U-233 & $1.40 \mathrm{E}-01$ & $4.47 \mathrm{E}-02$ & $-68.07 \%$ \\
\hline U-234 & $1.35 \mathrm{E}-01$ & $4.30 \mathrm{E}-02$ & $-68.15 \%$ \\
\hline U-235 & $1.30 \mathrm{E}-01$ & $4.14 \mathrm{E}-02$ & $-68.15 \%$ \\
\hline U-236 & $1.30 \mathrm{E}-01$ & $4.14 \mathrm{E}-02$ & $-68.15 \%$ \\
\hline U-237 & $7.23 \mathrm{E}-05$ & $2.30 \mathrm{E}-05$ & $-68.19 \%$ \\
\hline U-238 & $1.20 \mathrm{E}-01$ & $3.81 \mathrm{E}-02$ & $-68.25 \%$ \\
\hline W-187 & $7.28 \mathrm{E}-06$ & $2.32 \mathrm{E}-06$ & $-68.13 \%$ \\
\hline $\mathrm{Y}-90$ & $1.10 \mathrm{E}-04$ & $3.49 \mathrm{E}-05$ & $-68.27 \%$ \\
\hline Y-91 & $2.19 \mathrm{E}-03$ & $6.96 \mathrm{E}-04$ & $-68.22 \%$ \\
\hline Y-91M & $1.87 \mathrm{E}-18$ & 5.94E-19 & $-68.24 \%$ \\
\hline Y-92 & $9.98 \mathrm{E}-09$ & $3.18 \mathrm{E}-09$ & $-68.14 \%$ \\
\hline Y-93 & $1.91 \mathrm{E}-06$ & $6.09 \mathrm{E}-07$ & $-68.12 \%$ \\
\hline Zn-65 & $6.28 \mathrm{E}-03$ & $2.18 \mathrm{E}-03$ & $-65.29 \%$ \\
\hline Zr-93 & $8.32 \mathrm{E}-04$ & $2.65 \mathrm{E}-04$ & $-68.15 \%$ \\
\hline Zr-95 & $9.02 \mathrm{E}-04$ & $2.87 \mathrm{E}-04$ & $-68.18 \%$ \\
\hline Zr-97 & $1.17 \mathrm{E}-05$ & $3.72 \mathrm{E}-06$ & $-68.21 \%$ \\
\hline Total & $3.40 \mathrm{E}+01$ & $1.08 \mathrm{E}+01$ & $-68.24 \%$ \\
\hline
\end{tabular}


Table 40. MAXDOSE-SR Maximally Exposed Individual Milk Consumption Comparisons (mrem/yr)

\begin{tabular}{|c|c|c|c|}
\hline \multicolumn{4}{|c|}{ Maxdose Milk Pathway } \\
\hline Nuclide & Hamby & Jannik & \% Diff \\
\hline Ag-110M & $5.80 \mathrm{E}-03$ & $6.28 \mathrm{E}-03$ & $8.28 \%$ \\
\hline Am-241 & $2.87 \mathrm{E}-04$ & $2.75 \mathrm{E}-04$ & $-4.18 \%$ \\
\hline Am-243 & $2.87 \mathrm{E}-04$ & $2.75 \mathrm{E}-04$ & $-4.18 \%$ \\
\hline Ba-139 & $2.35 \mathrm{E}-20$ & $6.05 \mathrm{E}-20$ & $157.45 \%$ \\
\hline Ba-140 & $6.50 \mathrm{E}-06$ & $1.65 \mathrm{E}-05$ & $153.85 \%$ \\
\hline Be-7 & $5.80 \mathrm{E}-08$ & $9.40 \mathrm{E}-08$ & $62.07 \%$ \\
\hline Br-83 & $5.27 \mathrm{E}-14$ & $1.33 \mathrm{E}-13$ & $152.37 \%$ \\
\hline Br-84 & $9.32 \mathrm{E}-37$ & $2.36 \mathrm{E}-36$ & $153.22 \%$ \\
\hline C-14 & $1.64 \mathrm{E}-04$ & $1.64 \mathrm{E}-04$ & $0.00 \%$ \\
\hline Ce-141 & $5.72 \mathrm{E}-06$ & $1.15 \mathrm{E}-05$ & $101.05 \%$ \\
\hline Ce-143 & $3.66 \mathrm{E}-07$ & $9.42 \mathrm{E}-07$ & $157.38 \%$ \\
\hline Ce-144 & $1.26 \mathrm{E}-04$ & $1.35 \mathrm{E}-04$ & $7.14 \%$ \\
\hline Cf-252 & $5.66 \mathrm{E}-05$ & $5.60 \mathrm{E}-05$ & $-1.06 \%$ \\
\hline Cm-242 & $5.04 \mathrm{E}-06$ & $5.84 \mathrm{E}-06$ & $15.87 \%$ \\
\hline Cm-243 & $1.84 \mathrm{E}-04$ & $1.77 \mathrm{E}-04$ & $-3.80 \%$ \\
\hline Cm-244 & $1.46 \mathrm{E}-04$ & $1.40 \mathrm{E}-04$ & $-4.11 \%$ \\
\hline Cm-246 & $2.88 \mathrm{E}-04$ & $2.75 \mathrm{E}-04$ & $-4.51 \%$ \\
\hline Cm-248 & $1.02 \mathrm{E}-03$ & $9.78 \mathrm{E}-04$ & $-4.12 \%$ \\
\hline Co-57 & $1.15 \mathrm{E}-05$ & $1.23 \mathrm{E}-05$ & $6.96 \%$ \\
\hline Co-58 & $2.23 \mathrm{E}-05$ & $3.22 \mathrm{E}-05$ & $44.39 \%$ \\
\hline Co-60 & $3.23 \mathrm{E}-04$ & $3.14 \mathrm{E}-04$ & $-2.79 \%$ \\
\hline Cr-51 & $9.32 \mathrm{E}-07$ & $1.99 \mathrm{E}-06$ & $113.52 \%$ \\
\hline Cs-134 & $1.05 \mathrm{E}-02$ & $1.05 \mathrm{E}-02$ & $0.00 \%$ \\
\hline Cs-135 & $1.09 \mathrm{E}-03$ & $1.04 \mathrm{E}-03$ & $-4.59 \%$ \\
\hline Cs-137 & $7.64 \mathrm{E}-03$ & $7.33 \mathrm{E}-03$ & $-4.06 \%$ \\
\hline Cs-138 & $5.86 \mathrm{E}-37$ & $1.51 \mathrm{E}-36$ & $157.68 \%$ \\
\hline Eu-152 & $3.79 \mathrm{E}-07$ & $3.65 \mathrm{E}-07$ & $-3.69 \%$ \\
\hline Eu-154 & $5.71 \mathrm{E}-07$ & $5.52 \mathrm{E}-07$ & $-3.33 \%$ \\
\hline Eu-155 & $8.05 \mathrm{E}-08$ & $7.84 \mathrm{E}-08$ & $-2.61 \%$ \\
\hline Fe-55 & $8.39 \mathrm{E}-06$ & $8.29 \mathrm{E}-06$ & $-1.19 \%$ \\
\hline Fe-59 & $3.68 \mathrm{E}-05$ & $6.45 \mathrm{E}-05$ & $75.27 \%$ \\
\hline H-3 & $1.96 \mathrm{E}-07$ & $1.96 \mathrm{E}-07$ & $0.00 \%$ \\
\hline Hf-181 & $9.65 \mathrm{E}-08$ & $1.72 \mathrm{E}-07$ & $78.24 \%$ \\
\hline I-129 & $1.07 \mathrm{E}-01$ & $1.03 \mathrm{E}-01$ & $-3.74 \%$ \\
\hline I-131 & $2.23 \mathrm{E}-03$ & $5.72 \mathrm{E}-03$ & $156.50 \%$ \\
\hline I-132 & $1.25 \mathrm{E}-13$ & $3.22 \mathrm{E}-13$ & $157.60 \%$ \\
\hline I-133 & $1.51 \mathrm{E}-05$ & $3.89 \mathrm{E}-05$ & $157.62 \%$ \\
\hline I-134 & $2.48 \mathrm{E}-25$ & $6.38 \mathrm{E}-25$ & $157.26 \%$ \\
\hline La-135 140 & $2.61 \mathrm{E}-08$ & $6.72 \mathrm{E}-08$ & $157.47 \%$ \\
\hline & $8.06 \mathrm{E}-09$ & $2.07 \mathrm{E}-08$ & $156.82 \%$ \\
\hline
\end{tabular}


SRNL-STI-2010-00447, REVISION 0

Table 40. MAXDOSE-SR Maximally Exposed Individual Milk Consumption Comparisons (mrem/yr) (continued)

\begin{tabular}{|c|c|c|c|}
\hline \multicolumn{4}{|c|}{ Maxdose Milk Pathway } \\
\hline Nuclide & Hamby & Jannik & $\%$ Diff \\
\hline La-141 & $5.03 \mathrm{E}-14$ & $1.29 \mathrm{E}-13$ & $156.46 \%$ \\
\hline La-142 & $8.23 \mathrm{E}-21$ & $2.12 \mathrm{E}-20$ & $157.59 \%$ \\
\hline Mn-54 & $7.25 \mathrm{E}-06$ & $7.68 \mathrm{E}-06$ & $5.93 \%$ \\
\hline $\mathrm{Mn}-56$ & $9.52 \mathrm{E}-15$ & $2.45 \mathrm{E}-14$ & $157.35 \%$ \\
\hline Mo-99 & $9.84 \mathrm{E}-06$ & $2.52 \mathrm{E}-05$ & $156.10 \%$ \\
\hline $\mathrm{Na}-24$ & $1.09 \mathrm{E}-06$ & $2.79 \mathrm{E}-06$ & $155.96 \%$ \\
\hline $\mathrm{Nb}-95$ & $2.14 \mathrm{E}-05$ & $4.16 \mathrm{E}-05$ & $94.39 \%$ \\
\hline $\mathrm{Nb}-97$ & $1.97 \mathrm{E}-21$ & $5.06 \mathrm{E}-21$ & $156.85 \%$ \\
\hline $\mathrm{Ni}-59$ & $1.72 \mathrm{E}-05$ & $1.64 \mathrm{E}-05$ & $-4.65 \%$ \\
\hline Ni-63 & $4.63 \mathrm{E}-05$ & $4.44 \mathrm{E}-05$ & $-4.10 \%$ \\
\hline $\mathrm{Ni}-65$ & $1.24 \mathrm{E}-13$ & $3.20 \mathrm{E}-13$ & $158.06 \%$ \\
\hline $\mathrm{Np}-237$ & $2.49 \mathrm{E}-04$ & $2.38 \mathrm{E}-04$ & $-4.42 \%$ \\
\hline $\mathrm{Np}-238$ & $5.23 \mathrm{E}-09$ & $1.34 \mathrm{E}-08$ & $156.21 \%$ \\
\hline Np-239 & $5.26 \mathrm{E}-09$ & $1.35 \mathrm{E}-08$ & $156.65 \%$ \\
\hline Os-185 & $7.79 \mathrm{E}-05$ & $1.03 \mathrm{E}-04$ & $32.22 \%$ \\
\hline $\mathrm{P}-32$ & 4.34E-04 & $1.04 \mathrm{E}-03$ & $139.63 \%$ \\
\hline $\mathrm{Pa}-233$ & $5.27 \mathrm{E}-08$ & $1.14 \mathrm{E}-07$ & $116.32 \%$ \\
\hline Pd-109 & $3.32 \mathrm{E}-07$ & $7.69 \mathrm{E}-07$ & $131.63 \%$ \\
\hline Pm-147 & $5.72 \mathrm{E}-08$ & $5.66 \mathrm{E}-08$ & $-1.05 \%$ \\
\hline Pm-148 & $4.68 \mathrm{E}-08$ & $1.20 \mathrm{E}-07$ & $156.41 \%$ \\
\hline Pm-151 & $1.47 \mathrm{E}-09$ & $3.78 \mathrm{E}-09$ & $157.14 \%$ \\
\hline $\mathrm{Pu}-238$ & $9.69 \mathrm{E}-05$ & $9.28 \mathrm{E}-05$ & $-4.23 \%$ \\
\hline Pu-239 & $1.10 \mathrm{E}-04$ & $1.05 \mathrm{E}-04$ & $-4.55 \%$ \\
\hline $\mathrm{Pu}-240$ & $1.10 \mathrm{E}-04$ & $1.05 \mathrm{E}-04$ & $-4.55 \%$ \\
\hline $\mathrm{Pu}-241$ & $2.17 \mathrm{E}-06$ & $2.09 \mathrm{E}-06$ & $-3.69 \%$ \\
\hline $\mathrm{Pu}-242$ & $1.05 \mathrm{E}-04$ & $1.00 \mathrm{E}-04$ & $-4.76 \%$ \\
\hline Ra-224 & $1.72 \mathrm{E}-03$ & $4.41 \mathrm{E}-03$ & $156.40 \%$ \\
\hline Ra-228 & $1.19 \mathrm{E}-01$ & $1.16 \mathrm{E}-01$ & $-2.52 \%$ \\
\hline $\mathrm{Rb}-86$ & 7.02E-04 & $1.68 \mathrm{E}-03$ & $139.32 \%$ \\
\hline $\mathrm{Rb}-87$ & $1.89 \mathrm{E}-03$ & $1.81 \mathrm{E}-03$ & $-4.23 \%$ \\
\hline Rh-105 & $3.53 \mathrm{E}-06$ & $7.14 \mathrm{E}-06$ & $102.27 \%$ \\
\hline Rh-106M & $2.73 \mathrm{E}-14$ & $5.61 \mathrm{E}-14$ & $105.49 \%$ \\
\hline Ru-103 & $1.16 \mathrm{E}-08$ & $2.13 \mathrm{E}-08$ & $83.62 \%$ \\
\hline $\mathrm{Ru}-105$ & $2.21 \mathrm{E}-14$ & $5.67 \mathrm{E}-14$ & $156.56 \%$ \\
\hline Ru-106 & $2.33 \mathrm{E}-07$ & $2.43 \mathrm{E}-07$ & $4.29 \%$ \\
\hline S-35 & $9.09 \mathrm{E}-05$ & $1.20 \mathrm{E}-04$ & $32.01 \%$ \\
\hline Sb-122 & $4.18 \mathrm{E}-06$ & $1.07 \mathrm{E}-05$ & $155.98 \%$ \\
\hline Sb-124 & $8.01 \mathrm{E}-05$ & $1.23 \mathrm{E}-04$ & $53.56 \%$ \\
\hline Sb-125 & $4.72 \mathrm{E}-05$ & $4.66 \mathrm{E}-05$ & $-1.27 \%$ \\
\hline Sb-126 & $2.73 \mathrm{E}-05$ & $6.94 \mathrm{E}-05$ & $154.21 \%$ \\
\hline Se-75 & $3.98 \mathrm{E}-03$ & $4.75 \mathrm{E}-03$ & $19.35 \%$ \\
\hline Se-79 & $6.15 \mathrm{E}-03$ & $5.94 \mathrm{E}-03$ & $-3.41 \%$ \\
\hline
\end{tabular}


Table 40. MAXDOSE-SR Maximally Exposed Individual Milk Consumption Comparisons (mrem/yr) (continued)

\begin{tabular}{|c|c|c|c|}
\hline \multicolumn{5}{|c|}{ Maxdose Milk Pathway } \\
\hline Nuclide & Hamby & Jannik & $\mathbf{\% ~ D i f f ~}$ \\
\hline Sm-151 & $2.17 \mathrm{E}-08$ & $2.08 \mathrm{E}-08$ & $-4.15 \%$ \\
\hline Sn-123 & $1.63 \mathrm{E}-04$ & $1.98 \mathrm{E}-04$ & $21.47 \%$ \\
\hline Sn-126 & $5.43 \mathrm{E}-04$ & $5.19 \mathrm{E}-04$ & $-4.42 \%$ \\
\hline Sr-89 & $3.55 \mathrm{E}-05$ & $5.87 \mathrm{E}-05$ & $65.35 \%$ \\
\hline Sr-90 & $1.33 \mathrm{E}-03$ & $1.27 \mathrm{E}-03$ & $-4.51 \%$ \\
\hline Sr-91 & $7.57 \mathrm{E}-09$ & $1.95 \mathrm{E}-08$ & $157.60 \%$ \\
\hline Sr-92 & $1.33 \mathrm{E}-13$ & $3.43 \mathrm{E}-13$ & $157.89 \%$ \\
\hline Tc-99 & $4.38 \mathrm{E}-04$ & $4.20 \mathrm{E}-04$ & $-4.11 \%$ \\
\hline Tc-99 M & $3.61 \mathrm{E}-10$ & $9.24 \mathrm{E}-10$ & $155.96 \%$ \\
\hline Te-125M & $2.26 \mathrm{E}-05$ & $3.33 \mathrm{E}-05$ & $47.35 \%$ \\
\hline Te-127 & $2.13 \mathrm{E}-09$ & $5.32 \mathrm{E}-09$ & $149.77 \%$ \\
\hline Te-127M & $7.61 \mathrm{E}-05$ & $9.27 \mathrm{E}-05$ & $21.81 \%$ \\
\hline Te-129 & $2.20 \mathrm{E}-22$ & $5.50 \mathrm{E}-22$ & $150.00 \%$ \\
\hline Te-129M & $4.29 \mathrm{E}-05$ & $7.94 \mathrm{E}-05$ & $85.08 \%$ \\
\hline Te-131 & $0.00 \mathrm{E}+00$ & $1.40 \mathrm{E}-45$ & $\# \mathrm{DIV} / 0 !$ \\
\hline Te-131M & $1.88 \mathrm{E}-06$ & $4.67 \mathrm{E}-06$ & $148.40 \%$ \\
\hline Te-132 & $4.42 \mathrm{E}-06$ & $1.10 \mathrm{E}-05$ & $148.87 \%$ \\
\hline Te-134 & $2.30 \mathrm{E}-31$ & $5.75 \mathrm{E}-31$ & $150.00 \%$ \\
\hline Th-228 & $2.24 \mathrm{E}-05$ & $2.24 \mathrm{E}-05$ & $0.00 \%$ \\
\hline Th-230 & $3.39 \mathrm{E}-05$ & $3.24 \mathrm{E}-05$ & $-4.42 \%$ \\
\hline Th-232 & $1.79 \mathrm{E}-04$ & $1.71 \mathrm{E}-04$ & $-4.47 \%$ \\
\hline Th-234 & $1.91 \mathrm{E}-07$ & $4.30 \mathrm{E}-07$ & $125.13 \%$ \\
\hline U-232 & $8.29 \mathrm{E}-03$ & $7.94 \mathrm{E}-03$ & $-4.22 \%$ \\
\hline U-233 & $1.73 \mathrm{E}-03$ & $1.65 \mathrm{E}-03$ & $-4.62 \%$ \\
\hline U-234 & $1.66 \mathrm{E}-03$ & $1.59 \mathrm{E}-03$ & $-4.22 \%$ \\
\hline U-235 & $1.60 \mathrm{E}-03$ & $1.53 \mathrm{E}-03$ & $-4.38 \%$ \\
\hline U-236 & $1.60 \mathrm{E}-03$ & $1.53 \mathrm{E}-03$ & $-4.38 \%$ \\
\hline U-237 & $1.64 \mathrm{E}-06$ & $4.20 \mathrm{E}-06$ & $156.10 \%$ \\
\hline U-238 & $1.47 \mathrm{E}-03$ & $1.41 \mathrm{E}-03$ & $-4.08 \%$ \\
\hline W-187 & $9.30 \mathrm{E}-08$ & $2.39 \mathrm{E}-07$ & $156.99 \%$ \\
\hline Y-90 & $4.34 \mathrm{E}-08$ & $1.12 \mathrm{E}-07$ & $158.06 \%$ \\
\hline Y-91 & $5.01 \mathrm{E}-07$ & $7.80 \mathrm{E}-07$ & $55.69 \%$ \\
\hline Y-91M & $1.80 \mathrm{E}-30$ & $4.63 \mathrm{E}-30$ & $157.22 \%$ \\
\hline Y-92 & $4.68 \mathrm{E}-14$ & $1.20 \mathrm{E}-13$ & $156.41 \%$ \\
\hline Y-93 & $1.87 \mathrm{E}-10$ & $4.82 \mathrm{E}-10$ & $157.75 \%$ \\
\hline Zn-65 & $5.98 \mathrm{E}-03$ & $6.48 \mathrm{E}-03$ & $8.36 \%$ \\
\hline Zr-93 & $1.02 \mathrm{E}-07$ & $9.77 \mathrm{E}-08$ & $-4.22 \%$ \\
\hline Zr-97 & $1.03 \mathrm{E}-07$ & $1.53 \mathrm{E}-07$ & $48.54 \%$ \\
\hline $\mathbf{2 . 9 7 E - 0 1}$ & $\mathbf{2 . 9 9 E - 0 1}$ & $\mathbf{0 . 6 7 \%}$ \\
\hline & & $2.87 \mathrm{E}-09$ & $156.25 \%$ \\
\hline
\end{tabular}


Table 41. MAXDOSE-SR Maximally Exposed Individual Meat Consumption Comparisons (mrem/yr)

\begin{tabular}{|c|c|c|c|}
\hline \multicolumn{4}{|c|}{ Maxdose Meat Pathway } \\
\hline Nuclide & Hamby & Jannik & $\%$ Diff \\
\hline $\mathrm{Ag}-110 \mathrm{M}$ & $5.75 \mathrm{E}-04$ & $8.82 \mathrm{E}-04$ & $53.39 \%$ \\
\hline Am-241 & $3.18 \mathrm{E}-03$ & $4.47 \mathrm{E}-03$ & $40.57 \%$ \\
\hline $\mathrm{Am}-243$ & $3.18 \mathrm{E}-03$ & $4.47 \mathrm{E}-03$ & $40.57 \%$ \\
\hline Ba-139 & $9.37 \mathrm{E}-41$ & $2.41 \mathrm{E}-40$ & $157.20 \%$ \\
\hline Ba-140 & $1.96 \mathrm{E}-05$ & $5.00 \mathrm{E}-05$ & $155.10 \%$ \\
\hline $\mathrm{Be}-7$ & $1.97 \mathrm{E}-07$ & 4.01E-07 & $103.55 \%$ \\
\hline $\mathrm{Br}-83$ & $1.12 \mathrm{E}-26$ & $2.84 \mathrm{E}-26$ & $153.57 \%$ \\
\hline $\mathrm{C}-14$ & 1.49E-04 & $1.49 \mathrm{E}-04$ & $0.00 \%$ \\
\hline Ce-141 & $4.25 \mathrm{E}-06$ & $9.73 \mathrm{E}-06$ & $128.94 \%$ \\
\hline Ce-143 & $4.66 \mathrm{E}-08$ & $1.20 \mathrm{E}-07$ & $157.51 \%$ \\
\hline Ce-144 & $7.26 \mathrm{E}-05$ & $1.12 \mathrm{E}-04$ & $54.27 \%$ \\
\hline Cf-252 & $6.35 \mathrm{E}-04$ & $9.16 \mathrm{E}-04$ & $44.25 \%$ \\
\hline $\mathrm{Cm}-242$ & $5.98 \mathrm{E}-05$ & $9.76 \mathrm{E}-05$ & $63.21 \%$ \\
\hline $\mathrm{Cm}-243$ & $2.04 \mathrm{E}-03$ & $2.88 \mathrm{E}-03$ & $41.18 \%$ \\
\hline $\mathrm{Cm}-244$ & $1.62 \mathrm{E}-03$ & $2.28 \mathrm{E}-03$ & $40.74 \%$ \\
\hline $\mathrm{Cm}-246$ & $3.19 \mathrm{E}-03$ & $4.48 \mathrm{E}-03$ & $40.44 \%$ \\
\hline $\mathrm{Cm}-248$ & $1.13 \mathrm{E}-02$ & $1.59 \mathrm{E}-02$ & $40.71 \%$ \\
\hline Co-57 & $4.30 \mathrm{E}-05$ & $6.63 \mathrm{E}-05$ & $54.19 \%$ \\
\hline Co-58 & $9.38 \mathrm{E}-05$ & $1.78 \mathrm{E}-04$ & $89.77 \%$ \\
\hline Co-60 & $1.17 \mathrm{E}-03$ & $1.67 \mathrm{E}-03$ & $42.74 \%$ \\
\hline $\mathrm{Cr}-51$ & $3.86 \mathrm{E}-07$ & $9.13 \mathrm{E}-07$ & $136.53 \%$ \\
\hline Cs-134 & $9.88 \mathrm{E}-04$ & $1.44 \mathrm{E}-03$ & $45.75 \%$ \\
\hline Cs-135 & $1.01 \mathrm{E}-04$ & $1.41 \mathrm{E}-04$ & $39.60 \%$ \\
\hline Cs-137 & $7.07 \mathrm{E}-04$ & $9.94 \mathrm{E}-04$ & $40.59 \%$ \\
\hline Eu-152 & $1.01 \mathrm{E}-04$ & $1.43 \mathrm{E}-04$ & $41.58 \%$ \\
\hline Eu-154 & $1.52 \mathrm{E}-04$ & $2.16 \mathrm{E}-04$ & $42.11 \%$ \\
\hline Eu-155 & $2.15 \mathrm{E}-05$ & $3.07 \mathrm{E}-05$ & $42.79 \%$ \\
\hline Fe-55 & $7.83 \mathrm{E}-05$ & $1.13 \mathrm{E}-04$ & $44.32 \%$ \\
\hline $\mathrm{Fe}-59$ & $4.30 \mathrm{E}-04$ & $9.16 \mathrm{E}-04$ & $113.02 \%$ \\
\hline $\mathrm{H}-3$ & $8.30 \mathrm{E}-08$ & $8.30 \mathrm{E}-08$ & $0.00 \%$ \\
\hline Hf-181 & $2.73 \mathrm{E}-03$ & $5.88 \mathrm{E}-03$ & $115.38 \%$ \\
\hline $\mathrm{I}-129$ & $1.44 \mathrm{E}-02$ & $2.02 \mathrm{E}-02$ & $40.28 \%$ \\
\hline I-131 & $3.60 \mathrm{E}-04$ & $9.24 \mathrm{E}-04$ & $156.67 \%$ \\
\hline I-132 & $8.23 \mathrm{E}-27$ & $2.12 \mathrm{E}-26$ & $157.59 \%$ \\
\hline I-133 & $1.42 \mathrm{E}-07$ & $3.65 \mathrm{E}-07$ & $157.04 \%$ \\
\hline I-135 & $2.51 \mathrm{E}-13$ & $6.46 \mathrm{E}-13$ & $157.37 \%$ \\
\hline La-140 & $2.92 \mathrm{E}-08$ & $7.51 \mathrm{E}-08$ & $157.19 \%$ \\
\hline La-141 & $4.47 \mathrm{E}-20$ & $1.15 \mathrm{E}-19$ & $157.27 \%$ \\
\hline La-142 & $2.90 \mathrm{E}-38$ & $7.45 \mathrm{E}-38$ & $156.90 \%$ \\
\hline $\mathrm{Mn}-54$ & $6.67 \mathrm{E}-06$ & $1.01 \mathrm{E}-05$ & $51.42 \%$ \\
\hline Mn-56 & $8.32 \mathrm{E}-26$ & $2.14 \mathrm{E}-25$ & $157.21 \%$ \\
\hline Мo-99 & $1.81 \mathrm{E}-06$ & $4.64 \mathrm{E}-06$ & $156.35 \%$ \\
\hline
\end{tabular}


SRNL-STI-2010-00447, REVISION 0

Table 41. MAXDOSE-SR Maximally Exposed Individual Meat Consumption Comparisons (mrem/yr) (continued)

\begin{tabular}{|c|c|c|c|}
\hline \multicolumn{4}{|c|}{ Maxdose Meat Pathway } \\
\hline Nuclide & Hamby & Jannik & \% Diff \\
\hline Na-24 & $4.64 \mathrm{E}-09$ & $1.19 \mathrm{E}-08$ & $156.47 \%$ \\
\hline Nb-95 & $8.77 \mathrm{E}-04$ & $1.98 \mathrm{E}-03$ & $125.77 \%$ \\
\hline Nb-97 & $1.11 \mathrm{E}-43$ & $2.84 \mathrm{E}-43$ & $155.86 \%$ \\
\hline Ni-59 & $3.77 \mathrm{E}-06$ & $5.29 \mathrm{E}-06$ & $40.32 \%$ \\
\hline Ni-63 & $1.02 \mathrm{E}-05$ & $1.43 \mathrm{E}-05$ & $40.20 \%$ \\
\hline Ni-65 & $1.66 \mathrm{E}-25$ & $4.26 \mathrm{E}-25$ & $156.63 \%$ \\
\hline Np-237 & $2.76 \mathrm{E}-03$ & $3.88 \mathrm{E}-03$ & $40.58 \%$ \\
\hline Np-238 & $2.67 \mathrm{E}-08$ & $6.86 \mathrm{E}-08$ & $156.93 \%$ \\
\hline Np-239 & $3.07 \mathrm{E}-08$ & $7.89 \mathrm{E}-08$ & $157.00 \%$ \\
\hline Os-185 & $1.95 \mathrm{E}-03$ & $3.47 \mathrm{E}-03$ & $77.95 \%$ \\
\hline P-32 & $3.06 \mathrm{E}-04$ & $7.41 \mathrm{E}-04$ & $142.16 \%$ \\
\hline Pa-233 & $3.21 \mathrm{E}+00$ & $7.64 \mathrm{E}+00$ & $138.01 \%$ \\
\hline Pd-109 & $4.51 \mathrm{E}-10$ & $1.04 \mathrm{E}-09$ & $130.60 \%$ \\
\hline Pm-147 & $1.54 \mathrm{E}-05$ & $2.22 \mathrm{E}-05$ & $44.16 \%$ \\
\hline Pm-148 & $1.27 \mathrm{E}-05$ & $3.26 \mathrm{E}-05$ & $156.69 \%$ \\
\hline Pm-151 & $6.38 \mathrm{E}-08$ & $1.64 \mathrm{E}-07$ & $157.05 \%$ \\
\hline Pu-238 & $1.88 \mathrm{E}-04$ & $2.64 \mathrm{E}-04$ & $40.43 \%$ \\
\hline Pu-239 & $2.13 \mathrm{E}-04$ & $2.99 \mathrm{E}-04$ & $40.38 \%$ \\
\hline Pu-240 & $2.13 \mathrm{E}-04$ & $2.99 \mathrm{E}-04$ & $40.38 \%$ \\
\hline Pu-241 & $4.22 \mathrm{E}-06$ & $5.96 \mathrm{E}-06$ & $41.23 \%$ \\
\hline Pu-242 & $2.03 \mathrm{E}-04$ & $2.85 \mathrm{E}-04$ & $40.39 \%$ \\
\hline Ra-224 & $1.62 \mathrm{E}-03$ & $4.15 \mathrm{E}-03$ & $156.17 \%$ \\
\hline Ra-228 & $1.41 \mathrm{E}-01$ & $2.01 \mathrm{E}-01$ & $42.55 \%$ \\
\hline Rb-86 & $2.83 \mathrm{E}-04$ & $7.03 \mathrm{E}-04$ & $148.41 \%$ \\
\hline Rb-87 & $5.46 \mathrm{E}-04$ & $7.59 \mathrm{E}-04$ & $39.01 \%$ \\
\hline Rh-105 & $3.80 \mathrm{E}-08$ & $7.68 \mathrm{E}-08$ & $102.11 \%$ \\
\hline Rh-106M & $1.14 \mathrm{E}-28$ & $2.33 \mathrm{E}-28$ & $104.39 \%$ \\
\hline Ru-103 & $1.66 \mathrm{E}-03$ & $3.63 \mathrm{E}-03$ & $118.67 \%$ \\
\hline Ru-105 & $1.31 \mathrm{E}-15$ & $3.36 \mathrm{E}-15$ & $156.49 \%$ \\
\hline Ru-106 & $2.66 \mathrm{E}-02$ & $4.00 \mathrm{E}-02$ & $50.38 \%$ \\
\hline S-35 & $1.62 \mathrm{E}-04$ & $2.81 \mathrm{E}-04$ & $73.46 \%$ \\
\hline Sb-122 & $1.89 \mathrm{E}-06$ & $4.87 \mathrm{E}-06$ & $157.67 \%$ \\
\hline Sb-124 & $7.09 \mathrm{E}-05$ & $1.40 \mathrm{E}-04$ & $97.46 \%$ \\
\hline Sb-125 & $3.53 \mathrm{E}-05$ & $5.08 \mathrm{E}-05$ & $43.91 \%$ \\
\hline Se-126 & $2.73 \mathrm{E}-05$ & $6.97 \mathrm{E}-05$ & $155.31 \%$ \\
\hline Se-79 & $4.21 \mathrm{E}-04$ & $6.55 \mathrm{E}-04$ & $55.58 \%$ \\
\hline Sm-151 & $6.02 \mathrm{E}-04$ & $7.80 \mathrm{E}-04$ & $29.57 \%$ \\
\hline & $6.01 \mathrm{E}-06$ & $8.45 \mathrm{E}-06$ & $40.60 \%$ \\
\hline & & \\
\hline & & \\
\hline
\end{tabular}


Table 41. MAXDOSE-SR Maximally Exposed Individual Meat Consumption Comparisons (mrem/yr) (continued)

\begin{tabular}{|c|c|c|c|}
\hline \multicolumn{5}{|c|}{ Maxdose Meat Pathway } \\
\hline Nuclide & Hamby & Jannik & \% Diff \\
\hline & & & \\
\hline Sn-123 & $1.57 \mathrm{E}-03$ & $2.66 \mathrm{E}-03$ & $69.43 \%$ \\
\hline Sn-126 & $4.82 \mathrm{E}-03$ & $6.76 \mathrm{E}-03$ & $40.25 \%$ \\
\hline Sr-89 & $9.11 \mathrm{E}-06$ & $1.88 \mathrm{E}-05$ & $106.37 \%$ \\
\hline Sr-90 & $2.76 \mathrm{E}-04$ & $3.88 \mathrm{E}-04$ & $40.58 \%$ \\
\hline Sr-91 & $2.43 \mathrm{E}-12$ & $6.26 \mathrm{E}-12$ & $157.61 \%$ \\
\hline Sr-92 & $1.09 \mathrm{E}-24$ & $2.79 \mathrm{E}-24$ & $155.96 \%$ \\
\hline Tc-99 & $1.97 \mathrm{E}-03$ & $2.71 \mathrm{E}-03$ & $37.56 \%$ \\
\hline Tc-99 M & $4.38 \mathrm{E}-14$ & $1.12 \mathrm{E}-13$ & $155.71 \%$ \\
\hline Te-125M & $5.97 \mathrm{E}-04$ & $1.08 \mathrm{E}-03$ & $80.90 \%$ \\
\hline Te-127 & $6.35 \mathrm{E}-11$ & $1.58 \mathrm{E}-10$ & $148.82 \%$ \\
\hline Te-127M & $1.87 \mathrm{E}-03$ & $2.96 \mathrm{E}-03$ & $58.29 \%$ \\
\hline Te-129 & $1.40 \mathrm{E}-45$ & $2.80 \mathrm{E}-45$ & $100.00 \%$ \\
\hline Te-129M & $1.24 \mathrm{E}-03$ & $2.58 \mathrm{E}-03$ & $108.06 \%$ \\
\hline Te-131M & $7.43 \mathrm{E}-06$ & $1.85 \mathrm{E}-05$ & $148.99 \%$ \\
\hline Te-132 & $6.87 \mathrm{E}-05$ & $1.71 \mathrm{E}-04$ & $148.91 \%$ \\
\hline Th-228 & $2.52 \mathrm{E}-04$ & $3.68 \mathrm{E}-04$ & $46.03 \%$ \\
\hline Th-230 & $3.76 \mathrm{E}-04$ & $5.27 \mathrm{E}-04$ & $40.16 \%$ \\
\hline Th-232 & $1.98 \mathrm{E}-03$ & $2.79 \mathrm{E}-03$ & $40.91 \%$ \\
\hline Th-234 & $2.95 \mathrm{E}-06$ & $7.14 \mathrm{E}-06$ & $142.03 \%$ \\
\hline U-232 & $1.56 \mathrm{E}-03$ & $2.20 \mathrm{E}-03$ & $41.03 \%$ \\
\hline U-233 & $3.25 \mathrm{E}-04$ & $4.57 \mathrm{E}-04$ & $40.62 \%$ \\
\hline U-234 & $3.13 \mathrm{E}-04$ & $4.40 \mathrm{E}-04$ & $40.58 \%$ \\
\hline U-235 & $3.01 \mathrm{E}-04$ & $4.23 \mathrm{E}-04$ & $40.53 \%$ \\
\hline U-236 & $3.01 \mathrm{E}-04$ & $4.23 \mathrm{E}-04$ & $40.53 \%$ \\
\hline U-237 & $3.48 \mathrm{E}-07$ & $8.94 \mathrm{E}-07$ & $156.90 \%$ \\
\hline U-238 & $2.77 \mathrm{E}-04$ & $3.89 \mathrm{E}-04$ & $40.43 \%$ \\
\hline W-187 & $7.09 \mathrm{E}-09$ & $1.82 \mathrm{E}-08$ & $156.70 \%$ \\
\hline Y-90 & $3.34 \mathrm{E}-06$ & $8.60 \mathrm{E}-06$ & $157.49 \%$ \\
\hline Y-91 & $7.68 \mathrm{E}-05$ & $1.53 \mathrm{E}-04$ & $99.22 \%$ \\
\hline Y-92 & $7.69 \mathrm{E}-20$ & $1.98 \mathrm{E}-19$ & $157.48 \%$ \\
\hline Y-93 & $5.59 \mathrm{E}-11$ & $1.44 \mathrm{E}-10$ & $157.60 \%$ \\
\hline Zn-65 & $1.36 \mathrm{E}-03$ & $2.05 \mathrm{E}-03$ & $50.74 \%$ \\
\hline Zr-93 & $1.92 \mathrm{E}-04$ & $2.70 \mathrm{E}-04$ & $40.63 \%$ \\
\hline Zr-95 & $2.29 \mathrm{E}-04$ & $4.44 \mathrm{E}-04$ & $93.89 \%$ \\
\hline Zr-97 & $7.02 \mathrm{E}-08$ & $1.80 \mathrm{E}-07$ & $156.41 \%$ \\
\hline Total & $\mathbf{3 . 4 6 E + 0 0}$ & $\mathbf{8 . 0 0 E + 0 0}$ & $\mathbf{1 3 1 . 2 1 \%}$ \\
\hline & & & \\
\hline
\end{tabular}


Table 42. MAXDOSE-SR Maximally Exposed Individual Total All Pathways Comparisons (mrem/yr)

\begin{tabular}{|c|c|c|c|}
\hline \multicolumn{4}{|c|}{ Maxdose Total Pathway } \\
\hline Nuclide & Hamby & Jannik & $\%$ Diff \\
\hline Ar-41 & $1.66 \mathrm{E}-06$ & $1.57 \mathrm{E}-06$ & $-5.42 \%$ \\
\hline $\mathrm{Ag}-110 \mathrm{M}$ & $2.11 \mathrm{E}-02$ & $1.89 \mathrm{E}-02$ & $-10.43 \%$ \\
\hline Am-241 & $5.16 \mathrm{E}+00$ & $3.40 \mathrm{E}+00$ & $-34.11 \%$ \\
\hline Am-243 & $5.16 \mathrm{E}+00$ & $3.40 \mathrm{E}+00$ & $-34.11 \%$ \\
\hline Ba-139 & $2.49 \mathrm{E}-07$ & $2.35 \mathrm{E}-07$ & $-5.62 \%$ \\
\hline Ba-140 & $1.54 \mathrm{E}-03$ & $1.32 \mathrm{E}-03$ & $-14.29 \%$ \\
\hline $\mathrm{Ba}-141$ & $1.88 \mathrm{E}-09$ & $1.86 \mathrm{E}-09$ & $-1.06 \%$ \\
\hline Ba-142 & $1.35 \mathrm{E}-11$ & $1.35 \mathrm{E}-11$ & $0.00 \%$ \\
\hline $\mathrm{Be}-7$ & $1.18 \mathrm{E}-04$ & $1.02 \mathrm{E}-04$ & $-13.56 \%$ \\
\hline Br- 83 & $2.15 \mathrm{E}-07$ & $2.02 \mathrm{E}-07$ & $-6.05 \%$ \\
\hline Br- 84 & 5.39E-08 & 5.39E-08 & $0.00 \%$ \\
\hline $\mathrm{C}-14$ & $6.94 \mathrm{E}-04$ & $6.55 \mathrm{E}-04$ & $-5.62 \%$ \\
\hline Ce-141 & 5.37E-04 & $2.90 \mathrm{E}-04$ & $-46.00 \%$ \\
\hline Ce-143 & $5.01 \mathrm{E}-05$ & $3.66 \mathrm{E}-05$ & $-26.95 \%$ \\
\hline Ce-144 & $1.10 \mathrm{E}-02$ & $5.04 \mathrm{E}-03$ & $-54.18 \%$ \\
\hline Cf- 252 & $1.17 \mathrm{E}+00$ & $8.13 \mathrm{E}-01$ & $-30.51 \%$ \\
\hline $\mathrm{Cm}-242$ & $1.36 \mathrm{E}-01$ & $1.01 \mathrm{E}-01$ & $-25.74 \%$ \\
\hline $\mathrm{Cm}-243$ & $3.40 \mathrm{E}+00$ & $2.27 \mathrm{E}+00$ & $-33.24 \%$ \\
\hline $\mathrm{Cm}-244$ & $2.65 \mathrm{E}+00$ & $1.76 \mathrm{E}+00$ & $-33.58 \%$ \\
\hline $\mathrm{Cm}-246$ & $5.27 \mathrm{E}+00$ & $3.51 \mathrm{E}+00$ & $-33.40 \%$ \\
\hline Cm-248 & $1.86 \mathrm{E}+01$ & $1.24 \mathrm{E}+01$ & $-33.33 \%$ \\
\hline Co-57 & $1.10 \mathrm{E}-03$ & $8.07 \mathrm{E}-04$ & $-26.64 \%$ \\
\hline Co-58 & $3.27 \mathrm{E}-03$ & $2.74 \mathrm{E}-03$ & $-16.21 \%$ \\
\hline Co-60 & $2.55 \mathrm{E}-02$ & $1.73 \mathrm{E}-02$ & $-32.16 \%$ \\
\hline $\mathrm{Cr}-51$ & $5.30 \mathrm{E}-05$ & $4.40 \mathrm{E}-05$ & $-16.98 \%$ \\
\hline Cs-134 & 5.41E-02 & $3.06 \mathrm{E}-02$ & $-43.44 \%$ \\
\hline Cs-135 & $4.85 \mathrm{E}-03$ & $2.39 \mathrm{E}-03$ & $-50.72 \%$ \\
\hline Cs- 137 & $3.67 \mathrm{E}-02$ & $1.95 \mathrm{E}-02$ & $-46.87 \%$ \\
\hline Cs-138 & $7.18 \mathrm{E}-08$ & $7.21 \mathrm{E}-08$ & $0.42 \%$ \\
\hline Eu-152 & $9.46 \mathrm{E}-03$ & $7.41 \mathrm{E}-03$ & $-21.67 \%$ \\
\hline Eu-154 & $1.17 \mathrm{E}-02$ & $8.60 \mathrm{E}-03$ & $-26.50 \%$ \\
\hline Eu-155 & $1.21 \mathrm{E}-03$ & 7.64E-04 & $-36.86 \%$ \\
\hline $\mathrm{Fe}-55$ & $3.85 \mathrm{E}-04$ & $2.27 \mathrm{E}-04$ & $-41.04 \%$ \\
\hline Fe-59 & $3.54 \mathrm{E}-03$ & $3.19 \mathrm{E}-03$ & $-9.89 \%$ \\
\hline $\mathrm{H}-3$ & $1.56 \mathrm{E}-06$ & $1.48 \mathrm{E}-06$ & $-5.13 \%$ \\
\hline Hf-181 & $4.43 \mathrm{E}-03$ & $7.07 \mathrm{E}-03$ & $59.59 \%$ \\
\hline I-129 & $8.41 \mathrm{E}-01$ & $3.56 \mathrm{E}-01$ & $-57.67 \%$ \\
\hline I-131 & $1.10 \mathrm{E}-02$ & $9.56 \mathrm{E}-03$ & $-13.09 \%$ \\
\hline I-132 & $4.40 \mathrm{E}-06$ & $4.40 \mathrm{E}-06$ & $0.00 \%$ \\
\hline I-133 & $1.70 \mathrm{E}-04$ & 1.19E-04 & $-30.00 \%$ \\
\hline I-134 & $4.89 \mathrm{E}-07$ & $4.92 \mathrm{E}-07$ & $0.61 \%$ \\
\hline I-135 & $1.67 \mathrm{E}-05$ & $1.59 \mathrm{E}-05$ & $-4.79 \%$ \\
\hline $\mathrm{Kr}-83 \mathrm{M}$ & $1.19 \mathrm{E}-10$ & $1.12 \mathrm{E}-10$ & $-5.88 \%$ \\
\hline
\end{tabular}


SRNL-STI-2010-00447, REVISION 0

Table 42. MAXDOSE-SR Maximally Exposed Individual Total All Pathways Comparisons (mrem/yr) (continued)

\begin{tabular}{|c|c|c|c|}
\hline \multicolumn{4}{|c|}{ Maxdose Total Pathway } \\
\hline Nuclide & Hamby & Jannik & $\%$ Diff \\
\hline $\mathrm{Kr}-85$ & $7.70 \mathrm{E}-09$ & $7.27 \mathrm{E}-09$ & $-5.58 \%$ \\
\hline $\mathrm{Kr}-85 \mathrm{M}$ & $3.71 \mathrm{E}-07$ & $3.51 \mathrm{E}-07$ & $-5.39 \%$ \\
\hline Kr-87 & 7.19E-07 & $6.82 \mathrm{E}-07$ & $-5.15 \%$ \\
\hline $\mathrm{Kr}-88$ & $5.34 \mathrm{E}-06$ & $5.05 \mathrm{E}-06$ & $-5.43 \%$ \\
\hline $\mathrm{Kr}-89$ & $5.82 \mathrm{E}-21$ & $6.03 \mathrm{E}-21$ & $3.61 \%$ \\
\hline La-140 & $1.95 \mathrm{E}-04$ & $1.63 \mathrm{E}-04$ & $-16.41 \%$ \\
\hline La-141 & $2.00 \mathrm{E}-06$ & $1.87 \mathrm{E}-06$ & $-6.50 \%$ \\
\hline La-142 & $2.09 \mathrm{E}-06$ & $2.09 \mathrm{E}-06$ & $0.00 \%$ \\
\hline Mn-54 & $4.46 \mathrm{E}-03$ & $3.69 \mathrm{E}-03$ & $-17.26 \%$ \\
\hline Mn-56 & $3.94 \mathrm{E}-06$ & $3.92 \mathrm{E}-06$ & $-0.51 \%$ \\
\hline Mo-99 & $9.17 \mathrm{E}-05$ & $8.71 \mathrm{E}-05$ & $-5.02 \%$ \\
\hline $\mathrm{Na}-24$ & $7.96 \mathrm{E}-05$ & $8.08 \mathrm{E}-05$ & $1.51 \%$ \\
\hline Nb-95 & $2.23 \mathrm{E}-03$ & $3.13 \mathrm{E}-03$ & $40.36 \%$ \\
\hline $\mathrm{Nb}-97$ & $3.51 \mathrm{E}-07$ & $3.51 \mathrm{E}-07$ & $0.00 \%$ \\
\hline Ni-59 & $1.33 \mathrm{E}-04$ & $6.37 \mathrm{E}-05$ & $-52.11 \%$ \\
\hline Ni-63 & $3.50 \mathrm{E}-04$ & $1.64 \mathrm{E}-04$ & $-53.14 \%$ \\
\hline $\mathrm{Ni}-65$ & $1.47 \mathrm{E}-06$ & $1.44 \mathrm{E}-06$ & $-2.04 \%$ \\
\hline Np-237 & $4.69 \mathrm{E}+00$ & $3.15 \mathrm{E}+00$ & $-32.84 \%$ \\
\hline Np-238 & $2.32 \mathrm{E}-04$ & $2.02 \mathrm{E}-04$ & $-12.93 \%$ \\
\hline Np-239 & $5.50 \mathrm{E}-05$ & $3.60 \mathrm{E}-05$ & $-34.55 \%$ \\
\hline Os-185 & $4.63 \mathrm{E}-03$ & $5.72 \mathrm{E}-03$ & $23.54 \%$ \\
\hline $\mathrm{P}-32$ & $1.25 \mathrm{E}-03$ & $2.00 \mathrm{E}-03$ & $60.00 \%$ \\
\hline Pa-233 & $3.21 \mathrm{E}+00$ & $7.64 \mathrm{E}+00$ & $138.01 \%$ \\
\hline Pd-109 & $7.26 \mathrm{E}-06$ & $6.42 \mathrm{E}-06$ & $-11.57 \%$ \\
\hline Pm-147 & $6.59 \mathrm{E}-04$ & $3.46 \mathrm{E}-04$ & $-47.50 \%$ \\
\hline Pm-148 & $3.78 \mathrm{E}-04$ & $2.55 \mathrm{E}-04$ & $-32.54 \%$ \\
\hline Pm-151 & $3.28 \mathrm{E}-05$ & $2.59 \mathrm{E}-05$ & $-21.04 \%$ \\
\hline Pr-144 & $3.72 \mathrm{E}-10$ & $3.72 \mathrm{E}-10$ & $0.00 \%$ \\
\hline $\mathrm{Pu}-238$ & $4.32 \mathrm{E}+00$ & $2.98 \mathrm{E}+00$ & $-31.02 \%$ \\
\hline $\mathrm{Pu}-239$ & $4.84 \mathrm{E}+00$ & $3.32 \mathrm{E}+00$ & $-31.40 \%$ \\
\hline $\mathrm{Pu}-240$ & $4.84 \mathrm{E}+00$ & $3.32 \mathrm{E}+00$ & $-31.40 \%$ \\
\hline $\mathrm{Pu}-241$ & $9.54 \mathrm{E}-02$ & $6.52 \mathrm{E}-02$ & $-31.66 \%$ \\
\hline Pu-242 & $4.58 \mathrm{E}+00$ & $3.13 \mathrm{E}+00$ & $-31.66 \%$ \\
\hline Ra-224 & $2.29 \mathrm{E}-02$ & $2.47 \mathrm{E}-02$ & $7.86 \%$ \\
\hline Ra-228 & $8.92 \mathrm{E}-01$ & 5.32E-01 & $-40.36 \%$ \\
\hline $\mathrm{Rb}-86$ & $1.81 \mathrm{E}-03$ & $2.71 \mathrm{E}-03$ & $49.72 \%$ \\
\hline Rb-87 & $4.99 \mathrm{E}-03$ & $3.43 \mathrm{E}-03$ & $-31.26 \%$ \\
\hline Rb-88 & $1.36 \mathrm{E}-09$ & $1.36 \mathrm{E}-09$ & $0.00 \%$ \\
\hline Rb-89 & $6.37 \mathrm{E}-10$ & $6.37 \mathrm{E}-10$ & $0.00 \%$ \\
\hline Rh-105 & $2.09 \mathrm{E}-05$ & $1.97 \mathrm{E}-05$ & $-5.74 \%$ \\
\hline Rh-106M & $4.38 \mathrm{E}-07$ & $4.38 \mathrm{E}-07$ & $0.00 \%$ \\
\hline Ru-103 & $2.90 \mathrm{E}-03$ & $4.53 \mathrm{E}-03$ & $56.21 \%$ \\
\hline Ru-105 & $5.04 \mathrm{E}-06$ & $5.03 \mathrm{E}-06$ & $-0.20 \%$ \\
\hline
\end{tabular}


Table 42. MAXDOSE-SR Maximally Exposed Individual Total All Pathways Comparisons (mrem/yr) (continued)

\begin{tabular}{|c|c|c|c|}
\hline \multicolumn{4}{|c|}{ Maxdose Total Pathway } \\
\hline Nuclide & Hamby & Jannik & $\%$ Diff \\
\hline Ru-106 & $3.94 \mathrm{E}-02$ & $4.62 \mathrm{E}-02$ & $17.26 \%$ \\
\hline S-35 & $4.76 \mathrm{E}-04$ & $4.87 \mathrm{E}-04$ & $2.31 \%$ \\
\hline Sb-122 & $1.44 \mathrm{E}-04$ & $1.06 \mathrm{E}-04$ & $-26.39 \%$ \\
\hline Sb-124 & $6.05 \mathrm{E}-03$ & $4.57 \mathrm{E}-03$ & $-24.46 \%$ \\
\hline Sb-125 & $3.40 \mathrm{E}-03$ & $2.54 \mathrm{E}-03$ & $-25.29 \%$ \\
\hline Sb-126 & $1.85 \mathrm{E}-03$ & $1.62 \mathrm{E}-03$ & $-12.43 \%$ \\
\hline $\mathrm{Sb}-126 \mathrm{M}$ & $2.75 \mathrm{E}-09$ & $2.75 \mathrm{E}-09$ & $0.00 \%$ \\
\hline Se-75 & $9.20 \mathrm{E}-03$ & $8.07 \mathrm{E}-03$ & $-12.28 \%$ \\
\hline Se-79 & 1.19E-02 & $8.90 \mathrm{E}-03$ & $-25.21 \%$ \\
\hline Sm-151 & $3.31 \mathrm{E}-04$ & $2.13 \mathrm{E}-04$ & $-35.65 \%$ \\
\hline Sn-123 & $4.72 \mathrm{E}-03$ & $3.92 \mathrm{E}-03$ & $-16.95 \%$ \\
\hline Sn-126 & $1.49 \mathrm{E}-02$ & $1.08 \mathrm{E}-02$ & $-27.52 \%$ \\
\hline Sr-89 & $2.15 \mathrm{E}-03$ & $8.79 \mathrm{E}-04$ & $-59.12 \%$ \\
\hline Sr-90 & $7.57 \mathrm{E}-02$ & 2.99E-02 & $-60.50 \%$ \\
\hline Sr-91 & $1.51 \mathrm{E}-05$ & $1.44 \mathrm{E}-05$ & $-4.64 \%$ \\
\hline Sr-92 & $4.52 \mathrm{E}-06$ & $4.52 \mathrm{E}-06$ & $0.00 \%$ \\
\hline Tc-101 & $8.79 \mathrm{E}-11$ & $8.79 \mathrm{E}-11$ & $0.00 \%$ \\
\hline Tc-99 & $3.14 \mathrm{E}-03$ & $3.41 \mathrm{E}-03$ & $8.60 \%$ \\
\hline Tc-99 M & 1.14E-06 & $1.14 \mathrm{E}-06$ & $0.00 \%$ \\
\hline Te-125M & $1.60 \mathrm{E}-03$ & $1.53 \mathrm{E}-03$ & $-4.38 \%$ \\
\hline Te-127 & $1.52 \mathrm{E}-06$ & $1.36 \mathrm{E}-06$ & $-10.53 \%$ \\
\hline Te-127M & $5.09 \mathrm{E}-03$ & $4.35 \mathrm{E}-03$ & $-14.54 \%$ \\
\hline Te-129 & $1.01 \mathrm{E}-07$ & $1.01 \mathrm{E}-07$ & $0.00 \%$ \\
\hline Te-129M & $3.03 \mathrm{E}-03$ & $3.39 \mathrm{E}-03$ & $11.88 \%$ \\
\hline Te-131 & $9.45 \mathrm{E}-09$ & $9.45 \mathrm{E}-09$ & $0.00 \%$ \\
\hline $\mathrm{Te}-131 \mathrm{M}$ & $1.59 \mathrm{E}-04$ & $1.32 \mathrm{E}-04$ & $-16.98 \%$ \\
\hline Te-132 & 2.44E-04 & $2.84 \mathrm{E}-04$ & $16.39 \%$ \\
\hline Te-134 & $1.05 \mathrm{E}-07$ & $1.05 \mathrm{E}-07$ & $0.00 \%$ \\
\hline Th-228 & $1.77 \mathrm{E}+00$ & $1.64 \mathrm{E}+00$ & $-7.34 \%$ \\
\hline Th-230 & $1.91 \mathrm{E}+00$ & $1.72 \mathrm{E}+00$ & $-9.95 \%$ \\
\hline Th-232 & $9.63 \mathrm{E}+00$ & $8.64 \mathrm{E}+00$ & $-10.28 \%$ \\
\hline Th-234 & $1.55 \mathrm{E}-03$ & $6.21 \mathrm{E}-04$ & $-59.94 \%$ \\
\hline U-232 & $4.11 \mathrm{E}+00$ & $3.65 \mathrm{E}+00$ & $-11.19 \%$ \\
\hline $\mathrm{U}-233$ & $8.06 \mathrm{E}-01$ & $7.11 \mathrm{E}-01$ & $-11.79 \%$ \\
\hline U-234 & $8.01 \mathrm{E}-01$ & $7.09 \mathrm{E}-01$ & $-11.49 \%$ \\
\hline U-235 & $7.45 \mathrm{E}-01$ & $6.57 \mathrm{E}-01$ & $-11.81 \%$ \\
\hline U-236 & $7.45 \mathrm{E}-01$ & $6.56 \mathrm{E}-01$ & $-11.95 \%$ \\
\hline U-237 & 1.31E-04 & $8.47 \mathrm{E}-05$ & $-35.34 \%$ \\
\hline U-238 & 7.34E-01 & $6.53 \mathrm{E}-01$ & $-11.04 \%$ \\
\hline W-187 & $2.68 \mathrm{E}-05$ & $2.20 \mathrm{E}-05$ & $-17.91 \%$ \\
\hline Xe-131M & $2.77 \mathrm{E}-08$ & $2.77 \mathrm{E}-08$ & $0.00 \%$ \\
\hline Xe-133 & $1.13 \mathrm{E}-07$ & $1.13 \mathrm{E}-07$ & $0.00 \%$ \\
\hline $\mathrm{Xe}-133 \mathrm{M}$ & $9.34 \mathrm{E}-08$ & $9.34 \mathrm{E}-08$ & $0.00 \%$ \\
\hline
\end{tabular}


SRNL-STI-2010-00447, REVISION 0

Table 42. MAXDOSE-SR Maximally Exposed Individual Total All Pathways Comparisons (mrem/yr) (continued)

\begin{tabular}{|c|c|c|c|}
\hline \multicolumn{4}{|c|}{ Maxdose Total Pathway } \\
\hline Nuclide & Hamby & Jannik & \% Diff \\
\hline Xe-135 & $6.62 \mathrm{E}-07$ & $6.62 \mathrm{E}-07$ & $0.00 \%$ \\
\hline Xe-135M & $1.03 \mathrm{E}-09$ & $1.03 \mathrm{E}-09$ & $0.00 \%$ \\
\hline Xe-137 & $2.88 \mathrm{E}-19$ & $2.88 \mathrm{E}-19$ & $0.00 \%$ \\
\hline Xe-138 & $1.64 \mathrm{E}-09$ & $1.64 \mathrm{E}-09$ & $0.00 \%$ \\
\hline Y-90 & $1.54 \mathrm{E}-04$ & $8.43 \mathrm{E}-05$ & $-45.26 \%$ \\
\hline Y-91 & $2.50 \mathrm{E}-03$ & $1.08 \mathrm{E}-03$ & $-56.80 \%$ \\
\hline Y-91 M & $9.28 \mathrm{E}-08$ & $9.28 \mathrm{E}-08$ & $0.00 \%$ \\
\hline Y-92 & $2.68 \mathrm{E}-06$ & $2.68 \mathrm{E}-06$ & $0.00 \%$ \\
\hline Y-93 & $1.19 \mathrm{E}-05$ & $1.06 \mathrm{E}-05$ & $-10.92 \%$ \\
\hline Zn-65 & $1.57 \mathrm{E}-02$ & $1.28 \mathrm{E}-02$ & $-18.47 \%$ \\
\hline Zr-93 & $2.66 \mathrm{E}-03$ & $2.17 \mathrm{E}-03$ & $-18.42 \%$ \\
\hline Zr-95 & $4.40 \mathrm{E}-03$ & $4.00 \mathrm{E}-03$ & $-9.09 \%$ \\
\hline Zr-97 & $3.42 \mathrm{E}-05$ & $2.63 \mathrm{E}-05$ & $-23.10 \%$ \\
\hline *TOTAL $*$ & $\mathbf{9 . 1 6 E + 0 1 ~}$ & $\mathbf{7 . 1 5 E + 0 1}$ & $\mathbf{- 2 1 . 9 4 \%}$ \\
\hline
\end{tabular}


Table 43. POPDOSE-SR Collective Ground Exposure Comparisons (person-rem/yr)

\begin{tabular}{|c|c|c|c|}
\hline \multicolumn{5}{|c|}{ Popdose Ground Pathway } \\
\hline Nuclide & Hamby & Jannik & \% Diff \\
\hline Ag1 10M & $4.83 \mathrm{E}-01$ & $4.82 \mathrm{E}-01$ & $-0.21 \%$ \\
\hline Am241 & $6.87 \mathrm{E}-03$ & $6.85 \mathrm{E}-03$ & $-0.29 \%$ \\
\hline Am243 & $1.52 \mathrm{E}-02$ & $1.52 \mathrm{E}-02$ & $0.00 \%$ \\
\hline Ba139 & $1.65 \mathrm{E}-07$ & $1.63 \mathrm{E}-07$ & $-1.23 \%$ \\
\hline Ba140 & $5.31 \mathrm{E}-02$ & $5.30 \mathrm{E}-02$ & $-0.19 \%$ \\
\hline Ba141 & $1.73 \mathrm{E}-09$ & $2.03 \mathrm{E}-09$ & $14.78 \%$ \\
\hline Ba142 & $1.99 \mathrm{E}-11$ & $2.58 \mathrm{E}-11$ & $22.87 \%$ \\
\hline Be 7 & $4.50 \mathrm{E}-03$ & $4.49 \mathrm{E}-03$ & $-0.22 \%$ \\
\hline Br 83 & $1.82 \mathrm{E}-07$ & $1.80 \mathrm{E}-07$ & $-1.11 \%$ \\
\hline Br 84 & $9.55 \mathrm{E}-08$ & $9.91 \mathrm{E}-08$ & $3.63 \%$ \\
\hline Ce141 & $5.02 \mathrm{E}-03$ & $5.01 \mathrm{E}-03$ & $-0.20 \%$ \\
\hline Ce143 & $6.01 \mathrm{E}-04$ & $5.99 \mathrm{E}-04$ & $-0.33 \%$ \\
\hline Ce144 & $9.45 \mathrm{E}-03$ & $9.43 \mathrm{E}-03$ & $-0.21 \%$ \\
\hline Cf252 & $1.37 \mathrm{E}-04$ & $1.36 \mathrm{E}-04$ & $-0.74 \%$ \\
\hline Cm242 & $2.87 \mathrm{E}-04$ & $2.86 \mathrm{E}-04$ & $-0.35 \%$ \\
\hline Cm243 & $3.34 \mathrm{E}-02$ & $3.34 \mathrm{E}-02$ & $0.00 \%$ \\
\hline Cm244 & $1.89 \mathrm{E}-04$ & $1.88 \mathrm{E}-04$ & $-0.53 \%$ \\
\hline Cm246 & $1.69 \mathrm{E}-04$ & $1.69 \mathrm{E}-04$ & $0.00 \%$ \\
\hline Cm248 & $1.35 \mathrm{E}-04$ & $1.35 \mathrm{E}-04$ & $0.00 \%$ \\
\hline Co 57 & $2.62 \mathrm{E}-02$ & $2.61 \mathrm{E}-02$ & $-0.38 \%$ \\
\hline Co 58 & $1.06 \mathrm{E}-01$ & $1.06 \mathrm{E}-01$ & $0.00 \%$ \\
\hline Co 60 & $5.05 \mathrm{E}-01$ & $5.04 \mathrm{E}-01$ & $-0.20 \%$ \\
\hline Cr 51 & $1.68 \mathrm{E}-03$ & $1.68 \mathrm{E}-03$ & $0.00 \%$ \\
\hline Cs134 & $3.33 \mathrm{E}-01$ & $3.33 \mathrm{E}-01$ & $0.00 \%$ \\
\hline Cs137 & $1.32 \mathrm{E}-01$ & $1.32 \mathrm{E}-01$ & $0.00 \%$ \\
\hline Cs138 & $1.39 \mathrm{E}-07$ & $1.44 \mathrm{E}-07$ & $3.47 \%$ \\
\hline Eu152 & $2.53 \mathrm{E}-01$ & $2.52 \mathrm{E}-01$ & $-0.40 \%$ \\
\hline Eu154 & $2.72 \mathrm{E}-01$ & $2.72 \mathrm{E}-01$ & $0.00 \%$ \\
\hline Eu155 & $1.59 \mathrm{E}-02$ & $1.58 \mathrm{E}-02$ & $-0.63 \%$ \\
\hline Fe 55 & $4.74 \mathrm{E}-05$ & $4.73 \mathrm{E}-05$ & $-0.21 \%$ \\
\hline Fe 59 & $8.36 \mathrm{E}-02$ & $8.34 \mathrm{E}-02$ & $-0.24 \%$ \\
\hline Hf181 & $4.20 \mathrm{E}-02$ & $4.19 \mathrm{E}-02$ & $-0.24 \%$ \\
\hline I 129 & $5.06 \mathrm{E}-03$ & $5.05 \mathrm{E}-03$ & $-0.20 \%$ \\
\hline I 131 & $5.72 \mathrm{E}-03$ & $5.71 \mathrm{E}-03$ & $-0.18 \%$ \\
\hline I 132 & $4.87 \mathrm{E}-05$ & $4.81 \mathrm{E}-05$ & $-1.25 \%$ \\
\hline I 133 & $8.00 \mathrm{E}-04$ & $7.96 \mathrm{E}-04$ & $-0.50 \%$ \\
\hline I 134 & $1.86 \mathrm{E}-06$ & $1.85 \mathrm{E}-06$ & $-0.54 \%$ \\
\hline I 135 & $3.20 \mathrm{E}-04$ & $3.17 \mathrm{E}-04$ & $-0.95 \%$ \\
\hline La140 & $5.62 \mathrm{E}-03$ & $5.61 \mathrm{E}-03$ & $-0.18 \%$ \\
\hline La141 & $3.03 \mathrm{E}-06$ & $3.00 \mathrm{E}-06$ & $-1.00 \%$ \\
\hline Mn 54 99 & $1.54 \mathrm{E}-05$ & $1.51 \mathrm{E}-05$ & $-1.99 \%$ \\
\hline Mn 0101 & $1.58 \mathrm{E}-01$ & $-0.63 \%$ \\
\hline & $4.59 \mathrm{E}-05$ & $4.54 \mathrm{E}-05$ & $-1.10 \%$ \\
\hline $\mathrm{E}-03$ & $1.33 \mathrm{E}-03$ & $0.00 \%$ \\
\hline
\end{tabular}


Table 43. POPDOSE-SR Collective Ground Exposure Comparisons (person-rem/yr) (continued)

\begin{tabular}{|c|c|c|c|}
\hline \multicolumn{5}{|c|}{ Popdose Ground Pathway } \\
\hline Nuclide & Hamby & Jannik & \% Diff \\
\hline Na 24 & $2.79 \mathrm{E}-03$ & $2.77 \mathrm{E}-03$ & $-0.72 \%$ \\
\hline Nb 95 & $4.77 \mathrm{E}-02$ & $4.76 \mathrm{E}-02$ & $-0.21 \%$ \\
\hline Nb 97 & $1.78 \mathrm{E}-06$ & $1.76 \mathrm{E}-06$ & $-1.14 \%$ \\
\hline Ni 59 & $9.57 \mathrm{E}-05$ & $9.55 \mathrm{E}-05$ & $-0.21 \%$ \\
\hline Ni 65 & $1.35 \mathrm{E}-05$ & $1.33 \mathrm{E}-05$ & $-1.50 \%$ \\
\hline Np237 & $7.45 \mathrm{E}-03$ & $7.44 \mathrm{E}-03$ & $-0.13 \%$ \\
\hline Np238 & $1.85 \mathrm{E}-03$ & $1.84 \mathrm{E}-03$ & $-0.54 \%$ \\
\hline Np239 & $7.42 \mathrm{E}-04$ & $7.40 \mathrm{E}-04$ & $-0.27 \%$ \\
\hline Os185 & $9.14 \mathrm{E}-02$ & $9.14 \mathrm{E}-02$ & $0.00 \%$ \\
\hline P 32 & $0.00 \mathrm{E}+00$ & $0.00 \mathrm{E}+00$ & \\
\hline Pa233 & $1.13 \mathrm{E}-02$ & $1.13 \mathrm{E}-02$ & $0.00 \%$ \\
\hline Pd109 & $4.75 \mathrm{E}-07$ & $4.75 \mathrm{E}-07$ & $0.00 \%$ \\
\hline Pm147 & $8.81 \mathrm{E}-07$ & $8.81 \mathrm{E}-07$ & $0.00 \%$ \\
\hline Pm148 & $5.00 \mathrm{E}-03$ & $5.00 \mathrm{E}-03$ & $0.00 \%$ \\
\hline Pm151 & $6.27 \mathrm{E}-04$ & $6.27 \mathrm{E}-04$ & $0.00 \%$ \\
\hline Pr144 & $4.59 \mathrm{E}-11$ & $4.59 \mathrm{E}-11$ & $0.00 \%$ \\
\hline Pu238 & $1.96 \mathrm{E}-04$ & $1.96 \mathrm{E}-04$ & $0.00 \%$ \\
\hline Pu239 & $8.68 \mathrm{E}-05$ & $8.68 \mathrm{E}-05$ & $0.00 \%$ \\
\hline Pu240 & $1.88 \mathrm{E}-04$ & $1.88 \mathrm{E}-04$ & $0.00 \%$ \\
\hline Pu242 & $1.57 \mathrm{E}-04$ & $1.57 \mathrm{E}-04$ & $0.00 \%$ \\
\hline Ra224 & $6.85 \mathrm{E}-05$ & $6.85 \mathrm{E}-05$ & $0.00 \%$ \\
\hline Ra228 & $1.48 \mathrm{E}-10$ & $1.48 \mathrm{E}-10$ & $0.00 \%$ \\
\hline Rb 86 & $3.00 \mathrm{E}-03$ & $3.00 \mathrm{E}-03$ & $0.00 \%$ \\
\hline Rb 88 & $1.12 \mathrm{E}-09$ & $1.12 \mathrm{E}-09$ & $0.00 \%$ \\
\hline Rb 89 & $1.17 \mathrm{E}-09$ & $1.17 \mathrm{E}-09$ & $0.00 \%$ \\
\hline Rh105 & $1.90 \mathrm{E}-04$ & $1.90 \mathrm{E}-04$ & $0.00 \%$ \\
\hline Ru103 & $3.43 \mathrm{E}-02$ & $3.43 \mathrm{E}-02$ & $0.00 \%$ \\
\hline Ru105 & $8.22 \mathrm{E}-05$ & $8.22 \mathrm{E}-05$ & $0.00 \%$ \\
\hline Ru106 & $4.08 \mathrm{E}-02$ & $4.08 \mathrm{E}-02$ & $0.00 \%$ \\
\hline Sb122 & $2.04 \mathrm{E}-03$ & $2.04 \mathrm{E}-03$ & $0.00 \%$ \\
\hline Sb124 & $1.68 \mathrm{E}-01$ & $1.68 \mathrm{E}-01$ & $0.00 \%$ \\
\hline Sb125 & $9.65 \mathrm{E}-02$ & $9.65 \mathrm{E}-02$ & $0.00 \%$ \\
\hline Sb126 & $6.19 \mathrm{E}-02$ & $6.19 \mathrm{E}-02$ & $0.00 \%$ \\
\hline Sb126M & $4.76 \mathrm{E}-09$ & $4.76 \mathrm{E}-09$ & $0.00 \%$ \\
\hline Se 75 & $6.10 \mathrm{E}-02$ & $6.10 \mathrm{E}-02$ & $0.00 \%$ \\
\hline Sm151 & $1.19 \mathrm{E}-06$ & $1.19 \mathrm{E}-06$ & $0.00 \%$ \\
\hline Sn123 & $9.54 \mathrm{E}-04$ & $9.54 \mathrm{E}-04$ & $0.00 \%$ \\
\hline Sn126 & $1.42 \mathrm{E}-02$ & $1.42 \mathrm{E}-02$ & $0.00 \%$ \\
\hline Sr 89 & $1.13 \mathrm{E}-05$ & $1.13 \mathrm{E}-05$ & $0.00 \%$ \\
\hline Sr 90 & $0.00 \mathrm{E}+00$ & $0.00 \mathrm{E}+00$ & \\
\hline Sr 91 $92 \mathrm{E}-0.79 \mathrm{E}-04$ & $2.70 \mathrm{E}-04$ & $0.00 \%$ \\
\hline Tc 99 & $1.44 \mathrm{E}-07$ & $1.44 \mathrm{E}-07$ & $0.00 \%$ \\
\hline & $2.74 \mathrm{E}-05$ & $2.74 \mathrm{E}-05$ & $0.00 \%$ \\
\hline & & \\
\hline
\end{tabular}


Table 43. POPDOSE-SR Collective Ground Exposure Comparisons (person-rem/yr) (continued)

\begin{tabular}{|c|c|c|c|}
\hline \multicolumn{4}{|c|}{ Popdose Ground Pathway } \\
\hline Nuclide & Hamby & Jannik & $\mathbf{\% ~ D i f f ~}$ \\
\hline Tc101 & $1.33 \mathrm{E}-10$ & $1.33 \mathrm{E}-10$ & $0.00 \%$ \\
\hline Te125M & $1.95 \mathrm{E}-03$ & $1.95 \mathrm{E}-03$ & $0.00 \%$ \\
\hline Te127 & $1.99 \mathrm{E}-06$ & $1.99 \mathrm{E}-06$ & $0.00 \%$ \\
\hline Te127M & $9.07 \mathrm{E}-04$ & $9.07 \mathrm{E}-04$ & $0.00 \%$ \\
\hline Te129 & $1.28 \mathrm{E}-07$ & $1.28 \mathrm{E}-07$ & $0.00 \%$ \\
\hline Te129M & $2.16 \mathrm{E}-03$ & $2.16 \mathrm{E}-03$ & $0.00 \%$ \\
\hline Te131 & $7.08 \mathrm{E}-09$ & $7.08 \mathrm{E}-09$ & $0.00 \%$ \\
\hline Te131M & $2.64 \mathrm{E}-03$ & $2.64 \mathrm{E}-03$ & $0.00 \%$ \\
\hline Te132 & $1.36 \mathrm{E}-03$ & $1.36 \mathrm{E}-03$ & $0.00 \%$ \\
\hline Te134 & $2.26 \mathrm{E}-07$ & $2.26 \mathrm{E}-07$ & $0.00 \%$ \\
\hline Th228 & $5.81 \mathrm{E}-04$ & $5.81 \mathrm{E}-04$ & $0.00 \%$ \\
\hline Th230 & $2.09 \mathrm{E}-04$ & $2.09 \mathrm{E}-04$ & $0.00 \%$ \\
\hline Th232 & $1.53 \mathrm{E}-04$ & $1.53 \mathrm{E}-04$ & $0.00 \%$ \\
\hline Th234 & $4.33 \mathrm{E}-04$ & $4.33 \mathrm{E}-04$ & $0.00 \%$ \\
\hline U 232 & $2.37 \mathrm{E}-04$ & $2.37 \mathrm{E}-04$ & $0.00 \%$ \\
\hline U 233 & $1.15 \mathrm{E}-04$ & $1.15 \mathrm{E}-04$ & $0.00 \%$ \\
\hline U 234 & $1.85 \mathrm{E}-04$ & $1.85 \mathrm{E}-04$ & $0.00 \%$ \\
\hline U 235 & $3.92 \mathrm{E}-02$ & $3.92 \mathrm{E}-02$ & $0.00 \%$ \\
\hline U 236 & $1.68 \mathrm{E}-04$ & $1.68 \mathrm{E}-04$ & $0.00 \%$ \\
\hline U 237 & $1.90 \mathrm{E}-03$ & $1.90 \mathrm{E}-03$ & $0.00 \%$ \\
\hline U 238 & $1.48 \mathrm{E}-04$ & $1.48 \mathrm{E}-04$ & $0.00 \%$ \\
\hline W 187 & $7.02 \mathrm{E}-04$ & $7.02 \mathrm{E}-04$ & $0.00 \%$ \\
\hline Y 90 & $0.00 \mathrm{E}+00$ & $0.00 \mathrm{E}+00$ & \\
\hline Y 91 & $3.09 \mathrm{E}-04$ & $3.09 \mathrm{E}-04$ & $0.00 \%$ \\
\hline Y 91M & $3.09 \mathrm{E}-07$ & $3.09 \mathrm{E}-07$ & $0.00 \%$ \\
\hline Y 92 & $1.52 \mathrm{E}-05$ & $1.52 \mathrm{E}-05$ & $0.00 \%$ \\
\hline Y 93 & $3.75 \mathrm{E}-05$ & $3.75 \mathrm{E}-05$ & $0.00 \%$ \\
\hline Zn 65 & $9.79 \mathrm{E}-02$ & $9.79 \mathrm{E}-02$ & $0.00 \%$ \\
\hline Zr 95 & $1.54 \mathrm{E}-01$ & $1.54 \mathrm{E}-01$ & $0.00 \%$ \\
\hline Zr 97 & $1.59 \mathrm{E}-04$ & $1.59 \mathrm{E}-04$ & $0.00 \%$ \\
\hline & & & \\
TOTAL* & $\mathbf{3 . 5 2 E + 0 0}$ & $\mathbf{3 . 5 2 E + 0 0}$ & $\mathbf{0 . 0 0 \%}$ \\
\hline & & & \\
\hline & & & \\
\hline & & & \\
\hline
\end{tabular}


SRNL-STI-2010-00447, REVISION 0

Table 44. POPDOSE-SR Collective Plume Immersion Comparisons (person-rem/yr)

\begin{tabular}{|c|c|c|c|}
\hline \multicolumn{4}{|c|}{ Popdose Plume } \\
\hline Nuclide & Hamby & Jannik & \% Diff \\
\hline Ar 41 & $2.44 \mathrm{E}-05$ & $2.44 \mathrm{E}-05$ & $0.00 \%$ \\
\hline $\mathrm{Kr} 83 \mathrm{M}$ & $1.76 \mathrm{E}-09$ & $1.76 \mathrm{E}-09$ & $0.00 \%$ \\
\hline $\mathrm{Kr} 85$ & $6.15 \mathrm{E}-07$ & $6.15 \mathrm{E}-07$ & $0.00 \%$ \\
\hline $\mathrm{Kr} 85 \mathrm{M}$ & $1.28 \mathrm{E}-05$ & $1.28 \mathrm{E}-05$ & $0.00 \%$ \\
\hline $\mathrm{Kr} 87$ & $6.71 \mathrm{E}-06$ & $6.71 \mathrm{E}-06$ & $0.00 \%$ \\
\hline $\mathrm{Kr} 88$ & $1.26 \mathrm{E}-04$ & $1.26 \mathrm{E}-04$ & $0.00 \%$ \\
\hline $\mathrm{Kr} 89$ & $8.85 \mathrm{E}-19$ & $8.85 \mathrm{E}-19$ & $0.00 \%$ \\
\hline Xe131M & $2.30 \mathrm{E}-06$ & $2.30 \mathrm{E}-06$ & $0.00 \%$ \\
\hline Xe133 & $9.20 \mathrm{E}-06$ & $9.20 \mathrm{E}-06$ & $0.00 \%$ \\
\hline Xe133M & $7.28 \mathrm{E}-06$ & $7.28 \mathrm{E}-06$ & $0.00 \%$ \\
\hline Xe135 & $3.59 \mathrm{E}-05$ & $3.59 \mathrm{E}-05$ & $0.00 \%$ \\
\hline Xe135M & $2.84 \mathrm{E}-09$ & $2.84 \mathrm{E}-09$ & $0.00 \%$ \\
\hline Xe137 & $1.60 \mathrm{E}-17$ & $1.60 \mathrm{E}-17$ & $0.00 \%$ \\
\hline Xe138 & $4.82 \mathrm{E}-09$ & $4.82 \mathrm{E}-09$ & $0.00 \%$ \\
\hline *TOTAL* & $\mathbf{2 . 2 8 E - 0 4}$ & $\mathbf{2 . 2 8 E - 0 4}$ & $\mathbf{0 . 0 0 \%}$ \\
\hline
\end{tabular}


Table 45. POPDOSE-SR Collective Inhalation Dose Comparisons (person-rem/yr)

\begin{tabular}{|c|c|c|c|}
\hline \multicolumn{4}{|c|}{ Popdose Inhalation Pathway } \\
\hline Nuclide & Hamby & Jannik & \% Diff \\
\hline Ag110M & $2.61 \mathrm{E}-02$ & $1.79 \mathrm{E}-02$ & $-31.42 \%$ \\
\hline Am241 & $2.56 \mathrm{E}+02$ & $1.76 \mathrm{E}+02$ & $-31.25 \%$ \\
\hline Am243 & $2.56 \mathrm{E}+02$ & $1.76 \mathrm{E}+02$ & $-31.25 \%$ \\
\hline Ba139 & $3.00 \mathrm{E}-06$ & $2.04 \mathrm{E}-06$ & $-32.00 \%$ \\
\hline Ba140 & $1.74 \mathrm{E}-03$ & $1.20 \mathrm{E}-03$ & $-31.03 \%$ \\
\hline Ba141 & $2.79 \mathrm{E}-09$ & $2.20 \mathrm{E}-09$ & $-21.15 \%$ \\
\hline Ba142 & $2.73 \mathrm{E}-11$ & $2.36 \mathrm{E}-11$ & $-13.55 \%$ \\
\hline Be 7 & $1.32 \mathrm{E}-04$ & $9.10 \mathrm{E}-05$ & $-31.06 \%$ \\
\hline Br 83 & $4.91 \mathrm{E}-06$ & $3.35 \mathrm{E}-06$ & $-31.77 \%$ \\
\hline Br 84 & $5.81 \mathrm{E}-08$ & $4.10 \mathrm{E}-08$ & $-29.43 \%$ \\
\hline Ce141 & $4.15 \mathrm{E}-03$ & $2.86 \mathrm{E}-03$ & $-31.08 \%$ \\
\hline Ce143 & $1.32 \mathrm{E}-03$ & $9.08 \mathrm{E}-04$ & $-31.21 \%$ \\
\hline Ce144 & $1.72 \mathrm{E}-01$ & $1.18 \mathrm{E}-01$ & $-31.40 \%$ \\
\hline Cf252 & $6.40 \mathrm{E}+01$ & $4.40 \mathrm{E}+01$ & $-31.25 \%$ \\
\hline Cm242 & $8.35 \mathrm{E}+00$ & $5.75 \mathrm{E}+00$ & $-31.14 \%$ \\
\hline Cm243 & $1.72 \mathrm{E}+02$ & $1.19 \mathrm{E}+02$ & $-30.81 \%$ \\
\hline Cm244 & $1.33 \mathrm{E}+02$ & $9.14 \mathrm{E}+01$ & $-31.28 \%$ \\
\hline Cm246 & $2.66 \mathrm{E}+02$ & $1.83 \mathrm{E}+02$ & $-31.20 \%$ \\
\hline Cm248 & $9.35 \mathrm{E}+02$ & $6.44 \mathrm{E}+02$ & $-31.12 \%$ \\
\hline Co 57 & $3.69 \mathrm{E}-03$ & $2.54 \mathrm{E}-03$ & $-31.17 \%$ \\
\hline Co 58 & $3.48 \mathrm{E}-03$ & $2.40 \mathrm{E}-03$ & $-31.03 \%$ \\
\hline Co 60 & $7.38 \mathrm{E}-02$ & $5.08 \mathrm{E}-02$ & $-31.17 \%$ \\
\hline Cr 51 & $1.27 \mathrm{E}-04$ & $8.73 \mathrm{E}-05$ & $-31.26 \%$ \\
\hline Cs134 & $2.31 \mathrm{E}-02$ & $1.59 \mathrm{E}-02$ & $-31.17 \%$ \\
\hline Cs135 & $2.21 \mathrm{E}-03$ & $1.52 \mathrm{E}-03$ & $-31.22 \%$ \\
\hline Cs137 & $1.57 \mathrm{E}-02$ & $1.08 \mathrm{E}-02$ & $-31.21 \%$ \\
\hline Cs138 & $6.26 \mathrm{E}-08$ & $4.40 \mathrm{E}-08$ & $-29.71 \%$ \\
\hline Eu152 & $1.08 \mathrm{E}-01$ & $7.45 \mathrm{E}-02$ & $-31.02 \%$ \\
\hline Eu154 & $1.28 \mathrm{E}-01$ & $8.81 \mathrm{E}-02$ & $-31.17 \%$ \\
\hline Eu155 & $1.92 \mathrm{E}-02$ & $1.32 \mathrm{E}-02$ & $-31.25 \%$ \\
\hline Fe 55 & $1.28 \mathrm{E}-03$ & $8.80 \mathrm{E}-04$ & $-31.25 \%$ \\
\hline Fe 59 & $7.34 \mathrm{E}-03$ & $5.05 \mathrm{E}-03$ & $-31.20 \%$ \\
\hline H 3 & $8.40 \mathrm{E}-05$ & $5.79 \mathrm{E}-05$ & $-31.07 \%$ \\
\hline Hf181 & $6.36 \mathrm{E}-03$ & $4.38 \mathrm{E}-03$ & $-31.13 \%$ \\
\hline I 129 & $8.86 \mathrm{E}-02$ & $6.10 \mathrm{E}-02$ & $-31.15 \%$ \\
\hline I 131 & $1.53 \mathrm{E}-02$ & $1.05 \mathrm{E}-02$ & $-31.37 \%$ \\
\hline I 132 134 & $1.89 \mathrm{E}-05$ & $1.29 \mathrm{E}-05$ & $-31.75 \%$ \\
\hline I 133 & $2.01 \mathrm{E}-03$ & $1.38 \mathrm{E}-03$ & $-31.34 \%$ \\
\hline $5.38 \mathrm{E}-07$ & $3.67 \mathrm{E}-07$ & $-31.78 \%$ \\
\hline $2.34 \mathrm{E}-04$ & $1.60 \mathrm{E}-04$ & $-31.62 \%$ \\
\hline $1.87 \mathrm{E}-03$ & $1.29 \mathrm{E}-03$ & $-31.02 \%$ \\
\hline & & \\
\hline I 140 & & & \\
\hline
\end{tabular}


SRNL-STI-2010-00447, REVISION 0

Table 45. POPDOSE-SR Collective Inhalation Dose Comparisons (person-rem/yr) (continued)

\begin{tabular}{|c|c|c|c|}
\hline \multicolumn{4}{|c|}{ Popdose Inhalation Pathway } \\
\hline Nuclide & Hamby & Jannik & \% Diff \\
\hline La141 & $6.91 \mathrm{E}-05$ & $4.73 \mathrm{E}-05$ & $-31.55 \%$ \\
\hline La142 & $5.45 \mathrm{E}-06$ & $3.71 \mathrm{E}-06$ & $-31.93 \%$ \\
\hline Mn 54 & $3.15 \mathrm{E}-03$ & $2.17 \mathrm{E}-03$ & $-31.11 \%$ \\
\hline Mn 56 & $2.29 \mathrm{E}-05$ & $1.56 \mathrm{E}-05$ & $-31.88 \%$ \\
\hline Mo 99 & $1.62 \mathrm{E}-03$ & $1.11 \mathrm{E}-03$ & $-31.48 \%$ \\
\hline Na 24 & $3.19 \mathrm{E}-04$ & $2.19 \mathrm{E}-04$ & $-31.35 \%$ \\
\hline Nb 95 & $2.20 \mathrm{E}-03$ & $1.51 \mathrm{E}-03$ & $-31.36 \%$ \\
\hline Nb 97 & $9.30 \mathrm{E}-07$ & $6.33 \mathrm{E}-07$ & $-31.94 \%$ \\
\hline Ni 59 & $6.40 \mathrm{E}-04$ & $4.40 \mathrm{E}-04$ & $-31.25 \%$ \\
\hline Ni 63 & $1.48 \mathrm{E}-03$ & $1.02 \mathrm{E}-03$ & $-31.08 \%$ \\
\hline Ni 65 & $1.41 \mathrm{E}-05$ & $9.63 \mathrm{E}-06$ & $-31.70 \%$ \\
\hline Np237 & $2.41 \mathrm{E}+02$ & $1.66 \mathrm{E}+02$ & $-31.12 \%$ \\
\hline Np238 & $1.36 \mathrm{E}-02$ & $9.35 \mathrm{E}-03$ & $-31.25 \%$ \\
\hline Np239 & $9.76 \mathrm{E}-04$ & $6.71 \mathrm{E}-04$ & $-31.25 \%$ \\
\hline Os185 & $4.87 \mathrm{E}-03$ & $3.38 \mathrm{E}-03$ & $-30.60 \%$ \\
\hline P 32 & $6.24 \mathrm{E}-03$ & $4.33 \mathrm{E}-03$ & $-30.61 \%$ \\
\hline Pa233 & $4.16 \mathrm{E}-03$ & $2.89 \mathrm{E}-03$ & $-30.53 \%$ \\
\hline Pd109 & $3.51 \mathrm{E}-04$ & $2.43 \mathrm{E}-04$ & $-30.77 \%$ \\
\hline Pm147 & $1.66 \mathrm{E}-02$ & $1.15 \mathrm{E}-02$ & $-30.72 \%$ \\
\hline Pm148 & $4.67 \mathrm{E}-03$ & $3.24 \mathrm{E}-03$ & $-30.62 \%$ \\
\hline Pm151 & $6.36 \mathrm{E}-04$ & $4.41 \mathrm{E}-04$ & $-30.66 \%$ \\
\hline Pr144 & $1.30 \mathrm{E}-09$ & $8.98 \mathrm{E}-10$ & $-30.92 \%$ \\
\hline Pu238 & $2.25 \mathrm{E}+02$ & $1.56 \mathrm{E}+02$ & $-30.67 \%$ \\
\hline Pu239 & $2.49 \mathrm{E}+02$ & $1.73 \mathrm{E}+02$ & $-30.52 \%$ \\
\hline Pu240 & $2.49 \mathrm{E}+02$ & $1.73 \mathrm{E}+02$ & $-30.52 \%$ \\
\hline Pu241 & $4.88 \mathrm{E}+00$ & $3.39 \mathrm{E}+00$ & $-30.53 \%$ \\
\hline Pu242 & $2.34 \mathrm{E}+02$ & $1.63 \mathrm{E}+02$ & $-30.34 \%$ \\
\hline Ra224 & $1.32 \mathrm{E}+00$ & $9.18 \mathrm{E}-01$ & $-30.45 \%$ \\
\hline Ra228 & $2.05 \mathrm{E}+00$ & $1.42 \mathrm{E}+00$ & $-30.73 \%$ \\
\hline Rb 86 & $3.18 \mathrm{E}-03$ & $2.21 \mathrm{E}-03$ & $-30.50 \%$ \\
\hline Rb 87 & $1.61 \mathrm{E}-03$ & $1.12 \mathrm{E}-03$ & $-30.43 \%$ \\
\hline Rb 88 & $3.00 \mathrm{E}-09$ & $2.08 \mathrm{E}-09$ & $-30.67 \%$ \\
\hline Rb 89 & $5.16 \mathrm{E}-10$ & $3.58 \mathrm{E}-10$ & $-30.62 \%$ \\
\hline Rh105 & $3.68 \mathrm{E}-04$ & $2.55 \mathrm{E}-04$ & $-30.71 \%$ \\
\hline Rh106M & $1.02 \mathrm{E}-05$ & $7.07 \mathrm{E}-06$ & $-30.69 \%$ \\
\hline Ru103 & $3.79 \mathrm{E}-03$ & $2.63 \mathrm{E}-03$ & $-30.61 \%$ \\
\hline & & & \\
\hline
\end{tabular}


SRNL-STI-2010-00447, REVISION 0

Table 45. POPDOSE-SR Collective Inhalation Dose Comparisons (person-rem/yr) (continued)

\begin{tabular}{|c|c|c|c|}
\hline \multicolumn{4}{|c|}{ Popdose Inhalation Pathway } \\
\hline Nuclide & Hamby & Jannik & \% Diff \\
\hline Ru105 & $5.94 \mathrm{E}-05$ & $4.12 \mathrm{E}-05$ & $-30.64 \%$ \\
\hline Ru106 & $2.15 \mathrm{E}-01$ & $1.49 \mathrm{E}-01$ & $-30.70 \%$ \\
\hline S 35 & $1.12 \mathrm{E}-03$ & $7.77 \mathrm{E}-04$ & $-30.63 \%$ \\
\hline Sb122 & $2.10 \mathrm{E}-03$ & $1.45 \mathrm{E}-03$ & $-30.95 \%$ \\
\hline Sb124 & $1.02 \mathrm{E}-02$ & $7.08 \mathrm{E}-03$ & $-30.59 \%$ \\
\hline Sb125 & $4.78 \mathrm{E}-03$ & $3.32 \mathrm{E}-03$ & $-30.54 \%$ \\
\hline Sb126 & $4.79 \mathrm{E}-03$ & $3.32 \mathrm{E}-03$ & $-30.69 \%$ \\
\hline Sb126M & $1.49 \mathrm{E}-09$ & $1.04 \mathrm{E}-09$ & $-30.20 \%$ \\
\hline Se 75 & $4.00 \mathrm{E}-03$ & $2.77 \mathrm{E}-03$ & $-30.75 \%$ \\
\hline Se 79 & $4.35 \mathrm{E}-03$ & $3.01 \mathrm{E}-03$ & $-30.80 \%$ \\
\hline Sm151 & $1.42 \mathrm{E}-02$ & $9.82 \mathrm{E}-03$ & $-30.85 \%$ \\
\hline Sn123 & $1.46 \mathrm{E}-02$ & $1.01 \mathrm{E}-02$ & $-30.82 \%$ \\
\hline Sn126 & $4.20 \mathrm{E}-02$ & $2.91 \mathrm{E}-02$ & $-30.71 \%$ \\
\hline Sr 89 & $1.80 \mathrm{E}-02$ & $1.25 \mathrm{E}-02$ & $-30.56 \%$ \\
\hline Sr 90 & $6.35 \mathrm{E}-01$ & $4.40 \mathrm{E}-01$ & $-30.71 \%$ \\
\hline Sr 91 & $3.76 \mathrm{E}-04$ & $2.61 \mathrm{E}-04$ & $-30.59 \%$ \\
\hline Sr 92 & $5.75 \mathrm{E}-05$ & $3.99 \mathrm{E}-05$ & $-30.61 \%$ \\
\hline Tc 99 & $3.66 \mathrm{E}-03$ & $2.54 \mathrm{E}-03$ & $-30.60 \%$ \\
\hline Tc 99M & $6.24 \mathrm{E}-06$ & $4.33 \mathrm{E}-06$ & $-30.61 \%$ \\
\hline Tc101 & $1.41 \mathrm{E}-10$ & $9.75 \mathrm{E}-11$ & $-30.85 \%$ \\
\hline Te125M & $3.26 \mathrm{E}-03$ & $2.26 \mathrm{E}-03$ & $-30.67 \%$ \\
\hline Te127 & $7.72 \mathrm{E}-05$ & $5.36 \mathrm{E}-05$ & $-30.57 \%$ \\
\hline Te127M & $9.26 \mathrm{E}-03$ & $6.42 \mathrm{E}-03$ & $-30.67 \%$ \\
\hline Te129 & $8.92 \mathrm{E}-07$ & $6.19 \mathrm{E}-07$ & $-30.61 \%$ \\
\hline Te129M & $9.70 \mathrm{E}-03$ & $6.72 \mathrm{E}-03$ & $-30.72 \%$ \\
\hline Te131 & $2.22 \mathrm{E}-08$ & $1.54 \mathrm{E}-08$ & $-30.63 \%$ \\
\hline Te131M & $2.21 \mathrm{E}-03$ & $1.53 \mathrm{E}-03$ & $-30.77 \%$ \\
\hline Te132 & $3.49 \mathrm{E}-03$ & $2.42 \mathrm{E}-03$ & $-30.66 \%$ \\
\hline Te134 & $2.08 \mathrm{E}-07$ & $1.45 \mathrm{E}-07$ & $-30.29 \%$ \\
\hline Th228 & $1.51 \mathrm{E}+02$ & $1.05 \mathrm{E}+02$ & $-30.46 \%$ \\
\hline Th230 & $1.56 \mathrm{E}+02$ & $1.08 \mathrm{E}+02$ & $-30.77 \%$ \\
\hline Th232 & $7.81 \mathrm{E}+02$ & $5.42 \mathrm{E}+02$ & $-30.60 \%$ \\
\hline Th234 & $1.60 \mathrm{E}-02$ & $1.11 \mathrm{E}-02$ & $-30.63 \%$ \\
\hline & & & \\
\hline
\end{tabular}


SRNL-STI-2010-00447, REVISION 0

Table 45. POPDOSE-SR Collective Inhalation Dose Comparisons (person-rem/yr) (continued)

\begin{tabular}{|c|c|c|c|}
\hline \multicolumn{4}{|c|}{ Popdose Inhalation Pathway } \\
\hline Nuclide & Hamby & Jannik & \% Diff \\
\hline U 232 & $3.27 \mathrm{E}+02$ & $2.27 \mathrm{E}+02$ & $-30.58 \%$ \\
\hline U 233 & $6.35 \mathrm{E}+01$ & $4.40 \mathrm{E}+01$ & $-30.71 \%$ \\
\hline U 234 & $6.35 \mathrm{E}+01$ & $4.40 \mathrm{E}+01$ & $-30.71 \%$ \\
\hline U 235 & $5.86 \mathrm{E}+01$ & $4.06 \mathrm{E}+01$ & $-30.72 \%$ \\
\hline U 236 & $5.86 \mathrm{E}+01$ & $4.06 \mathrm{E}+01$ & $-30.72 \%$ \\
\hline U 237 & $1.55 \mathrm{E}-03$ & $1.08 \mathrm{E}-03$ & $-30.32 \%$ \\
\hline U 238 & $5.86 \mathrm{E}+01$ & $4.06 \mathrm{E}+01$ & $-30.72 \%$ \\
\hline W 187 & $2.03 \mathrm{E}-04$ & $1.41 \mathrm{E}-04$ & $-30.54 \%$ \\
\hline Y 90 & $3.65 \mathrm{E}-03$ & $2.53 \mathrm{E}-03$ & $-30.68 \%$ \\
\hline Y 91 & $2.14 \mathrm{E}-02$ & $1.48 \mathrm{E}-02$ & $-30.84 \%$ \\
\hline Y 91M & $1.24 \mathrm{E}-07$ & $8.61 \mathrm{E}-08$ & $-30.56 \%$ \\
\hline Y 92 & $6.87 \mathrm{E}-05$ & $4.76 \mathrm{E}-05$ & $-30.71 \%$ \\
\hline Y 93 & $5.83 \mathrm{E}-04$ & $4.05 \mathrm{E}-04$ & $-30.53 \%$ \\
\hline Zn 65 & $8.78 \mathrm{E}-03$ & $6.09 \mathrm{E}-03$ & $-30.64 \%$ \\
\hline Zr 93 & $1.56 \mathrm{E}-01$ & $1.08 \mathrm{E}-01$ & $-30.77 \%$ \\
\hline Zr 95 & $9.24 \mathrm{E}-03$ & $6.41 \mathrm{E}-03$ & $-30.63 \%$ \\
\hline Zr 97 & $1.39 \mathrm{E}-03$ & $9.62 \mathrm{E}-04$ & $-30.79 \%$ \\
\hline *TOTAL* & $\mathbf{5 . 0 2 E}+03$ & $\mathbf{3 . 4 6 E}+\mathbf{0 3}$ & $\mathbf{- 3 1 . 0 8 \%}$ \\
\hline
\end{tabular}


SRNL-STI-2010-00447, REVISION 0

Table 46. POPDOSE-SR Collective Vegetable Ingestion Comparisons (person-rem/yr)

\begin{tabular}{|c|c|c|c|}
\hline \multicolumn{4}{|c|}{ Popdose Vegetation Pathway } \\
\hline Nuclide & Hamby & Jannik & $\mathbf{\% ~ D i f f ~}$ \\
\hline Ag110M & $8.95 \mathrm{E}-02$ & $3.79 \mathrm{E}-03$ & $-95.77 \%$ \\
\hline Am241 & $3.93 \mathrm{E}+01$ & $1.66 \mathrm{E}+00$ & $-95.78 \%$ \\
\hline Am243 & $3.93 \mathrm{E}+01$ & $1.66 \mathrm{E}+00$ & $-95.78 \%$ \\
\hline Ba140 & $1.66 \mathrm{E}-02$ & $1.08 \mathrm{E}-03$ & $-93.49 \%$ \\
\hline Be 7 & $6.43 \mathrm{E}-04$ & $3.00 \mathrm{E}-05$ & $-95.33 \%$ \\
\hline C 14 & $9.45 \mathrm{E}-03$ & $1.27 \mathrm{E}-03$ & $-86.56 \%$ \\
\hline Ce141 & $1.20 \mathrm{E}-02$ & $5.98 \mathrm{E}-04$ & $-95.02 \%$ \\
\hline Ce143 & $2.51 \mathrm{E}-06$ & $5.80 \mathrm{E}-06$ & $131.08 \%$ \\
\hline Ce144 & $1.62 \mathrm{E}-01$ & $6.94 \mathrm{E}-03$ & $-95.72 \%$ \\
\hline Cf252 & $8.03 \mathrm{E}+00$ & $3.40 \mathrm{E}-01$ & $-95.77 \%$ \\
\hline Cm242 & $8.40 \mathrm{E}-01$ & $3.66 \mathrm{E}-02$ & $-95.64 \%$ \\
\hline Cm243 & $2.53 \mathrm{E}+01$ & $1.07 \mathrm{E}+00$ & $-95.77 \%$ \\
\hline Cm244 & $2.00 \mathrm{E}+01$ & $8.44 \mathrm{E}-01$ & $-95.78 \%$ \\
\hline Cm246 & $3.93 \mathrm{E}+01$ & $1.66 \mathrm{E}+00$ & $-95.78 \%$ \\
\hline Cm248 & $1.40 \mathrm{E}+02$ & $5.89 \mathrm{E}+00$ & $-95.79 \%$ \\
\hline Co 57 & $8.87 \mathrm{E}-03$ & $3.84 \mathrm{E}-04$ & $-95.67 \%$ \\
\hline Co 58 & $2.26 \mathrm{E}-02$ & $1.03 \mathrm{E}-03$ & $-95.44 \%$ \\
\hline Co 60 & $2.25 \mathrm{E}-01$ & $9.58 \mathrm{E}-03$ & $-95.74 \%$ \\
\hline Cr 51 & $5.42 \mathrm{E}-04$ & $2.78 \mathrm{E}-05$ & $-94.87 \%$ \\
\hline Cs134 & $6.28 \mathrm{E}-01$ & $2.67 \mathrm{E}-02$ & $-95.75 \%$ \\
\hline Cs135 & $6.21 \mathrm{E}-02$ & $2.62 \mathrm{E}-03$ & $-95.78 \%$ \\
\hline Cs137 & $4.37 \mathrm{E}-01$ & $1.84 \mathrm{E}-02$ & $-95.79 \%$ \\
\hline Eu152 & $5.22 \mathrm{E}-02$ & $2.20 \mathrm{E}-03$ & $-95.79 \%$ \\
\hline Eu154 & $7.90 \mathrm{E}-02$ & $3.34 \mathrm{E}-03$ & $-95.77 \%$ \\
\hline Eu155 & $1.12 \mathrm{E}-02$ & $4.75 \mathrm{E}-04$ & $-95.76 \%$ \\
\hline Fe 55 & $4.95 \mathrm{E}-03$ & $2.11 \mathrm{E}-04$ & $-95.74 \%$ \\
\hline Fe 59 & $3.58 \mathrm{E}-02$ & $1.71 \mathrm{E}-03$ & $-95.22 \%$ \\
\hline H 3 & $1.36 \mathrm{E}-05$ & $1.82 \mathrm{E}-06$ & $-86.62 \%$ \\
\hline Hf181 & $2.28 \mathrm{E}-02$ & $1.10 \mathrm{E}-03$ & $-95.18 \%$ \\
\hline I 129 & $1.22 \mathrm{E}+01$ & $5.16 \mathrm{E}-01$ & $-95.77 \%$ \\
\hline I 131 & $2.53 \mathrm{E}-01$ & $2.12 \mathrm{E}-02$ & $-91.62 \%$ \\
\hline I 133 & $2.73 \mathrm{E}-07$ & $6.74 \mathrm{E}-06$ & $2368.86 \%$ \\
\hline I 135 & $0.00 \mathrm{E}+00$ & $0.00 \mathrm{E}+00$ & $0.00 \%$ \\
\hline La140 & $1.97 \mathrm{E}-05$ & $2.24 \mathrm{E}-05$ & $13.71 \%$ \\
\hline La141 & $0.00 \mathrm{E}+00$ & $0.00 \mathrm{E}+00$ & $0.00 \%$ \\
\hline Mn 54 & $2.20 \mathrm{E}-02$ & $9.64 \mathrm{E}-04$ & $-95.62 \%$ \\
\hline Mn 56 & $0.00 \mathrm{E}+00$ & $0.00 \mathrm{E}+00$ & $0.00 \%$ \\
\hline Mo 99 & $1.15 \mathrm{E}-04$ & $3.62 \mathrm{E}-05$ & $-68.52 \%$ \\
\hline Na 24 & $6.88 \mathrm{E}-11$ & $1.96 \mathrm{E}-08$ & $28388.37 \%$ \\
\hline
\end{tabular}


Table 46. POPDOSE-SR Collective Vegetable Ingestion Comparisons (person-rem/yr) (continued)

\begin{tabular}{|c|c|c|c|}
\hline \multicolumn{5}{|c|}{ Popdose Vegetation Pathway } \\
\hline Nuclide & Hamby & Jannik & \% Diff \\
\hline Nb 95 & $1.06 \mathrm{E}-02$ & $5.22 \mathrm{E}-04$ & $-95.08 \%$ \\
\hline Ni 59 & $1.75 \mathrm{E}-03$ & $7.43 \mathrm{E}-05$ & $-95.75 \%$ \\
\hline Ni 63 & $4.73 \mathrm{E}-03$ & $2.00 \mathrm{E}-04$ & $-95.77 \%$ \\
\hline Ni 65 & $0.00 \mathrm{E}+00$ & $0.00 \mathrm{E}+00$ & $0.00 \%$ \\
\hline Np237 & $3.41 \mathrm{E}+01$ & $1.44 \mathrm{E}+00$ & $-95.78 \%$ \\
\hline Np238 & $3.65 \mathrm{E}-05$ & $2.09 \mathrm{E}-05$ & $-42.74 \%$ \\
\hline Np239 & $5.54 \mathrm{E}-05$ & $2.43 \mathrm{E}-05$ & $-56.14 \%$ \\
\hline Os185 & $1.46 \mathrm{E}-02$ & $6.51 \mathrm{E}-04$ & $-95.54 \%$ \\
\hline P 32 & $1.80 \mathrm{E}-02$ & $1.10 \mathrm{E}-03$ & $-93.89 \%$ \\
\hline Pa233 & $1.35 \mathrm{E}-02$ & $6.97 \mathrm{E}-04$ & $-94.84 \%$ \\
\hline Pd109 & $1.55 \mathrm{E}-11$ & $1.16 \mathrm{E}-08$ & $74738.71 \%$ \\
\hline Pm147 & $8.11 \mathrm{E}-03$ & $3.47 \mathrm{E}-04$ & $-95.72 \%$ \\
\hline Pm148 & $3.74 \mathrm{E}-03$ & $4.41 \mathrm{E}-04$ & $-88.21 \%$ \\
\hline Pm151 & $4.25 \mathrm{E}-07$ & $1.94 \mathrm{E}-06$ & $356.47 \%$ \\
\hline Pu238 & $3.32 \mathrm{E}+01$ & $1.40 \mathrm{E}+00$ & $-95.78 \%$ \\
\hline Pu239 & $3.76 \mathrm{E}+01$ & $1.58 \mathrm{E}+00$ & $-95.80 \%$ \\
\hline Pu240 & $3.76 \mathrm{E}+01$ & $1.58 \mathrm{E}+00$ & $-95.80 \%$ \\
\hline Pu241 & $7.48 \mathrm{E}-01$ & $3.15 \mathrm{E}-02$ & $-95.79 \%$ \\
\hline Pu242 & $3.58 \mathrm{E}+01$ & $1.51 \mathrm{E}+00$ & $-95.78 \%$ \\
\hline Ra224 & $4.07 \mathrm{E}-02$ & $7.76 \mathrm{E}-03$ & $-80.93 \%$ \\
\hline Ra228 & $1.04 \mathrm{E}+01$ & $4.40 \mathrm{E}-01$ & $-95.77 \%$ \\
\hline Rb 86 & $2.85 \mathrm{E}-02$ & $1.63 \mathrm{E}-03$ & $-94.28 \%$ \\
\hline Rb 87 & $4.27 \mathrm{E}-02$ & $1.87 \mathrm{E}-03$ & $-95.62 \%$ \\
\hline Rh105 & $1.65 \mathrm{E}-06$ & $2.50 \mathrm{E}-06$ & $51.52 \%$ \\
\hline Ru103 & $1.39 \mathrm{E}-02$ & $6.73 \mathrm{E}-04$ & $-95.16 \%$ \\
\hline Ru105 & $0.00 \mathrm{E}+00$ & $0.00 \mathrm{E}+00$ & $0.00 \%$ \\
\hline Ru106 & $1.74 \mathrm{E}-01$ & $7.40 \mathrm{E}-03$ & $-95.75 \%$ \\
\hline S 35 & $4.64 \mathrm{E}-03$ & $2.13 \mathrm{E}-04$ & $-95.41 \%$ \\
\hline Sb122 & $2.34 \mathrm{E}-04$ & $7.57 \mathrm{E}-05$ & $-67.65 \%$ \\
\hline Sb124 & $5.70 \mathrm{E}-02$ & $2.63 \mathrm{E}-03$ & $-95.39 \%$ \\
\hline Sb125 & $2.23 \mathrm{E}-02$ & $9.41 \mathrm{E}-04$ & $-95.78 \%$ \\
\hline Sb126 & $1.82 \mathrm{E}-02$ & $1.20 \mathrm{E}-03$ & $-93.41 \%$ \\
\hline Se 75 & $7.18 \mathrm{E}-02$ & $2.84 \mathrm{E}-03$ & $-96.04 \%$ \\
\hline Se 79 & $8.54 \mathrm{E}-02$ & $3.08 \mathrm{E}-03$ & $-96.39 \%$ \\
\hline
\end{tabular}


Table 46. POPDOSE-SR Collective Vegetable Ingestion Comparisons (person-rem/yr) (continued)

\begin{tabular}{|c|c|c|c|}
\hline \multicolumn{4}{|c|}{ Popdose Vegetation Pathway } \\
\hline Nuclide & Hamby & Jannik & $\mathbf{\% ~ D i f f ~}$ \\
\hline Sm151 & $2.97 \mathrm{E}-03$ & $1.25 \mathrm{E}-04$ & $-95.79 \%$ \\
\hline Sn123 & $5.68 \mathrm{E}-02$ & $2.50 \mathrm{E}-03$ & $-95.60 \%$ \\
\hline Sn126 & $1.49 \mathrm{E}-01$ & $6.26 \mathrm{E}-03$ & $-95.80 \%$ \\
\hline Sr 89 & $4.99 \mathrm{E}-02$ & $2.40 \mathrm{E}-03$ & $-95.19 \%$ \\
\hline Sr 90 & $1.14 \mathrm{E}+00$ & $5.02 \mathrm{E}-02$ & $-95.60 \%$ \\
\hline Sr 91 & $0.00 \mathrm{E}+00$ & $0.00 \mathrm{E}+00$ & $0.00 \%$ \\
\hline Sr 92 & $0.00 \mathrm{E}+00$ & $0.00 \mathrm{E}+00$ & $0.00 \%$ \\
\hline Tc 99 & $1.17 \mathrm{E}-02$ & $4.17 \mathrm{E}-03$ & $-64.36 \%$ \\
\hline Tc 99M & $0.00 \mathrm{E}+00$ & $0.00 \mathrm{E}+00$ & $0.00 \%$ \\
\hline Te125M & $2.23 \mathrm{E}-02$ & $9.63 \mathrm{E}-04$ & $-95.68 \%$ \\
\hline Te127 & $0.00 \mathrm{E}+00$ & $0.00 \mathrm{E}+00$ & $0.00 \%$ \\
\hline Te127M & $6.31 \mathrm{E}-02$ & $2.54 \mathrm{E}-03$ & $-95.97 \%$ \\
\hline Te129M & $4.94 \mathrm{E}-02$ & $2.33 \mathrm{E}-03$ & $-95.28 \%$ \\
\hline Te131M & $3.92 \mathrm{E}-06$ & $1.35 \mathrm{E}-05$ & $244.39 \%$ \\
\hline Te132 & $6.09 \mathrm{E}-04$ & $1.37 \mathrm{E}-04$ & $-77.50 \%$ \\
\hline Th228 & $3.22 \mathrm{E}+00$ & $1.37 \mathrm{E}-01$ & $-95.75 \%$ \\
\hline Th230 & $4.63 \mathrm{E}+00$ & $1.95 \mathrm{E}-01$ & $-95.79 \%$ \\
\hline Th232 & $2.45 \mathrm{E}+01$ & $1.03 \mathrm{E}+00$ & $-95.80 \%$ \\
\hline Th234 & $4.89 \mathrm{E}-02$ & $2.59 \mathrm{E}-03$ & $-94.70 \%$ \\
\hline U 232 & $1.14 \mathrm{E}+01$ & $4.79 \mathrm{E}-01$ & $-95.80 \%$ \\
\hline U 233 & $2.36 \mathrm{E}+00$ & $9.96 \mathrm{E}-02$ & $-95.78 \%$ \\
\hline U 234 & $2.27 \mathrm{E}+00$ & $9.59 \mathrm{E}-02$ & $-95.78 \%$ \\
\hline U 235 & $2.19 \mathrm{E}+00$ & $9.22 \mathrm{E}-02$ & $-95.79 \%$ \\
\hline U 236 & $2.19 \mathrm{E}+00$ & $9.22 \mathrm{E}-02$ & $-95.79 \%$ \\
\hline U 237 & $1.81 \mathrm{E}-03$ & $1.73 \mathrm{E}-04$ & $-90.44 \%$ \\
\hline U 238 & $2.01 \mathrm{E}+00$ & $8.49 \mathrm{E}-02$ & $-95.78 \%$ \\
\hline W 187 & $7.15 \mathrm{E}-08$ & $7.74 \mathrm{E}-07$ & $982.52 \%$ \\
\hline Y 90 & $3.49 \mathrm{E}-04$ & $1.16 \mathrm{E}-04$ & $-66.76 \%$ \\
\hline Y 91 & $5.39 \mathrm{E}-02$ & $2.49 \mathrm{E}-03$ & $-95.38 \%$ \\
\hline Y 92 & $0.00 \mathrm{E}+00$ & $0.00 \mathrm{E}+00$ & $0.00 \%$ \\
\hline Y 93 & $0.00 \mathrm{E}+00$ & $1.35 \mathrm{E}-09$ & \\
\hline Zn 65 & $1.17 \mathrm{E}-01$ & $5.10 \mathrm{E}-03$ & $-95.64 \%$ \\
\hline Zr 93 & $1.40 \mathrm{E}-02$ & $5.89 \mathrm{E}-04$ & $-95.79 \%$ \\
\hline Zr 95 & $2.14 \mathrm{E}-02$ & $9.81 \mathrm{E}-04$ & $-95.42 \%$ \\
\hline Zr 97 & $2.49 \mathrm{E}-09$ & $2.71 \mathrm{E}-07$ & $10783.53 \%$ \\
\hline *TOTAL* & $\mathbf{5 . 7 3 E + 0 2}$ & $\mathbf{2 . 4 2 E + 0 1}$ & $-95.78 \%$ \\
\hline
\end{tabular}


Table 47. POPDOSE-SR Collective Milk Ingestion Comparisons (person-rem/yr)

\begin{tabular}{|c|c|c|c|}
\hline \multicolumn{4}{|c|}{ Popdose Milk Pathway } \\
\hline Nuclide & Hamby & Jannik & $\%$ Diff \\
\hline Ag110M & $1.57 \mathrm{E}-01$ & $6.97 \mathrm{E}-03$ & $-95.56 \%$ \\
\hline Am241 & $7.82 \mathrm{E}-03$ & $8.26 \mathrm{E}-04$ & $-89.44 \%$ \\
\hline Am243 & $7.82 \mathrm{E}-03$ & $8.26 \mathrm{E}-04$ & $-89.44 \%$ \\
\hline Ba139 & $2.99 \mathrm{E}-25$ & $6.39 \mathrm{E}-25$ & $113.71 \%$ \\
\hline Ba140 & $1.65 \mathrm{E}-04$ & $2.20 \mathrm{E}-04$ & $33.33 \%$ \\
\hline Be 7 & $1.55 \mathrm{E}-06$ & $2.75 \mathrm{E}-08$ & $-98.23 \%$ \\
\hline $\mathrm{Br} 83$ & $2.53 \mathrm{E}-16$ & $4.63 \mathrm{E}-16$ & $83.00 \%$ \\
\hline C 14 & 7.33E-03 & $8.50 \mathrm{E}-03$ & $15.96 \%$ \\
\hline Ce141 & $1.52 \mathrm{E}-04$ & $1.33 \mathrm{E}-05$ & $-91.25 \%$ \\
\hline Ce143 & $5.18 \mathrm{E}-06$ & 5.99E-07 & $-88.44 \%$ \\
\hline Ce144 & $3.42 \mathrm{E}-03$ & $1.60 \mathrm{E}-04$ & $-95.32 \%$ \\
\hline Cf252 & $1.54 \mathrm{E}-03$ & $6.00 \mathrm{E}-04$ & $-61.04 \%$ \\
\hline $\mathrm{Cm} 242$ & $1.36 \mathrm{E}-04$ & 8.31E-04 & $511.03 \%$ \\
\hline $\mathrm{Cm} 243$ & $5.02 \mathrm{E}-03$ & $2.53 \mathrm{E}-02$ & $403.98 \%$ \\
\hline $\mathrm{Cm} 244$ & $3.96 \mathrm{E}-03$ & $2.00 \mathrm{E}-02$ & $405.05 \%$ \\
\hline $\mathrm{Cm} 246$ & $7.83 \mathrm{E}-03$ & $3.93 \mathrm{E}-02$ & $401.92 \%$ \\
\hline $\mathrm{Cm} 248$ & $2.78 \mathrm{E}-02$ & $1.40 \mathrm{E}-01$ & $403.60 \%$ \\
\hline Co 57 & $3.11 \mathrm{E}-04$ & $4.85 \mathrm{E}-05$ & $-84.41 \%$ \\
\hline Co 58 & $6.00 \mathrm{E}-04$ & $1.26 \mathrm{E}-04$ & $-79.00 \%$ \\
\hline Co 60 & $8.79 \mathrm{E}-03$ & $1.24 \mathrm{E}-03$ & $-85.89 \%$ \\
\hline Cr 51 & $2.46 \mathrm{E}-05$ & $1.35 \mathrm{E}-05$ & $-45.12 \%$ \\
\hline Cs134 & $2.87 \mathrm{E}-01$ & $1.44 \mathrm{E}-01$ & $-49.83 \%$ \\
\hline Cs135 & $2.97 \mathrm{E}-02$ & $1.43 \mathrm{E}-02$ & $-51.85 \%$ \\
\hline Cs137 & $2.08 \mathrm{E}-01$ & $1.00 \mathrm{E}-01$ & $-51.92 \%$ \\
\hline Eu152 & $1.03 \mathrm{E}-05$ & $7.83 \mathrm{E}-05$ & $660.19 \%$ \\
\hline Eu154 & $1.55 \mathrm{E}-05$ & $1.18 \mathrm{E}-04$ & $661.29 \%$ \\
\hline Eu155 & $2.19 \mathrm{E}-06$ & $1.68 \mathrm{E}-05$ & $667.12 \%$ \\
\hline Fe 55 & $2.28 \mathrm{E}-04$ & $8.66 \mathrm{E}-06$ & $-96.20 \%$ \\
\hline Fe 59 & $9.83 \mathrm{E}-04$ & $6.60 \mathrm{E}-05$ & $-93.29 \%$ \\
\hline H 3 & $8.77 \mathrm{E}-06$ & $1.53 \mathrm{E}-05$ & $74.46 \%$ \\
\hline Hf181 & $2.57 \mathrm{E}-06$ & $6.65 \mathrm{E}-07$ & $-74.12 \%$ \\
\hline I 129 & $2.92 \mathrm{E}+00$ & $3.31 \mathrm{E}+00$ & $13.36 \%$ \\
\hline I 131 & 5.41E-02 & $1.65 \mathrm{E}-01$ & $204.99 \%$ \\
\hline I 132 & $4.34 \mathrm{E}-16$ & $1.85 \mathrm{E}-15$ & $326.27 \%$ \\
\hline I 133 & $1.45 \mathrm{E}-04$ & $4.61 \mathrm{E}-04$ & $217.93 \%$ \\
\hline I 134 & $1.42 \mathrm{E}-33$ & $7.41 \mathrm{E}-33$ & $421.83 \%$ \\
\hline I 135 & $2.78 \mathrm{E}-08$ & $9.67 \mathrm{E}-08$ & $247.84 \%$ \\
\hline La140 & $1.28 \mathrm{E}-07$ & $1.77 \mathrm{E}-06$ & $1282.81 \%$ \\
\hline La141 & $6.46 \mathrm{E}-15$ & $1.08 \mathrm{E}-13$ & $1571.83 \%$ \\
\hline
\end{tabular}


Table 47. POPDOSE-SR Collective Milk Ingestion Comparisons (person-rem/yr) (continued)

\begin{tabular}{|l|l|l|l|}
\hline \multicolumn{5}{|c|}{ Popdose Milk Pathway } \\
\hline Nuclide & Hamby & Jannik & \% Diff \\
\hline La142 & $4.56 \mathrm{E}-25$ & $9.50 \mathrm{E}-24$ & $1983.33 \%$ \\
\hline Mn 54 & $1.97 \mathrm{E}-04$ & $4.52 \mathrm{E}-05$ & $-77.06 \%$ \\
\hline Mn 56 & $8.04 \mathrm{E}-17$ & $6.06 \mathrm{E}-17$ & $-24.63 \%$ \\
\hline Mo 99 & $1.93 \mathrm{E}-04$ & $9.64 \mathrm{E}-05$ & $-50.05 \%$ \\
\hline Na 24 & $7.03 \mathrm{E}-06$ & $8.17 \mathrm{E}-06$ & $16.22 \%$ \\
\hline Nb 95 & $5.67 \mathrm{E}-04$ & $2.38 \mathrm{E}-07$ & $-99.96 \%$ \\
\hline Nb 97 & $3.57 \mathrm{E}-27$ & $3.22 \mathrm{E}-30$ & $-99.91 \%$ \\
\hline Ni 59 & $4.68 \mathrm{E}-04$ & $8.34 \mathrm{E}-05$ & $-82.18 \%$ \\
\hline Ni 63 & $1.26 \mathrm{E}-03$ & $2.25 \mathrm{E}-04$ & $-82.14 \%$ \\
\hline Ni 65 & $9.10 \mathrm{E}-16$ & $5.96 \mathrm{E}-16$ & $-34.51 \%$ \\
\hline Np237 & $6.78 \mathrm{E}-03$ & $8.53 \mathrm{E}-03$ & $25.81 \%$ \\
\hline Np238 & $9.28 \mathrm{E}-08$ & $3.19 \mathrm{E}-07$ & $243.75 \%$ \\
\hline Np239 & $9.75 \mathrm{E}-08$ & $3.35 \mathrm{E}-07$ & $243.59 \%$ \\
\hline Os185 & $2.13 \mathrm{E}-03$ & $3.62 \mathrm{E}-03$ & $69.95 \%$ \\
\hline P 32 & $1.12 \mathrm{E}-02$ & $2.72 \mathrm{E}-02$ & $142.86 \%$ \\
\hline Pa233 & $1.40 \mathrm{E}-06$ & $3.94 \mathrm{E}-06$ & $181.43 \%$ \\
\hline Pd109 & $1.84 \mathrm{E}-06$ & $5.50 \mathrm{E}-06$ & $198.91 \%$ \\
\hline Pm147 & $1.58 \mathrm{E}-06$ & $1.22 \mathrm{E}-05$ & $672.15 \%$ \\
\hline Pm148 & $1.09 \mathrm{E}-06$ & $2.20 \mathrm{E}-05$ & $1918.35 \%$ \\
\hline Pm151 & $1.88 \mathrm{E}-08$ & $3.89 \mathrm{E}-07$ & $1969.15 \%$ \\
\hline Pu238 & $2.67 \mathrm{E}-03$ & $1.66 \mathrm{E}-02$ & $521.72 \%$ \\
\hline Pu239 & $3.03 \mathrm{E}-03$ & $1.88 \mathrm{E}-02$ & $520.46 \%$ \\
\hline Pu240 & $3.03 \mathrm{E}-03$ & $1.88 \mathrm{E}-02$ & $520.46 \%$ \\
\hline Pu241 & $5.99 \mathrm{E}-05$ & $3.74 \mathrm{E}-04$ & $524.37 \%$ \\
\hline Pu242 & $2.89 \mathrm{E}-03$ & $1.79 \mathrm{E}-02$ & $519.38 \%$ \\
\hline Ra224 & $3.69 \mathrm{E}-02$ & $5.91 \mathrm{E}-03$ & $-83.98 \%$ \\
\hline Ra228 & $3.29 \mathrm{E}+00$ & $1.97 \mathrm{E}-01$ & $-94.01 \%$ \\
\hline Rb 86 & $1.84 \mathrm{E}-02$ & $2.30 \mathrm{E}-02$ & $25.00 \%$ \\
\hline Rb 87 & $5.22 \mathrm{E}-02$ & $2.60 \mathrm{E}-02$ & $-50.19 \%$ \\
\hline Rh105 & $5.26 \mathrm{E}-05$ & $1.17 \mathrm{E}-04$ & $122.43 \%$ \\
\hline Rh106M & $6.00 \mathrm{E}-17$ & $1.91 \mathrm{E}-16$ & $218.33 \%$ \\
\hline Ru103 & $3.13 \mathrm{E}-07$ & $6.98 \mathrm{E}-06$ & $2130.03 \%$ \\
\hline Ru105 & $5.16 \mathrm{E}-15$ & $1.96 \mathrm{E}-13$ & $3698.45 \%$ \\
\hline Ru106 & $6.40 \mathrm{E}-06$ & $8.07 \mathrm{E}-05$ & $1160.94 \%$ \\
\hline S 35 & $2.48 \mathrm{E}-03$ & $1.80 \mathrm{E}-03$ & $-27.42 \%$ \\
\hline Sb122 & $8.24 \mathrm{E}-05$ & $7.06 \mathrm{E}-06$ & $-91.43 \%$ \\
\hline Sb124 & $2.17 \mathrm{E}-03$ & $1.10 \mathrm{E}-04$ & $-94.93 \%$ \\
\hline
\end{tabular}


Table 47. POPDOSE-SR Collective Milk Ingestion Comparisons (person-rem/yr) (continued)

\begin{tabular}{|c|c|c|c|}
\hline \multicolumn{4}{|c|}{ Popdose } \\
\hline \multicolumn{4}{|c|}{ Milk Pathway } \\
\hline Nuclide & Hamby & Jannik & $\%$ Diff \\
\hline Sb125 & $1.30 \mathrm{E}-03$ & $4.21 \mathrm{E}-05$ & $-96.76 \%$ \\
\hline Sb126 & $7.00 \mathrm{E}-04$ & $5.86 \mathrm{E}-05$ & $-91.63 \%$ \\
\hline Se 75 & $1.09 \mathrm{E}-01$ & $1.27 \mathrm{E}-02$ & $-88.35 \%$ \\
\hline Se 79 & $1.69 \mathrm{E}-01$ & $1.46 \mathrm{E}-02$ & $-91.36 \%$ \\
\hline Sm151 & $5.98 \mathrm{E}-07$ & $4.46 \mathrm{E}-06$ & $645.82 \%$ \\
\hline Sn123 & $4.45 \mathrm{E}-03$ & $2.81 \mathrm{E}-03$ & $-36.85 \%$ \\
\hline Sn126 & $1.50 \mathrm{E}-02$ & $7.43 \mathrm{E}-03$ & $-50.47 \%$ \\
\hline Sr 89 & $9.60 \mathrm{E}-04$ & $3.38 \mathrm{E}-03$ & $252.08 \%$ \\
\hline Sr 90 & $3.65 \mathrm{E}-02$ & $7.57 \mathrm{E}-02$ & $107.40 \%$ \\
\hline Sr 91 & $2.16 \mathrm{E}-08$ & $1.29 \mathrm{E}-07$ & $497.22 \%$ \\
\hline Sr 92 & $1.72 \mathrm{E}-15$ & $1.26 \mathrm{E}-14$ & $632.56 \%$ \\
\hline Тc 99 & $1.21 \mathrm{E}-02$ & $5.59 \mathrm{E}-03$ & $-53.80 \%$ \\
\hline Tc 99M & $2.88 \mathrm{E}-10$ & $1.06 \mathrm{E}-10$ & $-63.19 \%$ \\
\hline Te125M & $6.12 \mathrm{E}-04$ & $3.58 \mathrm{E}-04$ & $-41.50 \%$ \\
\hline Te127 & 5.89E-09 & $7.01 \mathrm{E}-09$ & $19.02 \%$ \\
\hline Te127M & $2.08 \mathrm{E}-03$ & $9.61 \mathrm{E}-04$ & $-53.80 \%$ \\
\hline Te129 & $2.26 \mathrm{E}-28$ & $4.01 \mathrm{E}-28$ & $77.43 \%$ \\
\hline Te129M & $1.15 \mathrm{E}-03$ & $8.77 \mathrm{E}-04$ & $-23.74 \%$ \\
\hline Te131M & $2.51 \mathrm{E}-05$ & $2.78 \mathrm{E}-05$ & $10.76 \%$ \\
\hline Te132 & $9.23 \mathrm{E}-05$ & $1.00 \mathrm{E}-04$ & $8.34 \%$ \\
\hline Te134 & $5.97 \mathrm{E}-42$ & $1.15 \mathrm{E}-41$ & $92.63 \%$ \\
\hline Th228 & $6.16 \mathrm{E}-04$ & $8.00 \mathrm{E}-04$ & $29.87 \%$ \\
\hline Th230 & $9.34 \mathrm{E}-04$ & $1.16 \mathrm{E}-03$ & $24.20 \%$ \\
\hline Th232 & $4.93 \mathrm{E}-03$ & $6.12 \mathrm{E}-03$ & $24.14 \%$ \\
\hline Th234 & $5.07 \mathrm{E}-06$ & $1.48 \mathrm{E}-05$ & $191.91 \%$ \\
\hline U 232 & $2.28 \mathrm{E}-01$ & $1.02 \mathrm{E}+00$ & $347.37 \%$ \\
\hline U 233 & $4.75 \mathrm{E}-02$ & $2.13 \mathrm{E}-01$ & $348.42 \%$ \\
\hline U 234 & $4.58 \mathrm{E}-02$ & $2.05 \mathrm{E}-01$ & $347.60 \%$ \\
\hline U 235 & $4.40 \mathrm{E}-02$ & $1.97 \mathrm{E}-01$ & $347.73 \%$ \\
\hline U 236 & 4.40E-02 & $1.97 \mathrm{E}-01$ & $347.73 \%$ \\
\hline U 237 & $3.94 \mathrm{E}-05$ & $4.76 \mathrm{E}-04$ & $1108.12 \%$ \\
\hline U 238 & $4.05 \mathrm{E}-02$ & $1.81 \mathrm{E}-01$ & $346.91 \%$ \\
\hline W 187 & $1.04 \mathrm{E}-06$ & $1.36 \mathrm{E}-06$ & $30.77 \%$ \\
\hline Y 90 & $8.52 \mathrm{E}-07$ & $5.76 \mathrm{E}-06$ & $576.06 \%$ \\
\hline Y 91 & $1.36 \mathrm{E}-05$ & $5.50 \mathrm{E}-05$ & $304.41 \%$ \\
\hline Y 91M & $3.32 \mathrm{E}-39$ & $3.84 \mathrm{E}-38$ & $1056.63 \%$ \\
\hline Y 92 & $3.48 \mathrm{E}-15$ & $2.94 \mathrm{E}-14$ & $744.83 \%$ \\
\hline Y 93 & $6.11 \mathrm{E}-10$ & 4.45E-09 & $628.31 \%$ \\
\hline Zn 65 & $1.64 \mathrm{E}-01$ & $1.54 \mathrm{E}-02$ & $-90.61 \%$ \\
\hline Zr 93 & $2.82 \mathrm{E}-06$ & $2.52 \mathrm{E}-06$ & $-10.64 \%$ \\
\hline Zr 95 & $2.80 \mathrm{E}-06$ & $3.90 \mathrm{E}-06$ & $39.29 \%$ \\
\hline Zr 97 & $8.55 \mathrm{E}-09$ & $2.17 \mathrm{E}-08$ & $153.80 \%$ \\
\hline *TOTAL* & $8.15 E+00$ & $6.50 \mathrm{E}+00$ & $-20.25 \%$ \\
\hline
\end{tabular}


Table 48. POPDOSE-SR Collective Meat Ingestion Comparisons (person- rem/yr)

\begin{tabular}{|c|c|c|c|c|c|c|c|}
\hline \multicolumn{8}{|c|}{ Popdose Meat Pathway } \\
\hline Nuclide & Hamby & Jannik & $\%$ Diff & Nuclide & Hamby & Jannik & $\%$ Diff \\
\hline Ag110M & $8.19 \mathrm{E}-03$ & $1.37 \mathrm{E}-03$ & $-83.3 \%$ & H 3 & $1.97 \mathrm{E}-06$ & $0.00 \mathrm{E}+00$ & $-100.0 \%$ \\
\hline Am241 & $4.54 \mathrm{E}-02$ & $1.01 \mathrm{E}-01$ & $122.5 \%$ & Hf181 & $3.87 \mathrm{E}-02$ & $4.22 \mathrm{E}-06$ & $-100.0 \%$ \\
\hline Am243 & $4.54 \mathrm{E}-02$ & $1.01 \mathrm{E}-01$ & $122.5 \%$ & I 129 & $2.05 \mathrm{E}-01$ & $4.20 \mathrm{E}-01$ & $104.9 \%$ \\
\hline Ba139 & $1.59 \mathrm{E}-40$ & $1.47 \mathrm{E}-41$ & $-90.8 \%$ & I 131 & $5.00 \mathrm{E}-03$ & $1.88 \mathrm{E}-02$ & $276.0 \%$ \\
\hline Ba140 & $2.75 \mathrm{E}-04$ & $1.94 \mathrm{E}-05$ & $-92.9 \%$ & I 132 & $2.60 \mathrm{E}-26$ & $1.22 \mathrm{E}-25$ & $369.2 \%$ \\
\hline Be 7 & $2.79 \mathrm{E}-06$ & $3.60 \mathrm{E}-06$ & $29.0 \%$ & I 133 & $1.62 \mathrm{E}-06$ & $6.30 \mathrm{E}-06$ & $288.9 \%$ \\
\hline Br 83 & $3.70 \mathrm{E}-26$ & $6.99 \mathrm{E}-26$ & $88.9 \%$ & I 135 & $1.88 \mathrm{E}-12$ & $7.81 \mathrm{E}-12$ & $315.4 \%$ \\
\hline C 14 & $3.55 \mathrm{E}-03$ & $2.08 \mathrm{E}-03$ & $-41.4 \%$ & La140 & $3.71 \mathrm{E}-07$ & $3.98 \mathrm{E}-07$ & $7.3 \%$ \\
\hline Ce141 & $6.02 \mathrm{E}-05$ & $1.45 \mathrm{E}-06$ & $-97.6 \%$ & La141 & $2.34 \mathrm{E}-19$ & $2.89 \mathrm{E}-19$ & $23.5 \%$ \\
\hline Ce143 & $5.77 \mathrm{E}-07$ & $1.60 \mathrm{E}-08$ & $-97.2 \%$ & La142 & $5.72 \mathrm{E}-38$ & $7.79 \mathrm{E}-38$ & $36.2 \%$ \\
\hline Ce144 & $1.03 \mathrm{E}-03$ & $1.67 \mathrm{E}-05$ & $-98.4 \%$ & Mn 54 & $9.50 \mathrm{E}-05$ & $6.88 \mathrm{E}-05$ & $-27.6 \%$ \\
\hline Cf252 & $9.05 \mathrm{E}-03$ & $1.65 \mathrm{E}-03$ & $-81.8 \%$ & Mn 56 & $2.95 \mathrm{E}-25$ & $4.42 \mathrm{E}-25$ & $49.8 \%$ \\
\hline $\mathrm{Cm} 242$ & $8.51 \mathrm{E}-04$ & $1.75 \mathrm{E}-04$ & $-79.4 \%$ & Mo 99 & $2.40 \mathrm{E}-05$ & $4.91 \mathrm{E}-06$ & $-79.5 \%$ \\
\hline $\mathrm{Cm} 243$ & $2.92 \mathrm{E}-02$ & $5.18 \mathrm{E}-03$ & $-82.3 \%$ & $\mathrm{Na} 24$ & $4.89 \mathrm{E}-08$ & $4.16 \mathrm{E}-08$ & $-14.9 \%$ \\
\hline $\mathrm{Cm} 244$ & $2.31 \mathrm{E}-02$ & $4.10 \mathrm{E}-03$ & $-82.3 \%$ & $\mathrm{Nb} 95$ & $1.24 \mathrm{E}-02$ & $1.64 \mathrm{E}-08$ & $-100.0 \%$ \\
\hline $\mathrm{Cm} 246$ & $4.55 \mathrm{E}-02$ & $8.05 \mathrm{E}-03$ & $-82.3 \%$ & Ni 59 & $5.38 \mathrm{E}-05$ & $4.49 \mathrm{E}-05$ & $-16.5 \%$ \\
\hline $\mathrm{Cm} 248$ & $1.62 \mathrm{E}-01$ & $2.86 \mathrm{E}-02$ & $-82.3 \%$ & $\mathrm{Ni} 63$ & $1.45 \mathrm{E}-04$ & $1.21 \mathrm{E}-04$ & $-16.6 \%$ \\
\hline Co 57 & $6.13 \mathrm{E}-04$ & $1.98 \mathrm{E}-05$ & $-96.8 \%$ & $\mathrm{Ni} 65$ & $5.77 \mathrm{E}-25$ & $1.09 \mathrm{E}-24$ & $88.9 \%$ \\
\hline Co 58 & $1.33 \mathrm{E}-03$ & $5.30 \mathrm{E}-05$ & $-96.0 \%$ & Np237 & $3.94 \mathrm{E}-02$ & $1.75 \mathrm{E}-01$ & $344.2 \%$ \\
\hline Co 60 & $1.67 \mathrm{E}-02$ & $4.97 \mathrm{E}-04$ & $-97.0 \%$ & Np238 & $3.47 \mathrm{E}-07$ & $2.86 \mathrm{E}-06$ & $724.2 \%$ \\
\hline Cr 51 & $5.47 \mathrm{E}-06$ & $3.06 \mathrm{E}-05$ & $459.4 \%$ & $\mathrm{~Np} 239$ & $4.03 \mathrm{E}-07$ & $3.31 \mathrm{E}-06$ & $721.3 \%$ \\
\hline Cs134 & $1.41 \mathrm{E}-02$ & $7.10 \mathrm{E}-02$ & $403.5 \%$ & Os185 & $2.77 \mathrm{E}-02$ & $3.11 \mathrm{E}-02$ & $12.3 \%$ \\
\hline Cs135 & $1.44 \mathrm{E}-03$ & $7.00 \mathrm{E}-03$ & $386.1 \%$ & P 32 & $4.30 \mathrm{E}-03$ & $7.66 \mathrm{E}-03$ & $78.1 \%$ \\
\hline Cs137 & $1.01 \mathrm{E}-02$ & $4.92 \mathrm{E}-02$ & $387.1 \%$ & $\mathrm{~Pa} 233$ & $4.55 \mathrm{E}+01$ & $3.84 \mathrm{E}-05$ & $-100.0 \%$ \\
\hline Eu152 & $1.44 \mathrm{E}-03$ & $5.35 \mathrm{E}-06$ & $-99.6 \%$ & Pd109 & $4.60 \mathrm{E}-09$ & $6.60 \mathrm{E}-09$ & $43.5 \%$ \\
\hline Eu154 & $2.17 \mathrm{E}-03$ & $8.10 \mathrm{E}-06$ & $-99.6 \%$ & Pm147 & $2.19 \mathrm{E}-04$ & $8.36 \mathrm{E}-07$ & $-99.6 \%$ \\
\hline Eu155 & $3.07 \mathrm{E}-04$ & $1.15 \mathrm{E}-06$ & $-99.6 \%$ & Pm148 & $1.74 \mathrm{E}-04$ & $1.19 \mathrm{E}-06$ & $-99.3 \%$ \\
\hline $\mathrm{Fe} 55$ & $1.12 \mathrm{E}-03$ & $3.57 \mathrm{E}-04$ & $-68.1 \%$ & Pm151 & $7.71 \mathrm{E}-07$ & $5.36 \mathrm{E}-09$ & $-99.3 \%$ \\
\hline Fe 59 & $6.11 \mathrm{E}-03$ & $2.88 \mathrm{E}-03$ & $-52.9 \%$ & Pu238 & $2.68 \mathrm{E}-03$ & $1.87 \mathrm{E}-04$ & $-93.0 \%$ \\
\hline
\end{tabular}


SRNL-STI-2010-00447, REVISION 0

Table 48. POPDOSE-SR Collective Meat Ingestion Comparisons (person- rem/yr) (continued)

\begin{tabular}{|c|c|c|c|c|c|c|c|}
\hline \multicolumn{8}{|c|}{ Popdose Meat Pathway } \\
\hline Nuclide & Hamby & Jannik & \% Diff & Nuclide & Hamby & Jannik & $\%$ Diff \\
\hline Pu239 & $3.04 \mathrm{E}-03$ & $2.12 \mathrm{E}-04$ & $-93.0 \%$ & $\mathrm{Te} 125 \mathrm{M}$ & $8.48 \mathrm{E}-03$ & $8.00 \mathrm{E}-04$ & $-90.6 \%$ \\
\hline Pu240 & $3.04 \mathrm{E}-03$ & $2.12 \mathrm{E}-04$ & $-93.0 \%$ & Te127 & $5.64 \mathrm{E}-10$ & $8.57 \mathrm{E}-11$ & $-84.8 \%$ \\
\hline Pu241 & $6.02 \mathrm{E}-05$ & $4.22 \mathrm{E}-06$ & $-93.0 \%$ & Te127M & $2.67 \mathrm{E}-02$ & $2.11 \mathrm{E}-03$ & $-92.1 \%$ \\
\hline $\mathrm{Pu} 242$ & $2.90 \mathrm{E}-03$ & $2.02 \mathrm{E}-04$ & $-93.0 \%$ & Te129M & $1.75 \mathrm{E}-02$ & $1.97 \mathrm{E}-03$ & $-88.7 \%$ \\
\hline Ra224 & $2.18 \mathrm{E}-02$ & $1.79 \mathrm{E}-03$ & $-91.8 \%$ & $\mathrm{Te} 131 \mathrm{M}$ & $9.07 \mathrm{E}-05$ & $1.30 \mathrm{E}-05$ & $-85.7 \%$ \\
\hline $\operatorname{Ra} 228$ & $2.01 \mathrm{E}+00$ & $9.06 \mathrm{E}-02$ & $-95.5 \%$ & Te132 & $9.23 \mathrm{E}-04$ & $1.30 \mathrm{E}-04$ & $-85.9 \%$ \\
\hline $\mathrm{Rb} 86$ & $4.00 \mathrm{E}-03$ & $2.02 \mathrm{E}-03$ & $-49.5 \%$ & Th228 & $3.59 \mathrm{E}-03$ & $3.80 \mathrm{E}-03$ & $5.8 \%$ \\
\hline $\mathrm{Rb} 87$ & $7.78 \mathrm{E}-03$ & $2.21 \mathrm{E}-03$ & $-71.6 \%$ & Th230 & $5.36 \mathrm{E}-03$ & $5.45 \mathrm{E}-03$ & $1.7 \%$ \\
\hline Rh105 & 4.74E-07 & $6.84 \mathrm{E}-07$ & $44.3 \%$ & Th232 & $2.83 \mathrm{E}-02$ & $2.88 \mathrm{E}-02$ & $1.8 \%$ \\
\hline Rh106M & $3.39 \mathrm{E}-28$ & $6.18 \mathrm{E}-28$ & $82.3 \%$ & Th234 & $4.17 \mathrm{E}-05$ & $7.34 \mathrm{E}-05$ & $76.0 \%$ \\
\hline Ru103 & $2.36 \mathrm{E}-02$ & $2.67 \mathrm{E}-04$ & $-98.9 \%$ & U 232 & $2.23 \mathrm{E}-02$ & $2.27 \mathrm{E}-02$ & $1.8 \%$ \\
\hline Ru105 & $7.54 \mathrm{E}-15$ & $1.16 \mathrm{E}-16$ & $-98.5 \%$ & U 233 & $4.64 \mathrm{E}-03$ & $4.72 \mathrm{E}-03$ & $1.7 \%$ \\
\hline Ru106 & $3.80 \mathrm{E}-01$ & $2.94 \mathrm{E}-03$ & $-99.2 \%$ & U 234 & $4.46 \mathrm{E}-03$ & $4.54 \mathrm{E}-03$ & $1.8 \%$ \\
\hline S 35 & $2.30 \mathrm{E}-03$ & $4.89 \mathrm{E}-03$ & $112.6 \%$ & U 235 & $4.29 \mathrm{E}-03$ & $4.37 \mathrm{E}-03$ & $1.9 \%$ \\
\hline Sb122 & $2.51 \mathrm{E}-05$ & $1.24 \mathrm{E}-05$ & $-50.6 \%$ & U 236 & $4.29 \mathrm{E}-03$ & $4.37 \mathrm{E}-03$ & $1.9 \%$ \\
\hline Sb124 & $1.01 \mathrm{E}-03$ & $3.77 \mathrm{E}-04$ & $-62.7 \%$ & U 237 & $4.82 \mathrm{E}-06$ & $9.01 \mathrm{E}-06$ & $86.9 \%$ \\
\hline Sb125 & $5.03 \mathrm{E}-04$ & $1.37 \mathrm{E}-04$ & $-72.8 \%$ & U 238 & $3.95 \mathrm{E}-03$ & $4.02 \mathrm{E}-03$ & $1.8 \%$ \\
\hline Sb126 & $3.83 \mathrm{E}-04$ & $1.86 \mathrm{E}-04$ & $-51.4 \%$ & W 187 & $8.33 \mathrm{E}-08$ & $4.29 \mathrm{E}-06$ & $5050.1 \%$ \\
\hline Se 75 & $5.99 \mathrm{E}-03$ & $5.08 \mathrm{E}-03$ & $-15.2 \%$ & Y 90 & $4.43 \mathrm{E}-05$ & $1.58 \mathrm{E}-05$ & $-64.3 \%$ \\
\hline Se 79 & $8.59 \mathrm{E}-03$ & $5.59 \mathrm{E}-03$ & $-34.9 \%$ & Y 91 & $1.09 \mathrm{E}-03$ & $2.98 \mathrm{E}-04$ & $-72.7 \%$ \\
\hline Sm151 & $8.57 \mathrm{E}-05$ & $4.87 \mathrm{E}-06$ & $-94.3 \%$ & Y 92 & $3.70 \mathrm{E}-19$ & $1.55 \mathrm{E}-19$ & $-58.1 \%$ \\
\hline Sn123 & $2.24 \mathrm{E}-02$ & $2.39 \mathrm{E}-02$ & $6.7 \%$ & Y 93 & $5.13 \mathrm{E}-10$ & $1.95 \mathrm{E}-10$ & $-62.0 \%$ \\
\hline Sn126 & $6.87 \mathrm{E}-02$ & $6.09 \mathrm{E}-02$ & $-11.4 \%$ & Zn 65 & $1.94 \mathrm{E}-02$ & $9.50 \mathrm{E}-02$ & $389.7 \%$ \\
\hline Sr 89 & $1.29 \mathrm{E}-04$ & $3.68 \mathrm{E}-04$ & $185.3 \%$ & Zr 93 & $2.75 \mathrm{E}-03$ & $8.59 \mathrm{E}-08$ & $-100.0 \%$ \\
\hline Sr 90 & $3.94 \mathrm{E}-03$ & $7.73 \mathrm{E}-03$ & $96.2 \%$ & $\mathrm{Zr} 95$ & $3.26 \mathrm{E}-03$ & $1.41 \mathrm{E}-07$ & $-100.0 \%$ \\
\hline Sr 91 & $2.18 \mathrm{E}-11$ & $8.28 \mathrm{E}-11$ & $279.8 \%$ & Zr 97 & $7.63 \mathrm{E}-07$ & $4.57 \mathrm{E}-11$ & $-100.0 \%$ \\
\hline Sr 92 & $4.07 \mathrm{E}-24$ & $1.75 \mathrm{E}-23$ & $330.0 \%$ & *TOTAL* & $4.90 \mathrm{E}+01$ & $1.43 E+00$ & $-97.1 \%$ \\
\hline Tc 99 & $2.81 \mathrm{E}-02$ & $1.69 \mathrm{E}-03$ & $-94.0 \%$ & & & & \\
\hline Tc 99M & $3.10 \mathrm{E}-13$ & $1.11 \mathrm{E}-14$ & $-96.4 \%$ & & & & \\
\hline
\end{tabular}


SRNL-STI-2010-00447, REVISION 0

Table 49. POPDOSE-SR Total Collective Dose Comparisons (person-rem/yr)

\begin{tabular}{|c|c|c|c|c|c|c|c|c|c|c|c|}
\hline \multicolumn{12}{|c|}{ Popdose Total Pathway } \\
\hline Nuclide & Hamby & Jannik & $\%$ Diff & Nuclide & Hamby & Jannik & $\%$ Diff & Nuclide & Hamby & Jannik & $\%$ Diff \\
\hline $\operatorname{Ar} 41$ & $2.48 \mathrm{E}-05$ & $2.44 \mathrm{E}-05$ & $-1.61 \%$ & Eu152 & $4.15 \mathrm{E}-01$ & $3.29 \mathrm{E}-01$ & $-20.72 \%$ & $\mathrm{Ni} 63$ & $7.61 \mathrm{E}-03$ & $1.56 \mathrm{E}-03$ & $-79.50 \%$ \\
\hline Ag110M & 7.64E-01 & $5.12 \mathrm{E}-01$ & $-32.98 \%$ & Eu154 & $4.82 \mathrm{E}-01$ & $3.63 \mathrm{E}-01$ & $-24.69 \%$ & $\mathrm{Ni} 65$ & $2.76 \mathrm{E}-05$ & $2.30 \mathrm{E}-05$ & $-16.67 \%$ \\
\hline Am241 & $2.95 \mathrm{E}+02$ & $1.78 \mathrm{E}+02$ & $-39.66 \%$ & Eu155 & $4.66 \mathrm{E}-02$ & $2.95 \mathrm{E}-02$ & $-36.70 \%$ & $\mathrm{~Np} 237$ & $2.75 \mathrm{E}+02$ & $1.68 \mathrm{E}+02$ & $-38.91 \%$ \\
\hline Am243 & $2.95 \mathrm{E}+02$ & $1.78 \mathrm{E}+02$ & $-39.66 \%$ & Fe 55 & $7.63 \mathrm{E}-03$ & $1.50 \mathrm{E}-03$ & $-80.34 \%$ & Np238 & $1.55 \mathrm{E}-02$ & $1.12 \mathrm{E}-02$ & $-27.74 \%$ \\
\hline Ba139 & $3.16 \mathrm{E}-06$ & $2.20 \mathrm{E}-06$ & $-30.38 \%$ & Fe 59 & $1.34 \mathrm{E}-01$ & $9.31 \mathrm{E}-02$ & $-30.52 \%$ & Np239 & $1.77 \mathrm{E}-03$ & $1.44 \mathrm{E}-03$ & $-18.64 \%$ \\
\hline Ba140 & 7.19E-02 & $5.55 \mathrm{E}-02$ & $-22.81 \%$ & H 3 & $1.08 \mathrm{E}-04$ & 7.49E-05 & $-30.65 \%$ & Os185 & $1.41 \mathrm{E}-01$ & $1.30 \mathrm{E}-01$ & $-7.80 \%$ \\
\hline Ba141 & $4.52 \mathrm{E}-09$ & $4.23 \mathrm{E}-09$ & $-6.42 \%$ & Hf181 & $1.10 \mathrm{E}-01$ & $4.74 \mathrm{E}-02$ & $-56.91 \%$ & P 32 & $3.98 \mathrm{E}-02$ & $4.03 \mathrm{E}-02$ & $1.26 \%$ \\
\hline Ba142 & $4.72 \mathrm{E}-11$ & $4.93 \mathrm{E}-11$ & $4.45 \%$ & I 129 & $1.55 \mathrm{E}+01$ & $4.31 \mathrm{E}+00$ & $-72.19 \%$ & $\mathrm{~Pa} 233$ & $4.55 \mathrm{E}+01$ & 1.49E-02 & $-99.97 \%$ \\
\hline Be 7 & $5.28 \mathrm{E}-03$ & $4.62 \mathrm{E}-03$ & $-12.50 \%$ & 131 & $3.33 \mathrm{E}-01$ & $2.21 \mathrm{E}-01$ & $-33.63 \%$ & Pd109 & $3.53 \mathrm{E}-04$ & $2.49 \mathrm{E}-04$ & $-29.46 \%$ \\
\hline $\mathrm{Br} 83$ & 5.09E-06 & $3.53 \mathrm{E}-06$ & $-30.65 \%$ & I 132 & $6.76 \mathrm{E}-05$ & $6.10 \mathrm{E}-05$ & $-9.76 \%$ & Pm147 & 2.49E-02 & 1.19E-02 & $-52.21 \%$ \\
\hline Br 84 & $1.54 \mathrm{E}-07$ & $1.40 \mathrm{E}-07$ & $-9.09 \%$ & I 133 & $2.96 \mathrm{E}-03$ & $2.65 \mathrm{E}-03$ & $-10.47 \%$ & Pm148 & $1.36 \mathrm{E}-02$ & $8.70 \mathrm{E}-03$ & $-36.03 \%$ \\
\hline $\begin{array}{ll}\text { C } & 14\end{array}$ & $2.03 \mathrm{E}-02$ & $1.19 \mathrm{E}-02$ & $-41.38 \%$ & I 134 & $2.40 \mathrm{E}-06$ & $2.21 \mathrm{E}-06$ & $-7.92 \%$ & Pm151 & $1.26 \mathrm{E}-03$ & $1.07 \mathrm{E}-03$ & $-15.08 \%$ \\
\hline Ce141 & $2.14 \mathrm{E}-02$ & $8.48 \mathrm{E}-03$ & $-60.37 \%$ & I 135 & $5.54 \mathrm{E}-04$ & $4.78 \mathrm{E}-04$ & $-13.72 \%$ & Pr144 & 1.34E-09 & $9.44 \mathrm{E}-10$ & $-29.55 \%$ \\
\hline Ce143 & $1.93 \mathrm{E}-03$ & $1.51 \mathrm{E}-03$ & $-21.76 \%$ & $\mathrm{Kr} 83 \mathrm{M}$ & 1.79E-09 & $1.76 \mathrm{E}-09$ & $-1.68 \%$ & $\mathrm{Pu} 238$ & $2.58 \mathrm{E}+02$ & $1.57 \mathrm{E}+02$ & $-39.15 \%$ \\
\hline Ce144 & $3.48 \mathrm{E}-01$ & $1.35 \mathrm{E}-01$ & $-61.21 \%$ & $\mathrm{Kr} 85$ & $6.19 \mathrm{E}-07$ & $6.15 \mathrm{E}-07$ & $-0.65 \%$ & I 132 & $6.76 \mathrm{E}-05$ & $6.10 \mathrm{E}-05$ & $-9.76 \%$ \\
\hline Cf252 & $7.20 \mathrm{E}+01$ & $4.44 \mathrm{E}+01$ & $-38.33 \%$ & $\mathrm{Kr} 85 \mathrm{M}$ & $1.30 \mathrm{E}-05$ & $1.28 \mathrm{E}-05$ & $-1.54 \%$ & I 133 & $2.96 \mathrm{E}-03$ & $2.65 \mathrm{E}-03$ & $-10.47 \%$ \\
\hline $\mathrm{Cm} 242$ & $9.20 \mathrm{E}+00$ & $5.79 \mathrm{E}+00$ & $-37.07 \%$ & Kr 87 & $6.84 \mathrm{E}-06$ & $6.71 \mathrm{E}-06$ & $-1.90 \%$ & I 134 & $2.40 \mathrm{E}-06$ & $2.21 \mathrm{E}-06$ & $-7.92 \%$ \\
\hline $\mathrm{Cm} 243$ & $1.98 \mathrm{E}+02$ & $1.20 \mathrm{E}+02$ & $-39.39 \%$ & Kr 88 & $1.28 \mathrm{E}-04$ & $1.26 \mathrm{E}-04$ & $-1.56 \%$ & I 135 & 5.54E-04 & $4.78 \mathrm{E}-04$ & $-13.72 \%$ \\
\hline $\mathrm{Cm} 244$ & $1.53 \mathrm{E}+02$ & $9.23 \mathrm{E}+01$ & $-39.67 \%$ & Kr 89 & $1.06 \mathrm{E}-18$ & $8.85 \mathrm{E}-19$ & $-16.51 \%$ & $\mathrm{Kr} 83 \mathrm{M}$ & $1.79 \mathrm{E}-09$ & $1.76 \mathrm{E}-09$ & $-1.68 \%$ \\
\hline $\mathrm{Cm} 246$ & $3.05 \mathrm{E}+02$ & $1.85 \mathrm{E}+02$ & $-39.34 \%$ & La140 & $7.52 \mathrm{E}-03$ & $6.92 \mathrm{E}-03$ & $-7.98 \%$ & $\mathrm{Kr} 85$ & $6.19 \mathrm{E}-07$ & $6.15 \mathrm{E}-07$ & $-0.65 \%$ \\
\hline $\mathrm{Cm} 248$ & $1.08 \mathrm{E}+03$ & $6.50 \mathrm{E}+02$ & $-39.81 \%$ & La141 & $7.22 \mathrm{E}-05$ & $5.03 \mathrm{E}-05$ & $-30.33 \%$ & $\mathrm{Kr} 85 \mathrm{M}$ & $1.30 \mathrm{E}-05$ & $1.28 \mathrm{E}-05$ & $-1.54 \%$ \\
\hline Co 57 & $3.97 \mathrm{E}-02$ & $2.91 \mathrm{E}-02$ & $-26.70 \%$ & La142 & $2.08 \mathrm{E}-05$ & $1.89 \mathrm{E}-05$ & $-9.13 \%$ & Kr 87 & $6.84 \mathrm{E}-06$ & $6.71 \mathrm{E}-06$ & $-1.90 \%$ \\
\hline Co 58 & $1.34 \mathrm{E}-01$ & $1.10 \mathrm{E}-01$ & $-17.91 \%$ & Mn 54 & $1.84 \mathrm{E}-01$ & $1.62 \mathrm{E}-01$ & $-11.96 \%$ & $\mathrm{Kr} 88$ & $1.28 \mathrm{E}-04$ & $1.26 \mathrm{E}-04$ & $-1.56 \%$ \\
\hline Co 60 & $8.29 \mathrm{E}-01$ & $5.66 \mathrm{E}-01$ & $-31.72 \%$ & Mn 56 & $6.88 \mathrm{E}-05$ & $6.10 \mathrm{E}-05$ & $-11.34 \%$ & $\mathrm{Kr} 89$ & $1.06 \mathrm{E}-18$ & $8.85 \mathrm{E}-19$ & $-16.51 \%$ \\
\hline Cr 51 & $2.38 \mathrm{E}-03$ & $1.84 \mathrm{E}-03$ & $-22.69 \%$ & Mo 99 & $3.28 \mathrm{E}-03$ & $2.58 \mathrm{E}-03$ & $-21.34 \%$ & La140 & 7.52E-03 & $6.92 \mathrm{E}-03$ & $-7.98 \%$ \\
\hline Cs134 & $1.29 \mathrm{E}+00$ & $5.90 \mathrm{E}-01$ & $-54.26 \%$ & $\mathrm{Na} 24$ & $3.11 \mathrm{E}-03$ & $3.00 \mathrm{E}-03$ & $-3.54 \%$ & La141 & 7.22E-05 & $5.03 \mathrm{E}-05$ & $-30.33 \%$ \\
\hline Cs135 & $9.55 \mathrm{E}-02$ & $2.54 \mathrm{E}-02$ & $-73.40 \%$ & $\mathrm{Nb} 95$ & $7.35 \mathrm{E}-02$ & $4.96 \mathrm{E}-02$ & $-32.52 \%$ & La142 & $2.08 \mathrm{E}-05$ & $1.89 \mathrm{E}-05$ & $-9.13 \%$ \\
\hline Cs137 & 8.03E-01 & $3.11 \mathrm{E}-01$ & $-61.27 \%$ & $\mathrm{Nb} 97$ & $2.71 \mathrm{E}-06$ & 2.39E-06 & $-11.81 \%$ & Mn 54 & 1.84E-01 & $1.62 \mathrm{E}-01$ & $-11.96 \%$ \\
\hline Cs138 & $2.01 \mathrm{E}-07$ & $1.88 \mathrm{E}-07$ & $-6.47 \%$ & Ni 59 & $3.01 \mathrm{E}-03$ & 7.38E-04 & $-75.48 \%$ & Mn 56 & $6.88 \mathrm{E}-05$ & $6.10 \mathrm{E}-05$ & $-11.34 \%$ \\
\hline
\end{tabular}


Table 49. POPDOSE-SR Total Collective Dose Comparisons (person-rem/yr) (continued)

\begin{tabular}{|c|c|c|c|c|c|c|c|c|c|c|c|}
\hline \multicolumn{12}{|c|}{ Popdose Total Pathway } \\
\hline Nuclide & Hamby & Jannik & $\%$ Diff & Nuclide & Hamby & Jannik & $\%$ Diff & Nuclide & Hamby & Jannik & $\%$ Diff \\
\hline Mo 99 & $3.28 \mathrm{E}-03$ & $2.58 \mathrm{E}-03$ & $-21.34 \%$ & Rh106M & $1.02 \mathrm{E}-05$ & 7.07E-06 & $-30.69 \%$ & Te134 & $4.34 \mathrm{E}-07$ & $3.70 \mathrm{E}-07$ & $-14.75 \%$ \\
\hline $\mathrm{Na} 24$ & $3.11 \mathrm{E}-03$ & $3.00 \mathrm{E}-03$ & $-3.54 \%$ & Ru103 & $7.56 \mathrm{E}-02$ & $3.79 \mathrm{E}-02$ & $-49.87 \%$ & Th228 & $1.55 \mathrm{E}+02$ & $1.05 \mathrm{E}+02$ & $-32.26 \%$ \\
\hline $\mathrm{Nb} 95$ & $7.35 \mathrm{E}-02$ & $4.96 \mathrm{E}-02$ & $-32.52 \%$ & Ru105 & $1.42 \mathrm{E}-04$ & $1.23 \mathrm{E}-04$ & $-13.38 \%$ & Th230 & $1.61 \mathrm{E}+02$ & $1.09 \mathrm{E}+02$ & $-32.30 \%$ \\
\hline $\mathrm{Nb} 97$ & $2.71 \mathrm{E}-06$ & $2.39 \mathrm{E}-06$ & $-11.81 \%$ & Ru106 & 8.09E-01 & $2.00 \mathrm{E}-01$ & $-75.28 \%$ & Th232 & $8.06 \mathrm{E}+02$ & $5.43 \mathrm{E}+02$ & $-32.63 \%$ \\
\hline Ni 59 & $3.01 \mathrm{E}-03$ & $7.38 \mathrm{E}-04$ & $-75.48 \%$ & S 35 & $1.05 \mathrm{E}-02$ & $7.69 \mathrm{E}-03$ & $-26.76 \%$ & Th234 & $6.53 \mathrm{E}-02$ & $1.42 \mathrm{E}-02$ & $-78.25 \%$ \\
\hline $\mathrm{Ni} 63$ & $7.61 \mathrm{E}-03$ & $1.56 \mathrm{E}-03$ & $-79.50 \%$ & Sb122 & $4.48 \mathrm{E}-03$ & $3.59 \mathrm{E}-03$ & $-19.87 \%$ & U 232 & $3.39 \mathrm{E}+02$ & $2.28 \mathrm{E}+02$ & $-32.74 \%$ \\
\hline Ni 65 & $2.76 \mathrm{E}-05$ & $2.30 \mathrm{E}-05$ & $-16.67 \%$ & Sb124 & $2.38 \mathrm{E}-01$ & $1.78 \mathrm{E}-01$ & $-25.21 \%$ & U 233 & $6.59 \mathrm{E}+01$ & $4.43 \mathrm{E}+01$ & $-32.78 \%$ \\
\hline Np237 & $2.75 \mathrm{E}+02$ & $1.68 \mathrm{E}+02$ & $-38.91 \%$ & Sb125 & $1.25 \mathrm{E}-01$ & $1.01 \mathrm{E}-01$ & $-19.20 \%$ & U 234 & $6.58 \mathrm{E}+01$ & $4.43 \mathrm{E}+01$ & $-32.67 \%$ \\
\hline Np238 & $1.55 \mathrm{E}-02$ & $1.12 \mathrm{E}-02$ & $-27.74 \%$ & Sb126 & $8.60 \mathrm{E}-02$ & $6.67 \mathrm{E}-02$ & $-22.44 \%$ & U 235 & $6.09 \mathrm{E}+01$ & $4.10 \mathrm{E}+01$ & $-32.68 \%$ \\
\hline Np239 & $1.77 \mathrm{E}-03$ & $1.44 \mathrm{E}-03$ & $-18.64 \%$ & $\mathrm{Sb} 126 \mathrm{M}$ & $6.25 \mathrm{E}-09$ & 5.79E-09 & $-7.36 \%$ & U 236 & $6.08 \mathrm{E}+01$ & $4.09 \mathrm{E}+01$ & $-32.73 \%$ \\
\hline Os185 & $1.41 \mathrm{E}-01$ & $1.30 \mathrm{E}-01$ & $-7.80 \%$ & Se 75 & $2.52 \mathrm{E}-01$ & $8.44 \mathrm{E}-02$ & $-66.51 \%$ & U 237 & $5.30 \mathrm{E}-03$ & $3.63 \mathrm{E}-03$ & $-31.51 \%$ \\
\hline P 32 & $3.98 \mathrm{E}-02$ & $4.03 \mathrm{E}-02$ & $1.26 \%$ & Se 79 & $2.68 \mathrm{E}-01$ & $2.63 \mathrm{E}-02$ & $-90.19 \%$ & U 238 & $6.07 \mathrm{E}+01$ & $4.09 \mathrm{E}+01$ & $-32.62 \%$ \\
\hline $\mathrm{Pa} 233$ & $4.55 \mathrm{E}+01$ & $1.49 \mathrm{E}-02$ & $-99.97 \%$ & Sm151 & $1.72 \mathrm{E}-02$ & $9.96 \mathrm{E}-03$ & $-42.09 \%$ & W 187 & $9.06 \mathrm{E}-04$ & 8.49E-04 & $-6.29 \%$ \\
\hline Pd109 & $3.53 \mathrm{E}-04$ & $2.49 \mathrm{E}-04$ & $-29.46 \%$ & Sn123 & $9.92 \mathrm{E}-02$ & $4.03 \mathrm{E}-02$ & $-59.38 \%$ & Xe131M & $2.30 \mathrm{E}-06$ & $2.30 \mathrm{E}-06$ & $0.00 \%$ \\
\hline Pm147 & $2.49 \mathrm{E}-02$ & $1.19 \mathrm{E}-02$ & $-52.21 \%$ & Sn126 & $2.88 \mathrm{E}-01$ & $1.18 \mathrm{E}-01$ & $-59.03 \%$ & Xe133 & $9.20 \mathrm{E}-06$ & $9.20 \mathrm{E}-06$ & $0.00 \%$ \\
\hline Pm148 & $1.36 \mathrm{E}-02$ & $8.70 \mathrm{E}-03$ & $-36.03 \%$ & Sr 89 & $6.90 \mathrm{E}-02$ & $1.86 \mathrm{E}-02$ & $-73.04 \%$ & Xe133M & $7.28 \mathrm{E}-06$ & $7.28 \mathrm{E}-06$ & $0.00 \%$ \\
\hline Pm151 & $1.26 \mathrm{E}-03$ & $1.07 \mathrm{E}-03$ & $-15.08 \%$ & Sr 90 & $1.81 \mathrm{E}+00$ & $5.74 \mathrm{E}-01$ & $-68.29 \%$ & $\mathrm{Xe} 135$ & $3.59 \mathrm{E}-05$ & $3.59 \mathrm{E}-05$ & $0.00 \%$ \\
\hline Pr144 & 1.34E-09 & $9.44 \mathrm{E}-10$ & $-29.55 \%$ & Sr 91 & $6.46 \mathrm{E}-04$ & $5.31 \mathrm{E}-04$ & $-17.80 \%$ & $\mathrm{Xe} 135 \mathrm{M}$ & $2.84 \mathrm{E}-09$ & $2.84 \mathrm{E}-09$ & $0.00 \%$ \\
\hline $\mathrm{Pu} 238$ & $2.58 \mathrm{E}+02$ & $1.57 \mathrm{E}+02$ & $-39.15 \%$ & Sr 92 & $9.64 \mathrm{E}-05$ & $7.88 \mathrm{E}-05$ & $-18.26 \%$ & Xe137 & $1.60 \mathrm{E}-17$ & $1.60 \mathrm{E}-17$ & $0.00 \%$ \\
\hline Pu239 & $2.87 \mathrm{E}+02$ & $1.74 \mathrm{E}+02$ & $-39.37 \%$ & Tc 99 & $5.56 \mathrm{E}-02$ & $1.40 \mathrm{E}-02$ & $-74.82 \%$ & Xe138 & $4.82 \mathrm{E}-09$ & $4.82 \mathrm{E}-09$ & $0.00 \%$ \\
\hline $\mathrm{Pu} 240$ & $2.87 \mathrm{E}+02$ & $1.74 \mathrm{E}+02$ & $-39.37 \%$ & Tc 99M & $3.36 \mathrm{E}-05$ & $3.17 \mathrm{E}-05$ & $-5.65 \%$ & Y 90 & $4.05 \mathrm{E}-03$ & $2.67 \mathrm{E}-03$ & $-34.07 \%$ \\
\hline $\mathrm{Pu} 241$ & $5.63 \mathrm{E}+00$ & $3.42 \mathrm{E}+00$ & $-39.25 \%$ & Tc101 & $2.73 \mathrm{E}-10$ & $2.30 \mathrm{E}-10$ & $-15.75 \%$ & Y 91 & $7.67 \mathrm{E}-02$ & $1.80 \mathrm{E}-02$ & $-76.53 \%$ \\
\hline $\mathrm{Pu} 242$ & $2.70 \mathrm{E}+02$ & $1.64 \mathrm{E}+02$ & $-39.26 \%$ & Te125M & $3.67 \mathrm{E}-02$ & $6.34 \mathrm{E}-03$ & $-82.72 \%$ & Y 91M & $4.33 \mathrm{E}-07$ & $3.95 \mathrm{E}-07$ & $-8.78 \%$ \\
\hline $\mathrm{Ra} 224$ & $1.42 \mathrm{E}+00$ & $9.34 \mathrm{E}-01$ & $-34.23 \%$ & Te127 & $7.92 \mathrm{E}-05$ & $5.56 \mathrm{E}-05$ & $-29.80 \%$ & Y 92 & 8.39E-05 & $6.29 \mathrm{E}-05$ & $-25.03 \%$ \\
\hline Ra228 & $1.77 \mathrm{E}+01$ & $2.15 \mathrm{E}+00$ & $-87.85 \%$ & Te127M & $1.02 \mathrm{E}-01$ & $1.29 \mathrm{E}-02$ & $-87.35 \%$ & Y 93 & $6.21 \mathrm{E}-04$ & $4.42 \mathrm{E}-04$ & $-28.82 \%$ \\
\hline $\mathrm{Rb} 86$ & $5.71 \mathrm{E}-02$ & $3.18 \mathrm{E}-02$ & $-44.31 \%$ & Te129 & $1.02 \mathrm{E}-06$ & $7.46 \mathrm{E}-07$ & $-26.86 \%$ & Zn 65 & 4.07E-01 & $2.20 \mathrm{E}-01$ & $-45.95 \%$ \\
\hline $\mathrm{Rb} 87$ & $1.04 \mathrm{E}-01$ & $3.12 \mathrm{E}-02$ & $-70.00 \%$ & Te129M & $8.00 \mathrm{E}-02$ & $1.41 \mathrm{E}-02$ & $-82.38 \%$ & Zr 93 & $1.73 \mathrm{E}-01$ & $1.09 \mathrm{E}-01$ & $-36.99 \%$ \\
\hline $\mathrm{Rb} 88$ & 4.12E-09 & $3.20 \mathrm{E}-09$ & $-22.33 \%$ & Te131 & $2.93 \mathrm{E}-08$ & $2.25 \mathrm{E}-08$ & $-23.21 \%$ & Zr 95 & $1.88 \mathrm{E}-01$ & $1.62 \mathrm{E}-01$ & $-13.83 \%$ \\
\hline $\mathrm{Rb} 89$ & $1.68 \mathrm{E}-09$ & $1.52 \mathrm{E}-09$ & $-9.52 \%$ & Te131M & $4.97 \mathrm{E}-03$ & $4.22 \mathrm{E}-03$ & $-15.09 \%$ & Zr 97 & $1.55 \mathrm{E}-03$ & $1.12 \mathrm{E}-03$ & $-27.74 \%$ \\
\hline Rh105 & $6.12 \mathrm{E}-04$ & $5.65 \mathrm{E}-04$ & $-7.68 \%$ & Te132 & $6.47 \mathrm{E}-03$ & $4.14 \mathrm{E}-03$ & $-36.01 \%$ & *TOTAL* & $5.65 E+03$ & $3.51 \mathrm{E}+03$ & $-37.88 \%$ \\
\hline
\end{tabular}


Table 50. LADTAP XL 2009 Annual Report Maximally Exposed Individual Dose Comparisons (mrem)

\begin{tabular}{|l|l|l|l|}
\hline \multirow{2}{*}{ Nuclide } & \multicolumn{2}{|c|}{ Maximally-Exposed-Individual Dose (50-Yr CEDE) (mrem) } & \multirow{2}{*}{ \% Diff } \\
\cline { 2 - 4 } & \multicolumn{1}{|c|}{$\mathbf{2 0 0 9}$ SRS Annual Report Doses } & \multicolumn{1}{|c|}{ Jannik 2010 Parameters (2009 Data) } & $0.28 \%$ \\
\hline H-3 & $1.3 \mathrm{E}-02$ & $1.3 \mathrm{E}-02$ & $68.57 \%$ \\
\hline Zn-65 & $5.3 \mathrm{E}-05$ & $8.9 \mathrm{E}-05$ & $-39.61 \%$ \\
\hline Sr-90 & $1.2 \mathrm{E}-03$ & $7.3 \mathrm{E}-04$ & $9.36 \%$ \\
\hline Tc-99 & $4.6 \mathrm{E}-06$ & $5.0 \mathrm{E}-06$ & $27.91 \%$ \\
\hline I-129 & $1.8 \mathrm{E}-03$ & $2.3 \mathrm{E}-03$ & $-0.09 \%$ \\
\hline Cs-137 & $4.7 \mathrm{E}-02$ & $4.7 \mathrm{E}-02$ & $-2.58 \%$ \\
\hline U-234 & $5.7 \mathrm{E}-06$ & $5.6 \mathrm{E}-06$ & $-3.04 \%$ \\
\hline U-235 & $7.7 \mathrm{E}-08$ & $7.5 \mathrm{E}-08$ & $-2.57 \%$ \\
\hline U-238 & $3.6 \mathrm{E}-06$ & $3.5 \mathrm{E}-06$ & $22.70 \%$ \\
\hline Np-237 & $5.8 \mathrm{E}-06$ & $7.1 \mathrm{E}-06$ & $338.90 \%$ \\
\hline Am-241 & $1.0 \mathrm{E}-04$ & $4.4 \mathrm{E}-04$ & $63.21 \%$ \\
\hline Pu-238 & $1.2 \mathrm{E}-03$ & $2.0 \mathrm{E}-03$ & $63.21 \%$ \\
\hline Pu-239 & $9.4 \mathrm{E}-05$ & $1.5 \mathrm{E}-04$ & $7.88 \%$ \\
\hline Cm-244 & $1.4 \mathrm{E}-05$ & $1.5 \mathrm{E}-05$ & $63.21 \%$ \\
\hline Unidentified alpha & $1.07 \mathrm{E}-02$ & $1.8 \mathrm{E}-02$ & $-39.61 \%$ \\
\hline Unidentified beta & $1.64 \mathrm{E}-03$ & $9.9 \mathrm{E}-04$ & $\mathbf{9 . 5 7 \%}$ \\
\hline TOTAL Dose & $\mathbf{7 . 7 0 E}-02$ & $\mathbf{8 . 4 E}-02$ & \\
\hline
\end{tabular}


SRNL-STI-2010-00447, REVISION 0

Table 51. LADTAP XL Annual Report Collective Dose Comparisons (person-rem)

\begin{tabular}{|c|c|c|c|}
\hline \multirow{2}{*}{ Nuclide } & \multicolumn{2}{|c|}{ All Pathways } & \multirow{2}{*}{$\%$ Diff } \\
\hline & 2009 SRS Annual Report Doses & Jannik 2010 Parameters (2009 Data) & \\
\hline $\mathrm{H}-3$ & $8.05 \mathrm{E}-01$ & $7.34 \mathrm{E}-01$ & $-8.84 \%$ \\
\hline $\mathrm{Zn}-65$ & 7.94E-03 & $7.93 \mathrm{E}-03$ & $-0.17 \%$ \\
\hline Sr-90 & $4.59 \mathrm{E}-02$ & $3.91 \mathrm{E}-02$ & $-14.74 \%$ \\
\hline Tc-99 & $2.37 \mathrm{E}-04$ & $2.01 \mathrm{E}-04$ & $-15.48 \%$ \\
\hline I-129 & 9.29E-02 & $8.78 \mathrm{E}-02$ & $-5.51 \%$ \\
\hline Cs-137 & $1.45 \mathrm{E}-01$ & $2.07 \mathrm{E}-01$ & $42.74 \%$ \\
\hline U-234 & $3.54 \mathrm{E}-04$ & $3.22 \mathrm{E}-04$ & $-8.80 \%$ \\
\hline $\mathrm{U}-235$ & 4.70E-06 & 4.28E-06 & $-8.97 \%$ \\
\hline $\mathrm{U}-238$ & $2.24 \mathrm{E}-04$ & $2.04 \mathrm{E}-04$ & $-8.80 \%$ \\
\hline $\mathrm{Np}-237$ & 2.99E-04 & 2.79E-04 & $-6.64 \%$ \\
\hline Am-241 & $1.38 \mathrm{E}-02$ & 8.29E-03 & $-40.02 \%$ \\
\hline $\mathrm{Pu}-238$ & $1.07 \mathrm{E}-01$ & $1.20 \mathrm{E}-01$ & $12.31 \%$ \\
\hline $\mathrm{Pu}-239$ & $8.25 \mathrm{E}-03$ & $9.27 \mathrm{E}-03$ & $12.31 \%$ \\
\hline $\mathrm{Cm}-244$ & $1.96 \mathrm{E}-03$ & $1.15 \mathrm{E}-03$ & $-41.33 \%$ \\
\hline Unidentified alpha & $9.42 \mathrm{E}-01$ & $1.06 \mathrm{E}+00$ & $12.31 \%$ \\
\hline Unidentified beta & $6.26 \mathrm{E}-02$ & $5.34 \mathrm{E}-02$ & $-14.74 \%$ \\
\hline TOTAL Dose & $2.23 \mathrm{E}+00$ & $2.33 E+00$ & $4.18 \%$ \\
\hline
\end{tabular}


SRNL-STI-2010-00447, REVISION 0

Table 52. LADTAP XL IRRIDOSE 2009 Annual Report Maximally Exposed Individual Dose Comparisons (mrem)

\begin{tabular}{|l|c|c|c|}
\hline \multirow{2}{*}{ Nuclide } & 2009 SRS Annual Report Doses & Jannik 2010 Parameters (2009 Data) & \multirow{2}{*}{ \% Diff } \\
\cline { 2 - 3 } & All Pathways & All Pathways & \multirow{2}{*}{$0.00 \%$} \\
\hline H-3 & $1.1 \mathrm{E}-02$ & $1.1 \mathrm{E}-02$ & $63.80 \%$ \\
\hline Zn-65 & $1.1 \mathrm{E}-05$ & $1.8 \mathrm{E}-05$ & $163.15 \%$ \\
\hline Sr-90 & $2.5 \mathrm{E}-03$ & $6.7 \mathrm{E}-03$ & $896.14 \%$ \\
\hline Tc-99 & $4.0 \mathrm{E}-04$ & $4.0 \mathrm{E}-03$ & $33.45 \%$ \\
\hline I-129 & $6.7 \mathrm{E}-03$ & $8.9 \mathrm{E}-03$ & $41.56 \%$ \\
\hline Cs-137 & $3.1 \mathrm{E}-03$ & $4.3 \mathrm{E}-03$ & $46.69 \%$ \\
\hline U-234 & $1.7 \mathrm{E}-05$ & $2.5 \mathrm{E}-05$ & $46.69 \%$ \\
\hline U-235 & $2.2 \mathrm{E}-07$ & $3.2 \mathrm{E}-07$ & $46.69 \%$ \\
\hline U-238 & $1.1 \mathrm{E}-05$ & $1.6 \mathrm{E}-05$ & $25.12 \%$ \\
\hline Np-237 & $1.4 \mathrm{E}-05$ & $1.7 \mathrm{E}-05$ & $21.19 \%$ \\
\hline Am-241 & $1.8 \mathrm{E}-04$ & $2.2 \mathrm{E}-04$ & $20.16 \%$ \\
\hline Pu-238 & $3.3 \mathrm{E}-03$ & $3.9 \mathrm{E}-03$ & $20.10 \%$ \\
\hline Pu-239 & $2.5 \mathrm{E}-04$ & $3.0 \mathrm{E}-04$ & $17.11 \%$ \\
\hline Cm-244 & $2.6 \mathrm{E}-05$ & $3.0 \mathrm{E}-05$ & $20.10 \%$ \\
\hline Unidentified alpha & $2.9 \mathrm{E}-02$ & $3.4 \mathrm{E}-02$ & $162.85 \%$ \\
\hline Unidentified beta & $3.5 \mathrm{E}-03$ & $9.1 \mathrm{E}-03$ & $\mathbf{3 9 . 3 3 \%}$ \\
\hline TOTAL Dose & $\mathbf{6 . 0 E}-02$ & $\mathbf{8 . 3 E}-\mathbf{0 2}$ & \\
\hline
\end{tabular}


Table 53. LADTAP XL IRRIDOSE Annual Report Collective Dose Comparisons (person-rem)

\begin{tabular}{|l|c|c|c|}
\hline \multirow{2}{*}{ Nuclide } & \multicolumn{2}{|c|}{ CALCULATION OF POPULATION DOSE } & \multirow{2}{*}{$\%$} \\
\cline { 2 - 4 } & 2009 SRS Annual Report Doses & Jannik 2010 Parameters (2009 Data) & \multirow{2}{*}{$-80.90 \%$} \\
\hline H-3 & $9.40 \mathrm{E}-01$ & $1.80 \mathrm{E}-01$ & $-89.46 \%$ \\
\hline Zn-65 & $1.08 \mathrm{E}-03$ & $1.13 \mathrm{E}-04$ & $12.99 \%$ \\
\hline Sr-90 & $1.53 \mathrm{E}-01$ & $1.73 \mathrm{E}-01$ & $285.68 \%$ \\
\hline Tc-99 & $2.60 \mathrm{E}-02$ & $1.00 \mathrm{E}-01$ & $-64.85 \%$ \\
\hline I-129 & $4.83 \mathrm{E}-01$ & $1.70 \mathrm{E}-01$ & $-72.75 \%$ \\
\hline Cs-137 & $2.54 \mathrm{E}-01$ & $6.91 \mathrm{E}-02$ & $-39.14 \%$ \\
\hline U-234 & $1.01 \mathrm{E}-03$ & $6.14 \mathrm{E}-04$ & $-39.14 \%$ \\
\hline U-235 & $1.30 \mathrm{E}-05$ & $7.90 \mathrm{E}-06$ & $-39.14 \%$ \\
\hline U-238 & $6.39 \mathrm{E}-04$ & $3.89 \mathrm{E}-04$ & $-40.85 \%$ \\
\hline Np-237 & $8.06 \mathrm{E}-04$ & $4.77 \mathrm{E}-04$ & $-42.08 \%$ \\
\hline Am-241 & $1.03 \mathrm{E}-02$ & $5.97 \mathrm{E}-03$ & $-41.95 \%$ \\
\hline Pu-238 & $1.88 \mathrm{E}-01$ & $1.09 \mathrm{E}-01$ & $-41.98 \%$ \\
\hline Pu-239 & $1.45 \mathrm{E}-02$ & $8.42 \mathrm{E}-03$ & $-43.54 \%$ \\
\hline Cm-244 & $1.50 \mathrm{E}-03$ & $8.47 \mathrm{E}-04$ & $-41.98 \%$ \\
\hline Unidentified alpha & $1.66 \mathrm{E}+00$ & $9.61 \mathrm{E}-01$ & $12.85 \%$ \\
\hline Unidentified beta & $2.09 \mathrm{E}-01$ & $2.36 \mathrm{E}-01$ & $\mathbf{- 4 8 . 8 6 \%}$ \\
\hline TOTAL Dose & $\mathbf{3 . 9 4 E + 0 0}$ & $\mathbf{2 . 0 1 E}+\mathbf{0 0}$ & \\
\hline
\end{tabular}


Table 54. MAXDOSE SR Annual Report Maximally Exposed Individual Dose Comparisons (mrem)

\begin{tabular}{|l|c|c|c|}
\hline \multirow{2}{*}{ Nuclide } & \multicolumn{2}{|c|}{ All Pathways } & \multirow{2}{*}{$\%$ Diff } \\
\cline { 2 - 4 } & 2009 SRS Annual Report Doses & Jannik 2010 Parameters (2009 Data) & \multirow{2}{*}{$-6.37 \%$} \\
\hline Ag-110M & $1.02 \mathrm{E}-13$ & $9.55 \mathrm{E}-14$ & $-27.93 \%$ \\
\hline Am-241 & $2.22 \mathrm{E}-05$ & $1.60 \mathrm{E}-05$ & $-25.41 \%$ \\
\hline C-14 & $5.51 \mathrm{E}-09$ & $4.11 \mathrm{E}-09$ & $0.00 \%$ \\
\hline Ce-144 & $4.47 \mathrm{E}-11$ & $4.47 \mathrm{E}-11$ & $-48.93 \%$ \\
\hline Cm-244 & $8.38 \mathrm{E}-11$ & $4.28 \mathrm{E}-11$ & $-27.78 \%$ \\
\hline Co-58 & $1.08 \mathrm{E}-05$ & $7.80 \mathrm{E}-06$ & $-15.45 \%$ \\
\hline Co-60 & $1.23 \mathrm{E}-13$ & $1.04 \mathrm{E}-13$ & $-4.96 \%$ \\
\hline Cs-134 & $1.21 \mathrm{E}-06$ & $1.15 \mathrm{E}-06$ & $-26.06 \%$ \\
\hline Cs-137 & $1.88 \mathrm{E}-08$ & $1.39 \mathrm{E}-08$ & $-11.49 \%$ \\
\hline Eu-154 & $1.74 \mathrm{E}-03$ & $1.54 \mathrm{E}-03$ & $-2.40 \%$ \\
\hline H-3 & $7.49 \mathrm{E}-09$ & $7.31 \mathrm{E}-09$ & $0.00 \%$ \\
\hline I-129 & $3.36 \mathrm{E}-02$ & $3.36 \mathrm{E}-02$ & $-56.03 \%$ \\
\hline Ni-63 & $3.98 \mathrm{E}-03$ & $1.75 \mathrm{E}-03$ & $-46.33 \%$ \\
\hline Np-237 & $1.09 \mathrm{E}-13$ & $5.85 \mathrm{E}-14$ & $-23.91 \%$ \\
\hline Np-239 & $1.38 \mathrm{E}-05$ & $1.05 \mathrm{E}-05$ & $-31.24 \%$ \\
\hline Pa-233 & $4.29 \mathrm{E}-12$ & $2.95 \mathrm{E}-12$ & $138.10 \%$ \\
\hline Pm-147 & $7.14 \mathrm{E}-06$ & $1.70 \mathrm{E}-05$ & $-42.55 \%$ \\
\hline Pu-238 & $1.02 \mathrm{E}-09$ & $5.86 \mathrm{E}-10$ & $-24.92 \%$ \\
\hline Pu-239 & $3.21 \mathrm{E}-04$ & $2.41 \mathrm{E}-04$ & $-27.01 \%$ \\
\hline Pu-240 & $3.48 \mathrm{E}-04$ & $2.54 \mathrm{E}-04$ & $-25.30 \%$ \\
\hline Pu-241 & $2.49 \mathrm{E}-05$ & $1.86 \mathrm{E}-05$ & $-25.35 \%$ \\
\hline
\end{tabular}


Table 54. MAXDOSE SR Annual Report Maximally Exposed Individual Dose Comparisons (mrem) (continued)

\begin{tabular}{|l|c|c|c|}
\hline \multirow{2}{*}{ Nuclide } & \multicolumn{2}{|c|}{ All Pathways } & \multirow{2}{*}{$\%$ Diff } \\
\cline { 2 - 4 } & 2009 SRS Annual Report Doses & Jannik 2010 Parameters (2009 Data) & \multirow{2}{*}{$-25.04 \%$} \\
\hline Pu-242 & $1.27 \mathrm{E}-07$ & $9.52 \mathrm{E}-08$ & $-39.79 \%$ \\
\hline Ra-228 & $3.82 \mathrm{E}-07$ & $2.30 \mathrm{E}-07$ & $55.56 \%$ \\
\hline Ru-106 & $2.34 \mathrm{E}-12$ & $3.64 \mathrm{E}-12$ & $15.66 \%$ \\
\hline Sb-124 & $7.09 \mathrm{E}-10$ & $8.20 \mathrm{E}-10$ & $-22.15 \%$ \\
\hline Sb-125 & $1.07 \mathrm{E}-10$ & $8.33 \mathrm{E}-11$ & $-4.73 \%$ \\
\hline Sn-126 & $8.45 \mathrm{E}-10$ & $8.05 \mathrm{E}-10$ & $-12.84 \%$ \\
\hline Sr-89 & $1.02 \mathrm{E}-11$ & $8.89 \mathrm{E}-12$ & $-56.80 \%$ \\
\hline Sr-90 & $5.37 \mathrm{E}-11$ & $2.32 \mathrm{E}-11$ & $-55.17 \%$ \\
\hline Tc-99 & $3.77 \mathrm{E}-05$ & $1.69 \mathrm{E}-05$ & $2.11 \%$ \\
\hline Th-228 & $1.42 \mathrm{E}-08$ & $1.45 \mathrm{E}-08$ & $-4.94 \%$ \\
\hline Th-230 & $2.43 \mathrm{E}-10$ & $2.31 \mathrm{E}-10$ & $-6.94 \%$ \\
\hline U-232 & $8.79 \mathrm{E}-11$ & $8.18 \mathrm{E}-11$ & $-7.95 \%$ \\
\hline U-233 & $5.03 \mathrm{E}-09$ & $4.63 \mathrm{E}-09$ & $-8.40 \%$ \\
\hline U-234 & $2.50 \mathrm{E}-10$ & $2.29 \mathrm{E}-10$ & $-9.35 \%$ \\
\hline U-235 & $3.21 \mathrm{E}-05$ & $2.91 \mathrm{E}-05$ & $-8.94 \%$ \\
\hline U-236 & $1.23 \mathrm{E}-06$ & $1.12 \mathrm{E}-06$ & $-8.47 \%$ \\
\hline U-238 & $2.36 \mathrm{E}-08$ & $2.16 \mathrm{E}-08$ & $-9.02 \%$ \\
\hline Zr-95 & $5.32 \mathrm{E}-05$ & $4.84 \mathrm{E}-05$ & $-8.08 \%$ \\
\hline Alpha & $2.60 \mathrm{E}-12$ & $2.39 \mathrm{E}-12$ & $-26.63 \%$ \\
\hline Beta & $9.05 \mathrm{E}-04$ & $6.64 \mathrm{E}-04$ & $-54.24 \%$ \\
\hline Total & $7.43 \mathrm{E}-04$ & $3.40 \mathrm{E}-04$ & $-\mathbf{7 . 8 6 \%}$ \\
\hline
\end{tabular}


Table 55. POPDOSE SR Annual Report Collective Dose Comparisons (person-rem)

\begin{tabular}{|l|l|l|l|}
\hline \multirow{2}{*}{ Nuclide } & 2009 SRS Annual Report Doses & Jannik 2010 Parameters (2009 Data) & \multirow{2}{*}{ \% Diff } \\
\cline { 2 - 3 } & All Pathways & All Pathways & $-16.81 \%$ \\
\hline Ag110M & $6.90 \mathrm{E}-12$ & $5.74 \mathrm{E}-12$ & $-43.04 \%$ \\
\hline Am241 & $1.25 \mathrm{E}-03$ & $7.12 \mathrm{E}-04$ & $-42.81 \%$ \\
\hline Am243 & $2.78 \mathrm{E}-07$ & $1.59 \mathrm{E}-07$ & $-41.33 \%$ \\
\hline C 14 & $6.63 \mathrm{E}-10$ & $3.89 \mathrm{E}-10$ & $-62.73 \%$ \\
\hline Ce144 & $3.22 \mathrm{E}-09$ & $1.20 \mathrm{E}-09$ & $-42.86 \%$ \\
\hline Cm244 & $6.09 \mathrm{E}-04$ & $3.48 \mathrm{E}-04$ & $-14.44 \%$ \\
\hline Co 58 & $8.31 \mathrm{E}-12$ & $7.11 \mathrm{E}-12$ & $-2.92 \%$ \\
\hline Co 60 & $9.26 \mathrm{E}-05$ & $8.99 \mathrm{E}-05$ & $-21.92 \%$ \\
\hline Cs134 & $1.04 \mathrm{E}-06$ & $8.12 \mathrm{E}-07$ & $-8.26 \%$ \\
\hline Cs137 & $1.21 \mathrm{E}-01$ & $1.11 \mathrm{E}-01$ & $-1.52 \%$ \\
\hline Eu154 & $5.94 \mathrm{E}-07$ & $5.85 \mathrm{E}-07$ & $-30.49 \%$ \\
\hline H 3 & $1.64 \mathrm{E}+00$ & $1.14 \mathrm{E}+00$ & $-70.44 \%$ \\
\hline I 129 & $1.14 \mathrm{E}-01$ & $3.37 \mathrm{E}-02$ & $-82.67 \%$ \\
\hline Ni 63 & $3.33 \mathrm{E}-12$ & $5.77 \mathrm{E}-13$ & $-42.13 \%$ \\
\hline Np237 & $7.05 \mathrm{E}-04$ & $4.08 \mathrm{E}-04$ & $-14.94 \%$ \\
\hline Np239 & $1.74 \mathrm{E}-10$ & $1.48 \mathrm{E}-10$ & $-99.97 \%$ \\
\hline Pa233 & $1.65 \mathrm{E}-04$ & $5.18 \mathrm{E}-08$ & $-58.56 \%$ \\
\hline Pm147 & $4.03 \mathrm{E}-08$ & $1.67 \mathrm{E}-08$ & $-42.59 \%$ \\
\hline Pu238 & $1.66 \mathrm{E}-02$ & $9.53 \mathrm{E}-03$ & $-42.50 \%$ \\
\hline Pu239 & $1.91 \mathrm{E}-02$ & $1.10 \mathrm{E}-02$ & $-42.70 \%$ \\
\hline Pu240 & $1.26 \mathrm{E}-03$ & $7.22 \mathrm{E}-04$ & $-42.86 \%$ \\
\hline Pu241 & $7.21 \mathrm{E}-04$ & $4.12 \mathrm{E}-04$ & \\
\hline
\end{tabular}

(continued)

\begin{tabular}{|l|l|l|l|}
\hline \multirow{2}{*}{ Nuclide } & \multicolumn{2}{|c|}{ All Pathways } & \multirow{2}{*}{ \% Diff } \\
\cline { 2 - 4 } & \multicolumn{1}{|c|}{ 2009 SRS Annual Report Doses } & \multicolumn{1}{|c|}{ Jannik 2010 Parameters (2009 Data) } & $-42.86 \%$ \\
\hline Pu242 & $6.44 \mathrm{E}-06$ & $3.68 \mathrm{E}-06$ & $-90.09 \%$ \\
\hline Ra228 & $1.13 \mathrm{E}-05$ & $1.12 \mathrm{E}-06$ & $-48.02 \%$ \\
\hline Ru103 & $9.83 \mathrm{E}-11$ & $5.11 \mathrm{E}-11$ & $-70.32 \%$ \\
\hline Ru106 & $2.21 \mathrm{E}-08$ & $6.56 \mathrm{E}-09$ & $-21.27 \%$ \\
\hline Sb124 & $6.91 \mathrm{E}-09$ & $5.44 \mathrm{E}-09$ & $-2.60 \%$ \\
\hline Sb125 & $6.53 \mathrm{E}-08$ & $6.36 \mathrm{E}-08$ & $-15.22 \%$ \\
\hline Sn126 & $5.65 \mathrm{E}-10$ & $4.79 \mathrm{E}-10$ & $-76.70 \%$ \\
\hline Sr 89 & $2.30 \mathrm{E}-09$ & $5.36 \mathrm{E}-10$ & $-63.44 \%$ \\
\hline Sr 90 & $1.45 \mathrm{E}-03$ & $5.29 \mathrm{E}-04$ & $146.46 \%$ \\
\hline Tc 99 & $3.81 \mathrm{E}-07$ & $9.39 \mathrm{E}-07$ & $-32.89 \%$ \\
\hline Th228 & $1.52 \mathrm{E}-08$ & $1.02 \mathrm{E}-08$ & \\
\hline
\end{tabular}


SRNL-STI-2010-00447, REVISION 0

\begin{tabular}{|l|l|l|l|}
\hline Th230 & $5.37 \mathrm{E}-09$ & $3.57 \mathrm{E}-09$ & $-33.52 \%$ \\
\hline U 232 & $3.05 \mathrm{E}-07$ & $2.03 \mathrm{E}-07$ & $-33.44 \%$ \\
\hline U 233 & $1.50 \mathrm{E}-08$ & $9.98 \mathrm{E}-09$ & $-33.47 \%$ \\
\hline U 234 & $2.25 \mathrm{E}-03$ & $1.50 \mathrm{E}-03$ & $-33.33 \%$ \\
\hline U 235 & $8.68 \mathrm{E}-05$ & $5.92 \mathrm{E}-05$ & $-31.80 \%$ \\
\hline U 236 & $1.42 \mathrm{E}-06$ & $9.41 \mathrm{E}-07$ & $-33.73 \%$ \\
\hline U 238 & $3.74 \mathrm{E}-03$ & $2.49 \mathrm{E}-03$ & $-33.42 \%$ \\
\hline Zr 95 & $1.82 \mathrm{E}-10$ & $1.62 \mathrm{E}-10$ & $-10.99 \%$ \\
\hline Alpha & $4.88 \mathrm{E}-02$ & $2.79 \mathrm{E}-02$ & $-42.71 \%$ \\
\hline Beta-Gamma & $2.39 \mathrm{E}-02$ & $8.72 \mathrm{E}-03$ & $-63.44 \%$ \\
\hline *TOTAL* & $\mathbf{2 . 0 0 E}+\mathbf{0 0}$ & $\mathbf{1 . 3 5 E}+\mathbf{0 0}$ & $\mathbf{- 3 2 . 3 5 \%}$ \\
\hline
\end{tabular}

